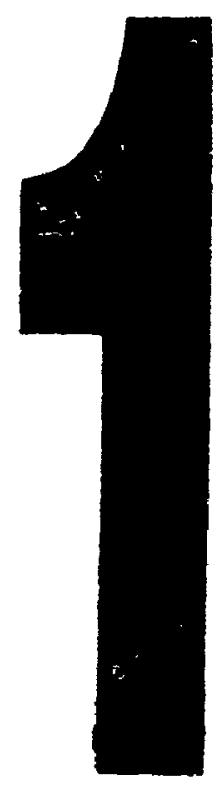

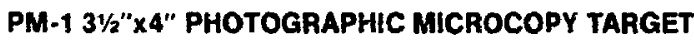
NES 1010a ANSI/ISO \#2 EOUIVALENT

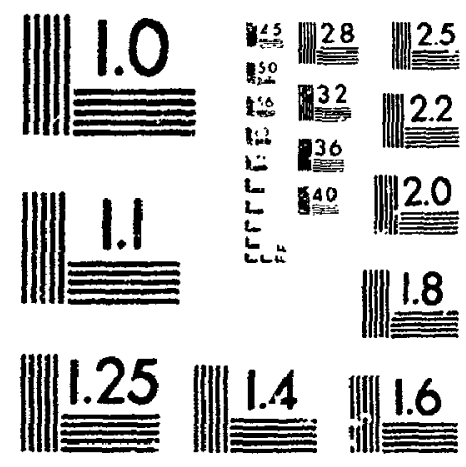

PRECISICNSM RESOLUTION TARGETS 
The quality of this microform is heavily dependent upon the quality of the original thesis submitted for microfilming. Every effort has been made to ensure the highest quality of reproduction possible.

If pages are missing, contact the university which granted the degree.

Some pages may have indistinct print especially if the original pages were typed with a poor typewriter ribbon or if the university sent us an inferior photocopy.
La qualité de cette microforme dépend grandement de lir qualité de la thèse soumise au microfilmage. Nous avons tout fait pour assurer une qualité supérieure de reproduction.
S'il manque des pages, veuillez communiquer avec l'université qui a conféré le grade.

La qualité d'impression de certaines pages peut laisser à désirer, surtout si les pages originales ont été dactylographiées à l'aide d'un ruban usé ou si l'université nous a fait parvenir une photocopie de qualité inférieure.

La reproduction, même partielle, de cette microforme est soumise à la Loi canadienne sur le droit d'auteur, SRC 1970, c. C-30, et ses amendements subséquents.
Reproduction in full or in part of this microform is governed by the Canadian Copyright Act, R.S.C. 1970, c. C-30, and subsequent amendments. 


\title{
On The Theory of FRESH-DFE and its Application in Digital Cellular Radio Systems
}

\author{
by

\section{Faramarz Hendessi, B. Sc., M. Sc.}

\author{
A thesis submitted to \\ the Faculty of Graduate Studies and Research \\ in partial fulfillment of \\ the requirements for degree of \\ Doctor of Philosophy
}

Ottawa-Carleton Institute for Electrical Engineering

Faculty of Engineering

Department of Systems and Computer Engineering

Carleton University

September, 1994

Copyright

1994, Faramarz Hendessi 
National Library

of Canada

Acquisitions and Bibliographic Services Branch

395 Wellington Street Ottawa. Ontario KIA ON4
Bibliotheque nationale

du Canada

Direction des acquisitions et des services bibliographiques

395. rue Wellington

Ottawa (Ontano)

Vour the volre reference

Our ble Notre ieference

THE AUTHOR HAS GRANTED AN IRREVOCABLE NON-EXCLUSIVE LICENCE ALLOWING THE NATIONAL LIBRARY OF CANADA TO REPRODUCE, LOAN, DISTRIBUTE OR SELL COPIES OF HIS/HER THESIS BY ANY MEANS AND IN ANY FORM OR FORMAT, MAKING THIS THESIS AVAILABLE TO INTERESTED PERSONS.
L'AUTEUR A ACCORDE UNE LICENCE IRREVOCABLE ET NON EXCLUSIVE PERMETTANT A LA BIBLIOTHEQUE NATIONALE DU CANADA DE REPRODUIRE, PRETER, DISTRIBUER OU VENDRE DES COPIES DE SA THESE DE QUELQUE M.ANIERE ET SOUS QUELQUE FORME QUE CE SOIT POUR METTRE DES EXEMPLAIRES DE CETTE THESE A LA DISPOSITION DES PERSONNE INTERESSEES.
THE AUTHOR RETAINS OWNERSHIP OF THE COPYRIGHT IN HIS/HER THESIS. NEITHER THE THESIS NOR SUBSTANTIAL EXTRACTS FROM IT MAY BE PRINTED OR OTHERWISE REPRODUCED WITHOUT HIS/HER PERMISSION.
L'AUTEUR CONSERVE LA PROPRIETE DU DROIT D'AUTEUR QUI PROTEGE SA THESE. NI LA THESE NI DES EXTRAITS SUBSTANTIELS DE CELLECI NE DOIVENT ETRE IMPRIMES OU AUTREMENT REPRODUITS SANS SON AUTORISATION. 
Name Faramarz Hewdess,

Dissertation Abstracts International is arranged by broad, general subject categories. Please select the one subject which most nearly describes the content of your dissertation. Enter the corresponding four-digit code in the spaces provided.

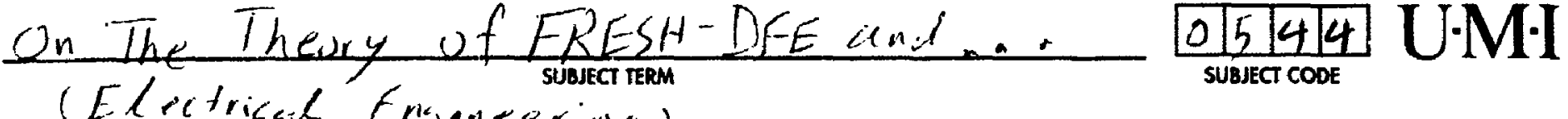

$$
\begin{aligned}
& \text { Subjact Categories }
\end{aligned}
$$

\section{THE MUMANITIES AND SOCIAL SCIENCES}

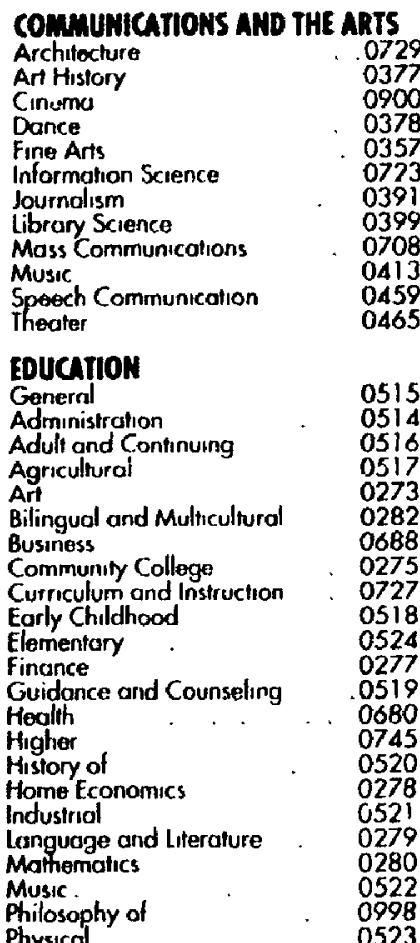

$\begin{array}{ll}\text { Physical } & 0598 \\ & . \quad 0523\end{array}$

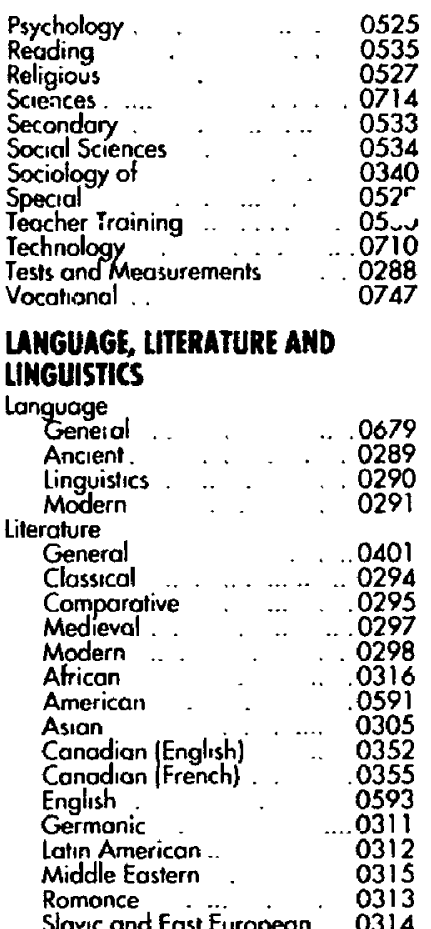

Slaric and East European. .0314

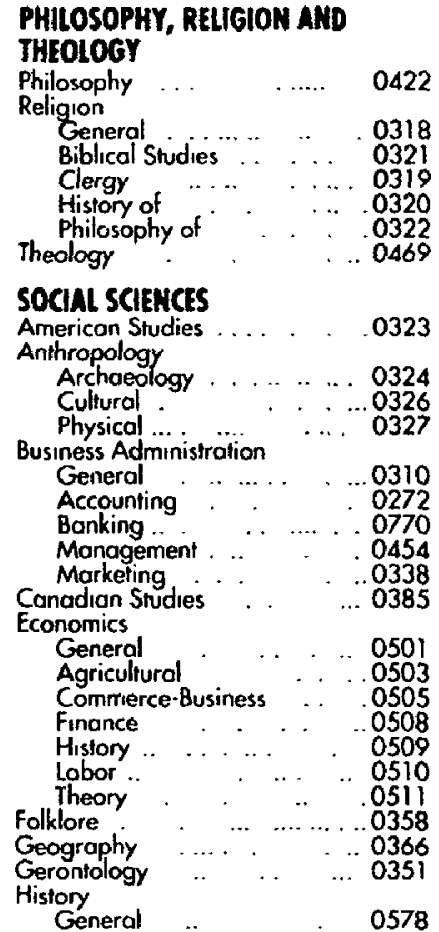

THE SCIENCIS AND ENGINEERINE

\section{DIOLOGICAL SCIENCES}

\begin{tabular}{|c|c|c|c|}
\hline $\begin{array}{l}\text { Geadesy .. . . } \\
\text { Geology } \\
\text { Geophysics } \\
\text { Hydrology } \\
\text { Muneralogy } \\
\text { Paleobotany } \\
\text { Paleoecology } \\
\text { Paleonlology } \\
\text { Paleozoology. . } \\
\text { Palynology } \\
\text { Physical Geogra } \\
\text { Physical Oceano }\end{array}$ & 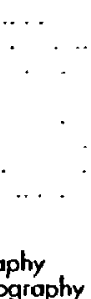 & 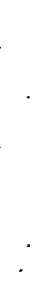 & \\
\hline
\end{tabular}

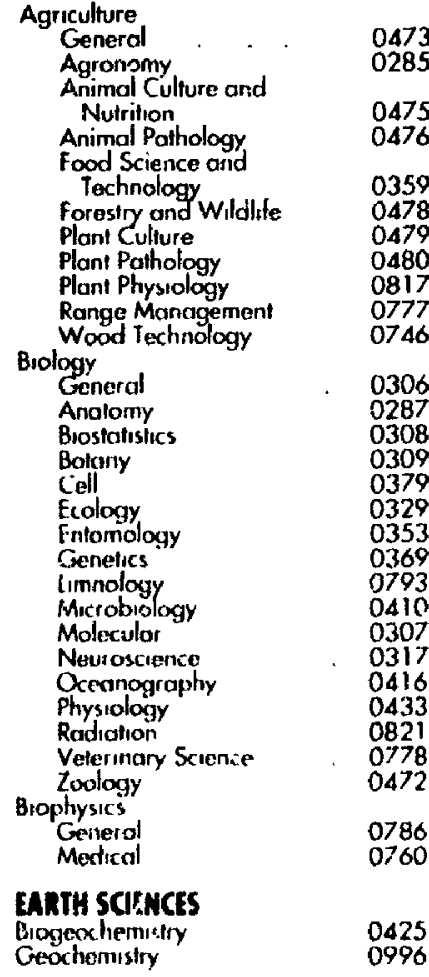

\section{HEATH AND EAVIRONGEHTAL}

\section{SCHences}

Environmental Sciences

0768

Health Sciences

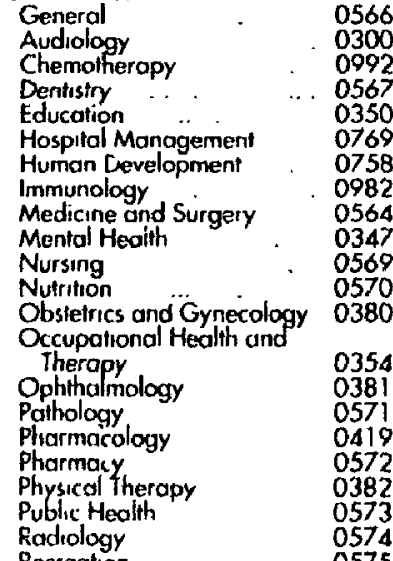

Radiology
Recreation
Speech Pathology $\quad . . \quad .0460$ $\begin{array}{rrr}\text { Toxicology } & \cdots & 0383 \\ \text { Home Economics . . } & .0386\end{array}$

\section{PHYSICAL SCIENCES}

Pure Sciences

Chemistry

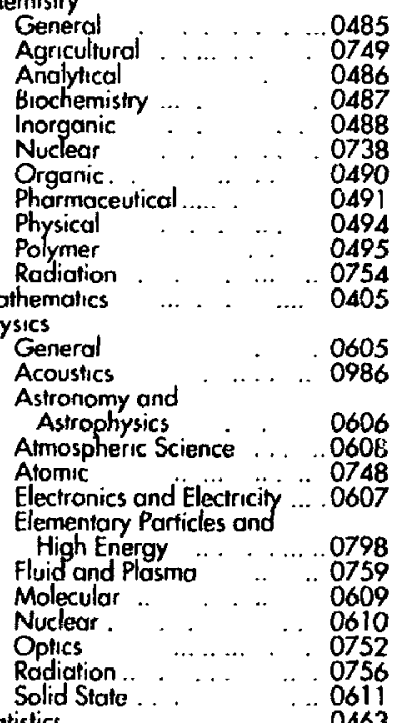

Statistics.

Applied Sciences

Applied Mechanics

Computer Science

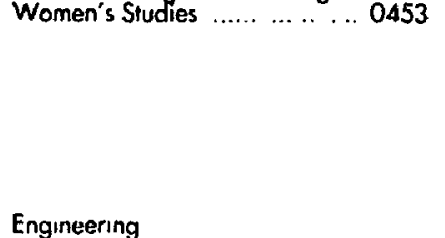

Engineering

Genera . 0537

Aerospace $\ldots \ldots \ldots \ldots \ldots . \ldots 0538$

Agricultural .

Aulomotive . . ... .....0540

Biomedical .......... 0541

Chemical . ............. 0542

Civil ............. .0543

Electronirs and Electrical .. . OSA4

Heat and Thermodynamics 0348

\begin{tabular}{llll} 
Hydraulic. ... &. & $\ldots$ & .0545 \\
\hline
\end{tabular}

Industrial $\ldots . . . \quad \ldots \quad \ldots .0546$

Morine ...... ...0547

Malerials Science .. ...... . 0794

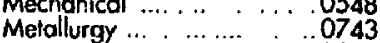

Mining.............

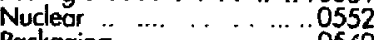

Packgging .... ....... .. OSC9

Petroleum ... .............. 0765

Sanitary and Municipal .... 0554

System Science . .............0790

Geotechnology .... ... ....... 0428

Operations Research ......... 0796

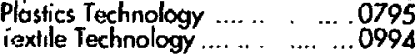

PSYCHOLOGY

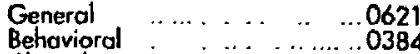

Clinical .................. 0622

Developmental . . .............. 0620

Experimental . ..

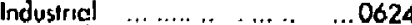

Personality. ............. 0625

$\begin{array}{lllll}\text { Physiological } & \ldots & \ldots \ldots . . . & 0989 \\ \text { Psychobiology } & \ldots & \ldots & \ldots & 0349\end{array}$

Psychobiology $\ldots \ldots$
Psychometrics . . . . . . . .

Social 
The undersigned hereby recommend to

the Faculty of Graduate Studies and Research acceptance of the thesis,

"On The Theory of FRESH-DFE and its Application in Digital Cellular Radio Systems"

submitted by Faramarz Hendessi, B. Sc., M. Sc.

in partial fulfillment of the requirements for

the degree of Doctor of Philosophy

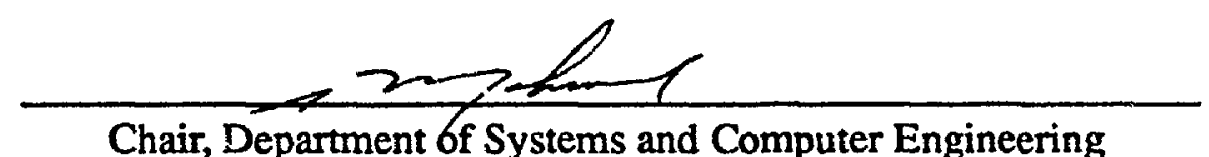

Professor Samy A. Mahmoud

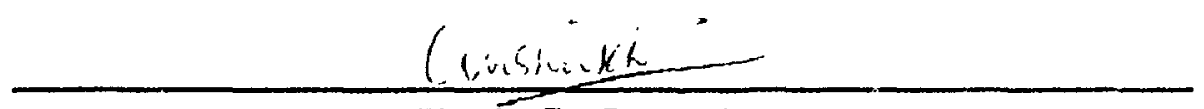

Thesis Co-Supervisor

Professor Asrar U. H. Sheikh
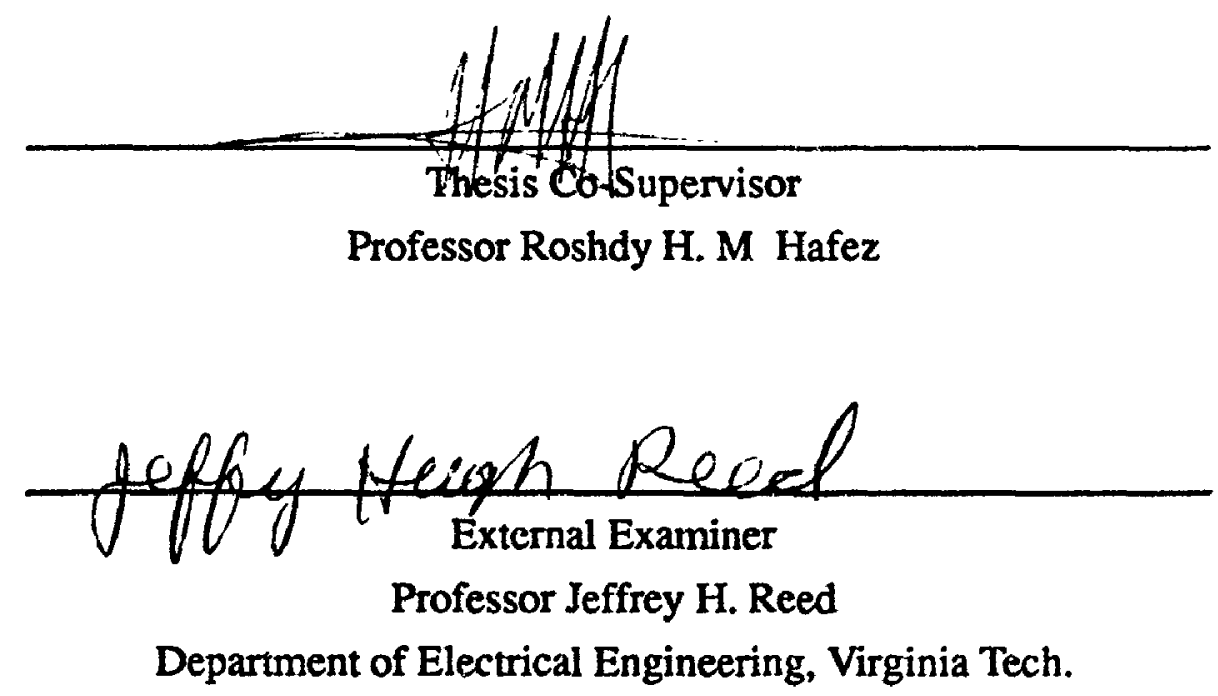

Carleton University

September, 1994 


\section{Abstract}

Modulated signals are known to be spectrally redundant and this redundancy can be exploited in the design of optimum receiver. This is the main topic of this thesis. It is shown that the structure of such a receiver is system-dependent and related to: channel spacing, signal excess bandwidth, number of available receiver antennas, modulation scheme, and users' symbol rates.

In terms of cyclostationarity, it is shown that modulation schemes can be classified into three categories. Binary Phase-Shift Keying (BPSK), Quadrature Phase-Shift Keying (QPSK), and Off-set QPSK (OQPSK) are representative of these three categories. An optimum receiver can be designed and analyzed for each of these three categories.

A complete analysis of decision feedback equalizer (DFE) in cyclostationary environment is presented. The analysis is based on minimum time averaged mean square error (MTAMSE) criterion. It is shown that the conventional DFE is not an optimum receiver for BPSK and OQPSK signals but is optimum for QPSK signals if and only if all user transmit at an identical symbol rate. Two different structures are suggested for DFE. The first is based on harmonic series representation (HSR) of a cyclostationary random process (CRP) and is called frequency-shift DFE (FRESH-DFE). The second is based on time series representation (TSR) of a CRP and is called generalized DFE (GDFE). It is shown that FRESH-DFE and GDFE are identical in performance, and in the case of "single symbol rate", their structures are also identical. We shall refer to FRESH-DFE (or GDFE) in single symbol rate systems as optimum DFE (ODFE). The results of analysis and simulation presented in this thesis show that the ODFE has a better performance than the conventional DFE, specially in high interference environment.

An optimum multi-antenna receiver is also considered, and it is shown that the bit error rate (BER) can be reduced by increasing the number of antennas. This reduction of BER can be converted into additional capacity if moderate BER is acceptable.

The indoor cellular mobile environment in the presence of high interference and multipath fading is chosen to evaluate the structure, complexity and performance of the optimum receiver. In this evaluation, the co-channel and adjacent channel interference ( $\mathrm{CCl}$ and $\mathrm{ACl}$ ) signals are treated separately and it is shown that the effect of $\mathrm{ACl}$ can be 
removed despite its considerable spectral overlaps with the signal of interest (SOI). It is also demonstrated that the ODFE with the same complexity as the conventional DFE can perform better in multipath fading environment.

The developed concepts were applied to cellular environment. To this end, the thesis studies signal to noise ratio (SNR), signal to interference ratio (SIR) and the capacity of cellular systems. It is shown that the capacity can be increased if moderate BER is chosen as a criterion.

Computer simulation results are presented to support the theoretical results. It is shown that for the same level of complexity, the performance of ODFE is superior to that of the conventional DFE. 


\section{Acknowledgments}

I wish to express my gratitude to my thesis supervisors, Dr. A. U. H. Sheikh and Dr. R. H. M. Hafez, for giving me freedom to explore new ideas, and for their guidance at times when I needed it. I am also grateful to Dr. W. A. Gardner for his kindness during the time 1 was visiting U.C. Davis.

I thank Dr. Brent Peterson and Dr. Majeed Abdulrahman for showing patience while listening to my ideas and interesting discussions that followed.

Moreover, I am appreciative of the faculty, alumni, staff and students of Carleton University who provided much support through the development of my thusis.

In addition, I am grateful to Minister of Higher Education of Islamic Republic of Iran, and the Natural Sciences and Engineering Research Council of Canada for their generous financial support.

Finally, I admire my wife and my parents for showing patience and support throughout the duration of this research. 


\section{Table of Contents}

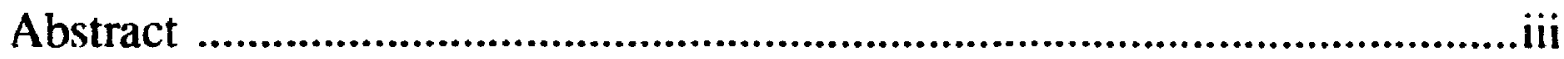

Acknowledgments .........................................................................................

Table of Contents ......................................................................................vi

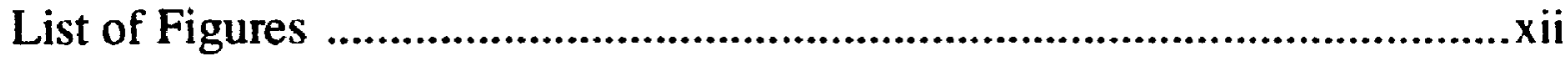

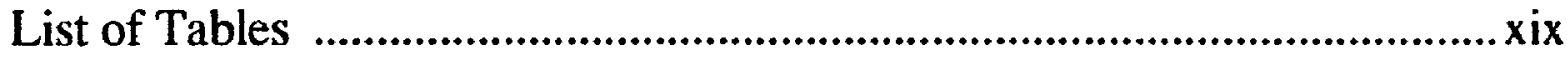

\section{Chapter 1}

Introduction ..............................................................................................

1.1 Thesis Motivation..........................................................................................

1.2 Thesis Contributions ......................................................................................

1.3 Thesis Organization......................................................................................

\section{Chapter 2}

Back ground :

Cyclostationary Random Process ................................................................6

2.1 Introduction ..............................................................................................

2.2 Continuous Time CRPs ..........................................................................6

2.2.1 Basic Functions and Definitions ........................................................6

2.2.2 The Analytic Signal and Complex Envelope ……………..................

2.2.3 Some Examples .....................................................................10

2.2.3.1 An Example of a Real Signal ........................................10

2.2.3.2 An Example of a Complex Signal ...................................12

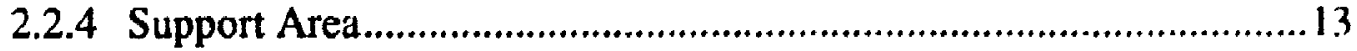


2.2.5 Linear Filtering a CRP.

2.2.5.1 Time Invariant Filtering …...................................................13

2.2.5.2 Time Variant Filtering ……................................................ 16

2.2.6 Stationary Representation ..................................................................17

2.2.6.1 Real Signal ........................................................................17

2.2.6.2 Analytical Signal and Complex Envelope ............................18

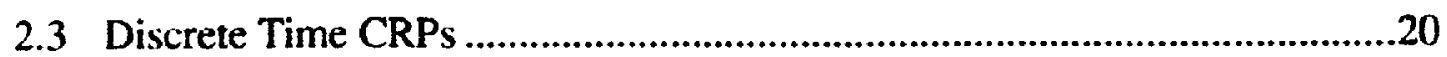

2.3.1 Stationary Representation ....................................................................22

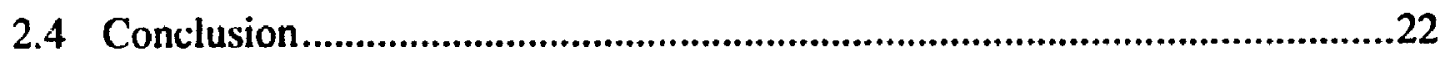

\section{Chapter 3}

Cyclic Spectra of Digitally Modulated Signals ......................................24

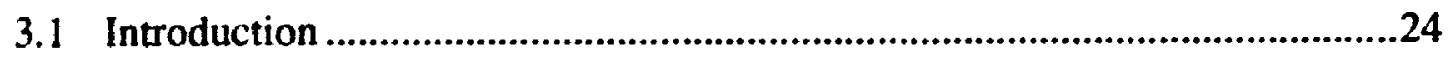

3.2 Digital Modulation Schemes ........................................................................24

3.2.1 Non-Carrier Modulation Schemes......................................................24

3.2.1.1 Binary Pulse Amplitude Modulation (Binary PAM) ...........26

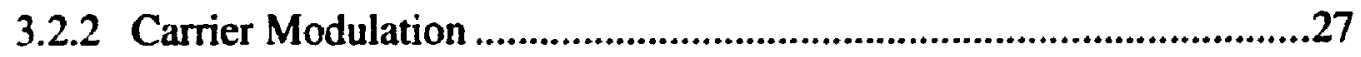

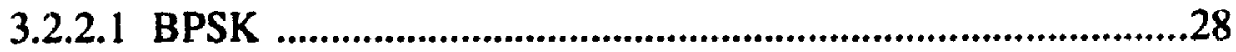

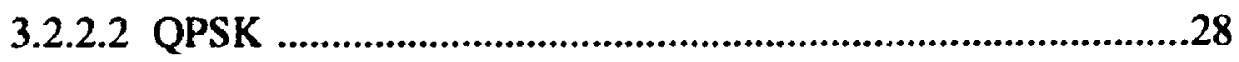

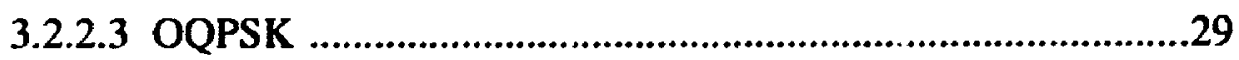

3.2.3 Differentially Modulation Techniques...............................................29

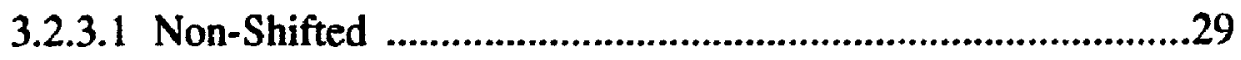

3.2.3.2 Shifted Schemes …..............................................................30

3.3 Categorizing The Digital Modulated Signals ..................................................31

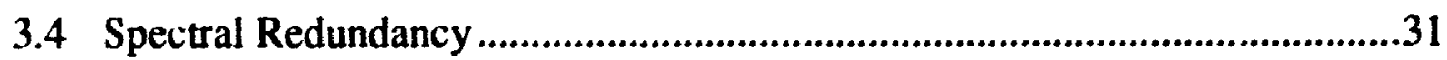

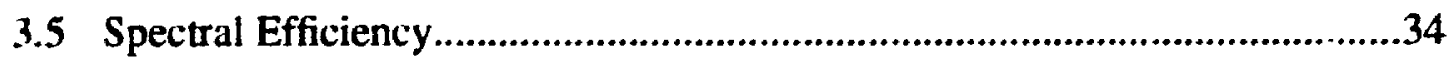

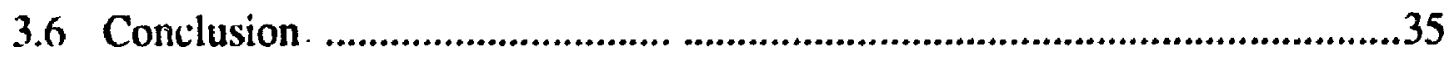




\section{Chapter 4}

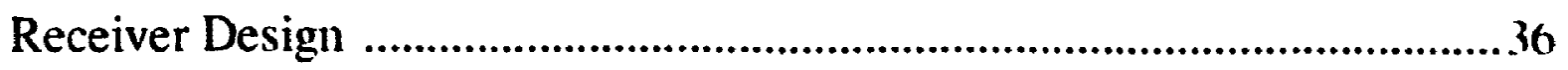

4.1 Introduction ................................................................................................

4.2 Digital Communication System (DCS) Model ……………………………......36

4.2.1 The Cyclic Spectral Density (CSD) and Conjugate CSD (CCSD) of The Received Signal ......................................................38

4.3 Receiver Design .......................................................................................42

4.3.1 Estimating The Entire SOI Waveform..............................................42

4.3.1.1 HSR Time Variant Filter (HSR-TVF) ................................42

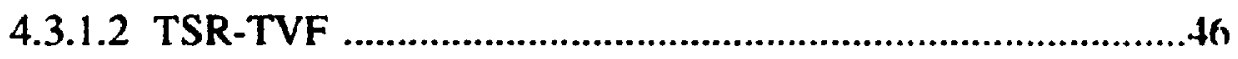

4.3.2 Estimating the Data Sequence .............................................................

4.3.2.1 The Aliasing Effect of a Sampler .........................................4X

4.3.2.2 Adding Sampler to The TSR-TVF ......................................49

4.3.2.3 Adding Sampler to The HSR-TVF ...................................53

4.4 Decision Feedback Receiver .......................................................................57

4.4.1 Conventional Decision Feedback Equalizer.........................................57

4.4.2 Conventional MMSE-DFE .............................................................59

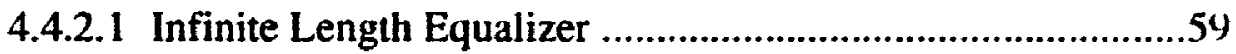

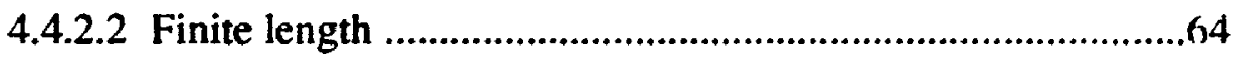

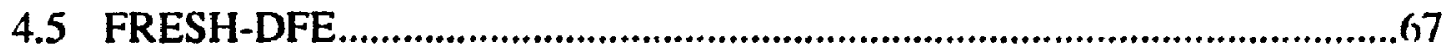

4.6 GDFE

4.7 ODFE

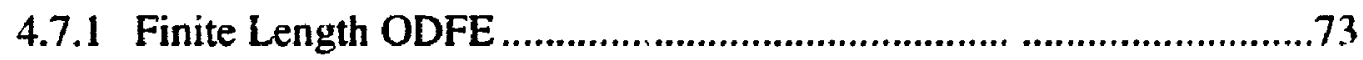

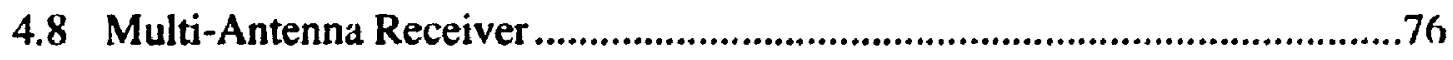

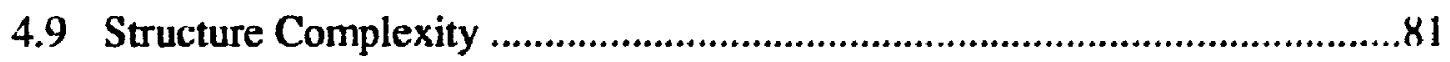

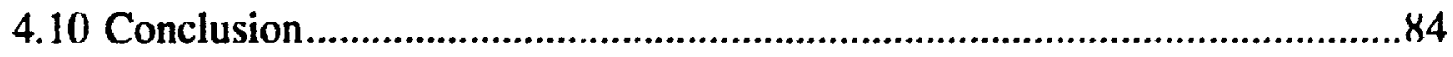




\section{Chapter 5}

Mobile Cellular Radio Systems ..................................................................8

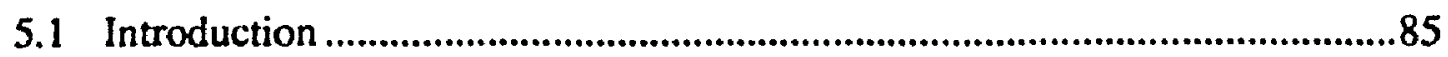

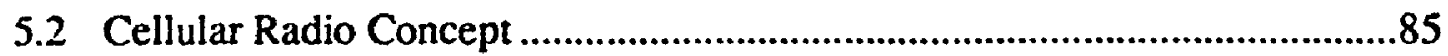

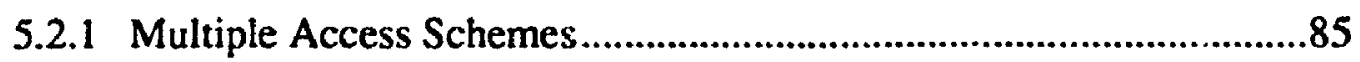

5.2.2 Frequency Reuse and Cell Splitting ……………................................86

5.3 Digital Mobile Radio Channel Properties . ........................................................89

5.3.1 Multipath Channel Properties................................................................89

5.3.2 Time-Variant Fading Channel Properties ............................................89

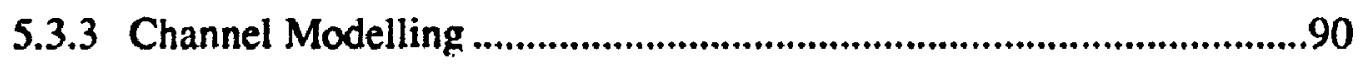

5.3.3.1 Tapped Delay Line Channel Modelling ...............................90

5.3.3.1.1 3-Rays Model .........................................................93

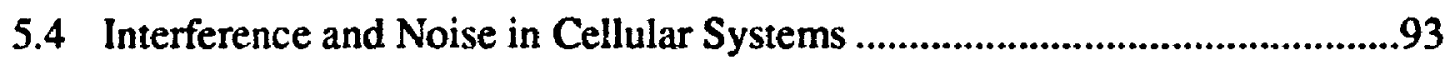

5.4.1 SNR in Cellular Systems ...................................................................96

5.4.2 Signal to CCI Ratio (SCCIR) in Cellular Systems ...........................98

5.4.3 Signal to ACI Ratio (SACIR) in Cellular Systems.............................104

5.4.4 SIR in Cellular Systems..............................................................107

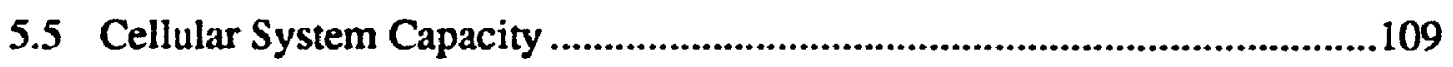

5.5.1 Multi-Symbol Rate Systems .....................................................113

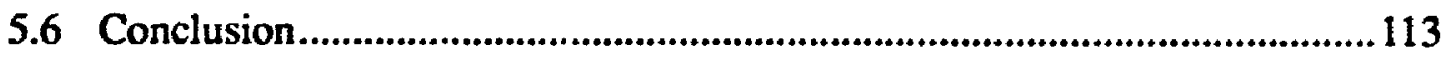

\section{Chapter 6}

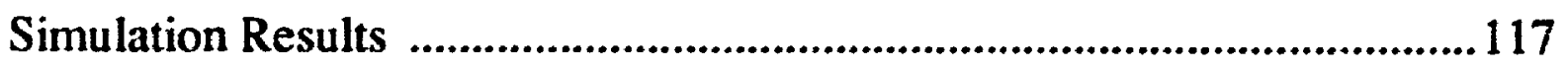

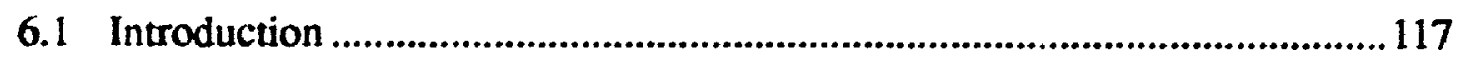

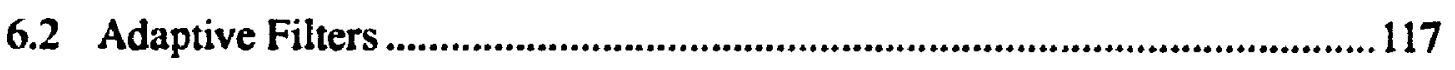

6.3 Adaptive Conventional DFE with Normalized Leart-Mean-Square

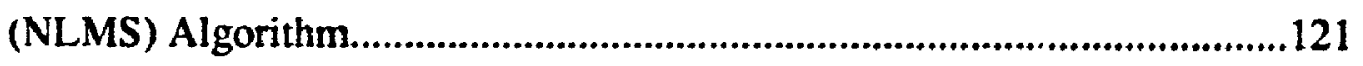

6.4 Adaptive Multi-Antenna Conventional DFE with NLMS Algorithm.........122 
6.5 Adaptive FRESH-DFE with NLMS Algorithm

6.6 Adaptive Muti-Antenna FRESH-DFE (MA-FRESH-DFE) with NLMS Algorithm 126

6.7 Noise Limiting Filter 127

6.8 Simulated Systems 127

6.8.1 No CCI-No ACI System 132

6.8.2 No CCI-6-ACI System 132

6.8.3 6-CCI-No ACI System 138

6.8.4 1-CCI-2-ACI System 1.38

6.8.5 6-CCI 6-ACI System 154

6.8.6 System with Channel Spacing Equals to Half The Symbol Rate. 154

6.8.7 2-Symbo! Rate System 154

6.9 Receivers with Limited Complexity. 167

6.10 Conclusion 181

\section{Chapter 7}

Conclusions 182

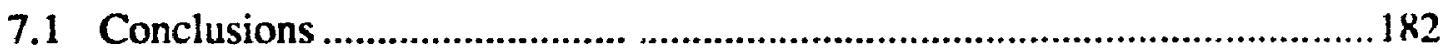

7.2 Recommendations for Future Research ..................................................183

7.2.1 Using Recursive Least Squares (RLS) Algorithm in Adaptive FRESH-DFE.

7.2.2 Considering The Effect of Sampling Phase on The Performance of The FRESH-DFE.

7.2.3 Considering The Effect of The Doppler Spread on The FRESH-DFE.

7.2.4 Investigating The Performance and Perhaps New Structure of Optimum DFE in Fast Fading Environment......................................184

7.2.5 Using Channel Estimator Besides ODFE 184 


\subsubsection{Investigating The Performance of The FRESH-DFE in Code}

Division Multiple Access (CDMA) Systems

Appendix A

Some Useful Cyclic Relations

Appendix B

Analysis of The FRESH-DFE

Appendix C

Analysis of GDFE

References 


\section{List of Figures}

Figure 2.1 (a) The Symmetric version of the Time-Dependent Autocorrelation (STDA), (b) The Time-Dependent Spectral Density (TDSD), (c) The Cyclic Autocorrelation (CA) and (d) 'The Cyclic Spectral Density (CSD) of the Amplitude Modulation (AM) example................... 11

Figure 2.2 Support area (shaded) for (a) Lowpass CRP (b) Bandpass CRP (c) Highpass CRP.

Figure 2.3 Support area for the analytic: signal. .....................................................14

Figure 2.4 Filtering a CRP by an ideal bandpass filter..........................................15

Figure 2.5 The Equivalent Baseband (EB) of the figure $2.4 \ldots \ldots \ldots \ldots \ldots \ldots \ldots \ldots \ldots \ldots \ldots \ldots . . .15$

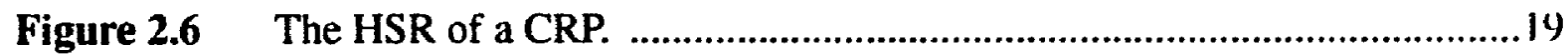

Figure 2.7 Separation of the sta' snary components of a CRP..................................23

Figure 2.8 Stationary component of interest. ....................................................23

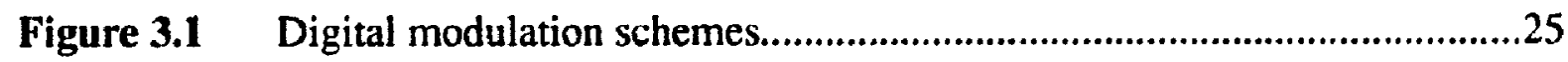

Figure 4.1 The equivalent baseband of a DCS........................................................37

Figure 4.2 The structure of a receiver using Linear-Conjugate-Linear FRESH (LCL-FRESH) filtering.................................................................44

Figure 4.3 The structure of a receiver using LCL-FRESH filtering with tinite number of frequency shifts. .44

Figure 4.4 The structure of a receiver using TSR-TVF...........................................47

Figure 4.5 Sampling the output of a filtered signal...............................................50

Figure 4.6 The equivalent procedures in equation (4.35). ......................................51 
Figure 4.7 The structure of Sampled TSR-TVF (STSR-TVF) .................................52

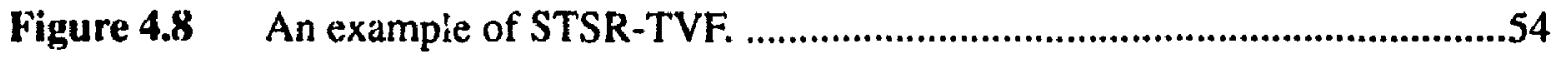

Figure 4.9 The structure of STSR-TVF for single symbol rate sysiems.....................55

Figure 4.10 The structure of STSR-TVF for balanced QPSK and single symbol rate systems.

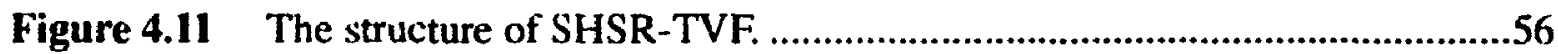

Figure 4.12 The structure of SHSR-TVF for single symbol rate systems. .................56

Figure 4.13 The structure of conventional DFE with infinite length filters...................58

Figure 4.14 Finite length conventional DFE.........................................................65

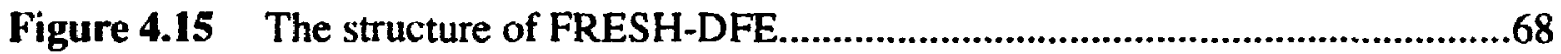

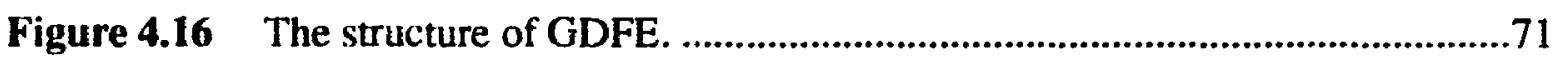

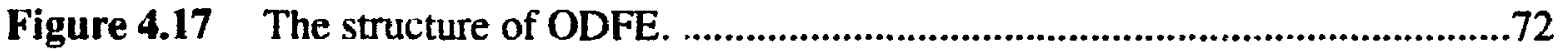

Figure 4.18 The structure of ODFE in systems with channel spacings multiple of half the symbol rate. ...............................................................................72

Figure 4.19 The finite length ODFE in the system with channel spacings mult:ple of half the symbol rate..................................................................74

Figure 4.20 Communication with a multi-antenna receiver.....................................78

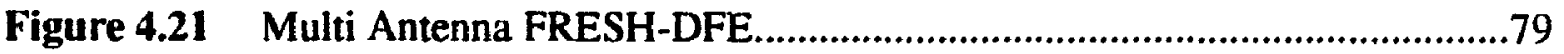

Figure 4.22 Real-Imag Synchronizer which can be used to reduce the structure of ODFE into the conventional DFE for OQPSK signal..........................8.

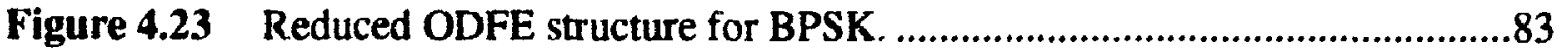

Figure 5.1 (a) A Cellular Radio System (CRS) with cluster size seven (b) A CRS with cluster size three.....................................................................87

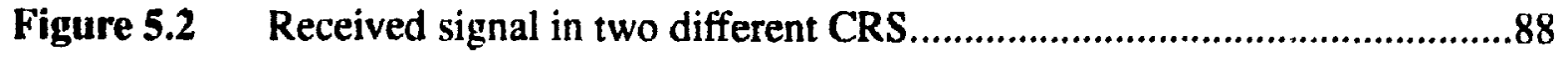




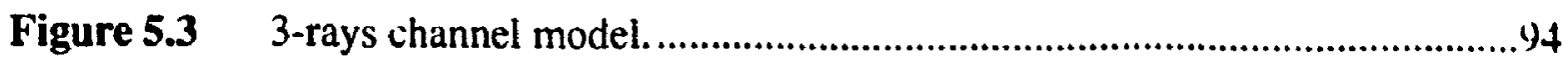

Figure 5.4 MultiPath Delay Protile (MPDP) for 3-rays model.................................94

Figure 5.5 An example of mobile position in a cellular system with cluster size three. .95

Figure 5.6 SNR-SNR tmint versus mobile position......................................................97

Figure 5.7 (a) cumulative distribution function (cdf) (b) probability density function (pdf) of SNR-SNR $\min _{\min }$ in a cellular system...............................99

Figure 5.8 SCCIR versus (a) mobile position (b) number of active CCI.................101

Figure 5.9 (a) cdf (b) pdf of SCCIR in cellular systems with cluster sizes three

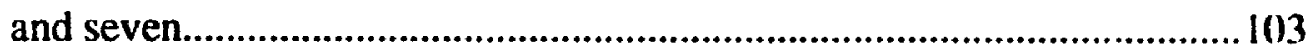

Figure 5.10 A system with channel spacing equals to symbol rate............................106

Figure 5.11 (a) $S A C I R_{\min }$ and (b) $S_{\min }$ vers's signal excess bandwidth..............10x

Figure 5.12 (a) $\mathrm{SACIR}_{\min }$ and (b) $\mathrm{SIR}_{\min }$ versus signal excess bandwidth.............110

Figure 5.13 (a) multi-symbol rate first tier $\mathrm{CCI}$ (b) multi-symbol rate system using three symbol rates. ....................................................................114

Figure 5.14 2-symbol rates system (a) cluster 3 (b) cluster $7 \ldots \ldots \ldots \ldots \ldots \ldots \ldots \ldots \ldots \ldots \ldots \ldots . . .115$

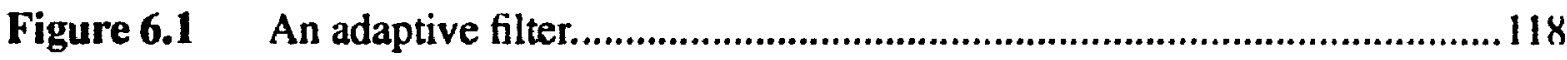

Figure 6.2 An example of Gear Shifting (GS) rule............................................. 20)

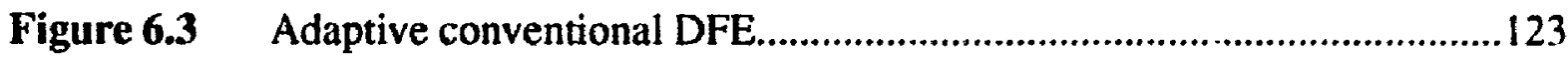

Figure 6.4 Adaptive multi-antenna conventional DFE.....................................123

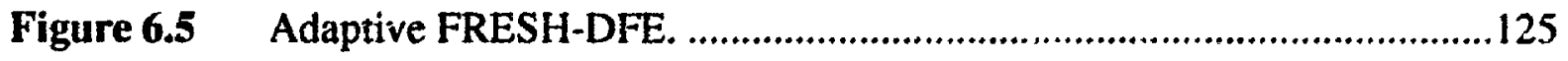

Figure 6.6 (a) A FDMA system with channel spacing equals to the symbol rate

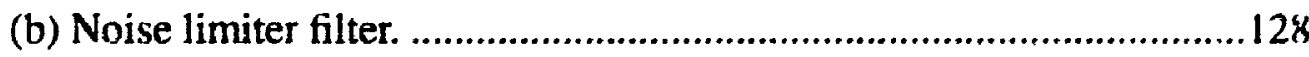

Figure 6.7 (a) The FDMA system and the noise limiter filter (b) The noise limiter filter in $\mathrm{dB}$ scale. 
Figure 6.8 (a) The BER and (b) The BER improvement for no CCI-no ACI system.

Figure 6.9 (a) The MMSE and (b) The MMSE improvement for no CCI-rio ACI system.

Figure 6.10 (a) The BER and (b) The BER improvement in no CCI-no ACI systems when OQPSK signals with excess bandwidths of 100 and 300 percent are used.

Figure 6.11 Forward filters frequency responses in no CCI-no $\mathrm{ACl}$ systems for (a) BPSK (b) OQPSK.

Figure 6.12 (a) The BER and (b) The BER improvement for no $\mathrm{CCl}-6 \mathrm{ACI}$ system.

Figure 6.13 (a) The MMSE and (b) The MMSE improvement for no CCI- 6 ACl system.

Figure 6.14 (a) The BER and (b) The BER improvement for $6 \mathrm{CCI}-$ no $\mathrm{ACl}$ system.

Figure 6.15 (a) The MMSE and (b) The MMSE improvement for $6 \mathrm{CCI}-$ no ACI system.

Figure 6.16 (a) The BER and (b) The BER improvement for $1 \mathrm{CCl}-2 \mathrm{ACl}$ system.

Figure 6.17 (a) The MMSE and (b) The MMSE improvement for $1 \mathrm{CCI}-2 \mathrm{ACI}$ system.

Figure 6.18 (a) The BER and (b) The BER improvement versus SCCIR for 1 CCI- 2 ACI OQPSK system. 144

Figure 6.19 (a) The MMSE and (b) The MMSE improvement versus SCCIR for $1 \mathrm{CCl}-2 \mathrm{ACI}$ OQPSK system. 145

Figure 6.20 (a) The BER and (b) The BER improvement versus SACIR for 1 CCI- 2 ACI OQPSK system. 146

Figure 6.21 (a) The MMSE and (b) The MMSE improvement versus SACIR 
Figure 6.22 (a) The BER and (b) The BER improvement versus SCCIR for 1 CCI- 2 ACI BPSK system.

Figure 6.23 (a) The MMSE and (b) The MMSE improvement versus SCCIR for 1 CCI- 2 ACl BPSK system.

Figure 6.24 (a) The BER and (b) The BER improvement versus SACIR for I CCI- 2 ACI BPSK system.

Figure 6.25 (a) The MMSE and (b) The MMSE improvement versus SACIR for 1 CCI- 2 ACI BPSK system.

Figure 6.26 (a) The BER and (b) The MMSE versus SCCIR for $1 \mathrm{CCI}-2 \mathrm{ACI}$ QPSK system.

Figure 6.27 (a) The BER and (b) The MMSE versus SACIR for $1 \mathrm{CCl} 2 \mathrm{ACI}$ QPSK system.

Figure 6.28 (a) The BER and (b) The BER improvement for $6 \mathrm{CCI}-6 \mathrm{ACI}$ system.

Figure 6.29 (a) The MMSE and (b) The MMSE improvement for $6 \mathrm{CCI}-6 \mathrm{ACI}$ system.

Figure 6.30 (a) The BER and (b) The BER improvement for multi-antenna receiver in $6 \mathrm{CCI}-6 \mathrm{ACI}$ OQPSK system.

Figure 6.31 (a) The BER and (b) The BER improvement for multi-antenna receiver in $6 \mathrm{CCI}-6 \mathrm{ACI}$ OQPSK system.

Figure 6.32 (a) The BER and (b) The BER improvement for multi-antenna receiver in $6 \mathrm{CCI}-6 \mathrm{ACI}$ BPSK system.

Figure 6.33 (a) The MMSE and (b) The MMSE improvement for multi-antenna receiver in $6 \mathrm{CCI}-6 \mathrm{ACl}$ BPSK system.

Figure 6.34 (a) The BER and (b) The MMSE for multi-antenna receiver in 6 CCI- 6 ACI QPSK system. 
Figure 6.35 (a) The BER and (b) The BER improvement for multi-antenna receiver in $6 \mathrm{CCI}-6 \mathrm{ACI}$ OQPSK system when channel spacing is half the symbol rate

Figure 6.36 (a) The MMSE and (b) The MMSE improvement for multiantenna receiver in $6 \mathrm{CCl}-6 \mathrm{ACI}$ OQPSK system when channel spacing is half the symbol rate.

Figure 6.37 (a) The BER and (b) The BER improvement for multi-antenna receiver in $6 \mathrm{CCl}-6 \mathrm{ACl}$ BPSK system when channel spacing is half the symbol rate.

Figure 6.38 (a) The MMSE and (b) The MMSE improvement for multiantenna receiver in $6 \mathrm{CCl}-6 \mathrm{ACI} \mathrm{BPSK}$ system when channel spacing is half the symbol rate.

Figure 6.39 (a) The BER and (b) The MMSE for multi-antenna receiver in 6 CCI- 6 ACI QPSK system when channel spacing is half the symbol rate.

Figure 6.40 (a) The BER and (b) The BER improvement for 2-symbol rate system.

Figure 6.41 (a) The MMSE and (b) The MMSE improvement for 2-symbol rate system.

Figure 6.42 (a) The BER and (b) The BER improvement for 2-symbol rate OQPSK system.

Figure 6.43 (a) The MMSE and (b) The MMSE improvement for 2-symbol rate OQPSK system.

Figure 6.44 (a) The BER and (b) The BER improvement for 2-symbol rate QPSK system.

Figure 6.45 (a) The MMSE and (b) The MMSE improvement for 2-symbol rate QPSK system.

Figure 6.46 (a) The BER and (b) The BER improvement for 2-symbol rate system and when $T_{0}=1$ and 2 seconds. 
Figure 6.47 (a) The MMSE and (b) The MMSE improvement for 2 -symid rate system when $T_{0}=1$ and 2 seconds.

Figure 6.48 (a) The BER and (b) The BER improvement for 2-symbol rate BPSK system when $T_{0}=1$ and 2 seconds.........................................176

Figure 6.49 (a) The MMSE and (b) The MMSE improvement for 2-symbol rate BPSK system when $T_{0}=1$ and 2 seconds.

Figure 6.50 (a) The BER and (b) The BER improvement for 2-symbol rate QPSK system when $T_{0}=1$ and 2 seconds...........................................178

Figure 6.51 (a) The BER and (b) The BER improvement for $1 \mathrm{CCl}-2 \mathrm{ACl}$ system when the complexity of DFE and ODFE are the same.

Figure 6.52 (a) The MMSE and (b) The MMSE improvement for $1 \mathrm{CCI}-2 \mathrm{ACI}$ system when the complexity of DFE and ODFE are the same. 


\section{List of Tables}

Table $3.1 \pi / 4$-shifting of constellation. .30

Table 3.2 Relations between cyclic frequencies with nonzero CSD (or CCSD) and signal excess bandwidth.

Table 4.1 A summary of cyclic spectral properties of BPSK, OQPSK and QPSK systems.

Table 5.1 Minimum SCCIK $(r=1)$.

Table 5.2 Overall cell average of SCCIR.

Table 5.3 Minimum SACIR $(r=1)$.

Table 5.4 Minimum SIR $(r=1)$

Table 6.1 The simulation parameters in all the simulated systems 


\section{List of Abbreviations and Acronyms}

Abbreviations

or
Acronym

$\mathrm{ACl}$

AM

ASK

AWGN

EB

BER

BPSK

B.Sc.

CA

CCA

CCI

CCSD

cdf

CDMA

CIR

CRP

CSD

dB

DCRS

DCS

DFE

DPAM

DQPSK

Dr.

DSP

e.g.

FDMA

FIR

FM
Meaning

Adjacent-Channel Interference

Amplitude Modulation

Amplitude-Shift Keying

Additive White Gaussian Noise

Equivalent Baseband

Bit-Error Rate

Binary Phase-Shift Keying

Bachelor of Science

Cyclic Autocorrelation

Conjugate Cyclic Autocorrelation

Co-Channel Interference

Conjugate Cyclic Spectral Density

cumulative distribution function

Code-Division Multiple Access

Channel Impulse Response

Cyclostationary Random Process

Cyclic Spectral Density

decibels

Digital Cellular Radio System

Digital Communication System

Decision-Feedback Equalizer

Differential Pulse Amplitude Modulation

Differential Quadrature Phase Shift Keying

Doctor

Digital Signal Processing

example

Frequency Division Multiple Access

Finite Impulse Response

Frequency Modulation 


\begin{tabular}{ll}
$\begin{array}{c}\text { Abbreviations } \\
\text { or } \\
\text { Acronym }\end{array}$ & Meaning \\
\hline FRESH & FRequency SHift \\
FRESH-DFE & FRequency SHift DFE \\
FSE & Fractionally Space Equalizer \\
FSK & Frequency Shift Keying \\
GDFE & Generalized DFE \\
GFSE & Generalized FSE \\
GS & Gear Shifting \\
HSR & Harmonic-Series Representation \\
HSR-TVF & HSR-Time Variant Filter \\
iff & if and only if \\
iid & independent identically distributed \\
ISDN & Integrated Services Digital Network \\
ISI & Inter-Symbol Interference \\
LCL & Linear-Conjugate-Linear \\
LCL-FRESH & Linear-Conjugate-Linear FRequency SHift \\
LMS & Least-Mean Square \\
LTI & Linear Time Invariant \\
LTV & Linear Time Variant \\
MA-DFE & Multi-Antenna DFE \\
MA-FRESH-DFE & Multi-Antenna FRESH-DFE \\
M.Sc. & Master of Science \\
MMSE & Minimum Mean Square Error \\
MPDP & Multipath Power Delay Protile \\
MCRS & Mobile Cellular Radio System \\
MSE & Mean Square Error \\
MTAMSE & Mirimum TAMSE \\
NLMS & Normalized LMS \\
ODFE & Optimum DFE \\
OQPSK & Off-set Quadrature Phase-Shift Keying \\
OTIF & Optimum Time-Invariant Filters \\
OTVF & Optimum Time-Variant Filters \\
pdf & probability density function \\
& \\
\hline
\end{tabular}


Abbreviations

\section{or} Acronym

PAM

PPM

PSD

PWM

QPSK

RLS

RMS

sec.

SACIR

SCCIR

SHSR-TVF

SIR

SNR

SINR

SOI

SONI

SRRC

STDA

STSR-TVF

TAMSE

TDF

TDL

TDMA

TDSA

TDSD

TIF

TSR

TVF

TSR-TVF

U.C.

WS

WSC

\section{Meaning}

Pulse Amplitude Modulation

Pulse Position Modulation

Power Spectral Density

Pulse Width Modulation

Quadrature Phase-Shift Keying

Recursive Least Squares

Root-Mean-Square

seconds

Signal to ACI Ratio

Signal to CCI Ratio

Sampled HSR-TVF

Signal to Interference Ratio

Signal-to Noise Ratio

Signal to Interference-plus-ivoise Ratio

Signal-Of-Interest

Signal-Of-No-Intei ast

Square Root Raised Cosine

Symmetric Version of the Time-Dependent Autocorrelation

Sampled TSR-TVF

Time-Average Mean Square Error

Time-Dependent Filter

Tapped Delay Line

Time Division Multiple Access

Time-Dependent Symmetric Autocorrelation

Time-Dependent Spectral Density

Time-Independent Filter

Time Series Representation

Time Variant Filter

TSR Time Variant Filter

University of California

Wide Sense

Wide Sense Cyclostationary 
Abbreviations

or Meaning

WSS

Wide Sense Stationary

VLSI

Very Large Scale Integrated

xxiii 


\section{Special Mathematical Notations}

Notation

$\pi$

e

$j$

$\infty$

$\equiv$

$\neq$

$\rightarrow$

$\geq$

$\leq$

$\forall$

$\delta(t)$

$u(t)$

$D_{B}(f, \alpha)$

$W_{r}(t)$

$\lim$

Real(.)

$\operatorname{Imag}($.

$\left\lceil\frac{n}{p}\right\rceil$
Meaning

is the ratio of the circumference of a circle to its diameter.

is the base of natural logarithms.

is an imaginary number satisfying $j^{2}=-1$.

infinity.

is uniquely equal to.

is not equal to.

tends to.

is greater than or equal to.

is less than or equal to.

for all.

is the notation for Dirac delta function defined by:

$\delta(t)=\left(\begin{array}{ll}1 & t=0 \\ 0 & t \neq 0\end{array}\right.$

is the notation for step function defined by: $u(t)=\left(\begin{array}{ll}1 & 1 \geq 0 \\ 0 & 1<0\end{array}\right.$

is defined by: $u\left(f+\frac{B-\alpha}{2}\right) u\left(-f-\frac{B-\alpha}{2}\right)$

is the ideal lowpass tilter with bandwioth $1 / T$.

is the limit function.

is the real function.

is the imaginary function.

is the integer part of the division of $n$ over $p$. 


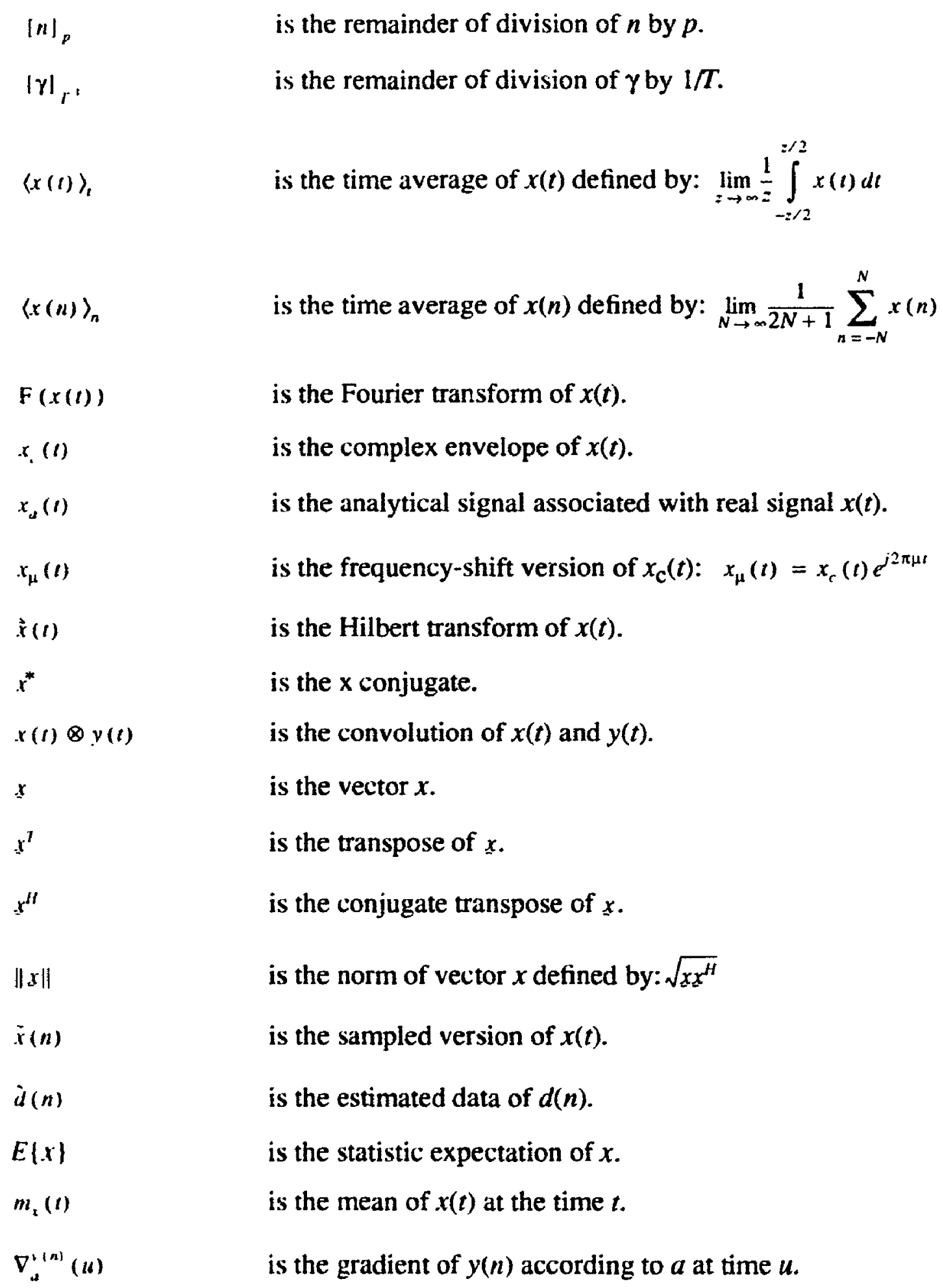

$[n]_{p}$

$|\gamma|_{r}$

$\langle x(t)\rangle$,

$\langle x(n)\rangle_{n}$

$F(x(t))$

$x(t)$

$x_{u}(1)$

$x_{\mu}(t)$

$\hat{x}(t)$

$x^{*}$

$x(t) \otimes y(\ell)$

$\underline{x}$

$x^{1}$

$x^{H}$

$\|x\|$

$\dot{r}(n)$

$\grave{d}(n)$

$E(x)$

$m_{\mathrm{x}}(t)$

$\nabla_{\triangleleft}^{\prime(n)}(u)$

is the remainder of division of $n$ by $p$.

is the remainder of division of $\gamma$ by $1 / T$.

is the time average of $x(t)$ defined by: $\lim _{z \rightarrow \infty} \frac{1}{z} \int_{-: / 2}^{: / 2} x(t) d t$

is the time average of $x(n)$ defined by: $\lim _{N \rightarrow \infty} \frac{1}{2 N+1} \sum_{n=-N}^{N} x(n)$

is the Fourier transform of $x(t)$.

is the complex envelope of $x(t)$.

is the analytical signal associated with real signal $x(t)$.

is the frequency-shift version of $x_{\mathrm{c}}(t): x_{\mu}(t)=x_{\mathrm{c}}(t) e^{j 2 \pi \mu t}$

is the Hilbert transform of $x(t)$.

is the $\mathrm{x}$ conjugate.

is the convolution of $x(t)$ and $y(t)$.

is the vector $x$.

is the transpose of $x$.

is the conjugate transpose of $x$.

is the norm of vector $x$ defined by: $\sqrt{x x^{H}}$

is the sampled version of $x(t)$.

is the estimated data of $d(n)$.

is the statistic expectation of $x$.

is the mean of $x(t)$ at the time $t$.

is the gradient of $y(n)$ according to $a$ at time $u$. 
Notation

$H_{q}^{\alpha}(f)$

$H_{q q^{*}}^{\alpha}(f)$

$s_{x}^{\alpha}(f)$

$S_{x x^{x}}^{\alpha}(f)$

$R_{x}^{\alpha}(\tau)$

$p_{x}^{\alpha}(f)$
Meaning

is defined by: $Q\left(f+\frac{\alpha}{2}\right) Q^{*}\left(f-\frac{\alpha}{2}\right)$ where $Q(f)$ is the Fourier transform of $q(t)$.

is defined by: $Q\left(f+\frac{\alpha}{2}\right) Q\left(f-\frac{\alpha}{2}\right)$

is the CSD of $x(t)$ at frequency $f$ and cyclic frequency $\alpha$.

is the CCSD of $x(t)$ at frequency $f$ and cyclic frequency $\alpha$.

is the CA of $x(t)$ at t.me-lag $\tau$ and cyclic frequency $\alpha$.

is the spectral autocoherence function at frequency $f$ and cyclic ferequincy $\alpha$.

xxvi 


\section{Chapter 1}

\section{Introduction}

\subsection{Thesis Motivation}

The demand for digital communication services has been rapidly increasing and is expected to continue to increase [16,4]. Digital technology brings many advantages, include inexpensive VLSI/DSP hardware, superior speech quality, reduced transmission noise sensitivity, greater data security and broadband services $[4,60]$. The high number of users requires more efficient use of the available bandwidth. To accomplish the efficient utilization of the limited bandwidth, researcher are developing low bit rate speech coders $[95,101]$, bandwidth efficient modulation techniques [3,78], multiple access schemes [65], frequency reuse and cell splitting [65]. However, some of these methods cause co-channel, adjacent-channel and inter-symbol interference (CCI, ACI, and ISI) [17]. In general, $\mathrm{ACI}$ and $\mathrm{CCl}$ limit the system capacity (number of users per unit area) while ISI limits the maximum data rate $[2,57]$. The presence of these impairments leads to a severe degradation in the performance of a digital communication systems. Since there is a trade-off between these impairments and the system capacity, suitable communication techniques and receiver structures are required to minimize the effects of these impairments and maximize the system capacity.

It is known that increasing receiver knowledge on the properties of the received signals, decreases the error probability. Therefore, for certain value of bit error rate (BER), the capacity of a system can be increased by using any information about the signal-ofinterest (SOI) and signal-of-no-interest (SONI) at the receiver.

The following examples illustrate techniques which improve the BER performance by exploiting the detailed structure of the received signal:

- In code division multiple access (CDMA) systems, knowing the users' codes at the receiver decreases the error probability [103].

- Estimating the channel at the receiver decreases the BER [74,75,98].

- Using a channel coding technique increases the redundancy of the transmitted data and lowers BER $[20,60]$. 
- Use of multi-antenna (either at the receiver or at transmitter) reduces BER $[6,7,102]$.

- Using time diversity increases the time redundancy of the received signal and consequently increases the knowledge of the receiver about transmitted signal and, thereby, decreases the BER [90].

- Any frequency diversity causes spectral redundan:y and, thereby, reduces the effect of channel fading and lowers the BER [84].

The cyclostationary nature of digitally modulated signal $[24,26,28,29]$ is another source of information which produces spectral redundancy and can be used to reduce the error rate or increase the capacity.

Recently much work has been done to design an optimum receiver for cyclostationary signals $[30,32,37,47,87,88]$. Most of this work considered analog signals. They are too complex to be practical. The motivation behind this thesis is to design an optimum receiver structure for digital communication systems which can exploit the specti il redundancy of digitally modulated signals and suppress the cyclostationary interference.

Another motivation behind this thesis is to design a practical receiver which exploits the spectral redundancy and works in a harsh environment. The mobile cellular radio environment is chosen to evaluate the performance of such a receiver. The reason is that mobile radio channels are inherently time-variant fading multipath transmission media $[59,63,84]$. Thus, the mobile radio communication is subject to signal dispersion (due to frequency-selectivity) and time-variant signal propagation (due to vehicular

motion). These two properties cause ISI and random Doppler fading of the received signal $[59,63,66,84]$.

\subsection{Thesis Contributions}

The contributions in this thesis in order of appearance are the following.

The main contributions in chapter 3 are:

3.a- Classifying digitally modulated signals into three categories according (") their cyclostationarity. BPSK, QPSK, and OQPSK are representatives of these three categories.

3.b- Introducing two parameters to measure the spectral redundancy and spectral 
efficiency of digitally modulated signals. It is show that there is a trade-off between the spectral redundancy and the spectral efficiency.

The main contributions in chapter 4 are:

4.a- Analyzing the conventional DFE with the MTAMSE criterion. It is shown that the conventional DFE is optimum for QPSK single rate systems.

4.b- Designing and analyzing a generic optimum receiver structure for digitally modulated signals. The optimum infinite length and finite length receiver are analyzed separately. It is shown that the structure of optimum receiver depends on the following system parameters: channel spacing. signal excess bandwidth, number of available antenna, modulation scheme, and users' symbol rates.

4.c- Designing and analyzing a multi-antenna optimum receiver. It is shown that the multi-antenna receiver can achieve very low minimum mean square error (MMSE) if the number of antennas is high.

The main contributions in chapter 5 are:

5.a- Deriving general formulas for signal to noise ratio (SNR), signal to CCI ratio (SCCIR), signal to ACI ratio (SACIR), and signal to total interference ratio (SIR) in a cellilar system. These formulas are derived by using curve fitting to simulation results.

5.b-Proposing a new definition for the capacity of a digital cellular radio system in terms of several design parameters.

5.c-Designing of a 2-symbol rate cellular system.

In chapter 6, a computer simulation study of a TDMA/FDM digital cellular radio system (DCRS) applicable to indoor wireless communications has demonstrated that:

6.a- The performance of FRESH-DFE is superior to that of conventional DFE.

6.b-The superiority of FRESH-SFE over conventional DFE increases when higher spectral redundant signals are used.

6.c- Multi-antenna receiver can achieve very low BER even though the channel spacing is less than the symbol rate. 
6.d-The superiority of FRESH-DFE over conventional DFE increases when multiantenna receiver is used.

6.e- The FRESH-DFE and the conventional DFE in 2-symbol rate system perform better when the SOI symbol rate is twice that of the SNOI.

\subsection{Thesis Organization}

This thesis describes a receiver and system design based on exploitation of cyclostationary properties of digitally modulated signals. Chapter 2 presents the concepts and basic cyclostationary properties of a random processes. The discrete time cyclostationary random process (CRP) is considered as well as continuous time CRP. Furthermore, the stationary representations of a CRP are considered.

Chapter 3 introduces the cyclostationary nature of digitally modulated signals. It is shown that digitally modulated signals can be classified into three categories. BPSK. QPSK, and OQPSK are used as the representatives of these cutegories. The spectral redundancy and spectral efficiency of a modulated signal is also considered and two parameters for measuring the spectral redundancy and spectral efficiency are introduced.

The structure of the receiver is designed in chapter 4 . The fractionally spaced equalizer (FSE) [8] is considered and developed. It is shown that two different structures, called FREquency SHift FSE (FRESH-FSE) and generalized FSE (GFSE), may be proposed to replace the FSE. On the basis of these structures, two other structures for decision feedback equalizer (DFE), called FRESH-DFE [53] and GDFE, are suggested. In the case of single symbol rate system, it is shown that the structures of FRESH-DFE and GDFE are identical. We refer to this structure as optimum DFE (ODFE). It is also shown that the conventional DFE uses all the spectral redundancy related to the SOI symbol rate while the FRESH-DFE (or GDFE) uses all the spectral redundancy related to all users' symbol rates and carrier frequencies. It is also concluded that the conventional DFE and FRESH-DFE (or GDFE) are equivalent for QPSK single symbol rate systems.

Chapter 5 presents a basic review of mobile cellular radio system (MCRS) wherein the properties of the MCRS channels are reviewed and the channel model used in this thesis is detailed. The frequency reuse and cell splitting are discussed in the context of cellular systems. The interference properties in a MCRS are studied and results which characterizes $\mathrm{CCI}$ and $\mathrm{ACI}$ in a cellular system are obtained. A new definition for capacity 
in terms of several design parameters is introduced and used in determining the overall cellular capacity. Furthermore, a multi-symbol rate system is proposed and it is shown that the impact of $\mathrm{CCl}$ is reduced in this system.

Chapter 6 presents the simulation results for investigating the analytical results of chanter 4 and 5. The performance of FRESH-DFE and the conventional DFE are compared. It is shown that FRESH-DFE always has better performance. It is also shown that multi-antenna receiver can achieve low BER even if the channel spacing is smaller than the symbol rate. A 2-symbol rate cellular system is simulated and it is shown that the performance of FRESH-DFE and that of the conventional DFE is better when the SOI symbol rate is twice the SNOI symbol rate. It is also shown that for the same receiver complexity, the ODFE has better performance than the conventional DFE.

Chapter 7 consists of conclusions, discussions and recommendations for further research. 


\section{Chapter 2 \\ Back ground : \\ Cyclostationary Random Process}

\subsection{Introduction}

In this Chapter, the properties of cyclostationary random processes (CRPs) are described. Continuous and discrete time CRPs are considered separately. First, continuous time CRPs are reviewed and the basic functions and definitions applied to these processes are discussed. This is followed by a discussion on sampled (discrete time) CRPs, the basic functions, and definitions applied to these processes.

More details on topics discussed in this chapter are be found in $[9,10,15,23$ to 42 , $44,54,58,62,69,79,86,96,105$ ].

\subsection{Continuc us Time CRPs}

\subsubsection{Basic Functions and Definitions}

A continuous time random process, say $x(t)$, is said to be cyclostationary in the wide sense if its mean and autocorrelation functions are periodic with a certain perind, $T$ : [77]

$$
\begin{aligned}
m_{x}(t+T) & =m_{x}(t) \quad \forall t \\
R_{x}(t+T, u+T) & =R_{x}(t, u) \quad \forall(t, u)
\end{aligned}
$$

where the mean and autocorrelation are defined as the follows :

$$
\begin{gathered}
m_{x}(t)=E\{x(t)\} \\
R_{x}(t, u)=\frac{1}{2} E\left\{x(t) x^{*}(u)\right\}
\end{gathered}
$$

where $E\{\cdot \mid$ is the expectation function. Equation (2.1) can be re-expressed using the symmetric version of the time-dependent autocorrelation (STDA) function as follows :

$$
R_{x}\left(t+\frac{\tau}{2}+T, t-\frac{\tau}{2}+T\right)=R_{x}\left(t+\frac{\tau}{2}, t-\frac{\tau}{2}\right)
$$


Since $R_{x}\left(t+\frac{\tau}{2}, t-\frac{\tau}{2}\right)$ is periodic in $t$ with period $T$ for each $\tau$, it can be expressed by the Fourier series representation as :

$$
R_{x}\left(t+\frac{\tau}{2}, t-\frac{\tau}{2}\right)=\sum_{\alpha} R_{x}^{\alpha}(\tau) e^{j 2 \pi \alpha t}
$$

where $\left\{R_{x}^{\alpha}(\tau)\right\}$ are the fourier coefficients,

$$
R_{x}^{\alpha}(\tau)=\frac{1}{T} \int_{-T / 2}^{T / 2} R_{x}\left(t+\frac{\tau}{2}, t-\frac{\tau}{2}\right) e^{-j 2 \pi \alpha t} d t
$$

and $\alpha$ ranges over all integer multiples of the fundamental frequency $\frac{1}{T}$.

If a random process contains other cyclostationary processes with different incommensurate periods $\left\{T_{i}: \mathrm{i}=1, \ldots, M\right\}$ where $M$ is the number of cyclostationary components, it is said to be almost cyclostationary with periods $\left\{T_{i}\right\}$. In this case $R_{x}^{\alpha}(\tau)$ is defined as :

$$
R_{x}^{\alpha}(\tau)=\lim _{z \rightarrow \infty} \frac{1}{z} \int_{-z / 2}^{z / 2} R_{x}\left(t+\frac{\tau}{2}, t-\frac{\tau}{2}\right) e^{-j 2 \pi \alpha t} d t
$$

where $\alpha$ ranges over all integer multiples of all fundamental frequencies $\left\{\frac{1}{T_{i}}\right\} . R_{x}^{\alpha}(\tau)$ is referred to as the cyclic autocorrelation (CA) function, and $\alpha$ is called the cycle frequency parameter. Letting $\alpha=0$ in (2.6) yields the CA at $\alpha=0$ which is the time average autocorrelation function,

$$
R_{x}^{0}(\tau)=\left\langle R_{x}(t, \tau)\right\rangle_{t}
$$

where $\langle\bullet\rangle_{\mathrm{t}}$ denotes averaging over $\mathrm{t}$.

Similarly, conjugate cyclic autocorrelation (CCA) function is defined as follows :

$$
R_{x x^{*}}^{\alpha}(\tau)=\lim _{z \rightarrow \infty} \frac{1}{z} \int_{-z / 2}^{z / 2} E\left\{x\left(t+\frac{\tau}{2}\right) x\left(t-\frac{\tau}{2}\right)\right\} e^{-j 2 \pi \alpha t} d t
$$

A complex valued time series $x(t)$ is said to exhibit cyclostationarity if either its CA or its CCA is not identically zero for $\alpha \neq 0$.

The time-dependent spectral density (TDSD) function of a random process $x(t)$ can be defined by the Fourier transforms of the STDA. 


$$
S_{x}(t, f)=\int_{\infty}^{\infty} R_{x}\left(t+\frac{\tau}{2}, t-\frac{\tau}{2}\right) e^{-j 2 \pi / \tau} d \tau
$$

The representative Fourier coefficients of TDSD is given by

$$
S_{x}^{\alpha}(f)=\lim _{z \rightarrow \infty} \frac{1}{z} \int_{-z / 2}^{=/ 2} S_{x}(t, f) e^{-\jmath 2 \pi \alpha t} d t
$$

and called the cyclic spectrum or cyclic spectral density (CSD) function. Letting $\alpha=0$ in (2.10) yields the time average spectral density function,

$$
S_{x}^{0}(f)=\left\langle S_{x}(t, f)\right\rangle_{t}
$$

It is easy to show that CSD and CA functions form a Fourier transform pair [27],

$$
S_{x}^{\alpha}(f)=\int_{\infty}^{\infty} R_{x}^{\alpha}(\tau) e^{-j 2 \pi f t} d \tau
$$

Conjugate cyclic spectral density (CCSD) function is defined as the Fourier transform of the CCA,

$$
S_{x x^{*}}^{\alpha}(f)=\int_{\infty}^{\infty} R_{x x^{*}}^{\alpha}(\tau) e^{-j 2 \pi f t} d \tau
$$

For a real time series random process, the CA and the CSD are equal to the CCA and the CCSD respectively.

The spectral autocoherence function for $x(t)$ is defined by

$$
\begin{aligned}
\rho_{x}^{\alpha}(f) & =\frac{S_{x}^{\alpha}(f)}{\sqrt{\left\langle S_{x}\left(t, f+\frac{\alpha}{2}\right)\right\rangle\left\langle S_{x}\left(t, f-\frac{\alpha}{2}\right)\right\rangle}} \\
& =\frac{S_{x}^{\alpha}(f)}{\sqrt{S_{x}^{0}\left(f+\frac{\alpha}{2}\right) S_{x}^{0}\left(f-\frac{\alpha}{2}\right)}}
\end{aligned}
$$

where we have used $\left\langle S_{x}\left(t, f+\frac{\alpha}{2}\right)\right\rangle=S_{x}^{0}\left(f+\frac{\alpha}{2}\right)$ and $\left\langle S_{x}\left(t, f-\frac{\alpha}{2}\right)\right\rangle=S_{x}^{\prime \prime}\left(f-\frac{\alpha}{2}\right)$. It is easy to show that $\rho_{x}^{\alpha}(f)$ is the time-averaged correlation coefficient for the two frequency 
components at frequencies $f+\frac{\alpha}{2}$ and $f-\frac{\alpha}{2}$ [27]. A process $x(t)$ is said to be completely coherent at spectral frequency $f$ and cycle frequency $\alpha$ if and only if (iff) the spectral autocoherence magnitude is unity, $\left|\rho_{x}^{\alpha}(f)\right|=1$. Also a process $x(t)$ is said to be completely incoherent at spectral frequency $f$ and cycle frequency $\alpha$ iff the spectral autocoherence is zero, $p_{x}^{\prime \prime}(f)=0$. It is conceptually (also from (2.14)) apparent that $\rho_{x}^{0}(f)=1$ for any process $x(t)$ and any $f$.

\subsubsection{The Analytic Signal and Complex Envelope}

The analytic signal associated with a real waveform $x(t)$ is defined according to :

$$
x_{a}(t)=x(t)+j \vec{x}(t)=\int \gamma(u) x(t-u) d u
$$

where $j=\sqrt{-1}, \vec{x}(t)$ is the Hilbert transform of $\mathrm{x}(\mathrm{t})$ and $\gamma(t)=\delta(t)+j \frac{1}{\pi t}$. It can be shown that the Fourier transform of $\gamma(t)$ is $\mathrm{F}[\gamma(t)]=\Gamma(f)=2 u(f)$, where $u(f)$ is the step function, and therefore $x_{a}(t)$ has strictly positive frequencies. The CSD and CCSD of the analytic signal $x_{a}(t)$ can be found from the CSD of $x(t)$ according to : [10]

$$
\begin{gathered}
S_{x_{u}}^{\alpha}(f)=4 u\left(f+\frac{\alpha}{2}\right) u\left(f-\frac{\alpha}{2}\right) S_{x}^{\alpha}(f) \\
S_{x_{u} x_{\alpha}^{*}}^{\alpha}(f)=4 u\left(f+\frac{\alpha}{2}\right) u\left(\frac{\alpha}{2}-f\right) S_{x}^{\alpha}(f)
\end{gathered}
$$

Inversely, the CSD and CCSD of $x(t)$ can be found from its analytic signal according to : [10]

$$
S_{x}^{\alpha}(f)=\frac{1}{4}\left[S_{x_{\alpha}}^{\alpha}(f)+S_{x_{u}}^{\alpha}(-f)+S_{x_{u} x_{u}^{*}}^{\alpha}(f)+S_{x_{\Delta} x_{\alpha}^{*}}^{-\alpha}(f)^{*}\right]
$$

The complex envelope of $x(t)$ relative to $f_{0}$ is defined as the down-shifted analytic signal :

$$
x_{r}(t)=x_{a}(t) e^{-j 2 \pi f_{0} t}
$$

If $x(t)$ is bandlimited to ||$A-f_{0} \mid<B / 2$, the SCD of the complex signal $x_{c}(t)$ can be found from the SCD of the analytic signal according to : [10]

$$
\begin{gathered}
S_{x_{c}}^{\alpha}(f)=S_{x_{\downarrow}}^{\alpha}\left(f+f_{0}\right) \\
S_{x_{c} x_{c} x^{*}}^{\alpha}(f)=S_{x_{s} x_{s}^{*}}^{\alpha+2 f_{0}}(f)
\end{gathered}
$$


Substituting (2.16) in (2.19) yields

$$
\begin{gathered}
S_{x_{i}}^{\alpha}(f)=4 D_{B}(f, \alpha) S_{x}^{\alpha}\left(f+f_{0}\right) \\
S_{x_{c} x_{c}^{*}}^{\alpha}(f)=4 D_{B}(f, \alpha) S_{x}^{\alpha+2 f_{10}}(f)
\end{gathered}
$$

where

$$
D_{B}(f, \alpha)=u\left(f+\frac{B-\alpha}{2}\right) u\left(-f-\frac{B-\alpha}{2}\right)
$$

inversely, the SCD of the real signal $x(t)$ can be found from its complex envelope according to : [10]

$$
S_{x}^{\alpha}(f)=\frac{1}{4}\left[S_{x_{c}}^{\alpha}\left(f-f_{0}\right)+S_{x_{r}}^{\alpha}\left(-f-f_{0}\right)+S_{x_{r} x_{c}^{*}}^{\alpha-2 f_{0}}(f)+S_{x_{c} x_{c}^{*}}^{-\alpha f_{0}}(f){ }^{*}\right]
$$

\subsubsection{Some Examples}

\subsubsection{An Example of a Real Signal}

As an example of a real signal, an amplitude modulating (AM) signal is considered. Let $y(t)$ be a zero-mean wide sense stationary (WSS) process with autocorrelation function $R_{y}(\tau)$ and spectral density function $S_{y}(f)$. Assume that $x(t)$ is AM modulated by $y(t)$. The carrier frequency is $f_{0}$ and the initial phase is $\phi_{0}$.

$$
x(t)=\sqrt{2} y(t) \cos \left(2 \pi f_{0} t+\phi_{0}\right)
$$

STDA, TDSD, CA, and CSD functions can be calculated using (2.2), (2.9), (2.5) and (2.12) as follows:

$$
\begin{gathered}
R_{x}\left(t+\frac{\tau}{2}, t-\frac{\tau}{2}\right)=R_{y}(\tau)\left(\cos \left(2 \pi f_{0} \tau\right)+\cos \left(4 \pi f_{0} t+2 \phi_{0}\right)\right) \\
S_{x}(t, f)=\frac{1}{2}\left(S_{y}\left(f-f_{0}\right)+S_{y}\left(f+f_{0}\right)\right)+\cos \left(4 \pi f_{0} t+2 \phi_{0}\right) S_{y}(f) \\
R_{x}^{\alpha}(\tau)=R_{y}(\tau)\left(\cos \left(2 \pi f_{0} \tau\right) \delta(\alpha)+\frac{1}{2}\left(e^{j 2 \phi_{11}} \delta\left(\alpha-2 f_{0}\right)+e^{-j 2} " \delta\left(\alpha+2 f_{0}\right)\right)\right) \\
S_{x}^{\alpha}(f)=\frac{1}{2}\left(S_{y}\left(f-f_{0}\right)+S_{y}\left(f+f_{0}\right)\right) \delta(\alpha)+ \\
\frac{1}{2} S_{y}(f)\left(e^{j 2 \phi_{0}} \delta\left(\alpha-2 f_{0}\right)+e^{-j 20_{0}} \delta\left(\alpha+2 f_{0}\right)\right)
\end{gathered}
$$

Figure 2.1 shows STDA, TDSD, CA, and CSD functions from (2.24) for 
Chaptor 2 Cyclostationary Random Procass

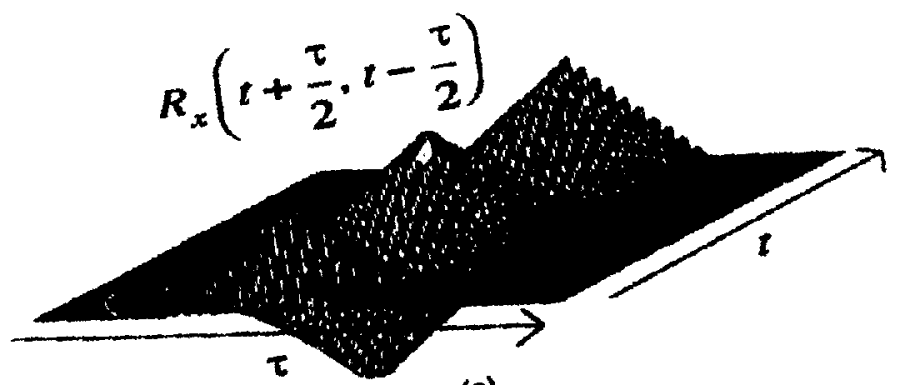

(a)

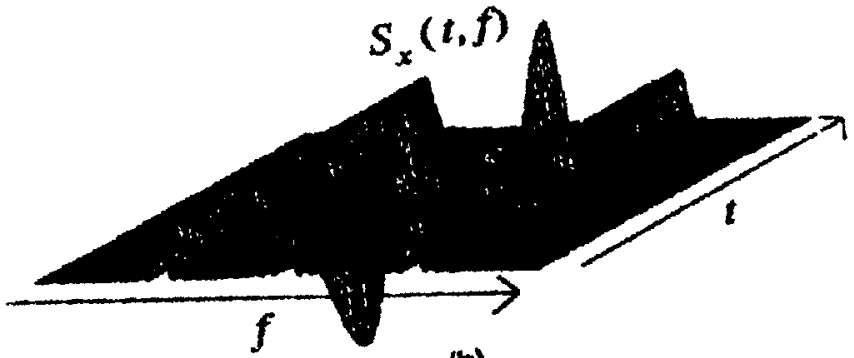

(b)
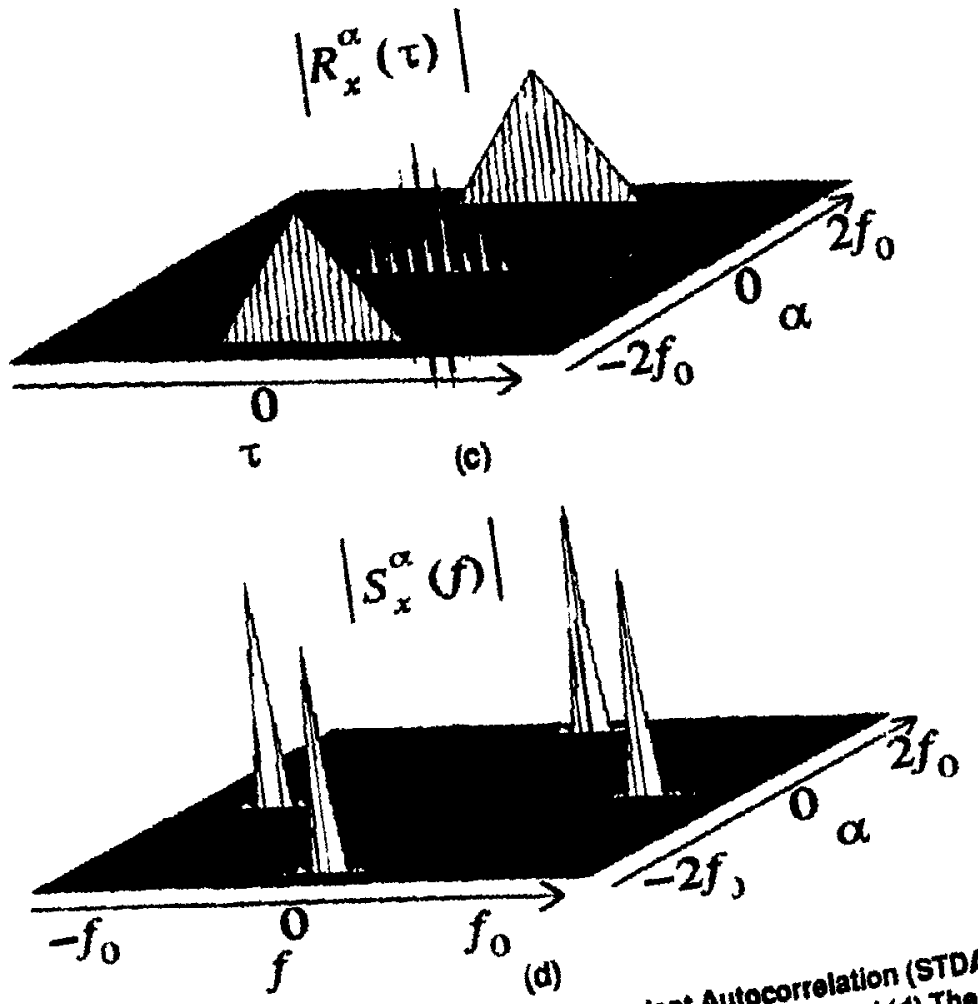

(b) The Time-

(d) Indent Autocorrelation (STDA), (b) Tho Trectral

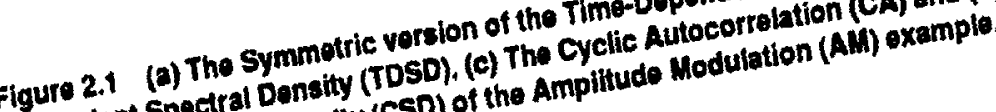




$$
R_{y}(\tau)=\left\{\begin{array}{cr}
1-|\tau| & |\tau| \leq 1 \\
0 & \text { elsewhere }
\end{array}\right.
$$

and $f_{0}=10$. It is apparent from the tigure and $(2.24)$ that

$$
\begin{gathered}
S_{x}^{ \pm 2 f_{0}}(f)=\frac{1}{2} S_{v}(f) e^{ \pm 20_{0}} \\
S_{x}^{\prime \prime}(f)=\left\langle S_{x}(t, f)\right\rangle_{t}=\frac{1}{2}\left(S_{y}\left(f-f_{0}\right)+S_{v}\left(f+f_{0}^{\prime}\right)\right)
\end{gathered}
$$

This example clearly shows that the frequency representation of a signal can be viewed as a (complex) height of a surface above the bifrequency $(f, \alpha)$ plane. Therefore some information about the signal can be found at $\alpha \neq 0$ as well as at $\alpha=0$.

For this example,

$$
\rho_{x}^{\alpha}(f)= \begin{cases}1 & \alpha=0, \pm 2 f_{0} \\ 0 & \text { elsewhere }\end{cases}
$$

Therefore, an AM signal is completely coherent at $\alpha=0, \pm 2 f_{0}$ and for all values of $f$.

\subsubsection{An Example of a Complex Signal}

We consider now the complex envelope of the AM signal relative to an arbitrary frequency $f_{1}$. If $x_{a}(t)$ is the analytic signal and $x_{r}(t)$ is the complex envelope relative to the frequency $f_{1}$, the following relations exist :

$$
\begin{gathered}
x_{a}(t)=\sqrt{2} y(t) e^{j\left(2 \pi f_{1} t+o_{1}\right)} \\
x_{c}(t)=x_{a}(t) e^{-2 \pi f_{1} t}=\sqrt{2} y(t) e^{j\left(2 \pi \Delta f t+o_{1}\right)} \\
x(t)=\operatorname{Real}\left\{x_{a}(t)\right\}=\operatorname{Real}\left\{x_{c}(t) e^{j 2 \pi f_{1} t}\right\}
\end{gathered}
$$

where $\Delta f=f_{0}-f_{1}$. In this case, the CSD and the CCSD of $x_{a}(t)$ and $x,(t)$ can be found as follows :

$$
\begin{aligned}
& S_{x_{u}}^{\alpha x}(f)=2 S_{v}\left(f-f_{0}\right) \delta(\alpha) \\
& S_{x_{t}}^{(\ell)}(f)=2 S_{y}(\alpha-\Delta f) \delta(\alpha) \\
& S_{x_{u^{\prime} x_{\lrcorner}}{ }^{\alpha}}(f)=2 S_{y}(f) e^{\prime 2 \theta_{10}} \delta\left(\alpha-2 f_{0}\right) \\
& S_{x_{1} x_{i}}^{\alpha}(f)=2 S_{y}(f) e^{\prime 2 o_{11}} \delta(\alpha-2 \Delta f)
\end{aligned}
$$


The CSD of $x(t)$ can be found from (2.17) (or (2.22)) and (2.29) and the result will be the same as (2.24). If $\Delta f=0, x_{c}(t)$ is called the equivalent baseband (EB) of $x(t)$. In this example, although the EB of $x(t)$ is not a CRP, $x(t)$ is a CRP. This shows that the EB of a signal contains all the information of the signal including its cyclic properties.

\subsubsection{Support Area}

It follows from the fait that the magnitude of spectral autocoherence function in (2.14) is bounded by unity, the following inequality exists for any $f$ and $\alpha$,

$$
\left|S_{x}^{\alpha}(f)\right| \leq \sqrt{\left\langle S_{x}\left(t, f+\frac{\alpha}{2}\right)\right\rangle\left\langle S_{x}\left(t, f-\frac{\alpha}{2}\right)\right\rangle}
$$

If $x(t)$ is bandlimited so that

$$
\left\langle S_{x}(t, f)\right\rangle_{t} \equiv 0 \text { for }|A| \leq b \text { or } \quad|A| \geq B
$$

where $b<B$, then (2.30) reveals that the cyclic spectrum is constrained to satisfy

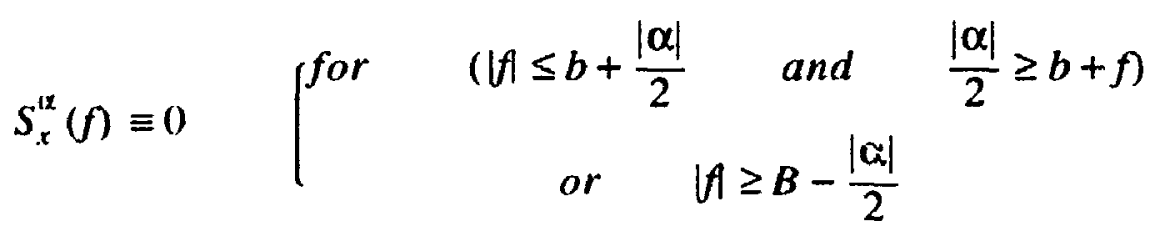

The area of bifrequency $(f, \alpha)$ plane where CSD (or CCSD) is not uniquely zero is called CSD (or CCSD) support area. Figure 2.2 shows this area for different kinds of mean-square bandlimited processes and Figure 2.3 shows the support area for the analytic signal.

\subsubsection{Linear Filtering a CRP}

\subsubsection{Time Invariant Filtering}

If a CRP, $x(t)$, is filtered by a time invariant linear filter, $h(t)$, its CSD is filtered along the $f$ and $\alpha$ axis. Figure 2.4 shows an example of this filtering. In this example, a CRP is filtered by a linear time-invariant ideal bandpass filter. The CSD of the output is given by : $\{27,10]$

$$
S_{y}^{\alpha}(f)=H\left(f+\frac{\alpha}{2}\right) S_{x}^{\alpha}(f) H^{*}\left(f-\frac{\alpha}{2}\right)
$$

Defining 


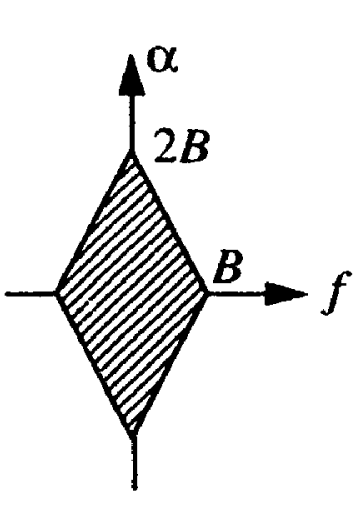

(a)

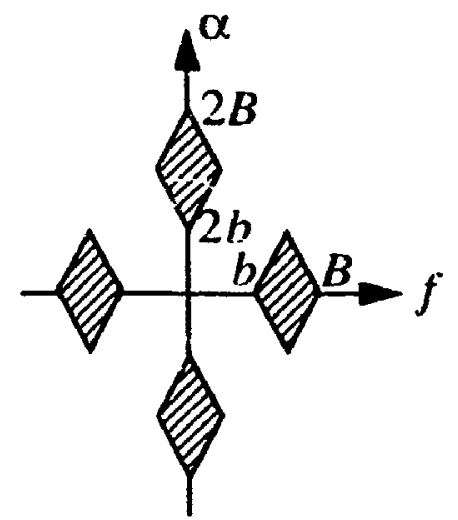

(b)

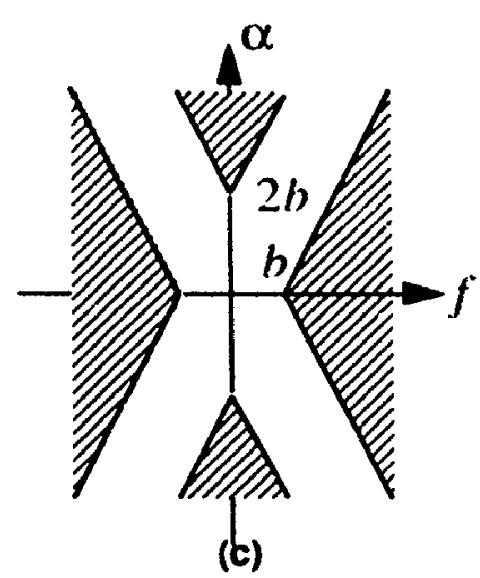

(c)

Figure 2.2 Support area (shaded) fer (a) Lowpass CRP (b) Bandpass CRP (c) Highpass CRP.

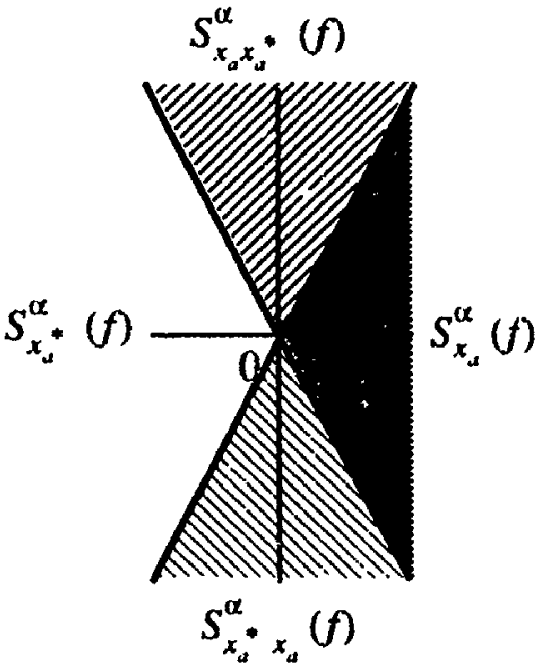

Figure 2.3 Support area for the analytic siznai. 


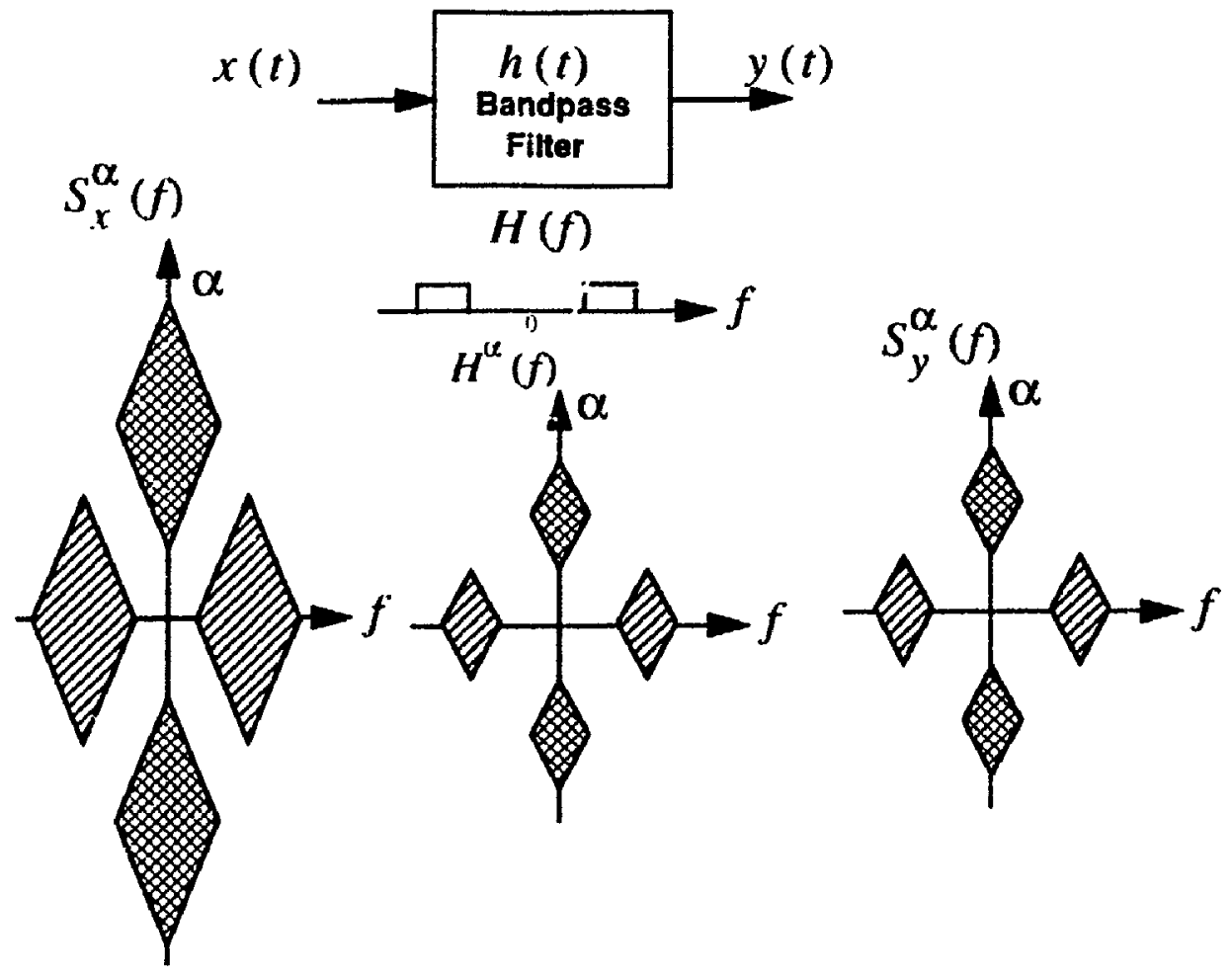

Figure 2.4 Filtering a CRP by an ideal bandpass filter.
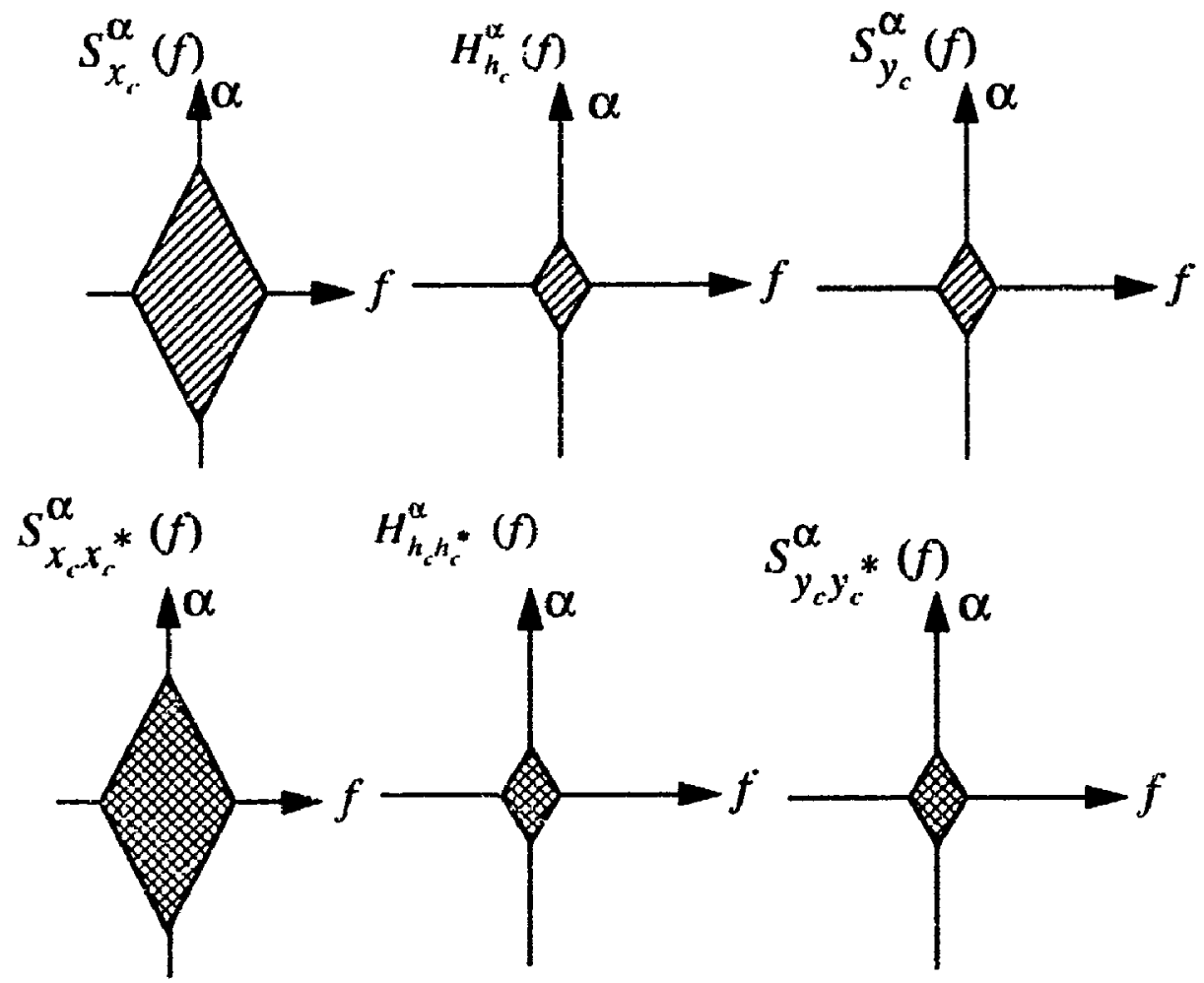

Figure 2.5 The Equivalent Baseband (EB) of the figure 2.4. 


$$
H_{h}^{\alpha}(f)=H\left(f+\frac{\alpha}{2}\right) H^{*}\left(f-\frac{\alpha}{2}\right)
$$

and substituting it in (2.33) yields :

$$
S_{v}^{\alpha}(f)=S_{x}^{\alpha}(f) H_{h}^{\alpha}(f)
$$

We refer to $H_{h}^{\alpha}(f)$ as the cyclic filter. The EB of the cyclic filter is defined based on the $\mathrm{EB}$ of the filter by the following equation :

$$
\begin{gathered}
H_{h_{\iota}}^{\alpha}(f)=H_{r}\left(f+\frac{\alpha}{2}\right) H_{c}^{*}\left(f-\frac{\alpha}{2}\right) \\
H_{h_{c} h_{c}^{*}}^{\alpha}(f)=H_{c}\left(f+\frac{\alpha}{2}\right) H_{c}\left(-f+\frac{\alpha}{2}\right)
\end{gathered}
$$

Were $H_{c}(f)$ is the fourier transform of the EB of the filter. Then, the CSD and CCSD of the EB of the output signal $y(t)$ can be found from the EB of the input signal $x(t)$ according to :

$$
\begin{gathered}
S_{y_{c}}^{\alpha}(f)=S_{x_{c}}^{\alpha}(f) H_{h_{c}}^{\alpha}(f) \\
S_{y_{c} y_{c}^{*}}^{\alpha}(f)=S_{x_{c} x_{c}^{*}}^{\alpha}(f) H_{h_{c} h_{c}^{*}}^{\alpha}(f)
\end{gathered}
$$

Figure 2.5 shiws the EB filtering of Figure 2.4.

\subsubsection{Time Variant Filtering}

A time variant filtering of CRP $x(t)$ can be expressed by the convolution equation as :

$$
y(t)=\int_{-\infty}^{\infty} h(t, l) x(u) d u
$$

If $h(t, u)$ is periodic (or almost periodi: vit' period $T_{h}$, it can be expressed by its Fourier series as follows:

$$
\cdot(t, u)=\sum_{p} \cdot(t-u) e^{\prime 2 \pi \mu u}
$$

Substituting (2.39) in (2.38) yields :

$$
\begin{gathered}
y(t)=\sum_{\mu} \int_{-\infty}^{\infty} g_{\mu}(t-u) x(u) e^{j 2 \pi \mu u} d u \\
=\sum_{\mu} g_{\mu}(t) \otimes x_{\mu}(t)
\end{gathered}
$$


where $\otimes$ denotes convolution and $x_{\mu}(u)=x_{c}(u) e^{j 2 \pi \mu u}$ is the frequency-shifted version of $x_{r}(t)$.The CSD of the output signal can be found according to : $[10,87,88,89]$

$$
S_{y}^{\alpha}(f)=\sum_{\lambda, \mu} G_{\mu}\left(f+\frac{\alpha}{2}\right) S_{x}^{\alpha+\lambda-\mu}\left(f-\frac{\mu+\lambda}{2}\right) G_{\lambda}\left(f-\frac{\alpha}{2}\right)^{*}
$$

where $G_{\mu}(f)$ is the Fourier transform of the $g_{\mu}(t)$.

Equation (2.41) shows that if the CSD of $x(t)$ is uniquely zero for $\alpha \neq 0$, the CSD of $y(t)$ is not uniquely zero for $\alpha \neq 0$. Thus, if a stationary random process passes through a periodic filter, the output will be a CRP with the periodicity of the filter.

Generally, the EB of (2.38) is given according to the following equation called linear-conjugate-linear (LCL) filtering : $[10,11,12,32,37]$

$$
\begin{gathered}
y_{c}(t)=\sum_{\mu} \int_{-\infty}^{\infty} a_{\mu}(t-u) x_{r}(u) e^{j 2 \pi \mu u} d u+\sum_{\beta} \int_{-\infty}^{\infty} b_{\beta}(t-u) x_{c}^{*}(u) e^{j 2 \pi \beta u} d u \\
=\sum_{\mu} a_{11}(t) \otimes x_{\mu}(t)+\sum_{\mu} b_{\mu}(t) \otimes x_{-\beta}{ }^{*}(t)
\end{gathered}
$$

where $x_{i}^{*}(u)=x_{c}^{*}(u) e^{j 2 \pi \beta u}$ is the frequency-shi! $d$ version of $x_{c}^{*}(t)$ and the Fourier transforms of $a_{\mu}(t)$ and $b_{\beta}(t)$ are given by :

$$
\begin{gathered}
A_{\mu}(f)=\frac{1}{2} \Gamma\left(f+f_{0}\right) G_{\mu}\left(f+f_{0}\right) \\
B_{\beta}(f)=\frac{1}{2} \Gamma\left(f+f_{0}\right) G_{\mu+2 f_{0}}\left(f+f_{0}\right)
\end{gathered}
$$

\subsubsection{Stationary Representation}

\subsubsection{Real Signal}

A continuous time cyclostationary random process $x(t)$ with period $T$ can be represented by a set of jointly stationary processes $\left\{A_{p}(t)=0, \pm 1, \pm 2, \ldots\right\}$ as follows :

$$
x(t)=\sum_{p=-\infty}^{\infty} A_{p}(t) e^{j 2 \pi p \frac{t}{T}}
$$

where. $A_{p}(t)$ is the $p$ th stationary component, and can be found from $x(t)$ by : $[23,27]$ 


$$
A_{p}(t)=\left[x(t) e^{-j 2 \pi p \frac{t}{T}}\right] \otimes{u_{T}^{\prime}}_{T}(t)
$$

where $w_{T}(t)$ is the ideal lowpass filter with bandwidth $1 / T$. This representation is referred to as the harmonic-series representation (HSR) [26]. Figure 2.6 shows how stationary components of a CRP can be separated. From (2.44) the TDA can be easily calculated as :

$$
R_{x}(t, u)=\sum_{p, q=-\infty}^{\infty} R_{p q}(t-u) e^{j 2 \pi(p t-q u) / T}
$$

where

$$
R_{p q}(t-u)=E\left\{A_{p}(t) A_{q}^{*}(u)\right\}
$$

Therefore $\mathrm{CA}$ can be found from its stationary components as :

$$
R_{x}^{n T}(\tau)=\sum_{p=-\infty}^{\infty} R_{p(p-n)}(\tau) e^{j \pi(2 p-n) \tau / T}
$$

For the AM example, $T=1 / f_{0}$ and the stationary components are :

$$
A_{ \pm 1}(t)=\frac{\sqrt{2}}{2} y(t) e^{ \pm j \phi_{0}}
$$

Using (2.48) yields the following CA for this AM signal :

$$
\begin{gathered}
R_{x}^{0}(\tau)=\frac{1}{2} R_{y}(\tau)\left(e^{j 2 \pi_{\bar{T}}^{\tau}}+e^{-j 2 \pi} \frac{\tau}{T}\right) \\
R_{x}^{ \pm 2 / T}(\tau)=\frac{1}{2} R_{y}(\tau) e^{ \pm j 2 \sigma_{i}}
\end{gathered}
$$

which is the same result as (2.24).

\subsubsection{Analytical Signal and Complex Envelope}

The analytical signal associated with $x(t)$ can be represented by a set of jointly stationary processes $\left\{B_{p}(t):=0, \pm 1, \pm 2 \ldots.\right\}$ as follows :

$$
x_{a}(t)=\sum_{p=-\infty}^{\infty} B_{p}(t) e^{2 \pi p \frac{t}{T}}
$$




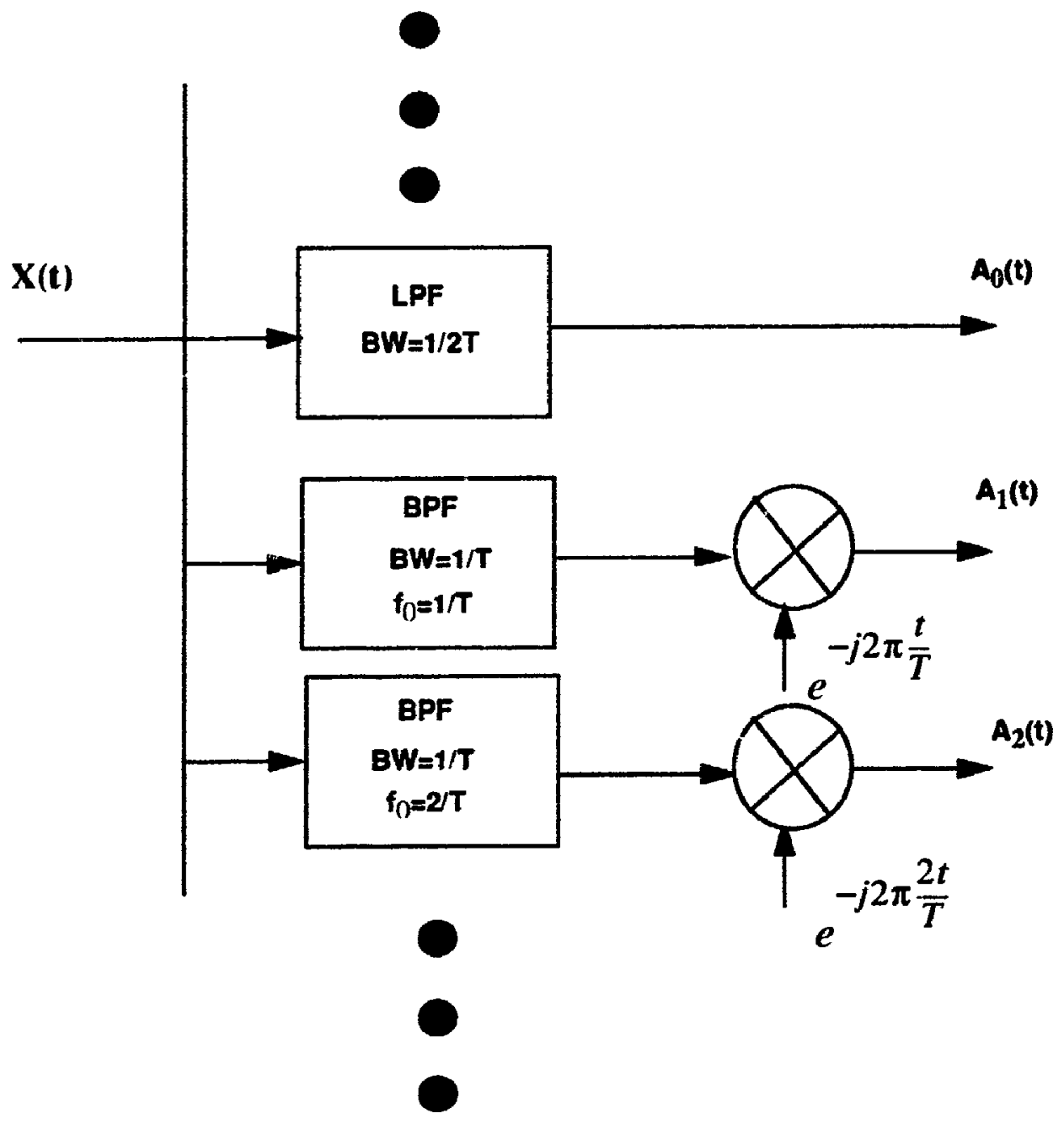

Figure 2.6 The HSR of a CRP. 
The relation between $A_{p}(t)$ and $B_{p}(t)$ can be found by substituting $(2.44)$ in $(2.15)$ :

$$
\begin{gathered}
x_{a}(t)=\int \gamma(u) x(t-u) d u=\int \gamma(u) \sum_{p=-\infty}^{\infty} A_{p}(t-u) e^{j 2 \pi p \frac{t-u}{T}} d u \\
=\sum_{p=-\infty}^{\infty}\left[\int A_{p}(t-u) \gamma(u) e^{-j 2 \pi p \frac{u}{T}} d u\right] e^{j 2 \pi p \frac{t}{T}} \\
B_{p}(t)=\int A_{p}(t-u) \gamma(u) e^{-j 2 \pi p \frac{u}{T}} d u=A_{p}(t) \otimes \gamma_{-p}(t)
\end{gathered}
$$

The stationary representation of the complex envelope of $x(t)$ relative to $f_{0}$ can be found by substituting $(2.18)$ in $(2.51)$ :

$$
\begin{gathered}
x_{c}(t)=x_{a}(t) e^{-j 2 \pi f_{0} t}=\sum_{p=-\infty}^{\infty}\left[B_{p}(t) e^{-j 2 \pi f_{0} t} \mid e^{j 2 \pi p \frac{t}{T}}\right. \\
x_{c}(t)=\sum_{p=-\infty}^{\infty} C_{p}(t) e^{j 2 \pi p \frac{t}{T}} \quad \text { where } \quad C_{p}(t)=B_{p}(t) e^{-j 2 \pi f_{1} t}
\end{gathered}
$$

In the example 2.2.3.2 the stationary components of the analytic and the envelope signals are :

$$
\begin{gathered}
B_{1}(t)=\sqrt{2} y(t) e^{j \varphi_{0}} \\
C_{1}(t)=\sqrt{2} y(t) e^{-j 2 \pi f_{1} s} e^{j \varphi_{0}}
\end{gathered}
$$

\subsection{Discrete Time CRPs}

A discrete random process can be assumed to be a sampled version of a continuous time random process. If a continuous time CRP (say $x(t)$ ) with periodicity $T$ is sampled at every $T_{s}$, the result will be a discrete time CRP (say $\tilde{x}(n)=x\left(n T_{s}\right)$ ) with periodicity $\left\lceil T / T_{s}\right\rceil$ (where $\lceil\bullet\rceil$ is the integer value of its argument). Since $\tilde{x}(n / 2)$ does not exist for odd integer $n$, the definition of the symmetric version of the CA (equation (2.6)) can not be directly extended to the discrete time CRP. Therefore, the CA for the discrete time CRP $\tilde{x}(n)$ is defined by :

$$
R_{\dot{x}}^{\alpha}(k)=\lim _{N \rightarrow \infty} \frac{1}{2 N+1} \sum_{-N}^{N} R_{\bar{x}}(n+k, n) e^{-\jmath 2 \pi \alpha(n+k / 2) T_{s}}
$$


the CSD of a discrete time CRP is detined by the Fourier transform of its CA,

$$
S_{\bar{x}}^{\alpha}(f)=\sum_{k=-\infty}^{\infty} R_{\bar{x}}^{\alpha}(k) e^{-j 2 \pi k T f}
$$

Applying the following equation (called the synchronized averaging identity [27]) to the definition (2.56) and using the fact that $\bar{x}(n)=x\left(n T_{s}\right)$,

$$
\lim _{N \rightarrow \infty} \frac{1}{2 N+1} \sum_{-N}^{N} R_{x}\left(t+n T_{s}, u+n T_{s}\right) \equiv \sum_{m=-\infty}^{\infty} \lim _{z \rightarrow \infty} \frac{1}{z} \int_{-z / 2}^{z / 2} R_{x}(t+v, u+v) e^{-\jmath 2 \pi m v / T} d v
$$

yields the following relationship between the CA of a continuous time CRP and the CA of its sampled version.

$$
R_{\dot{x}}^{\alpha}(k)=\sum_{m=-\infty}^{\infty} R_{x}^{\alpha+m / T_{s}}\left(k T_{s}\right) e^{j \pi m k}
$$

Taking the Fourier transform of the above equation yields :

$$
S_{\bar{x}}^{\alpha}(f)=\frac{1}{T_{s}} \sum_{n, m=-\infty}^{\infty} S_{x}^{\alpha+m / T_{s}}\left(f-\frac{m}{2 T_{s}}-\frac{n}{T_{s}}\right)
$$

The above equation shows that $S_{\bar{x}}^{\alpha}(f)$ exhibits the following periodicity properties :

$$
S_{i}^{\alpha}(f)=S_{\dot{x}}^{\alpha}\left(f+\frac{1}{T_{s}}\right)=S_{\dot{x}}^{\alpha+2 / T_{s}}(f)=S_{\dot{x}}^{\alpha+1 / T_{s}}\left(f-\frac{1}{2 T_{s}}\right)
$$

This periodicity properties are called aliasing effects in $\alpha$ and $f$ [27]. Due to this aliasing effects, the CSD of $x(t)$ is, in general, not obtainable from the CSD of $\tilde{x}(n)$. However, if $f(n)$ has been sampled at a rate higher than the Nyquist rate, its CSD can be recovered from the CSD $n$. sampled version by : [27]

$$
\begin{array}{cc}
S_{x}^{\alpha}(f)=S_{x}^{\alpha}(f) & |f|<\frac{1}{2}\left[\frac{1}{T_{s}}-|\alpha|\right] \\
S_{x}^{\alpha}(f)=0 & |f| \geq \frac{1}{2}\left[\frac{1}{T_{s}}-|\alpha|\right]
\end{array}
$$

Even though in this case, the CA of $x(n)$ can not be obtained from the CSD of the sampled version by the inverse Fourier transform over the bandwidth. In this case, the inversion formula needed is : 


$$
R_{x}^{\alpha}(f)=\int_{-B+|\alpha| / 2}^{B-|\alpha| / 2} S_{x}^{\alpha}(f) e^{j 2 \pi f t} d f
$$

where $-B$ to $B$ is the bandwidth of $x(t)$. However, if $x(n)$ has been sampled at twice the Nyquist rate, its CA can be recovered from the CSD of sampled version by simply applying the inverse Fourier transform over the bandwidth [27].

\subsubsection{Stationary Representation}

A discrete time CRP $\tilde{x}(n)$ with period $P$ can be represented by a set of jointly stitionary processes $\left\{Z_{k}(m): k=0,1,2, \ldots, P-1\right\}$ as follows : $[27 \mid$

$$
\tilde{x}(n)=\sum_{k=0}^{P-1} Z_{k}\left(\left\lceil\frac{n}{P}\right\rceil\right) \delta\left([n]_{p}-k\right)
$$

where $[n]_{p}$ is the remainder of $n$ divided by $P$ and $Z_{k}(m)$. called $k t h$ stationary compo$n e n t$, can be found from $\tilde{x}(n)$ by:

$$
Z_{k}(m)=\tilde{x}(m P+k)
$$

This representation is referred to as the time-series representation (TSR) [27|. Simply, each member of this representation is a subsequence of $\tilde{x}(n)$ obtained by sampling $\tilde{x}(n)$ once per period. Figure 2.7 shows how the stationary components of a cyclostacionary discrete signal can be provided from the signal. If we are interested only in one set of the stationary components, the switch in Figure 2.7 can be replaced by a sample and hold (Figure 2.8). In this case, the phase of the sampler (initial sampling time) indicates which set of stationary components has been taken.

\subsection{Conclusion}

In this chapter. the cyclostationary properties of a CRP were presented. The basic definitions for continuous and discrete time CRP were reviewed. Two stationary representation for CRP were detailed. Real CRPs and their complex envelopes were studied and it was found that all cyclic spectra of a real signal can be found from its complex envelope cyclic spectra. 


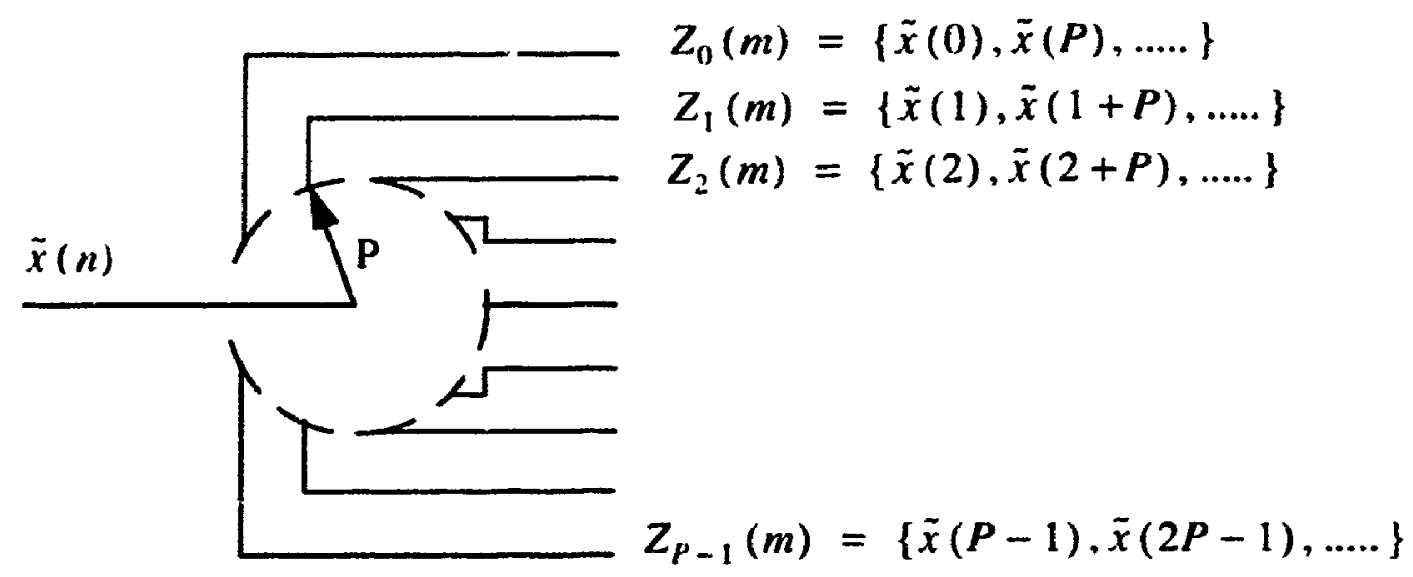

Figure 2.7 Separation of the stationary componer.ts of a CRP.

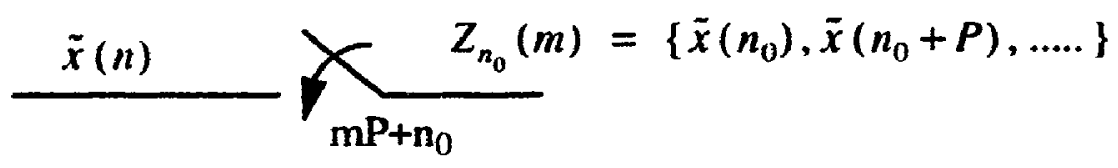

Figure 2.8 Stationary component of interest. 


\section{Chapter 3 \\ Cyclic Spectra of Digitally Modulated Signals}

\subsection{Introduction}

In this Chapter, the cyclostationarity of digitally modulated signals are considered. It is shown that the cyclostationary properties of all digital modulation schemes can be classified into three categories. Binary phase shift keying (BPSK), quartemary PSK (QPSK) and Off-set QPSK (OQPSK) are chosen to represent these ciategories. The spectral redundancy and spectral efficiency of a digital modulated signal are studied and two measurement parameters for measuring the spectral redundancy and spectral efficiency are introduced.

\subsection{Digital Modulation Schemes}

Digital modulation techniques may be classified into either carrier modulation or non-carrier modulation. Both carrier and non-carrier modulation schemes are sub-divided into two techniques, differential modulation and non-differential modulation. In non-differential techniques, data itself is modulated while in differential techniques, a combination of data is modulated. Differentially modulation techniques can be further divided into shifted and non-shifted techniques. In shifted techniques, the signal constellation is shifted by some phase during the modulation while in non-shifted techniques, the constellation remain the same. Figure 3.1 shows an overview of digital modulation techniques.

\subsubsection{Non-Carrier Modulation Schemes}

In non-carrier digital communication systems, a sequence of data is carried by a signal pulse which is shaped to avoid ISI. Generally a pulse modulated signal can be represented by the following formula :

$$
x(t)=\sum_{n=-\infty}^{\infty} \sum_{m=1}^{M} \delta_{m}(n) q_{m}(t-n T)
$$

where $M$ is the alphabet size. $\left\{q_{1}(t), q_{2}(t), \ldots, q_{M}(t)\right\}$ are the pulse shapes, $T$ is the 


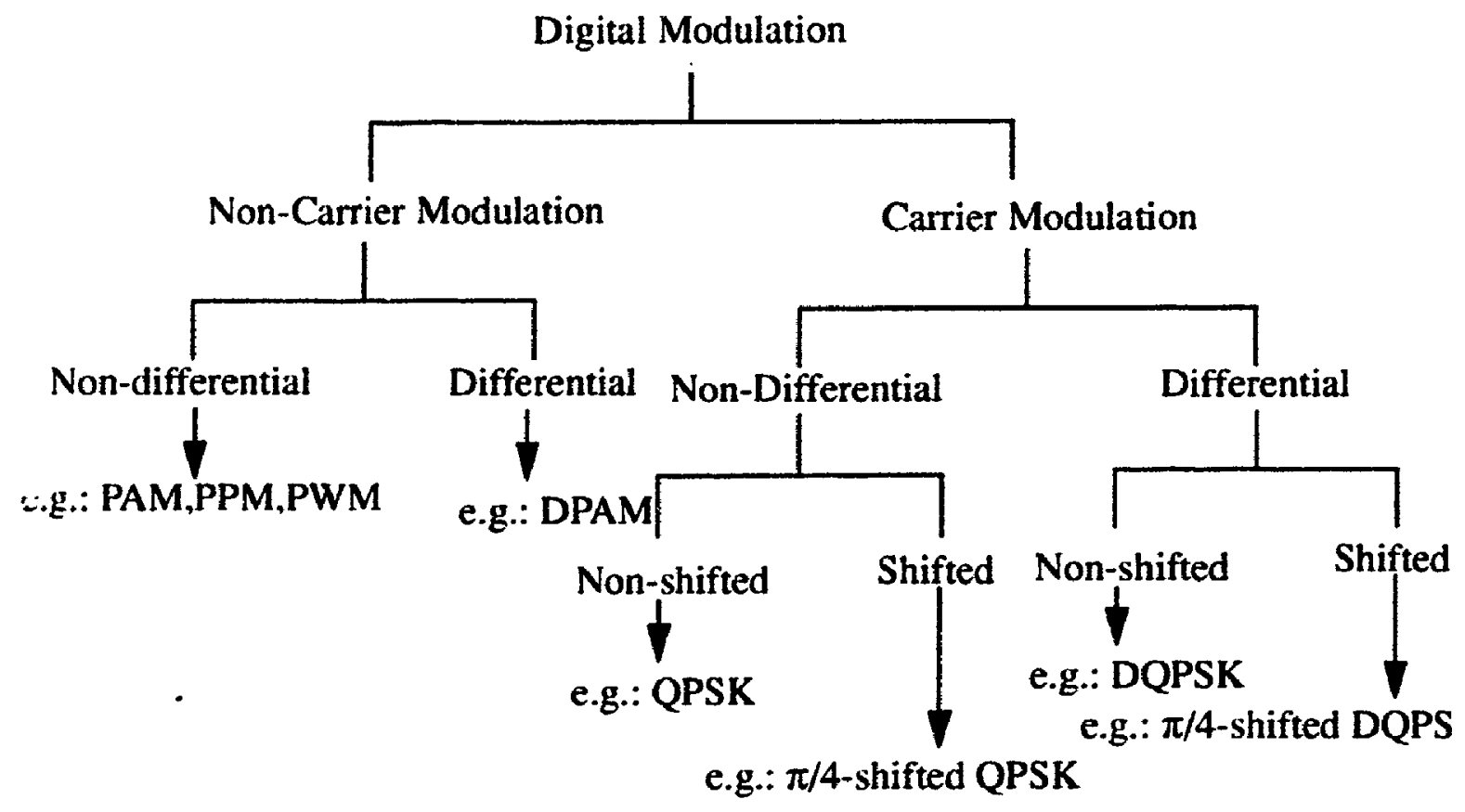

Figure 3.1 Digital modulation schomes. 
symbol rate and $\delta_{m}(n)$ is defined as follows:

$$
\delta_{m}(n)=\left\{\begin{array}{cc}
1 & \text { if } m \text { th symbol is generated in time } n T(d(n)=m \text { th symbol }) \\
0 & \text { otherwise }
\end{array}\right.
$$

The data sequences $\{d(n)\}$ are assumed to be real and uncorrelated in the rest of this thesis. It is shown in [2')] that the CSD of $y(t)$ in this case is :

$$
S_{x}^{\alpha}(f)=\left(\begin{array}{lc}
\frac{1}{M T} \sum_{m=1}^{M} \sigma_{d}^{2} Q_{m}\left(f+\frac{\alpha}{2}\right) Q_{m}^{*}\left(f-\frac{\alpha}{2}\right)-\frac{i}{M^{2} T}\left[\sum_{-m=1}^{M} Q_{m}{ }^{*}\left(f-\frac{\alpha}{2}\right)\right] \\
\cdot\left[\sum_{m=1}^{M} Q_{m}^{*}\left(f-\frac{\alpha}{2}\right)\right]\left[1-\frac{1}{T} \sum_{n=-\infty}^{\infty} \delta\left(f+\frac{\alpha}{2}-\frac{n}{T}\right)\right], & \alpha=\frac{k}{T} \\
0 & \alpha \neq \frac{k}{T}
\end{array}\right.
$$

Equation (3.3) indicates that the CSD of the signal can be nonzero only at cyclic frequencies equal to multiple of the symbol rate. As an example of non-carrier modulated signals, binary pulse amplitude modulated signals is detailed.

\subsubsection{Binary Pulse Amplitude Modulation (Binary PAM)}

In Binary PAM signal

$$
q_{m}(t)=\left(\begin{array}{cc}
q(t) & m=1 \\
-q(t) & m=2
\end{array}\right.
$$

therefore, a PAM signal can be represented by the following equation :

$$
x(t)=\sum_{n=-\infty}^{\infty} d(n) q\left(t-n T-t_{0}\right)
$$

where $\{d(n)\}$ takes values $\pm 1, q(t)$ is the pulse shape, $t_{1}$ is the initial time of $q(t)$ and $T$ is the symbol time. In this case (3.3) is reduced to the following equation :

$$
\begin{gathered}
S_{x}^{\alpha}(f)=\frac{\sigma_{d}^{2}}{T} Q\left(f+\frac{\alpha}{2}\right) Q^{*}\left(f-\frac{\alpha}{2}\right) e^{-j 2 \pi u t_{t}} \delta\left(\alpha-\frac{k}{T}\right) \\
=\frac{\sigma_{d}^{2}}{T} H_{4}^{\alpha}(f) e^{-j 2 \pi \alpha \ell_{1}} \delta\left(\alpha-\frac{k}{T}\right)
\end{gathered}
$$


where $Q(f)$ is the Fourier transform of $q(t), \sigma_{d_{m}}^{2}$ is the variance of the data and $k$ is an integer number. As it is apparent from (3.6), the CSD of $x(t)$ is not equal to zero for $\alpha=m / T$ iff $H_{q}^{m / l}(f) \neq 0$. Ori the other hand, $H_{q}^{m / \tau}(f) \neq 0$ iff the excess bandwidth of $q(t)$ is greater than $(m-1) 100$ percent [28]. Therefore for having a BPAM signal with nonzero CSD at $\alpha=m / T$, a pulse shape with an excess bandwidth larger than $(m-1) 100$ percent has to be used. For example, to have a CSD with cyclic spectrum $1 / T$, the excess bandwidth has to be greater than 0 . Therefore, PAM with zero excess bandwidth pulse shape is stationary.

\subsubsection{Carrier Modulation}

A carrier modulated signal is either a bandpass version of a non-carrier modulated signal or else it is a frequency modulated (e.g. FSK). The frequency modulation techniques are not considered in this thesis. However, it is shown in [29] that these modulation schemes also generate nonzero CSD at multiple of the symbol rate.

Assuming binary data, a general form of linear carrier modulation schemes can be expressed with the following equation :

$$
\begin{aligned}
x(t)= & \sum_{-\infty}^{\infty} d_{r}(n) q\left(t-n T-t_{r}\right) \cos \left(2 \pi f_{0} t+\phi_{r}\right)- \\
& \sum_{-\infty}^{\infty} d_{i}(n) p\left(t-n T-t_{i}\right) \sin \left(2 \pi f_{0} t+\phi_{i}\right)
\end{aligned}
$$

where $p(t)$ and $q(t)$ are the pulse shapes of the inphase and quadrature components, respectively. The CSD and CCSD of the complex envelope of the modulated signal relative to the frequency $f_{1}$ is given by the following equation :

$$
\begin{aligned}
& S_{x_{i}}^{\alpha}(f)=\left(\frac{\sigma_{d_{r}}^{2}}{T} H_{q}^{\alpha}(f-\Delta f) e^{-j 2 \pi \alpha t_{t}}+\frac{\sigma_{d_{1}}^{2}}{T} H_{p}^{\alpha}(f-\Delta f) e^{-j 2 \pi \alpha t_{i}}\right) \delta\left(\alpha-\frac{k}{T}\right) \\
& S_{x_{c_{c} x_{c}^{*}}^{\alpha}}^{\alpha}(f)=\left(\frac{\sigma_{d}^{2}}{T} H_{4 \dot{q}^{*}}^{\alpha}(f) e^{-j\left(2 \pi\left(\alpha-2 \Delta \pi t_{r}-2 \phi_{r}\right)\right.}-\right. \\
& \left.\frac{\sigma_{d}^{2}}{T} H_{p p^{*}}^{\alpha}(f) e^{-\jmath\left(2 \pi(\alpha-2 \Delta) t_{1}-2 \phi_{1}\right)}\right) \delta\left(\alpha-2 \Delta f+\frac{k}{T}\right)
\end{aligned}
$$

where $\Delta f=f_{1}-f_{1}$ and is referred to as the complex carrier. This class of signals can be divided into many subgroups depending upon the relationship between $p(t), q(t), t_{i}, t_{r}, \phi_{r}$ $\phi_{i} \sigma_{d}^{2}$ and $\sigma_{d i}^{2}$. However all these subgroups can be categorized into three categories. In 
the first category, the CSD can be non-zero at cyclic frequencies equal to plus and minus the harmonics of the symbol rate and the CCSD can be non-zero at cyclic freyuencies equal to twice the complex carrier plus and minus the harmonics of the symbol rate. BPSK is an example of this category. In the second category, the CSD can be nonzero only at cyclic frequencies equal to plus and minus the even harmonics of the symbol rate while the CCSD can be nonzero at cyclic frequencies equal to twice the complex envelope carrier plus and minus the odd harmonics of the symbol rate. OQPSK is an example of these signals. In the third category, the CSD can be non-zero at cyclic frequencies equal to plus and minus the harmonics of the symbol rate while the CCSD is uniquely zero. QPSK is an example of these signals. BPSK, OQPSK and QPSK are considered in details to illustrate these categories.

\subsubsection{BPSK}

A BPSK signal can be considered as a binary PAM modulated by an AM modulator :

$$
x(t)=\sum_{-\infty}^{\infty} d(n) q\left(t-n T-t_{0}\right) \cos \left(2 \pi f_{0} t+\phi_{0}\right)
$$

The CSD and CCSD of the complex envelope of the BPSK signal relative to the frequency $f_{1}$ is :

$$
\begin{gathered}
S_{x_{c}}^{\alpha}(f)=\frac{\sigma_{d}^{2}}{T} H_{q}^{\alpha}(f-\Delta f) e^{-j 2 \pi \alpha t_{0}} \delta\left(\alpha-\frac{k}{T}\right) \\
S_{x_{c} x_{c}^{*}}^{\alpha}(f)=\frac{\sigma_{d}^{2}}{T} H_{q q^{*}}^{\alpha}(f) e^{-j\left(2 \pi\left(\alpha-2 \Delta \rho t_{0}-2 \phi_{0}\right)\right.} \delta\left(\alpha-2 \Delta f+\frac{k}{T}\right)
\end{gathered}
$$

It is apparent from (3.10) that the CSD is nonzero for $\alpha$ equals to plus and minus the symbol rate while the CCSD includes $\alpha$ equals to twice the complex envei spe carrier plus and minus the symbol rate and its harmonics.

\subsubsection{QPSK}

In balanced QPSK signals the following relationship exist :

$$
P(t)=q(t), \quad t_{i}=t_{r}, \quad \phi_{i}=\phi_{r}, \quad \sigma_{d}^{2}=\sigma_{d,}^{2}
$$

In this case, (3.8) is simplified to : 


$$
\begin{gathered}
S_{x_{r}}^{\alpha}(f)=\left(\frac{2 \sigma_{d}^{2}}{T} H_{q}^{\alpha}(f-\Delta f) e^{-\jmath 2 \pi \alpha t_{r}}\right) \delta\left(\alpha-\frac{k}{T}\right) \\
S_{x_{r} x_{r}^{*}}^{\alpha}(f) \equiv 0
\end{gathered}
$$

It is apparent from (3.12) that the CCSD of a balanced QPSK does not contain any cyclic spectrum related to the carrier frequency.

\subsubsection{OQPSK}

In OQPSK, the time delays $t_{i}$ and $t_{r}$ are off from each other by half the symbol rate. The following relationship exists in this case :

$$
P(t)=q(t), \quad t_{i}=t_{r}+\frac{T}{2}, \quad \phi_{i}=\phi_{r}, \quad \sigma_{d_{1}}^{2}=\sigma_{d_{r}}^{2}
$$

Therefore (3.8) is simplified to :

$$
\begin{aligned}
& S_{k_{r}}^{\alpha}(f)=\left(\frac{\sigma_{d_{r}}^{2}\left(1+e^{-j \pi \alpha k T}\right)}{T} H_{q}^{\alpha}(f-\Delta f) e^{-j 2 \pi \alpha t_{r}}\right) \delta\left(\alpha-\frac{k}{T}\right) \\
& =\left(\frac{2 \sigma_{d_{r}}^{2}}{T} H_{q}^{\alpha}(f-\Delta f) e^{-j 2 \pi \alpha t_{r}}\right) \delta\left(\alpha-\frac{2 k}{T}\right) \\
& S_{x_{,}, x_{r}}^{\alpha}(f)=\left(\frac{\sigma_{d_{r}}^{2}\left(1-e^{-j \pi \alpha k T}\right)}{T} H_{q q^{*}}^{\alpha}(f) e^{-j\left(2 \pi\left(\alpha-2 \Delta \Omega_{r}-2 \phi_{r}\right)\right.}\right) \delta\left(\alpha-2 \Delta f+\frac{k}{T}\right) \\
& =\left(\frac{2 \sigma_{d_{r}}^{2}}{T} H_{d q^{*}}^{\alpha}(f) e^{-j\left(2 \pi\left(\alpha-2 \Delta f t_{r}-2 \phi_{r}\right)\right.}\right) \delta\left(\alpha-2 \Delta f+\frac{2 k+1}{T}\right)
\end{aligned}
$$

The above equations show that the CSD of OQPSK contains the even harmonics while the CCSD contains twice the complex carrier plus and minus the odd harmonics of the symbol rate.

\subsubsection{Differentially Modulation Techniques}

\subsubsection{Non-Shifted}

A non-shifted differentially modulated signal can be viewed as a non-differentially modulated signal with a combinational data sequence. For example, differential BPSK (DBPSK) signal is expressed as : 


$$
x(t)=\sum_{-\infty}^{\infty} d(n) q\left(t-n T-t_{0}\right) \cos \left(2 \pi f_{0} t+\phi_{0}\right)
$$

where $d(n)$ is a data sequence $\{-1.1\}$ created from the following binary sequence $\{0,1\}$ : [51]

$$
c(n)=c(n-1) b(n) \oplus \overline{c(n-1)} \overline{b(n)}
$$

where $\oplus$ is an XOR function and $b(n)$ is the binary data sequence $(0,1)$. The CSD and CCSD of DBPSK can be obtained from (3.10) where $\sigma_{d}^{2}=1$. Therefore DBPSK can be categorized in the same category with BPSK, and this is true for other differentially modulation schemes. For example differential QPSK (DQPSK) is categorized with QPSK. and DOQPSK is categorized with OQPSK.

\subsubsection{Shifted Schemes}

$\pi / 4-s h i f t e d-D Q P S K$ is considered as an example of this family of modulations. $\pi / 4$-shifted-DQPSK can be represented by the following equation :

$$
\begin{aligned}
x(t)= & \sum_{-\infty}^{\infty} \cos \left(\theta_{n}\right) q\left(t-n T-t_{0}\right) \cos \left(2 \pi f_{0} t+\phi_{0}\right)- \\
& \sum_{-\infty}^{\infty} \sin \left(\theta_{n}\right) q\left(t-n T-t_{0}\right) \sin \left(2 \pi f_{0} t+\phi_{0}\right)
\end{aligned}
$$

where $\theta_{n}=\theta_{n-1}+\Delta \theta_{n} \cdot \Delta \theta_{n}$ is the $\pi / 4$ shift to the signal constellation and it is calculated according to the Table 3.1 [14]. In this table, $b_{1 n}$ and $b_{2 n}$ are two transmitted bits at $t=n T+t_{0}$. Assuming $c_{r}(n)=\cos \left(\theta_{n}\right)$ and $c_{i}(n)=\sin \left(\theta_{n}\right)$, equation (3.17) is similar to (3.7). It is easy to show that in this case, the CSD of the signal can be found by the following equation :

Table 3.1 $\pi / 4$-shifting of constellation.

\begin{tabular}{|c|c|c|}
\hline$b_{2 n}$ & $b_{1 n}$ & $\Delta \theta_{n}$ \\
\hline 0 & 0 & $\pi / 4$ \\
\hline 0 & 1 & $3 \pi / 4$ \\
\hline 1 & 1 & $5 \pi / 4$ \\
\hline 1 & 0 & $7 \pi / 4$ \\
\hline
\end{tabular}




$$
S_{x}^{\alpha}(f)=\frac{2}{T} H_{q}^{\alpha}(f-\Delta f) e^{-j 2 \pi \alpha t_{0}} S_{c_{r}}\left(f+\frac{\alpha}{2}\right) \delta\left(\alpha-\frac{k}{T}\right)
$$

and the CCSD of the signal is uniquely zero. Therefore $\pi / 4$-shifted-DQPSK can be categorized with QPSK.

\subsection{Categorizing The Digital Modulated Signals}

It is concluded from previous sections that the complex envelope of all digitally modulated signals can be categorized into three category :

Category 1: Signals which have nonzero CSD at harmonics of the symbol rate and nonzero CCSD at twice the complex envelop carrier plus and minus harmonics of the symbol rate.

\section{Examples: BPSK, DBPSK.}

Category 2: Signals which have nonzero CSD at even harmonics of the symbol rate and nonzero CCSD at twice the complex envelop carrier plus and minus odd harmonics of the symbol rate.

Examples: OQPSK, DOPQSK, $\pi / 4$-shifted OQPSK, $\pi / 4$-shifted DOQPSK.

3. Signals which have nonzero CSD at harmonics of the symbol rate and uniquely zero CCSD.

Examples: PAM, PWM, PPM, FSK, QPSK, DQPSK, $\pi / 4$-shifted DQPSK, $\pi / 4$ shifted QPSK.

Therefore studying the cyclostationary properties of BPSK, QPSK, and OQPSK is enough to cover all digital modulation schemes.

In the rest of this chapter, the spectral redundancy and spectral efficiency of these three category are considered.

\subsection{Spectral Redundancy}

Table 1 summarizes the relationships between the cyclic frequencies with nonzero CSD (or CCSD) and signal excess bandwidth. It is clear from the table that both balanced QPSK and OQPSK signals are cyclostationary iff their excess bandwidths are greater than 
Table 3.2 Relations between cyclic frequencies with nonzero CSD (or CCSD) and signal excess bandwidth.

\begin{tabular}{|c|c|c|}
\hline Excess bandwidth $(\varepsilon)$ & The nonzero CSD (or CCSD) cyclic frequencios & $\begin{array}{l}\text { Modulation } \\
\text { scheme }\end{array}$ \\
\hline \multirow{3}{*}{$\begin{array}{c}100(k-1) \%<\varepsilon \leq 100(k) \% \\
k=1.2 \ldots\end{array}$} & $\left\{0 . \pm \frac{1}{T}, \pm \frac{2}{T}, \ldots . \pm \frac{k}{T}\right\},\left\{2 f .2 f \pm \frac{1}{T}, 2 f \pm \frac{2}{T}, \ldots .2 f \pm \frac{k}{T}\right\}$ & BIPSK \\
\hline & $\left\{\begin{array}{c}\left\{0 . \pm \frac{2}{T}, \pm \frac{4}{T}, \ldots . \pm \frac{k}{T}\right\},\left\{2 f \pm \frac{1}{T}, 2 f \pm \frac{3}{T}, \ldots, 2 f \pm \frac{k-1}{T}\right\} \\
\text { even k } \\
\left\{0 . \pm \frac{2}{T}, \pm \frac{4}{T}, \ldots . \pm \frac{k}{T}\right\},\left\{2 f \pm \frac{1}{T}, 2 f \pm \frac{3}{T}, \ldots, 2 f \pm \frac{k-1}{T}\right\} \\
\text { odd } k\end{array}\right.$ & (XUPSK \\
\hline & 10. $\left.\pm \frac{1}{T}, \pm \frac{2}{T}, \ldots . \pm \frac{k}{T}\right\}$ & QHSK \\
\hline
\end{tabular}


zero. Nevertheless, BPSK signals have a nonzero CCSD at twice the carrier frequency regardless of their excess bandwidths. However, for all digital signals, the greater is the excess bandwidth, the more the cyclic frequencies with nonzero CSD (or CCSD) exist. On the other hand, the signal excess bandwidth represents the spectral redundancy of the signal. Therefore nonzero CSD and CCSD of the signal can be translated to spectral redundancy. A redundancy factor can be defined as :

$$
r_{x}=\left(\frac{\sum_{n} \int_{-\infty}^{\infty}\left|S_{x}^{\alpha_{n}}(f)\right| d f+\sum_{n} \int\left|S_{-\infty}^{\beta_{n}}(f)\right| d f}{\int_{-\infty}^{\infty}\left|S_{x}^{0}(f)\right| d f}-1\right) 100 \%
$$

As it is apparent from (3.19), the presence of any cyclic frequency with nonzero CSD (or CCSD) indicates a spectral redundancy of the signal. The stronger the CSD (or (CSD) is, the more redundant the signal is. As an example, consider a balanced QPSK signal and a BPSK signal. If the excess bandwidth of the signal is zero, the balanced QPSK is a stationary signal and its spectrum has no redundancy.This can be shown by (3.19) as well :

$$
r_{x}=\left(\frac{\int_{-\infty}^{\infty}\left|S_{x}^{0}(f)\right| d f}{\int_{-\infty}^{\infty}\left|s_{x}^{0}(f)\right| d f}-1\right) 100 \%=0 \%
$$

However, a BPSK signal with zero excess bandwidth is redundant with a factor 100 percent :

$$
\begin{aligned}
& r_{x_{B}}=\left(\frac{\int_{-\infty}^{\infty}\left|S_{x}^{0}(f)\right| d f+\int_{-\infty}^{\infty}\left|S_{x x^{*}}^{0}(f)\right| d f}{\int_{-\infty}^{\infty}\left|S_{x}^{0}(f)\right| d f}-1\right) 100 \% \\
& =\left(\frac{\iint_{-\infty}^{\infty}\left|S_{x}^{0}(f)\right| d f+\int_{-\infty}^{\infty}\left|S_{x}^{0}(f)\right| d f}{\int_{-\infty}^{\infty}\left|S_{x}^{0}(f)\right| d f}-1\right) 100 \%=100 \%
\end{aligned}
$$


Generally. a QPSK signal with excess bandwidth $1(x) k \%<\varepsilon=1(k)(k+1) \%$ (where $k$ is an integer number) has a redundancy equals to :

$$
r_{x_{g}}=\left(\frac{\sum_{n=-k}^{k} \int_{-\infty}^{\infty}\left|S_{x}^{n T}(f)\right| d f}{\int_{-\infty}^{\infty}\left|S_{x}^{0}(f)\right| d f}-1\right)\left(00 \%=\left(\frac{2 \sum_{n=1}^{k} \int_{-\infty}^{\infty}\left|S_{x}^{n T}(f)\right| d f}{\int_{-\infty}^{\infty}\left|S_{x}^{0}(f)\right| d f}\right) 1(x) \%\right.
$$

with the same signal parameter, the redundancy of BPSK would be :

$$
\begin{aligned}
r_{x_{B}}= & \left(\frac{\sum_{n=-k}^{k} \int_{-\infty}^{\infty}\left|S_{x}^{n T}(f)\right| d f+\sum_{n=-k-\infty}^{k} \int_{-\infty}^{\infty}\left|S_{x x^{*}}^{2 f+\frac{n}{T}}(f)\right| d f}{\int\left|S_{x}^{0}(f)\right| d f}-1\right) 100 \% \\
& =\left(\begin{array}{c}
4 \sum_{-\infty}^{k} \int\left|S_{x}^{n T}(f)\right| d f \\
1+\frac{\int_{-\infty}^{\infty}\left|S_{x}^{0}(f)\right| d f}{-\infty} \\
=2 r_{x_{Q}}+100 \%
\end{array}\right) 100 \%
\end{aligned}
$$

It can be shown that the spectral redundancies of OQPSK and QPSK signals are equal, (i.e. $r_{x_{o}}=r_{x_{Q}}$ ).

\subsection{Spectral Efficiency}

The ratio of stationary signal bandwidth to the signal bandwidth when both are carrying the same information is defined as the efficiency factor $\mu$ :

$$
\mu=\frac{W_{0}}{W} 100 \%
$$

where $W$ is the signal bandwidth and $W_{0}$ is the stationary signal bandwidth. For example, the efficiency factor of a QPSK (or OQPSK) signal with an excess bandwidth $100 k \%<\varepsilon \leq 100(k+1) \%$ is :

$$
\mu_{Q}=\frac{W_{0}}{(\varepsilon+1) ! W_{0}} 100=\frac{100}{\varepsilon+1} \%
$$


and for BPSK signal is :

$$
\begin{gathered}
\mu_{B}=\frac{W_{0}}{2(\varepsilon+1) W_{0}} 100=\frac{100}{2(\varepsilon+1)} \% \\
=\frac{\mu_{Q}}{2}
\end{gathered}
$$

There is a trade-off between spectral efficiency and spectral redundancy. A signal with high redundancy is less efficient while a signal with high efficiency has lower redundancy. The efficiency and redundancy have some advantages and disadvantages relative to each other. An efticient signal can carry more information per unit time, but it is easier to recover a redundant signal in an environment impaired by interference, noise and fading. Therefore, redundancy (and spectral efficiency) should be chosen to suit the environment.

\subsection{Conclusion}

In this chapter, cyclostationary properties of signals have been considered. It has been shown that all digital modulated signals can be classified into three categories. BPSK, QPSK, OQPSK are representative of these three categories. Furthermore, the spectral redundancy and efficiency of a modulated signal are defined and the trade off between spectral redundancy and spectral efficiency is identified. The higher, spectral redundancy yields better performance over severe channels and high interference environment, while higher spectral efficiency yields higher system capacity. 


\section{Chapter 4 \\ Receiver Design}

\subsection{Introduction}

In this chapter, the optimum receiver structures, in the sense of exploiting the cyclostationarity, for digital communication systems (DCSs) are obtained and analyzed. It is shown that the structure of these receivers depend on (1) symbol rate, (2) channel spacing, (3) modulation scheme, (4) number of available antennas. The general structure of such a receiver is built on the basis of two stationary representations of the received cyclostationary signal, HSR and TSR. The structure based on HSR is referred to as FRESH-DFE and the structure based on TSR is referred to as GDFE. In addition to the FRESH-DFE and GDFE, the conventional DFE is analyzed in detail and it is shown that the conventional DFE is optimum only for balanced QPSK and single-symbol rate systems.

\subsection{Digital Communication System (DCS) Model}

The DCS studied in this chapter is a FDMA system operating in a quasi-static channel. Figure 4.1 shows the block diagram of the equivalent baseband model of the DCS. In the figure, the signal of interest (SOI) is indicated by the index zero, $\mathrm{CCl}$ signals are indicated by indices $0 l$ and $\mathrm{ACl}$ signals are indicated by indices either $l$ or $-l$. The negative indices are used for lower $\mathrm{ACl}$ signals and the positive indices are used for upper ACI signals. $M_{c}$ and $M_{a}$ are the number of $\mathrm{CCl}$ and $\mathrm{ACI}$ signals respectively. The scales $g_{l}$ (or $g_{0 l}$ ) represent distance dependent propagation loss. The pulse shaping filters, $q_{l}(t)$ (or $q_{o l}(t)$ ), and the symbol intervals, $T_{l}$ (or $T_{0 l}$ ) are usually equal for all the signals, however, we consider the general case that they can be different. We assume also that (1) all the channels are time-invariant over a time frame, (2) the data sequences, $\left\{d_{l}\right\}$ (or $\left\{d_{01}\right\}$ ), are complex and uncorrelated (3) the noise $n(t)$ is complex white Gaussian, (4) the modulation schemes are in the class of BPSK, OQPSK, and QPSK. Furthermore, some overlapping of $\mathrm{SOI}$ and $\mathrm{ACI}$ spectra is assumed.

The received signal can be expressed by the following equation : 


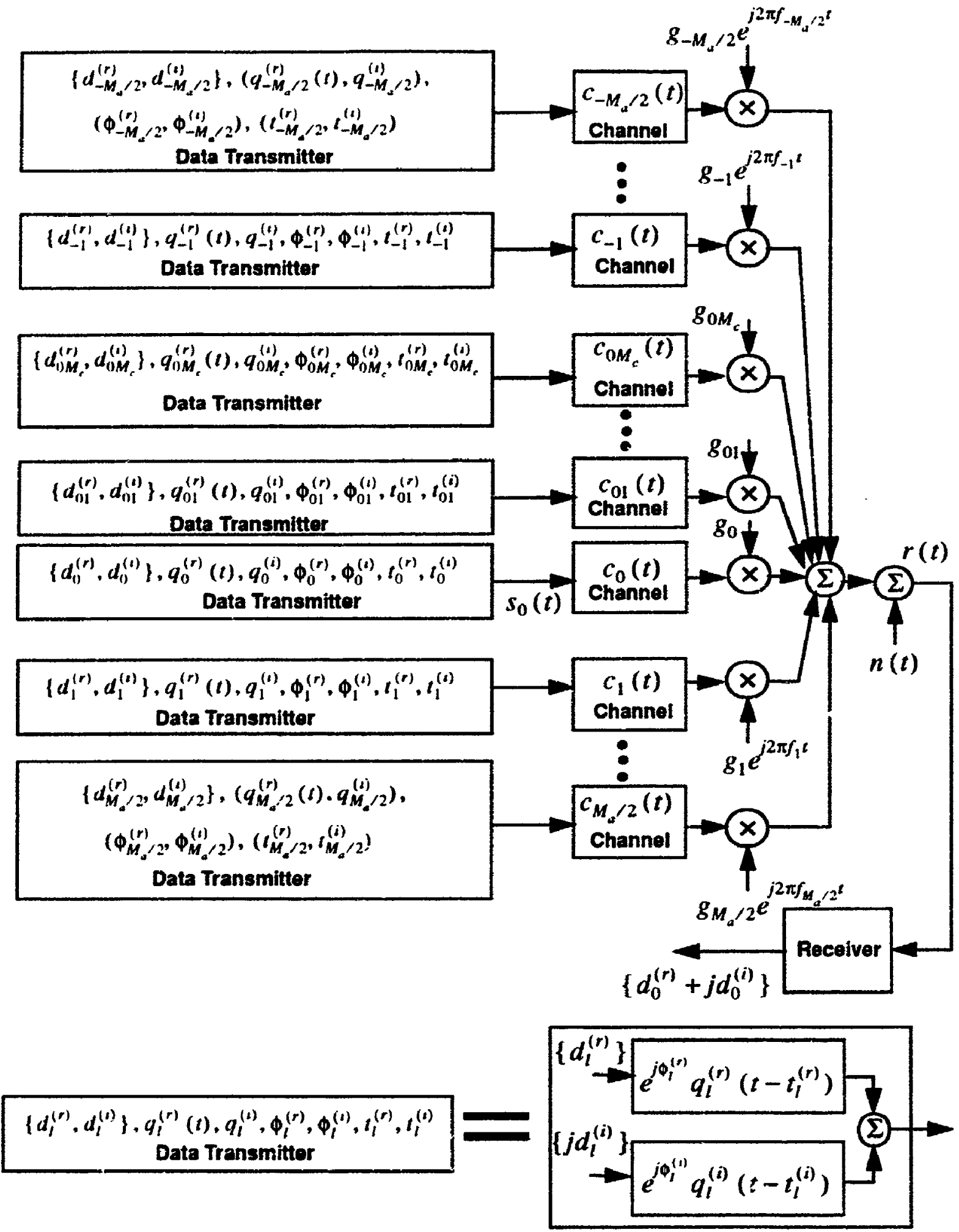

Figure 4.1 The equivalent baseband of a DCS. 


$$
r(t)=s_{0}(t)+s_{c}(t)+s_{a}(t)+n(t)
$$

where $n(t)$ is the additive white Gaussian noise (AWGN), $s_{0}(t), s_{12}(t)$ and $s_{t}(t)$ are the SOI, $\mathrm{ACI}$. and $\mathrm{CCI}$, respectively, and can be represented by the following equations :

$$
\begin{aligned}
& s_{0}(t)=\sum_{k=-\infty}^{\infty} d_{0}^{(r)}(k) p_{0}^{(1)}\left(t-k T_{0}-t_{0}^{(r)}\right) e^{\left(2 \pi \phi_{0}^{(r)}\right.}+ \\
& j d_{0}^{(i)}(k) p_{0}^{(2)}\left(t-k T_{0}-t_{0}^{(i)}\right) e^{j 2 \pi \phi_{0}^{(1)}} \\
& s_{c}(t)=\sum_{l=l}^{M_{c}} \sum_{k=-\infty}^{\infty} d_{0 l}^{(r)}(k) p_{0 l}^{(1)}\left(t-k T_{0 l}-t_{0 l}^{(r)}\right) e^{j 2 \pi \phi_{l i l}^{(n)}}+ \\
& j d_{0 l}^{(i)}(k) p_{0 l}^{(2)}\left(t-k T_{0 l}-t_{0 l}^{(i)}\right) e^{j 2 \pi \phi_{0 l}^{(1)}} \\
& s_{a}(t)=\sum_{\substack{l=-M_{\alpha} / 2 k \\
l \neq 0}}^{M_{\alpha} / 2} \sum_{k=-\infty}^{\infty}\left(d_{l}^{(r)}(k) p_{l}^{(1)}\left(t-k T_{l}-t_{l}^{(r)}\right) e^{j 2 \pi \varphi_{l}^{(n)}}+\right. \\
& \left.j d_{i}^{(i)}(k) p_{l}^{(2)}\left(t-k T_{l}-t_{i}^{(i)}\right) e^{j 2 \pi \phi_{l}^{(1)}}\right) e^{j 2 \pi f_{l} t}
\end{aligned}
$$

where $p_{0 l}^{(1)}(t), p_{0 l}^{(2)}(t), p_{l}^{(1)}(t), p_{l}^{(2)}(t), p_{0}^{(1)}(t)$, and $p_{0}^{(2)}(t)$ are detined by :

$$
\begin{aligned}
& p_{0 l}^{(1)}(t)=g_{0 l} q_{0 l}^{(r)}(t) \otimes c_{0 l}(t) \\
& p_{0 l}^{(2)}(t)=g_{0 l} q_{0 l}^{(i)}(t) \otimes c_{0 l}(t) \\
& p_{l}^{(1)}(t)=g_{l} q_{l}^{(r)}(t) \otimes c_{l}(t) \\
& p_{l}^{(2)}(t)=g_{1} q_{l}^{(i)}(t) \otimes c_{l}(t) \\
& p_{0}^{(1)}(t)=g_{0} q_{0}^{(r)}(t) \otimes c_{0}(t) \\
& p_{0}^{(2)}(t)=g_{0} q_{0}^{(i)}(t) \otimes c_{0}(t)
\end{aligned}
$$

\subsubsection{The Cyclic Spectral Density (CSD) and Conjugate CSD (CCSD) of The Received Signal}

Due to the assumption of uncorrelated data sequences, the CSD and CCSD of the received signal can be found by the following equations :

$$
\begin{aligned}
& S_{r}^{\alpha}(f)=S_{s_{0}}^{\alpha}(f)+S_{s_{c}}^{\alpha}(f)+S_{s_{u}}^{\alpha}(f)+N_{0} \delta(\alpha) \\
& S_{r r^{*}}^{\alpha}(f)=S_{s_{1}, s_{0}^{*}}^{\alpha}(f)+S_{s_{t} s_{c}^{*}}^{\alpha}(f)+S_{s_{u} s_{s}^{*}}^{\alpha}(f)
\end{aligned}
$$


where $N_{0}$ is the power spectral density (PSD) of the noise and

$$
\begin{aligned}
& S_{s_{i}}^{(x)}(f)=\left(\frac{\sigma_{d_{0}^{(r)}}^{2}}{T_{0}} H_{p_{0}^{(1)}}^{\alpha}(f) e^{-j 2 \pi \alpha t_{0}^{(1)}}+\frac{\sigma_{d_{0}^{(1)}}^{2}}{T_{0}} H_{p_{0}^{(2)}}^{\alpha}(f) e^{-j 2 \pi \alpha t_{0}^{(1)}}\right) \delta\left(\alpha-\frac{k}{T_{0}}\right) \\
& S_{s_{0} s_{0}}^{\alpha} \cdot(f)=\left(\frac{\sigma_{d_{0}^{(n)}}^{2}}{T_{0}} H_{p_{0}^{(1)} p_{0}^{(1) *}}^{\alpha}(f) e^{-j\left(2 \pi \alpha t_{0}^{(r)}-2 \sigma_{0}^{(r)}\right)}-\right. \\
& \left.\frac{\sigma_{d_{0}^{(1)}}^{2}}{T_{0}} H_{p_{01}^{(2)} p_{0}^{(2)}}^{\alpha}(f) e^{-j\left(2 \pi \alpha t_{0}^{(1)}-2 \sigma_{0}^{(1)}\right)}\right) \delta\left(\alpha-\frac{k}{T_{0}}\right) \\
& S_{s c}^{(\alpha)}(f)=\sum_{l=1}^{M_{c}}\left(\frac{\sigma_{d_{0 l}^{(r)}}^{2}}{T_{0 l}} H_{p_{0 l}^{(1)}}^{\alpha}(f) e^{-j 2 \pi \alpha l_{0 l}^{(r)}}+\frac{\sigma_{d_{0 l}^{(1)}}^{2}}{T_{0 l}} H_{P_{0 l}^{(2)}}^{\alpha}(f) e^{-j 2 \pi \alpha t_{0 l}^{(1)}}\right) \delta\left(\alpha-\frac{k}{T_{0 l}}\right) \\
& S_{s_{t} s_{c}^{*}}^{(\alpha)}(f)=\sum_{i=1}^{M_{c}}\left(\frac{\sigma_{d_{0 l}^{(n)}}^{2}}{T_{0 l}} H_{p_{0 l}^{(1)} p_{0 l}^{(i)}}^{\alpha}(f) e^{-j\left(2 \pi \alpha t_{0 i}^{(p)}-2 \sigma_{0 l}^{(r)}\right)}-\right. \\
& \left.\frac{\sigma_{d_{0 l}^{(1)}}^{2}}{T_{0 l}} H_{p_{0 \ell}^{(2)} p_{0 \ell}^{(2) *}}^{\alpha}(f) e^{-j\left(2 \pi \alpha t_{0 I}^{(1)}-2 \phi_{0 \ell}^{(1)}\right)}\right) \delta\left(\alpha-\frac{k}{T_{0 I}}\right) \\
& S_{s_{s}}^{\alpha}(f)=\sum_{\substack{l=-M_{+}^{\prime \prime 2} \\
l \neq 0}}^{M_{\alpha}^{\prime 2}}\left(\frac{\sigma_{d_{l}^{(\prime)}}^{2}}{T_{l}} H_{p_{l}^{(1)}}^{\alpha}\left(f-f_{l}\right) e^{-j 2 \pi \alpha t_{l}^{(\prime)}}+\frac{\sigma_{d_{l}^{(1)}}^{2}}{T_{l}} H_{p_{l}^{(2)}}^{\alpha}\left(f-f_{l}\right) e^{-j 2 \pi \alpha t_{l}^{(1)}}\right) \delta\left(\alpha-\frac{k}{T_{l}}\right)
\end{aligned}
$$

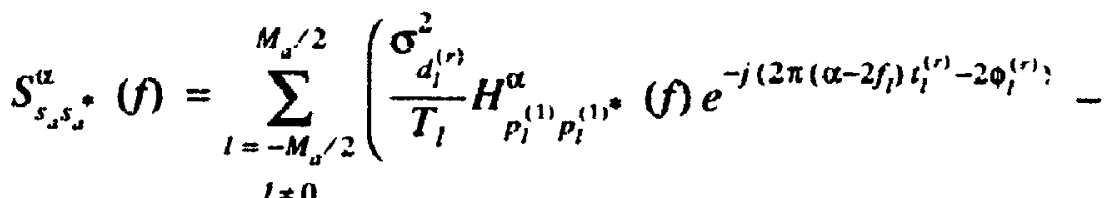

$$
\begin{aligned}
& \left.\frac{\sigma_{d}^{2}}{T_{l}^{(1)}} H_{p_{l}^{(2)} p_{l}^{(2) *}}^{\alpha}(f) e^{-j\left(2 \pi\left(\alpha-2 f_{l}\right) l_{l}^{(1)}-2 \phi_{l}^{(1)}\right)}\right) \delta\left(\alpha-2 f_{l}-\frac{k}{T_{l}}\right)
\end{aligned}
$$

From (4.6), we ste that the noise is only present on the CSD of the received signal and only effective at $\alpha=0$, suggesting that the CCSD of the received signal is noise free for all the cyclic spectra while the CSD of the received signal is noise free on the nonzero cyclic spectra. It should be made clear at this point that, although there is a noise free cyclic spectra, it does not mean that the data sequence can be estimated noise free. This is 
because the CSD or CCSD are the second order statistics of the signal and does not carry the information about data phase.

In the case of balanced BPSK $\left(\sigma_{d^{(1)}}^{2}=(1)\right.$ system. (4.7) to (4.9) are simplitied to the following equations :

$$
\begin{aligned}
& S_{s_{0}}^{\alpha}(f)=\frac{\sigma_{d_{0}^{(n)}}^{2}}{T_{0}} e^{-j 2 \pi \alpha s_{i}^{(n)}} H_{p_{0}^{\prime \prime \prime}}^{\alpha}(f) \delta\left(\alpha-\frac{k}{T_{0}}\right) \\
& S_{s_{0} s_{0}^{*}}^{\alpha}(f)=\frac{\sigma_{d_{0}^{(1)}}^{2}}{T_{0}} H_{p_{0}^{(1)} p_{0}^{(1)}}^{\alpha}(f) e^{-j\left(2 \pi \alpha \delta_{0}^{(1)}-20_{0}^{(n)}\right)} \\
& S_{s_{r}}^{(\alpha)}(f)=\sum_{l=1}^{M_{\varepsilon}} \frac{\sigma^{2}}{T_{0 l}^{(n)}} H_{p_{0 l}^{(1)}}^{(\alpha)}(f) e^{-j 2 \pi \alpha s_{0 l}^{(n)}} \delta\left(\alpha-\frac{k}{T_{0 l}}\right)
\end{aligned}
$$

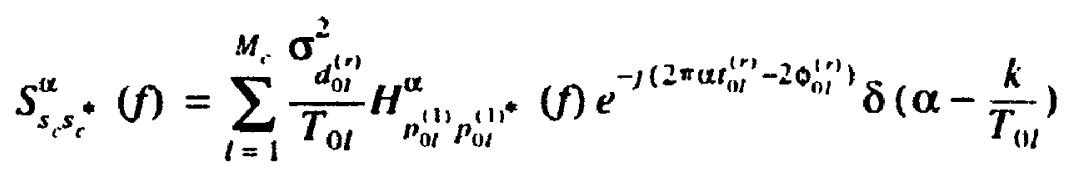

$$
\begin{aligned}
& S_{s_{\alpha}}^{\alpha}(f)=\sum_{\substack{l=-M_{d} / 2 \\
l \neq 0}}^{M_{s} / 2} \frac{\sigma_{d_{l}^{(r)}}^{2}}{T_{l}} H_{p_{l}^{(1)}}^{\alpha}\left(f-f_{l}\right) e^{-j 2 \pi \alpha f_{l}^{(r)}} \delta\left(\alpha-\frac{k}{T_{l}}\right)
\end{aligned}
$$

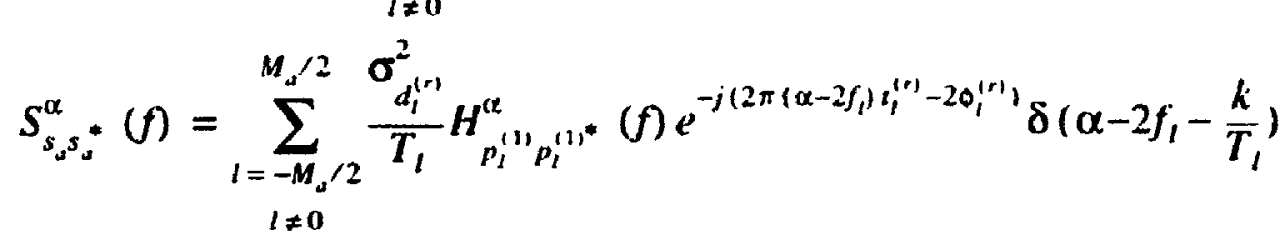

If the bit intervals are the same, the CSD of the SOI is corrupted by the CSD of the CCI and ACl signals on all the cyclic spectral components. However, the CCSD of SOI is corrupted on all the cyclic spectra by only the CCSD of CCI signals and it is free of ACI on some cyclic spectra. 't does mean that for single symbol rate BPSK systems, there exist some cyclic spectra of the CCSD of SOI where ACI does not exist, however CCI exists at all the cyclic spectra. Again ii should be mentioned that the $\mathrm{CCI}$ or $\mathrm{ACI}$ free cyclic spectra does not mean that the data sequence can be estimated $\mathrm{CCl}$ or $\mathrm{ACI}$ free.

In the case of conventional QPSK where $\sigma_{d^{(n)}}^{2}=\sigma_{d^{(1)}}^{2}, t^{(n)}=t^{(t)}$, $q^{(r)}(t)=q^{(i)}(t), \phi^{(r)}=\phi^{(t)},(4.7),(4.8)$ and (4.9) are simplitied to : 


$$
\begin{aligned}
& S_{s_{0}}^{\alpha}(f)=\frac{2 \sigma_{d_{0}^{(r)}}^{2}}{T_{0}} e^{-j 2 \pi \alpha t_{l}^{(n)}} H_{p_{0}^{(1)}}^{\alpha}(f) \delta\left(\alpha-\frac{k}{T_{0}}\right) \\
& S_{s_{0} s_{0}}^{(2)}(f)=0 \\
& S_{s_{c}}^{\alpha}(f)=\sum_{l=1}^{M_{i}} \frac{2 \sigma_{d_{0 I}^{(r)}}^{2}}{T_{0 l}} H_{p_{0 l}^{(1)}}^{\alpha}(f) e^{-ر 2 \pi \alpha l_{0 l}^{(n)}} \delta\left(\alpha-\frac{k}{T_{0 l}}\right) \\
& S_{s_{r} s_{c}^{*}}^{\alpha}(f)=0 \\
& S_{s_{*}}^{(t)}(f)=\sum_{\substack{l=M_{d} / 2 \\
l \neq 0}}^{M_{\alpha}^{\prime} / 2} \frac{2 \sigma_{d_{l}^{(n)}}^{2}}{T_{l}} H_{p_{l}^{(1)}}^{\alpha}\left(f-f_{l}\right) e^{-j 2 \pi \alpha t_{l}^{(r)}} \delta\left(\alpha-\frac{k}{T_{l}}\right) \\
& S_{s_{u} s_{a}^{*}}^{\alpha}(f)=0
\end{aligned}
$$

Equations (4.13) to (4.15) indicates that, in the case of balanced QPSK there is no information on the CCSD of the received signal. Therefore, if the symbol rates are the same, there is no $\mathrm{ACI}$ free cyclic spectrum.

In the case of balanced OQPSK where $\sigma_{d^{(r)}}^{2}=\sigma_{d^{(1)}}^{2}, t^{(r)}=\left(T_{0} / 2\right)+t^{(i)}$, $q^{(r)}(t)=q^{(t)}(t), \phi^{(r)}=\phi^{(i)},(4.7),(4.8)$ and (4.9) are simplified to :

$$
\begin{aligned}
& S_{s_{0}}^{\alpha}(f)=\frac{2 \sigma_{d_{0}^{(r)}}^{2}}{T_{0}} e^{-j 2 \pi \alpha t_{i}^{(r)}} H_{p_{0}^{(1)}}^{\alpha}(f) \delta\left(\alpha-\frac{2 k}{T_{0}}\right) \\
& S_{s_{0} s_{0}}^{\alpha}(f)=\frac{2 \sigma_{d_{0}^{(r)}}^{2}}{T_{0}} H_{p_{0}^{(1)} \rho_{0}^{(1)}}^{\alpha}(f) e^{-j\left(2 \pi \alpha t_{0}^{(p)}-2 \phi_{0}^{(r)}\right)} \delta\left(\alpha-\frac{2 k+1}{T_{0}}\right) \\
& S_{s_{r}}^{\alpha}(f)=\sum_{t=1}^{M_{c}} \frac{2 \sigma_{d_{0 l}^{(r)}}^{2}}{T_{0 l}} H_{p_{0 l}^{\prime \prime l}}^{\alpha}(f) e^{-j 2 \pi \alpha t_{0 l}^{(n)}}:\left(\alpha-\frac{2 k}{T_{0 l}}\right) \\
& S_{s_{1} s^{*}}^{\alpha}(f)=\sum_{l=1}^{M_{c}} \frac{2 \sigma_{d_{0 l}^{(r)}}^{2}}{T_{0 l}} H_{p_{0 t}^{(1)} p_{0 l}^{(1)}}^{\alpha}(f) e^{-J\left(2 \pi \alpha \alpha_{0 l}^{(r)}-2 \phi_{0}^{(r)}\right)} \delta\left(\alpha-\frac{2 k+1}{T_{0 l}}\right)
\end{aligned}
$$




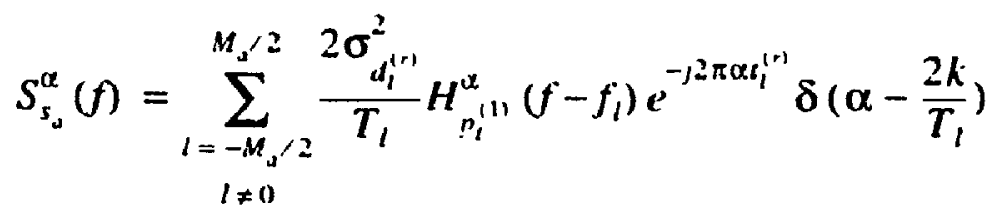

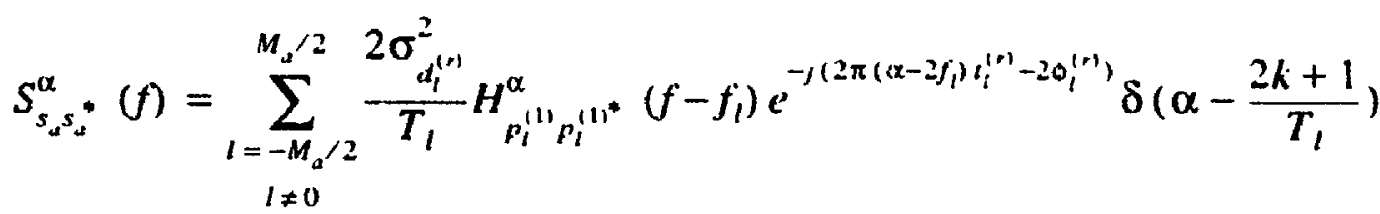

Equations (4.16) to (4.18) show that, the CCSD of the received signal in the balanced OQPSK systems have some ACI free cyclic spectrum.

The above equations show that in a DCS, the CCSD of the received signal includes some information about the SOI and interference signals. The above equations ai::o show that the CSD and CCSD of the SOI are separable from the CCl signals in some cyclic spectrum iff the symbol rate of the SOI is different from the CCI symbol rates. However. the CCSD of the SOI can be separated from $1 \mathrm{CI}$ signals even though they have the same symbol rate. Balanced QPSK signals are the exceptions. Table 4.1 summarizes the spectral properties of a received signal for the three modulation schemes.

\subsection{Receiver Design}

\subsubsection{Estimating The Entire SOI Waveform}

It is well known that the optimum receiver for a stationary signal is a time invariant filter (TIF). However, for a cyclostationary signal, the optimum receiver is a time-variant filter (TVF) $[87,88]$. The only way to implement an optimal time-variant tilter (OTVF) using optimal time-invariant filters (OTIFs) is to filter the stationary components of the signal by OTIFs. Therefore, two structures are fu.ad for OTVF due to two representations, HSR and TSR, of a CRP.

\subsubsection{HSR Time Variant Filter (HSR-TVF)}

The HSR-TVF explicitly exploits the spectral correlation that exists in cyclostationary signals to obtain a lower mean square error (MSE). The receiver which use HSR of the signal is called the linear-conjugate-linear frequency shift (LCL_FRESH) filter $[10,32,37,38]$. Figure 4.2 shows the structure of the LCL-FRESH filter. In this figure, $\left\{\alpha_{m}\right\}$ is the set of all the cyclic frequencies for which the CSD of the received signal is nonzero and $\left\{\beta_{n}\right\}$ is the set of all the cyctic frequencies for which the CCSD of the 
Table 4.1 A summary of cyclic spectral properties of BPSK, OQPSK and QPSK systems.

\begin{tabular}{|c|c|c|}
\hline Modulation & The CSD & The CCSD \\
\hline BPSK & $\begin{array}{l}\text { 1-nonzero at cyclic frequencies equal } \\
\text { to multiple of symbol rate. } \\
\text { 2-noise free at nonzero cyclic frequen- } \\
\text { cies. } \\
\text { 3-ACI and } \mathrm{CCI} \text { free in some nonzero } \\
\text { cyclic frequencies only for multi-sym- } \\
\text { bol rates systems. }\end{array}$ & $\begin{array}{l}\text { 1-nonzero at cyclic frequencies equal } \\
\text { to nultiple of symbol rate. } \\
\text { 2-noise free at all the cyclic frequen- } \\
\text { cies. } \\
\text { 3-CCI free in some nonzero cyclic fre- } \\
\text { quencies only for multi-symbol rates } \\
\text { systems and ACI free in some nonzero } \\
\text { cyclic frequencies even though for sin- } \\
\text { gle symbol rates systems }\end{array}$ \\
\hline OQPSK & $\begin{array}{l}\text { 1-nonzero at cyclic frequencies equal } \\
\text { to even harmonics of symbol rates. } \\
\text { 2-noise free at nonzero cyclic frequen- } \\
\text { cies. } \\
\text { 3-ACI and } \mathrm{CCl} \text { free in some nonzero } \\
\text { cyclic frequencies only for multi-sym- } \\
\text { bol rates systems. }\end{array}$ & $\begin{array}{l}\text { 1-nonzero at cyclic frequencies equal } \\
\text { to odd harmonics of symbol rates. } \\
\text { 2-noise free at all the cyclic frequen- } \\
\text { cies. } \\
\text { 3-CCI free in some nonzero cyclic fre- } \\
\text { quencies only for multi-symbol rates } \\
\text { systems and ACI free in some nonzero } \\
\text { cyclic frequencies even though for sin- } \\
\text { gle-symbol rates systems }\end{array}$ \\
\hline QPSK & $\begin{array}{l}\text { 1-nonzero at cyclic frequencies equal } \\
\text { to multiple of symbol rate. } \\
2 \text {-noise free at nonzero cyclic fre- } \\
\text { quency. } \\
\text { 3-ACI and } \mathrm{CCl} \text { free in some nonzero } \\
\text { cyclic frequencies only for multi-sym- } \\
\text { bol rates systems. }\end{array}$ & uniquely zero \\
\hline
\end{tabular}




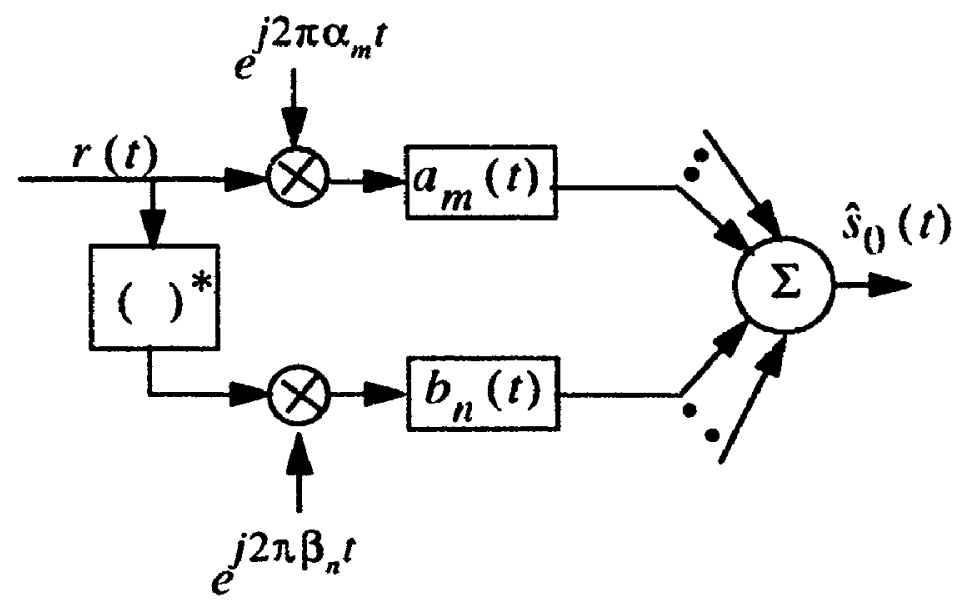

Figure 4.2 The structure of a receiver using Linear-Conjugate-Linear FRESH (LCL-FRESH) filtering.

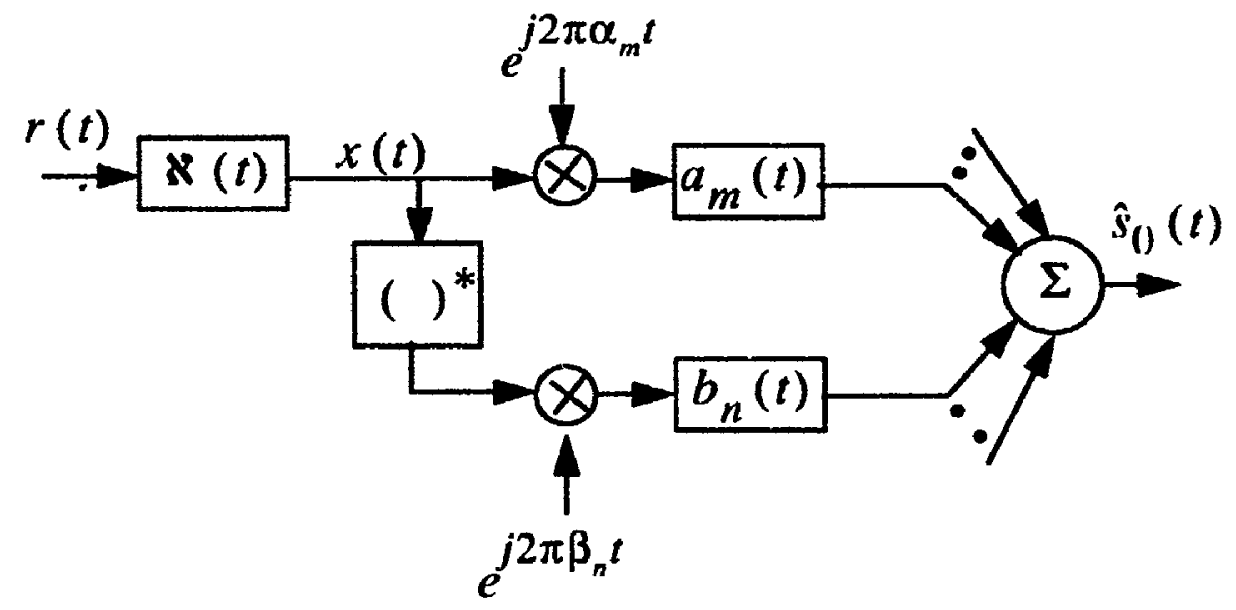

Figure 4.3 The structure of a receiver using LCL-FRESH fittering with finite number of frequency shifts. 
received signal is nonzero.

The estimated signal can be found by the following equation :

$$
\hat{s}_{n}(t)=\sum_{m=1}^{M} a_{m}(t) \otimes\left[r(t) e^{\prime 2 \pi \alpha_{m} t}\right]+\sum_{n=1}^{N} b_{n}(t) \otimes\left[r^{*}(t) e^{j 2 \pi \beta_{n} t}\right]
$$

where $M$ and $N$ are the maximum number of filters in the linear and conjugate paths respectively. The optimum solution of the LCL-FRESH filters with the criterion of minimum mean square of the error $\left(e(t)=\hat{s}_{0}(t)-s_{0}(t)\right)$ are called cyclic Wiener filters and can be found by the following equations [32] :

$$
\begin{gathered}
\sum_{m=1}^{M} A_{m}(f) S_{r}^{\alpha_{k}-\alpha_{m}}\left(f-\frac{\alpha_{m}+\alpha_{k}}{2}\right)+\sum_{\substack{n=1 \\
k=1,2, \ldots, M}}^{N} B_{n}(f)\left[S_{r r}^{\beta_{n}-\alpha_{k}}\left(f-\frac{\beta_{n}+\alpha_{k}}{2}\right)\right]^{*}=S_{s_{0} r}^{\alpha_{k}}\left(f-\frac{\alpha_{k}}{2}\right) \\
\sum_{m=1}^{M} A_{m}(f) S_{r r^{*}}^{\beta_{k}-\alpha_{m}}\left(f-\frac{\alpha_{m}+\beta_{k}}{2}\right)+\sum_{\substack{n=1 \\
k=1,2, \ldots, N}}^{N} B_{n}(f) S_{r}^{\beta_{k}-\beta_{n}}\left(-f+\frac{\beta_{n}+\beta_{k}}{2}\right)=S_{s_{0} r}^{\beta_{k}}\left(f-\frac{\beta_{k}}{2}\right)
\end{gathered}
$$

where $S_{s_{0} r}^{\alpha}(f)$ and $S_{s_{0} r^{*}}^{\alpha}(f)$ are the cyclic cross spectrum and conjugate cyclic cross spectrum between the SOI and the received signal respectively.

The spectrum of error can be found by the following equation [32] :

$$
S_{c}(f)=S_{d}(f)-\left(\sum_{m=1}^{M} A_{m}(f)\left[S_{s_{0} r}^{\alpha_{m}}\left(f-\frac{\alpha_{m}}{2}\right)\right]^{*}-\sum_{n=1}^{N} B_{n}(f)\left[S_{s_{0} r^{*}}^{\beta_{n}}\left(f-\frac{\beta_{n}}{2}\right)\right]^{*}\right)
$$

It is apparent from (4.20) that the best set of $\left\{\alpha_{m}\right\}$ contains all $\alpha_{m}$ [32] for which either

$$
S_{r}^{\alpha_{k}-\alpha_{m}}\left(f-\frac{\alpha_{m}+\alpha_{k}}{2}\right) \neq 0
$$

and $\alpha_{k}$ for which

$$
S_{s_{0^{r}}}^{\alpha_{k}}\left(f-\frac{\alpha_{k}}{2}\right) \neq 0
$$

or 


$$
S_{r r^{*}}^{\beta_{k}-\alpha_{m}}\left(f-\frac{\beta_{k}+\alpha_{m}}{2}\right) \neq 0
$$

and $\beta_{k}$ for which

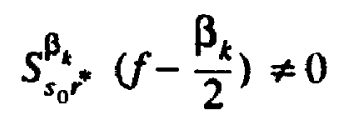

and the best set of $\left\{\beta_{n}\right\}$ contains all $\beta_{k}$ which either

$$
S_{r r^{*}}^{\beta_{n}-\alpha_{k}}\left(f-\frac{\beta_{n}+\alpha_{k}}{2}\right) \neq 0
$$

and $\alpha_{k}$ for which

$$
S_{s_{0} r}^{\alpha_{k}}\left(f-\frac{\alpha_{k}}{2}\right) \neq 0
$$

or

$$
S_{r}^{\beta_{k}-\beta_{n}}\left(-f+\frac{\beta_{n}+\beta_{k}}{2}\right) \neq 0
$$

and $\beta_{k}$ for which

$$
S_{s_{0^{*}}}^{\beta_{k^{*}}}\left(f-\frac{\beta_{k}}{2}\right) \neq 0
$$

Since the received signal is not band limited, the optimum number of frequency shifts is infinite. Nevertheless, when the received signal is passed through a noise limiting filter only a finite number of frequency shifts will be needed (Figure 4.3). If the SOI and ACI signals are not overlapped, the best choice for this filter is a filter matched to the transmitter pulse shape. However, in the case of overlapping signals, the best noise limiter filter is a filter which passes all the overlapped signals. Nevertheless, a sub-optimum filter which can passes only a portion of ACI signals may be considered.

\subsubsection{TSR-TVF}

Although the FRESH-filter is the optimum linear filter for cyclostationary signals, it is too complex. Another TVF can be built on the basis of TSR of a cyclostationary signal. In this receiver, the signal is sampled after passing through the receiver filter. Figure 4.4 shows the structure of this receiver. In this figure, $T_{0}$ is the symbol rate of SOI, $\left\{T_{0 l}\right\}$ are the incommensurate symbol rates of the $\mathrm{CCl}$ signals, $M_{0 l}=\left[T_{0, l} / T_{s}\right\},\left\{T_{l}\right\}$ 


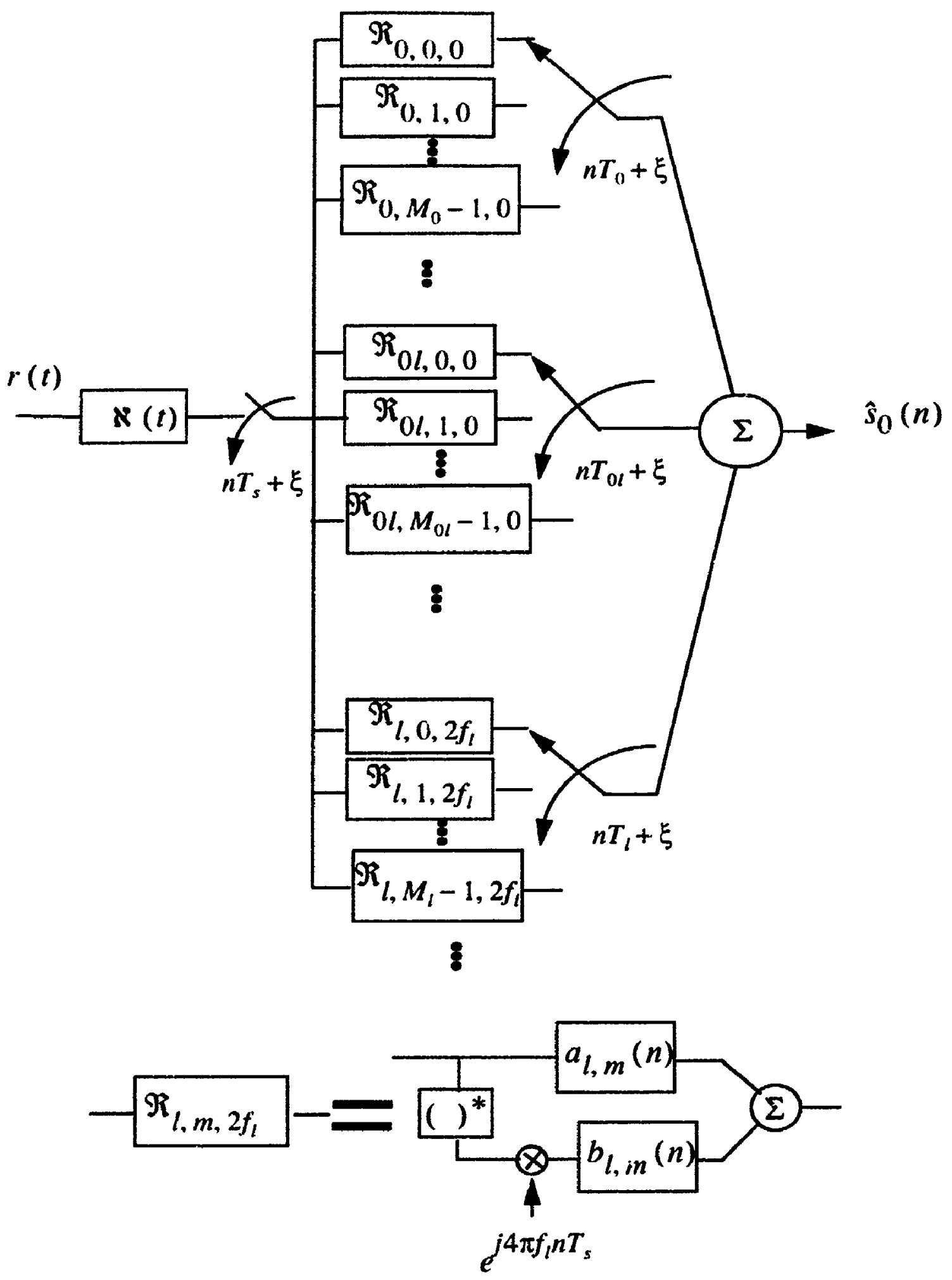

Figure 4.4 The structure of a recoiver using TSR-TVF. 
are the symbol rates of the ACI signals, and $M_{l}=\left\lceil T_{l} / T_{s}\right\rceil$. Each tilter of the branch $\Re_{l, m, f}$ will be updated at $m T_{s}+n T_{l}+\xi$ where $n$ is a positive integer and $\xi$ is the sampler delay time. In the case of $S_{s_{r} s_{i}^{*}}(f)=0$, the conjugate path (c-path) in $\Re_{l, m, l_{1}}$, will be removed. The linear path (l-path) of $\Re_{l, m, f_{l}}$ will be removed if $T_{l}$ is not incommensurate periodicity.

In general, the minimum sampling rate of the receiver has to be groater than the Nyquist rate. However, in order to exploit all periodicities, the minimum sampling rate has to be greater than the least common multiple of the fundamental cyclic frequencies in the received signal.

It can be shown that the TSR-TVF and the HSR-TVF are equivalent Appendix C].

\subsubsection{Estimating the Data Sequence}

In a digital communication system, the objective is to extract the data. Therefore, the output of the TSR-TVF and HSR-TVF should be sampled. The sampling rate has to be at least equal to the symbol rate. To understand how a sampler can simplify the structure of TSR-TVF and HSR-TVF, the aliasing effect of a sampler is considered in the following section.

\subsubsection{The Allasing Effect of a Sampler}

The following equality is valid for any signal $x(t)$ and any $L$ and $M$ :

$$
x(t)=\frac{1}{L+M+1} \sum_{m=-L}^{M} x(t) e^{j 2 \pi m}
$$

Multiplying $m$ by $n T / T$ where $n$ is an integer does not change the equality at (4.3()):

$$
x(t)=\frac{1}{L+M+1} \sum_{m=-L}^{M} x(t) e^{j 2 \pi \frac{m}{T}(n T)}
$$

If $x(t)$ is sampled at the rate $1 / T$, the above equation yields :

$$
\tilde{x}(n)=x(n T)=\left.\left[\frac{1}{L+M+1} \sum_{m=-L}^{M} x(t) e^{j 2 \pi \frac{m}{T}}\right]\right|_{t=n T}
$$

Equation (4.32) shows that sampling a signal with the rate of $1 / T$ is equivalent to adding 
all versions of the signals shifted by the frequencies $m / M$ where $m$ is an integer.

Now, we assume $x(t)$ is filtered by $h(t)$ and the output is sampled at the rate $1 / \pi$, then

$$
y(n)=\left.x(t) \otimes h(t)\right|_{t=n} T
$$

The above equation is equivalent to the following equation :

$$
\tilde{y}(n)=\left.\left[\frac{1}{L+M+1} \sum_{m=-L}^{M} x(t) e^{j 2 \pi m \frac{t}{T}} \otimes h(t) e^{j 2 \pi m \frac{t}{T}}\right]\right|_{t=n T}
$$

Equation (4.34) shows that sampling a filtered signal with the rate of $1 / T$ is equivalent to sampling the output of a bank of filters, which filters all frequency shifted versions of the signal (Figure 4.5).

If $x(t)$ is frequency shifted by $\alpha$ and passed through a filter $h(t)$ followed by a sampler with the rate of $1 / T$, it can be shown that :

$$
\begin{gathered}
\tilde{y}(n)=\left.\left[x(t) e^{j 2 \pi \alpha t} \otimes h(t)\right]\right|_{t=n T} \\
=\left[\left.\left\{x(t) \otimes h(t) e^{-j 2 \pi \Gamma \alpha T] t}\right]\right|_{t=n T}\right] e^{j 2 \pi \mid \alpha]}{T^{-1}}^{n T} \\
=\left.\left[x(t) e^{j 2 \pi[\alpha]} T_{r^{-3} t} \otimes h(t) e^{-j 2 \pi \Gamma \alpha T\rceil t}\right]\right|_{t=n T}
\end{gathered}
$$

Figure 4.6 illustrates the above equalities. Comparing (4.34) and (4.35) with (4.19) concludes that a filter followed by a sampler is equivalent to the FRESH-filter with the set of frequency shifts $\{0, \pm 1 / T, \pm 2 / T, \ldots\}$. It is clear that, if $x(t)$ is shifted by a frequency $\mu$ before entering $h(t)$, the set of equivalent frequency shifts will have an addition shift $\mu$ (i.e. $\{\mu, \mu \pm 1 / T, \mu \pm 2 / T, \ldots\})$. Therefore, to achieve an arbitrary frequency shifting, $\gamma$, it is enough to shift the frequency by the remainder of the division of $\gamma$ by $T^{-1},[\gamma]_{T^{-1}}$.

\subsubsection{Adding Sampler to The TSR-TVF}

Adding the symbol rate sampler to the structure of TSR-TVF creates the structure of sampled TSR-TVF (STSR-TVF) shown in Figure 4.7. In the figure, $M_{0 l}^{0}$ (or $M_{l}^{0}$ ) is the least common multiple of $M_{0 l}$ (or $M_{l}$ ) and $M_{0}$, and $T_{0 l}^{0}=M_{0 l}^{0} T_{s}$ (or $T_{l}^{0}=M_{l}^{0} T_{s}$ ). The structure of STSR-TVF is a general one, and it covers the cornmensurate periodicities as well as incommensurate periodicities. 


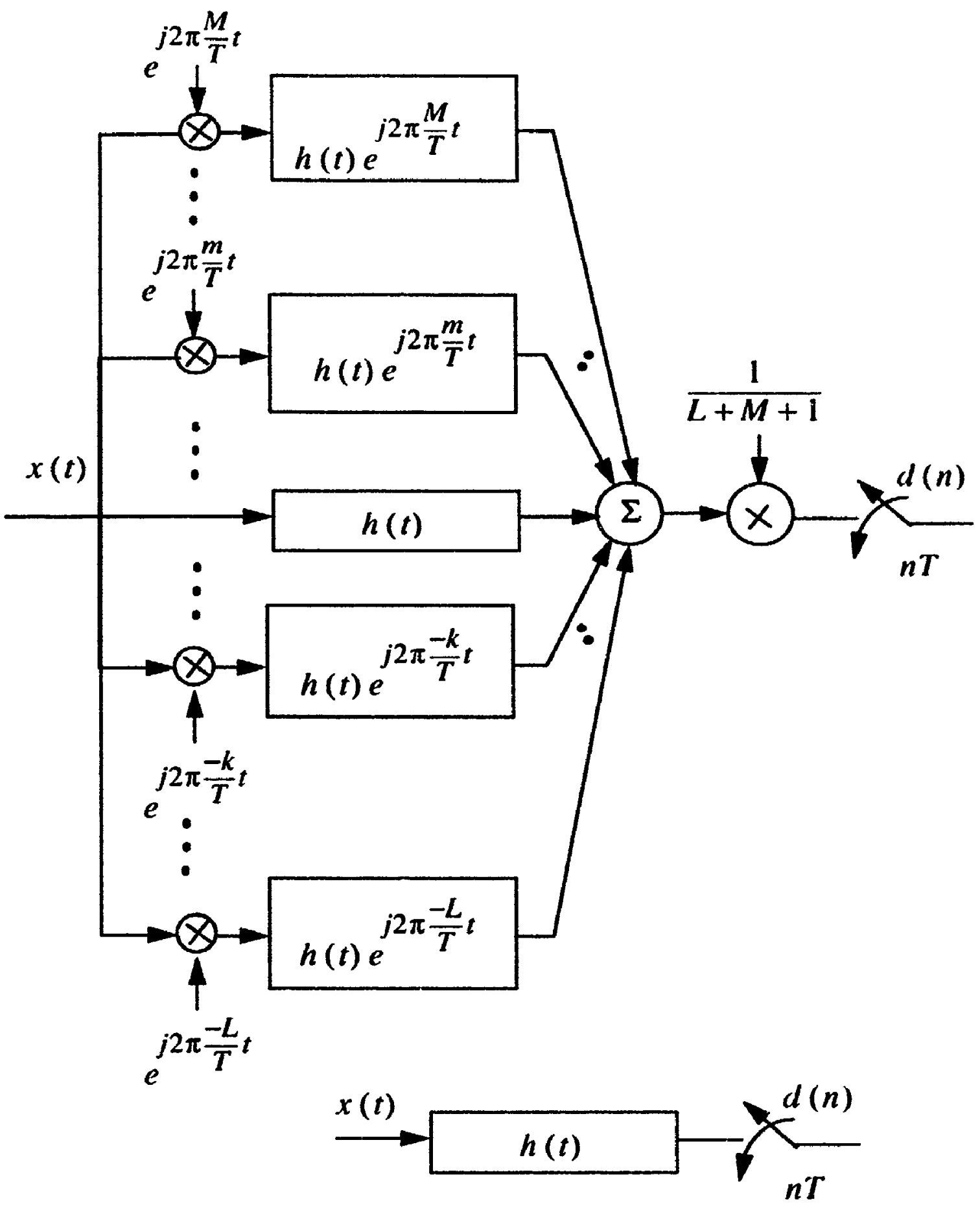

Figure 4.5 Sampling the output of a fittored signal. 

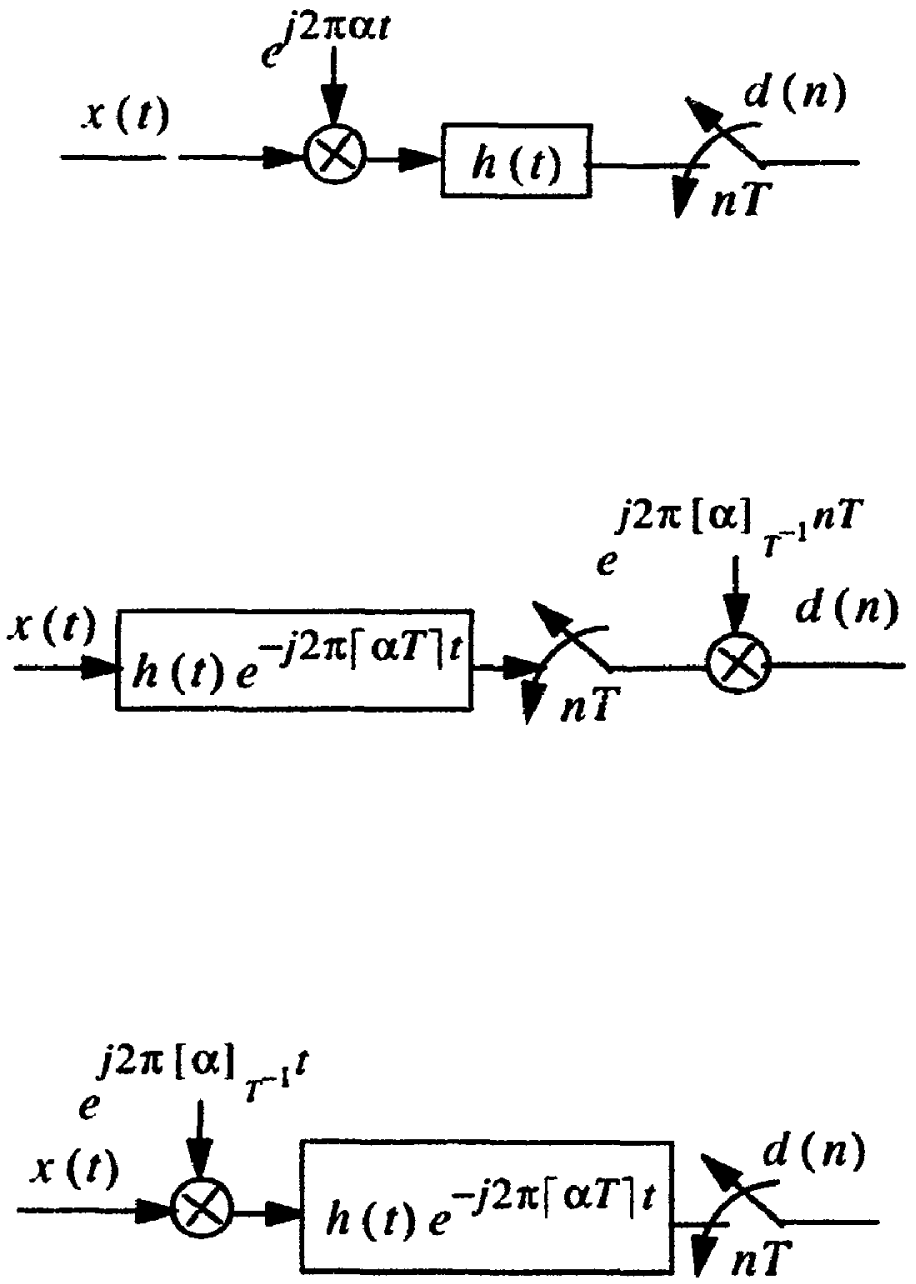

Figure 4.6 The equivalent procedures in equation (4.35). 


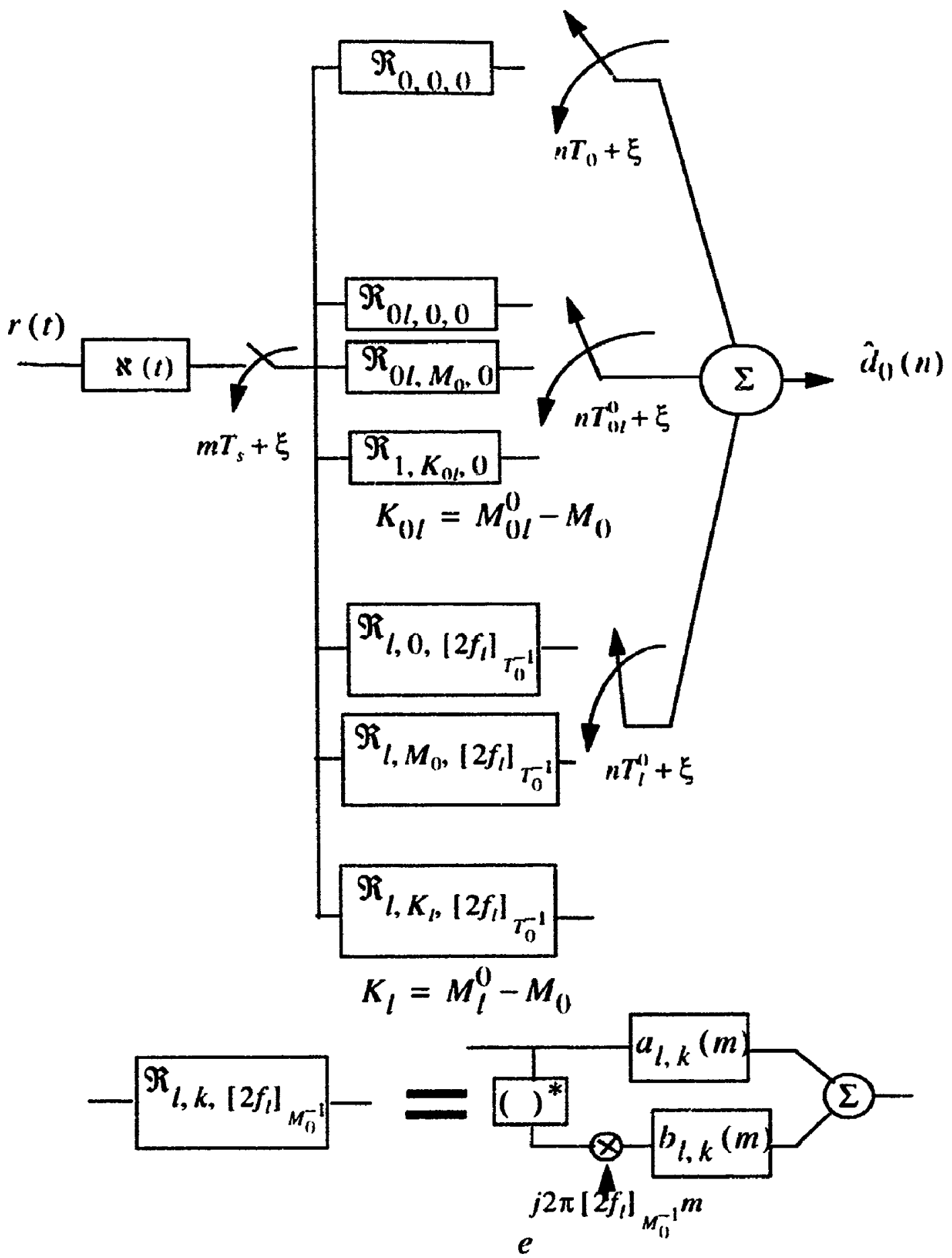

Figure 4.7 The structure of Sampled TSR-TVF (STSR-TVF). 
As an example, a three symbol rate balanced QPSK system with incommensurate symbol rates $1 / 2,1 / 3,1 / 4 \mathrm{~Hz}$ is considered. Assuming the symbol rate of $\mathrm{SOI}$ is $1 / 3 \mathrm{~Hz}$. In this case, the following parameters are calculated :

$$
\begin{gathered}
\left\{T_{11}=3 \text { sec., } T_{1}=2 \text { sec., } T_{2}=4 \text { sec. }\right\} \Rightarrow T_{s}=1 \text { sec. } \\
M_{1}=\left\lceil\left[\frac{T_{0}}{T s}\right\rceil=3, M_{1}=\left\lceil\frac{T_{1}}{T s}\right\rceil=2, M_{2}=\left\lceil\frac{T_{2}}{T s}\right\rceil=4\right. \\
M_{1}^{0}=M_{0} \times M_{1}=6, M_{2}^{0}=M_{0} \times M_{2}=12 \\
T_{1}^{\prime \prime}=M_{1}^{0} \times T_{s}=6 \text { sec., } T_{2}^{0}=M_{2}^{0} \times T_{s}=12 \text { sec. }
\end{gathered}
$$

Figure 4. $X$ shows the structure of STSR-TVF for this example where (k Hz, $\phi$ sec.) represents a sampler with the rate $\mathrm{k} \mathrm{Hz}$ and initial time $\phi$.

In the case of a single symbol rate system, the STSR-TVF is simplified to Figure 4.9. In the case of a single symbol rate balanced QPSK system, STSR-TVF is simplified to Figure 4.10 which is the conventional fractionally space equalizer (FSE). Therefore, STSR-TVF can be considered as the generalized FSE (GFSE). From now on, we will refer to STSR-TVF as GFSE.

\subsubsection{Adding Sampler to The HSR-TVF}

Adding a symbol rate sampler to the HSR-TVF simplifies the structure of HSRTVF to the one shown in Figure 4.11 called sampled HSR-TVF (SHSR-TVF). Although at first sight, it does not seem to show a great simplification, yet, we will see that the structure of HSR-TVF has became considerably simplified. This can be illustrated if we consider a set of frequency shiftings in HSR-TVF i.e. :

$$
\begin{gathered}
\{\alpha\}=\left\{\frac{1}{T_{0}}\{\ldots,-1,0,1,2, \ldots\}, \frac{1}{T_{01}}\{\ldots,-1,1,2, \ldots\}, \ldots, \frac{1}{T_{0 M_{c}}}\{\ldots,-1,1,2, \ldots\},\right. \\
\left.\quad \frac{1}{T_{1}}\{\ldots,-1,1,2, \ldots\}, \ldots, \frac{1}{T_{M_{a}}}\{\ldots,-1,1,2, \ldots\}\right\} \\
\{\beta\}=\left\{\frac{1}{T_{1}}\{\ldots-1,0,1,2, \ldots\}, \frac{1}{T_{01}}\{\ldots,-1,1,2, \ldots\}, \ldots, \frac{1}{T_{0 M_{c}}}\{\ldots,-1,1,2, \ldots\},\right. \\
\left.2 f_{1}+\frac{1}{T_{1}}\{\ldots,-1,1,2, \ldots\}, \ldots 2 f_{M_{a}} \div \frac{1}{T_{M_{a}}}\{\ldots,-1,1,2, \ldots\}\right\}
\end{gathered}
$$

and the set of frequency shifting in SHSR-TVF are: 


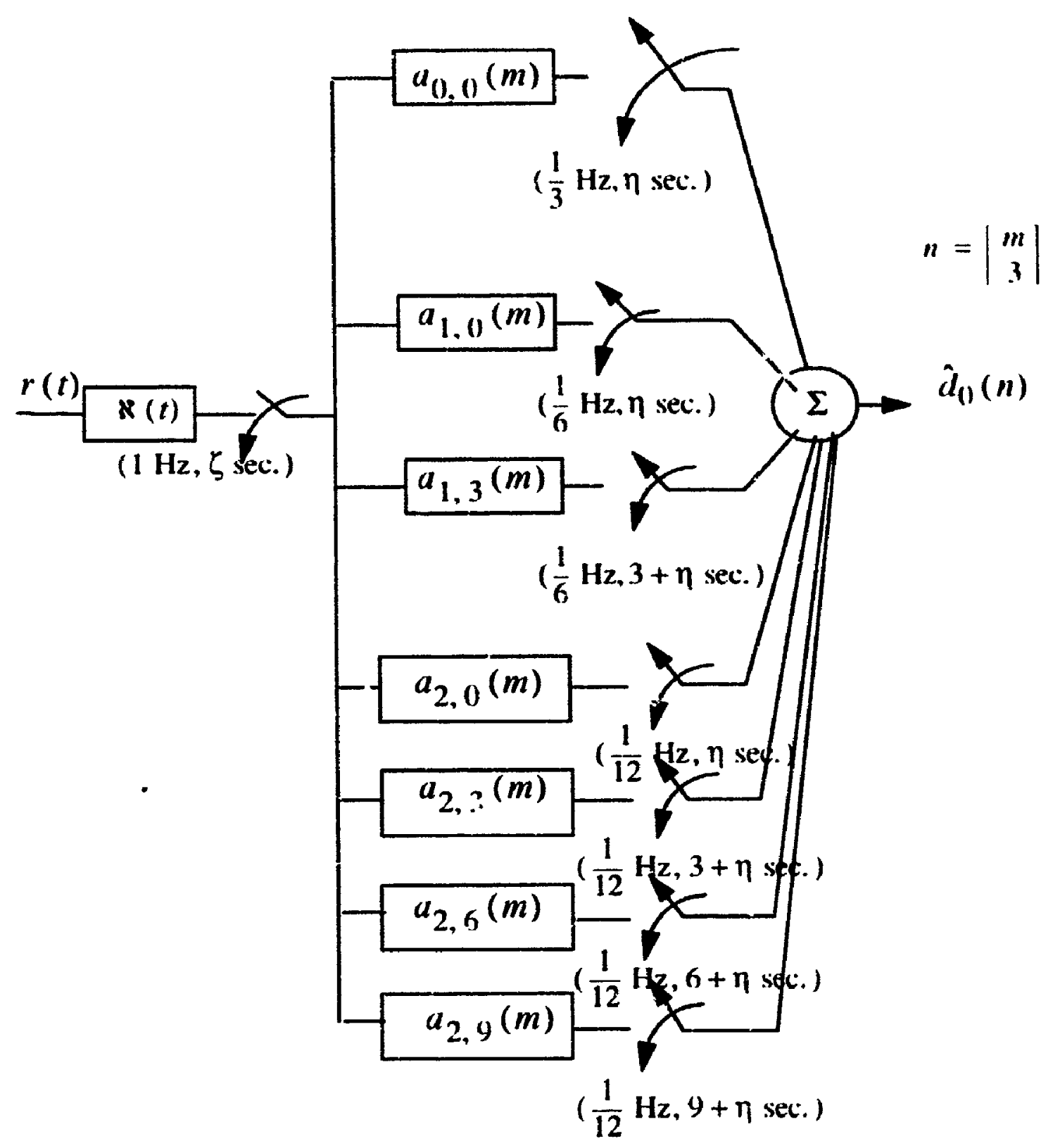

Figure 4.8 An example of SiiSR-TVF. 


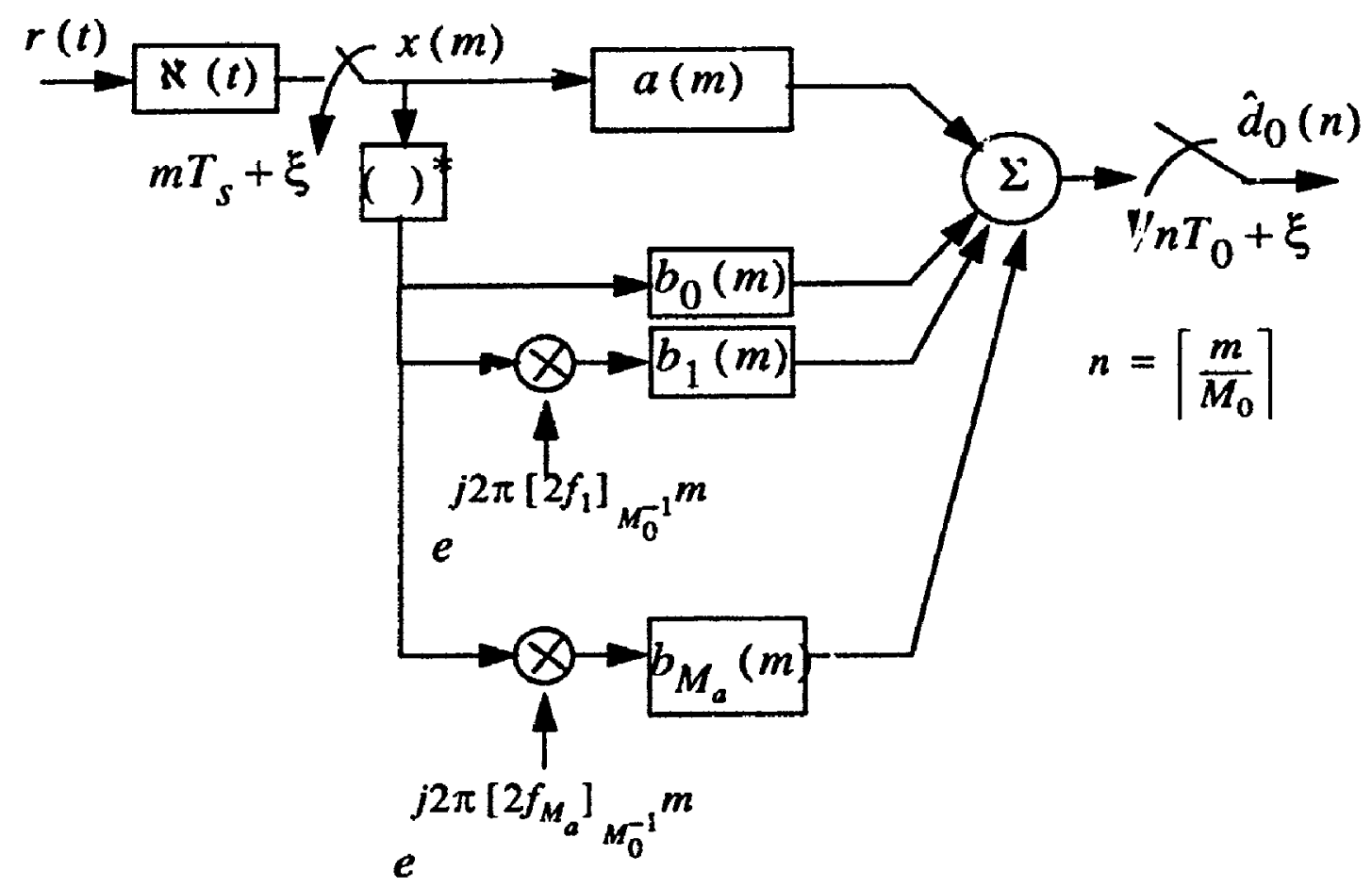

Flgure 4.9 The structure of STSR-TVF for single symbol rate systems.

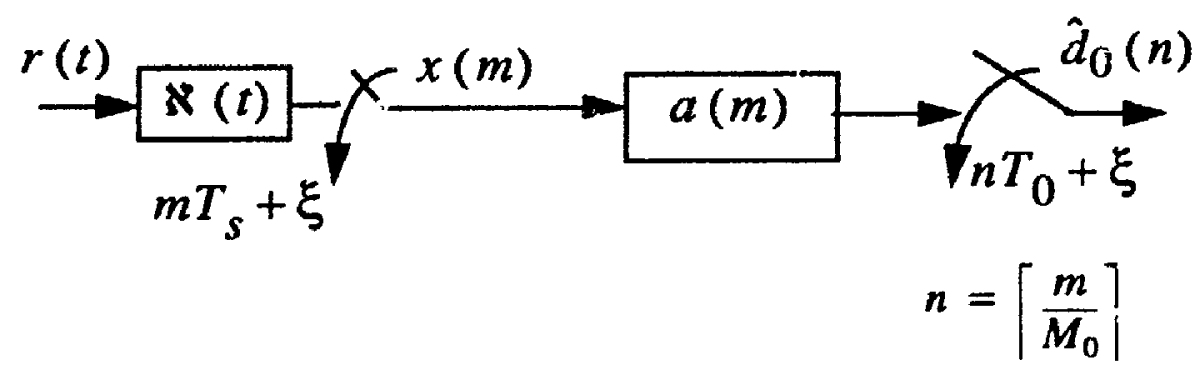

Figure 4.10 The structure of STSR-TVF for balanced QPSK and single symbol rate systems. 


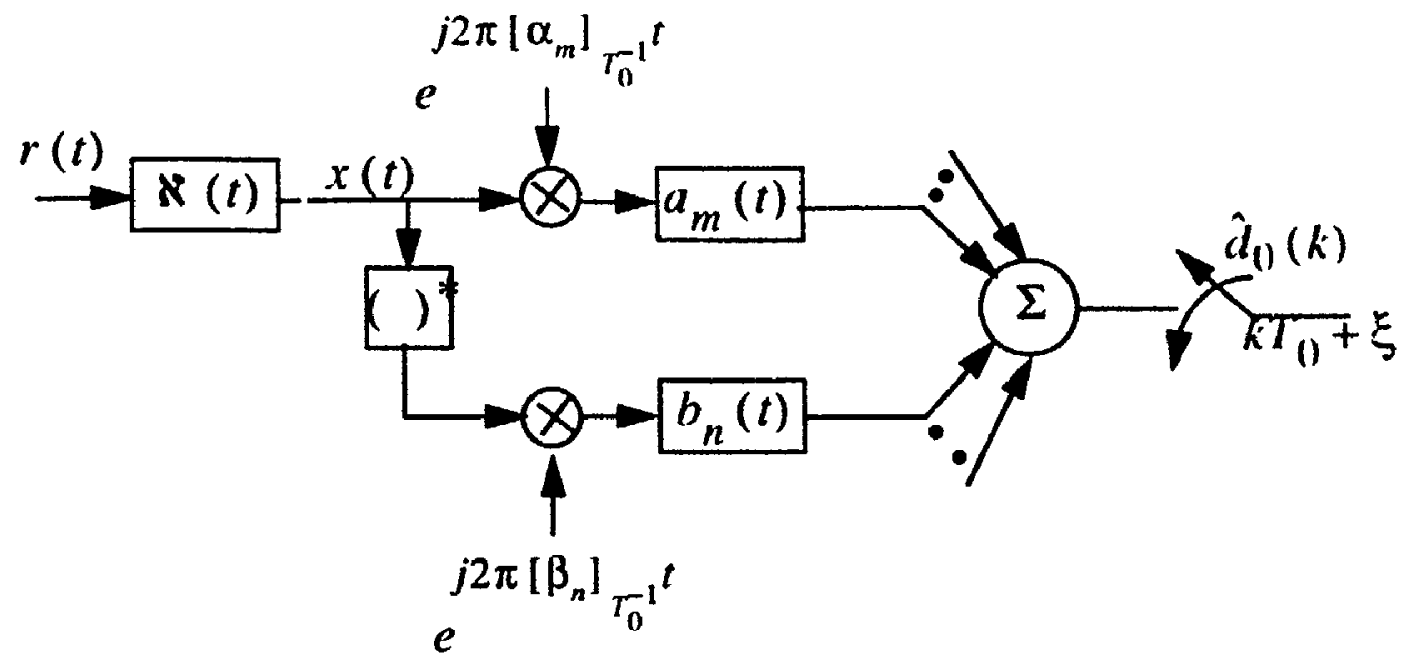

Figure 4.11 The structure of SHSR-TVF.

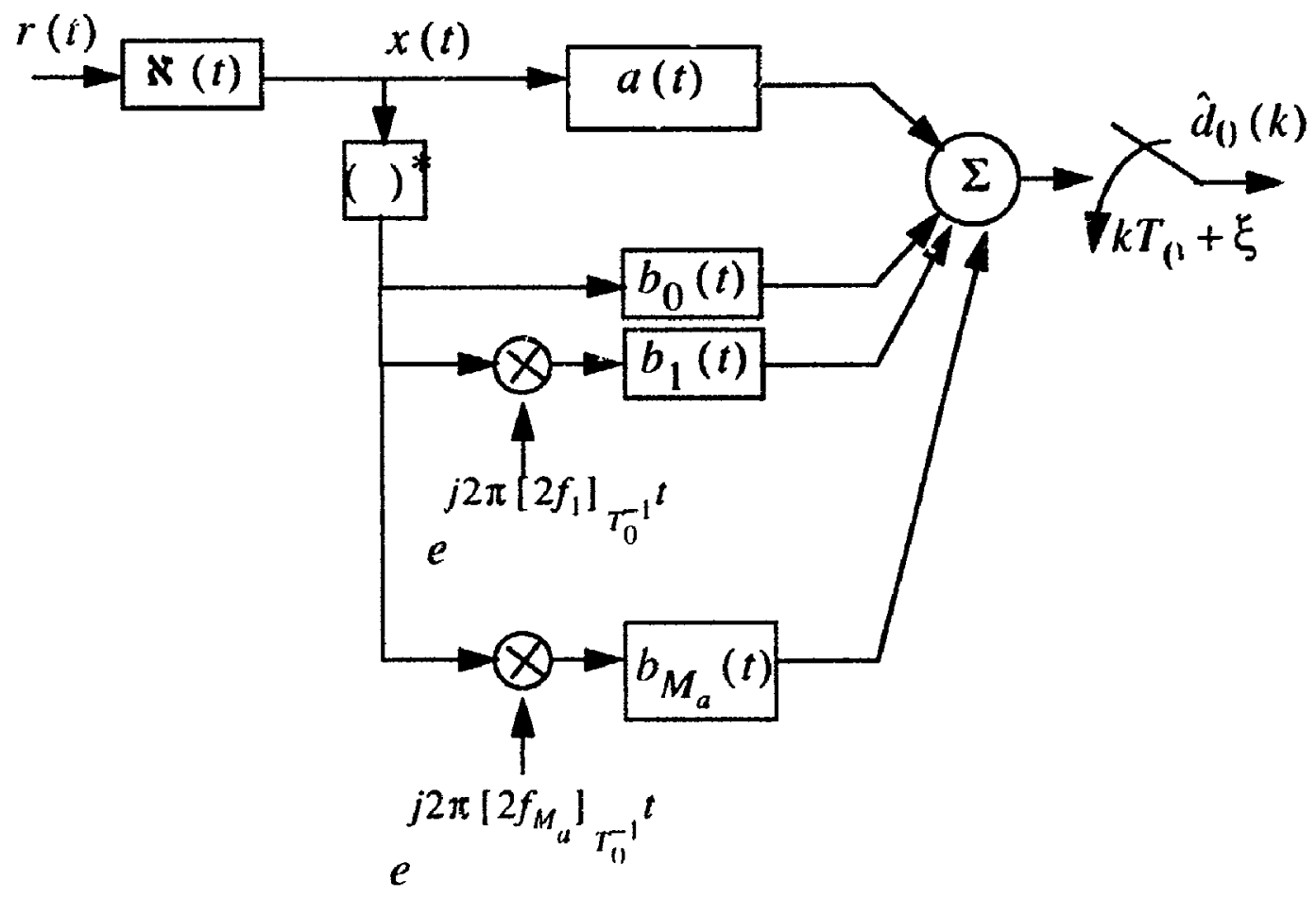

Figure 4.12 The structure of SHSR-TVF for single symbol rate systems. 


$$
\begin{aligned}
& \left\{|\alpha|_{T_{0}{ }^{1}}\right\}=\{\mid 0\}, \frac{1}{T_{01}^{0}}\left\{-\left\lceil\frac{T_{01}}{2}\right\rceil, \ldots,-1,1, \ldots,\left\lceil\frac{T_{01}}{2}\right\rceil\right\}, \ldots \\
& \frac{1}{T_{1 M}^{0}}\left\{-\left\lceil\frac{T_{0 M_{1}}}{2}\right\rceil, \ldots,-1,1, \ldots,\left\lceil\frac{T_{0 M_{c}}}{2}\right\rceil\right\}, \frac{1}{T_{1}^{0}}\left\{-\left\lceil\frac{T_{1}}{2}\right\rceil, \ldots,-1,1, \ldots,\left\lceil\frac{T_{1}}{2}\right\rceil\right\}, \ldots, \\
& \left.\frac{1}{T_{M_{u}}^{\prime}}\left\{-\left\lceil\frac{T_{M_{u}}}{2}\right\rceil, \ldots,-1,1, \ldots,\left\lceil\frac{T_{M_{d}}}{2}\right\rceil\right\} \quad\right\} \\
& \left\{[\beta]_{T_{0}^{-1}}\right\}=\left\{\{0\}, \frac{1}{T_{01}^{0}}\left\{-\left\lceil\frac{T_{01}}{2}\right\rceil, \ldots,-1,1, \ldots,\left\lceil\frac{T_{01}}{2}\right\rceil\right\}, \ldots\right. \\
& \frac{1}{T_{O M_{r}}^{\prime \prime}}\left\{-\left\lceil\frac{T_{O M_{c}}}{2}\right\rceil, \ldots,-1,1, \ldots,\left\lceil\frac{T_{O M_{c}}}{2}\right\rceil\right\},\left[2 f_{1}\right]_{T_{0}^{-1}}+\frac{1}{T_{1}^{0}}\left\{-\left\lceil\frac{T_{1}}{2}\right\rceil, \ldots,-1,1, \ldots,\left\lceil\frac{T_{1}}{2}\right\rceil\right\}, \ldots \\
& \left.\left[2 f_{M_{\lrcorner}}\right]_{T_{0}^{-1}}+\frac{1}{T_{M_{u}}^{0}}\left\{-\left\lceil\frac{T_{M_{a}}}{2}\right\rceil, \ldots,-1,1, \ldots,\left\lceil\frac{T_{M_{u}}}{2}\right\rceil\right\}\right\}
\end{aligned}
$$

Equations (4.37) and (4.38) show that, the amount and number of frequency shifts in SHSR-TVF are much lower than those for HSR-TVF.

In the case of a single symbol rate system, SHSR-TVF is simplified to Figure 4.12. Comparing Figure 4.9 and Figure 4.12 indicates that, the GFSE is the sampled version of SHSR-TVF, or in other words, SHSR-TVF is an infinite length GFSE. Therefore, SHSRTVF is an alternative structure for FSE. Since SHSR-TVF is built on the basis of shifting the frequency of the input data, we refer to SHSR-TVF as FRESH-FSE. Although FKESH-FSE and GFSE are equivalent, their structure complexities are different and prefexence of one over the other depends on the system under consideration.

\subsection{Decision Feedback Receiver}

\subsubsection{Conventional Decision Feedback Equalizer}

The conventional fractionally spaced decision feed back equalizer (conventional DFE) is created by adding a feedback path to an FSE. Figure 4.13 shows the structure of conventional DFE. In the figure, $x(t)$ is the received signal after passing through the receiver filter. The feedback is added to decrease the ISI caused by the previous symbols.

There are two criteria that can be used as basis for conventional DFE design (1) the zero forcing criterion and (2) the minimum MSE criterion. The zero forcing DFE and 


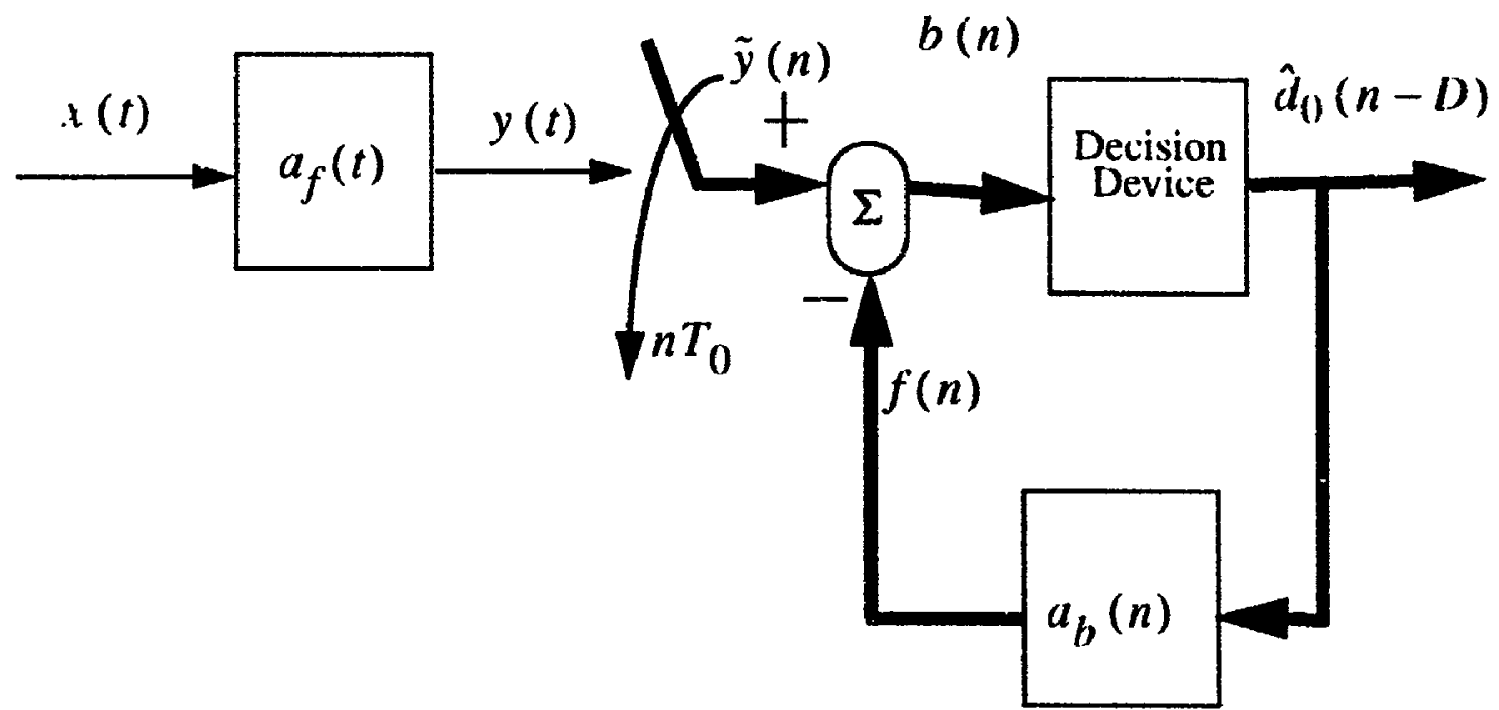

Sampled data line

Continuous time line

Figure 4.13 The structure of conventional DFE with infinite length filters. 
MMSE-DFE are already analyzed in $[1,19,21,22,61,67,70,72,73,80,81,85,93,99]$. However, the MMSE-DFE is analyzed in a different way in the following section.

\subsubsection{Conventional MMSE-DFE}

\subsubsection{Infinite Length Equalizer}

The MMSE-DFE minimizes the time average mean square of the error defined by the following equation :

$$
T A M S E=\left\langle E\left\{\left|b(n)-d_{0}(n-D)\right|^{2}\right\}\right\rangle_{n}
$$

where $D$ is the overall delay. To simplify the notations, we remove the index $n$ from \langle\rangle$_{n}$. Replacing $b(n)$ by $\tilde{y}(n)-f(n)$ in (4.39) and assuming the correct decisions for previous symbols, yields :

$$
\begin{gathered}
T A M S E=\left\langle E\left\{\left|d_{0}(n-D)\right|^{2}\right\}\right\rangle+\left\langle E\left\{|f(n)|^{2}\right\}\right\rangle_{n}+\left\langle E\left\{|\tilde{y}(n)|^{2}\right\}\right\rangle+ \\
\left\langle E\left\{d_{0}(n-D) f^{*}(n)\right\}\right\rangle+\left\langle E\left\{d_{0}^{*}(n-D) f(n)\right\}\right\rangle \\
-\left\langle E\left\{d_{0}(\eta-D) \tilde{y}^{*}(n)\right\}\right\rangle-\left\langle E\left\{d_{0}^{*}(n-D) \tilde{y}(n)\right\}\right\rangle \\
-\left\langle E\left\{f(n) \tilde{y}^{*}(n)\right\}\right\rangle-\left\langle E\left\{f^{*}(n) \tilde{y}(n)\right\}\right\rangle
\end{gathered}
$$

Assuming that the data are uncorrelated and the decisions are correct, the firs ${ }^{\dagger}$ term of the right hand side is :

$$
\sigma_{d_{0}}^{2}=\sigma_{d_{0}^{(2)}}^{2}+\sigma_{d_{0}^{(\prime)}}^{2}
$$

and 4 th and 5 th terms are zero.

The optimum feedback and forward filters can be achieved by letting the gradient of TAMSE equal to zero. The gradient of TAMSE according to the feedback filter coeffcients can be found by tha following equation :

$$
\nabla_{a_{b}}^{T A M S E}(m)=\left\langle E\left\{[f(n)-\tilde{y}(n)] \nabla_{a_{b}}^{f^{(n)}}(m)\right\}\right\rangle
$$

eplacing $f(n)$ by:

$$
f(n)=\sum_{m=1}^{\infty} d_{0}(n-D-m) a_{b}(m)
$$

and iaking gradient of it, 


$$
\nabla_{a_{b}}^{f(n)}(m)=d_{0}^{*}(n-D-m)
$$

yields :

$$
\begin{gathered}
\nabla_{a_{b}}^{T A M S E}(m)=\left\langle\sum_{k=1}^{\infty} E\left\{d_{0}^{*}(n-D-m) d_{0}(n-D-k)\right\} a_{b}(k)\right\rangle- \\
\left\langle E\left\{\tilde{y}(n) d_{0}^{*}(n-D-m)\right\}\right\rangle
\end{gathered}
$$

The first term of the above equation is nonzero iff $m=k$.

$$
\nabla_{a_{b}}^{T A M S E}(m)=\sigma_{d_{0}}^{2} a_{b}(m)+\left\langle E\left\{\tilde{y}(n) d_{0}^{*}(n-D-m)\right\}\right\rangle
$$

Replacing $\tilde{y}(n)$ by :

$$
\tilde{y}(n)=\int_{-\infty}^{\infty} a_{f}(u) x\left(n T_{0}-u\right) d(u)
$$

yields :

$$
\nabla_{a_{b}}^{T A M S E}(m)=\sigma_{d_{0}}^{2} a_{b}(m)-\int_{-\infty}^{\infty} a_{f}(u)\left\langle E\left\{d_{0}^{*}(n-D-m) x\left(n T_{0}-u\right)\right\}\right\rangle d(u)
$$

substituting $x(t)$ by $x(t)=r(t) \otimes *(t)$ and using (4.1) to (4.4) yields:

$$
\begin{gathered}
E\left\{d_{0}^{*}(n-D-m) x(n T-u)\right\}=\sigma_{d_{0}^{(r)}}^{2} z_{0}^{(1)}\left((D+m) T_{0}-t_{0}^{(r)}-u\right) e^{j 2 \pi \phi_{0}^{(\prime)}}+ \\
\sigma_{d_{0}^{(1)}}^{2} z_{0}^{(2)}\left((D+m) T_{0}-t_{0}^{(i)}-u\right) e^{j 2 \pi \phi_{0}^{(1)}}
\end{gathered}
$$

where :

$$
\begin{aligned}
& z_{0}^{(1)}=p_{0}^{(1)}(t) \otimes \aleph(t) \\
& z_{0}^{(2)}=p_{0}^{(2)}(t) \otimes \aleph(t)
\end{aligned}
$$

substituting (4.49) in (4.48) yields :

$$
\begin{aligned}
& \nabla_{a_{b}}^{\text {TAMSE }}(m)=\sigma_{d_{b}}^{2} a_{b}(m)-\int_{-\infty}^{\infty} a_{f}(u)\left(\sigma_{d_{0}^{(r)}}^{2} z_{0}^{(1)}\left((D+m) T_{0}-t_{0}^{(n)}-u\right) e^{\prime 2 \pi \varphi_{1,}^{(n)}}+\right. \\
& \left.\sigma_{\substack{(1) \\
t_{1}}}^{2}=_{0,0}^{(2)}\left((D+m) T_{0}-t_{0}^{(i)}-u\right) e^{j 2 \pi \varphi_{1 j}^{(1)}}\right) d(u)
\end{aligned}
$$


Therefore the optimum coefficient for feed back filters are :

$$
\begin{aligned}
& a_{b}(m)= \begin{cases}\left(\frac{1}{\sigma_{d_{0}}^{2}}\right) \int_{-\infty}^{\infty} a_{f}(u)\left(\sigma_{d_{0}^{(r)}}^{2} z_{0}^{(1)}\left((D+m) T_{0}-t_{0}^{(r)}-u\right) e^{j 2 \pi \phi_{0}^{(r)}}+\right. \\
\left.\sigma_{d_{0}^{(1)}}^{2} z_{0}^{(2)}\left((D+m) T_{0}-t_{0}^{(i)}-u\right) e^{j 2 \pi \phi_{0}^{(1)}}\right) d(u) & m>0 \\
0 & m \leq 0\end{cases} \\
& a_{b}(m)=\left\{\begin{array}{cc}
\left(\frac{1}{\sigma_{d_{0}}^{2}}\right)\left[a _ { f } ( t ) \otimes \left(\sigma_{d_{0}^{(r)}}^{2} z_{0}^{(1)}\left(t-t_{0}^{(r)}\right) e^{j 2 \pi \phi_{0}^{(r)}}+\right.\right. & m>0 \\
\sigma_{\left.\left.d_{0}^{(1)} z_{0}^{(2)}\left(t-t_{0}^{(i)}\right) e^{j 2 \pi \phi_{0}^{(1)}}\right)\right]\left.\right|_{t=(D+m) T_{0}}}^{2} & m \leq 0
\end{array}\right.
\end{aligned}
$$

For BPSK and balanced QPSK, (4.53) is simplified to the following equation :

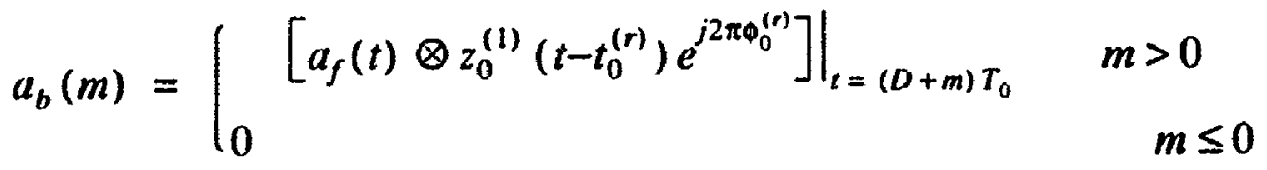

and for OQPSK, it is simplified to :

$a_{b}(m)=\left\{\begin{array}{cc}{\left.\left[a_{f}(t) \otimes\left(z_{0}^{(1)}\left(t-t_{0}^{(r)}\right)+z_{0}^{(1)}\left(t-t_{0}^{(r)}-\frac{T_{0}}{2}\right)\right) e^{j 2 \pi \omega_{0}^{(r)}}\right]\right|_{f=(D+m) T_{0}}} & m>0 \\ 0 & m \leq 0\end{array}\right.$

The same procedure has to be repeated to determine the optimum forward filter. The gradient of TAMSE according to $a_{f}(t)$ in the time $\mathrm{u}$ is :

$$
\nabla_{a_{f}}^{\text {TAMSE }}(u)=\left\langle E\left\{\left(\tilde{y}(n)-\left(d_{0}(n-D)+f(n)\right)\right) \nabla_{a_{f}}^{\vec{y}^{*}(n)}(u)\right\}\right\rangle
$$

From (4.47)

$$
\nabla_{t_{f}^{*}}^{y^{*}(n)}(u)=x^{*}\left(n T_{0}-u\right)
$$

Replacing the above equation in (4.56) and also considering (4.49), second and 3th term of (4.56) will be : 


$$
\begin{gathered}
\left\langle E\left\{d_{0}(n-D) \nabla_{a_{f}}^{*}(n)(u)\right\}\right\rangle=\sigma_{d_{0}^{(r)}}^{2} z_{0}^{(1)^{*}}\left(D T_{0}-t_{0}^{(r)}-u\right) e^{-j 2 \pi \phi_{0}^{(n)}}+ \\
\sigma_{d_{0}^{(1)}}^{2} z_{0}^{(2)^{*}}\left(D T_{0}-t_{0}^{(1)}-u\right) e^{-j 2 \pi \phi_{0}^{(1)}} \\
\left\langle E\left\{f(n) \nabla_{a_{f}}^{\vec{v}(n)}(u)\right\}\right\rangle=\sigma_{d_{0}^{(n)}}^{2} e^{-j 2 \pi \phi_{0}^{(n)}} \sum_{m=1}^{\infty} z_{0}^{(1)^{*}}\left((D+m) T_{0}-u-t_{0}^{(r)}\right) a_{b}(m)+ \\
\sigma_{d_{0}^{(1)}}^{2} e^{-j 2 \pi \phi_{0}^{(1)}} \sum_{m=1}^{\infty} z_{0}^{(2)^{*}}\left((D+m) T_{0}-u-t_{0}^{(i)}\right) a_{b}(m)
\end{gathered}
$$

The first term of $(4.56)$ is :

$$
\begin{gathered}
\left\langle E\left\{\tilde{y}(n) \nabla_{a_{f}}^{y^{*}(n)}(u)\right\}\right\rangle=\int_{-\infty}^{\infty} a_{f}(v)\left\langle E\left\{x\left(n T_{0}-v\right) x^{*}\left(n T_{0}-u\right)\right\}\right\rangle d v \\
=\int_{-\infty}^{\infty} a_{f}(v)\left\langle R_{x}\left(n T_{0}-v, n T_{0}-u\right)\right\rangle d v
\end{gathered}
$$

Using (2.58) yields :

$$
\left\langle R_{x}\left(n T_{0}-v, n T_{0}-u\right)\right\rangle=\sum_{m=-\infty}^{\infty} e^{-j \pi\left(\frac{m}{T_{0}}\right)(v+u)} R_{x}^{\frac{m}{T_{0}}}(u-v)
$$

Replacing (4.61) in (4.60) yields :

$$
\begin{gathered}
\left\langle E\left\{\tilde{y}(n) \nabla_{a_{f}}^{*}(n)(u)\right\}\right\rangle=\sum_{m=-\infty}^{\infty} \int a_{f}(v) R_{x x}^{\frac{m}{T_{0}}}(u-v) e^{-j \pi \frac{m}{T_{0}}(u+v)} d v \\
=\sum_{m=-\infty}^{\infty} e^{-j 2 \pi \frac{m}{T_{0}} u}\left[a_{f}(u) \otimes R_{x x}^{\frac{m}{T_{0}}}(u) e^{j \pi \frac{m}{T_{0}} u}\right]
\end{gathered}
$$

Substituting (4.59) and (4.62) in (4.56) and tending the gradient to zero yields the, following equation for the optimum filters : 


$$
\begin{gathered}
\sum_{m=-\infty}^{\infty} e^{-\rho 2 \pi \frac{m}{T_{0}} u}\left[a_{f}(u) \otimes R_{x x}^{\frac{m}{T_{0}}}(u) e^{j \pi\left(\frac{m}{T_{0}}\right) u}\right]= \\
\sigma_{d_{0}^{(r)}}^{2} e^{-\rho 2 \pi \phi_{0}^{(n)}}\left(z_{0}^{(1)^{*}}\left(D T_{0}-u-t_{0}^{(r)}\right)+\sum_{m=1}^{\infty} z_{0}^{(1)^{*}}\left((D+m) T_{0}-u-t_{0}^{(r)}\right) a_{b}(m)\right)+ \\
\sigma_{d_{0}^{(1)}}^{2} e^{-f 2 \pi \phi_{0}^{(1)}}\left(z_{0}^{(2)^{*}}\left(D T_{0}-u-t_{0}^{(i)}\right)+\sum_{m=1}^{\infty} z_{0}^{(2)^{*}}\left((D+m) T_{0}-u-t_{0}^{(i)}\right) a_{b}(m)\right)
\end{gathered}
$$

Taking the Fourier transform of (4.63) with respect to $u$ yields :

$$
\begin{aligned}
& \sum_{m=-\infty}^{\infty} A_{f}\left(f+\frac{m}{T_{0}}\right) S_{x x}^{\frac{m}{T_{0}}}\left(f+\frac{m}{2 T_{0}}\right) \\
& =\sigma_{d_{0}^{(f)}}^{2} e^{-j 2 \pi \theta_{0}^{(n)}}\left[1+\sum_{m=1}^{\infty} a_{b}(m) e^{-j 2 \pi m T_{0} f}\right] e^{-j 2 \pi\left(D T_{0}-t_{0}^{(p)}\right) f_{Z_{0}}^{(1)}}{ }^{*}(f)+ \\
& \sigma_{d_{0}^{(1)}}^{2} e^{-j 2 \pi \phi_{0}^{(1)}}\left[1+\sum_{m=1}^{\infty} a_{b}(m) e^{-j 2 \pi m T_{0} f}\right] e^{-j 2 \pi\left(D T_{0}-\varepsilon_{0}^{(1)}\right) f_{Z_{0}^{(2)}}^{(2)}}(f) \\
& =\left(\sigma_{d_{0}^{(r)}}^{2} e^{-j 2 \pi\left(\phi_{0}^{(n)}+\left(D T_{0}-t_{0}^{(n)}\right) f\right.} Z_{0}^{(1)^{*}}(f)+\sigma_{d_{0}^{(1)}}^{2} e^{-j 2 \pi\left(\phi_{0}^{(1)}+\left(D T_{0}-t_{0}^{(1)}\right) f\right)} Z_{0}^{(2)^{*}}(f)\right) \text {. } \\
& \left(A_{b}\left(e^{j 2 \pi f T_{0}}\right)+1\right)
\end{aligned}
$$

where $A_{f}(f)$ and $A_{b}\left(e^{j 2 \pi f}\right)$ are the Fourier transforms of $a_{f}(t)$ and $a_{b}(t)$ respectively. Equation (4.64) is simplified to the following equation for BPSK and balanced QPSK :

$$
\begin{gathered}
\sum_{m=-\infty}^{\infty} A_{f}\left(f+\frac{m}{T_{0}}\right) S_{x x}^{\frac{m}{T_{0}}}\left(f+\frac{m}{2 T_{0}}\right) \\
=\left(\sigma_{d_{0}}^{2} e^{-j 2 \pi \mid \phi_{0}^{(n)}+\left(D I_{0}-t_{0}^{(n)}\right) / n} Z_{0}^{(1)^{*}}(f)\right) \cdot\left(A_{b}\left(e^{j 2 \pi f T_{0}}\right)+1\right)
\end{gathered}
$$

and simplified to :

$$
\begin{gathered}
\sum_{m=-\infty}^{\infty} A_{f}\left(f+\frac{m}{T_{0}}\right) S_{x x}^{\frac{m}{T_{0}}}\left(f+\frac{m}{2 T_{0}}\right) \\
=\left(\sigma_{d_{0}}^{2} e^{-j 2 \pi \mid \varphi_{0}^{(s)}+\left(D T_{0}-t_{0}^{(r)}\right) / n} Z_{0}^{(1)^{*}}(f)\right)\left(1+e^{j \pi f T_{0}}\right)\left(A_{b}\left(e^{j 2 \pi f T_{0}}\right)+1\right)
\end{gathered}
$$

for OQPSK. 
The minimum TAMSE (MTAMSE) is the correlation between the error and data [88], therefore :

$$
\begin{aligned}
\text { MTAMSE } & =\left\langle E\left\{\left(d_{0}(n-D)-b(n)\right) d_{0}^{*}(n-D)\right\}\right\rangle \\
& =\sigma_{d_{0}}^{2}-\left\langle E\left\{d_{0}^{*}(n-D) \bar{y}(n)\right\}\right\rangle
\end{aligned}
$$

Using (4.49), the MTAMSE which can be reached is equal to the following equation :

$$
\begin{gathered}
\text { MTAMSE }=\sigma_{d_{0}}^{2}-\int_{-\infty}^{\infty} a_{f}(u)\left(\sigma_{d_{0}^{(1)}}^{2} e^{j 2 \pi \phi_{0}^{(r)}} z_{0}^{(1)}\left(D T_{0}-u-t_{0}^{(r)}\right)+\right. \\
\left.\sigma_{d_{0}^{(j)}}^{2} e^{j 2 \pi \phi_{0}^{(1)}} z_{0}^{(2)}\left(D T_{0}-u-t_{0}^{(i)}\right)\right) d(u) \\
=\sigma_{d_{0}}^{2}-\left.\left[a_{f}(t) \otimes\left(\sigma_{d_{0}^{(r)}}^{2} e^{j 2 \pi \phi_{0}^{(r)}} z_{0}^{(1)}\left(t-t_{0}^{(r)}\right)+\sigma_{d_{0}^{(1)}}^{2} e^{j 2 \pi \phi_{0}^{(1)}} z_{0}^{(2)}\left(t-t_{0}^{(i)}\right)\right)\right]\right|_{t=D T_{u}}
\end{gathered}
$$

\subsubsection{Finite length}

If the FSE and the feedback filter are modeled by finite length tap delay lines, the DFE structure will be as Figure 4.14. In the figure, the output of DFE can be found according to the following equation :

$$
b(n)=a_{f} \tilde{x}\left(n M_{0}\right)^{T}-a_{b} d(n-D-1)
$$

where (.) ${ }^{T}$ denotes the transpose of the argument,

$$
\begin{gathered}
\underline{a}_{f}^{T}=\left[\begin{array}{ll}
a_{f}(0) & a_{f}(1) \ldots a_{f}\left(N_{f}\right)
\end{array}\right] \\
\underline{a}_{b}^{T}=\left[\begin{array}{ll}
a_{b}(1) & a_{b}(2) \ldots a_{b}\left(N_{b}\right)
\end{array}\right] \\
\tilde{x}^{T}\left(n M_{0}\right)=\left[\begin{array}{lll}
\tilde{x}\left(n M_{0}\right) & \tilde{x}\left(n M_{0}-1\right) \ldots \tilde{x}\left(n M_{0}-N_{f}\right)
\end{array}\right] \\
\underline{d}^{T}(n-D-1)=\left[\begin{array}{ll}
d_{0}(n-D-1) & d_{0}(n-D-2) \ldots d_{0}\left(n-D-N_{b}\right)
\end{array}\right]
\end{gathered}
$$

where $\mathbf{N}_{f}$ and $\mathbf{N}_{b}$ are the number of taps in the forward and feedback filters, respectively. Defining :

$$
\begin{gathered}
\underline{W}^{T}=\left[\begin{array}{ll}
\underline{a}_{f}^{T} & -\underline{a}_{b}^{T}
\end{array}\right] \\
\underline{V}^{T}(n)=\left[\begin{array}{ll}
\tilde{x}^{T}\left(n M_{0}\right) & d^{T}(n-D-1)
\end{array}\right]
\end{gathered}
$$

and substituting in (4.69) yields : 

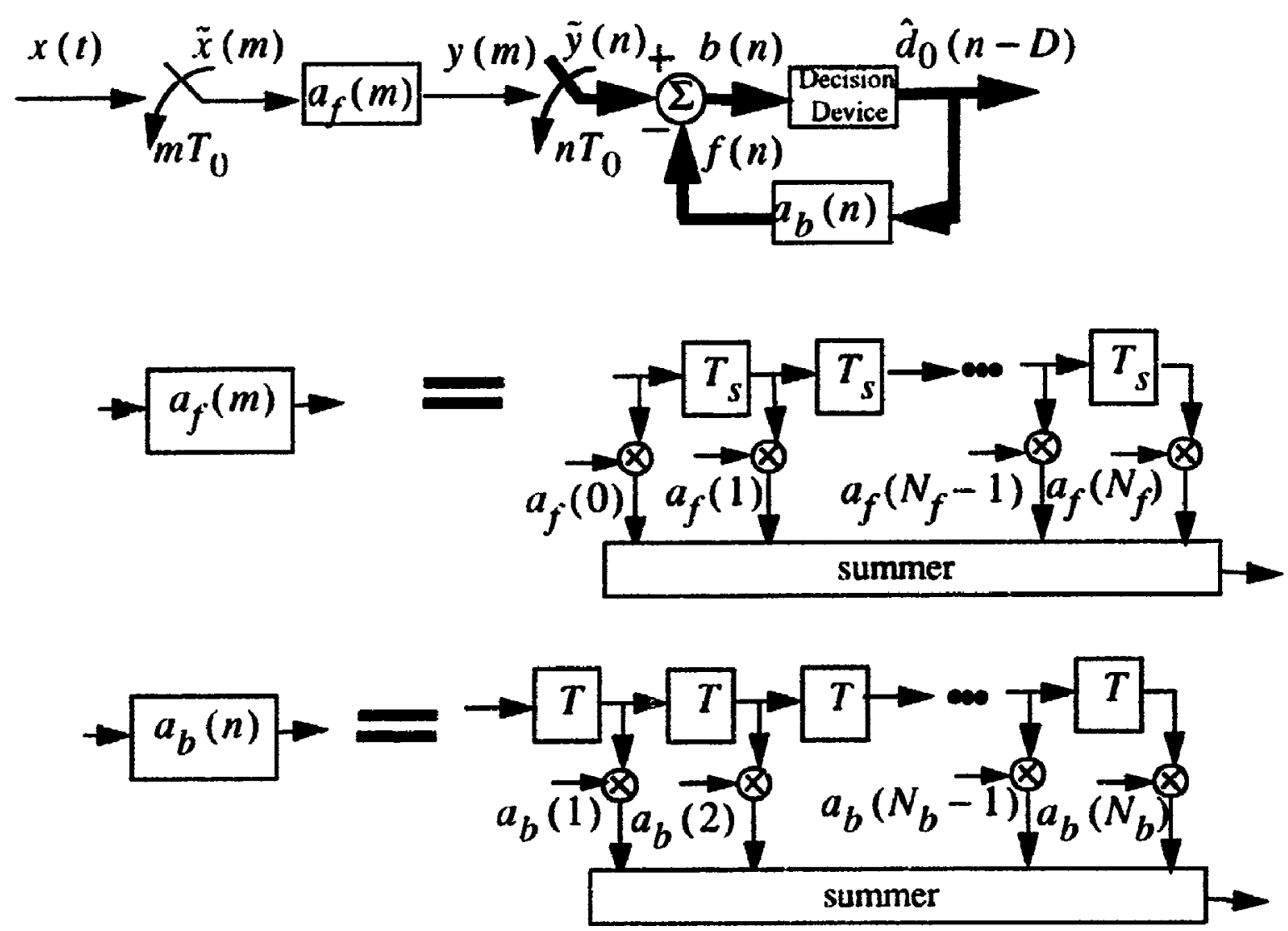

Figure 4.14 Finite length conventional DFE. 


$$
b(n)=\underline{W}^{r} \underline{V}(n)
$$

Therefore the optimum Weiner tilter coefficients can be found by the following equation [52] :

where

$$
\underline{W}_{0}=\underline{R}^{-1} P
$$

$$
\begin{gathered}
R=\left\langle E\left\{\left[\underline{V}^{T}(n) \underline{V}^{*}(n)\right]\right\}\right\rangle \\
P=\left\langle E\left\{\left[\underline{V}^{T}(n) d_{0}^{*}(n-D)\right]\right\}\right\rangle
\end{gathered}
$$

Substituting (4.71) in (4.74) yields :

$$
\begin{array}{r}
R=\left[\begin{array}{cc}
\left\langle E\left\{\tilde{x}^{T}\left(n M_{0}\right) \tilde{x}^{*}\left(n M_{0}\right)\right\}\right\rangle & \left\langle E\left\{\tilde{x}^{T}\left(n M_{0}\right) d^{*}(n-D-1)\right\}\right\rangle \\
\left\langle E\left\{d^{T}(n-D-1) \vec{x}^{*}\left(n M_{0}\right)\right\}\right\rangle & \left\langle E\left\{d^{T}(n-D-1) d^{*}(n-D-1)\right\}\right\rangle
\end{array}\right] \\
=\left[\begin{array}{cc}
R_{\tilde{x}} & R_{\tilde{x} d} \\
R_{d \tilde{x}} & \sigma_{d_{0}}^{2} I_{N_{b} \times N_{b}}
\end{array}\right]
\end{array}
$$

where $I_{N_{b} \times N_{b}}$ is the $N_{b}$ by $N_{b}$ unit matrix. $\underline{R}_{\tilde{x}}$ can be found by :

$$
\begin{aligned}
\underline{R}_{\underline{x}}(i, k) & =\left\langle E\left\{\tilde{x}\left(n M_{0}-i-1\right) \tilde{x}^{*}\left(n M_{0}-k-1\right)\right\}\right\rangle \\
& =\left\langle R_{\bar{x}}\left(n M_{0}-i-1, n M_{0}-k-1\right)\right\rangle \\
& =\sum_{m=-\infty}^{\infty} R_{\bar{x}}^{\frac{m}{T_{0}}}\left((k-i) M_{0}\right) e^{j \pi m(k-\imath)} \\
=\sum_{l=-\infty}^{\infty} \sum_{m=-\infty}^{\infty} R_{x}^{\frac{m}{T_{0}}+\frac{l}{T_{s}}}\left((k-i) M_{0} T_{s}\right) e^{j \pi m(k-i)} e^{j \pi l(k-\imath) M_{0}} & \\
= & \sum_{l=-\infty}^{\infty} \sum_{m=-\infty}^{\infty} R_{x}^{\frac{m+l M_{0}}{T_{0}}}\left((k-i) T_{0}\right) e^{j \pi\left(m+l M_{0}\right)(k-l)}
\end{aligned}
$$

and $\underline{R}_{x d}$ can be found by :

$$
\begin{aligned}
& B_{\text {sqd }}(i, k)=\left\langle E\left\{\bar{x}\left(n M_{0}-i-1\right) d^{*}(n-D-k)\right\}\right\rangle \\
& =\sigma_{d_{0}^{(n)}}^{2} z_{0}^{(1)}\left((D+k) T_{0}-(i+1) T_{s}-t_{0}^{(r)}\right) e^{f 2 \pi \theta_{01}^{(n)}}+ \\
& \sigma_{d_{01}^{(1)}}^{2} z_{0}^{(2)}\left((D+k) T_{0}-(i+1) T_{5}-t_{0}^{(i)}\right) e^{j 2 \pi 0_{10}^{(1)}}
\end{aligned}
$$


and consequently $\boldsymbol{B}_{d x}$

$$
\begin{aligned}
& \underline{R}_{d x}(i, k)=\left\langle E\left\{d(n-D-i) \vec{x}^{*}\left(n M_{0}-k-1\right)\right\}\right\rangle \\
= & \sigma_{d_{01}^{(1)}}^{2} z_{(1)}^{(1) *}\left((D+i) T_{0}-(k+1) T_{s}-t_{0}^{(r)}\right) e^{-j 2 \pi 0_{0}^{(n)}}+ \\
& \sigma_{d_{01}^{(1)}}^{2} z_{0}^{(2) *}\left((D+i) T_{0}-(k+1) T_{s}-t_{0}^{(i)}\right) e^{-j 2 \pi 0_{01}^{(1)}}
\end{aligned}
$$

On the other hand, the vector $P$ can be calculated by the following procedure :

$$
\begin{gathered}
P=\left\langle E\left\{\left[\underline{V}^{T}(n) d_{0}^{*}(n-D)\right]\right\}\right\rangle \\
=\left[\begin{array}{c}
\left\langle E\left\{\underline{x}^{T}\left(n M_{0}\right) d_{0}^{*}(n-D)\right\}\right\rangle \\
\left\langle E\left\{\underline{d}^{T}(n-D-1) d_{0}^{*}(n-D)\right\}\right\rangle
\end{array}\right] \\
=\left[\begin{array}{c}
P_{j u d} \\
0_{N_{k} \times 1}
\end{array}\right]
\end{gathered}
$$

where ${ }^{0} N_{r, \times 1}$ is the $N_{b}$ by one zero vector and

$$
\begin{gathered}
\underline{P}_{j d}(i)=\sigma_{d_{0}^{(n)}}^{2} z_{0}^{(1)}\left(D T_{0}-(i+1) T_{s}-t_{0}^{(r)}\right) e^{j 2 \pi 0_{0}^{(r)}}+ \\
\sigma_{d_{0}^{(1)}}^{2} z_{0}^{(2)}\left(D T_{0}-(i+1) T_{s}-t_{0}^{(i)}\right) e^{j 2 \pi \theta_{0}^{(n)}}
\end{gathered}
$$

The corresponding MTAMSE can be calculated from the optimum coefficient oy the following equation :

$$
M T A M S E=\sigma_{d_{0}}^{2}-\underline{P}^{*} \underline{W}_{0}
$$

It is apparent from (4.64) and (4.76) that the conventional DFE does not use the intormation of the CCSD of the received signal. Furthermore, it does not use the information of incommensurate periodicities of the CSD. Therefore, to obtain the optimum DFE, we should use GFSE or FILESH-FSE as the forward tilter of DFF. We shall refer to these structures as generalized DFE (GDFE) and frequency shift DFE (FRESH-DFE).

\subsection{FRESH-DFE}

Figure 4.15 shows the structure of FRESH-DFE. This structure is created by FRESH-FSE followed by a teedback path. Generally FRESH-DFE can be divided into 


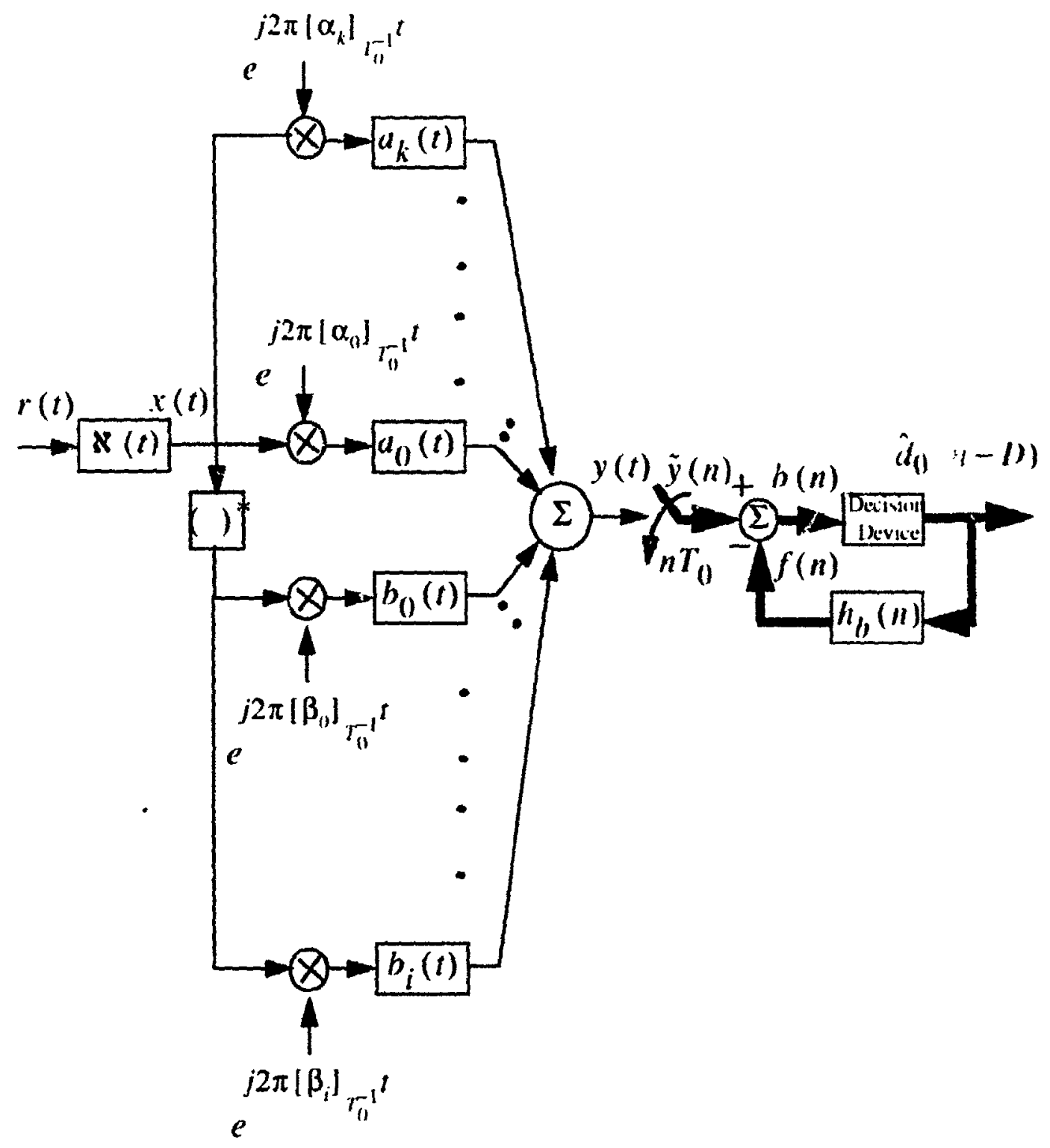

Figure 4.15 The :---ucture of FRESH-DFE. 


$$
\frac{2}{\underline{\underline{W}}}
$$


three parts. The tirst part is a LCL- FRESH filter, the second part is a baud rate simpler and the third part is a feedback filter. The first part exploits the information of the CSD and CCSD at incommensurate cyclic frequencies, the second part extracts the information at the symbol rate and it's harmonics and the third part removes the residual ISI caused by earlier symbols.

Similar to the conventional DFE, FRESH-DFE minimizes the TAMSE too. In Appendix B, FRESH-DFE is analyzed by the method used for the conventional DFE. The results show that the optimuis forward and backward tilters must satisfy the following equations :

$$
\begin{aligned}
& \int \frac{\sigma_{d_{0}^{(r)}}^{2}}{\sigma_{d_{0}}^{2}}\left[\left(a_{0}(t) \otimes z_{0}^{(1)}\left(t-t_{0}^{(r)}\right) e^{\left.j 2 \pi 0_{0}^{(r)}\right)+}\right.\right. \\
& h_{b}(m)=\left\{\begin{array}{l}
\left.\left(b_{0}(t) \otimes z_{0}^{(1) *}\left(t-t_{0}^{(r)}\right) e^{-22 \pi \phi_{0}^{(1)}}\right)\right]\left.\right|_{t=(D+m) r_{10}}+ \\
\frac{\sigma_{d_{0}^{(1)}}^{2}}{\sigma_{d_{0}}^{2}}\left[\left(a_{0}(t) \otimes z_{0}^{(2)}\left(t-t_{0}^{(i)}\right) e^{j 2 \pi \phi_{0}^{(1)}}\right)-\right.
\end{array} \quad m>0\right. \\
& \left.\left(b_{0}(t) \otimes z_{0}^{(2) *}\left(t-t_{0}^{(i)}\right) e^{-j 2 \pi \phi_{0}^{(1)}}\right)\right]\left.\right|_{t=(D+m) T_{0}} \\
& \text { 0 } \quad m \leq 0
\end{aligned}
$$

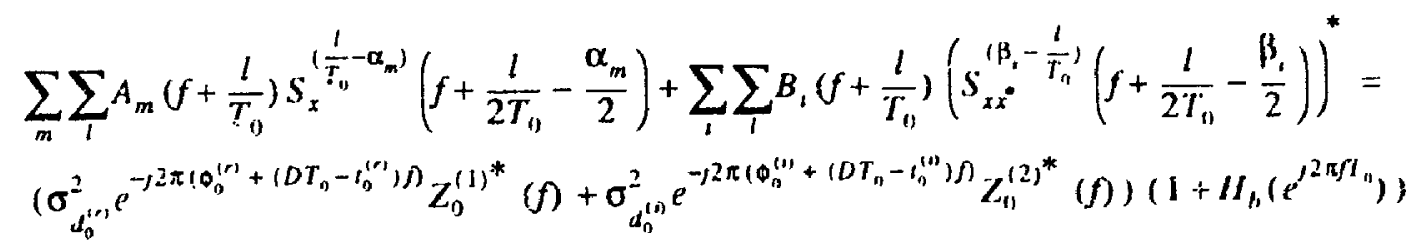

$$
\begin{aligned}
& \sum_{m} \sum_{l} A_{m}\left(f+\frac{l}{T_{1}}\right) S_{x}^{\left(\frac{1}{T_{1}}+\alpha_{k}-\alpha_{m}\right)}\left(f+\frac{l}{2 T_{10}}-\frac{\alpha_{m}+\alpha_{k}}{2}\right)+ \\
& \sum_{1} \sum_{1} B_{1}\left(f+\frac{1}{T_{0}}\right)\left(s_{x \pi^{*}}^{\left(\beta_{1}-u_{k}-\frac{1}{\left.T_{n}\right)}\right.}\left(f+\frac{1}{2 T_{n}}-\frac{\beta_{1}+\alpha_{k}}{2}\right)\right)^{*}=0 \quad\left|\alpha_{k}\right|_{1,1^{\prime}} \neq 0
\end{aligned}
$$

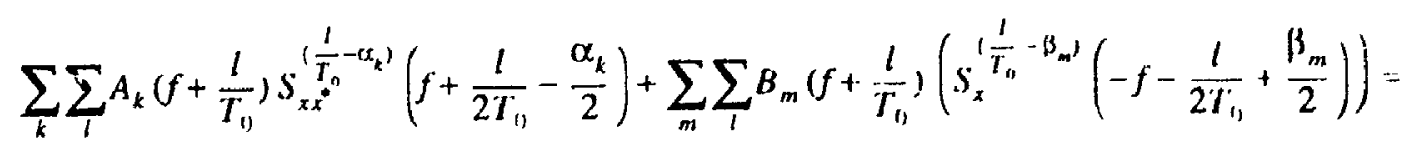

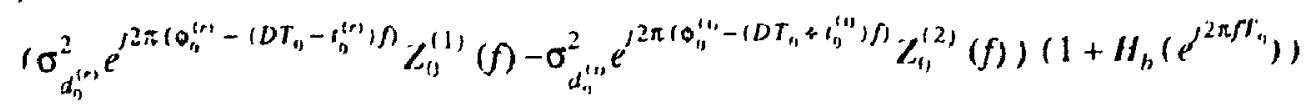




$$
\begin{aligned}
& \sum_{k} \sum_{l} A_{k}\left(f+\frac{l}{T_{0}}\right) S_{x i^{\prime \prime}}^{\left(\frac{l}{T_{0}}+\beta_{1}-\alpha_{k}\right)}\left(f+\frac{l}{2 T_{01}}-\frac{\beta_{1}+\alpha_{k}}{2}\right)+ \\
& \sum_{m} \sum_{j} B_{m}\left(f+\frac{l}{\left.T_{i}\right)}\right)\left(S_{x}^{\left(\beta_{1}-\alpha_{k}-\frac{l}{T_{n}}\right)^{\prime}}\left(-f-\frac{l}{2 T_{t}}+\frac{\beta_{1}+\beta_{m}}{2}\right)\right)=0 \quad\left[\beta_{l}\right]_{T_{0}^{-1}} \neq 0
\end{aligned}
$$

It is alsu shown in Appendix B that the achievable MTAMSE is :

$$
\begin{gathered}
\text { MTAMSE }=\sigma_{d_{0}}^{2}- \\
{\left.\left[a_{0}(t) \otimes\left(\sigma_{d_{0}^{(r)}}^{2} e^{j 2 \pi \sigma_{0}^{(n)}} z_{0}^{(1)}\left(t-t_{0}^{(r)}\right)+\sigma_{d_{0}^{(i)}}^{2} e^{j 2 \pi \theta_{0}^{(1)}} z_{0}^{(2)}\left(t-t_{0}^{(i)}\right)\right)\right]\right|_{t=D T_{0}}-} \\
{\left.\left[b_{0}(t) \otimes\left(\sigma_{d_{0}^{(r)}}^{2} e^{-j 2 \pi 0_{0}^{(r)}} z_{0}^{(1) *}\left(t-t_{0}^{(r)}\right)-\sigma_{d_{0}^{(1)}}^{2} e^{-j 2 \pi \phi_{0}^{(1)}} z_{0}^{(2)^{*}}\left(t-t_{0}^{(i)}\right)\right)\right]\right|_{t=D T_{0}}}
\end{gathered}
$$

Although all the forward filters do not effect (4.87) directly, their influences in $a_{0}(t)$ and $b_{0}(t)$ effect (4.87) indirectly. According to (4.83) to (4.87), FRESH-DFE uses both CSD and CCSD of the received signal.

It is easy to show that equations (4.82), (4.83), and (4.87) are reduced to equations (4.54), (4.65) and (4.68), respectively, for balanced QPSK systems. Thus, the conventional DFE and FRESH-DFE are equivalent for balanced QPSK systems.

\subsection{GDFE}

Figure 4.16 shows the structure of GDFE. This structure is created by a GFSE followed by a feedback filter. Since the GFSE and FRESH-FSE are equivalent [Appendix C], GDFE and FRESH-DFE are equivalent tco. Therefore, the analysis for FRESH-DFE is valid for GDFE. However, it should be noted that the optimum feed-forward filters of FRESH-DFE and GDFE are the frequency-time dual. The optimum feedback filter of GDFE and FRESH-DFE are equivalent.

In the single symbol rate systems, the structure of GDFE and FRESH-DFE will be identical (Figure 4.17). We refer to this structure as the optimum DFE (ODFE). The complexity of ODFE is minimized if the channel spacings are multiple of half the symbol rate (Figure 4.18). In this case the structure of ODFE is almost as simple as DFE. 


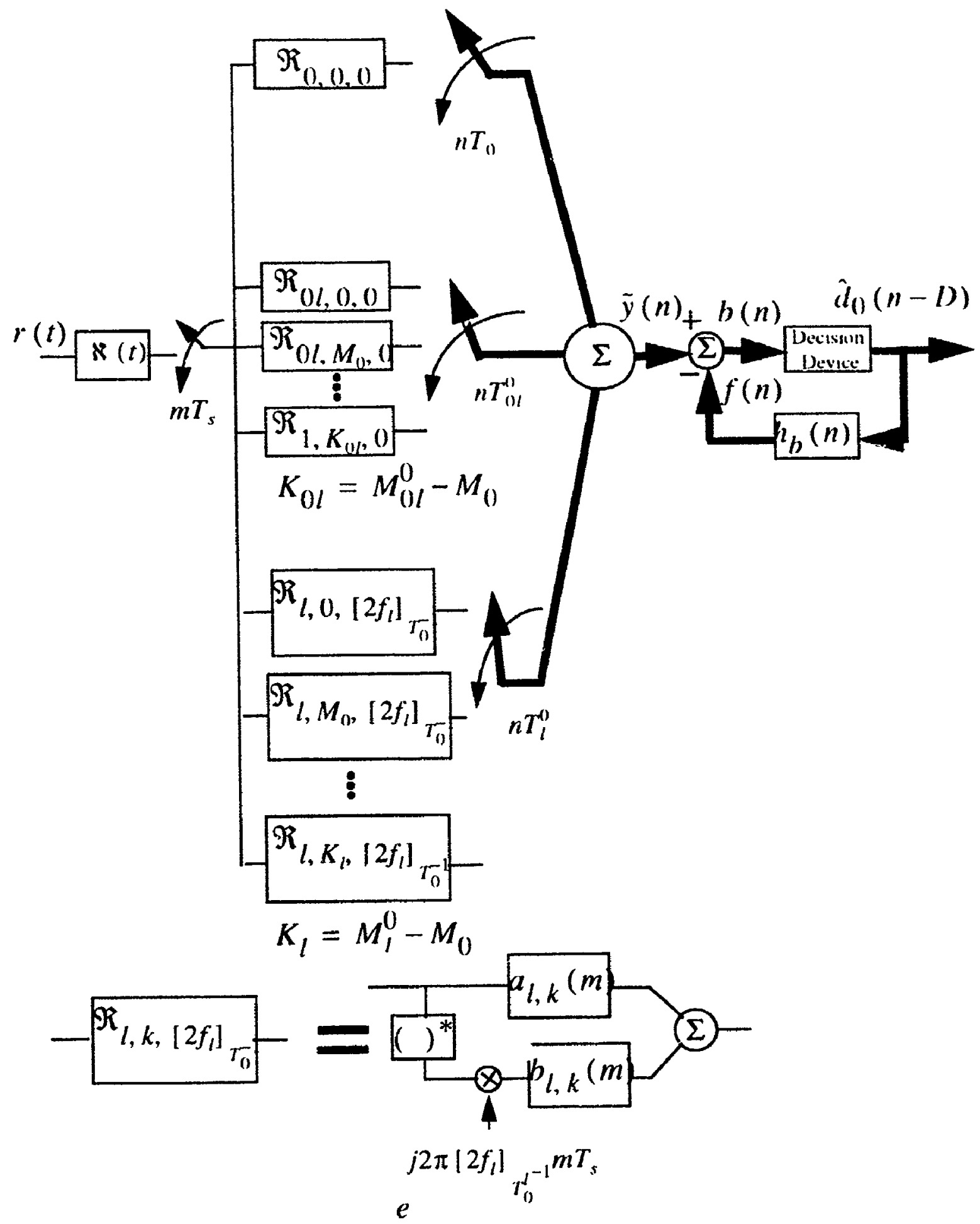

Figure 4.16 The structure of GDFE. 


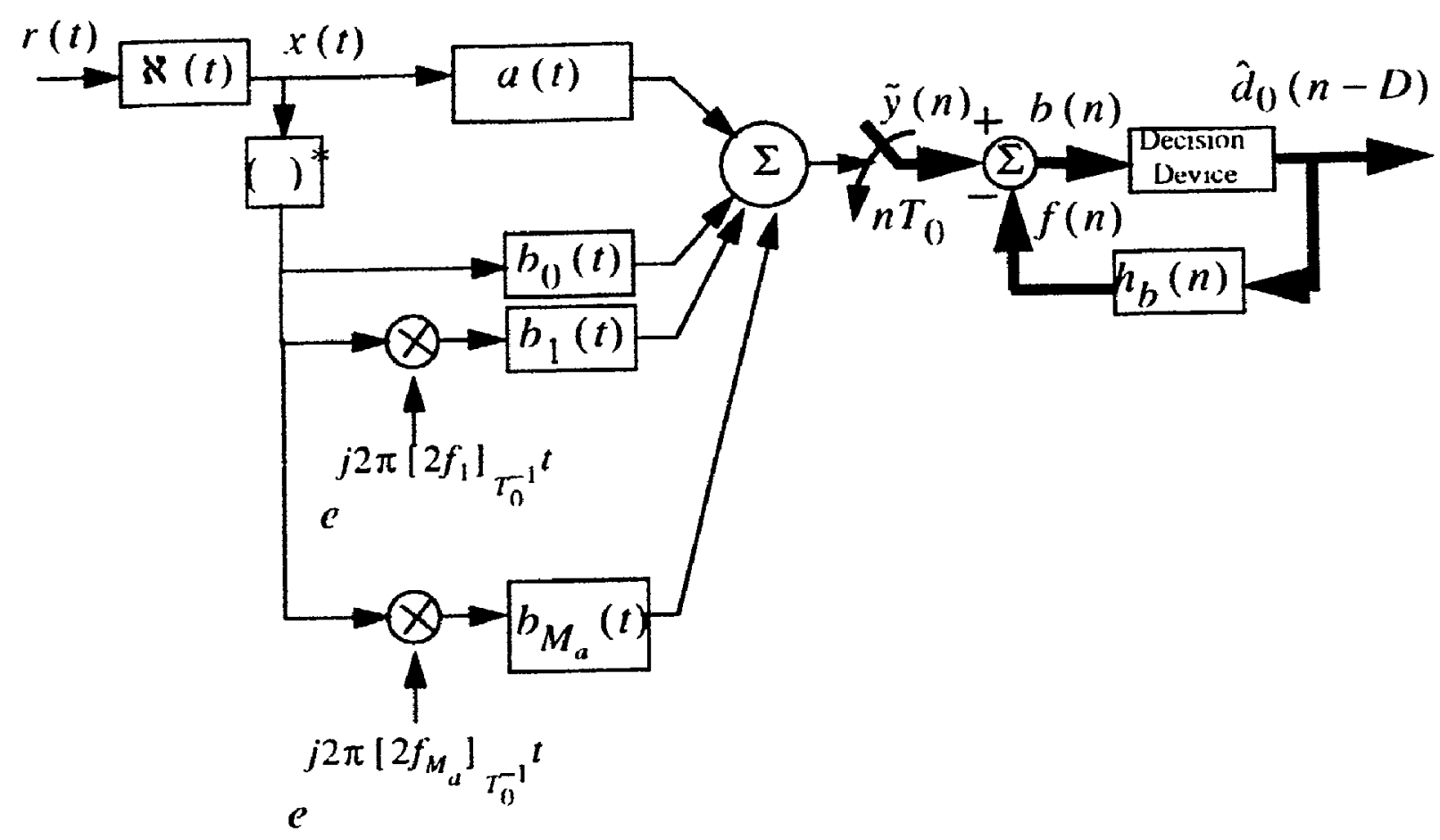

Figure 4.17 The structure of ODFE.

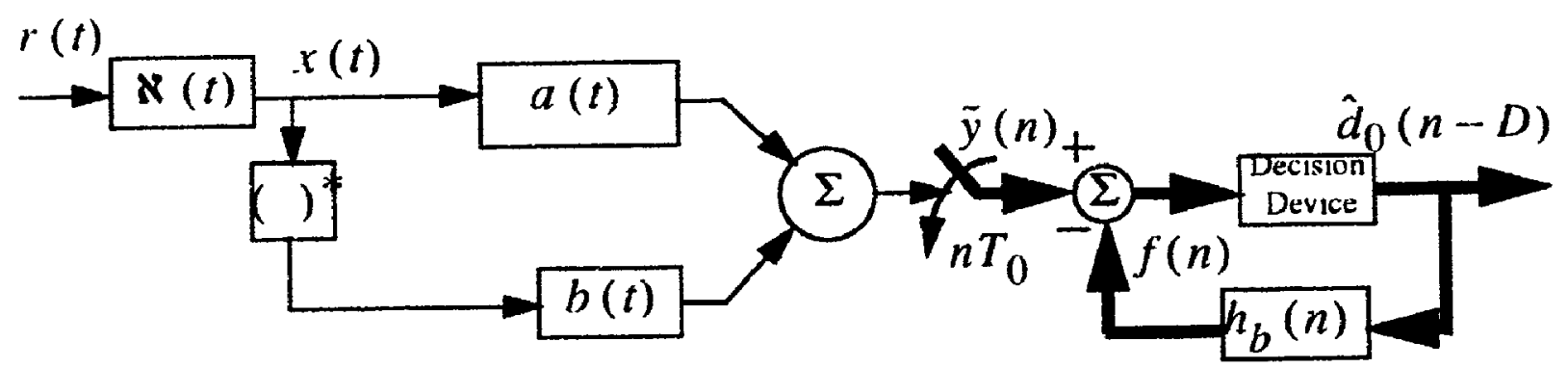

Figure 4.18 The structure of ODFE in systems with channel spacings multiple of half the symbol rate. 


\subsection{ODFE}

Since ODFE is a special case of FRESH-DFE (or GDFE), the analysis of intinite length FRESH-DFE (Appendix B) is valid for ODF too. However, the tinite length ODFE is analyzed in the next section

\subsubsection{Finite Length ODFE}

The analysis is simpler if we consider the special case in which the channel spacings are multiple of half the symbol rate. However, the analysis can be generalized for any other cases. Figure 4.19 shows the structure of the finite length ODFE. In the tigure, the output of DFE can be found according to the following equation :

$$
b(n)=\underline{a}_{l} \dot{x}\left(n M_{0}\right)^{T}+\underline{a}_{c} \tilde{x}\left(n M_{0}\right)^{\prime \prime}-\underline{a}_{b} \underline{d}(n-D-1)
$$

where $(.)^{H}$ denotes the conjugate transpose of the argument,

$$
\begin{gathered}
\underline{a}_{l}^{T}=\left[\begin{array}{ll}
a_{l}(0) & a_{l}(1) \ldots a_{l}\left(N_{f}\right)
\end{array}\right] \\
\underline{a}_{c}^{T}=\left[\begin{array}{ll}
a_{c}(0) & a_{c}(1) \ldots a_{c}\left(N_{f}\right)
\end{array}\right] \\
\underline{a}_{b}^{T}=\left[\begin{array}{ll}
a_{b}(1) & a_{b}(2) \ldots a_{b}\left(N_{b}\right)
\end{array}\right] \\
\tilde{x}^{T}\left(n M_{0}\right)=\left[\begin{array}{lll}
\tilde{x}\left(n M_{0}\right) & \tilde{x}\left(n M_{0}-1\right) \ldots \tilde{x}\left(n M_{0}-N_{j}\right)
\end{array}\right] \\
\underline{d}^{T}(n-D-1)=\left[\begin{array}{ll}
d_{0}(n-D-1) & d_{0}(n-D-2) \ldots d_{0}\left(n-D-N_{b}\right)
\end{array}\right]
\end{gathered}
$$

where $N_{f}$ and $N_{b}$ are the number of taps in the forward and feedback filters respectively. Defining :

$$
\begin{gathered}
\underline{W}^{T}=\left[\underline{a}_{l}^{T} \underline{a}_{c}^{T}-\underline{a}_{b}^{T}\right] \\
\underline{V}^{T}(n)=\left[\underline{\tilde{x}}^{T}\left(n M_{0}\right) \underline{\tilde{x}}^{H}\left(n M_{0}\right) \underline{d}^{T}(n-D-1)\right]
\end{gathered}
$$

and substituting in (4.88) yields :

$$
b(n)=\underline{W}^{T} \underline{V}(n)
$$

Therefore, the optimum Weiner filter coefficients can be found by the following equation :

$$
\underline{W}_{0}=\underline{R}^{-1} \underline{P}
$$

where 

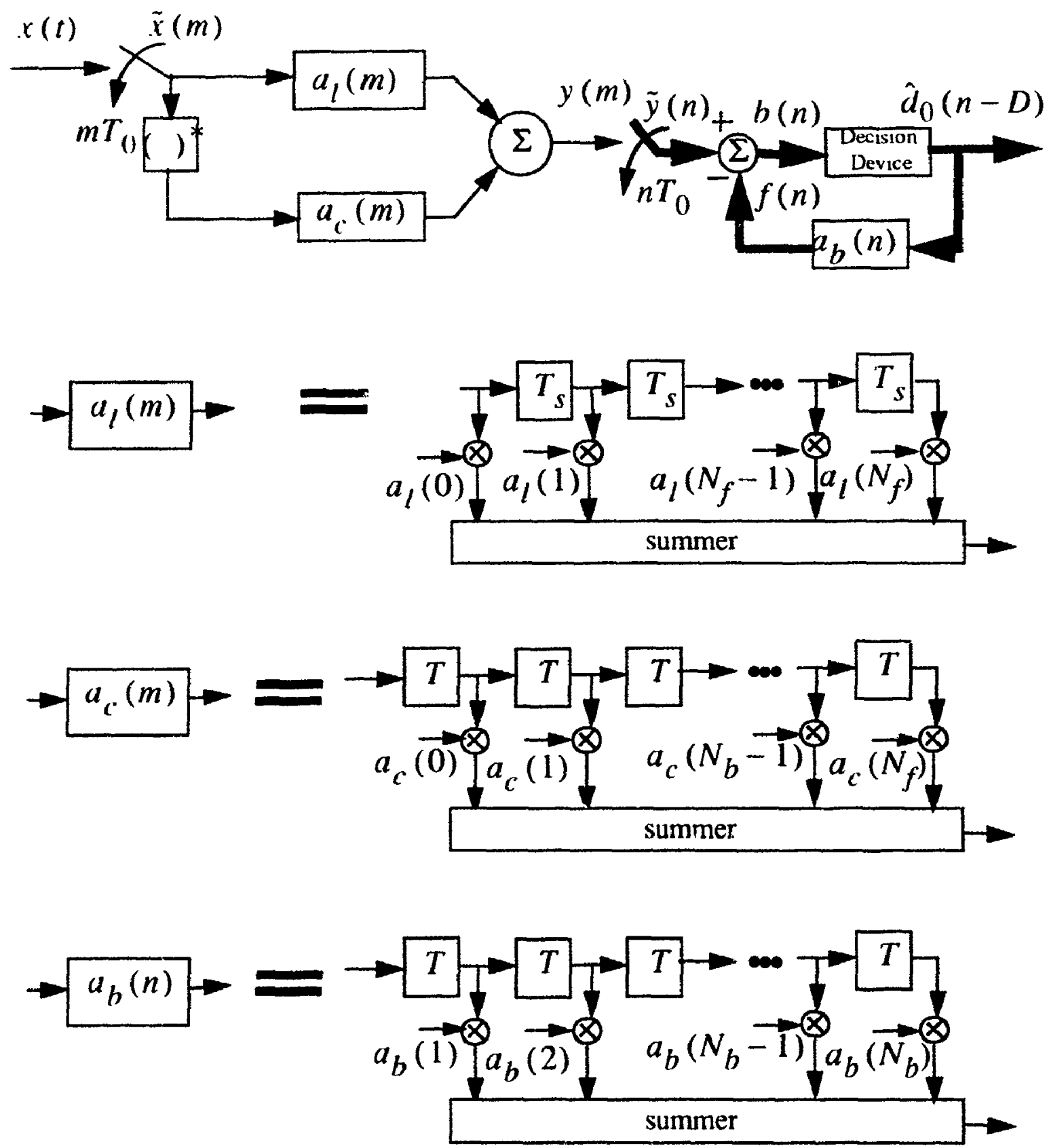

Figure 4.19 The finite length ODFE in the system with channel spacings multiple of half the symbol rate. 


$$
\begin{gathered}
\underline{R}=\left\langle E\left\{\left|\underline{V}^{r}(n) \underline{L}^{*}(n)\right|\right\}\right\rangle \\
\underline{P}=\left\langle E\left\{\left[\underline{V}^{r}(n) d_{0}^{*}(n-D)\right]\right\}\right\rangle
\end{gathered}
$$

Substituting $(4.90)$ in $(4.93)$ yields :

$$
\begin{aligned}
& \underline{R}=
\end{aligned}
$$

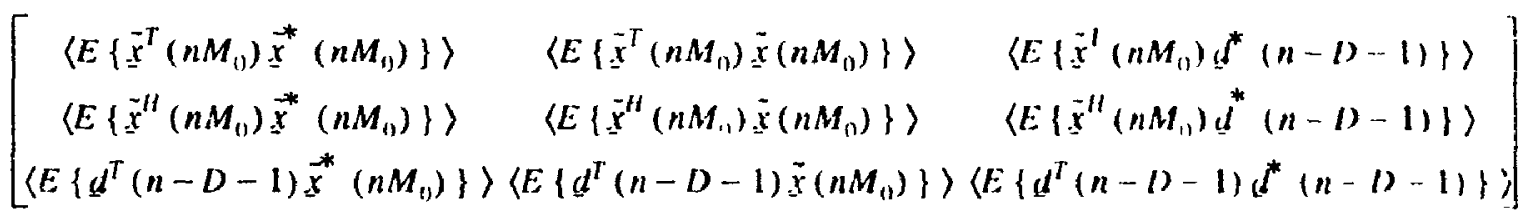

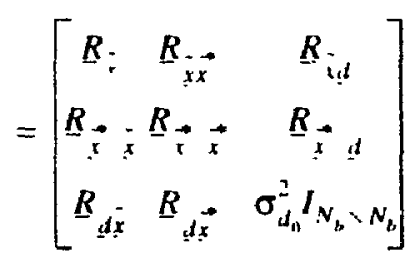

$\underline{R}_{\tilde{x}}, \underline{R}_{\tilde{x} d}$ and $\underline{R}_{d \tilde{x}}$ can be found by (4.76) (4.77)and (4.78). The same procedure as the one used in (4.76), (4.77) and (4.78) can be used to find $\underline{R}_{x_{x}^{*}}, \underline{R}_{x_{x}^{*}}, \underline{R}_{x_{*}^{*}}, \underline{R}_{*}^{*}$ and $\underline{R}_{\underline{d x}}:$

$$
\begin{aligned}
& \underline{R}_{x_{x}^{*} x^{*}}(i, k)=\sum_{l=-\infty m=-\infty}^{\infty} \sum_{x^{*} x^{*}}^{\frac{m+l M_{0}}{T_{0}}}\left((k-i) T_{0}\right) e^{j \pi\left(m+l M_{0}\right)(k-t)} \\
& =\sum_{l=-\infty}^{\infty} \sum_{m=-\infty}^{\infty}\left(R_{x}^{-\frac{m+l M_{0}}{T_{0}}}\left((k-i) T_{0}\right)\right)^{*} e^{j \pi\left(m+l M_{0}\right)(k-l)} \\
& \underline{R}_{\underline{x} x^{*}}(i, k)=\sum_{l=-\infty}^{\infty} \sum_{m=-\infty}^{\infty} R_{x x^{*}}^{\frac{m+l M_{0}}{\Gamma_{0}}}\left((k-i) T_{0}\right) e^{j \pi\left(m+l M_{i, j}\right)(l-l)} \\
& \underline{R}_{x_{x}}(i, k)=\sum_{i=-\infty m=-\infty}^{\infty} \sum_{x^{*}}^{\infty} \frac{m+i M_{0}}{T_{0}}\left((k-i) T_{0}\right) e^{j\left(m+i M_{i}\right)(k-i)} \\
& =\sum_{l=-\infty}^{\infty} \sum_{m=-\infty}^{\infty}\left(R_{x x^{*}}^{-\frac{m+l M_{0}}{T_{0}}}\left((k-i) T_{0}\right)\right)^{*} e^{j \pi\left(m+l M_{0,}\right)(k-l)}
\end{aligned}
$$




$$
\begin{aligned}
& \underline{R}_{\vec{*} d}(i, k)=\sigma_{u_{0}^{(r)}}^{2} z_{i t}^{(1)^{*}}\left((D+k) T_{0}-(i+1) T_{s}-t_{0}^{(r)}\right) e^{-j 2 \pi \theta_{0}^{(r)}}- \\
& \sigma_{d_{t i}^{(i)}}^{2} z_{1)}^{(2) *}\left((D+k) T_{0}-(i+1) T_{s}-t_{0}^{(i)}\right) e^{-j 2 \pi 0_{0}^{(1)}} \\
& \underline{k}_{d \underline{x}}(i, k)=\sigma_{d_{n}^{(r)}}^{2} z_{0}^{(1)}\left((D+i) T_{0}-(k+1) T_{s}-t_{0}^{(r)}\right) e^{j 2 \pi \theta_{0}^{(r)}}- \\
& \sigma_{d_{0}^{(1)}}^{2} z_{0}^{(2)}\left((D+i) T_{0}-(k+1) T_{s}-t_{0}^{(i)}\right) e^{2 \pi \phi_{0}^{(1)}}
\end{aligned}
$$

On the other hand the vector $\underline{P}$ can be calculated by the following procedure :

$$
\begin{gathered}
\underline{P}=\left\langle E\left\{\left[\underline{V}^{T}(n) d_{0}^{*}(n-D)\right]\right\}\right\rangle \\
=\left[\begin{array}{c}
\left\langle E\left\{\underline{\tilde{x}}^{T}\left(n M_{0}\right) d_{0}^{*}(n-D)\right\}\right\rangle \\
\left\langle E\left\{\tilde{x}^{H}\left(n M_{0}\right) d_{0}^{*}(n-D)\right\}\right\rangle \\
\left\langle E\left\{\underline{d}^{T}(n-D-1) d_{0}^{*}(n-D)\right\}\right\rangle
\end{array}\right] \\
=\left[\begin{array}{c}
\underline{P}_{\underline{x} d} \\
\underline{P}_{*}^{*} d \\
0_{N_{b} \times 1}
\end{array}\right]
\end{gathered}
$$

where

$$
\begin{gathered}
\underline{P}_{* a}(i)=\sigma_{d_{0}^{(1)}}^{2} z_{0}^{(1)^{*}}\left(D T_{0}-(i+1) T_{s}-t_{0}^{(r)}\right) e^{-j 2 \pi \varphi_{0}^{(r)}}- \\
\sigma_{d_{0}^{(i)}}^{2} z_{0}^{(2)^{*}}\left(D T_{0}-(i+1) T_{s}-t_{0}^{(i)}\right) e^{-j 2 \pi 0_{0}^{(1)}}
\end{gathered}
$$

The corresponding MTAMSE can be calculated from the optimum coefficients by the following equation :

$$
M T A M S E=\sigma_{d_{0}}^{2}-\underline{P}^{*} \underline{W}_{。}
$$

As it is appareni iron (4.92) and (4.94) that the ODFE uses the information of the CCSD of the received signal as well as CSD.

\subsection{Multi-Antenna Receiver}

Spectral redundancy of digitally modulated signals can be further exploited by 
using multi-antenna receiver. Figure 4.20 shows a block diagram of a multi-antenna receiver. In this figure, superscript $(l)$ represents all the related signal to the $/$ th antenna. If $L$ is the number of antenna, there exist $L$ received signals which can be represented by the following equation :

$$
r_{l}(t)=s_{0}^{(l)}(t)+s_{r}^{(l)}(t)+s_{u}^{(l)}(t)+n^{(l)}(t) \quad l=1,2, \ldots, L
$$

where $n^{(l)}(t), s_{0}^{(l)}(t), s_{u}^{(l)}(t)$ and $s_{\varepsilon^{\prime}}^{(l)}(t)$ are the AWGN, SOl, ACl. and CCl at the input of $l$ th antenna respectively, and can be represented by the following equations :

$$
\begin{aligned}
& s_{0}^{(l)}(t)=\sum_{k=-\infty}^{\infty} d_{0}^{(r)}(k) p_{0}^{(1, l)}\left(t-k T_{0}-t_{0}^{(r)}\right) e^{j 2 \pi \theta_{10}^{(n)}}+ \\
& j d_{0}^{(t)}(k) p_{0}^{(2, l)}\left(t-k T_{0}-t_{0}^{(1)}\right) e^{j 2 \pi 0_{0}^{(1)}} \\
& s_{r}^{(l)}(t)=\sum_{m=1 k}^{M_{c}} \sum_{k=-\infty}^{\infty} d_{0 m}^{(r)}(k) p_{0 m}^{(1, l)}\left(t-k T_{0 m}-t_{0 m}^{(r)}\right) e^{j 2 \pi \theta_{(l m}^{(n)}}+ \\
& j d_{0 m}^{(i)}(k) p_{0 m}^{(2, l)}\left(t-k T_{0 m}-t_{0 m}^{(i)}\right) e^{j 2 \pi \varphi_{0 m m}^{(1)}} \\
& s_{a}^{(l)}(t)=\sum_{m=-M_{d} / 2 k=-\infty}^{M_{d} / 2} \sum_{l \neq 0}^{\infty}\left(d_{m}^{(r)}(k) p_{m}^{(1, l)}\left(t-k T_{m}-t_{m}^{(r)}\right) e^{\left(2 \pi \Phi_{m}^{(n)}\right.}+\right. \\
& \left.j d_{m}^{(i)}(k) p_{m}^{(2, l)}\left(t-k T_{m}-t_{m}^{(i)}\right) e^{j 2 \pi \Phi_{m}^{(1)}}\right) e^{j 2 \pi f_{m} t}
\end{aligned}
$$

where $p_{0 m}^{(1, l)}(t), p_{0 m}^{(2, l)}(t), p_{m}^{(1, l)}(t), p_{m}^{(2, l)}(t), p_{0}^{(1, l)}(t)$, and $p_{0}^{(2, l)}(t)$ are defined by :

$$
\begin{aligned}
& p_{0 m}^{(1, l)}(t)=g_{0 m}^{(l)} q_{0 m}^{(r)}(t) \otimes c_{0 l}^{(l)}(t) \\
& p_{0 m}^{(2, l)}(t)=g_{0 m}^{(l)} q_{0 m}^{(i)}(t) \otimes c_{(1 l)}^{(l)}(t) \\
& p_{m}^{(1, l)}(t)=g_{m}^{(l)} q_{m}^{(r)}(t) \otimes c_{l}^{(l)}(t) \\
& p_{m}^{(2, l)}(t)=g_{m}^{(l)} q_{m}^{(t)}(t) \otimes c_{l}^{(l)}(t) \\
& p_{0}^{(1, l)}(t)=g_{0}^{(l)} q_{0}^{(r)}(t) \otimes c_{0}^{(l)}(t) \\
& p_{0}^{(2, l)}(t)=g_{0}^{(l)} q_{0}^{(i)}(t) \otimes c_{0}^{(l)}(t)
\end{aligned}
$$

The optimum multi-antenna receiver is a generalization of FRESH-DFE (or GDFE). Figure 4.21 shows an optimum multi-antenna FRESH-DFE (MA-FRESH-DFE). 
mth $\mathrm{ACl}$ source

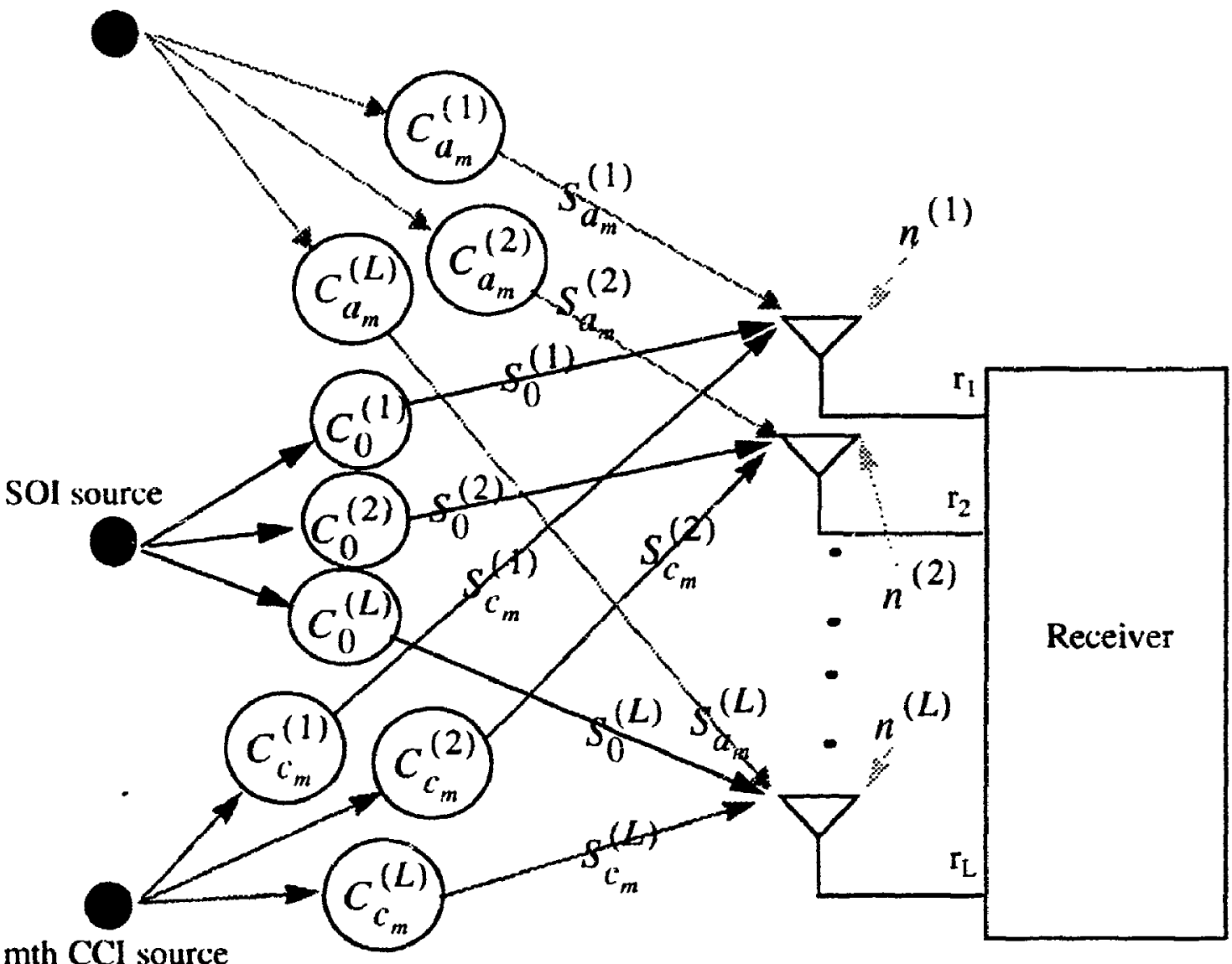

mth $\mathrm{CCl}$ source

Figure 4.20 Communication with a mulit-antenna receiver. 


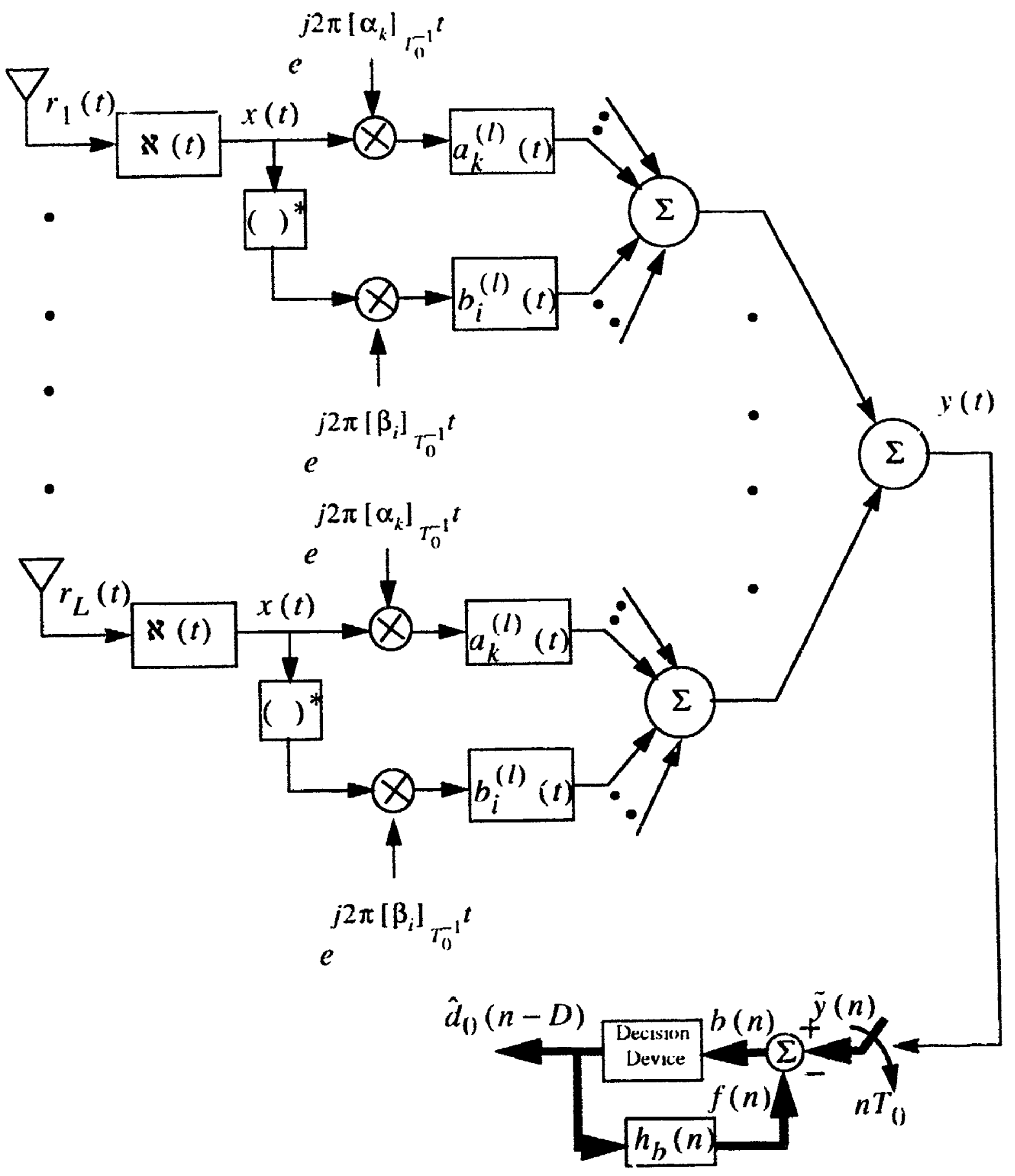

Figure 4.21 Multi Antenna FRESH-DFE. 
In this tigure, L FRESH-filter are used in forward filtering to exploit all the spectral redundancy of the $L$ received signals and a backward filter is used to reduce the effect of ISI.

The optimum solution for MA-FRESH-DFE filters can be found by generalizing (4.82) to (4.86) as follows :

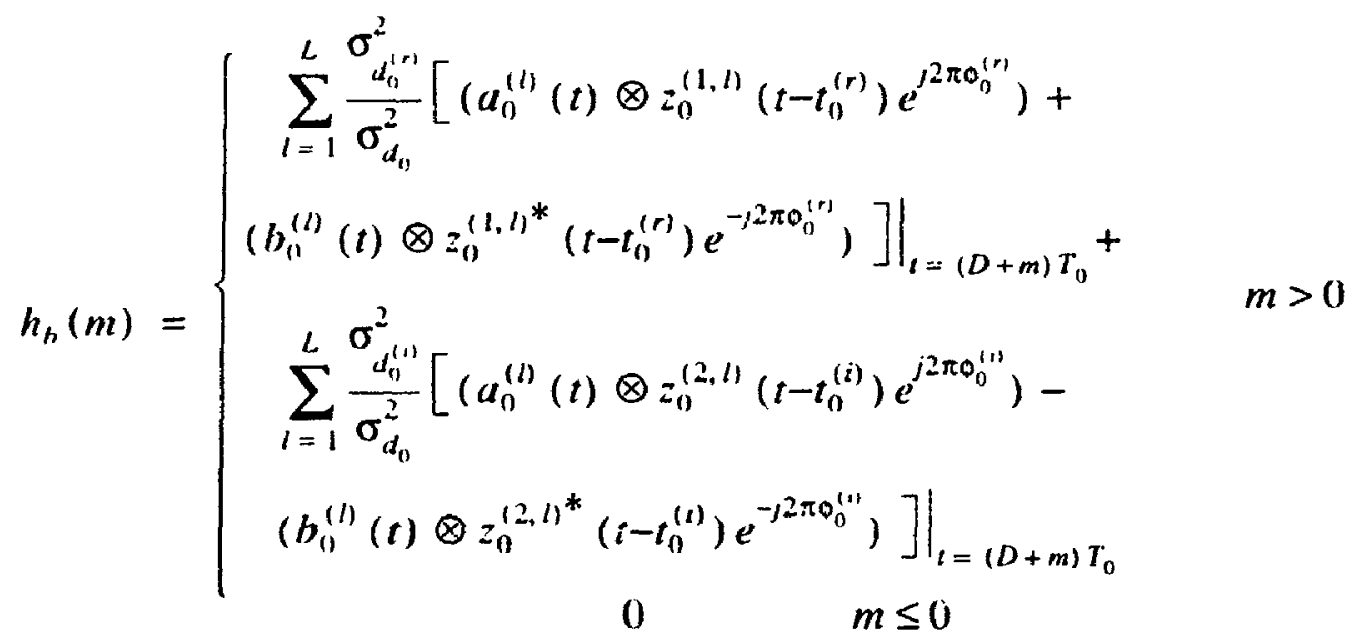

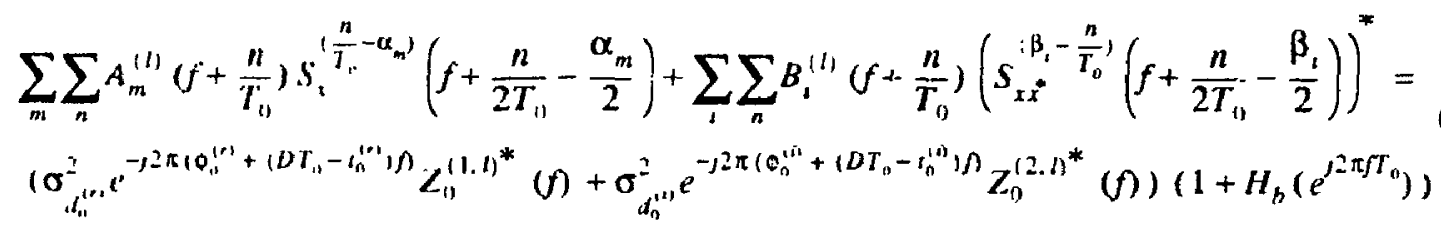

$$
\begin{aligned}
& \sum_{m} \sum_{n} A_{m}^{(n)}\left(f+\frac{n}{T_{0}}\right) S_{x_{i}}^{\left(\frac{n}{T_{n}}+\alpha_{k}-\alpha_{m}\right)}\left(f+\frac{n}{2 T_{0}}-\frac{\alpha_{m}+\alpha_{k}}{2}\right)+ \\
& \sum_{1} \sum_{i} B_{1}^{(\prime)}\left(f+\frac{n}{T_{1}}\right)\left(s_{x_{1} x_{i}^{*}}^{\left(\beta_{3}-\alpha_{k}-\frac{3}{T_{11}}\right.}\left(f+\frac{n}{2 T_{0}}-\frac{\beta_{1}+\alpha_{k}}{2}\right)\right)^{*}=0 \quad\left[\alpha_{k}\right]_{T_{0}{ }^{1}} \neq 0 \\
& \sum_{i} \sum_{n} A_{k}^{(l)}\left(f+\frac{n}{T_{n}}\right) s_{\alpha_{i} s_{i}^{(}}^{\left(\frac{n}{T_{2}}-u_{k}\right)}\left(f+\frac{n}{2 T_{0}}-\frac{\alpha_{k}}{2}\right)+\sum_{m} \sum_{i} B_{m}^{(l)}\left(f+\frac{n}{T_{0}}\right) S_{x_{j}}^{\left(\frac{n}{T_{0}}-\beta_{m}\right)}\left(-f-\frac{n}{2 T_{0}}+\frac{\beta_{m}}{2}\right)=
\end{aligned}
$$

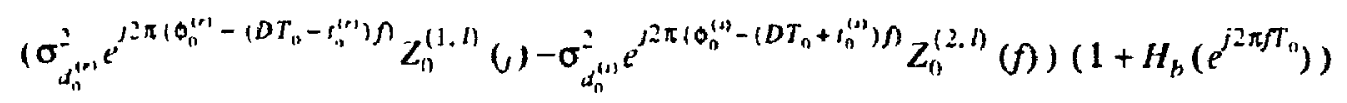

$$
\begin{aligned}
& \sum_{k} \sum_{n} A_{k}^{(1)}\left(f+\frac{n}{T_{n}}\right) S_{x_{i} z_{j}^{2}}^{\left(\frac{n}{T_{2}}+\beta_{1}-\alpha_{k}\right)}\left(f+\frac{n}{2 T_{0}}-\frac{\beta_{1}+\alpha_{k}}{2}\right)+
\end{aligned}
$$

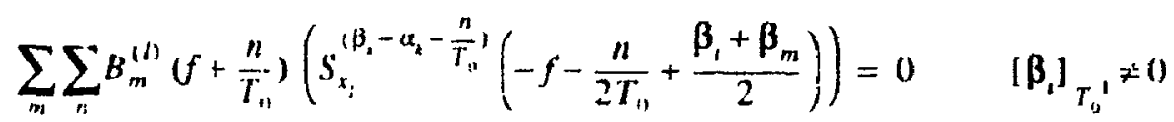

where: 


$$
\begin{aligned}
& z_{0}^{(1, n)}=p_{0}^{(1, n)}(t) \otimes N(t) \\
& z_{0}^{(2, n)}=p_{0}^{(2, n)}(t) \otimes \times(t)
\end{aligned}
$$

The achievable MTAMSE for MA-FRESH-DFE can be tound by generalizing (4.87) as follows :

$$
\begin{aligned}
& M T A M S E=\sigma_{a}^{2}- \\
& \left.\sum_{t=1}^{L}\left[a_{0}^{(l)}(t) \otimes\left(\sigma_{d_{0}^{(2)}}^{2} e^{j 2 \pi \sigma_{1}^{(r)}} z_{0}^{(1, l)}\left(t-t_{0}^{(r)}\right)+\sigma_{d_{0}^{(1)}}^{2} e^{j 2 \pi \sigma_{11}^{(1)}} z_{0}^{(2, n)}\left(t-t_{0}^{(1)}\right)\right)\right]\right|_{t-1 l_{1}}-
\end{aligned}
$$

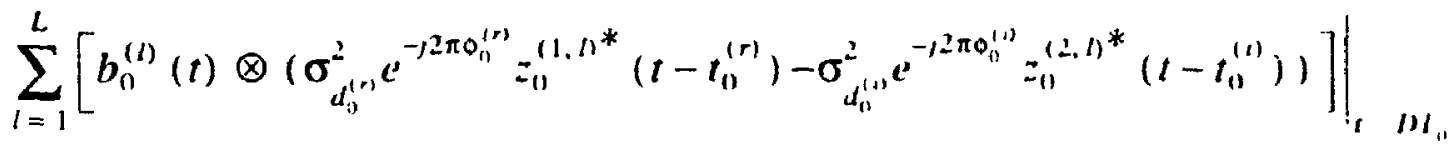

It is apparent from (4.114) that the MTAMSE of MA-FRESH-DFE can be reduced by increasing the number of antennas. The MTAMSE approaches zero if the number of antenna tends to infinity.

\subsection{Structure Complexity}

The stuctures introduced in this chapter might be more complex than needed for practical implementation. These structures might be reduced under certain conditions. For examples:

- The stricture of MA-FRESH DFE can bs: reduced for narrowband channels. In this case, the number of filters in a branch antenna can be reduced because some hilters can be simply created from combination of others.

- For OQPSK in a stationary environment (added AWGN), the cyclic spectrum in CCSD of the received signal can be easily transferred into CSD if the real and imaginary parts are synchronized (Figure 4.22). In this case, the structure of ODFE is rerfuced to the conventional DFE because there is no CCSD to be exploited. However, in the casse of cyclostationary environment, this synchronization can only be done if the imaginary and real parts are off-setted by $T / 2$. For example if a QPSK signal exist as at interference. using real-imaginary synchronizer block. transfers the interference QPSK irsto OOPSK while it is transferring SOI OQPSK into QPSK

- For BPSK signals, ODFE can be simplitied into Figure 4.23 $|5|$ if the channel 


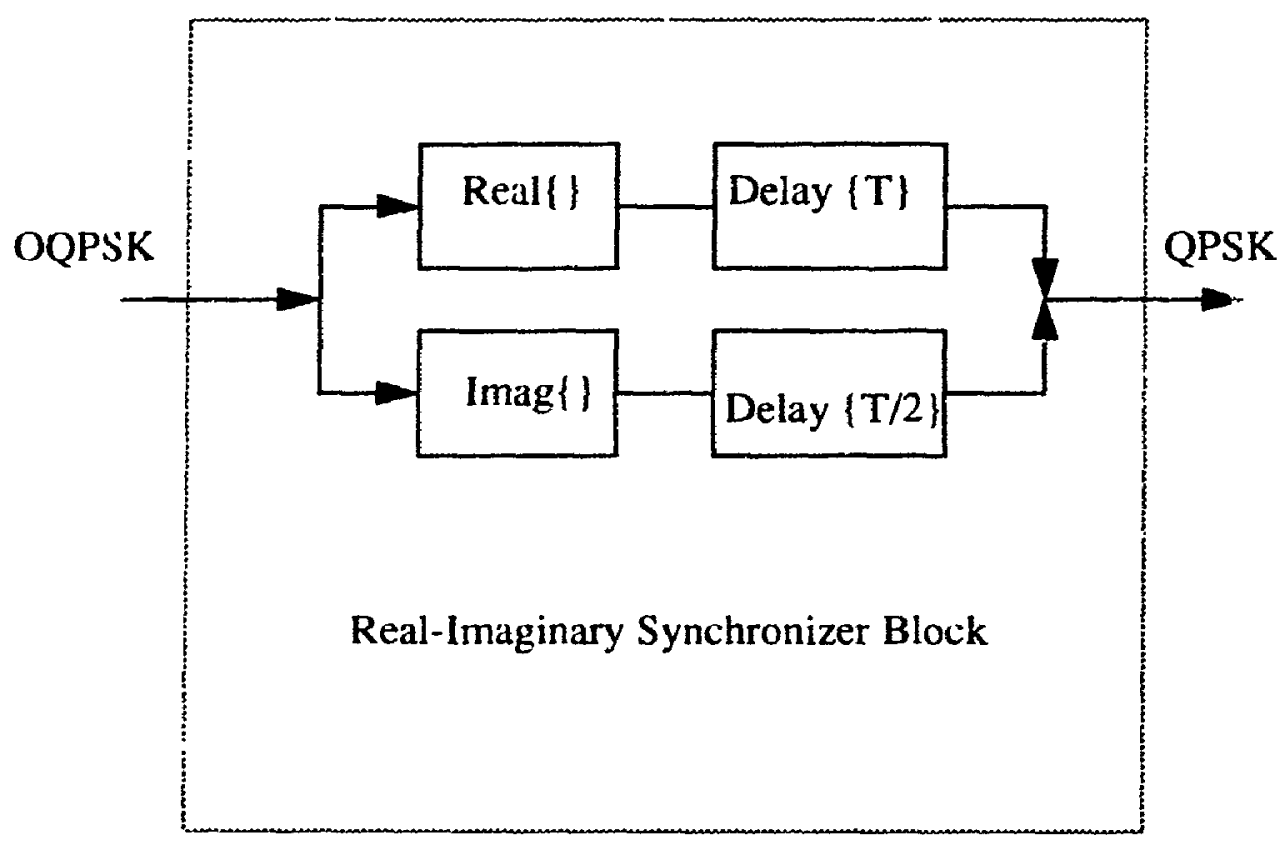

Figure 4.22 Real-Imag Syrichrorizer which can be used to reduce the structure of ODFE into the conventional DFE for OQPSK signa:. 


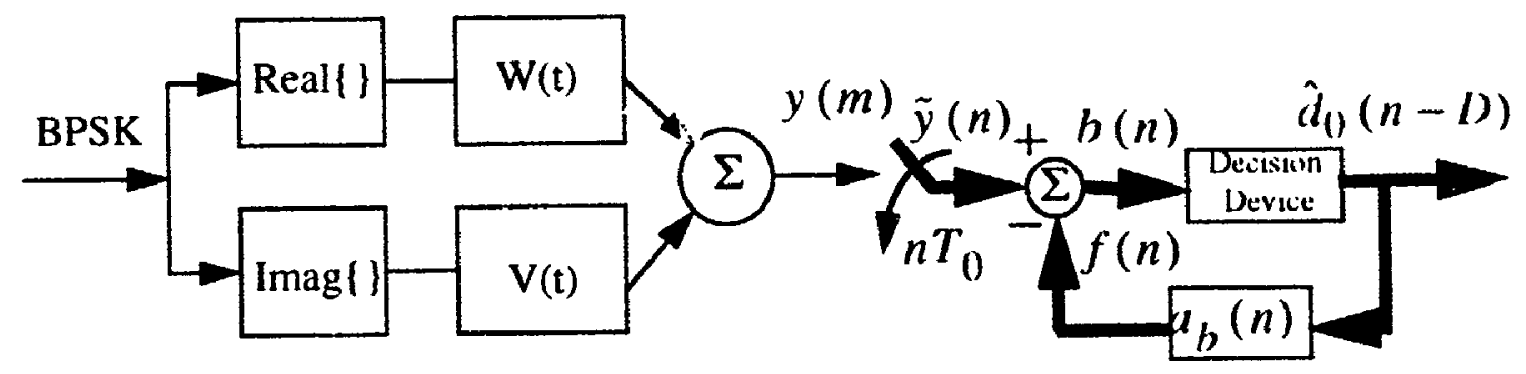

Figure 4.23 Reduced ODFE structure for BPSK. 
spacing is a multiple of half the symbol rate. In this structure, the inputs of the filters are real and therefore the complexity is reduced.

\subsection{Conclusion}

In this chapter, the optimum receiver structures for DCSs have been obtained and analyzed. It has been shown that the proposed equalizers (FRESH-DFE and GDFE) can exploit the information of the cyclic spectra of the received signal. In addition, the conventional DFE has been analyzed, and it has been shown that the conventional DFE is optimum only for balanced QPSK in a single symbol rate system. A structure for multiantenna receiver was also proposed and it was shown that the MTAMSE can approach zero if the number of antenna goes to infinity. 


\section{Chapter 5 \\ Mobile Cellular Radio Systems}

\subsection{Introduction}

The fundamentals of mobile cellular radio systems (MCRS) are reviewed in this chapter. First, the concepts of cellular radio systems relating to multiple access techniques, frequency reuse and cell splitting are described. This is followed by a review of the properties of digital mobile cellular radio channels with a particular emphasis on multipath fading and modelling. The interference properties in a MCRS are studied and results which characterize $\mathrm{CCl}$ and $\mathrm{ACl}$ in a cellular system are obtained. A new detinition for capacity in terms of several design parameters is introduced and later used in cellular system capacity evaluation. The chapter concludes with an example on a multi rate cellular system design.

\subsection{Cellular Radio Concept}

The first generation of MCRS employed analog FM modulation technology [66\}|. The second generation of MCRS employ digital modulation schemes $[16,45,65]$. The second generation digital cellular radio systems (DCRS) achieve significantly higher capacity than existing analog systems. Furthermore, digital technology brings many advantages including but not limited to inexpensive VLSI/DSP hardware, superior speech quality. reduced transmission noise sensitivity, greater data security and a natural extension to future ISDN implementation $[4,60]$.

One important design objective of a DCRS is to maximize the number of users in a given bandwidth. Several techniques are available to achieve this objective $|3,43,91,92|$. The multiple access schemes, frequency reuse and cell splitting strategies are the most common.

\subsubsection{Multiple Access Schemes}

There are essentially three basic multiple access schemes: code division multiple access (CDMA), time division multiple access (TDMA), and frequency division multiple 
access (FDMA) |84\}. A combination of these multiple access techniques are also used in some cases.

In a TDMA system, each user has access to full bandwidth for a part of the time known as a time slot while in a FDMA system, each user has access to only a part of the bandwidth all the time. In a CDMA system users share the bandwidth simultaneously. Therefore, TDMA and FDMA systems avoid interference by time and frequency filtering respectively, while the CDMA cleans multi user interference by users' codes. FDMA and TDMA are generally regarded as noise limited systems while CDMA is interference limiied [91]. Each multiple access scheme has some advantages or disadvantages relative to others, details of which are found in [84].

\subsubsection{Frequency Reuse and Cell Splitting}

Frequency reuse is the core concept of the cellular system. Users in different geographic locations (different cells) may simultaneously use the same frequency channel provided that they are sufficiently separated in distance. The frequency reuse can tremendously increase the total number of system users. However, if the frequency reuse is not properly designed, serious interference may result. $\mathrm{CCI}$, the co-hannel interference due channel reuse, is a major concern in cellular systems.

In a cellular system, regularly shaped cells are grouped into cell clusters, repeated to fill the given geographic service area. The total assigned spectrum, in FDMA or TDMA/FDM system, is divided into $K$ frequency reuse patterns where $K$ is the cluster size. $K$ is chosen to minimize the CCI, and is determined as $K=i^{2}+i m+m^{2}$ where $i$ and $m$ are integer numbers [63]. Thus, a cellular system can be organized into cluster sizes of one, three, four, seven, nine, twelve or more cells. The smaller the cluster size, the higher is the number of users. However, a smaller cluster size results in higher interference. Figure 5.1 shows two systems with $K=3$ and $K=7$. It is apparent from the figure that under the condition of equal transmit power, $\mathrm{CCI}$ and $\mathrm{ACI}$ is higher when $K=3$. This is because of shorter reuse distance for $K=3$. $\mathrm{CCl}$ is the interference between signals with similar carrier frequencies, whereas $\mathrm{ACl}$ is the interference between signals with different carrier frequencies which are close enough to cause overlaps in the spectrum. In fully equipped hexagonal-shaped cellular system. there are always six co-channel interfering cells in the first tier [65] while the number of adjacent channel interfering cells depends on the frequency reuse pattern (cluster size). channel spacing and excess bandwidth. Figure 5.2 shows the 


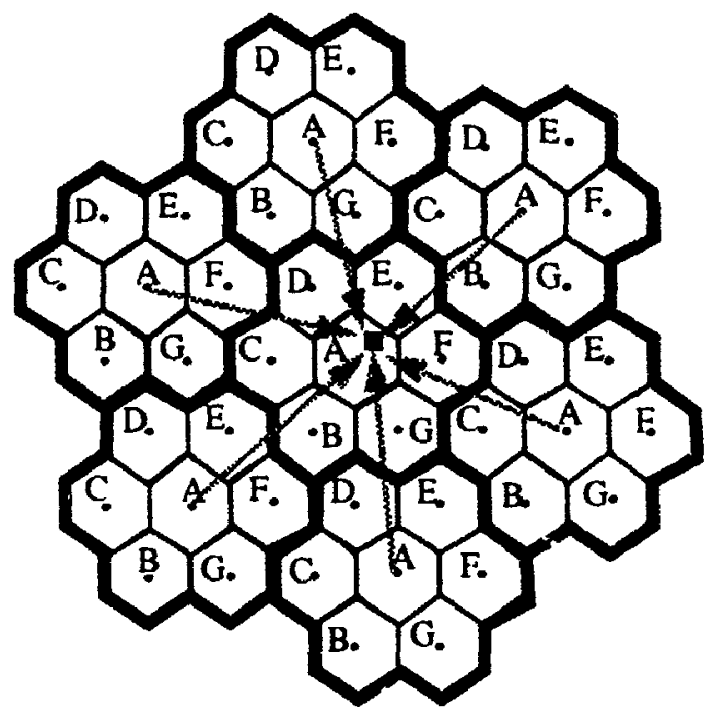

a.1) $\mathrm{CCI}$ in a system with cluster size seven.

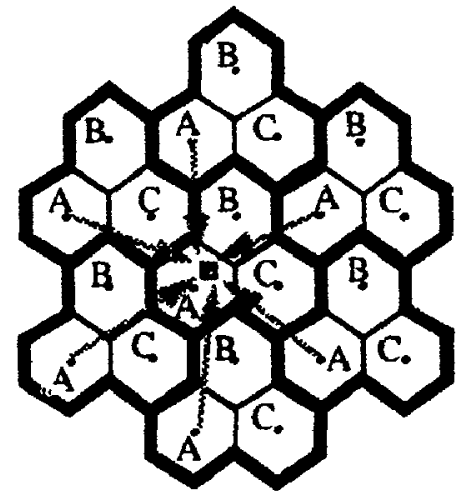

b.1) $\mathrm{CCI}$ in a system with cluster size three.

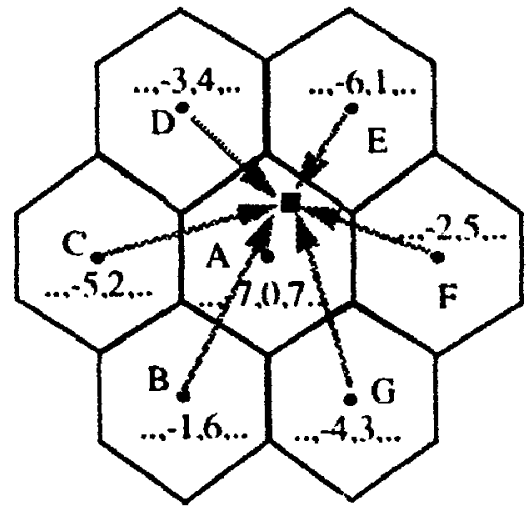

a.2) $\mathrm{ACl}$ in a system with cluster size seven

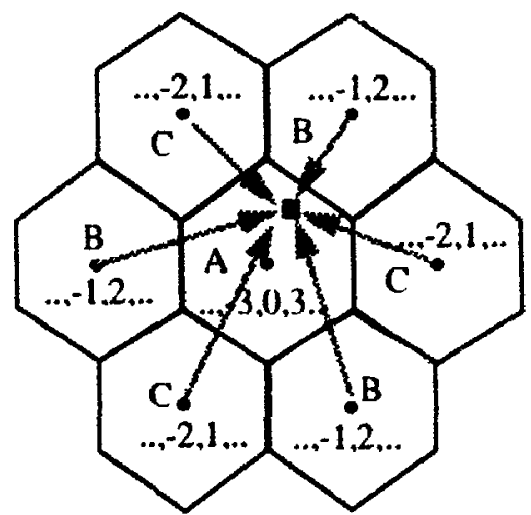

b.2) $\mathrm{ACl}$ in a system with cluster size three.

Figure 5.1 (a) A Cellular Radio System (CRS) with cluster size seven (b) A CRS with clustor size three. 


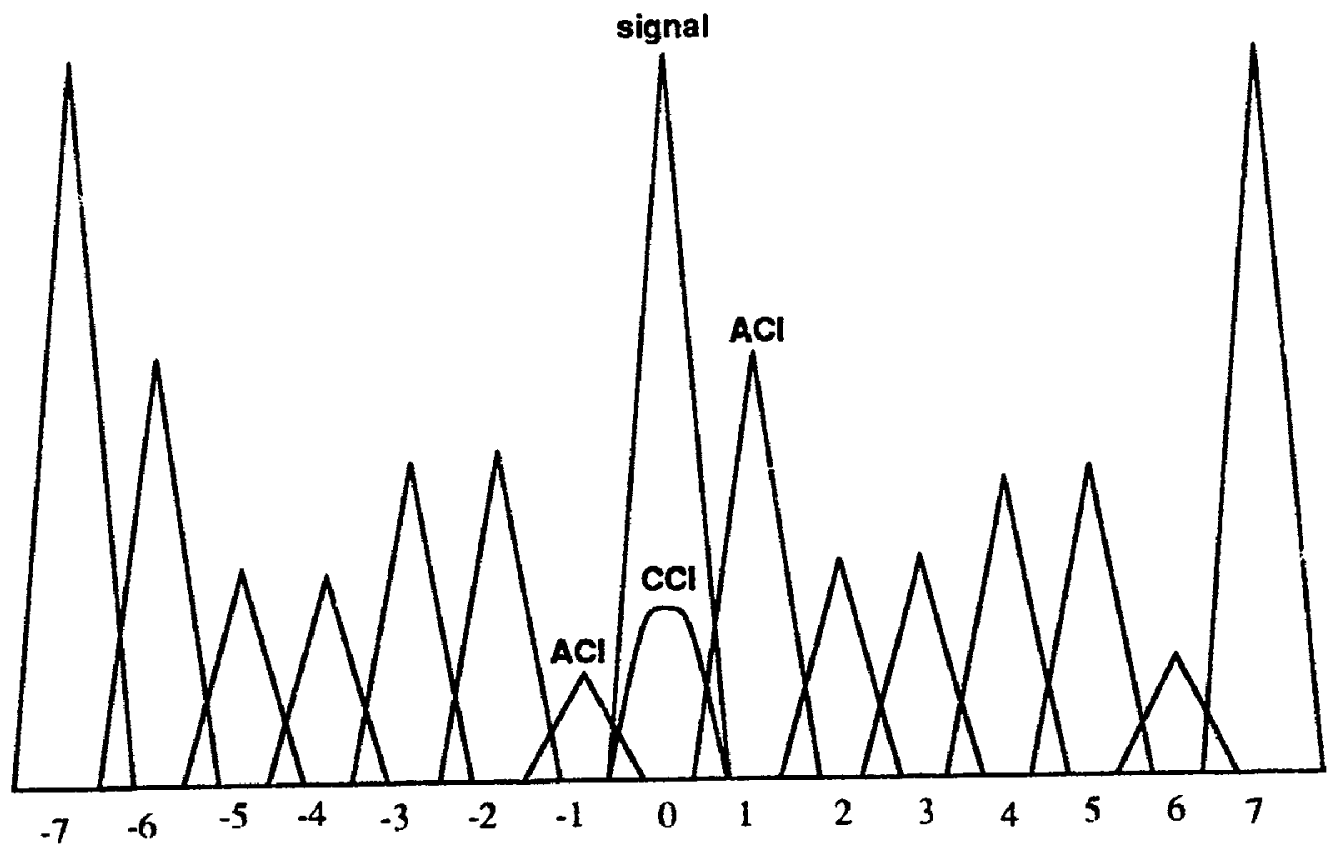

a) received signal by a user in a system with cluster size seven.

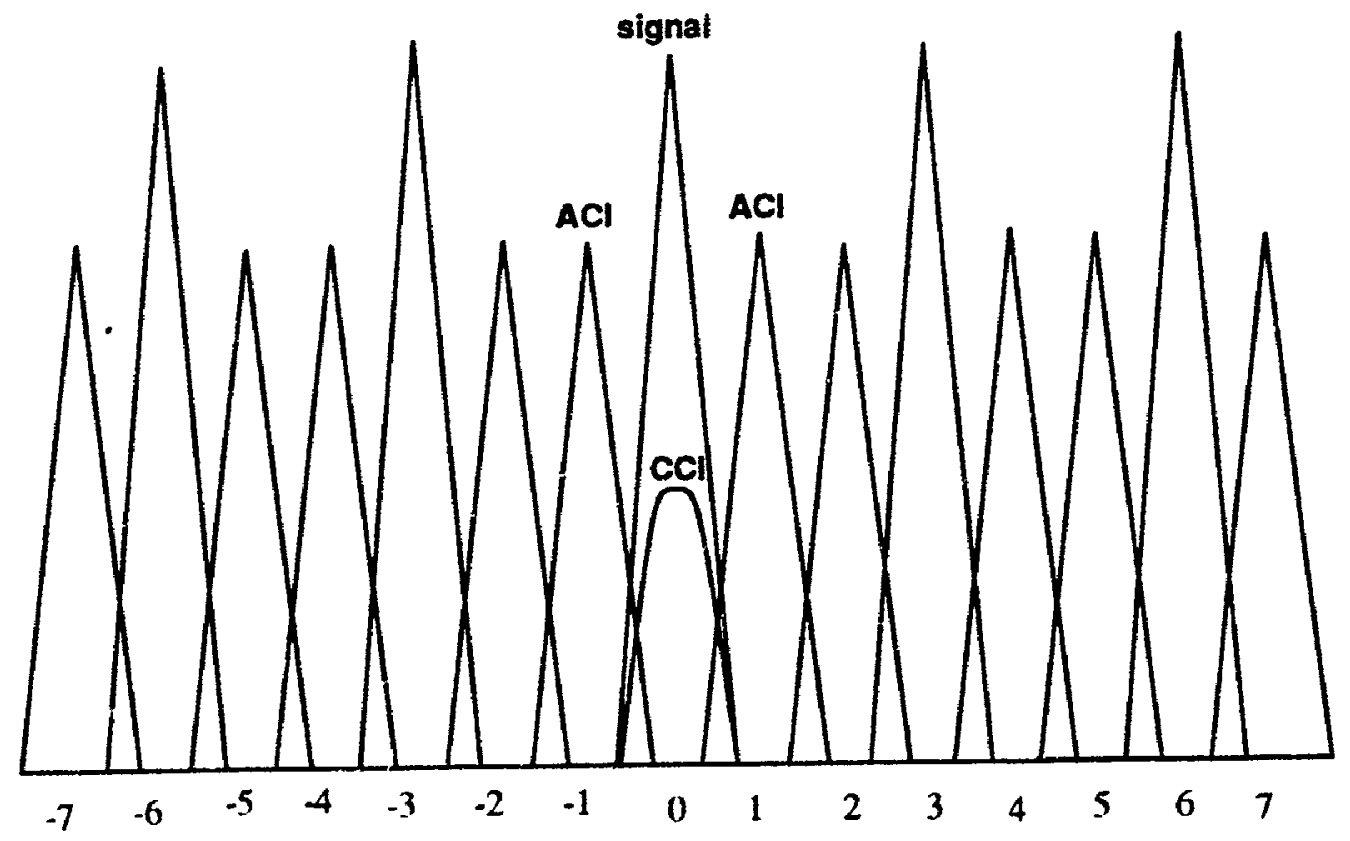

b) received signal by a user in a system with cluster size three.

Figure 5.2 Aeceived signal in two different CRS. 
spectrum of the signal received by one user in the systems of Figure 5.1. As it is apparent from the figure, the $\mathrm{ACl}$ and $\mathrm{CCl}$ signals are stronger in the system with cluster size three.

\subsection{Digital Mobile Radio Channel Properties}

\subsubsection{Multipath Channel Properties}

Mohile radio communication channels typically are multipath fading channels $[16,59,66,97]$. In a multipath channel, a transmitted signal arrives at the receiver via several paths with random delays and attenuations. This multipath propagation can be characterized in the time delay domain by multipath power delay protile (MPDP) which describes the average received power as a function of the propagation delay. The maximum propagation delay which MPDP is essentially nonzero is called the multipath delay spread of the channel. It is well known that if the ratio of the root-mean-syuare (RMS) delay spread to the symbol period is greater than about 0.3 , then an adaptive equalization technique must be used to reduce the inter-symbol interference (ISI) caused by neighboring data symbols at sampling time [84]. The inverse of the RMS delay spread is called the "coherence bandwidth" of the channel. Two sinusoids with frequency separation greater than the coherence bandwidth are affected independently by the channel. A fading channel is said to be frequency-selective if its coherence bandwidth is less than the signal bandwidth. In this case, although the signal is severely distorted, the multipatt are resolvable and an implicit diversity gain can be achieved [83]. On the other hand, if the coherence bandwidth is large (in comparison to the signal bandwidth), the channel is said to be frequency-nonselective. In this case, the channel exhibits spectrally flat fading in which all signal components fade simultaneously, the multipath rays are unresolvable and equalization is unnecessary [84]. A digital mobile radio channel typically is a frequency-selective fading channel $[46,59,97]$.

\subsubsection{Time-Variant Fading Channel Properties}

As the signal receiving mobile moves through an approximately stationary spatial pattern, the received signal comes through a time variant-channel. The time variations in the channel are evidenced as a Doppler broadening and Doppler shift of a spectral line. The fading signal power distribution over the range of the Doppler frequencies is called Doppler power spectrum. The range of the Doppler frequencies over which the Doppler power spectrum is essentially nonzero is called the Doppler spread of the channel. The 
severity of the time variations in the channel due to multipath fading can be quantified by the RMS Doppler spread. However, the multiplication of the maximum Doppler frequency, $f_{\text {nux }}$, by the symbol period time, $T$, can characterize the severity of a mobile channel. A multipath fading channel witi high $f_{\text {max }} T$ is called fast fading channel and a multipath fading channel with low $f_{\text {mux }} T$ is called slow fading channel. Due to slow mobile speed and high symbol rate, the slow fading indoor wireless communication channel does not suffer from significant time variations.

\subsubsection{Channel Modelling}

The equivalent baseband of a multipath fading channel can be modelled as a complex time-varying signal with random attenuation and phase $g(\tau, t)$, where $\tau$ is the propagation time delay and $t$ is the time dependence of the channel : [84]

$$
g(\tau, t)=\alpha(\tau, t) e^{-j 2 \pi f_{c} \tau}
$$

where $\alpha$ is the attenuation factor, and $f_{c}$ is the signal carrier frequency.

Assuming that $g(\tau, t)$ is made up of a finite number of statistically independent propagation paths, (5.1) is written as :

$$
g(\tau, t)=\sum_{m} \alpha_{m}(t) e^{-j 2 \pi f_{c} \tau_{m}(t)} \delta\left(\tau-\tau_{m}(t)\right)
$$

where $\alpha_{m}(t)$ is the $m$ th path attenuation, $\tau_{m}(t)$ is its propagation delay and $\delta(\tau)$ is dirac delta function.

Assuming a sufficiently large signal bandwidth such that the individual propagation paths can not be resolved, then each component of $g(\tau, t)$ is comprised of a sum of a large number of multipath signals. Therefore, from the central limit theorem [77], each componest of $g(\tau, t)$ has independent real and imaginary parts, identically distributed (iid) jointly zero mean Gaussian random processes. In this case, the envelope $|g(\tau, t)|$ at any time instant $t$ is Rayleigh-distributed and the channel is said to be a Rayleigh fading channel.

\subsubsection{Tapped Delay Line Channel Modelling}

The tapped delay line (TDL) channel model composed of evenly spaced tap coefficients represents multipath channel with district number of paths. The TDL should 
be sufficiently long to span the significant portion of the channel MPDP. Furthermore, the tap spacing should be tine enough to represent the channel adequately. Specitically, the complex baseband input-output relationship of the frequency selective channel can be written as the following convolution summation : $[84]$

$$
r\left(k \frac{T}{n}\right)=\sum_{m=0}^{M-1} g_{m}\left(k \frac{T}{n}\right) s\left(k \frac{T}{n}-m T_{r}\right)
$$

where $r(k T / n)$ is the channel output. $g_{m}(k T / n)$ is the mth $T / n$-spaced channel impulse response (CIR) coefficient, $s\left(k T / n-m T_{r}\right)$ is the channel input signal, $T_{r}$ is the time resolution, and $T$ is the symbol period. This TDL channel model distinguishes multipath to a time resolution of $T_{r}$ which has to be less than or equal to $T /(1+\varepsilon)$ and a multiple of $T / n$. where $\varepsilon$ is the excess bandwidth parameter.

For this discrete channel model, the $m$ th tap coefficient of the TDL can be represented as :

$$
g_{m}\left(k \frac{T}{n}\right)=\alpha_{m} d_{m}\left(k \frac{T}{n}\right)
$$

where $\alpha_{m}$ is the RMS attenuation factor of the $(m+1)$ th multipath component, and $\mathrm{d}_{m}(k(T / n))$ is the normalized Doppler fading factor of this path. The normalized Doppler fading factor in a Rayleigh fading environment can be modeled as a sum of weighted sinusoids with random phases and discrete frequencies spanning the Doppler spectrum [59]. Specifically, the fading component of the $m$ th tap coefficient of the TDL channel model is :

$$
d_{m}\left(k \frac{T}{n}\right)=\sum_{n=0}^{N_{s}} d_{m, n} \cos \left(2 \pi \delta_{n} f_{\max } k \frac{T}{n}+\theta_{m, n}\right)
$$

where $d_{m n}$ is the complex-valued amplitude of the nth Doppler frequency component, $\delta_{n}$ is the relative scaling factor for the $n$th Doppler frequency component, $f_{\max }$ is the maximum Doppler frequency, $N_{s}+1$ is the number of weighted Doppler sinusoids and $\boldsymbol{\theta}_{m, n}$ is the initial phase of the nth Doppler frequency component at $k=0$.

The relative scaling factor for $n$th Doppler frequency component is : 


$$
\delta_{n}=\cos \left(\pi \frac{n}{2 N_{s}+1}\right)
$$

The inphase and quadrature components of the amplitude for the $n$th Doppler frequency are, respectively : $|59|$

$$
\text { Real }\left\{d_{m, n}\right\}=\left\{\begin{array}{cc}
\sqrt{2} \cos \left(\frac{\pi}{4}\right) & n=0 \\
2 \cos \left(\frac{\pi n}{N_{s}}\right) & 1 \leq n \leq N_{s}
\end{array}\right.
$$

and

$$
I \operatorname{mag}\left\{d_{m, n}\right\}=\left\{\begin{array}{cc}
\sqrt{2} \sin \left(\frac{\pi}{4}\right) & n=0 \\
2 \sin \left(\frac{\pi n}{N_{s}}\right) & 1 \leq n \leq N_{s}
\end{array}\right.
$$

The initial phase $\theta_{m, n}$ is a random, uniformly distributed variable between $-\pi$ and $\pi$.

The accuracy of the sum of sines model improves with increasing number of weighted Doppler sinusoids. However, it has been shown that a model using nine discrete Doppler sinusoids yields sufficient accuracy [59].

In the case of low $f_{\text {max }}$ that is slow fading, the normalized Doppler fading of the $(m+1)$ th path can be assumed to be constani for a period of the time. In this period of the time, it is comprised of a sum of a large number of signals uniformly distributed between -1 and 1 . Therefore, from central limit theorem, its real and imaginary parts are iid jointly zero mean Gaussian random processes. In this case, the envelope of the $m$ th tap coefficient of the TDL is a Rayleigh distributed random process. Thus, in the case of slow fading and over a short period of time. TDL channel may be modelled as follows :

$$
r\left(k \frac{T}{n}\right)=\sum_{m=0}^{M-1} g_{m} s\left(k \frac{T}{n}-m T_{r}\right)
$$

where $g_{m}$ is a complex random variable with zero mean Gaussian distributed real and imaginary parts. 


\subsection{3-Rays Model}

As an example of TDL channel model. a 3-rays model (Figure 5.3) is considered. In this model, the TDL contains three nonzero complex tap coefticients. $\left(g_{n}\right.$ : $m=1,2,3)$, each of which represents a distinct path. The tap coefficients $g_{m}$ are characterized by the MPDP which is basically a measure of the variance of the channel impulse response (CIR) as a function of the propagation delay. For example, the "mountainous terrain" MPDP of the channel is specified in decibels, $d B$, relative to the tirst three paths : [68]

$$
g_{0}=0 \mathrm{~dB}, g_{1}=-5 \mathrm{~dB}, g_{2}=-15 \mathrm{~dB}
$$

Figure 5.4 shows the MPDP for $T / 4$ sampling interval. Regardless of sampling time $(T / n)$ the resolution time is $T_{r}=T / 2$ in this model. Therefore, this model is valid for signals, with less than 100 percent excess bandwidth, sampled at $T / n$ where $n$ is even. This model is used in all simulations reported in this thesis.

\subsection{Interference and Noise in Cellular Systems}

In a cellular system. mobile position and propagation loss factor determines the SOI, $\mathrm{CCI}$ and $\mathrm{ACl}$ powers while cluster size affect $\mathrm{CCl}$ and $\mathrm{ACl}$ powers. Figure 5.5 shows the desired signal, $\mathrm{CCI}$ and $\mathrm{ACl}$ for a system with a cluster size of three. The following relations can be directly found from the figute :

$$
\begin{aligned}
& r_{c}^{2}=9 R^{2}+r^{2}+6 R r \cos \left(\theta+m \frac{\pi}{3}-\frac{\pi}{2}\right) \\
& r_{A}^{2}=4 R^{2}+r^{2}+4 R r \cos \left(\theta+(m-1) \frac{\pi}{3}\right)
\end{aligned}
$$

Where $R$ is the cell size, $r$ is the mobile distance from base station, $r_{A}$ is the mobile distance from one of the $\mathrm{ACl}$ source, $r_{C}$ is the mobile distance from one of the first tier $\mathrm{CCl}$ source. Generally, the following relations are valid in any hexagonal cellular system :

$$
\begin{gathered}
r_{c m m}^{2}=(3 K) R^{2}+r^{2}+2(\sqrt{3 K}) R r \cos \left(\theta+m \frac{\pi}{3}-a \sin \left(\frac{3}{\sqrt{3 K}}\right)\right) \\
r_{a m}^{2}=4 R^{2}+r^{2}+4 R r \cos \left(\theta+(m-1) \frac{\pi}{3}\right)
\end{gathered}
$$

Where $K$ is the cluster size, $r_{c m}$ is the mobile's distance from mth source of first tier C(') 


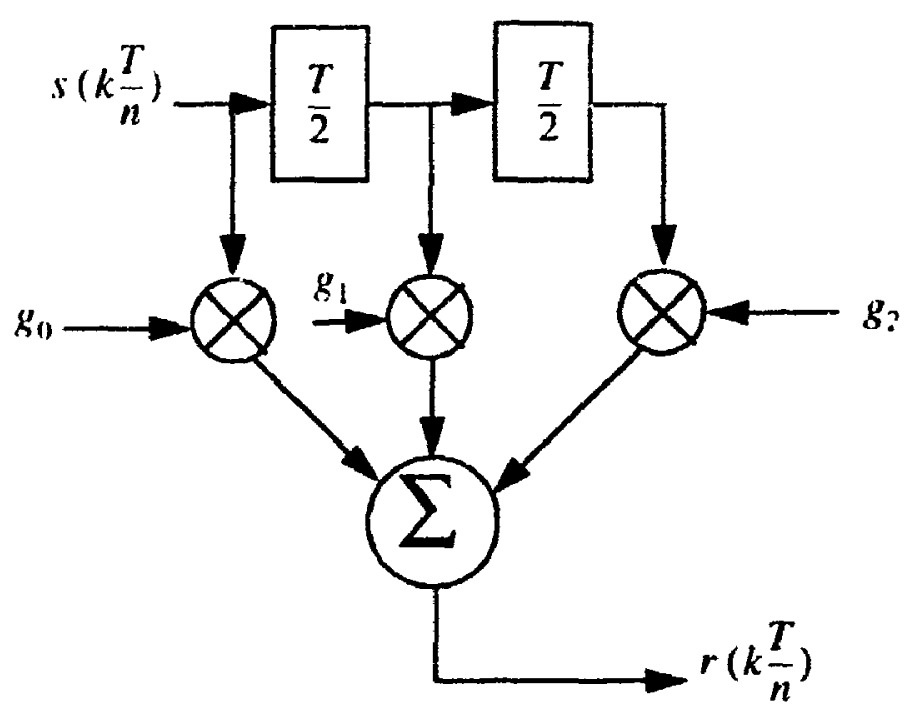

Figure 5.3 3-rays c nannel model.

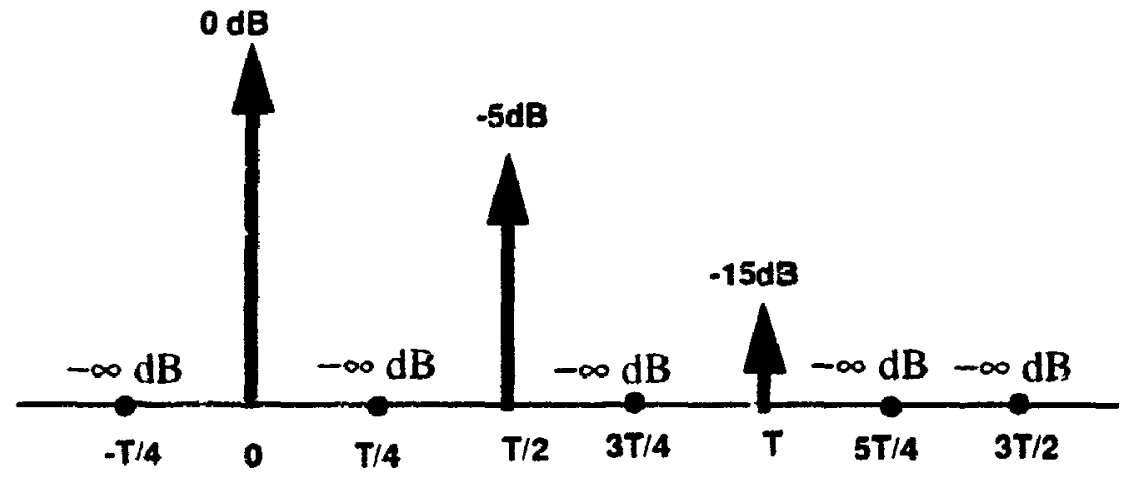

Figure 5.4 MultiPath Delay Profile (MPDP) for 3-rays model. 


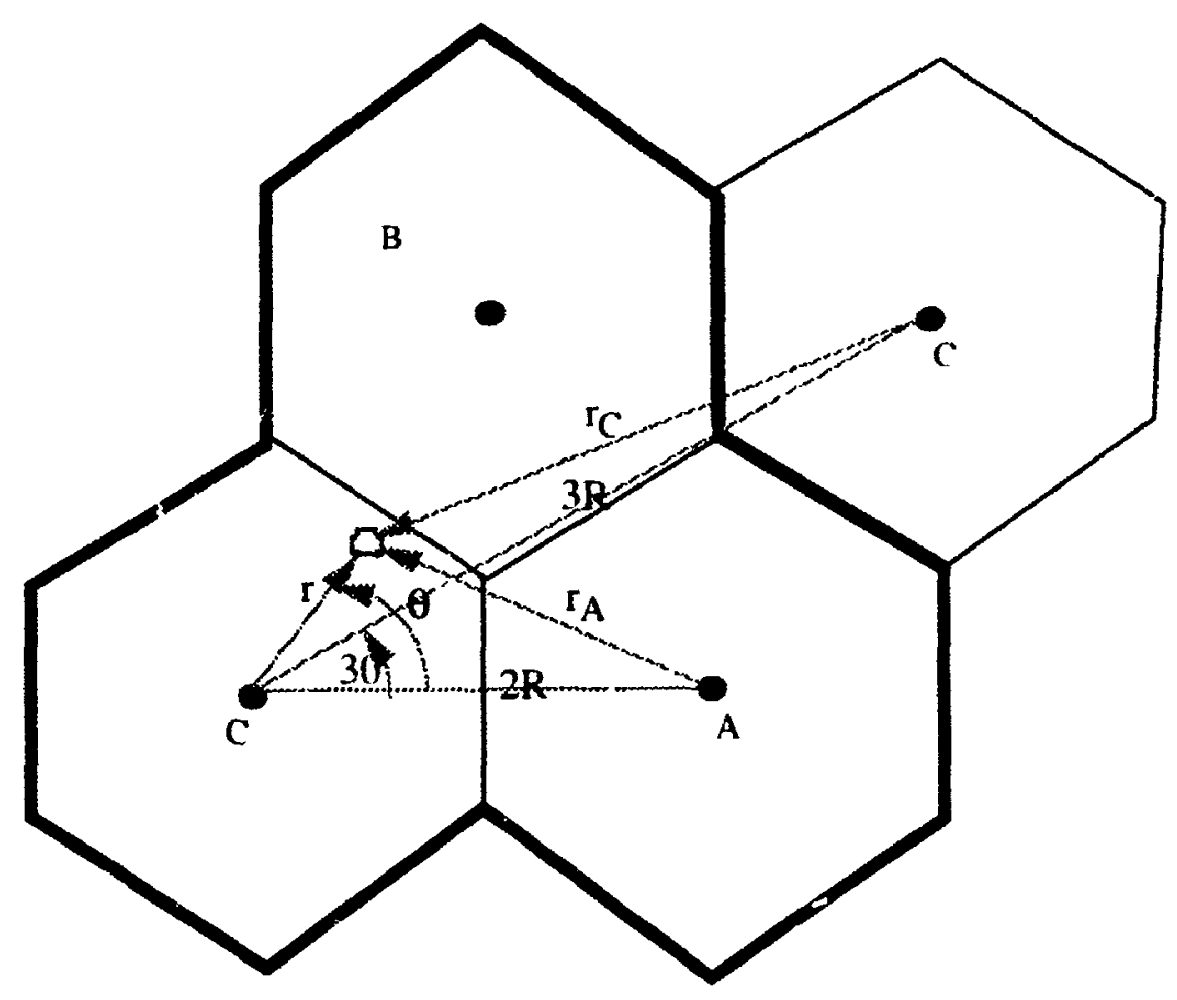

Figure 5.5 An example of mobile position in a cellular system with cluster size three. 
and $r_{a m}$ is the mobile'; distance from $m$ th source of first tier ACI. If the channel MPDPs used by interference and signal are similar except some attenuation caused by distances, signal to noise ratio (SNR), signal to CCI ratio (SCCIR), the signal to ACI ratio (SACIR), and signai to total interference ratio (SIR) can be easily found. In this case. cell size is not important and for simplicity we assume $R=100$ meters in the rest of this thesis.

\subsubsection{SNR in Cellular Systems}

The average signal power at the receiver at distance $R_{1}$ from the base station is found as:

$$
S=\frac{A}{\left(R_{\mathrm{l}} / R\right)^{\gamma}}=\frac{A}{r^{\gamma}} \quad 1 \geq r \geq \frac{1}{R}=0.01
$$

where $\gamma$ is the environmentally dependent propagation loss factor and usually lies between 2 and $5, r$ is the normalized distance and $A$ is the power of the signal at $r=1$. The above equation is valid for $R_{1}$ greater or equal to one meter. Therefore the minimum value for $r$ ; $1 / R$. If the variance of the noise $\left(\sigma_{n}^{2}\right)$ is assumed to be constant over the cell, the signal to noise ratio (SNR) at the receiver input can be calculated as follows :

$$
\begin{gathered}
\frac{S}{N}=\frac{A}{r^{\gamma} \sigma_{n}^{2}} \\
S N R=\left[\operatorname{lol} \log (A)-20 \log \left(\sigma_{n}\right)\right]-10 \gamma \log (r) \\
S N R=S N R_{\text {max }}-10 \gamma \log (R)-\operatorname{lo} \gamma \log (r)=S N R_{\text {min }}-10 \gamma \log (r) \quad 1 \geq r \geq \frac{1}{R}
\end{gathered}
$$

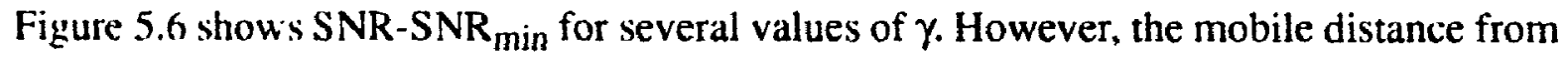
base station. $r$, is a random process and its cumulative distribution finction (cdf) and probability density function (pdf) can be found as follows :

$$
\begin{gathered}
F_{r}(Y)=p(r<Y)=\frac{\pi Y^{2}}{\pi}=Y^{2} \quad 0 \leq Y \leq 1 \\
f_{r}(Y)=\frac{d F}{d Y}=2 Y \quad 0 \leq Y \leq 1
\end{gathered}
$$

where $F_{x}$ and $f_{1}$ represent cdf and pdf of random variable $x$ respectively. Therefore, the cdf and pulf of average SNR can be found as follows : 


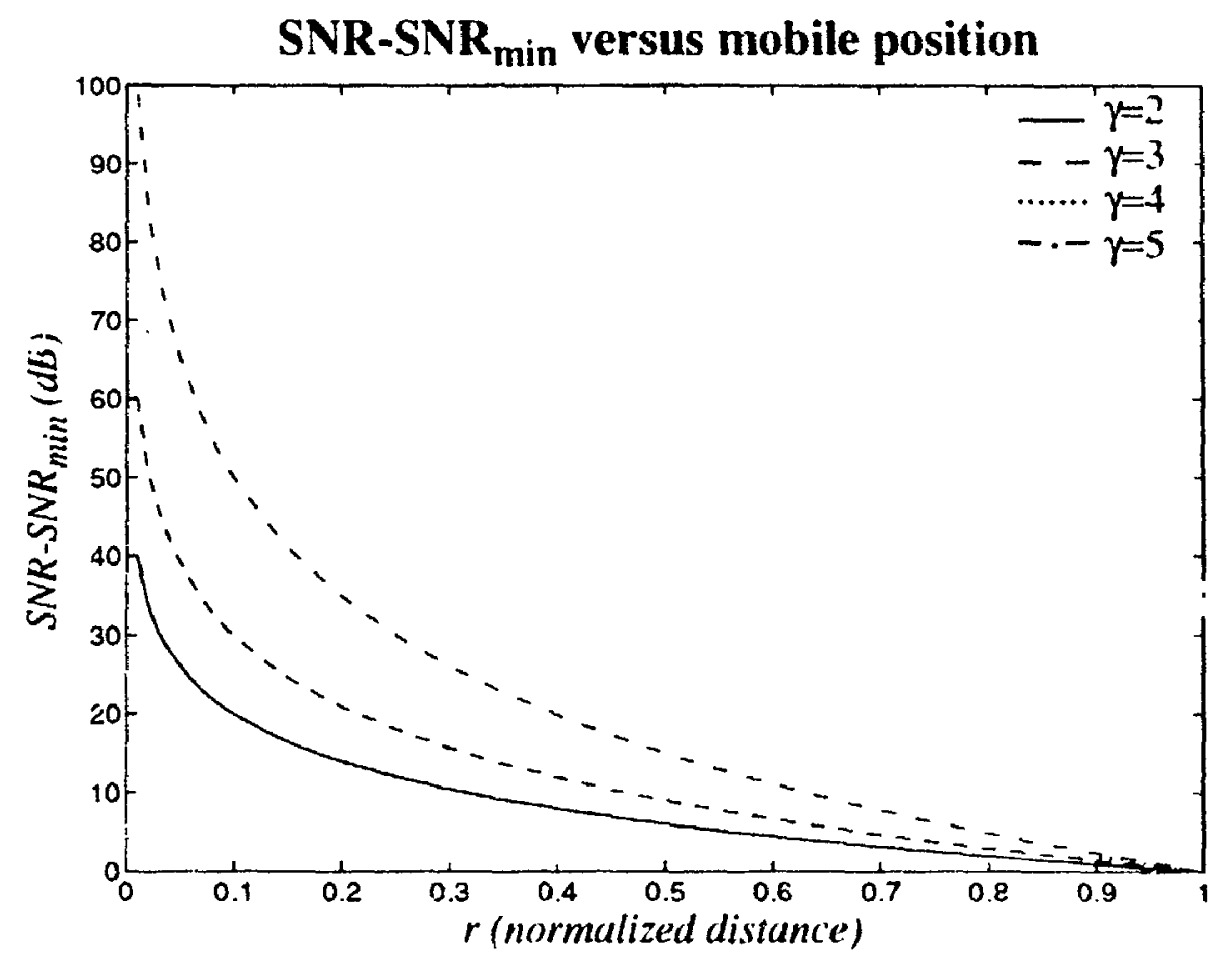

Figure 5.6 SNR-SNR Snn versus mobile position. 


$$
\begin{aligned}
& F_{S N R}(Y)=p(S N R<Y)=p\left(r>10^{-\left(\frac{Y-S N R_{\text {min }}}{10 \gamma}\right)}\right) \\
& F_{S N K}(Y)=1-F_{r}\left(10^{-\left(\frac{\gamma-S N R_{m i n}}{10 Y}\right)}\right)=1-10^{-2\left(\frac{Y-S N R_{m+r}}{10 \gamma}\right)} \\
& f_{S N R}(Y)=\frac{2}{10 \gamma} 10^{-2\left(\frac{Y-S N R_{\min }}{10 \gamma}\right)} \quad S N R_{\max } \geq Y \geq S N R_{\min }
\end{aligned}
$$

Figure $5.7(a, b)$ show cdf and pdf of SNR-SNR $\min$ for several values of $\boldsymbol{\gamma}$. As it is apparent from these curves, the probability of having $S N R$ around $S N R_{\min }$ is higher when $\gamma$ is low. The average SNR over a cell is found by taking another average of SNR as follows :

$$
\begin{gathered}
\overline{S N R}=\int_{S N R_{\min }}^{S N R_{\max }} Y \frac{2}{10 \gamma} 10^{-2\left(\frac{Y-S N R_{\min }}{10 \gamma}\right)} d Y \\
\overline{S N R}=S N R_{\min }+0.9421 \gamma
\end{gathered}
$$

As it is apparent from the above equation that the overall average SNR in $\mathrm{dB}$ has one to one increase with $\gamma$.

\subsubsection{Signal to CCl Ratio (SCCIR) in Cellular Systems}

The first tier CCI which contains a maximum of $6 \mathrm{CCI}$ signals is most serious interference in cellular systems. The power of this interference, $l_{c}$, at the receiver input is found as follows :

$$
\begin{gathered}
i_{c m}=\frac{A}{r_{c m}^{\gamma}} \\
I_{c}=\sum_{m=1}^{M} i_{c m}=A \sum_{m=1}^{6} \frac{1}{r_{c m}^{\gamma}} \\
I_{1}=A \sum_{m=1}^{M} E\left\{\frac{1}{\left(3 K+r^{2}+2(\sqrt{3 K}) r \cos \left(\theta+m \frac{\pi}{3}-\operatorname{asin}\left(\frac{3}{\sqrt{3 K}}\right)\right)\right)^{\gamma / 2}}\right\} r>0.01
\end{gathered}
$$

where $M$ is the number of active interference and expectation applies on $\theta$. The above 


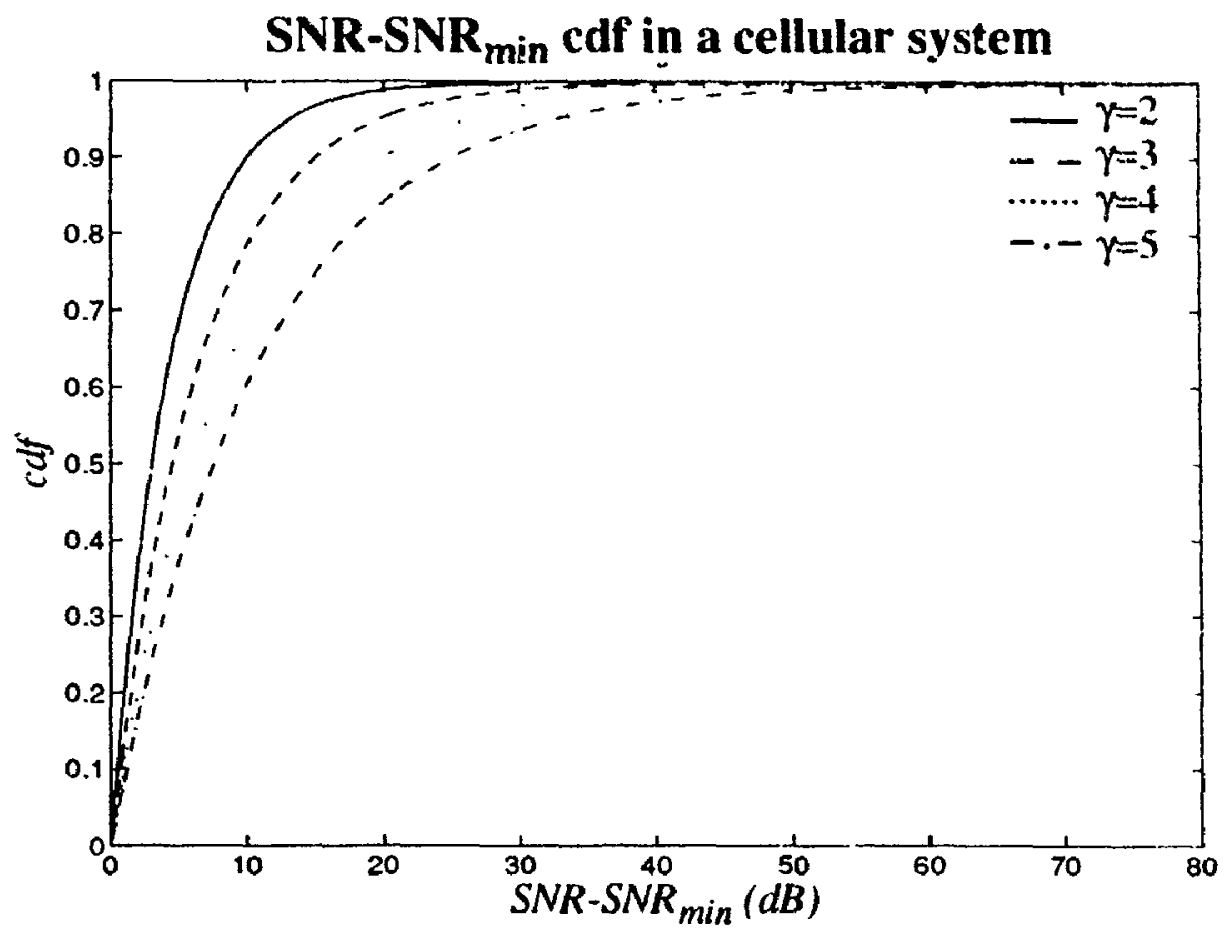

(a)

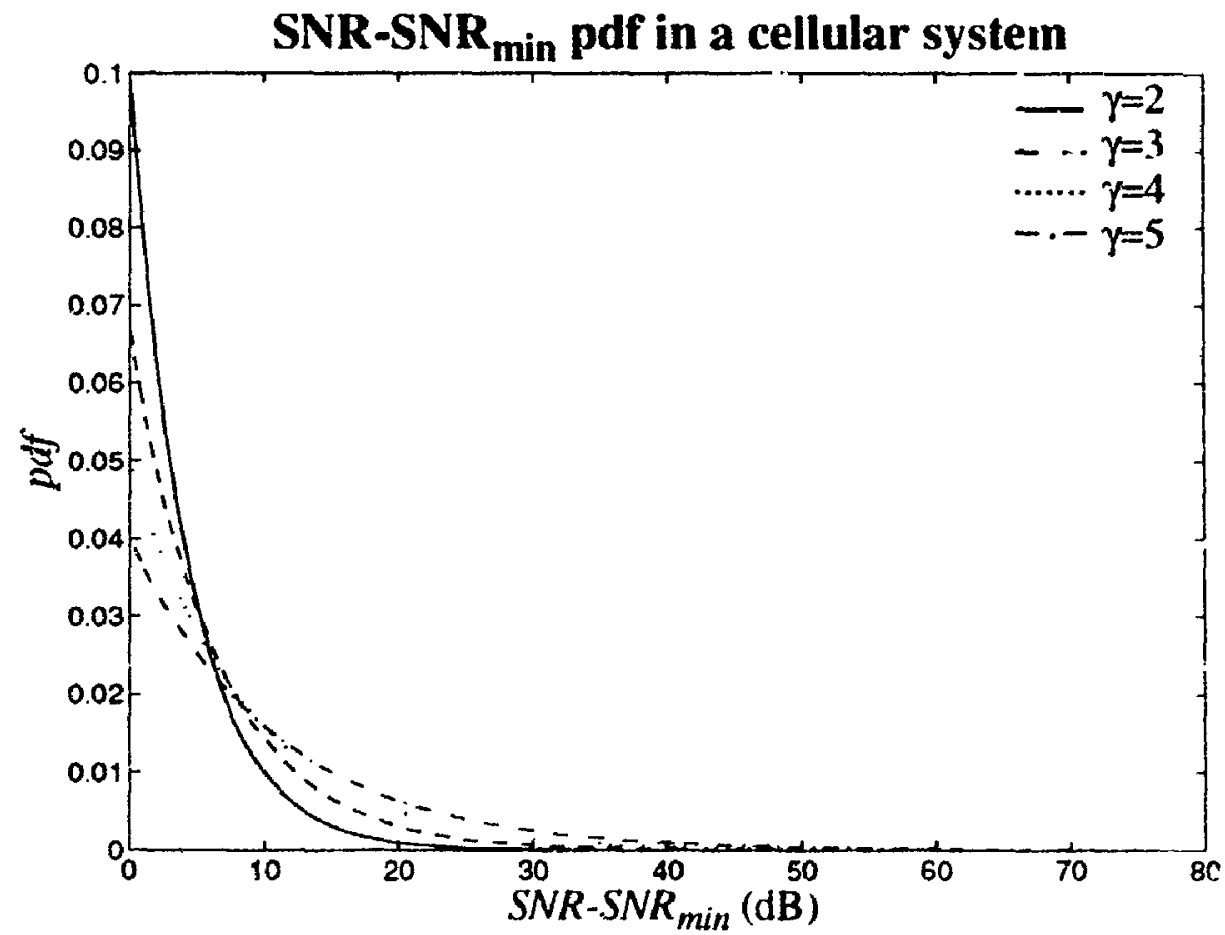

(b)

Figure 5.7 (a) cumulative distribution function (cdf) (b) probability density funcilon (pdf) of SNRSis $R_{\min }$ in a collular systom. 
average is difficult to calculate, however, the computer simulation and subsequent curve fitting shows that $(5.21)$ can be rewritten as :

$$
I_{r}=\frac{M A}{\left(3 K-0.5 \gamma r^{2}\right)^{\frac{\gamma}{2}}} \quad 1 \geq r \geq 0.01
$$

Therefore, the signal to first tier CCI ratio can be found according to :

$$
\begin{gathered}
\frac{S}{I_{c}}=\frac{\left(3 K-0.5 \gamma r^{2}\right)^{\frac{\gamma}{2}}}{r^{\gamma} M} \\
S C C I R=5 \gamma \log \left(3 K-0.5 \gamma r^{2}\right)-10 \gamma \log (r)-10 \log (M) \quad 1 \geq r \geq 0.01
\end{gathered}
$$

Figure 5.8 (a) shows the SCCIR versus $r$ and Figure 5.8 (b) shows the SCCIR versus the number of active CCI, $M$, for several values of $\gamma$ and $K$. Maximum and minimum SCCIR can be easily calculated from (5.23):

$$
\begin{gathered}
S C C I R_{\min }=5 \gamma \log (3 K-0.5 \gamma)-10 \log (M) \\
S C C I R_{\max }=10 \gamma \log (R)+5 \log (3 K)-10 \log (M)
\end{gathered}
$$

Table 5.1 summarizes the minimum SCCIR for different values of $M, \gamma$ and $K$. As it is apparent from the figures and the table, the minimum SCCIR for $K=3$ is significantly lower than for $K=7$. The cdf and pdf of SCCIR can be found using same method explained in previous section :

$$
\begin{gathered}
F_{S C C I R}(Y)=1-\frac{3 K}{M^{2 / \gamma} 10^{Y / 5 \gamma}+0.5 \gamma} \\
f_{S C C I R}(Y)=\frac{3 K M^{2 / \gamma} 10^{Y / 5 \gamma}}{5 \gamma\left(M^{2 / \gamma} 10^{Y / 5 \gamma}+0.5 \gamma\right)^{2}} \quad S C C I R_{\max } \geq Y \geq S C C I R_{\min }
\end{gathered}
$$

Figure $5.9(a, b)$ shows cdf and pdf of SCCIR for several values of $\gamma$ and for cluster sizes 3 and 7. As it is apparent from these curves, the probability of high SCCIR decreases as $\gamma$ decreases. The average SCCIR over a cell can be found by using the following integration:

$$
\overline{S C C I R}=\int_{S C C / R_{\min }}^{S C C / R_{\operatorname{mux}}} \frac{\left.3 Y K M^{2 / \gamma} 10^{\gamma / 5 \gamma}+0.5 \gamma\right)^{2 / 5 \gamma}}{5 Y}
$$


SCCIR versus mobile position

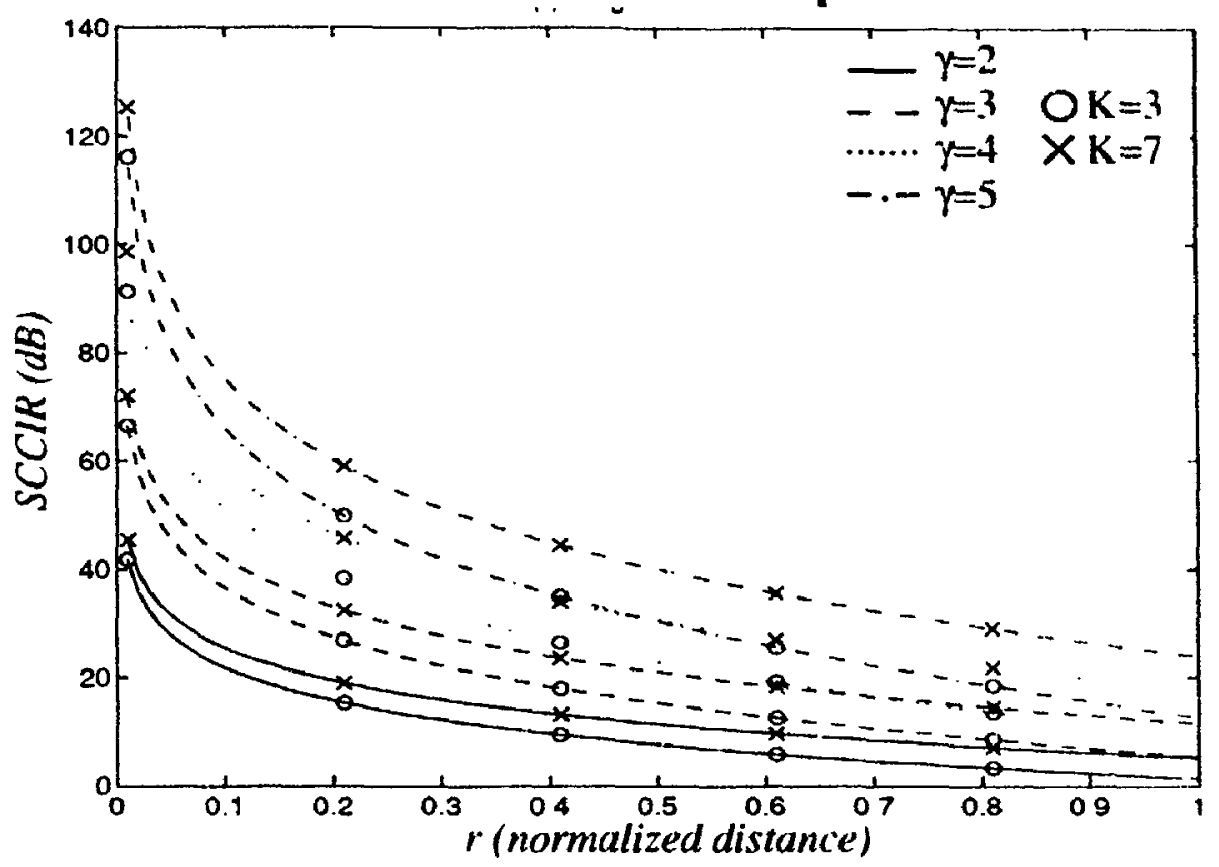

(a)

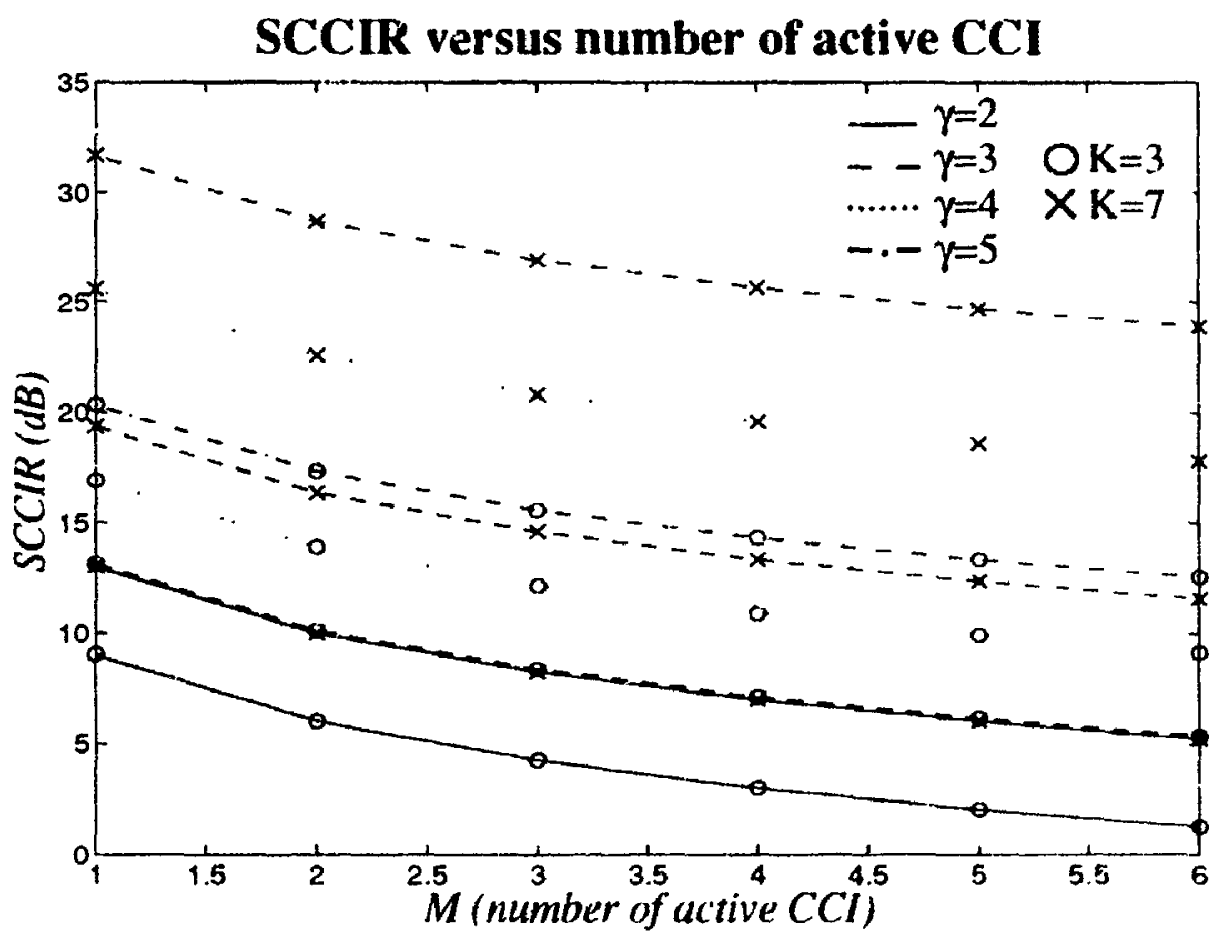

(b)

Figure 5.8 SCCIR versus (a) mobile position (b) number of active $\mathrm{CCl}$. 
Table 5.1 Minimum SCCIR ( $r=1)$.

\begin{tabular}{|c|c|c|}
\cline { 2 - 3 } \multicolumn{1}{c|}{2 active CCI } & $K=3$ & $K=7$ \\
\hline$\gamma=2$ & $6.0206 \mathrm{~dB}$ & $10.0000 \mathrm{~dB}$ \\
\hline$\gamma=3$ & $10.1156 \mathrm{~dB}$ & $16.3402 \mathrm{~dB}$ \\
\hline$\gamma=4$ & $13.8917 \mathrm{~dB}$ & $22.5648 \mathrm{~dB}$ \\
\hline$\gamma=5$ & $17.3125 \mathrm{~dB}$ & $28.6690 \mathrm{~dB}$ \\
\hline
\end{tabular}

\begin{tabular}{|c|c|c|}
\cline { 2 - 3 } \multicolumn{1}{c|}{3 active $\mathrm{CCI}$} & $\mathrm{K}=3$ & $\mathrm{~K}=7$ \\
\hline$\gamma=2$ & $4.2597 \mathrm{~dB}$ & $8.2319 \mathrm{~dB}$ \\
\hline$\gamma=3$ & $8.3547 \mathrm{~dB}$ & $14.5793 \mathrm{~dB}$ \\
\hline$\gamma=4$ & $12.1307 \mathrm{~dB}$ & $20.8039 \mathrm{~dB}$ \\
\hline$\gamma=5$ & $15.5516 \mathrm{~dB}$ & $26.9081 \mathrm{~dB}$ \\
\hline
\end{tabular}

\begin{tabular}{|c|c|c|}
\cline { 2 - 3 } \multicolumn{1}{c|}{4 active CCI } & $K=3$ & $K=7$ \\
\hline$\gamma=2$ & $3.0103 \mathrm{~dB}$ & $6.9897 \mathrm{~dB}$ \\
\hline$\gamma=3$ & $7.1053 \mathrm{~dB}$ & $13.3299 \mathrm{~dB}$ \\
\hline$\gamma=4$ & $10.8814 \mathrm{~dB}$ & $19.5545 \mathrm{~dB}$ \\
\hline$\gamma=5$ & $14.3022 \mathrm{~dB}$ & $26.9081 \mathrm{~dB}$ \\
\hline
\end{tabular}

\begin{tabular}{|c|c|c|}
\cline { 2 - 3 } 5 active CCI & $K=3$ & $K=7$ \\
\hline$\gamma=2$ & $2.0412 \mathrm{~dB}$ & $6.0206 \mathrm{~dB}$ \\
\hline$\gamma=3$ & $6.1362 \mathrm{~dB}$ & $12.3608 \mathrm{~dB}$ \\
\hline$\gamma=4$ & $9.9123 \mathrm{~dB}$ & $18.5854 \mathrm{~dB}$ \\
\hline$\gamma=5$ & $13.3331 \mathrm{~dB}$ & $24.6896 \mathrm{~dB}$ \\
\hline
\end{tabular}

\begin{tabular}{|c|c|c|}
\cline { 2 - 3 } \multicolumn{1}{c|}{6 active CCI } & $K=3$ & $K=7$ \\
\hline$\gamma=2$ & $1.2494 \mathrm{~dB}$ & $5.2288 \mathrm{~dB}$ \\
\hline$\gamma=3$ & $5.3444 \mathrm{~dB}$ & $11.5690 \mathrm{~dB}$ \\
\hline$\gamma=4$ & $9.1204 \mathrm{~dB}$ & $17.7936 \mathrm{~dB}$ \\
\hline$\gamma=5$ & $12.5413 \mathrm{~dB}$ & $23.8978 \mathrm{~dB}$ \\
\hline
\end{tabular}




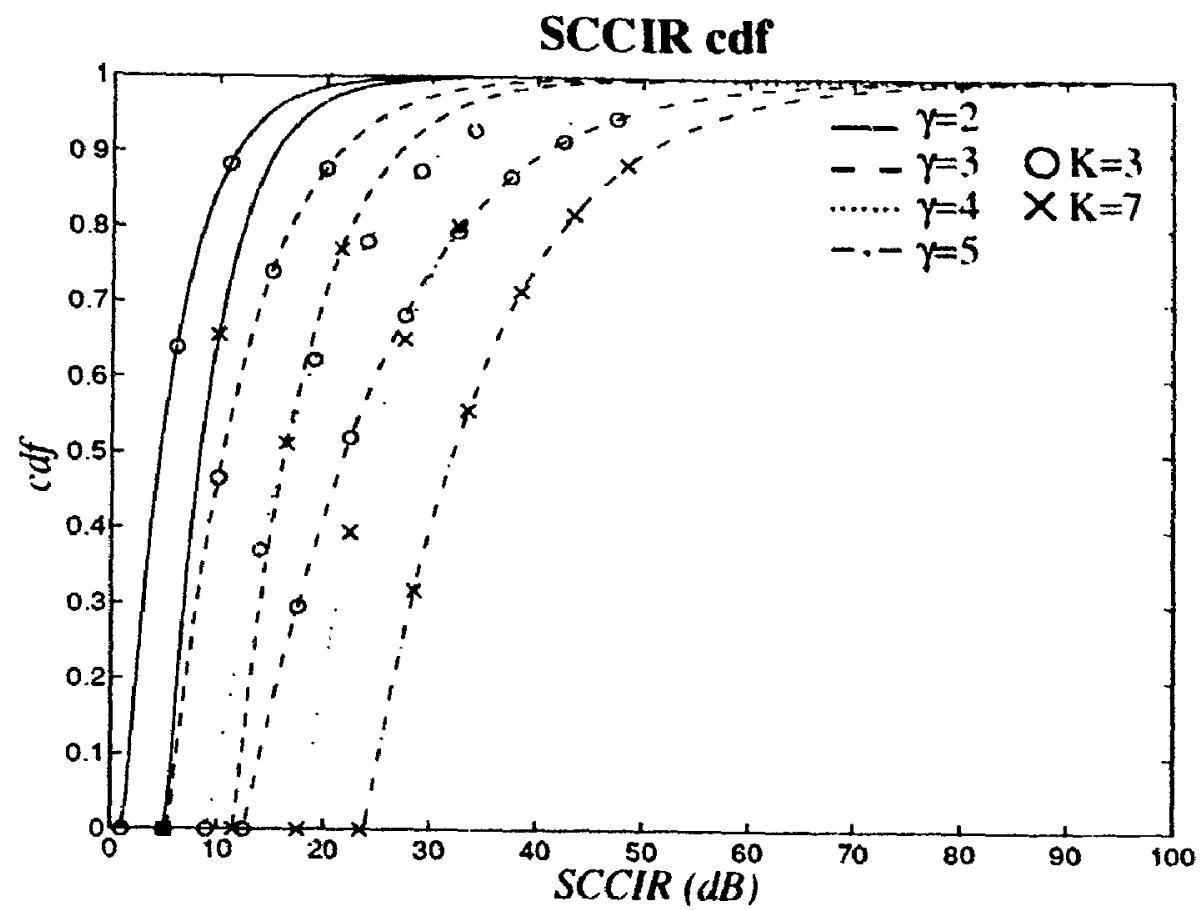

(a)

SCCIR pdf

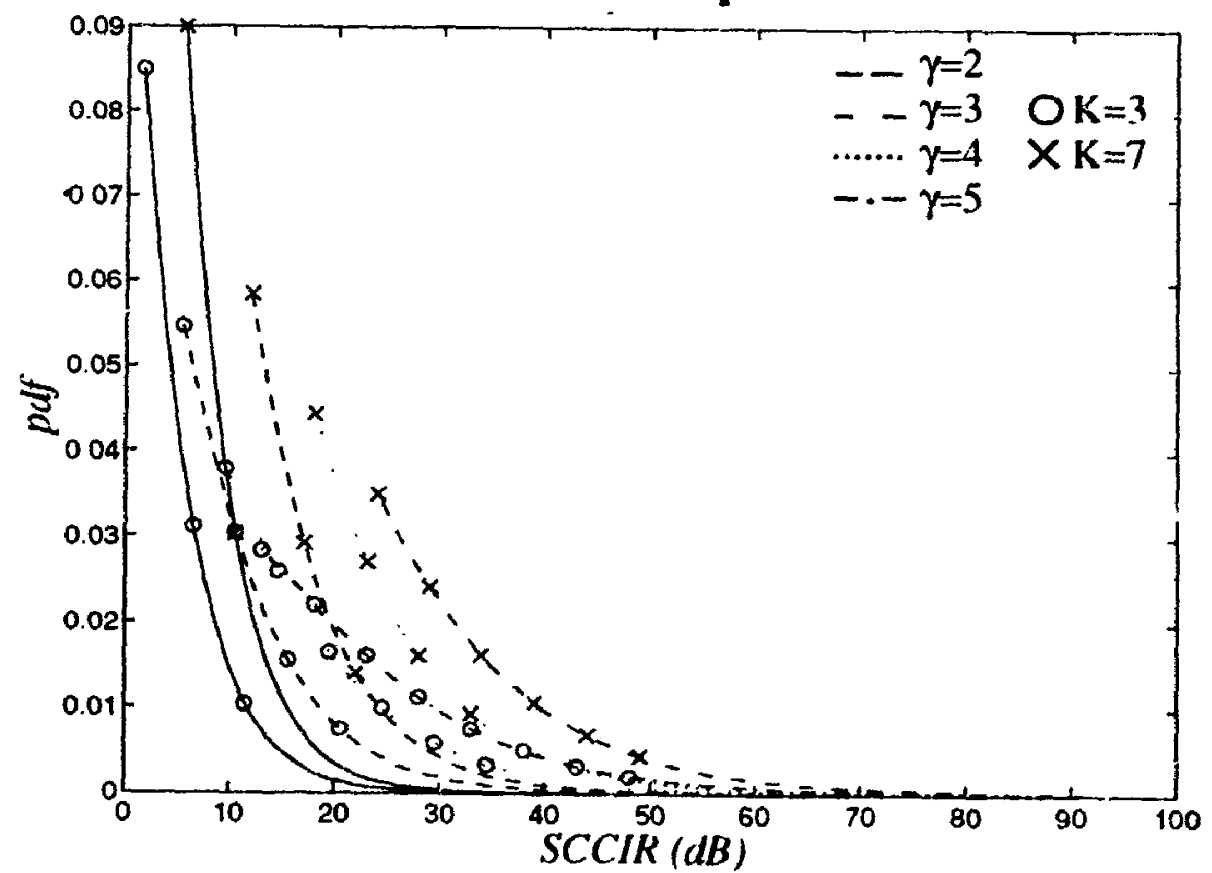

(b)

Figure 5.9 (a) edf (b) pdf of SCCIR in cellular systems with cluster sizes three and seven. 
Table 5.2 summarizes the average SCCIR over a cell. Comparing Table 5.1 and Table 5.2 yields that the overall average SCCIR values are close to minimum SCCIR values specially when cluster size is three.

\subsubsection{Signal to ACI Ratio (SACIR) in Cellular Systems}

In addition to mobile position, propagation loss factor and cluster size, the $\mathrm{ACI}$ power depends on channel spacing, excess bandwidth and pulse spectrum. The following equation presents the average power of $m$ th $\mathrm{ACI}$ at the receiver input :

$$
i_{a m}=\frac{A_{m}}{r_{a m}^{\gamma}}
$$

where $A_{m}$ is the power in the part of $\mathrm{ACl}$ spectrum overlapping SOI. The total $\mathrm{ACl}$ power can be found as follows :

$$
\begin{gathered}
I_{A}=\sum_{m=1}^{M} i_{a m}=\sum_{m=1}^{M} \frac{A_{m}}{r_{a m}^{\gamma}} \\
I_{A}=\sum_{m=1}^{M} A_{m} E\left(\frac{1}{\left(4+r^{2}+4 r \cos \left(\theta+(m-1) \frac{\pi}{3}\right)\right)^{\gamma / 2}}\right)
\end{gathered}
$$

where $M$ is the number of active $\mathrm{ACl}$. The total ACI, obviously, depends on how channels are assigned. The general form of above equation is not easy to find. However, for the special case where excess bandwidth is less than 100 percent and the channel spacing is equal to the symbol rate (Figure 5.10), (5.28) is simplified as :

$$
I_{A}=A_{\mathrm{i}} \sum_{m=1}^{M} E\left[\frac{1}{\left(4+r^{2}+4 r \cos \left(\theta+(m-1) \frac{\pi}{3}\right)\right)^{\gamma / 2}}\right)
$$

where $A_{1}$ is the power in the overlapped spectrum between first ACI and SOI. The computer simulation shows that the above equation may be empirically equivalent to the following equation :

$$
I_{A} \cong \frac{M A_{1}}{\left(4-0.4 \gamma r^{2}\right)^{0.5 y} r^{\gamma}}
$$


Table 5.2 Overall cell average of SCCIR.

\begin{tabular}{|c|c|c|}
\cline { 2 - 3 } \multicolumn{1}{c|}{2 active CCI } & $K=3$ & $K=7$ \\
\hline$\gamma=2$ & $6.8741 \mathrm{~dB}$ & $16 .(1396 \mathrm{~dB}$ \\
\hline$\gamma=3$ & $11.2760 \mathrm{~dB}$ & $26.3106 \mathrm{~dB}$ \\
\hline$\gamma=4$ & $14.9369 \mathrm{~dB}$ & $34.8527 \mathrm{~dB}$ \\
\hline$\gamma=5$ & $18.0093 \mathrm{~dB}$ & $42.0218 \mathrm{~dB}$ \\
\hline
\end{tabular}

\begin{tabular}{|c|c|c|}
\cline { 2 - 3 } \multicolumn{1}{c|}{3 active CCI } & $K=3$ & $K=7$ \\
\hline$\gamma=2$ & $4.8776 \mathrm{~dB}$ & $11.3812 \mathrm{~dB}$ \\
\hline$\gamma=3$ & $9.2046 \mathrm{~dB}$ & $21.4774 \mathrm{~dB}$ \\
\hline$\gamma=4$ & $13.0109 \mathrm{~dB}$ & $30.3588 \mathrm{~dB}$ \\
\hline$\gamma=5$ & $16.2635 \mathrm{~dB}$ & $37.9482 \mathrm{~dB}$ \\
\hline
\end{tabular}

\begin{tabular}{|c|c|c|}
\cline { 2 - 3 } \multicolumn{1}{c|}{4 active CCI } & $K=3$ & $K=7$ \\
\hline$\gamma=2$ & $3.7835 \mathrm{~dB}$ & $8.8283 \mathrm{~dB}$ \\
\hline$\gamma=3$ & $7.9178 \mathrm{~dB}$ & $18.4749 \mathrm{~dB}$ \\
\hline$\gamma=4$ & $11.7489 \mathrm{~dB}$ & $27.4141 \mathrm{~dB}$ \\
\hline$\gamma=5$ & $15.0881 \mathrm{~dB}$ & $35.2056 \mathrm{~dB}$ \\
\hline
\end{tabular}

\begin{tabular}{|c|c|c|}
\cline { 2 - 3 } 5 active $\mathrm{CCI}$ & $\mathrm{K}=3$ & $\mathrm{~K}=7$ \\
\hline$\gamma=2$ & $3.0915 \mathrm{~dB}$ & $7.2134 \mathrm{~dB}$ \\
\hline$\gamma=3$ & $7.0203 \mathrm{~dB}$ & $16.38(17 \mathrm{~dB}$ \\
\hline$\gamma=4$ & $10.8301 \mathrm{~dB}$ & $25.2703 \mathrm{~dB}$ \\
\hline$\gamma=5$ & $14.2135 \mathrm{~dB}$ & $33.1649 \mathrm{~dB}$ \\
\hline
\end{tabular}

\begin{tabular}{|c|c|c|}
\cline { 2 - 3 } \multicolumn{1}{c|}{6 active CCI } & $K=3$ & $K=7$ \\
\hline$\gamma=2$ & $2.6138 \mathrm{~dB}$ & $6.0989 \mathrm{~dB}$ \\
\hline$\gamma=3$ & $6.3495 \mathrm{~dB}$ & $14.8154 \mathrm{~dB}$ \\
\hline$\gamma=4$ & $10.1184 \mathrm{~dB}$ & $23.60997 \mathrm{~dB}$ \\
\hline$\gamma=5$ & $13.5234 \mathrm{~dB}$ & $31.5547 \mathrm{~dB}$ \\
\hline
\end{tabular}



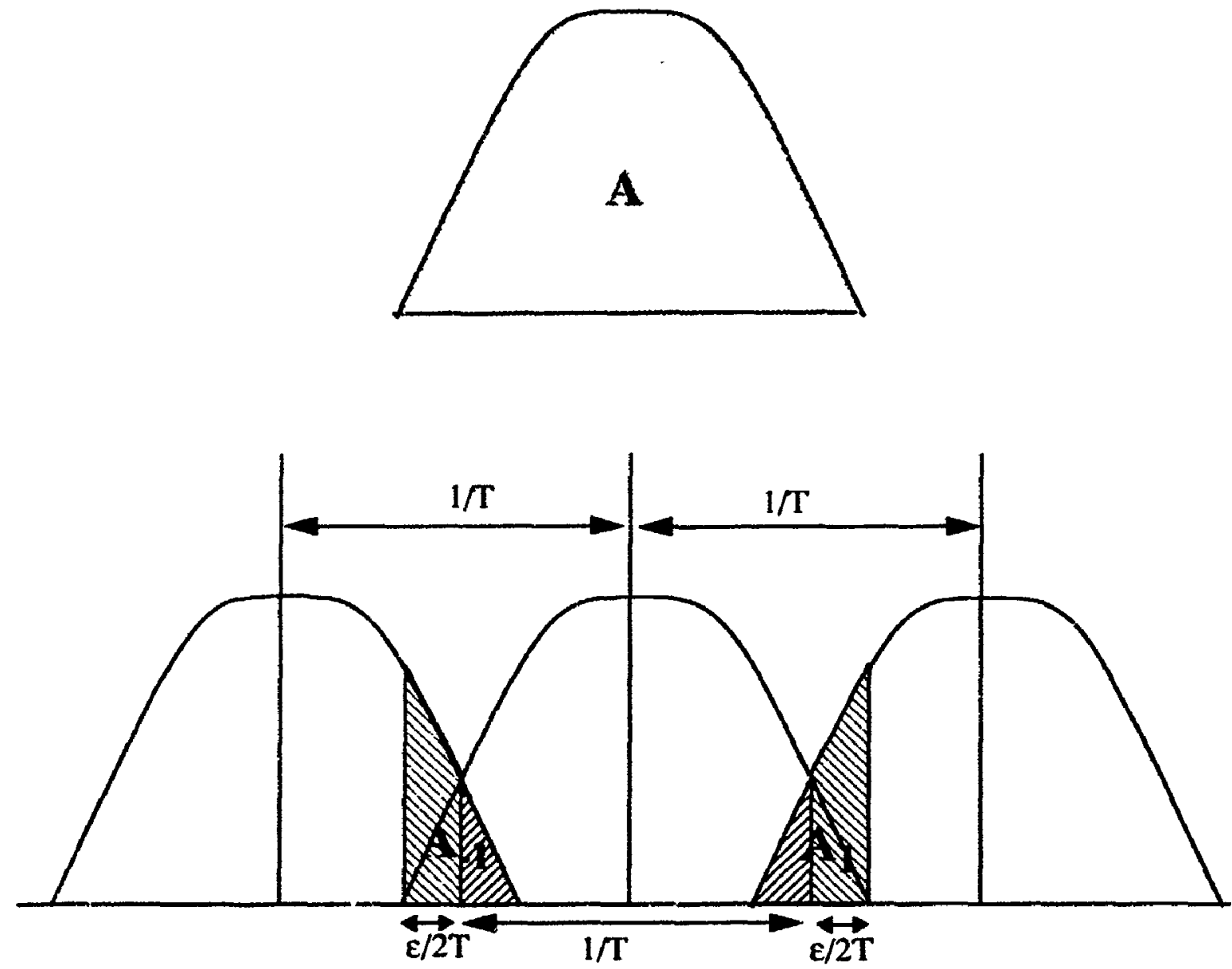

Figure 5.10 A system with channel spacing equals to symbol rate. 
and therefore,

$$
\frac{S}{I_{A}} \equiv \frac{\left(A / A_{1}\right)\left(4-0.4 \gamma r^{2}\right)^{0.5 \gamma}}{M r^{Y}}
$$

$A / A_{1}$ is related to pulse shape and for raised cosine filter $[84]$ with fractional excess bandwidth of $\varepsilon$, it is equal to $2 / \varepsilon$ :

$$
\begin{gathered}
A=\frac{1}{T} \quad A_{1}=\frac{\varepsilon}{2 T} \\
\frac{A}{A_{1}}=\frac{2}{\varepsilon}
\end{gathered}
$$

Therefore, the SACIR can be represented by the following equation :

$$
S A C I R \cong 3+5 \gamma \log \left(4-0.4 \gamma r^{2}\right)-10 \log (M \varepsilon)-1(\operatorname{lol} \log (r)
$$

Figure 5.11 (a) shows the SACIR versus $r$ when the number of active ACI is maximum. Minimum SACIR can be easily calculated by letting $r=1$ in the above equation :

$$
S A C I R_{\min } \cong 3+5 \gamma \log (4-0.4 \gamma)-10 \log (M \varepsilon)
$$

Figure 5.11 (b) shows the minimum SACIR versus excess bandwidth for several values of $\gamma$ and $K$ in the case of all ACI sources are active. It is evident from the tigures that SACIR is quite sensitive to excess bandwidth, cluster size, and propagation loss factor. Also, as the mobile position changes between $r=0.01$ and $r=1$ (edge of the cell), SACIR changes up to $120 \mathrm{~dB}$. Table 5.3 summarizes the minimum SACIR for 100 percent excess bandwidth. pdf and cdf and overall average of SACIR can be found in a manner similar to tuat for SCCIR.

\subsubsection{SIR in Cellular Systems}

Total interference can be calculated by adding ACI and $\mathrm{CCI}$ if they are uncorrelated :

$$
I=\frac{M A}{\left(3 K-0.5 \gamma r^{2}\right)^{0.5 \gamma}}+\frac{M A_{1}}{\left(4-0.4 \gamma r^{2}\right)^{0.5 \gamma} r^{\gamma}} \quad 1 \geq r \geq 0.01
$$

Therefore, 


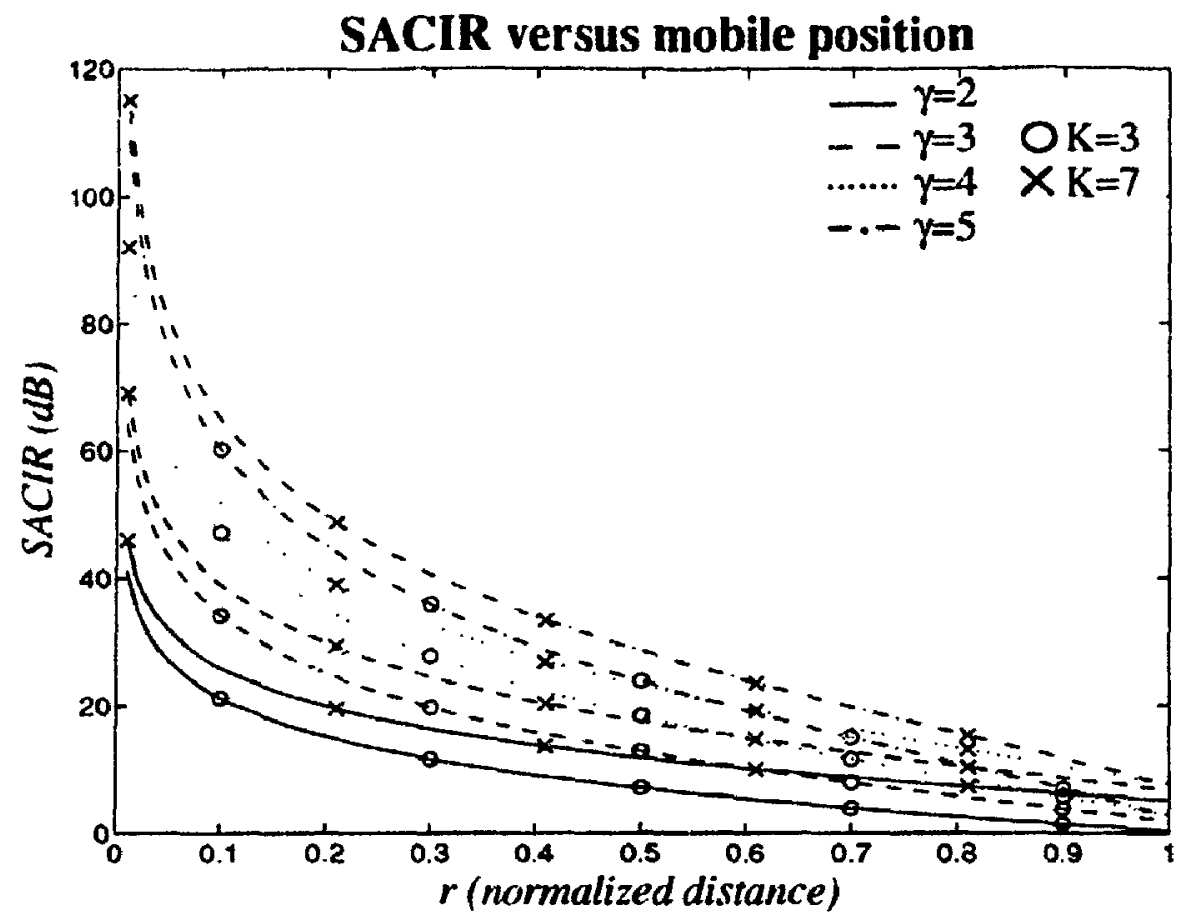

(a)

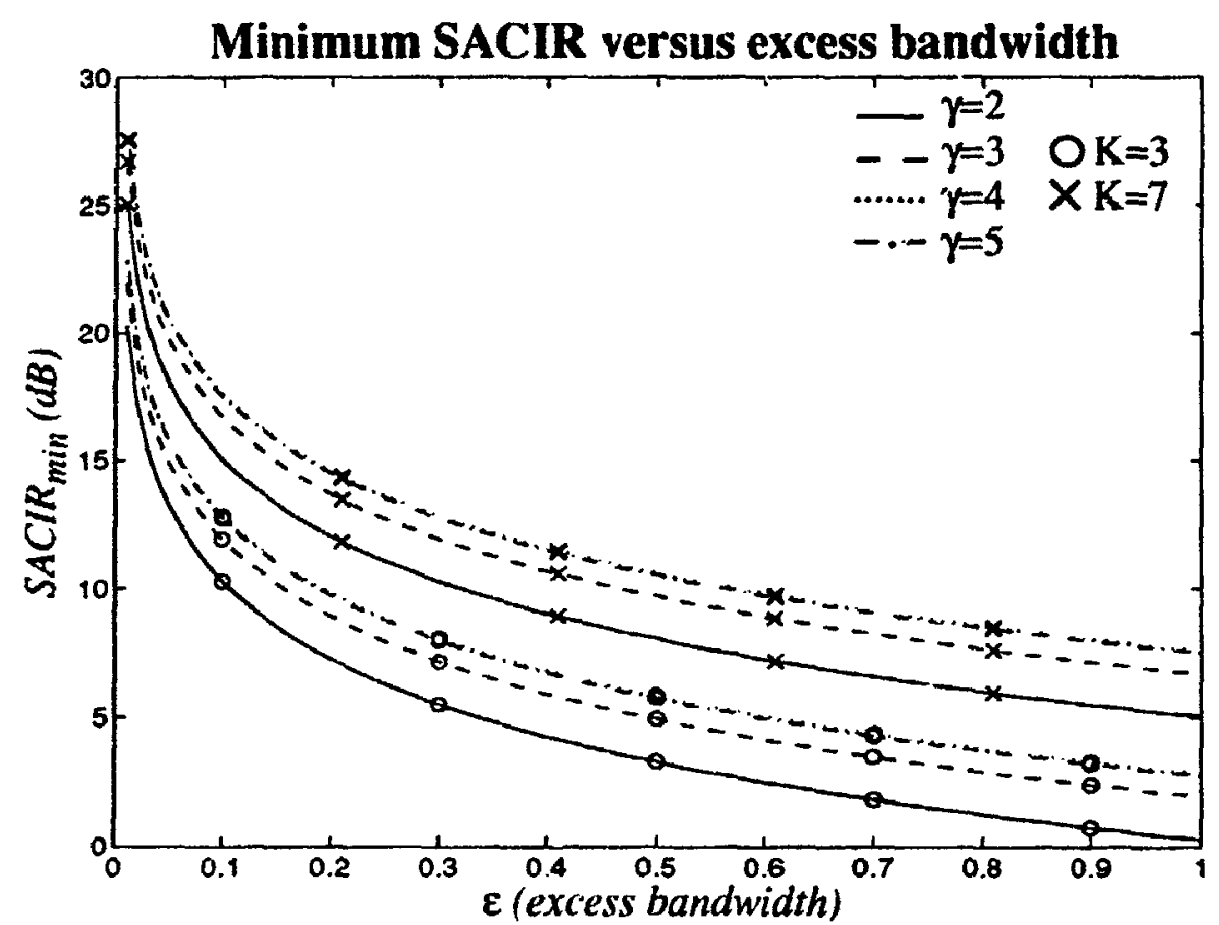

(b)

Figure 5.11 (a) $S A C I R_{\min }$ and (b) $S I R_{\min }$ versus signal excess bandwidth. 


$$
\frac{S}{i}=\frac{1}{r^{\gamma}\left(\frac{M_{c}}{\left(3 K-0.5 \gamma r^{2}\right)^{0.5 !}}+\frac{M_{u} \varepsilon}{2\left(4-0.4 \gamma r^{2}\right)^{11.5 \gamma}}\right)} \quad 1 \geq r \geq 0.01
$$

where $M_{c}$ and $M_{a}$ are the number of active $\mathrm{CCl}$ and active $\mathrm{ACl}$ sources respectively. The SIR can be presented by the following equation :

$$
S I R=-10 \gamma \log \left(\frac{M_{c}}{\left(3 K-0.5 \gamma r^{2}\right)^{0.5 \gamma}}+\frac{M_{c} \varepsilon}{2\left(4-0.4 \gamma r^{2}\right)^{0.5 \gamma}}\right)-10 \gamma \log (r)
$$

Figure 5.12 (a) shows SIR for maximum number of active interferers. The minimum SIR is obtained from (5.37) by setting $r=1$ :

$$
S I R_{\min }=-10 \gamma \log \left(\frac{M_{c}}{(3 K-0.5 \gamma)^{0.5 \gamma}}+\frac{M_{a d} \varepsilon}{2(4-0.4 \gamma)^{1.5 \gamma}}\right)
$$

Figure 5.12 (b) shows minimurn SIR versus signal excess bandwidth. It is observed that when excess bandwidth is low, $\mathbf{C C I}$ is dominant while $\mathrm{ACl}$ is significant for high excess bandwidth. Table 5.4 summarizes minimum SIR for 100 percent excess bandwidth when all $\mathrm{CCI}$ and $\mathrm{ACI}$ are active. The results given in the table indicate that, the minimum SIR is low and even can be negative in the case of cluster size three. The pdf and idf of SIR can also be found by the method similar to that of SCCIR.

\subsection{Cellular System Capacity}

The capacity and spectral efficiency of a cellular system has been defined in [49,50,64]. Although these definitions are adequate for analog cellular systems, we propose a better definition for digital cellular systems capacity. We define the capacity of a digital cellular system by the maximum number of bits per second which can be transmitted over a cell area for a given average bit error rate (BER) and total available bandwidth. The maximum number of users over total available bandwidth in a FDMA system can be found according to :

$$
N_{1}=\frac{W-B+\Delta f}{\Delta f}
$$

where $W$ is the total available bandwidth, $B$ is the user bandwidth and $\Delta f$ is the channel spacing. The maximum number of users per cell is $1 / K$ of $N_{1}$ where $K$ is the cluster size : 
SIR versus mobile position

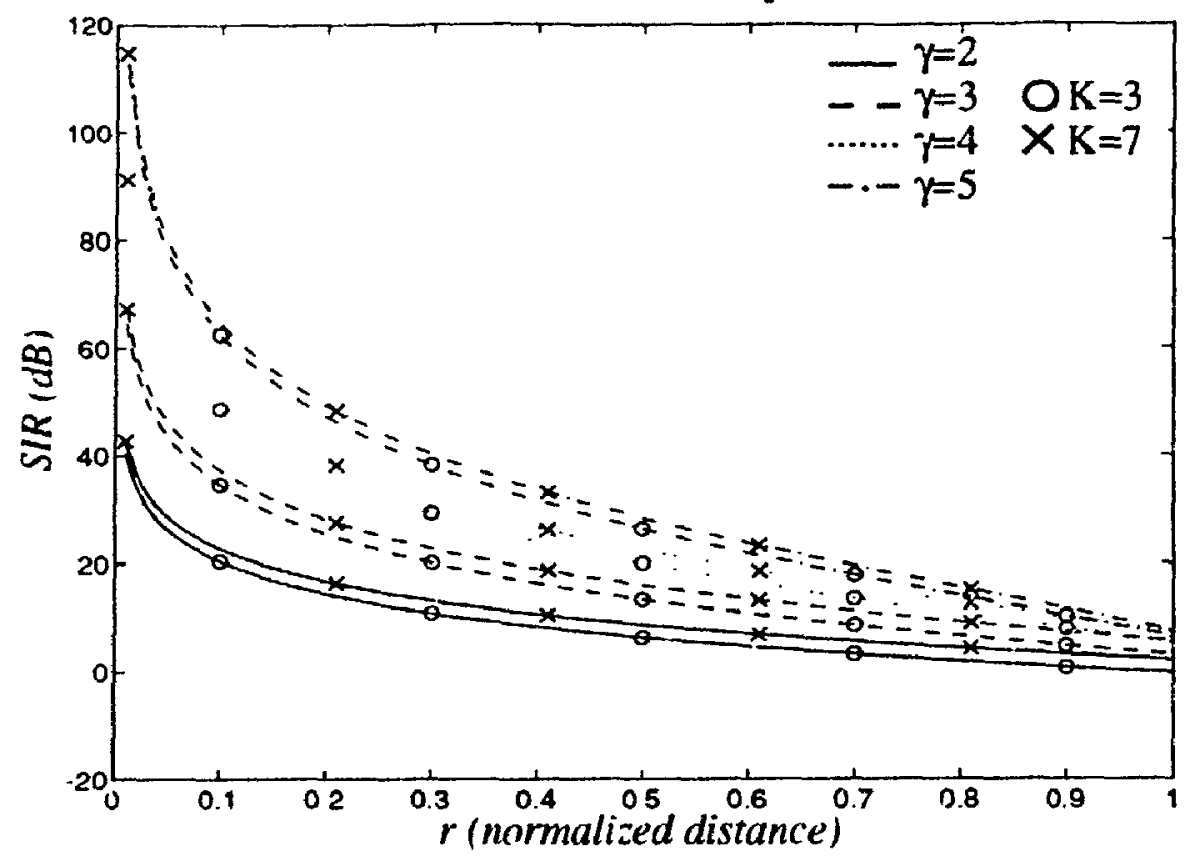

(a)

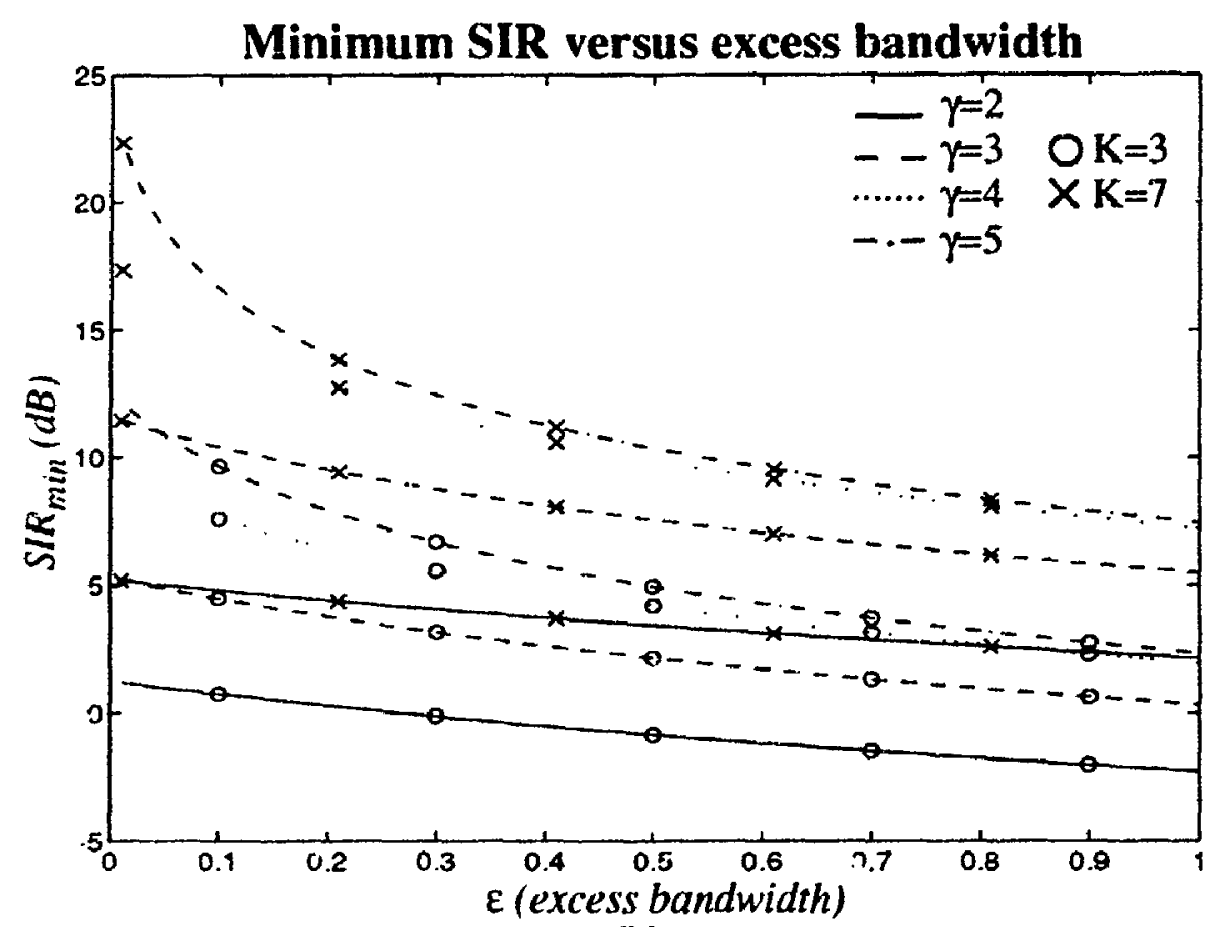

(b)

Figure 5.12 (a) $S_{A C I R_{\min }}$ and (b) SIR $_{\min }$ versus signal excess bandwidth. 
Table 5.3 Minimum SACIK $(r=1)$.

\begin{tabular}{|c|c|c|}
\cline { 2 - 3 } \multicolumn{1}{c|}{$E=1$} & $K=3$ & $K=7$ \\
\hline$\gamma=2$ & $0.2700 \mathrm{~dB}$ & $5.0515 \mathrm{~dB}$ \\
\hline$\gamma=3$ & $1.9259 \mathrm{~dB}$ & $6.7074 \mathrm{~dB}$ \\
\hline$\gamma=4$ & $2.8227 \mathrm{~dB}$ & $7.6042 \mathrm{~dB}$ \\
\hline$\gamma=5$ & $3.2685 \mathrm{~dB}$ & $7.8257 \mathrm{~dB}$ \\
\hline
\end{tabular}

Table 5.4 Minimum SIR $(r=1)$.

\begin{tabular}{|c|c|c|}
\cline { 2 - 3 } \multicolumn{1}{c|}{$\varepsilon=1, M=6$} & $K=3$ & $K=7$ \\
\hline$\gamma=2$ & $-2.2724 \mathrm{~dB}$ & $2.1289 \mathrm{~dB}$ \\
\hline$\gamma=3$ & $0.3039 \mathrm{~dB}$ & $5.4804 \mathrm{~dB}$ \\
\hline$\gamma=4$ & $1.9160 \mathrm{~dB}$ & $7.2072 \mathrm{~dB}$ \\
\hline$\gamma=5$ & $2.3208 \mathrm{~dB}$ & $7.4268 \mathrm{~dB}$ \\
\hline
\end{tabular}




$$
N=\frac{N_{1}}{K}=\frac{W-B+\Delta f}{K \Delta f}
$$

Therefore the capacity is :

$$
C=\left.\max \left(\frac{M N}{A T}\right)\right|_{B E R=b_{0}, w=W_{0}}=\left.\max \left(\frac{M\left(W_{0}-B+\Delta f\right)}{K A T \Delta f}\right)\right|_{B E R=b_{0}}
$$

where $M$ is the number of bits per symbol, $T$ is the symbol rate and $A$ is the cell area. With the above definition, the capacity is a function of $b_{0}$ and $W_{0}$.

It is apparent from (5.41) that the smaller cell area yields larger capacity. Therefore, in general, the micro-cellular systems have higher capacity than other cellular systems. However, the smaller cell area needs larger number of base stations for the same coverage area. A larger number of base stations leads to greater system complexity.

It is also apparent form (5.41) that the capacity goes to infinity when channel spacing reduces to zero. In this case, either the bit error rate goes to one for single antenna receiver, or infinite number of antennas must be used. For single antenna receiver, the BER is a function of channel spacing, cluster size, excess bandwidth and power of the signal (or SNR), and therefore, the capacity becomes a function of these variables as well.

When the channel spacing is grater than or equal to signal bandwidth,

$$
\Delta f \geq B=\frac{1+\varepsilon}{T}
$$

there is no $\mathrm{ACl}$, and $\mathrm{CCl}$ can be suppressed by using large excess bandwidth $[80,81]$. To suppress the first tier $\mathrm{CCl}$, excess bandwidth should be larger than 600 percent $(\varepsilon=6)$. In this case, a very low BER is achievable in single antenna system by increasing the signal power. However, the capacity is lower than $M W_{0} / K A(1+\varepsilon)$ bits $/ \mathrm{sec} / \mathrm{km}^{2}$. This limitation is calculated by substituting (5.42) in (5.41).

If the channel spacing is less than signal bandwidth, the ACI exists and the BER tinally settles to an irrefucable value in a single antenna receiver. The amount of this irreducable BER is related to the cluster size, excess bandwidth and channel spacing.

If the chann spacing is fixed to the symbol rate, the capacity is : 


$$
C=\left.\max \left(\frac{M\left(W_{0}-\varepsilon / T\right)}{K A}\right)\right|_{B E R=b_{0}}
$$

Since the $\varepsilon / T$ is much less than $W_{0}^{\prime}$, the above equation can be approximated to :

$$
\left.C \equiv \max \left(\frac{M W_{0}}{K A}\right)\right|_{B E R=b_{0}}
$$

It is apparent from the above equation that, for a fixed $A$, the maximum condition is satisfied when $K=1$. However, the achievable BER with this condition is expected to be very high. For $K=3$, the Table 5.4 shows that the SIR is lower than zero dB and in this condition the BER is still high. For $K=7$, the capacity will be $M W_{0} / 7 A$ bits $/ \mathrm{sec} / \mathrm{km}^{2}$ but the SIR is around $5.5 \mathrm{~dB}$ (for $\varepsilon=1$ ) which suggests that a reasonable BER is achievable. In the next chapter we will simulate a cellular system with cluster size seven and channel spacing equaling symbol rate to find out what BER can is achievable for $M W_{1} / 7 A$ bits $/ \mathrm{sec} / \mathrm{km}^{2}$.

\subsubsection{Minti-Symbol Rate Systems}

From the previous chapter, we sbserve that the singl' symbol rate systems do not create $\mathrm{CC}$ i free yclic spectra while the multi-symbol rate systems do. In this section, we will consider how a multi-symbol rate system can be designed. Figure 5.13 (a) shows that

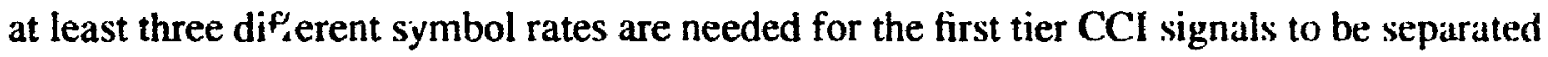
in the cyclic spectra. Figure 5.13 (b) shows a design of three symbol rate cellular system. Although the SOI has cyclic spectra free of $\mathrm{CCI}$ and $\mathrm{ACI}$, the system management and the receiver structure become complicated.

Consider Figure 5.14 to. $;$ in a syomivol rates system. In this system the number of nirst tier CCI with sa. e syinb.

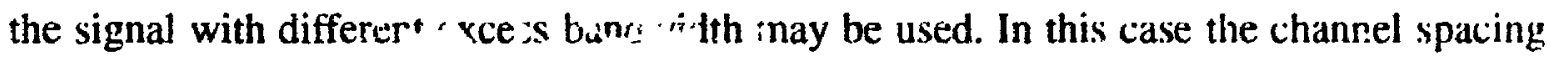
remain the same. How :er, the " par " y c $t$ the system is reduced. For example if 0.5 and 1 $\mathrm{Hz}$ are the two chosen symbol rates, the excess bandwidths of $(1+2 \varepsilon)$ and $\varepsilon$ will result in the same signal bandwidth for two different symbol rate signals. For channel spacing equals to one, the capacity is redurced to $3 M W_{0} / 28 A \mathrm{bits} / \mathrm{sec} / \mathrm{km}^{2}$. In next chapter this system is simulaied and the results are compared with single symbol rate system.

\subsection{Conclusion}

Mobile radio cellular systems have been considered in this chapter. Frequency 


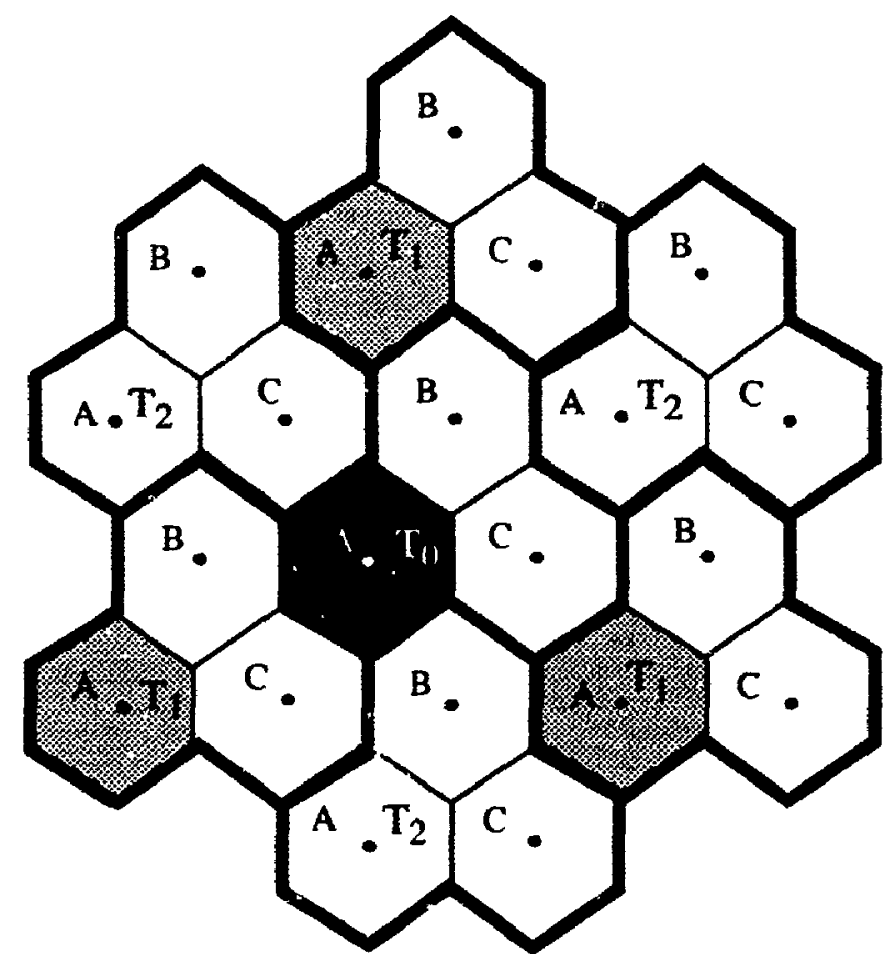

(a)

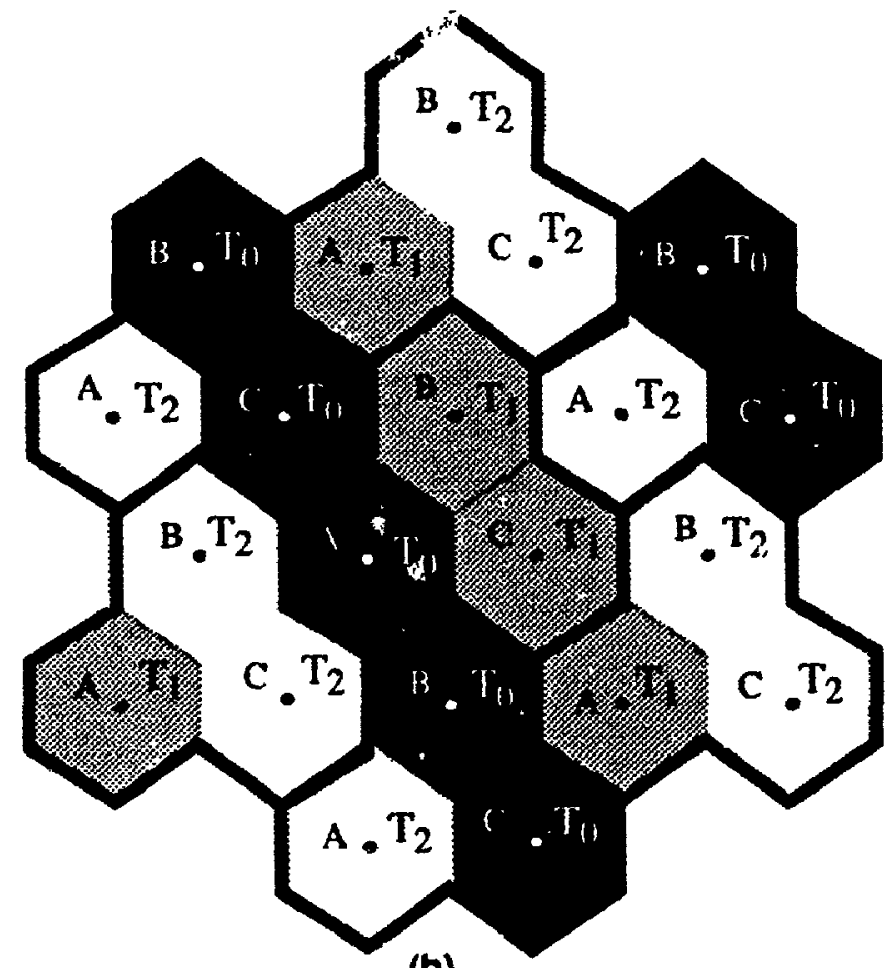

(b)

Figure 5.13 (a) multi-symbol rate first tier $\mathrm{CCl}$ (b) multi-symbol rate system using three symbol rates. 


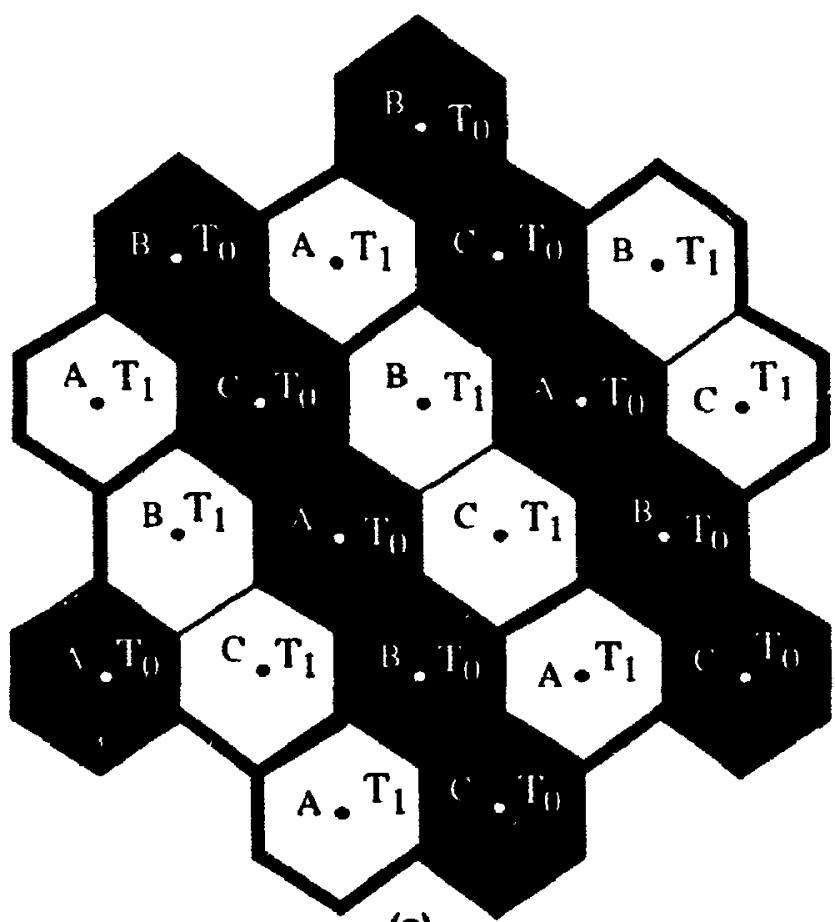

(a)

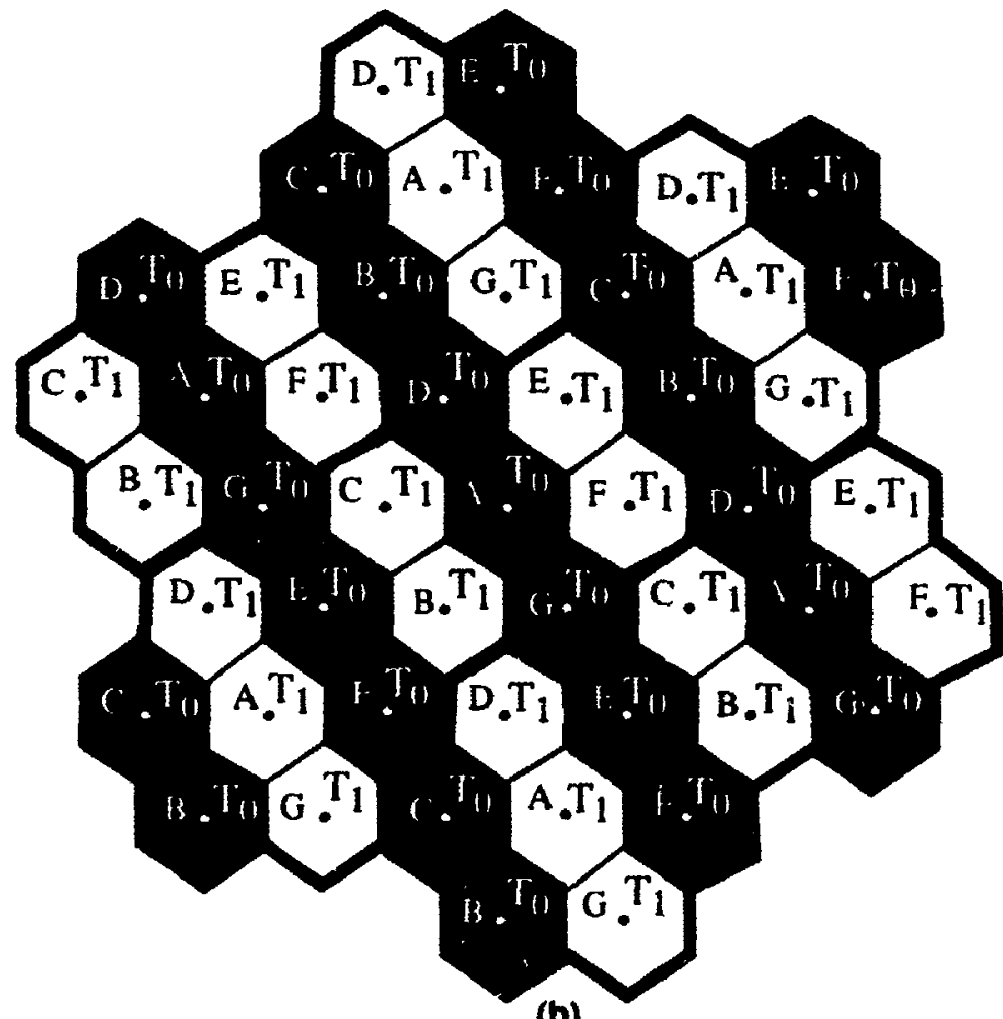

(b)

Figure 5.14 2-symbol rates system (a) cluster 3 (b) cluster 7. 
reuse, cell splitting and multiple access techniques have been discussed. The properties of digital mobile radio channels have been investigated. Multipath, and fading channels have been reviewed and the channel models have been considered. Expressions SNR, SCCIR, SACIR, and SIR in cellular system have been derived. The capacity of cellular systems have been defined and analyzed. A multi-symbol rate cellular system has been proposed. 


\section{Chapter 6 \\ Simulation Results}

\subsection{Introduction}

In this chapter, results of many simulations experiments to validate the analytical results in chapter 4 and 5 are presented. The performance of FRESH-DFE and conventional DFE are compared and it is shown that FRESH-DFE has better performance. It is also shown that the multi-antenna receiver can achieve low bit error rate even though the channel spacing is smaller than the symbol rate. The 2-symbol rate cellular system shown in Figure 5.14 is also simulated and it is shown that the performances of FRESH-DFE and conventional DFE are better in this system than the system with single symbol rate. It is concluded that for the same receiver complexity, the ODFE has better performance than DFE.

\section{Adaptive Filters}

An adaptive filter is a practical method of implementing an optimal filter that can adjust to slowly changing signal statistics. The adaptive filter schematic which is shown in Figure 6.1, has two inputs, the observed signal, $x_{k}$, and the training or desired signal, $y_{k}$. The observed signal is filtered and subtracted from training signal to produce an error signal. The adaptive filter is updated to minimize some error criterion, typically mean square error (MSE).

The most common adaptive filter is the finite length impulse response (FIR) leastmean-square (LMS) adaptive filter. The FIR LMS output is given by :

$$
\hat{y}_{n}=\left.\underline{W}_{k}^{T} \underline{X}_{k}\right|_{n=k M}
$$

where $\hat{y}_{n}$ is the filter output at the time $n=k M, M$ is the ratio of the input data rate to the desired data rate, $\underline{X}_{k}$ is a vector of observed data within the filter,

$$
\underline{X}_{k}^{T}=\left[\begin{array}{lll}
x_{k}(1) & x_{k}(2) \ldots & \left.x_{k}(N)\right]
\end{array}\right.
$$

and $\underline{W}_{k}$ is the vector of adjustable filter weights at the time $k$, 

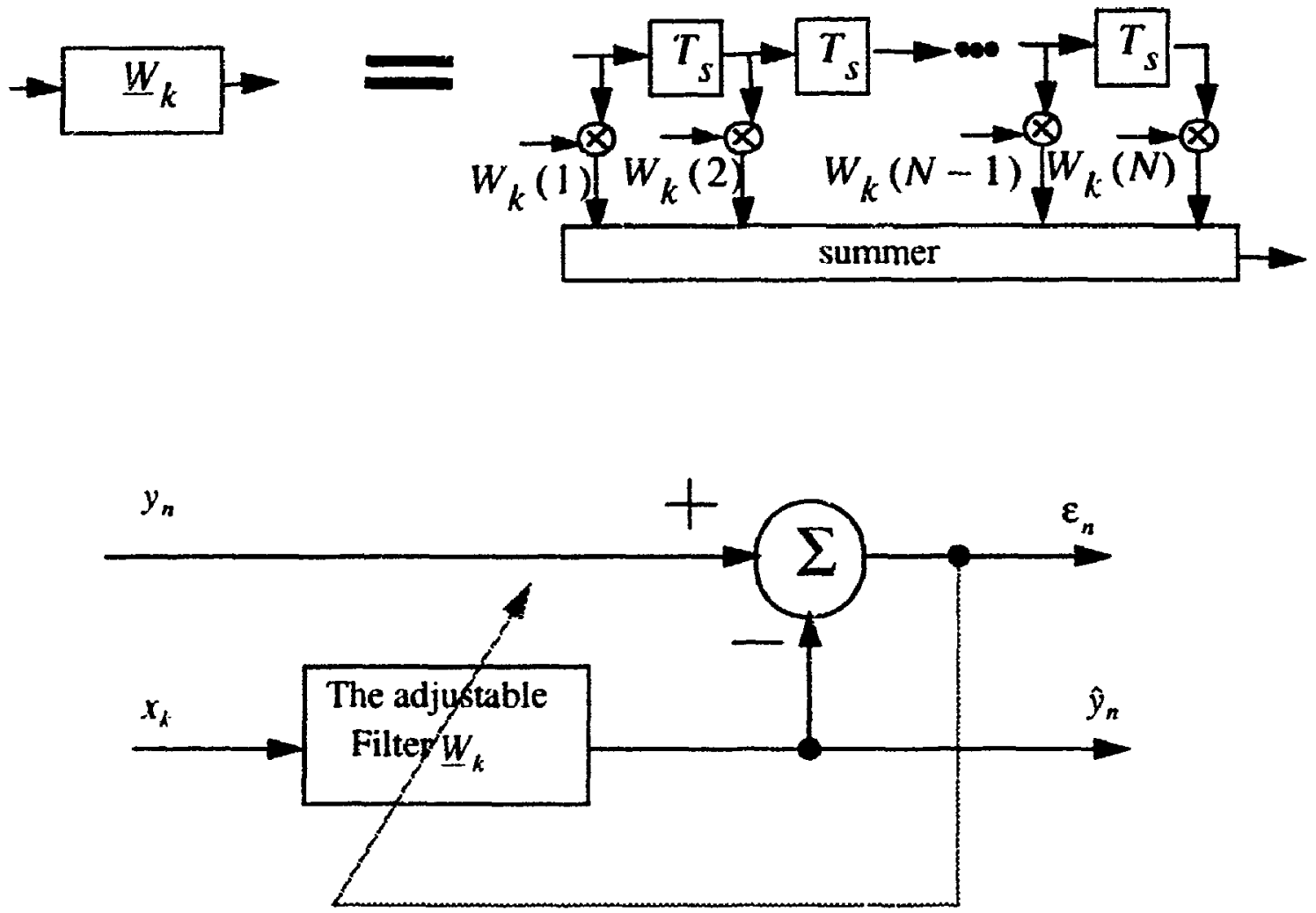

Figure 6.1 An adaptive filter. 


$$
\underline{W}_{k}^{T}=\left[W_{k}(1) W_{k}(2) \ldots \quad W_{k}(N)\right]
$$

The weights are adjusted using the LMS algorithm at the time $n=k M$.

$$
\underline{W}_{n+1}=\underline{W}_{n}+2 \mu \varepsilon_{n} X_{n}^{*}
$$

$\mu$ is the adaptive step size, and $\varepsilon_{n}$ is the error

$$
\varepsilon_{n}=y_{n}-\hat{y}_{n}
$$

where $y_{n}$ is the training or desired signal.

For LMS algorithm, the step size has to satisfy the following condition : [52]

$$
0<\mu<\frac{1}{\left\|X_{n}\right\|^{2}}
$$

where $\left\|\underline{X}_{n}\right\|$ is the norm of input vector at the time $n$. Since the above condition has to be satisfied at all the time, the step size will be related to the power of the input vector. To avoid this problem, equation (6.4) can be changed to the following equation :

$$
\underline{W}_{n+1}=\underline{W}_{n}+2 \mu \varepsilon_{n} \frac{X_{n}}{\left\|X_{n}\right\|^{2}}
$$

This is called normalized LMS (NLMS) [52]. In this case the condition (6.6) is changed to:

$$
0<\mu<1
$$

A smaller step size results in slower convergence but lower minimum MSE (MMSE), while a larger step size yields faster convergence but higher MMSE. This tradeoff leads to variable step size [52]. In variable step size LMS algorithm, the step size $\mu$ is time-variant and according to a certain rule is started with almost maximum value and decreases to the minimum value approaching convergence. Changing the step size can be function of time or the error. This function can be linear or non-linear. Therefore, there are many possible variable step size algorithms.

One of the most common variable step size rule is gear-shifting (GS) [52]. In GS algorithm, the step size is changed between several values during the convergence time. Figure 6.2 shows an example of GS algorithm which is used in our simulation. $P_{0}$ in the 


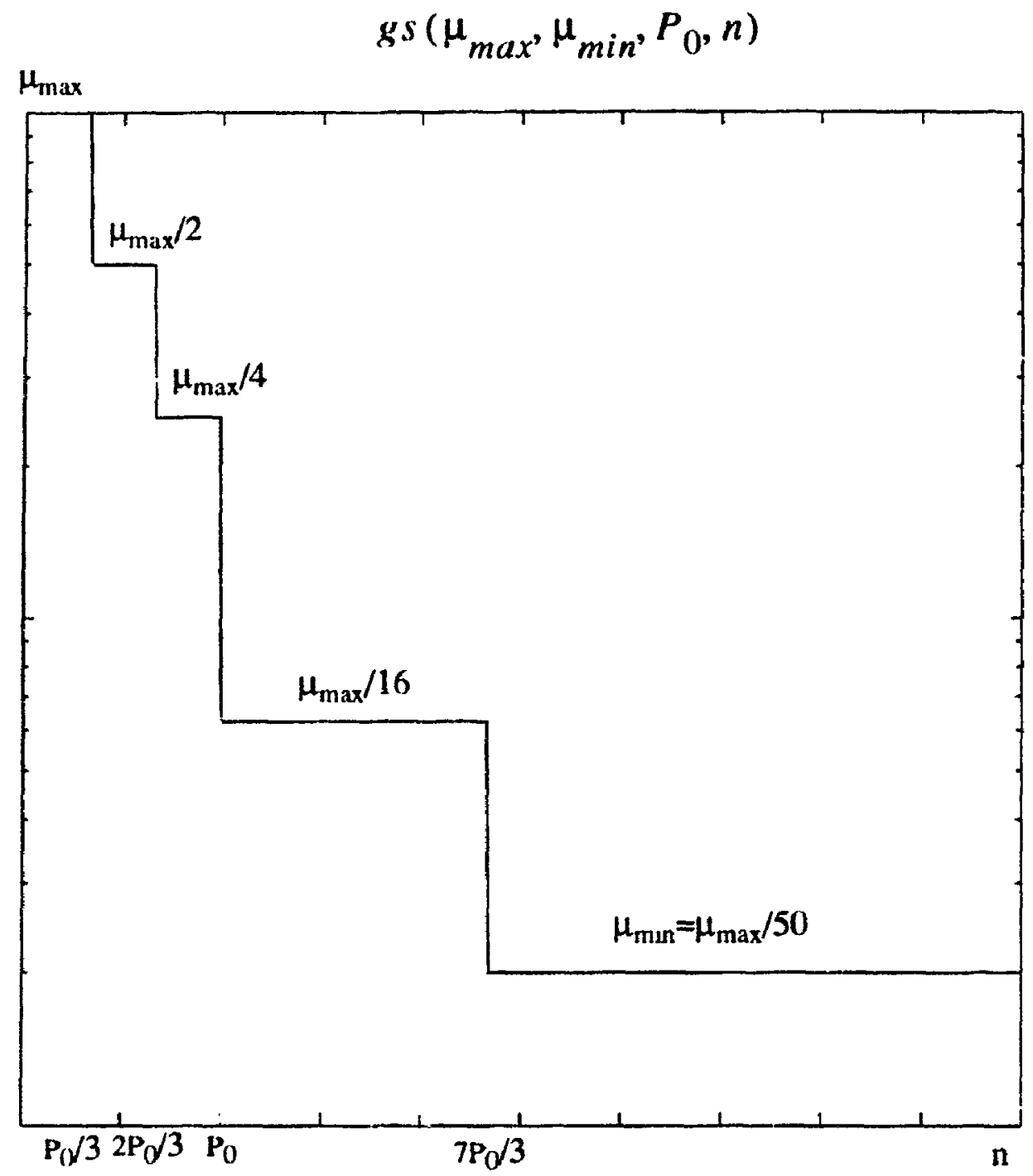

Figure 6.2 An example of Gear Shifting (GS) rule. 
figure designates the end of training sequence. At this time the adaptive filter either stop the adaptation or uses output decisions as the desired value. We will represent GS with the following notation :

$$
\mu_{n}=g s\left(\mu_{m a s}, \eta_{m e n}, P_{(n,} n\right)
$$

\subsection{Adaptive Conventional DFE with Normalized Least- Mean-Square (NLMS) Algorithm}

The NLMS updating-equation for the conventional DFE coefficicnts can be written as :

$$
\underline{W}_{n+1}=\underline{W}_{n}+\mu_{n} \underline{V}(n) 2 \mu_{n} \varepsilon_{n}{\frac{\underline{V}(n)}{\|\underline{V}(n)\|^{2}}}^{*}
$$

where $\underline{V}(n)$ is defined by $(4.71), \underline{W}_{n}$ and $\varepsilon_{n}$ are defined by :

$$
\begin{gathered}
\underline{W}_{n}^{T}=\left[\begin{array}{ll}
a_{f_{n}}^{T} & -\underline{a}_{b_{n}}^{T}
\end{array}\right] \\
\varepsilon_{n}=d_{0}(n-D)-\underline{W}_{n}^{T} V(n)
\end{gathered}
$$

$\mu_{n}$ is the step size at the time $t=n T_{0}$. Substituting (4.71) and (6.11) in (6.10) yields:

$$
\left[\underline{a}_{f_{n+1}}-\underline{a}_{b_{n+1}}\right]=\left[\underline{a}_{f_{n}}-\underline{a}_{b_{n}}\right]+2 \mu_{n} \varepsilon_{n} \frac{\left[\underline{\tilde{x}}\left(n M_{0}\right) \underline{d}(n-D-1)\right]^{*}}{\left\|\left[\tilde{\tilde{r}}\left(n M_{0}\right) d(n-D-1)\right]\right\|^{2}}
$$

Separating the elements of the abov: equation yields the following equations for updating the conventional DFE coefficients :

$$
\begin{aligned}
& \underline{a}_{f_{n+1}}=\underline{a}_{f_{n}}+2 \mu_{n} \varepsilon_{n} \frac{\underline{\vec{x}}^{*}\left(n M_{0}\right)}{\| \underline{\underline{x}\left(n M_{0}\right) \|^{2}+N_{b} \sigma_{d_{n}}^{2}}} \\
& \underline{a}_{b_{n+1}}=\underline{a}_{b_{n}}-2 \mu_{n} \varepsilon_{n} \frac{\underline{d}^{*}(n-D-1)}{\left\|\underline{\tilde{x}}\left(n M_{0}\right)\right\|^{2}+N_{b} \sigma_{d_{1}}^{2}}
\end{aligned}
$$

where the following simplification has been used : 


$$
\begin{gathered}
\left\|\left[\tilde{x}\left(n M_{0}\right) \underline{d}(n-D-1)\right]\right\|^{2}=E\left\{\left[\tilde{x}\left(n M_{0}\right) \underline{d}(n-D-1)\right]\left[\tilde{x}\left(n M_{0}\right) \underline{d}(n-D-1)\right]^{H}\right\} \\
=E\left\{\left[\underline{x}\left(n M_{0}\right) \mid\left[\left.\tilde{x}\left(n M_{0}\right)\right|^{H}\right\}+E\{\mid \underline{d}(n-D-1)][\underline{d}(n-D-1)]^{H}\right\}\right. \\
=\left\|\left[\tilde{x}\left(n M_{0}\right)\right]\right\|^{2}+N_{b} \sigma_{d_{0}}^{2}
\end{gathered}
$$

Figure 6.3 shows the structure of the adaptive conventional DFE used in simulations. In the conventional DFE two modes of operation exist. In mode one, called training mode, a training sequence is used to adjust the coefficients to achieve an acceptable MSE, and in mode 2, called tracking mode, DFE uses the data decisions to update its coefficients.

The decision device box in this structure is a hard decision device [84] which simply quantizes the real and imaginary parts of the input to two level, -1 and 1 . If BPSK is used, only the real part is used.

\subsection{Adaptive Multi-Antenna Conventional DFE with NLMS Algorithm}

Figure 6.4 shows the structure of multi-antenna conventional DFE used in the simulation. In this figure, superscript $(l)$ represents the $/$ th antenna branch signals. The NLMS updating equation for this structure can be also represented by $(6.10)$ where :

$$
\begin{aligned}
& \underline{\underline{v}}^{T}(n)=\left[\underline{x}^{(1)^{T}}\left(n M_{0}\right) \underline{\tilde{x}}^{(2)^{T}}\left(n M_{0}\right) \ldots \underline{\tilde{x}}^{(L)^{T}}\left(n M_{0}\right) \underline{d}^{T}(n-D-1)\right] \\
& \underline{W}_{n}^{T}=\left[\begin{array}{llll}
\underline{a}_{f_{n}}^{(1)^{T}} & \underline{a}_{f_{n}}^{(2)} & \ldots & \underline{a}_{f_{n}}^{(L)}
\end{array}\right. \\
& \varepsilon_{n}=d_{0}(n-D)-\underline{W}_{n}^{T} \underline{V}(n)
\end{aligned}
$$

Substituting (6.15) in (6.10) and separating the elements of the resulted equation yields : 


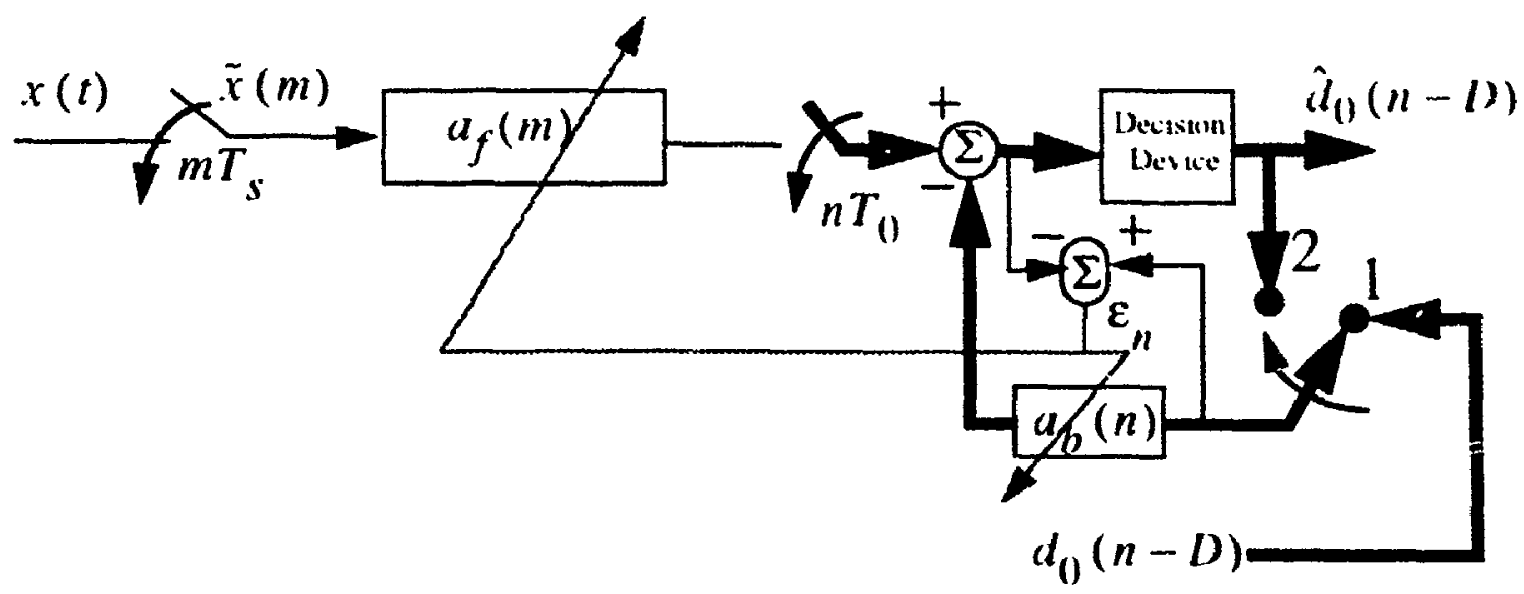

Figure 6.3 Adaptive conventional DFE.

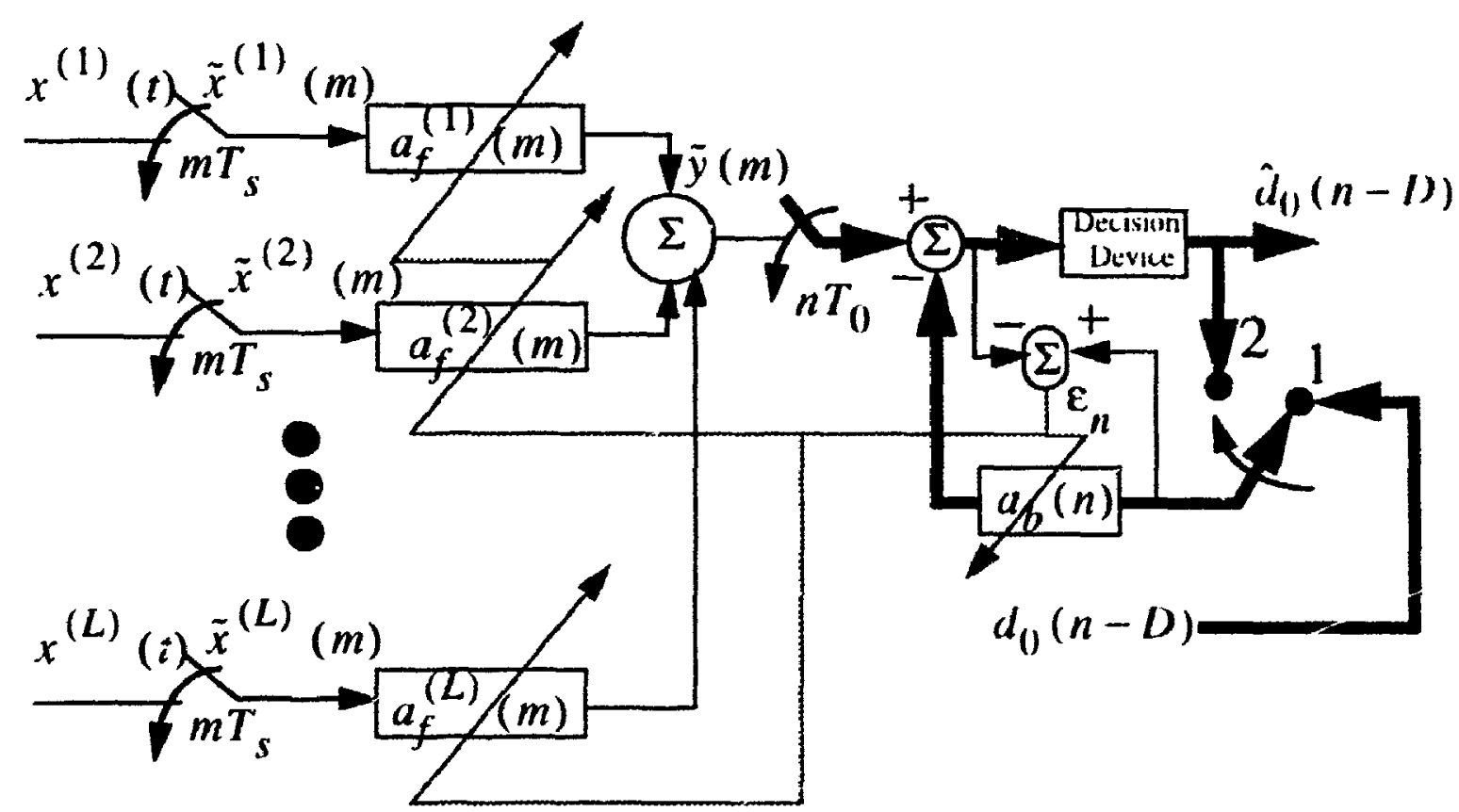

Figure 6.4 Adaptive multi-antenna conventional DFE. 


$$
\begin{gathered}
\underline{u}_{l_{n}, 1}^{\prime \prime)}=u_{l_{n}}^{(\prime)}+2 \mu_{n} \varepsilon_{n} \frac{\tilde{x}^{(l)^{*}}\left(n M_{0}\right)}{\sum_{m=1}^{L}\left\|\tilde{x}^{(m)}\left(n M_{0}\right)\right\|^{2}+N_{b} \sigma_{d_{0}}^{2}} l=1,2, \ldots, \mathrm{L} \\
\underline{u}_{h_{n},}=\underline{a}_{h_{n}}-2 \mu_{n} \varepsilon_{n} \frac{\underline{d}^{*}(n-D-1)}{\sum_{m=1}^{L}\left\|\underline{\tilde{x}}^{(m)}\left(n M_{0}\right)\right\|^{2}+N_{b} \sigma_{d_{0}}^{2}}
\end{gathered}
$$

where $\mu_{n}$ is defined by $(6.9)$.

\subsection{Adaptive FRESH-DFE with NLMS Algorithm}

The schematic of the adaptive FRESH-DFE used in the simulations is shown in Figure 6.5. Equation (6.10) is also valid for updating the coefficient of this equalizer. However. $\underline{V}(n) . \underline{W}_{n}$ and $\varepsilon_{n}$ are defined by :

$$
\begin{gathered}
\underline{V}^{T}(n)=\left[\begin{array}{ll}
\underline{X}^{(T)}(n) & \underline{d}^{T}(n-D-1)
\end{array}\right] \\
\underline{W}_{n}^{(1)}=\left[\begin{array}{lll}
\underline{a}_{0)_{n}}^{T} \underline{a}_{1_{n}}^{T} \ldots \underline{a}_{M_{n}}^{T} \underline{b}_{0_{n}}^{T} \underline{b}_{1_{n}}^{T} \ldots \underline{b}_{N_{n}}^{T}
\end{array}\right] \\
\varepsilon_{n}=d_{0}(n-D)-\underline{W}_{n}^{T} \underline{V}(n)
\end{gathered}
$$

where:

$$
\left.\underline{x}^{l}(n)=\left[\tilde{x}_{0}^{T}\left(n M_{0}\right) \underline{\tilde{x}}_{\alpha_{1}}^{T}\left(n M_{0}\right) \ldots \tilde{\tilde{x}}_{\alpha_{M}}^{T}\left(n M_{0}\right) \underline{x}_{0}^{* T}\left(n M_{0}\right) \tilde{x}_{-\beta_{1}}{ }^{T T}\left(n M_{0}\right) \ldots \tilde{x}_{-\beta_{N}}^{* T}(k 4 A\}\right)\right]
$$

Substituting (6.17) in (6.10) and separating elements of the resulted equation yields :

$$
\begin{gathered}
a_{k_{n}, 1}=\underline{a}_{k_{n}}+2 \mu_{n} \varepsilon_{n} \frac{\tilde{x}_{\alpha_{k}}^{*}\left(n M_{0}\right)}{\sum_{m=0}^{M}\left\|\tilde{x}_{\alpha_{m}}\left(n M_{0}\right)\right\|^{2}+\sum_{m=0}^{N}\left\|\tilde{x}_{\beta_{m}}\left(n M_{0}\right)\right\|^{2}+N_{b} \sigma_{d_{0}}^{2}} \\
k=1, \ldots, \mathrm{M} \\
b_{k_{n+1}}=\underline{b}_{k_{n}}+2 \mu_{n} \varepsilon_{n} \frac{\tilde{x}_{-\beta_{k}}\left(n M_{0}\right)}{\sum_{m=0}^{M}\left\|\tilde{x}_{\alpha_{m}}\left(n M_{0}\right)\right\|^{2}+\sum_{m=0}^{N}\left\|\tilde{x}_{\beta_{m}}\left(n M_{0}\right)\right\|^{2}+N_{b} \sigma_{d_{0}}^{2}} \\
k=1, \ldots, \mathrm{N}
\end{gathered}
$$




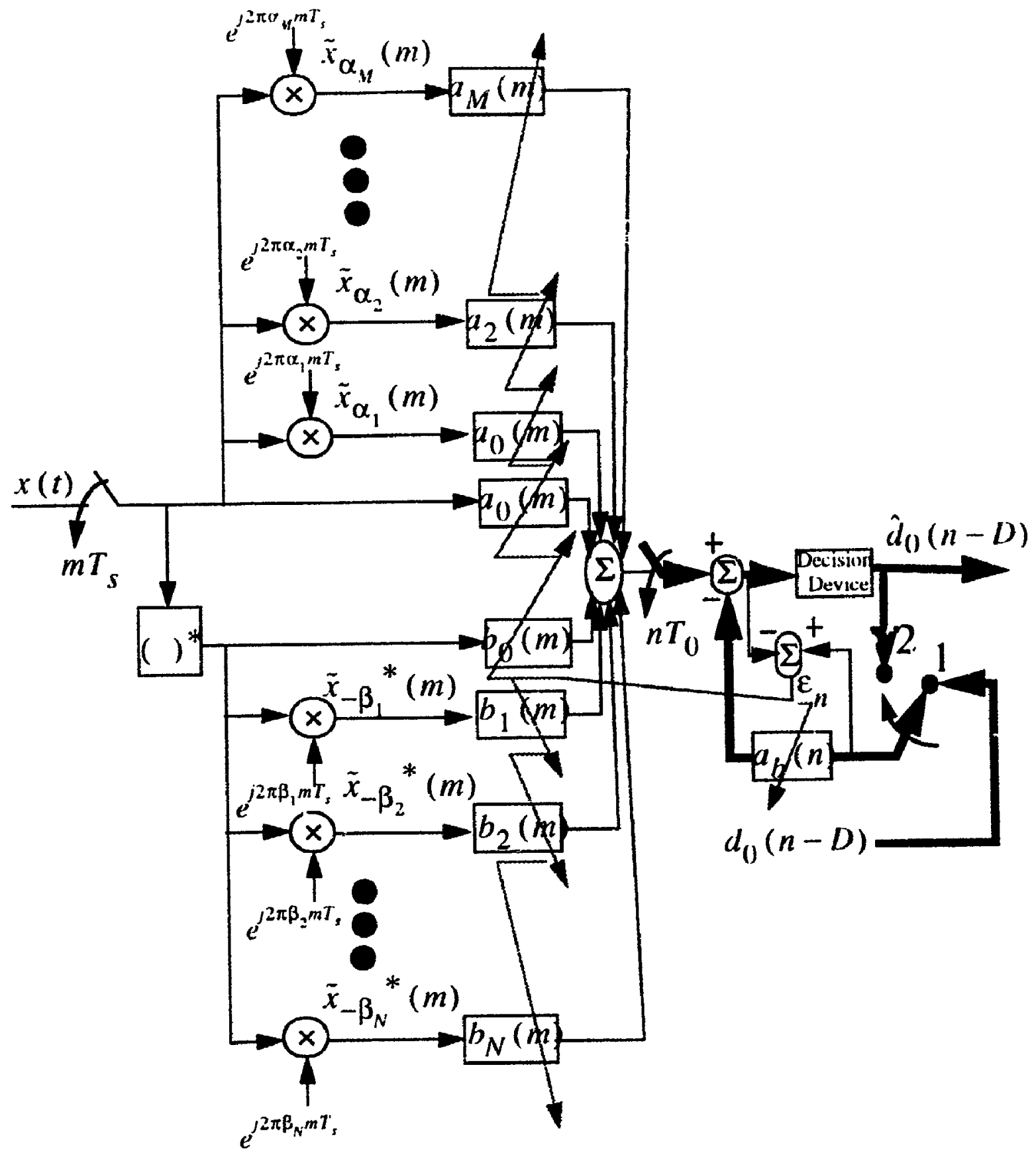

Figure 6.5 Adaptive FRESH-DFE. 


$$
\underline{a}_{h_{n+1}}=\underline{a}_{h_{n}}-2 \mu_{n} \varepsilon_{n} \frac{\underline{d}^{k^{*}}(n-D-1)}{\sum_{m=1}^{M}\left\|\tilde{\tilde{x}}_{\alpha_{m}}\left(n M_{0}\right)\right\|^{2}+\sum_{m=0}^{N}\left\|\tilde{x}_{\beta_{m}}\left(n M_{0}\right)\right\|^{2}+N_{b} \sigma_{d_{0}}^{2}}
$$

Same as the adaptive conventional DFE, the adaptive FRESH-DFE has two modes, training and tracking and uses the GS given in Figure 6.2.

\subsection{Adaptive Muti-Antenna FRESH-DFE (MA-FRESH-DFE) with NLMS Algorithm}

Adaptive MA-FRESH-DFE can be found by using an adaptive FRESH-DFE for every antenna. Therefore, $\underline{V}(n), \underline{W}_{n}$ and $\varepsilon_{n}$ are defined by :

$$
\begin{gathered}
\underline{V}^{T}(n)=\left[\underline{X}^{(1)^{T}}(n) \ldots \underline{X}^{(L)^{T}}(n) \underline{Y}^{(1)^{T}}(n) \ldots \underline{Y}^{(L) T}(n) \underline{d}^{T}(n-D-1)\right] \\
\underline{W}_{n}^{T}=\left[\underline{w}_{n}^{(1)^{T}} \underline{w}_{n}^{(2)} \ldots \underline{w}_{n}^{(L)}-\underline{a}_{b}(n)\right] \\
\varepsilon_{n}=d_{0}(n-D)-\underline{W}_{n}^{T} V(n)
\end{gathered}
$$

where :

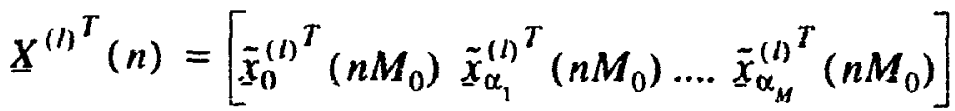

$$
\begin{aligned}
& \underline{Y}^{(l)}(n)=\left[\tilde{x}_{0}^{*(l)^{T}}\left(n M_{0}\right) \tilde{x}_{-\beta_{1}}{ }^{*(l)^{T}}\left(n M_{0}\right) \ldots \underline{\tilde{x}}_{-\beta_{N}}^{*(l)(T)}\left(n M_{0}\right)\right] \\
& \underline{w}_{n}^{(l)^{T}}=\left[\begin{array}{lllll}
\underline{a}_{0_{n}}^{(l)^{T}} & \underline{a}_{1_{n}}^{(l)} & \ldots & \underline{a}_{M_{n}}^{(l)^{T}} \underline{b}_{0_{n}}^{(l)^{T}} \underline{b}_{1_{n}}^{(l)^{T}} \ldots \underline{b}_{N_{n}}^{(l)}
\end{array}\right]
\end{aligned}
$$

Substituting $(6.21)$ in $(6.10)$ yields :

$$
\begin{aligned}
& \underline{a}_{a_{n+1}}^{(l)}=\underline{a}_{k_{n}}^{(l)}+2 \mu_{n} \varepsilon_{n} \frac{\tilde{\tilde{x}}_{\alpha_{k}}^{(l)}\left(n M_{0}\right)}{\sum_{l=1}^{L}\left[\sum_{m=0}^{L}\left\|\tilde{x}_{\alpha_{m}}^{(l)}\left(n M_{0}\right)\right\|^{2}+\sum_{m=0}^{N}\left\|\tilde{x}_{B_{m}}^{(l)}\left(n M_{0}\right)\right\|^{2}\right]+N_{b} \sigma_{d_{0}}^{2}} \\
& k=1, \ldots, \mathrm{M} \\
& l=1, \ldots, L
\end{aligned}
$$




$$
\begin{aligned}
& \underline{b}_{k_{n+1}}^{(l)}=\underline{b}_{k_{n}}^{(l)}+2 \mu_{n} \varepsilon_{n} \frac{\tilde{y}_{-\beta_{k}}^{(l)}\left(n M_{0}\right)}{\sum_{l=1}^{L}\left[\sum_{m=0}^{M}\left\|\tilde{x}_{\alpha_{m}}^{(l)}\left(n M_{0}\right)\right\|^{2}+\sum_{m=0}^{N}\left\|\tilde{x}_{\beta_{m}}^{(l)}\left(n M_{0}\right)\right\|^{2}\right]+N_{b} \sigma_{d_{n}}^{2}} \\
& k=1, \ldots . \mathrm{N} \\
& l=1, \ldots, 1 \\
& \underline{a}_{b_{n+1}}=\underline{a}_{b_{n}}-2 \mu_{n} \varepsilon_{n} \frac{\underline{d}^{*}(n-D-1)}{\sum_{l=1}^{L}\left[\sum_{m=0}^{M}\left\|\underline{\tilde{x}}_{\alpha_{m}}^{(l)}\left(n M_{0}\right)\right\|^{2}+\sum_{m=1}^{N}\left\|\tilde{x}_{\beta_{m}}^{(l)}\left(n M_{0}\right)\right\|^{2}\right]+N_{b} \sigma_{d_{11}^{2}}^{2}}
\end{aligned}
$$

\subsection{Noise Limiting Filter}

It is well known that a filter matched to SOI is the best choice to limit the noise provided there is no ACI. However, in the case of overlapping signal spectra, the best filter is one which passes all the overlapped signal spectra. But passing all the overlapping signals in a single channel spacing system means using an all pass filter before the equalizer which yields an infinite noise power at the equalizer input. Nevertheless, a sub-optimum noise limiter filter which passes spectra of SOI and overlapping interference may be considered. As an example, Figure 6.6 (a) shows a FDMA system which uses a channel spacing equals to the symbol rate. As it is clear from this figure, only first up-channel and down-channel signals are overlapped with SOI. Therefore, the noise limiter filter passes these two signals and partially other ACI signals. Figure $6.6(\mathrm{~b})$ shows a noise limiter filter for this system which is created by adding three filter responses matched to SOI, first up$\mathrm{ACI}$ and first down-ACI. This filter passes all the $\mathrm{CCI}$ signals, and the strongest $\mathrm{ACI}$ signals. Therefore there is enough information about $\mathrm{CCl}$ and $\mathrm{ACl}$ at the input of the equalizer. Figure 6.7 (a) shows that the noise limiter filter can cut the second, third,... overlapping signal. Figure 6.7 (b) shows this filter in $\mathrm{dB}$ scale. In all the simulation of this chapter such a noise limiter filter is used.

\subsection{Simulated Systems}

A cluster size 7 DMCS with channel spacing equals to the symbol rate is simulated to evaluate the performance of DFE and FRESH-DFE. Since the channel spacing is equal to the symbol rate, in a single symbol rate BPSK (or OQPSK) system, the ODFE needs one forward filter in the conjugate path and one forward filter in the linear path 
Spectrum

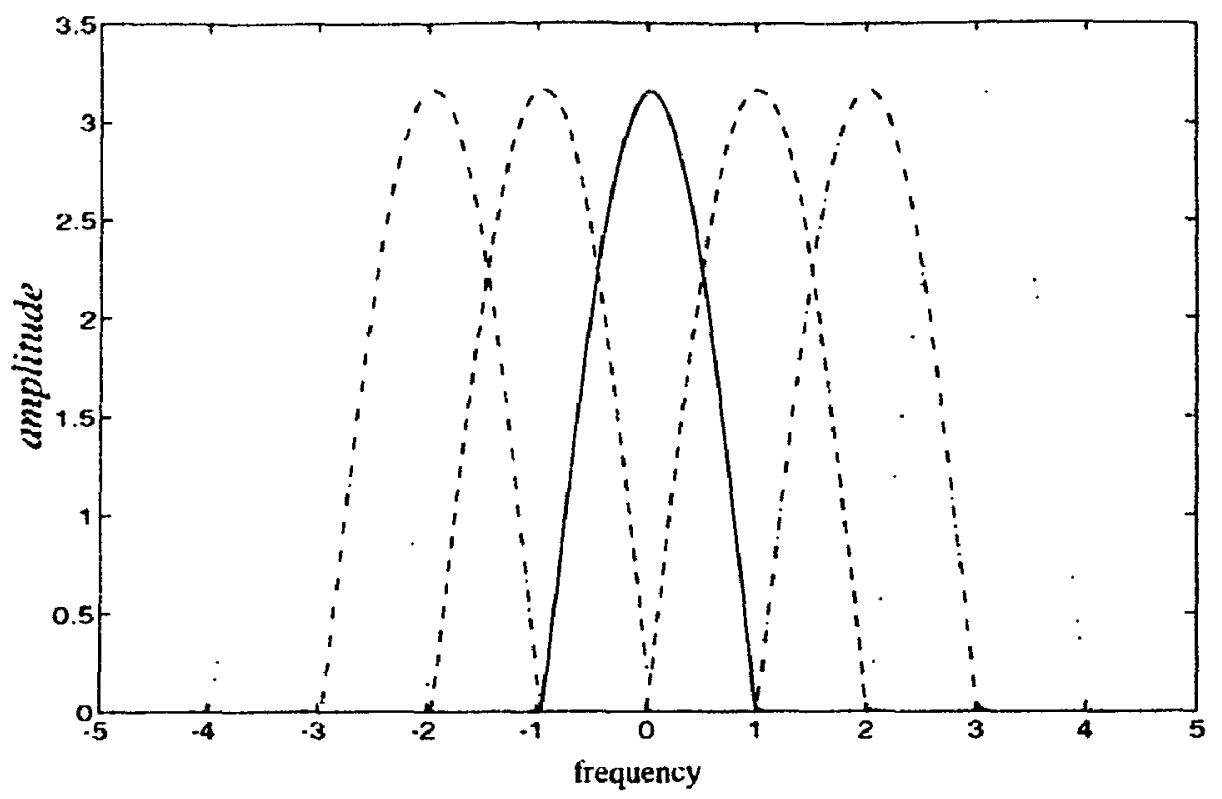

(a)

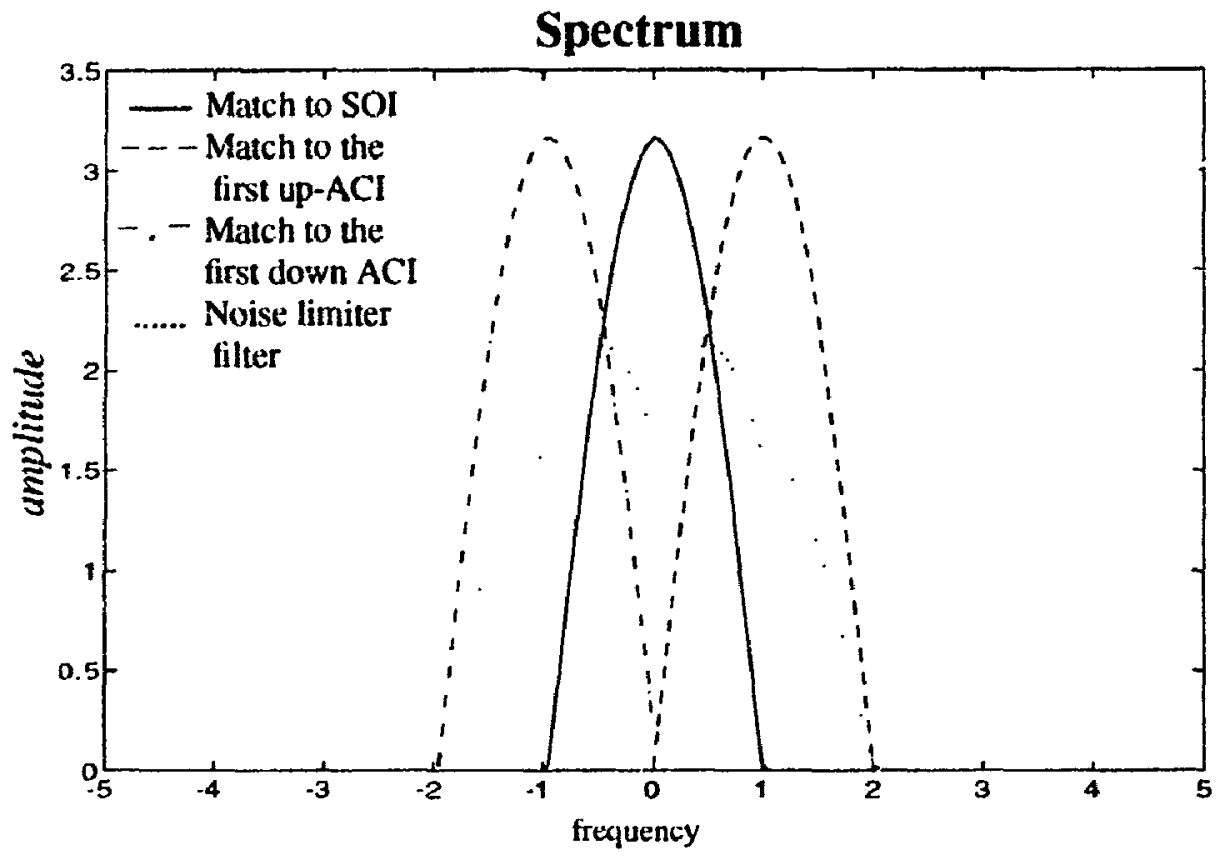

(b)

Figure 6.6 (a) A FDMA system with channel spacing equals to the symbol rate (b) Noise limiter filter. 


\section{Spectrum}

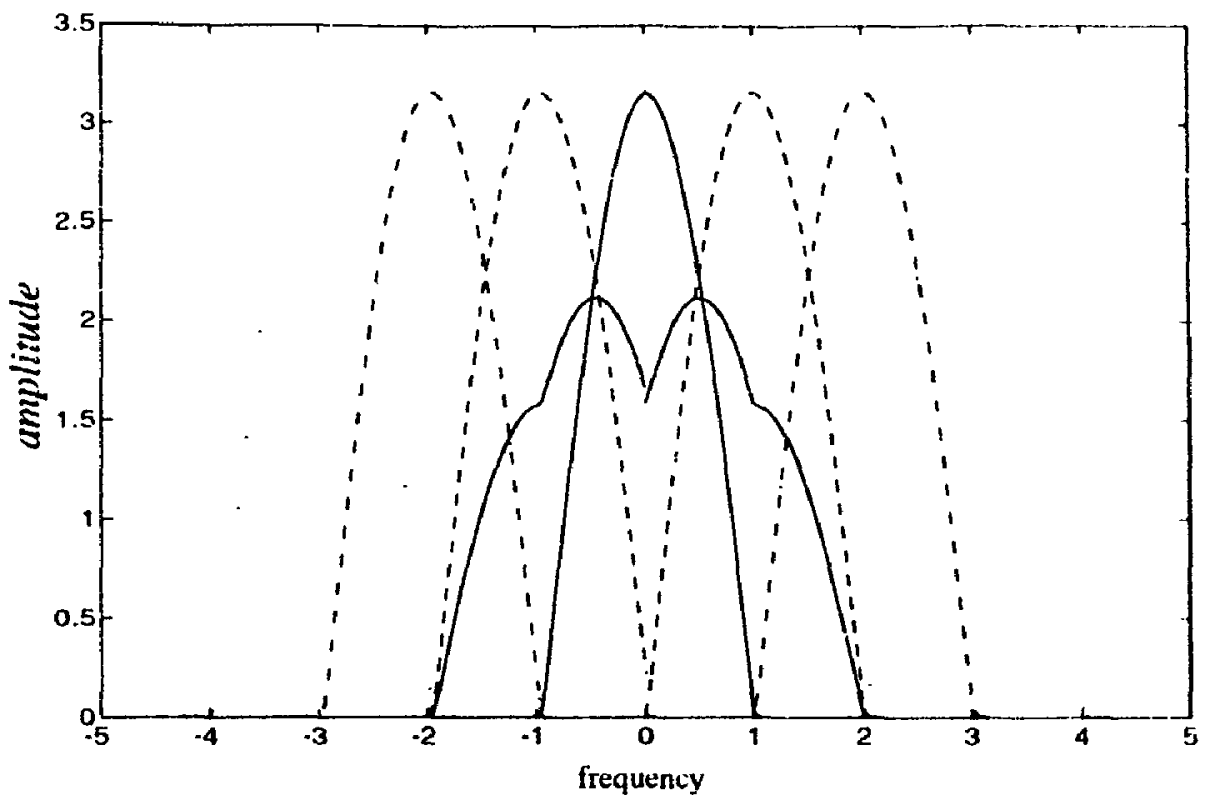

(a)

\section{Spectrum}

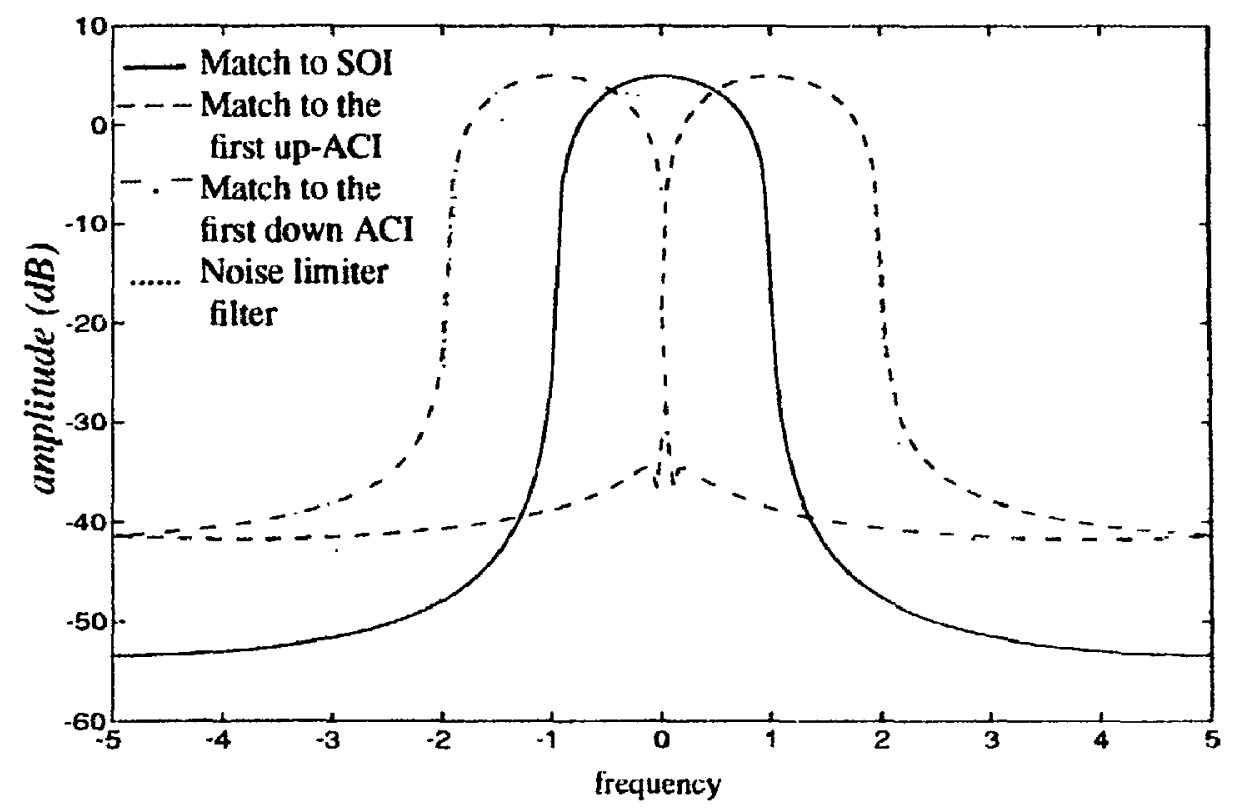

(b)

Figure 6.7 (a) The FDMA system and the noise limiter filter (b) The noise limiter filter in dB scale. 
$\{(\beta\}=\{0\},\{\alpha\}=\{0\})$. The simulation is run for the worst case that is when the mobile is at the cell boundary, $r=1$, and the channel MPDP for SOI, $\mathrm{CCl}$ and $\mathrm{ACl}$ signals has three rays at $t=(), T / 2, T$ (which $T$ is the symbol rate) regardless of sampling rate.

In the simulations, the following assumptions are made :

1- Regardless of attenuations caused by distances the channel profiles of SOI, CCI and $\mathrm{ACl}$ sources are the same.

2- The channels are multipath slow Rayleigh fading and they can be assumed invariant over one frame duration.

3- The 3-Rays "mountainous terrain" MPDP (Figure 5.4) is used to model all the channels.

4- The channels are uncorrelated.

4-All base stations use square root raised cosine with the same transmit power.

6- The cellular system use hexagonal shape celis with identical dimensions.

7- First tier $\mathrm{CCI}$ signals are enough to represent $\mathrm{CCl}$ effect.

8 - The data are uncorrelated with variance of 1.

9- The mobile position is at the boundary, i.e. $r=1$.

10- Running the simulation over 200 channels is sufficient to include the fading effect of the channels.

11 - The noise assume to be complex, white and Gaussian with variance $N_{0}$.

12- The equalizers are assumed to be sufficiently large to cover the effect of channel multipath.

13- The header symbols for training in each frame are assumed to be enough to achieve more than 70 percent of MMSE at the end of training.

14- Shadowing is not considered in the simulations.

In addition to the above assumptions, the parameters given in Table 6.1 are used in 
Table 6.1 The simulation parameters in all the simulated systems.

\begin{tabular}{|c|c|}
\hline Frame length & $50(0)$ symbols \\
\hline Train length & 500 symbols \\
\hline Total number of transmitted data for each run & $1.000,(10)($ symbols \\
\hline Number of taps in forward filters & 15 symbols \\
\hline Number of taps in backward filter & 3 symbols \\
\hline SOI symbol rate & normalized to $1 \mathrm{~Hz}$ \\
\hline SOI excess bandwidth & 100 percent \\
\hline Sampling rate at the input of equalizers & $5 \mathrm{~Hz}$ \\
\hline Propagation loss factor & 3 \\
\hline Mobile position $(r)$ & at the boundary of cell $(r=1)$ \\
\hline Variance of the real part $\left(\sigma^{2}{ }^{(r)}\right)$ & 1 \\
\hline Variance of the imaginary part $\left(\sigma^{2}{ }^{(1)}\right)$ & 1 \\
\hline SCCIR (measured) & $11.5 \mathrm{~dB}$ \\
\hline SACIR (measured) & $7.14 \mathrm{~dB}$ \\
\hline SIR (measured) & $5.88 \mathrm{~dB}$ \\
\hline
\end{tabular}


all the simulation. In this table, SCCIR, SACIR, and SIR are the measured values in the simulations. As it is clear, these values are close to the values computed in chapter 5 . It is also good to mention that since the number of symbols in one run is $\mathbf{1}^{6}$, the minimum contidence BER is $10^{-4}$.

With the above parameters and assumptions the following cases are investigated.

\subsubsection{No CCl-No ACl System}

As a simple case, first we assume that all the $\mathrm{CCl}$ and $\mathrm{ACI}$ sources are inactive. In this case, the only effect of the noise is sought. Figure 6.8 (a) shows the BER for such a system. As it is clear from the figure there is no saturation in BER and it can be reduced to zero if $E_{b} / N_{0}$ increases to infinity. Figure 6.8 (b) shows the ratio of conventional DFE BER to the ODFE BER. This ratio can be considered as the BER improvement of ODFE over the conventional DFE. As it is apparent from Figure 6.8 (b) that for $E_{b} / N_{0}>18 \mathrm{~dB}$, the BER improvernent can be more than 2.5 times for OQPSK. Figure 6.9 (a) shows the MMSE versus $E_{b} / N_{0}$. In this figure, each point of MMSE curves is obtained by averaging over 200 channels and in each channel by averaging over last 4000 square errors. Figure 6.9 (b) shows the difference between the MMSEs achieved by the conventional DFE and by the ODFE. This amount can be considered as the MMSE improvement of ODFE over conventional DFE. As it is apparent from Figure 6.9 (b), the ODFE can achieve more than 3 dB MMSE improvement over conventional DFE.

Figure 6.10 (a) shows the BER for OQPSK and for two values of excess bandwidth. 100 percent and 300 percent. As it is clear from the figure, the BER in the case of ODFE is improved by an order of magnitude when larger excess bandwidth is used. Corresponding improvement in conventional DFE is much lower. Figure 6.10 (b) shows the BER improvement of ODFE over the conventional DFE.

Figure 6.11 shows an example of the forward filters frequency responses of DFE and ODFE for BPSK and OQPSK systems when $E_{b} / N_{0}=23 \mathrm{~dB}$. The figures show that the conjugate path response in ODFE contributes to an improvement in BER and MMSE.

\subsubsection{No CCl-6-ACl System}

Figure 6.12 (a) shows the BER for the case when all $\mathrm{ACI}$ are active and $\mathrm{CCI}$ sources are inactive. Irreducible error rates for QPSK and OQPSK are indicated. It means 


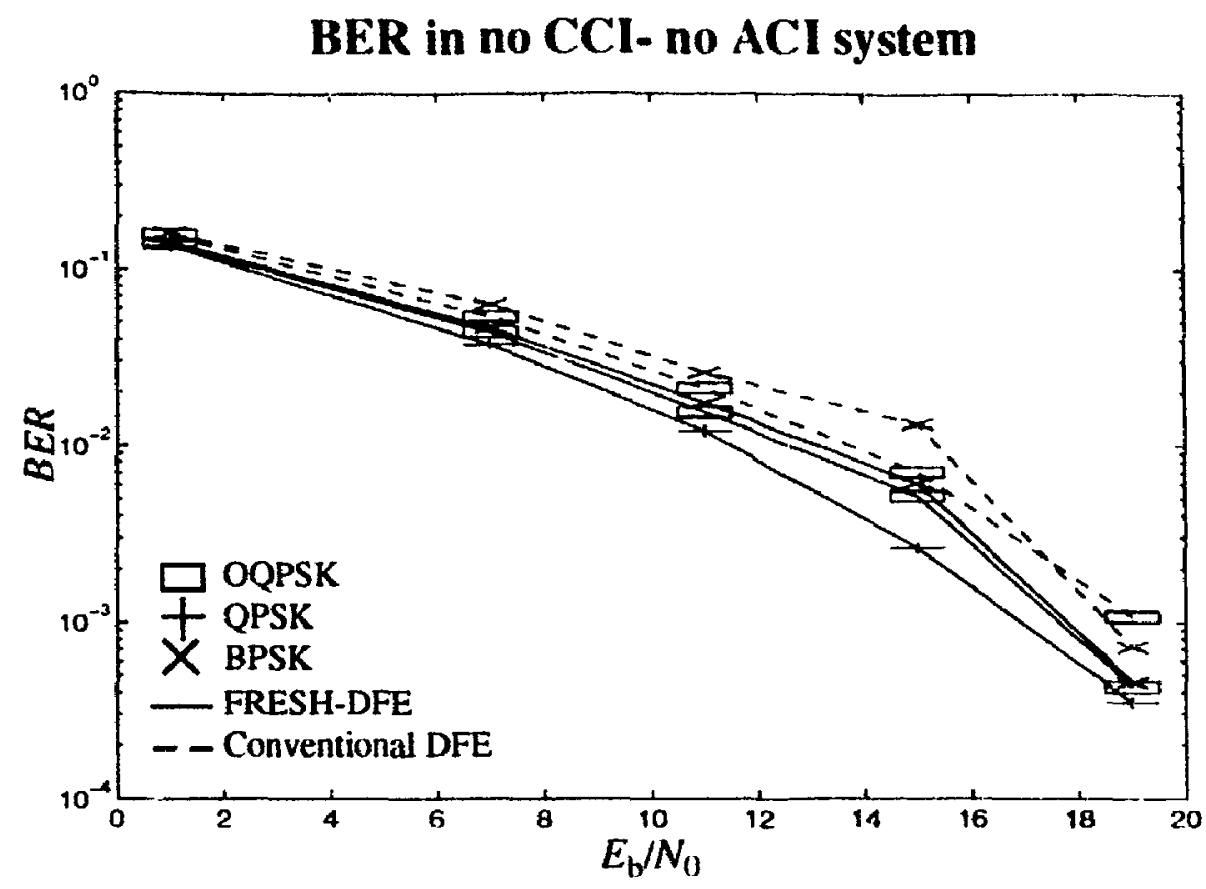

(a)

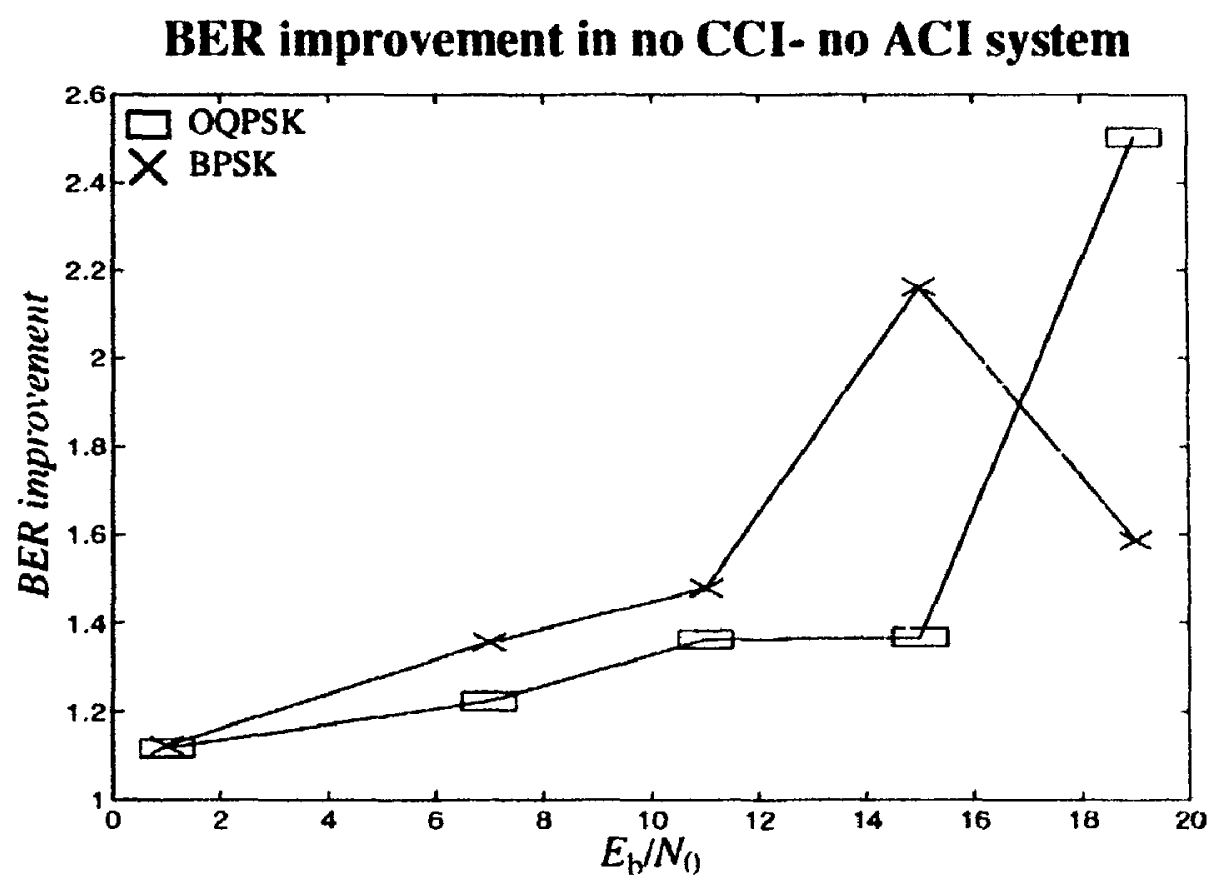

(b)

Figure 6.8 (a) The BER and (b) The BER improvement for no CCI-no ACl system. 


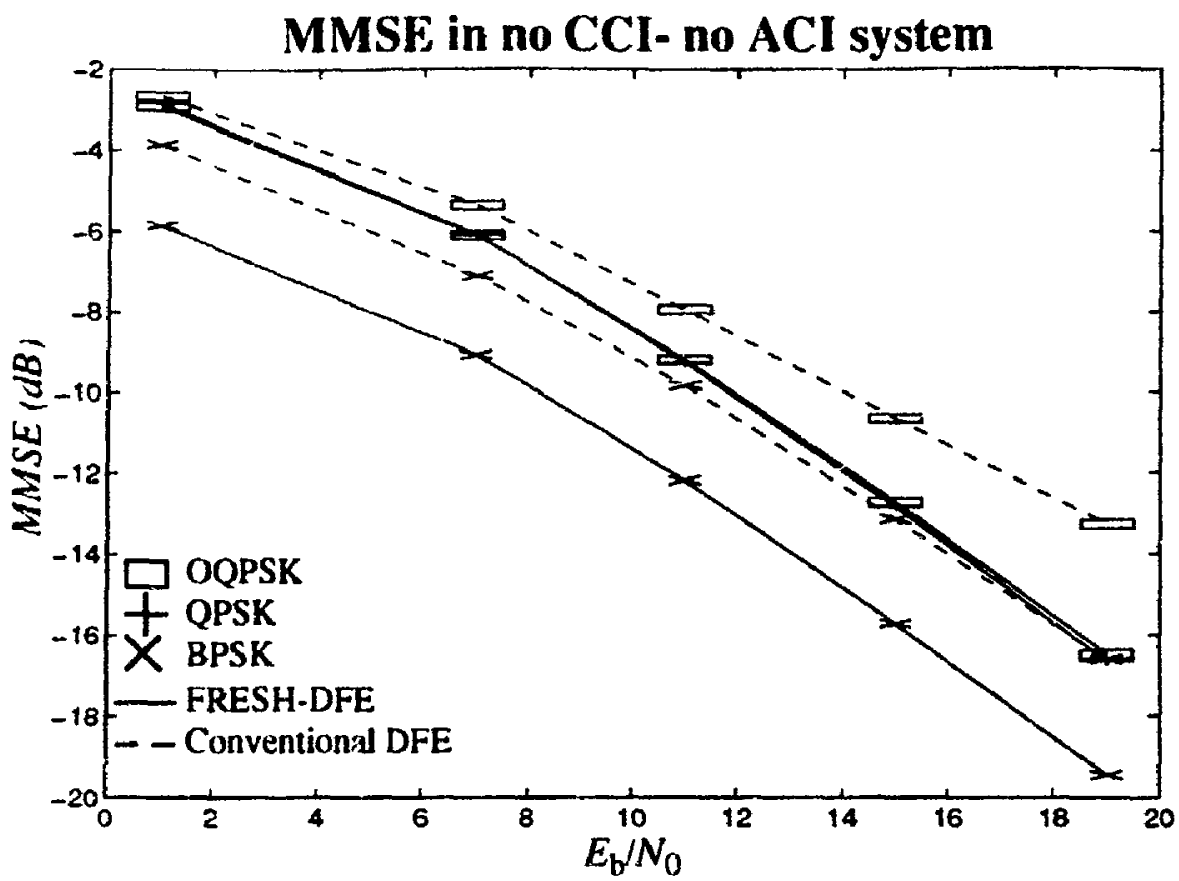

(a)

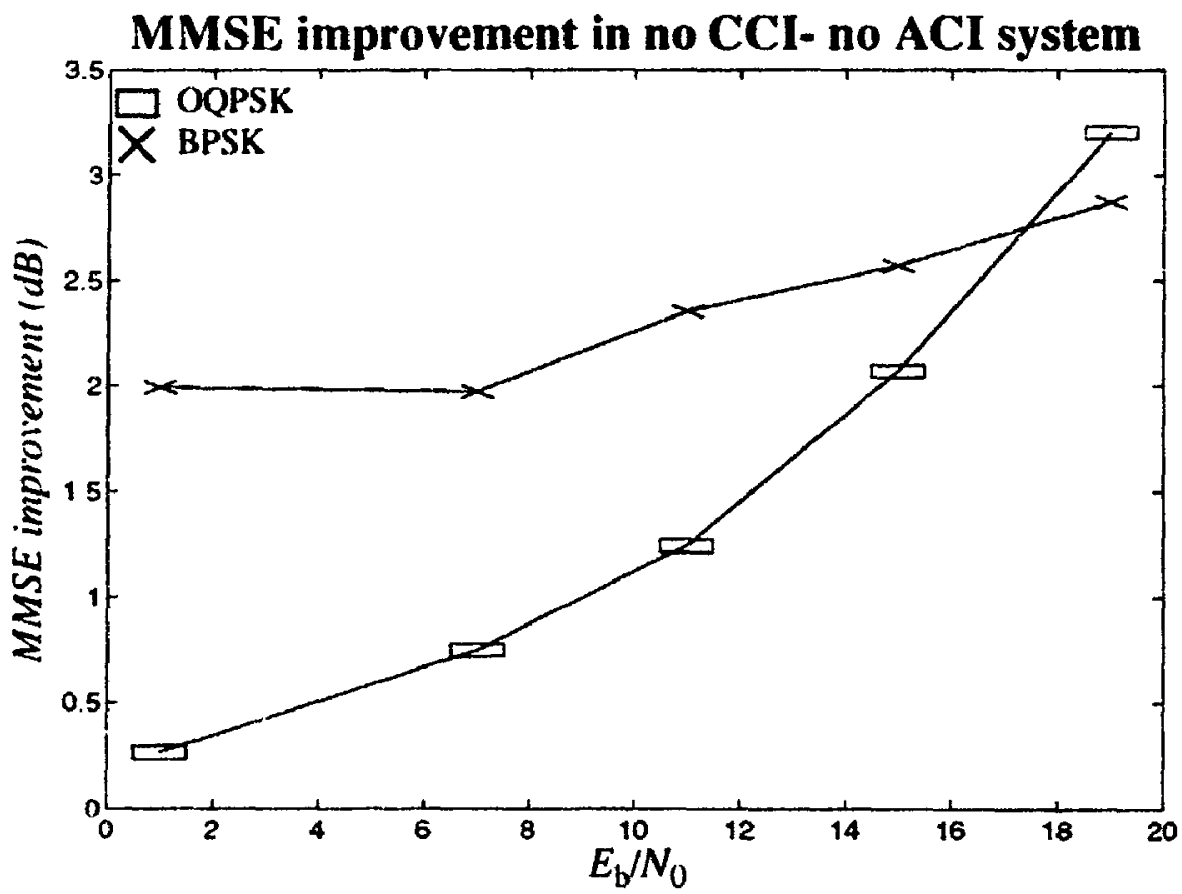

(b)

Figure 6.9 (a) The MMSE and (b) The MMSE improvement for no CCl-no ACl system. 


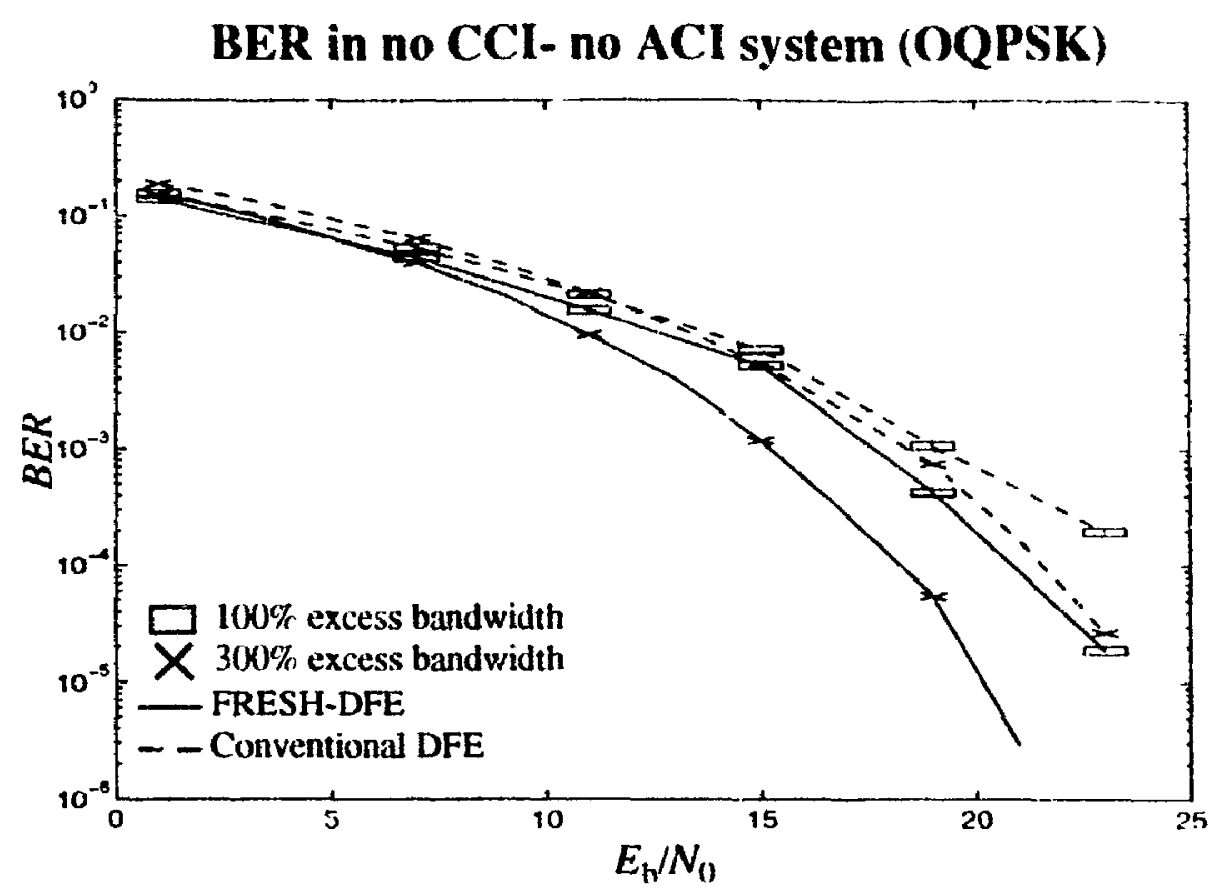

(a)

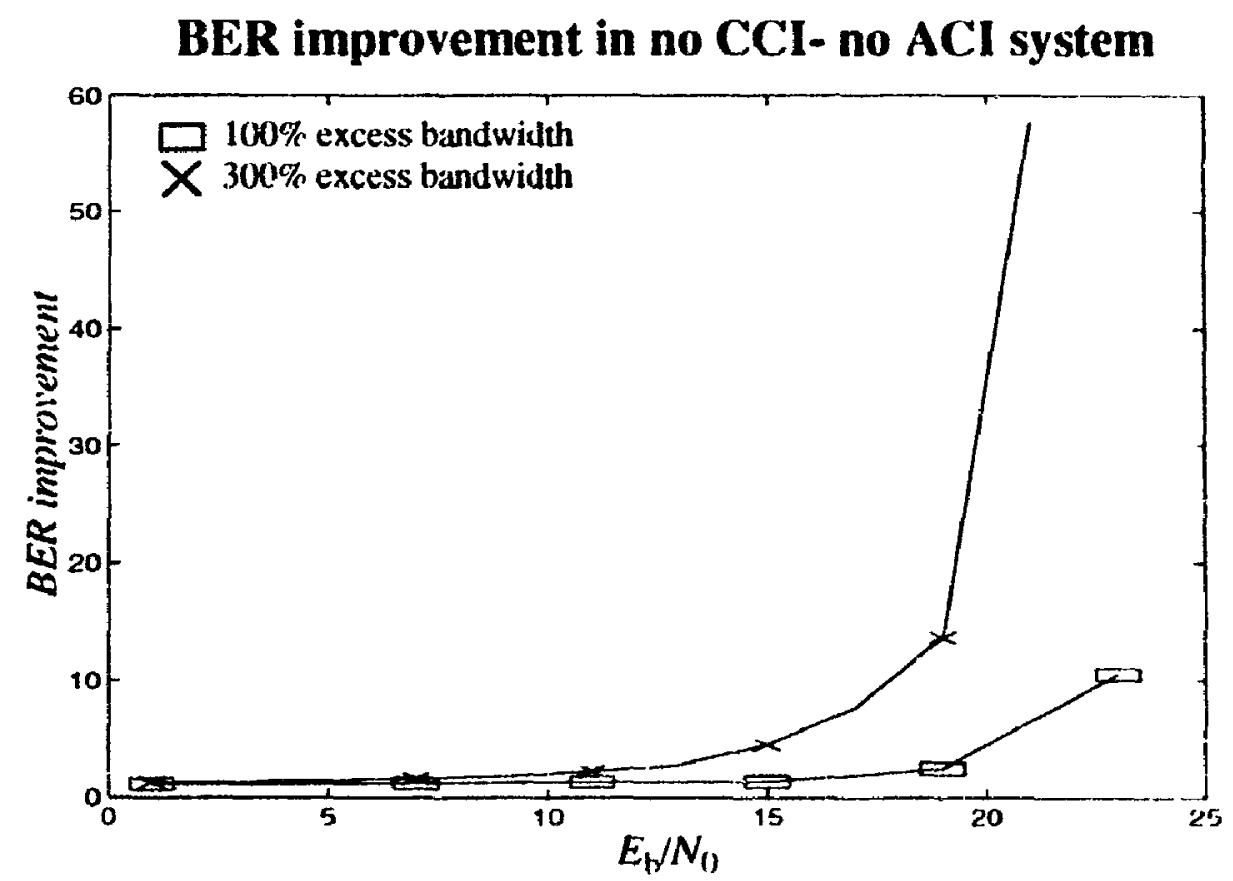

(b)

Figure 6.10 (a) The BER and (b) The BER improvement in no CCl-no ACl systems when OQPSK signals with excess bandwidths of 100 and 300 percent are used. 


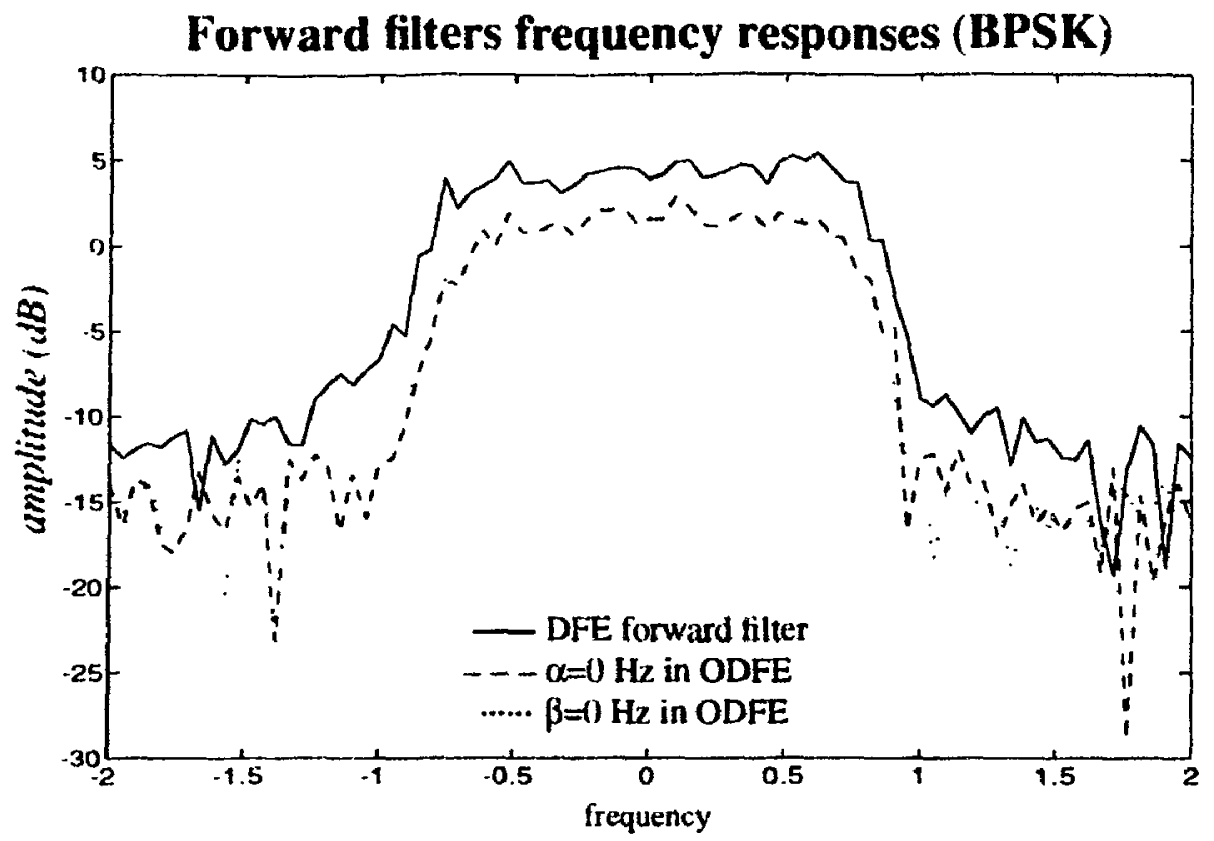

(a)

Forward filters frequency responses (OQPSK)

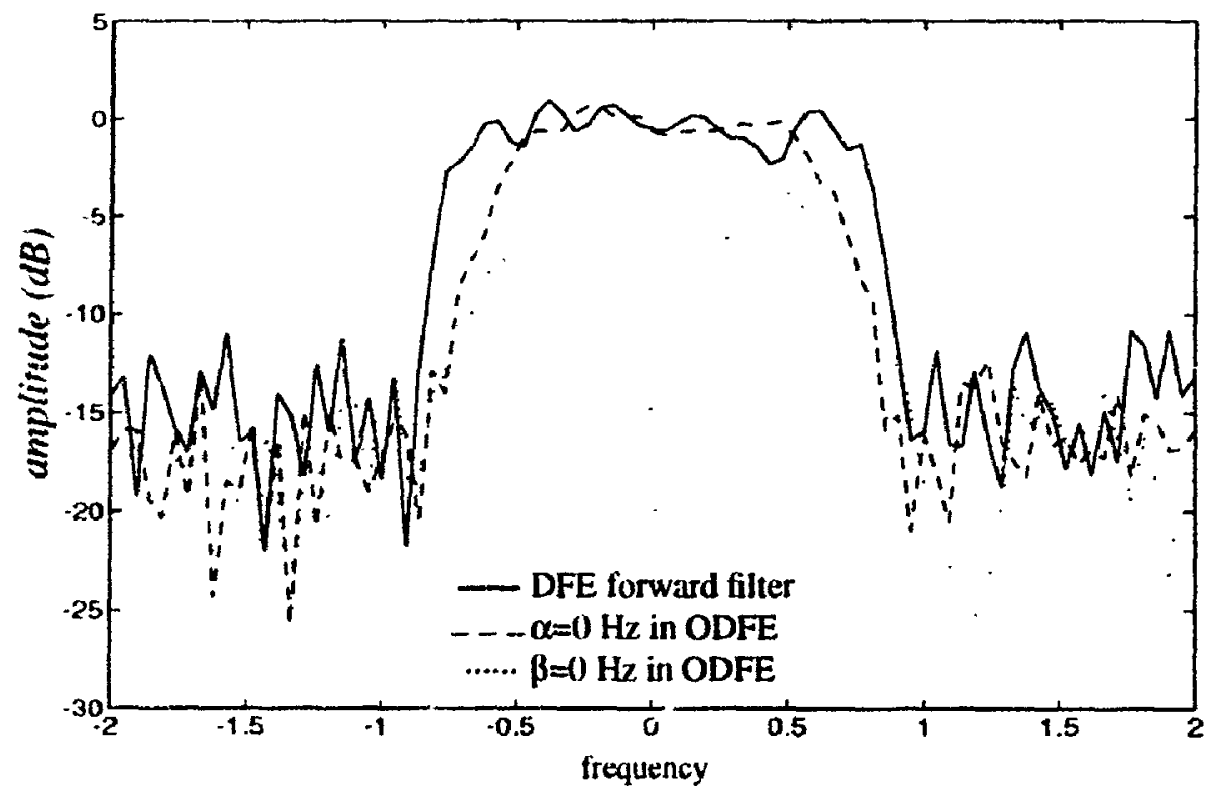

(b)

Figure 6.11 Forward filters frequency responses in no CCl-no ACl systems for (a) BPSK OOPSK. 


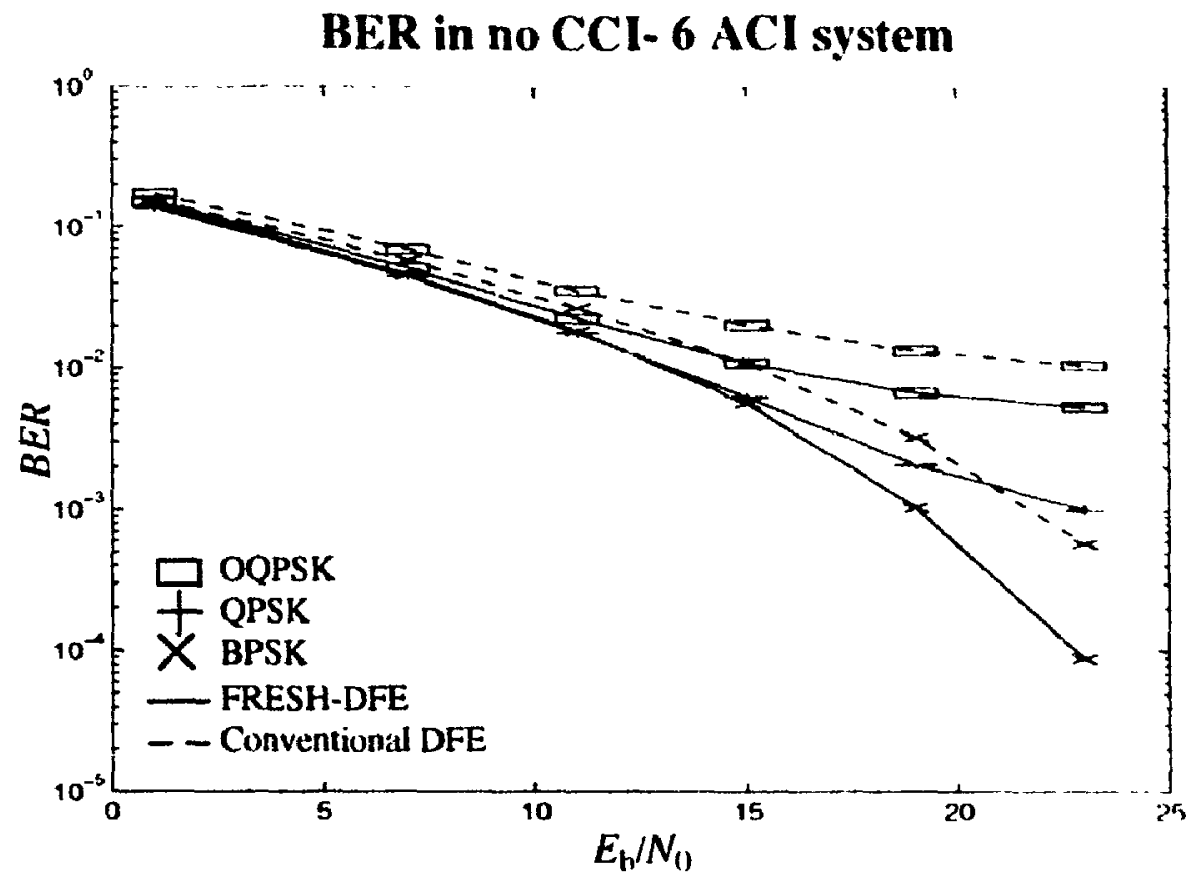

(a)

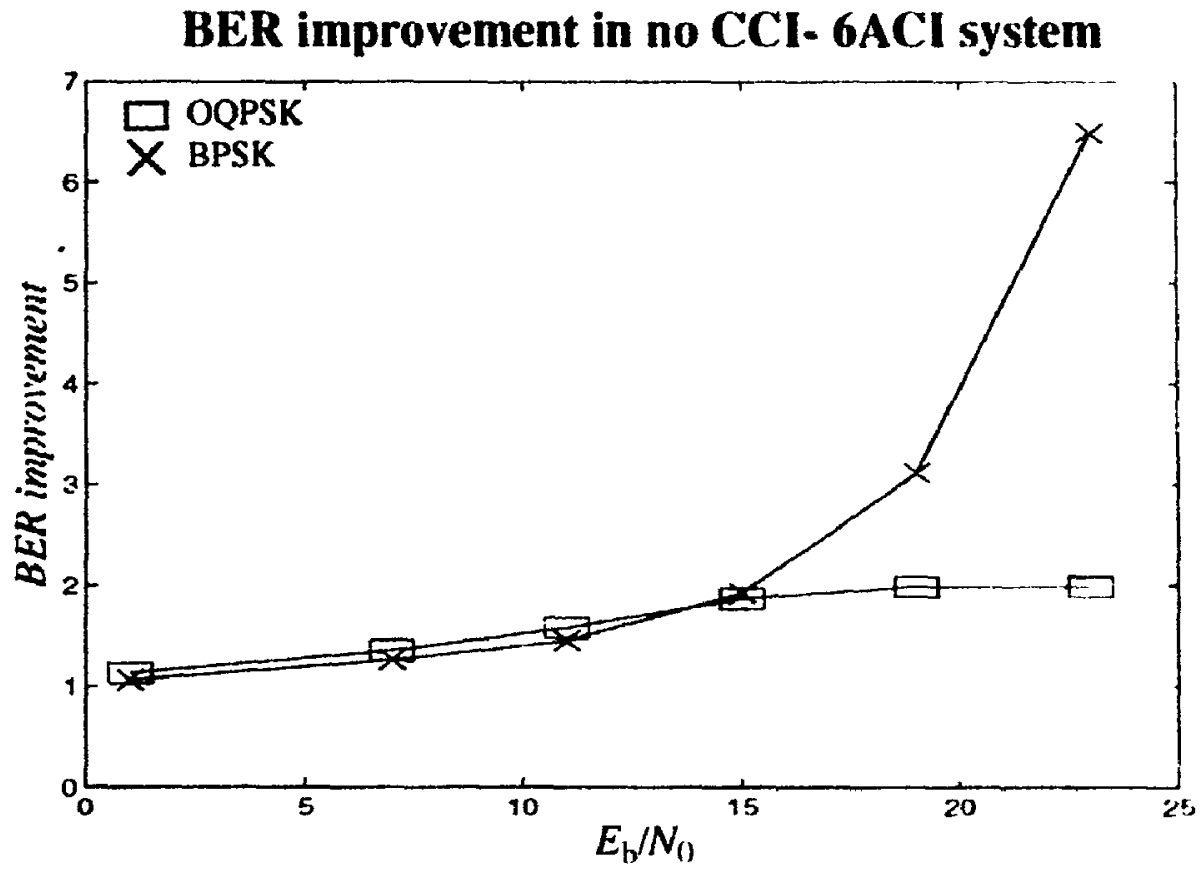

(b)

Figure 6.12 (a) The BER and (b) The BER improvement for no $\mathrm{CCl}-6 \mathrm{ACl}$ system. 
that QPSK and OQPSK can not cancel ACI in this system while BPSK can. Figure 6.12 (b) shows the BER improvement in this system. The performance of ODFE is better by several $\mathrm{dB}$ if more redundant signal (ex. BPSK) is used. Figure 6.13 shows the MMSE and MMSE improvement in this system.

\subsubsection{6-CCl-No ACl System}

If all $\mathrm{CCI}$ are active and $\mathrm{ACl}$ sources are inactive, the BER is changed to Figure 6.14 (a). The MMSE and MMSE improvement are sown in Figure 6.15. Comparing Figure 6.14 and Figure 6.15 with Figure 6.12 and Figure 6.13, it is observed that the CCI effect is nore serious than $\mathrm{ACI}$ when the channel spacing equals the symbol rate.

It can be concluded by comparing Figure 6.12 (a) and Figure 6.14 (a) with Figure 6.8 (a) that the interference causes irreducible BER.

\subsubsection{1-CCl-2-ACl System}

It is assumed that only one $\mathrm{CCl}$ and two $\mathrm{ACl}$, upper channel $\mathrm{ACl}$ and lower channel $\mathrm{ACl}$, sources are active. The power of $\mathrm{CCl}$ and $\mathrm{ACl}$ signals are adjusted to achieve the same SCCIR as in the system with 6 active $\mathrm{CCI}$ sources $(\mathrm{SCCIR}=11.5 \mathrm{~dB})$ and the same SACIR as in the system with 6 active $A C l$ sources (SACIR=7.14 dB). Figure 6.16 and Figure 6.17 show the BER, BER improvement. MMSE and MMSE improvement for this simulation. As it is clear from comparing these figures with Figure 6.14 and Figure 6.15, the performance of ODFE for one strong CCI signals is better than the performance of ODFE for 6 weak CCI signals. The reason is that. the summation of 6 Cyclostationary signals with random phases is close to a stationary signal.

Now we vary the power of $\mathrm{CCI}$ and $\mathrm{ACl}$ sources to plot the BER and MMSE versus SCCIR and SACIR. Figure 6.18 to Figure 6.27 show the BER, BER improvement, MMSE and MMSE improvement for different values of SCCIR and SACIR, and for different modulation scheme. The $E_{b} / N_{0}$ is equal to $28 \mathrm{~dB}$ in all these cases. It is observed from the tigures that,

1- the BER and MMSE can be reduced by reducing the interference.

2- the BER and MMSE are more sensitive to $\mathrm{CCI}$ than $\mathrm{ACI}$ for a system with channel spacing equals to the symbol rate. 


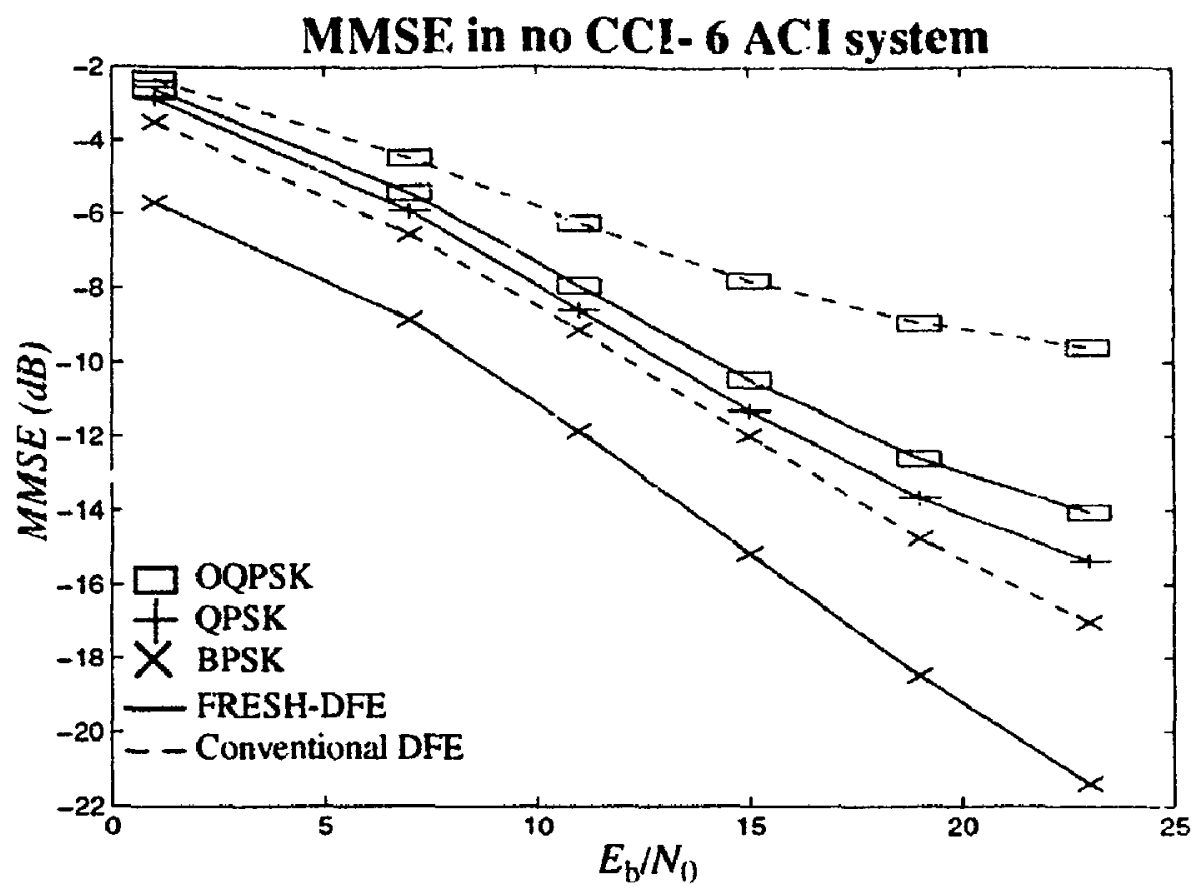

(a)

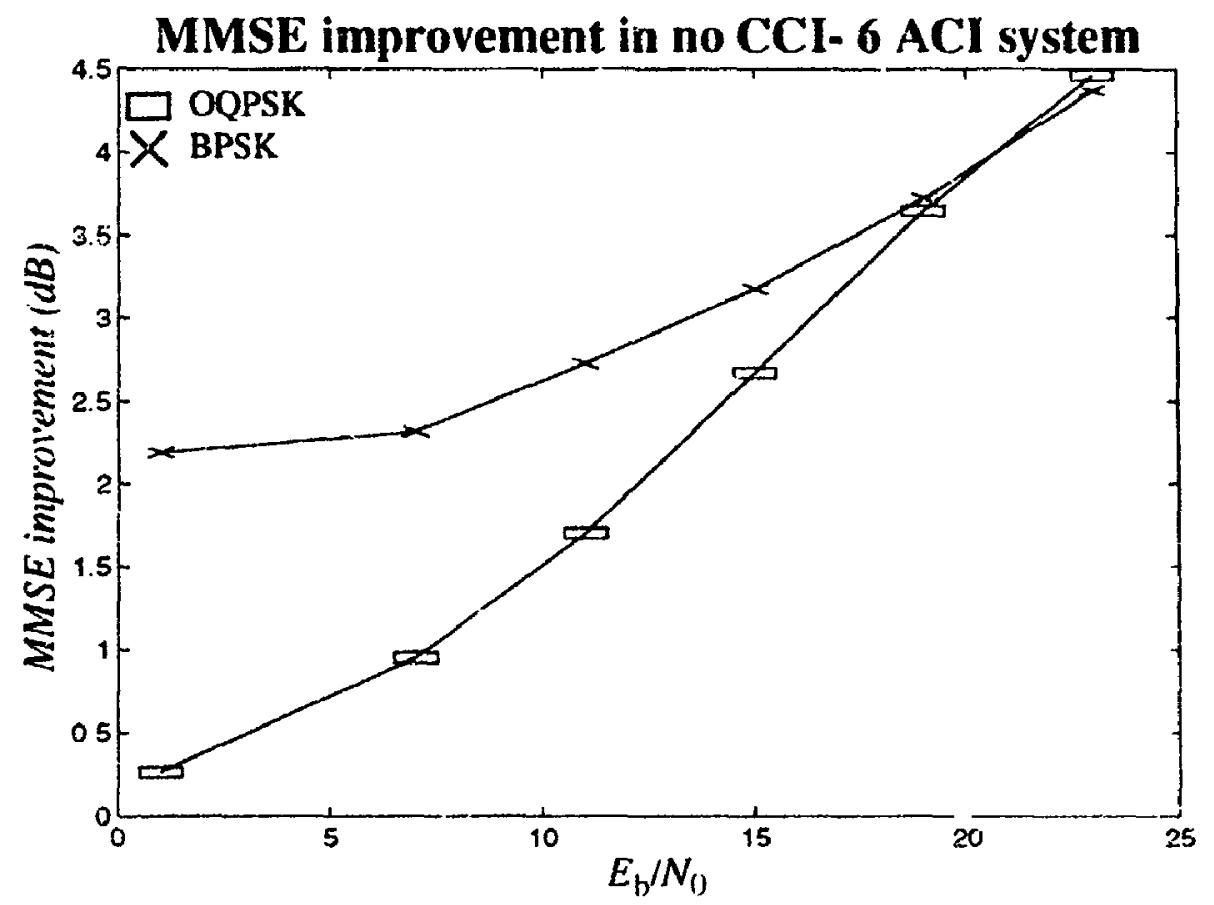

(b)

Figure 6.13 a) The MMSE and (b) The MMSE improvernent for no $\mathrm{CCl}-6 \mathrm{ACl}$ system. 


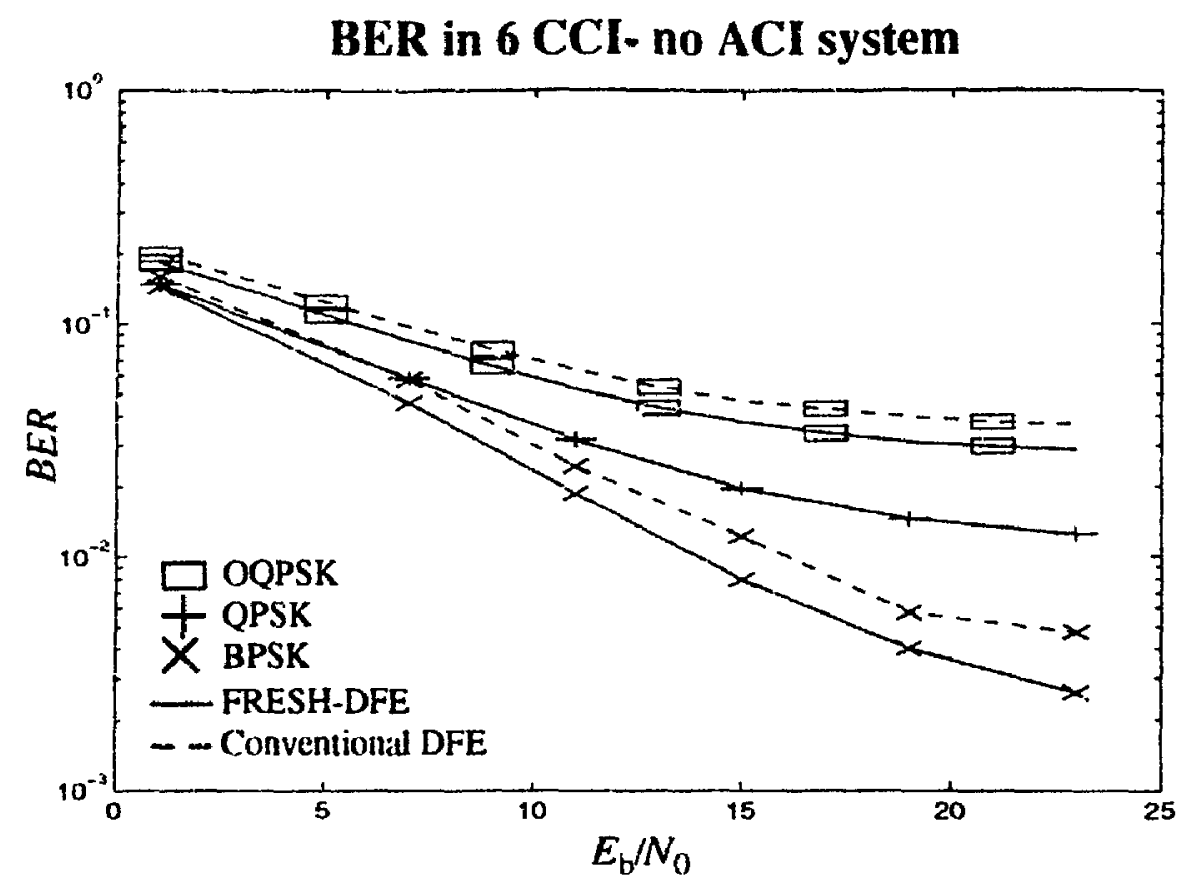

(a)

\section{BER improvement in $6 \mathrm{CCI}$ - no ACI system}

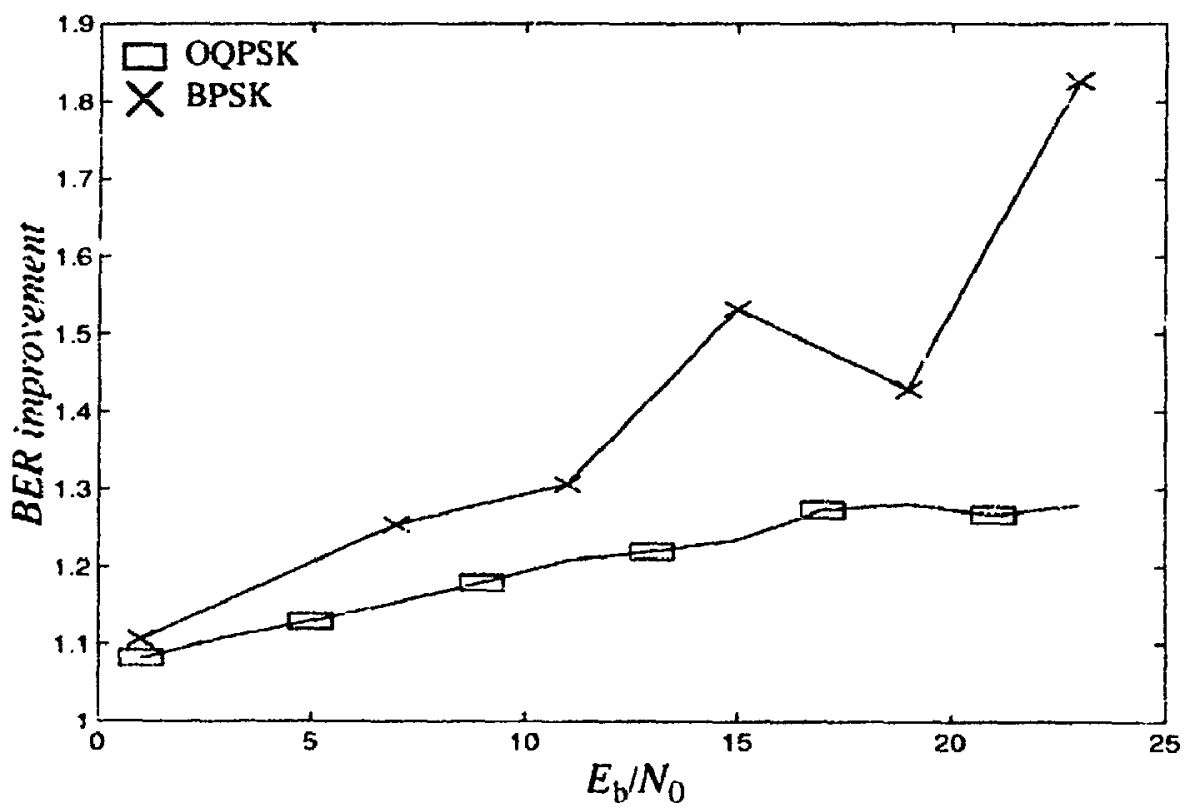

(b)

Figure 6.14 (a) The BEF and (b) The BER improvement for $6 \mathrm{CCi}$ - no ACl system. 


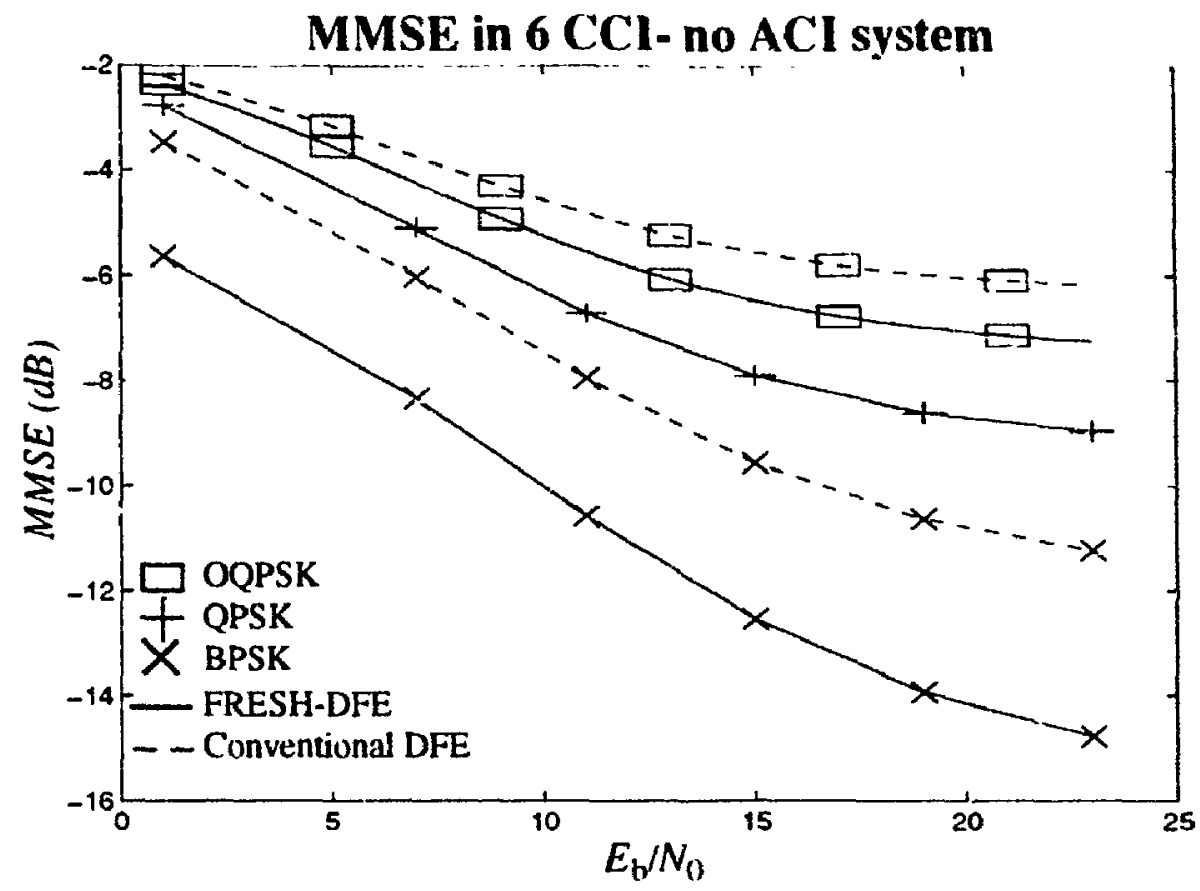

(a)

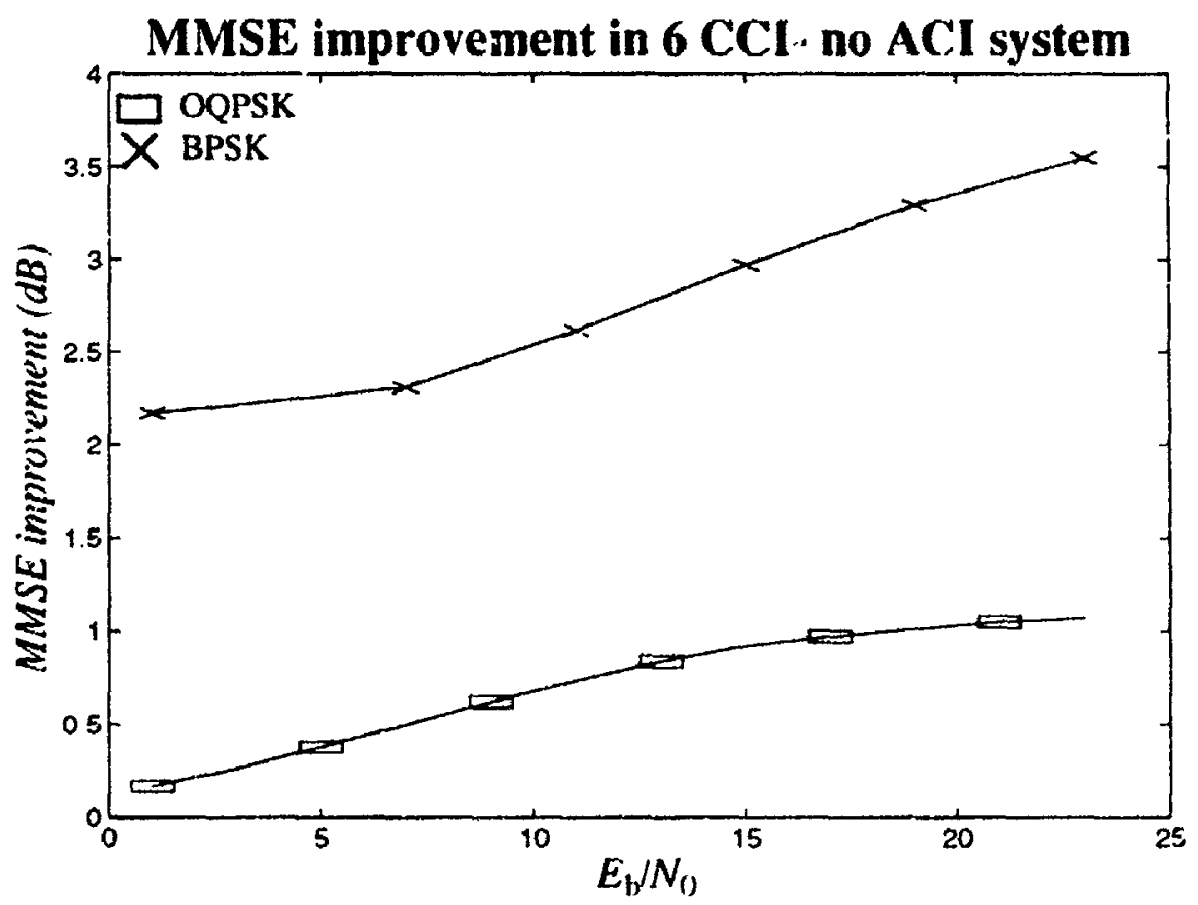

(b)

Figure 6.15 (a) The MMSE and (b) The MMSE improvement for $6 \mathrm{CCl}$. no ACl system. 
BER in $1 \mathrm{CCI}$ - 2 ACI system

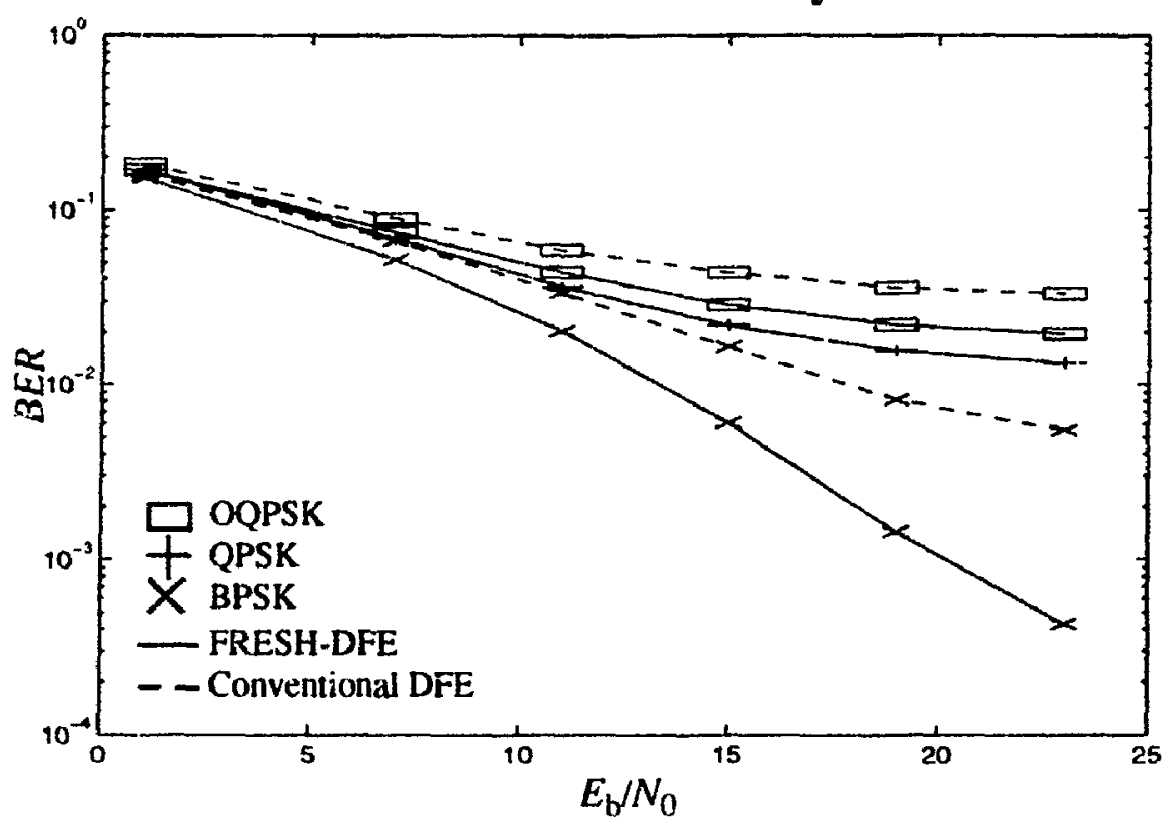

(a)

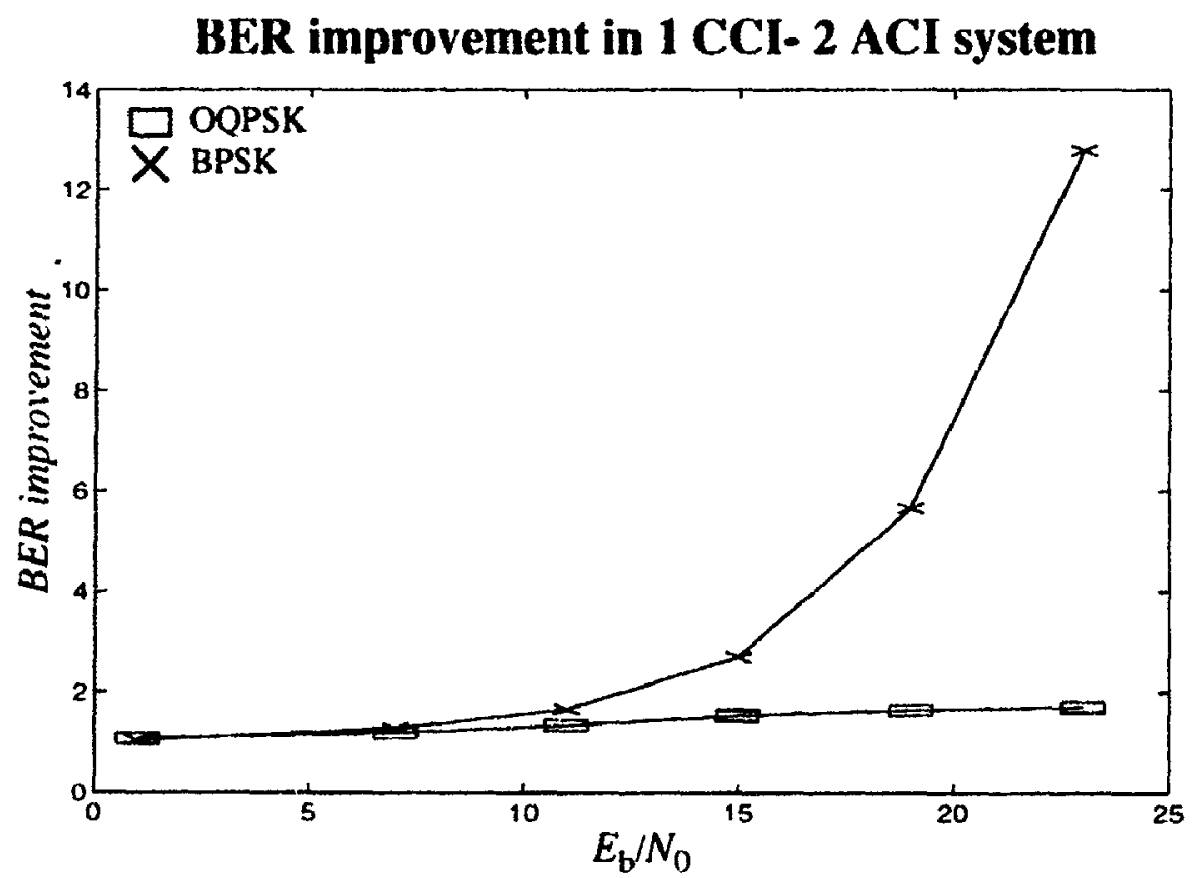

(b)

Figure 6.16 (a) The BER and (b) The BER improvement for $1 \mathrm{CCl} 2 \mathrm{ACl}$ system. 


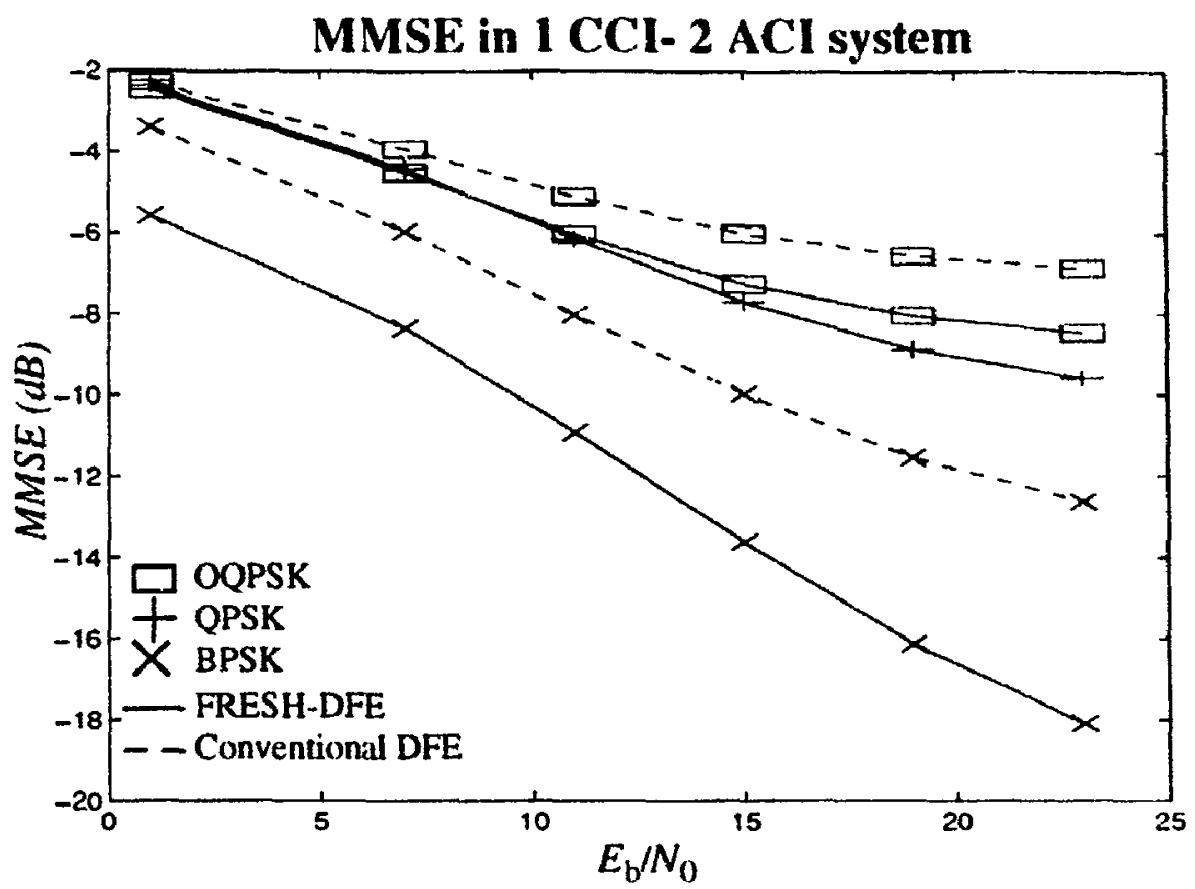

(a)

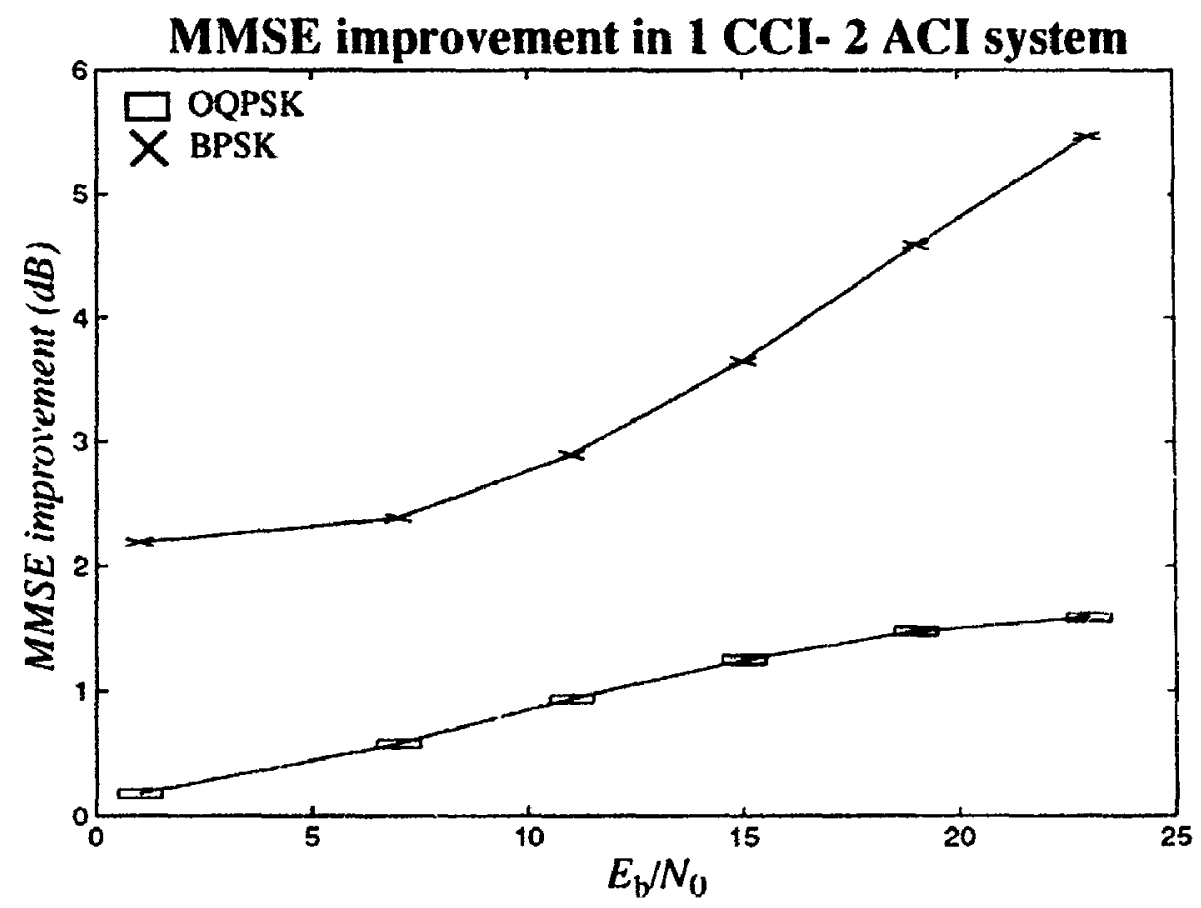

(b)

Figure 5.17 (a) The MMSE and (b) The MMSE improvement for $1 \mathrm{CCl}-2 \mathrm{ACl}$ system. 
BER in 1 CCI - 2 ACI system

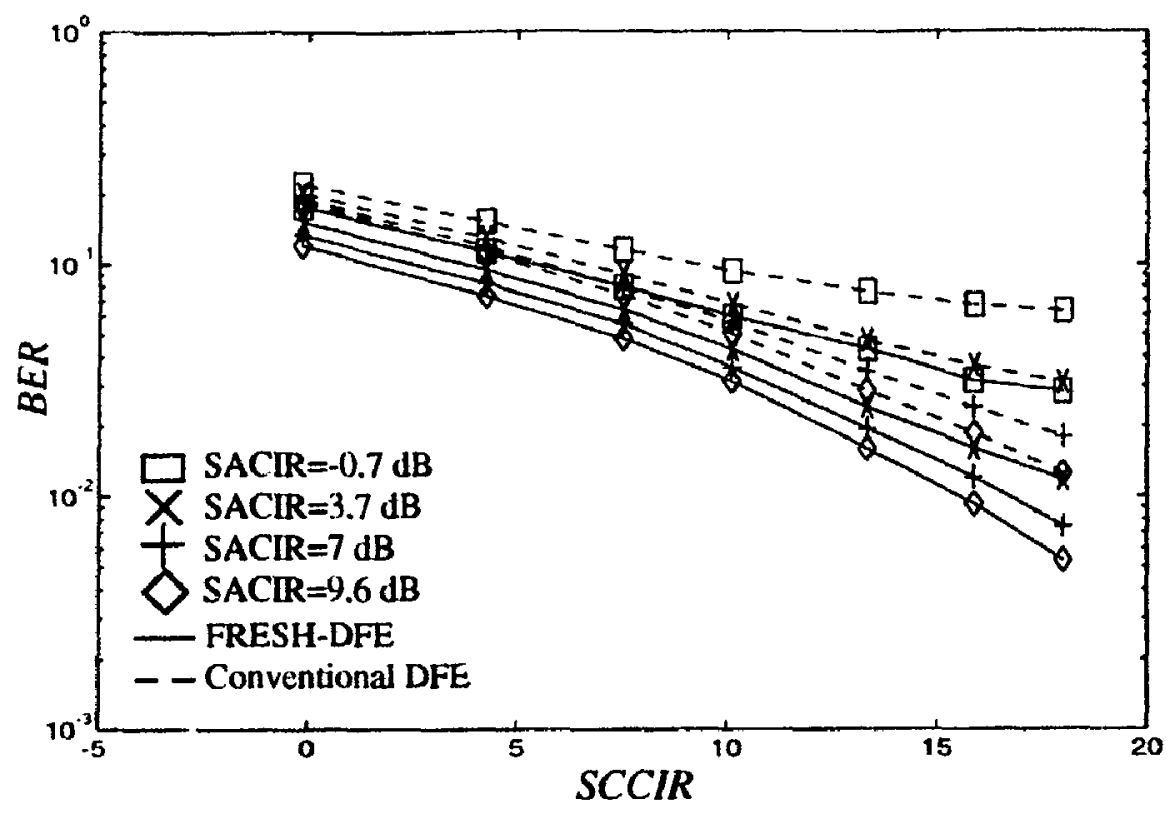

(a)

BER improvement in $1 \mathrm{CCI}-2 \mathrm{ACI}$ system

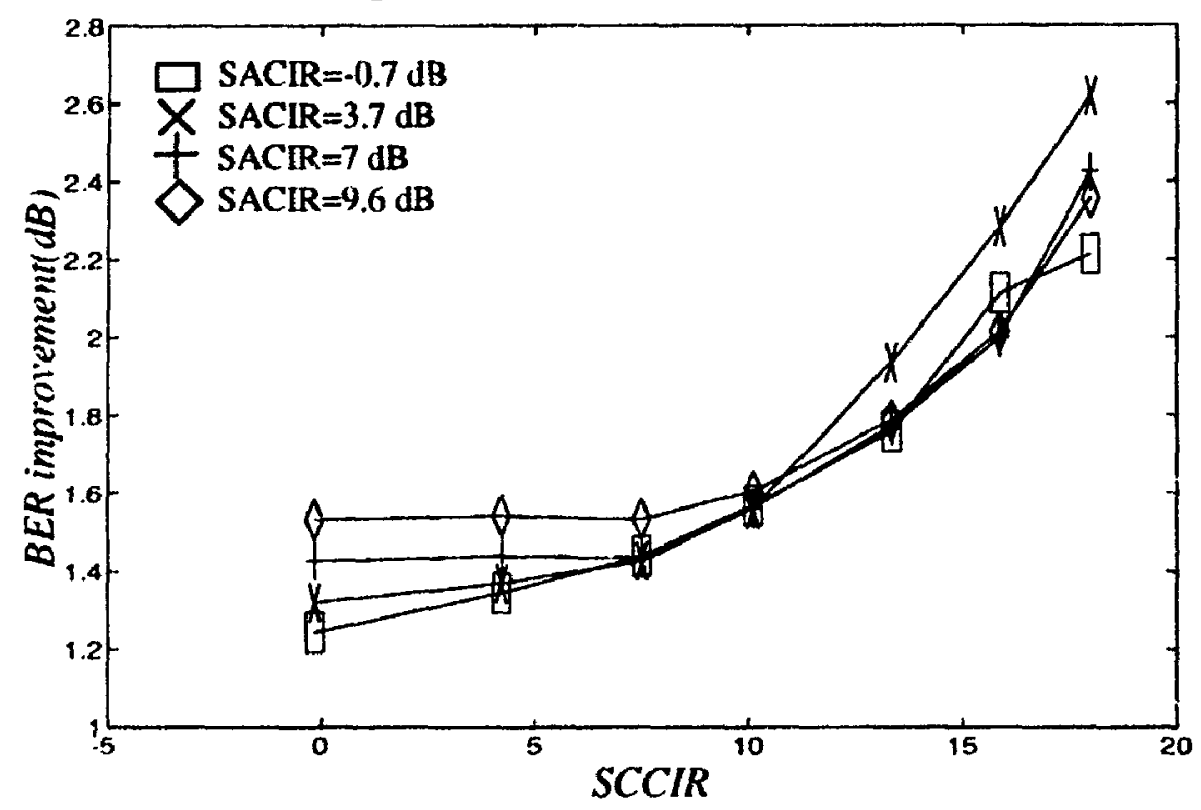

(b)

Figure 6.18 (a) The BEF and (b) The BER improvement versus SCCIR for $1 \mathrm{CCl} 2 \mathrm{ACl}$ OOPSK system. 


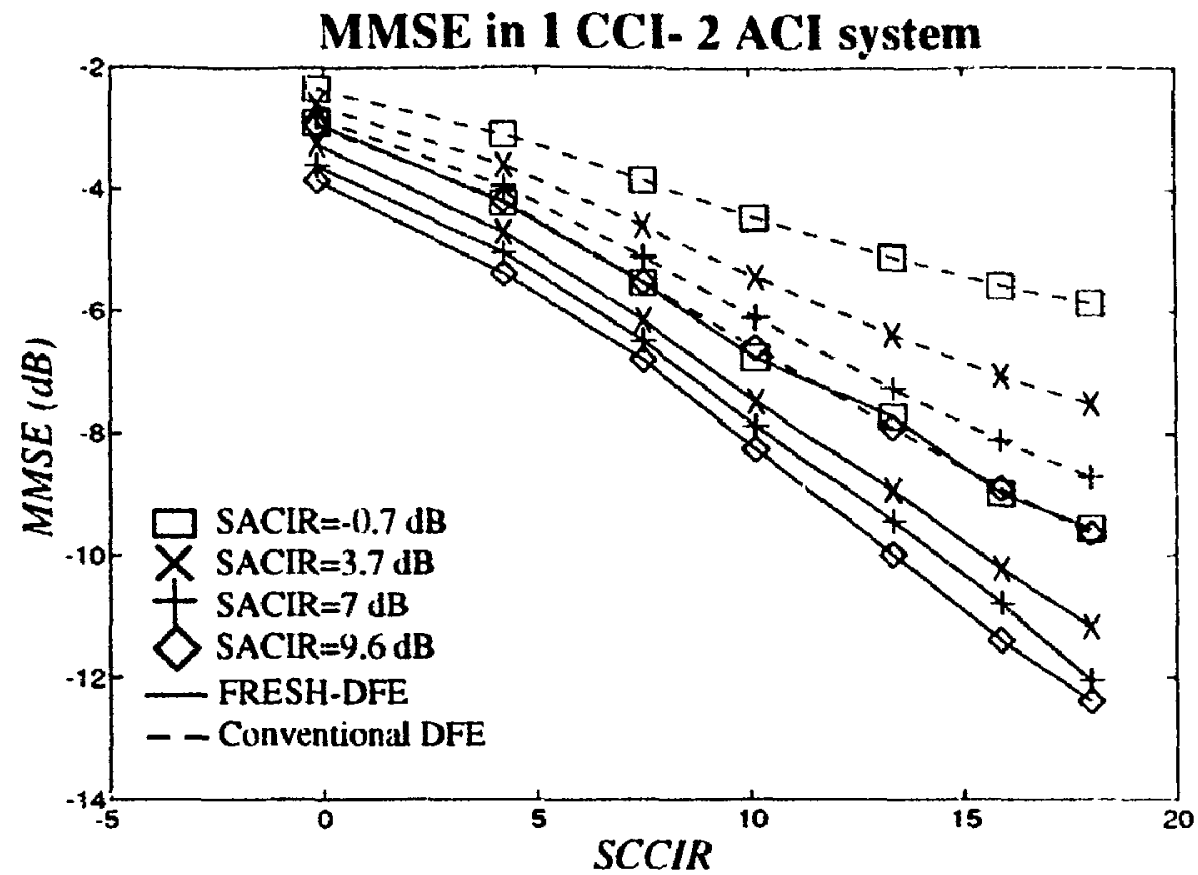

(a)

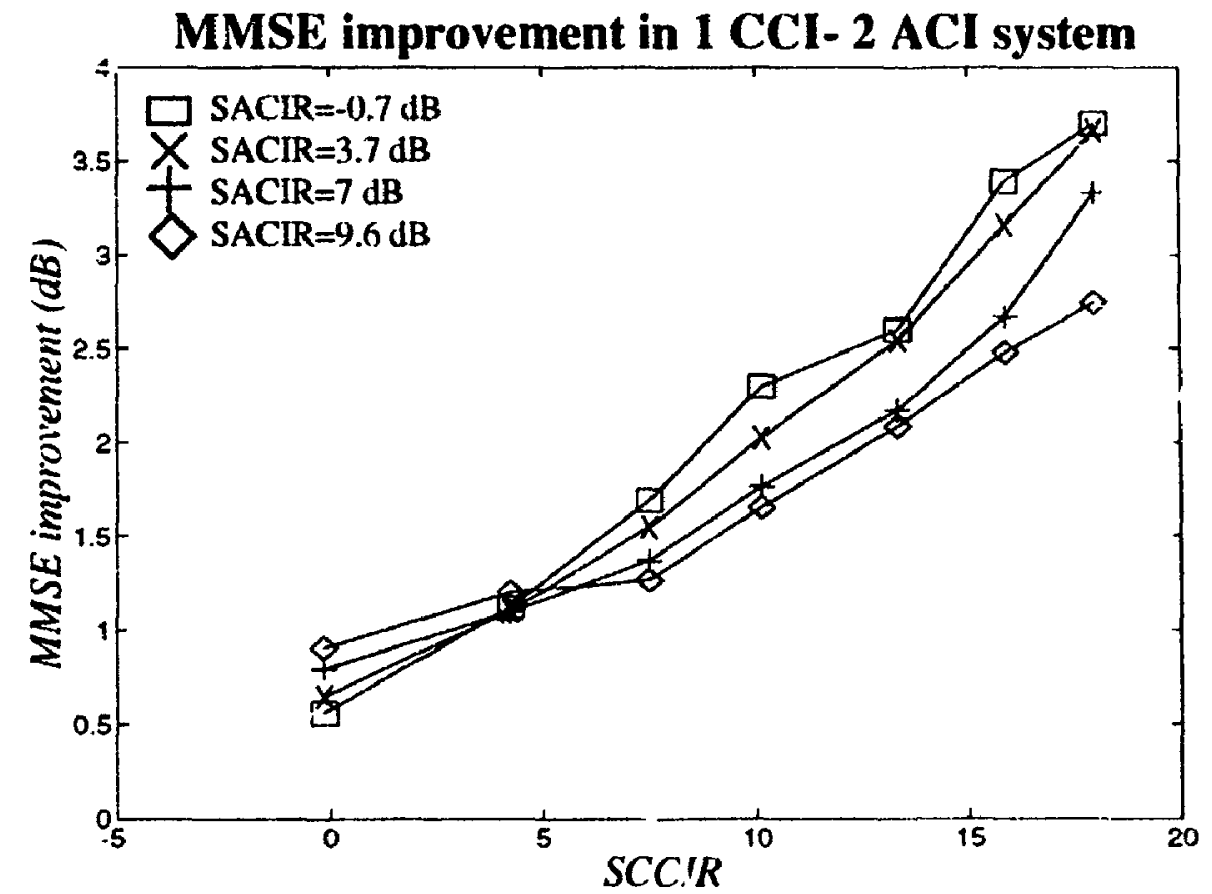

(b)

Figure 6.19 (a) The MMSE and (b) The MMSE im.Jrovement versus SCCIR for $1 \mathrm{CCl} 2 \mathrm{ACI}$ OOPSK systim. 
BER in 1 CCI- 2 ACI system

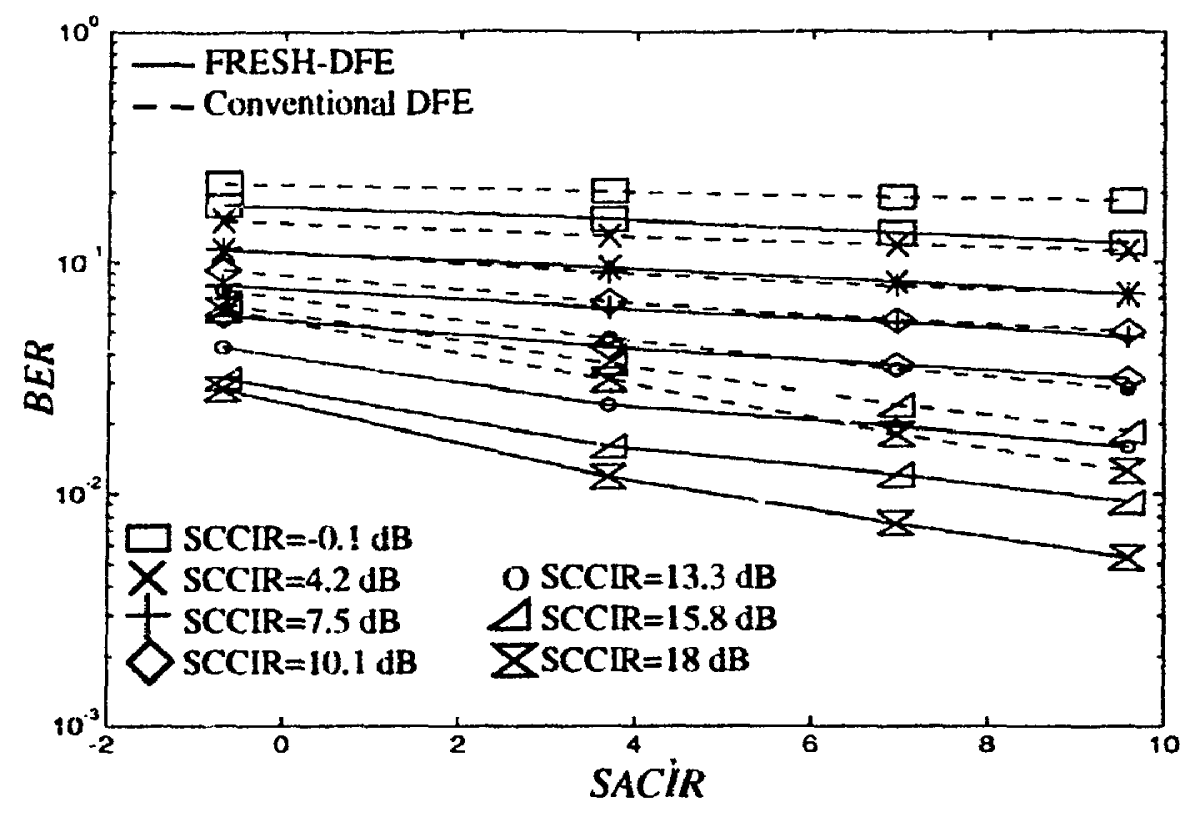

(a)

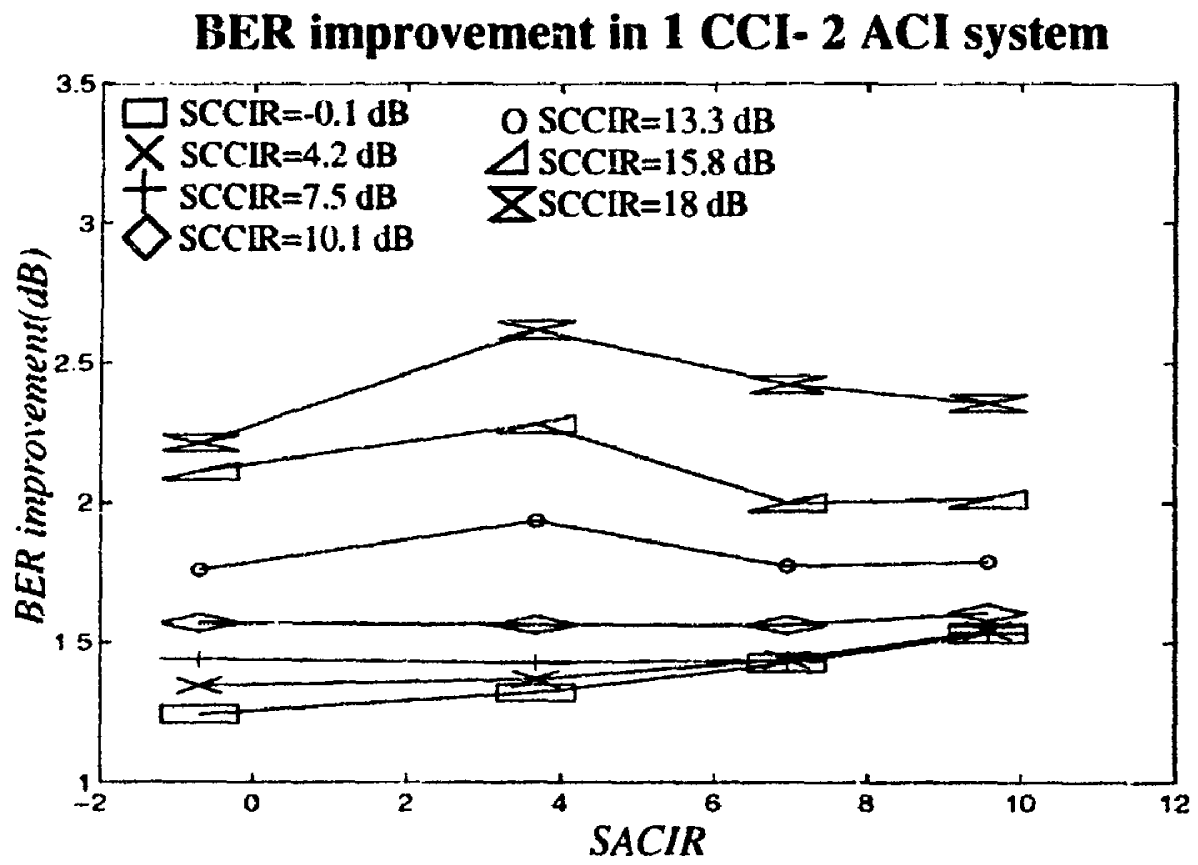

(b)

Figure 6.20 (a) The BER and (b) The BER improvement versus SACIR for 1 CCI- 2 ACI OQPSK system. 


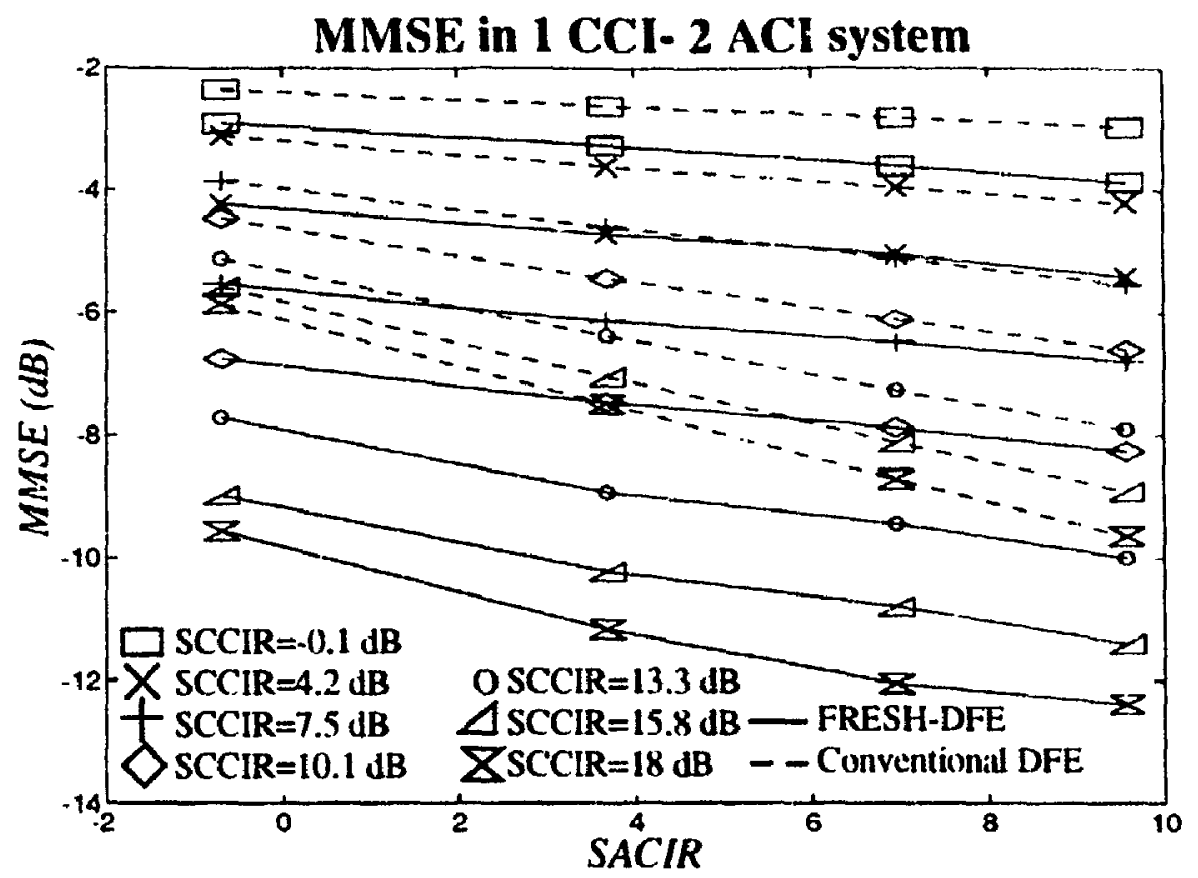

(a)

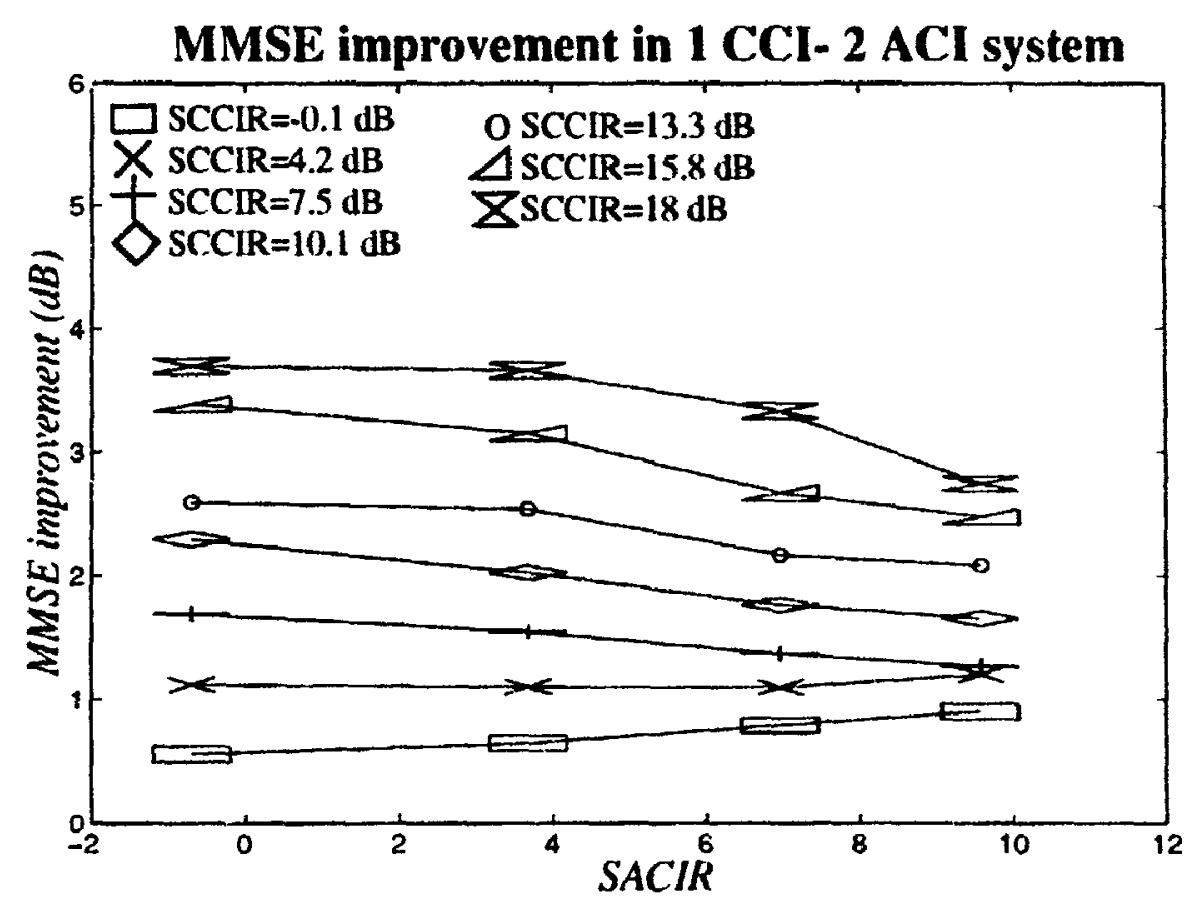

(b)

Figure 6.21 (a) The MMSE and (b) The MMSE improvemont vorsus SACIR for $1 \mathrm{CCl} 2 \mathrm{ACI}$ OQPSK system. 
BER in 1 CCI- 2 ACI system

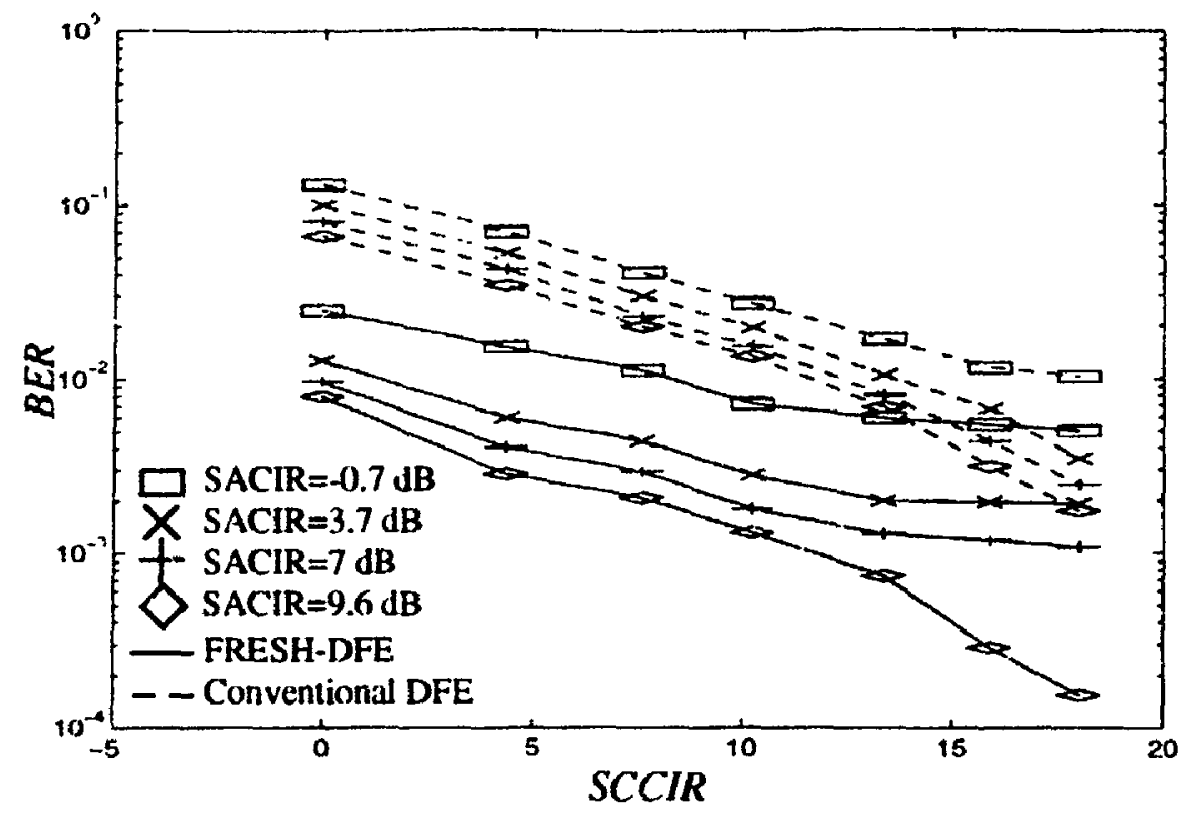

(a)

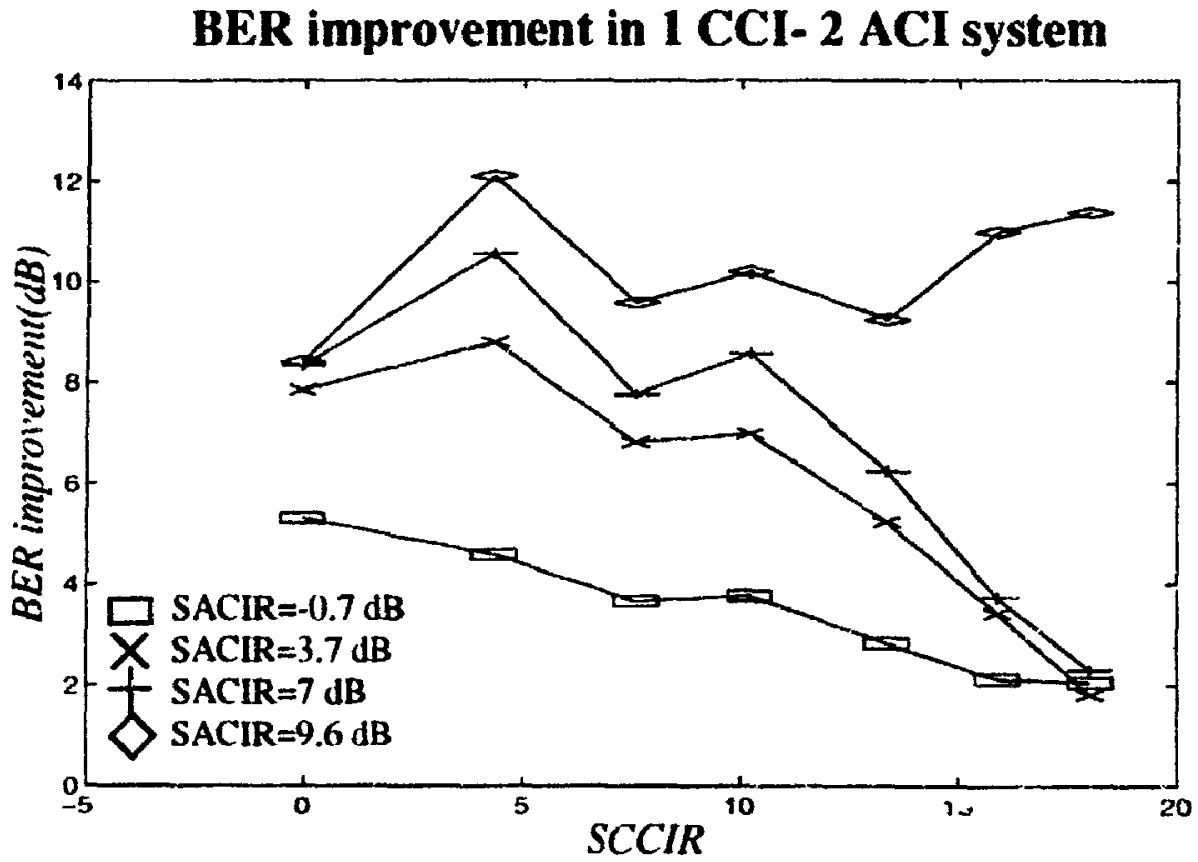

(b)

Figure 6.22 (a) The BER and (b) The BER improvement versus SCCIR for $1 \mathrm{CCI}-2$ ACI BPSK system. 


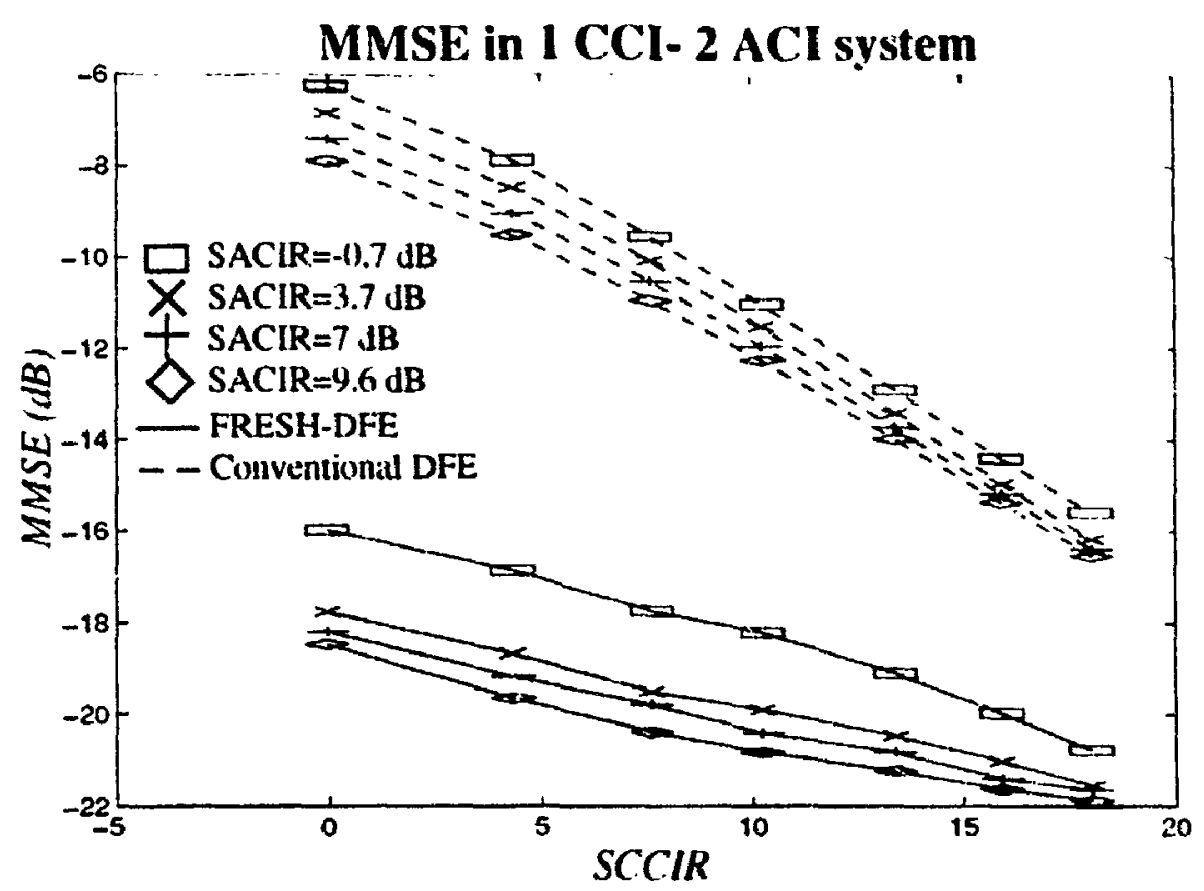

(a)

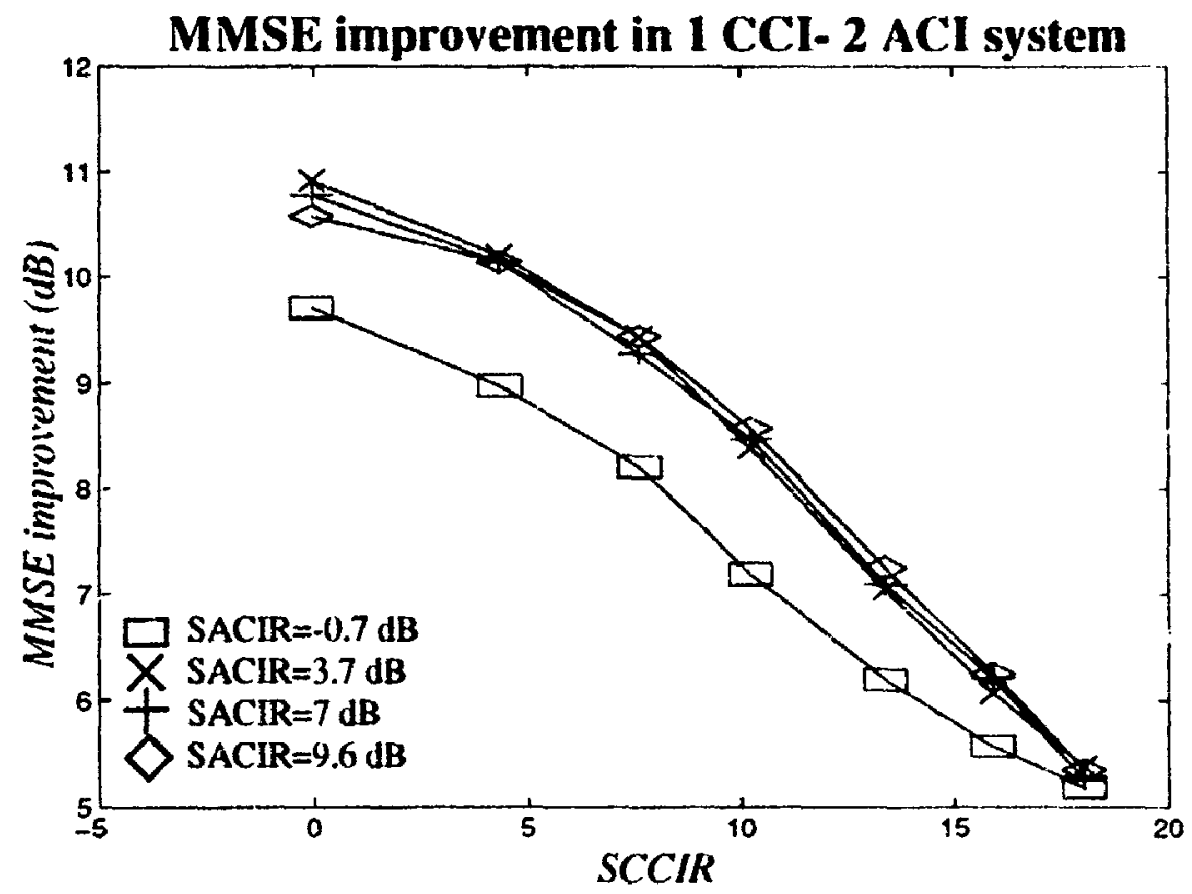

(b)

Figure 6.23 (a) The MMSE and (b) The MMSE imprevement versus SCCIR for $1 \mathrm{CCl}-2 \mathrm{ACI}$ BPSK system. 
BER in 1 CCI- 2 ACI system

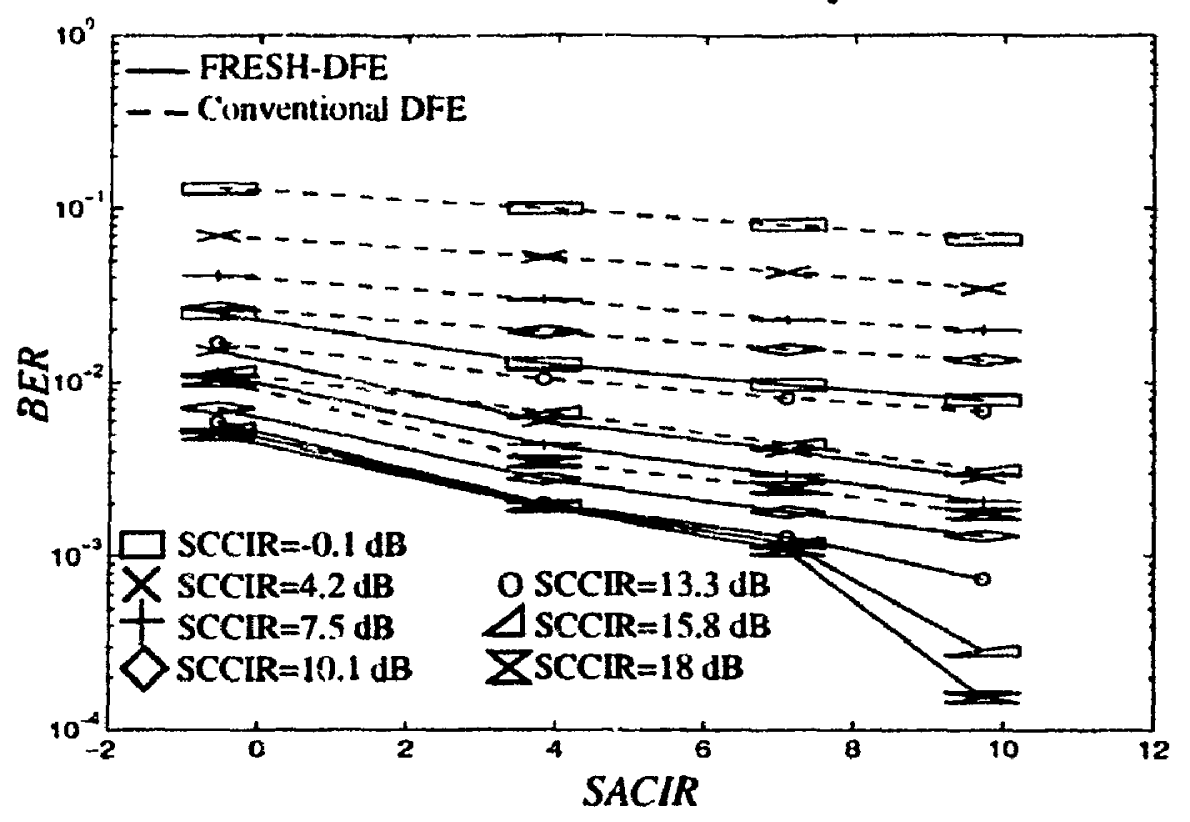

(a)

BER improvement in 1 CCI- 2 ACI system

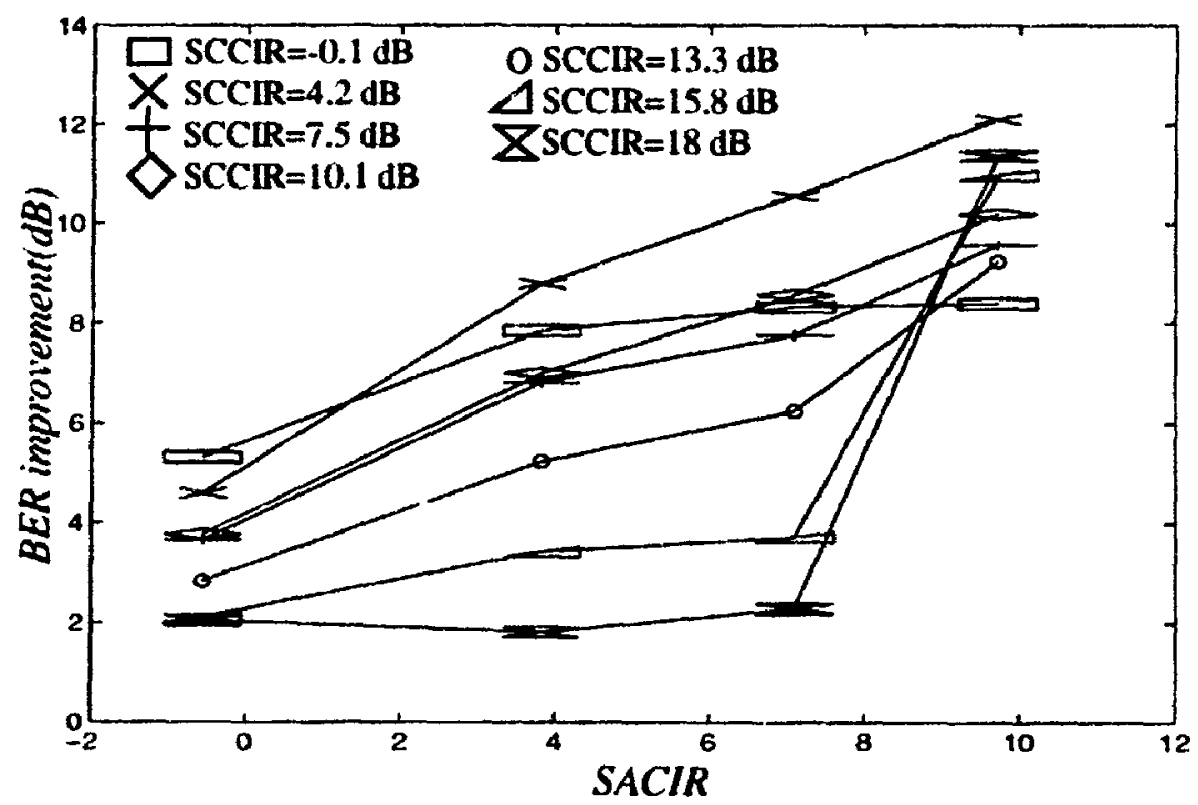

(b)

Figure 6.24 (a) The BER and (b) The BER improvement versus SACIR for $1 \mathrm{CCl}-2$ ACI BPSK system. 


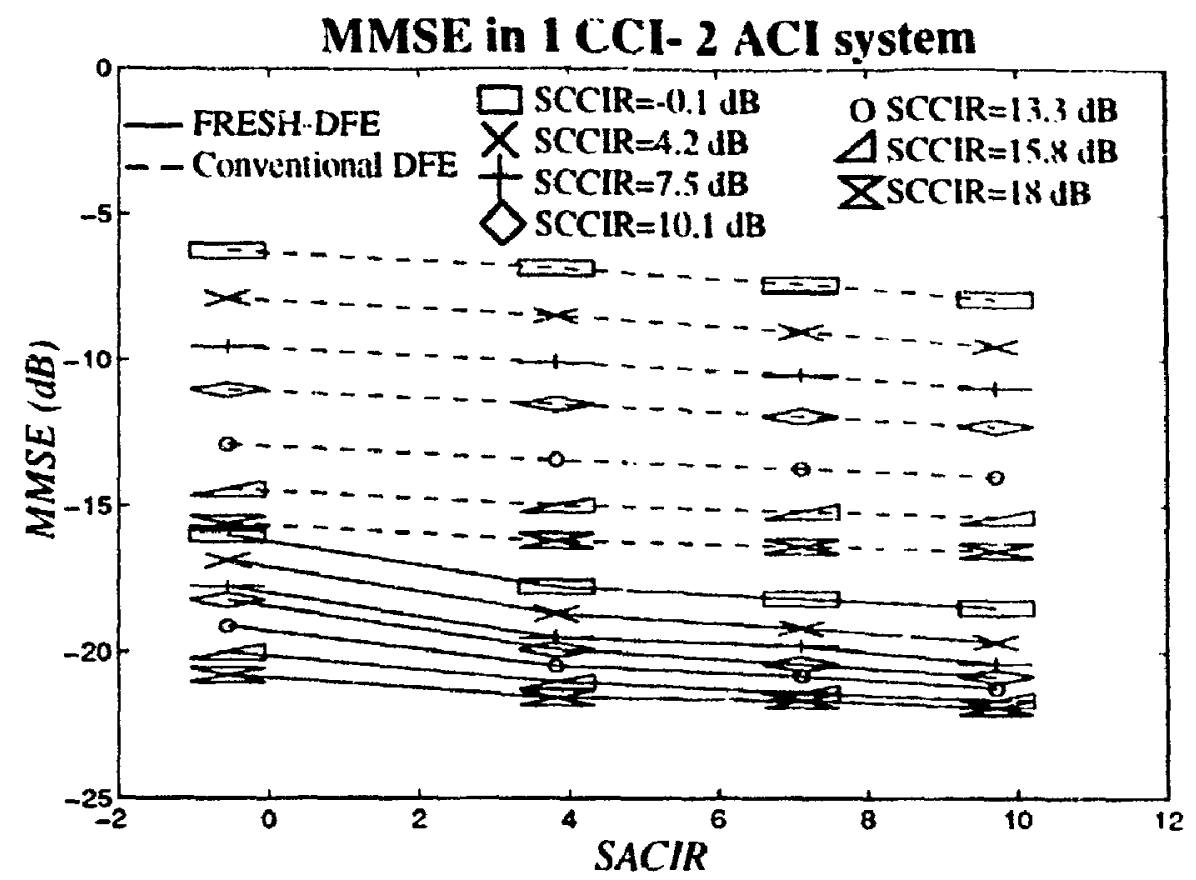

(a)

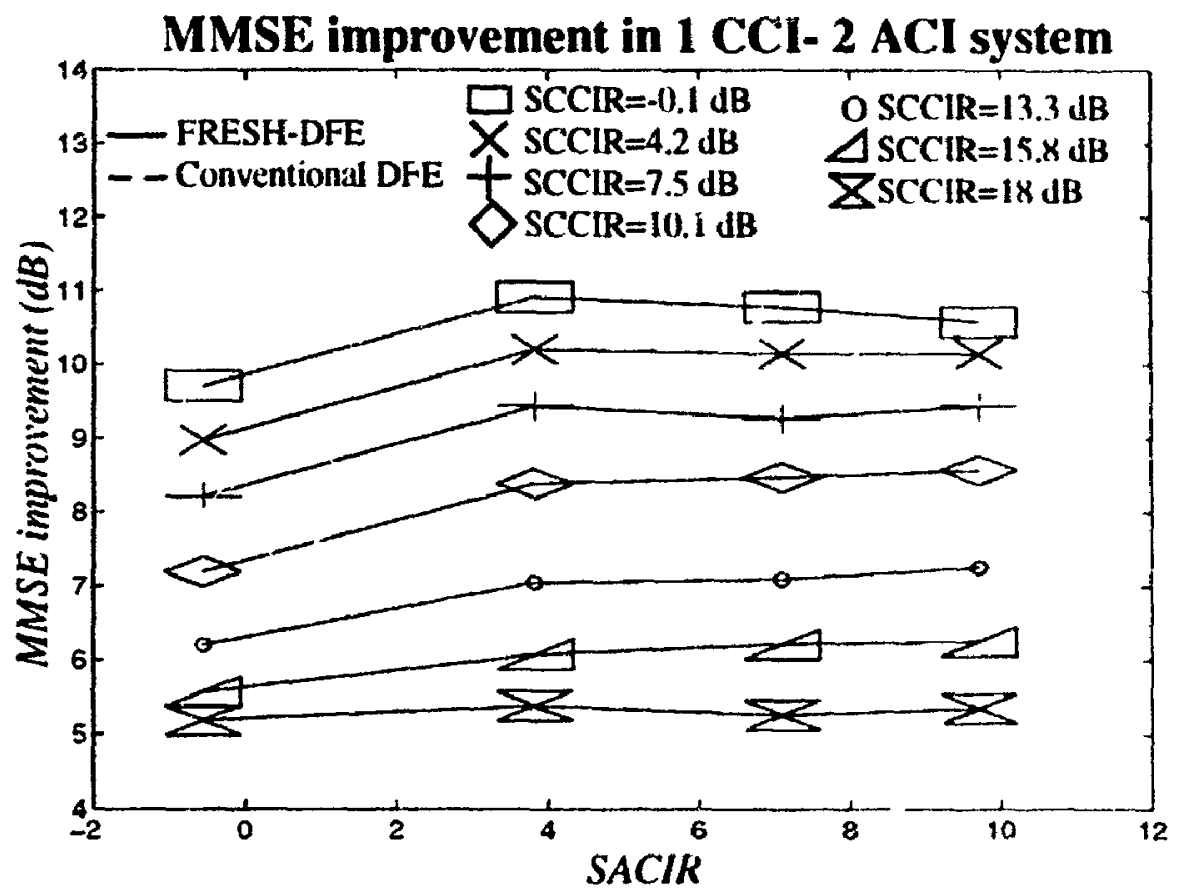

(b)

Figure 6.25 (a) The MMSE and (b) The MMSE improverrient versus SACIR for $1 \mathrm{CCL} 2 \mathrm{ACI}$ BPSK system. 


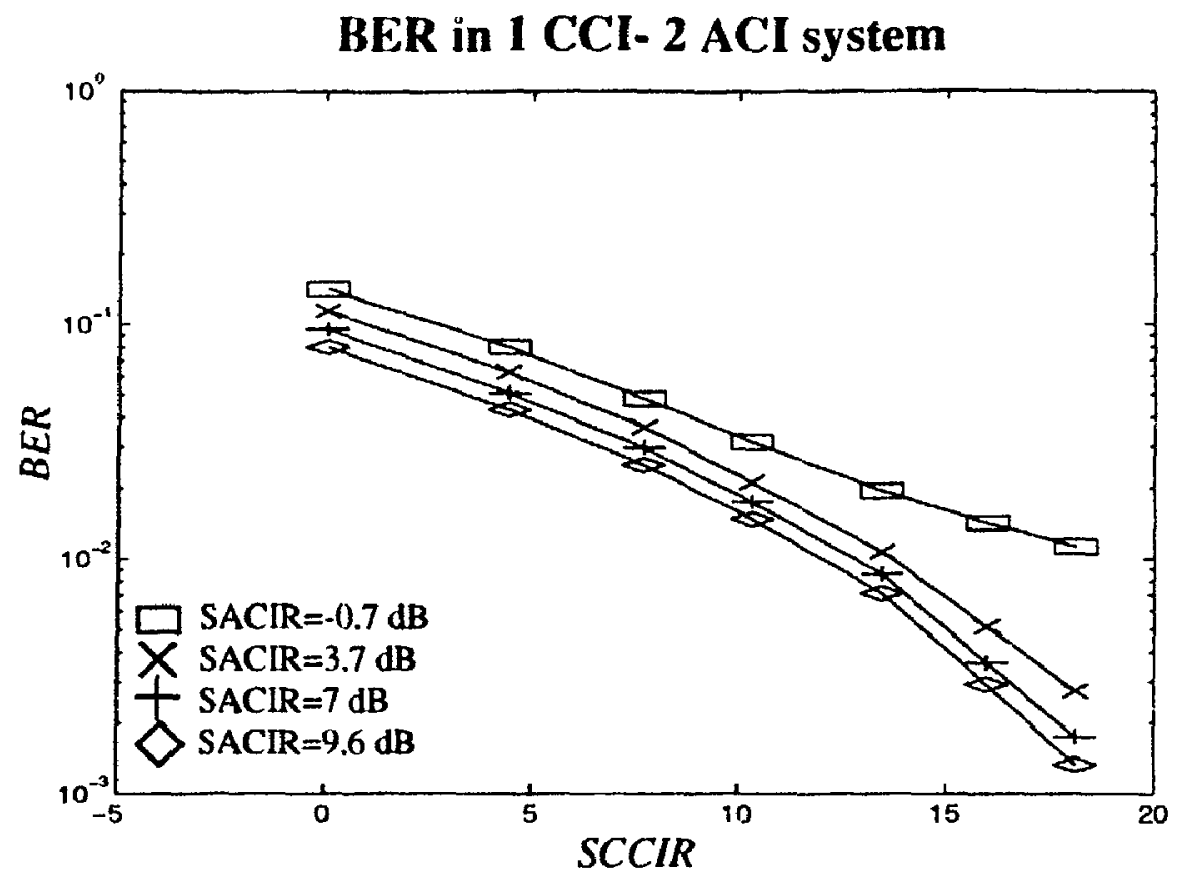

(a)

BER improvement in 1 CCI- 2 ACI system

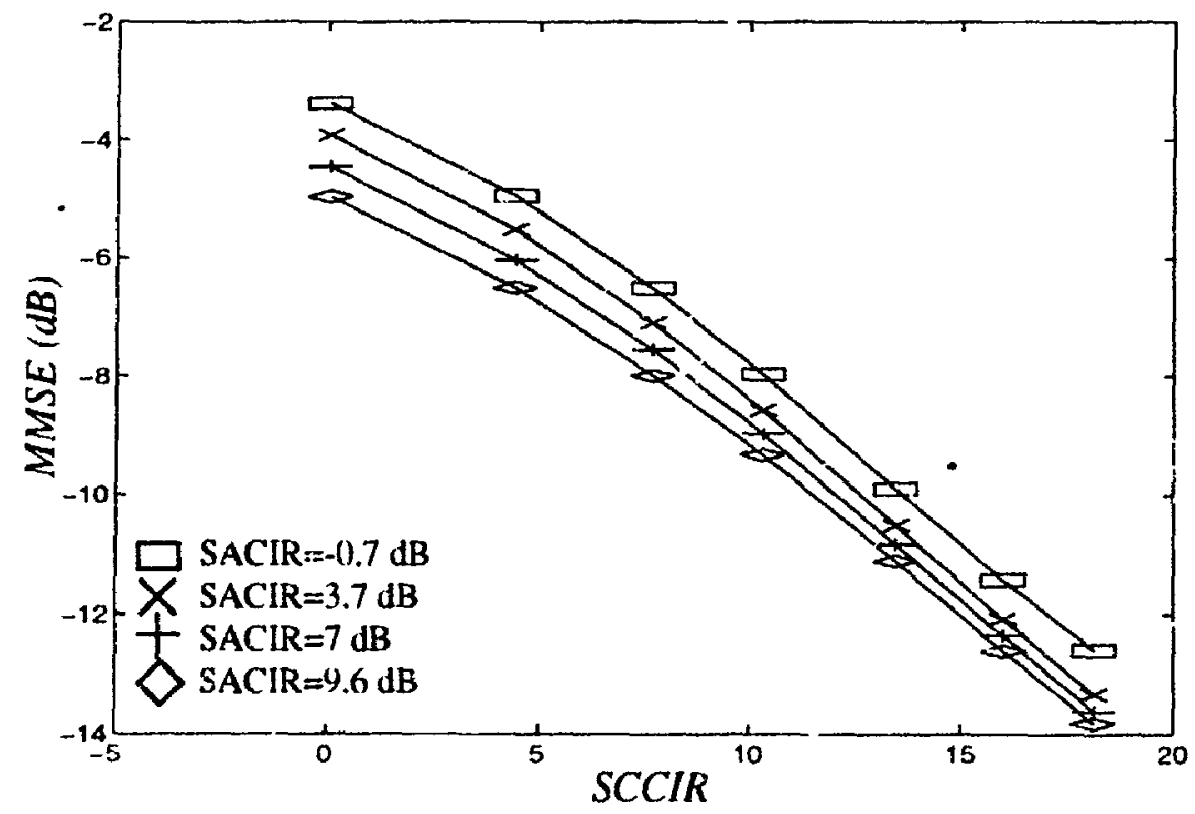

(b)

Figure 6.26 (a) The BER and (b) The MMSE versus SCCIR for $1 \mathrm{CCl} 2 \mathrm{ACI}$ QPSK system. 
BER in 1 CCI- 2 ACl system

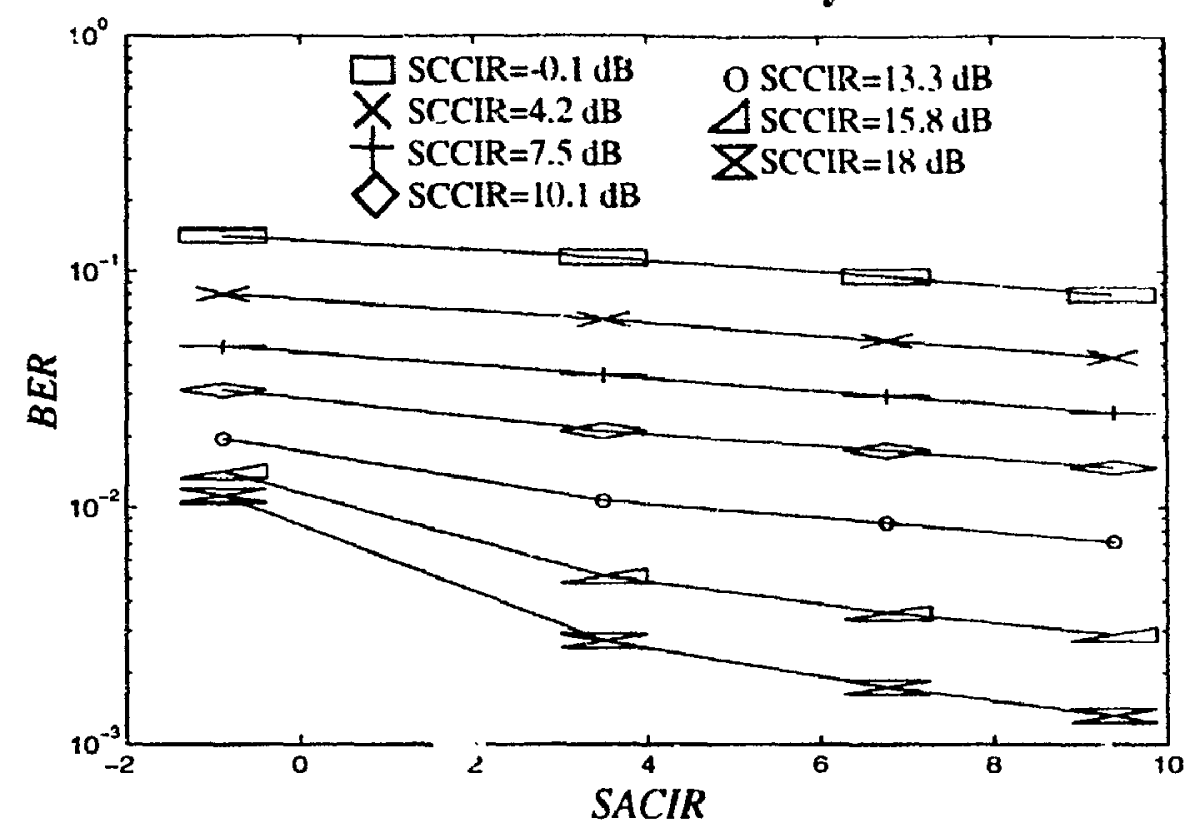

(a)

BER improvement in $1 \mathrm{CCI}-2$ ACI system

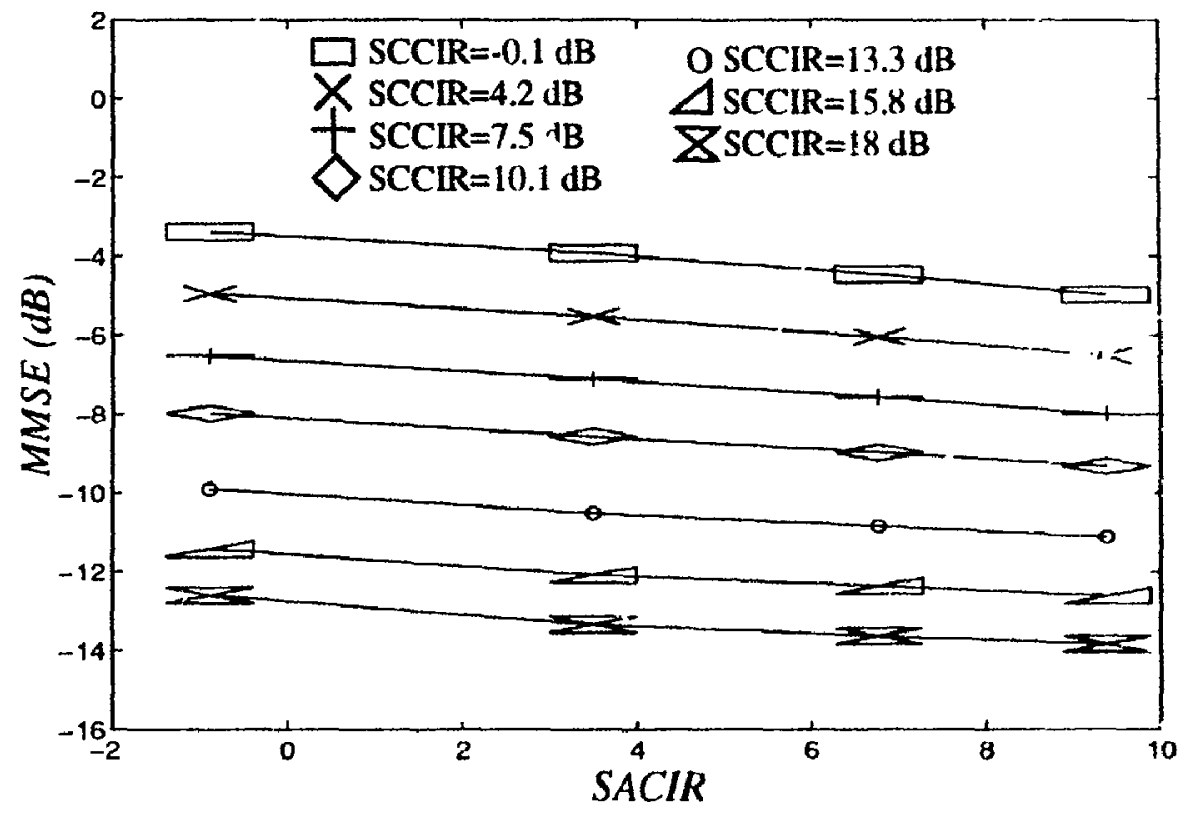

(b)

Figure 6.27 (a) The BER and (b) The MMSE versus SACIR for $1 \mathrm{CCl} 2 \mathrm{ACl}$ QPSK system. 
3- In OQPSK system, the BER improvement and MMSE improvement increases when SCCIR decreases while in BPSK system, the BER improvement and MMSE improvement decreases when SCCIR decreases.

\subsubsection{6-CCl 6-ACl System}

Figure 6.28 and Figure 6.29 show the BER and MMSE for different modulation schemes when all the $\mathrm{CCI}$ and $\mathrm{ACI}$ sources are active. As it is apparent form the figures, the QPSK technique gives better performance over OQPSK. The reason is that, the ODFE structure for OQPSK is bigger than the ODFE structure for QPSK and this cause slower convergence and more error. However, it is possible to achieve same performance for QPSK and OQPSK if Real-Imaginary synchronizer (Figure 4.22) is used.

The performance is improved if a multi-antrana receiver is used. Figure 6.30 to Figure 6.34 show the BER and MMSE when multi-antennas receivers are used. The branches assumed to be uncorrelated in this simulation. As it is clear from the figure, the BER can be lower than $10^{-5}$ for a SNR greater than $5 \mathrm{~dB}$ if number of antenna increases. These figures also show that the superiority of ODFE over conventional DFE increases when the number of antenna increases.

\subsubsection{System with Channel Spacing Equals to Half The Symbol Rate}

If the channel spacing decreases by factor of two, the capacity increases by factor of two provided interference does not have a negative influence. Figure 6.35 to Figure 6.39 show the simulation results when the channel spacing equals to half the symbol rate and all the $\mathrm{CCl}$ and $\mathrm{ACl}$ sources are active. It is observed from these figures that, the BER lower than $10^{-2}$ (which is speech quality limit [95]) may be achieved with two antenna receiver regardless of modulation scheme. Moreover, the performance of a BPSK system with channel spacing equals to half the symbol rate is as good as the performance of a QPSK system with channel spacing equals to the symbol rate using ODFE. Consequently, the capacities of these two systems are also equal.

\subsubsection{2-Symbol Rate System}

The 2-symbol rate system shown in Figure 5.14 (b) is simulated. $T_{0}$ (the SOI symbol interval) and $T_{1}$ are 1 and 2 seconds respectively. The excess bandwidth of the signal with symbol duration of 1 second is 100 percent aid for signal with symbol duration 0.2 


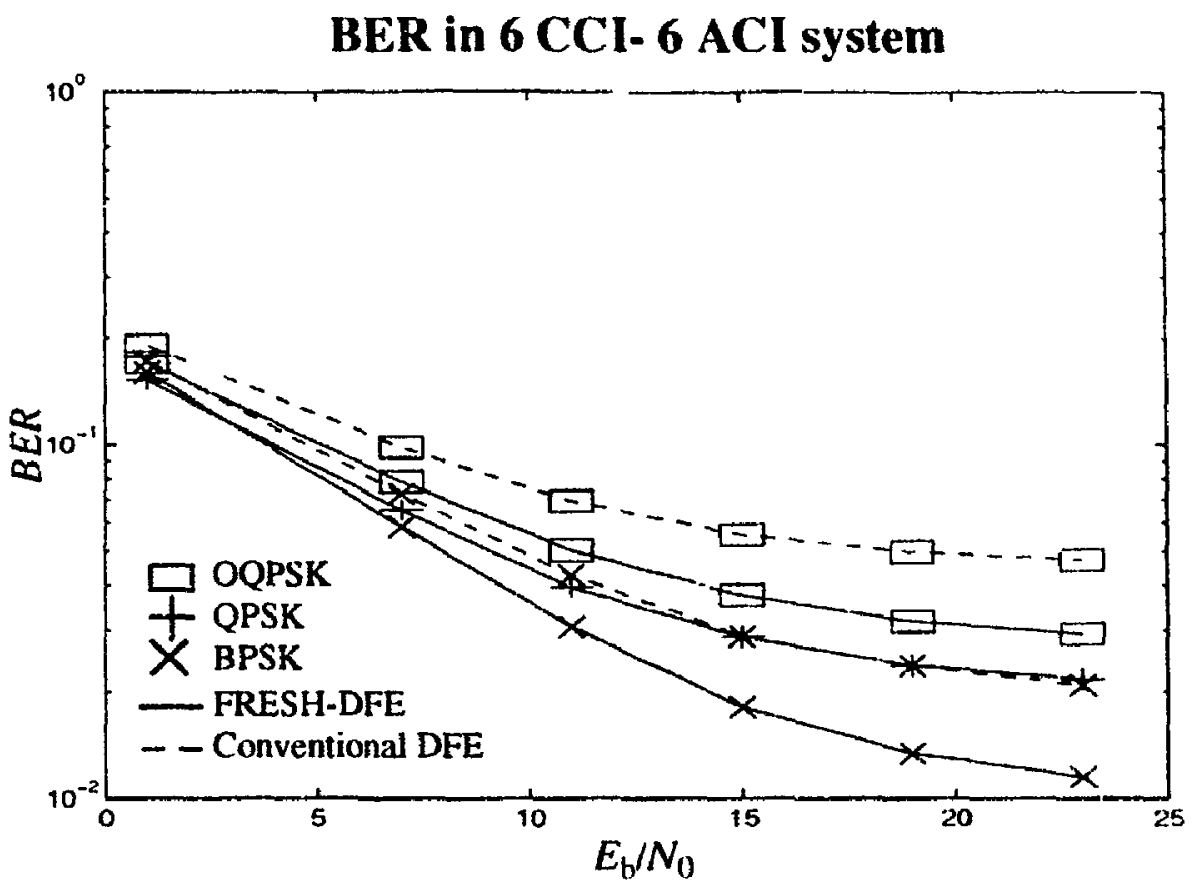

(a)

\section{BER improvement in $6 \mathrm{CCI}-6 \mathrm{ACI}$ system}

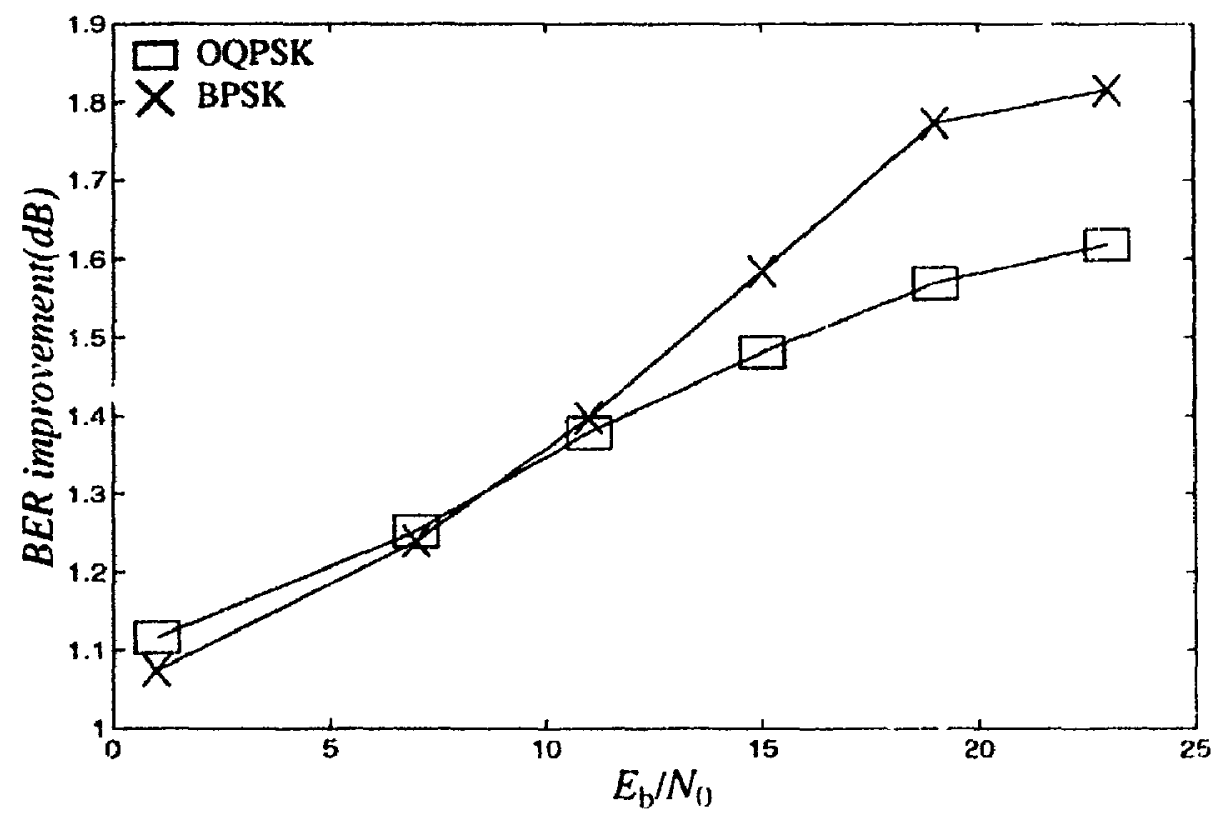

(b)

Figure 6.28 (a) The BER and (b) The BER improvement for $6 \mathrm{CCl}-6 \mathrm{ACl}$ system. 


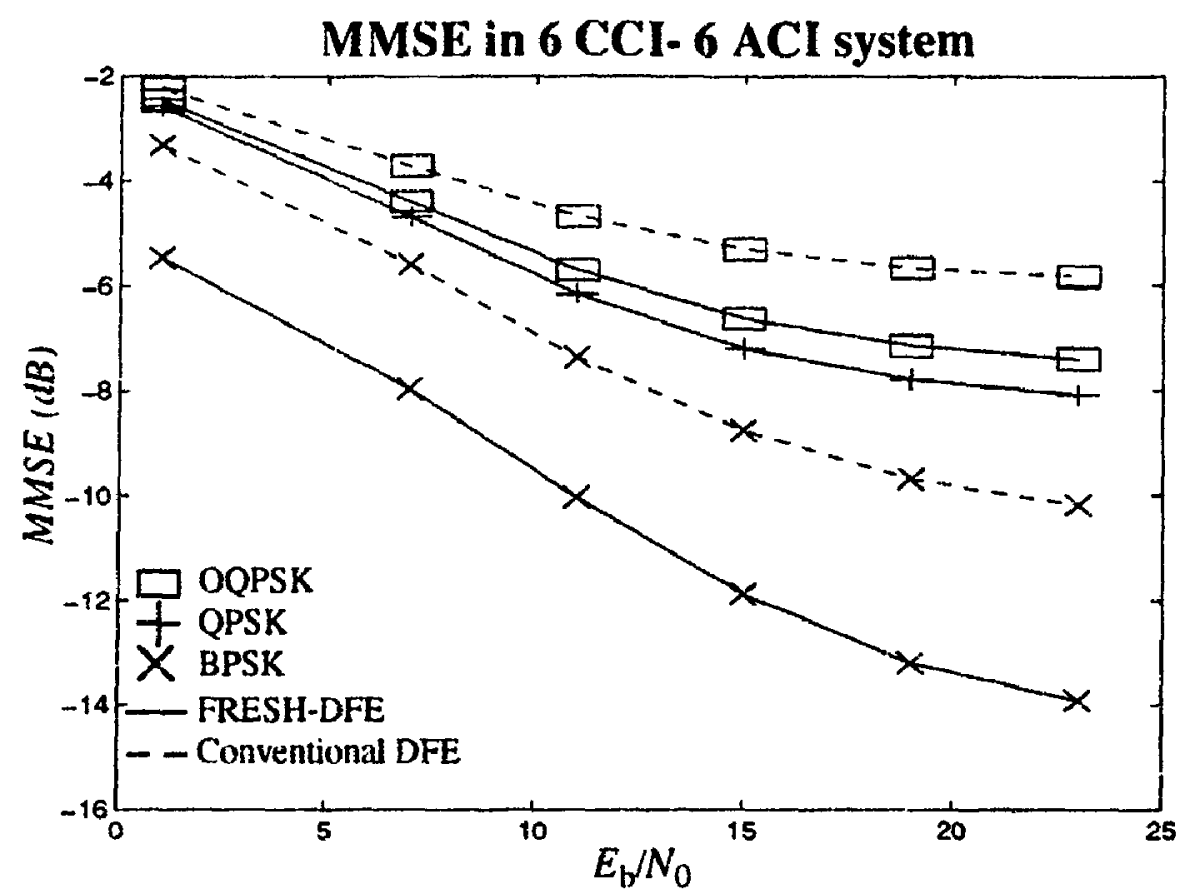

(a)

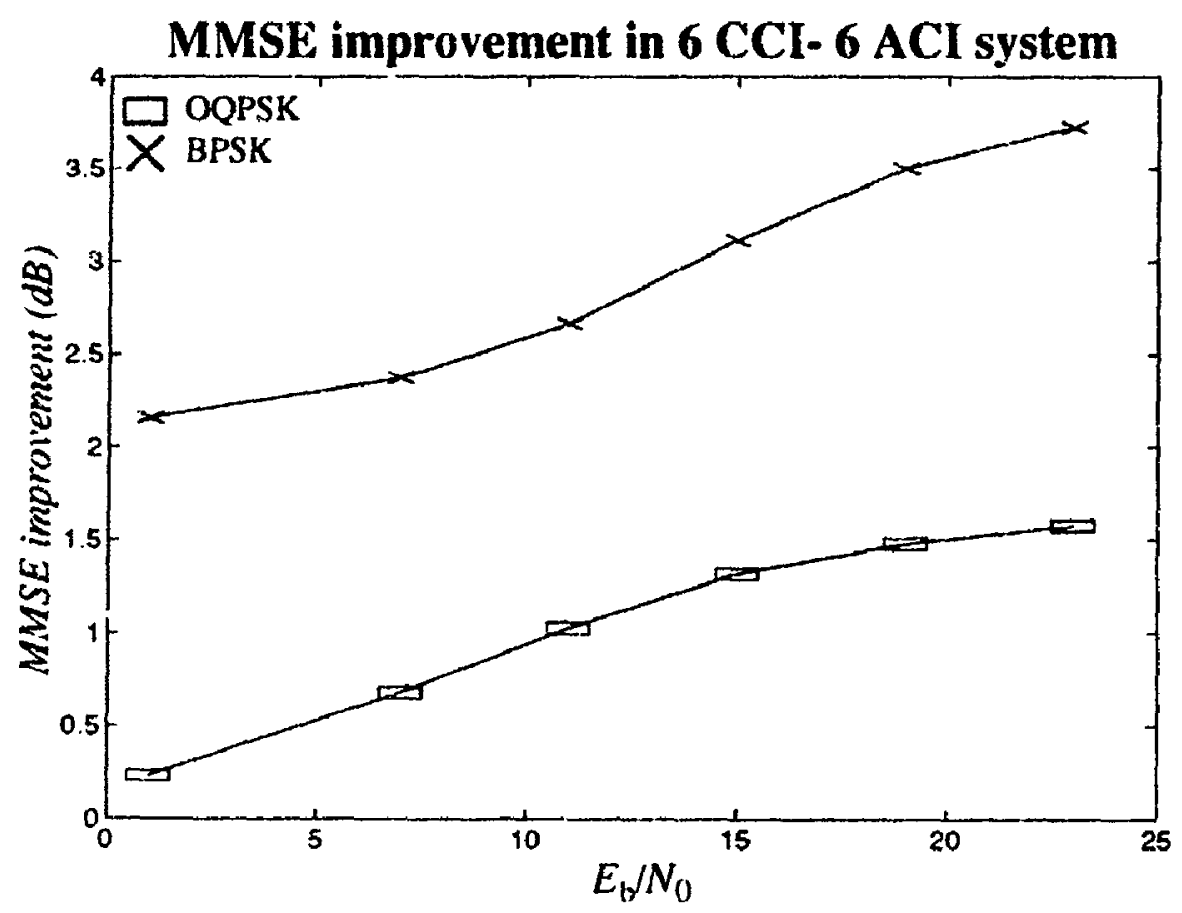

(b)

Figure 6.22 (a) The MMSE and (b) The MMSE improvement for $6 \mathrm{CCl}-6$ A.Cl system. 


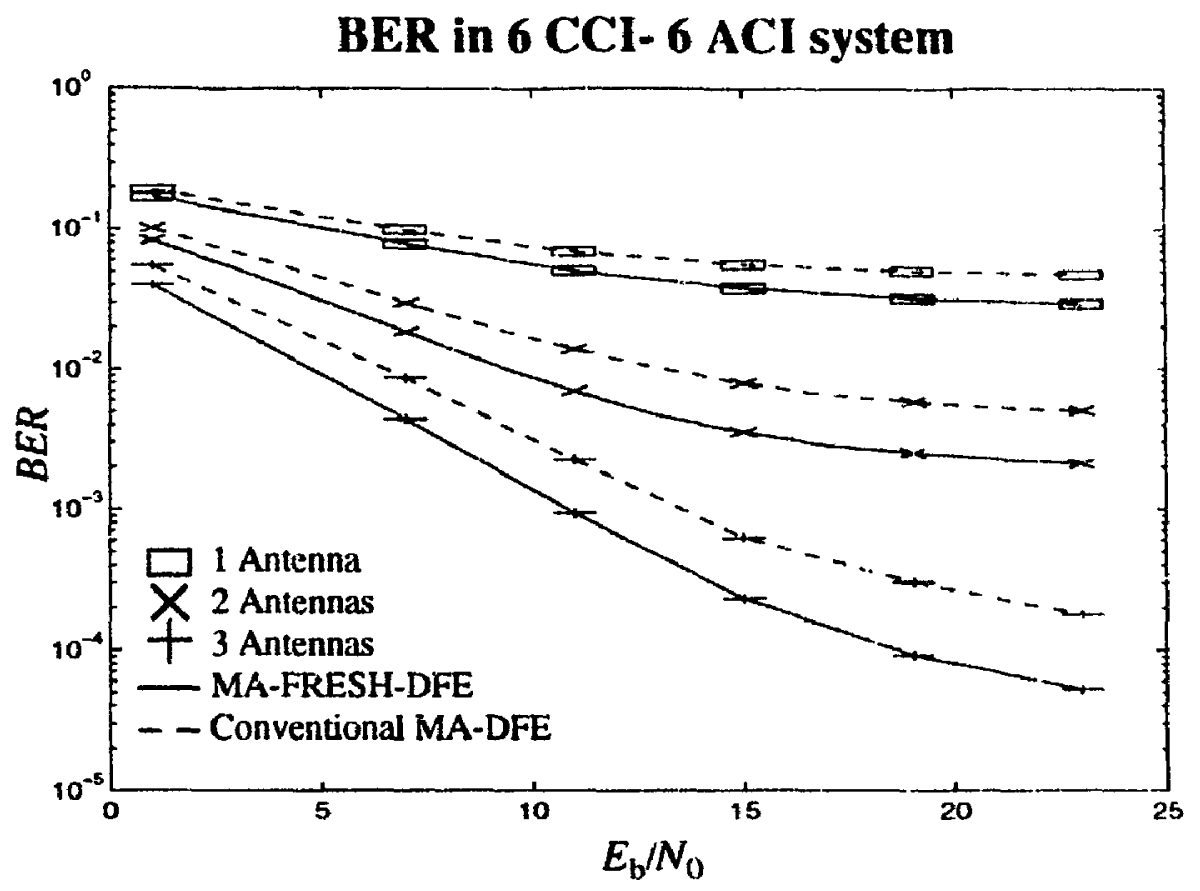

(a)

BER improvement in 6 CCI- 6 ACI system

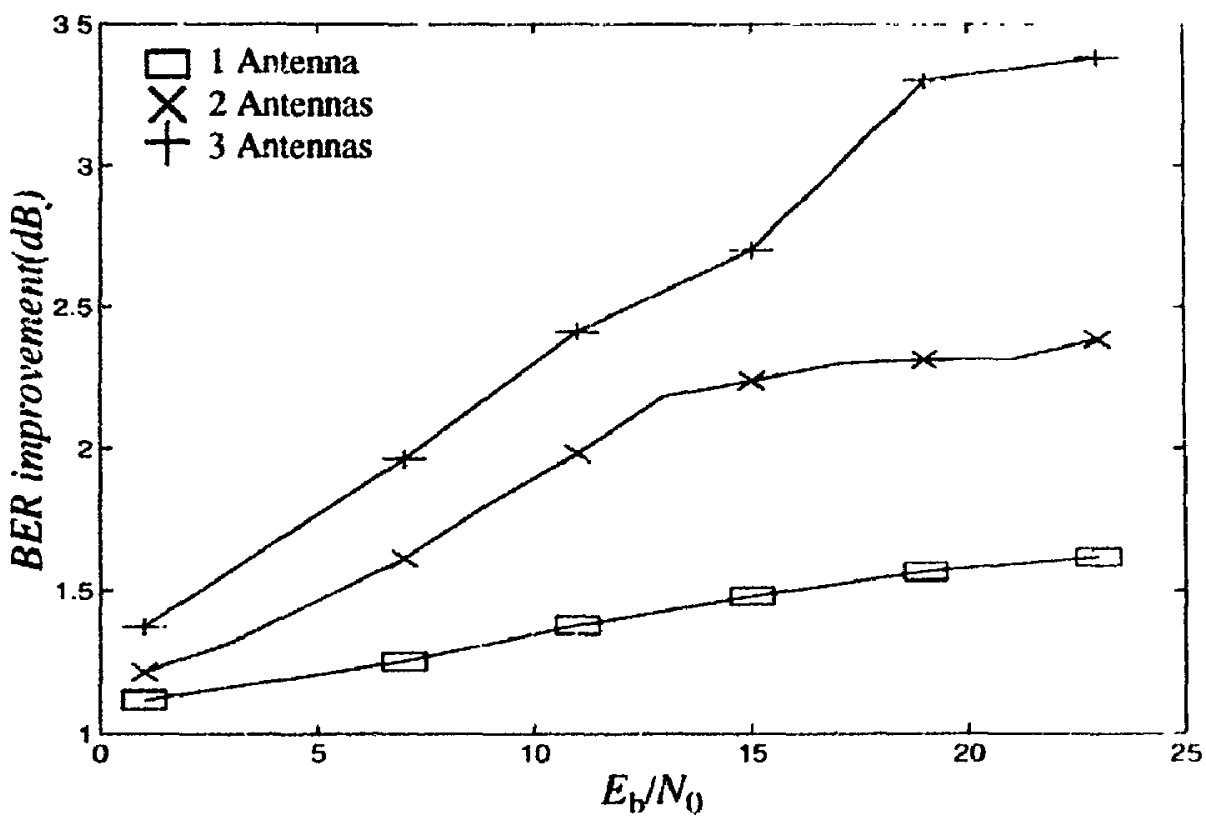

(b)

Figture 6.30 (a) The BER and (b) The BER improvement for multi-antenna recoiver in $6 \mathrm{CCl}-6 \mathrm{ACl}$ OOPSK system. 


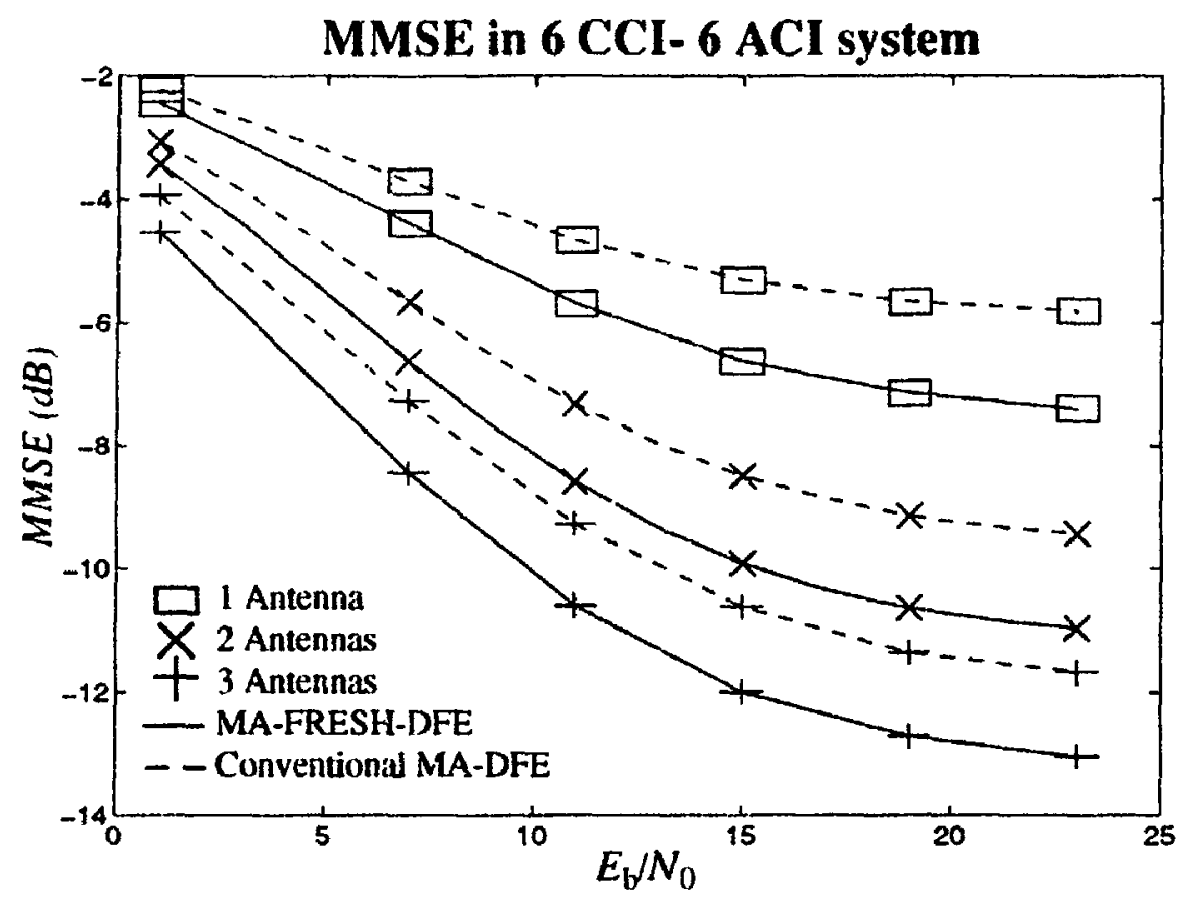

(a)

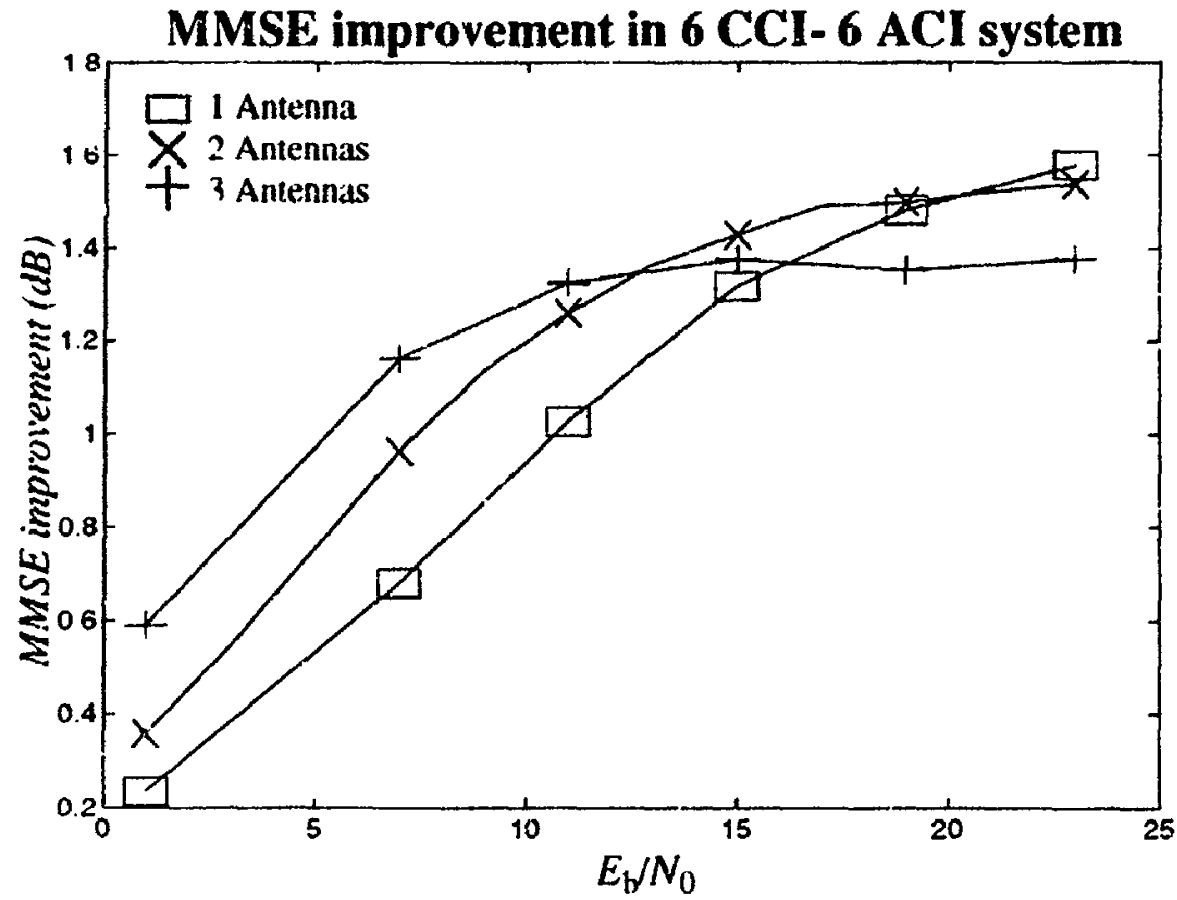

(b)

Figure 6.31 (a) The BER and (b) The BER improvement for multi-antenna receiver in $6 \mathrm{CCl}-6 \mathrm{ACl}$ OOPSK system. 


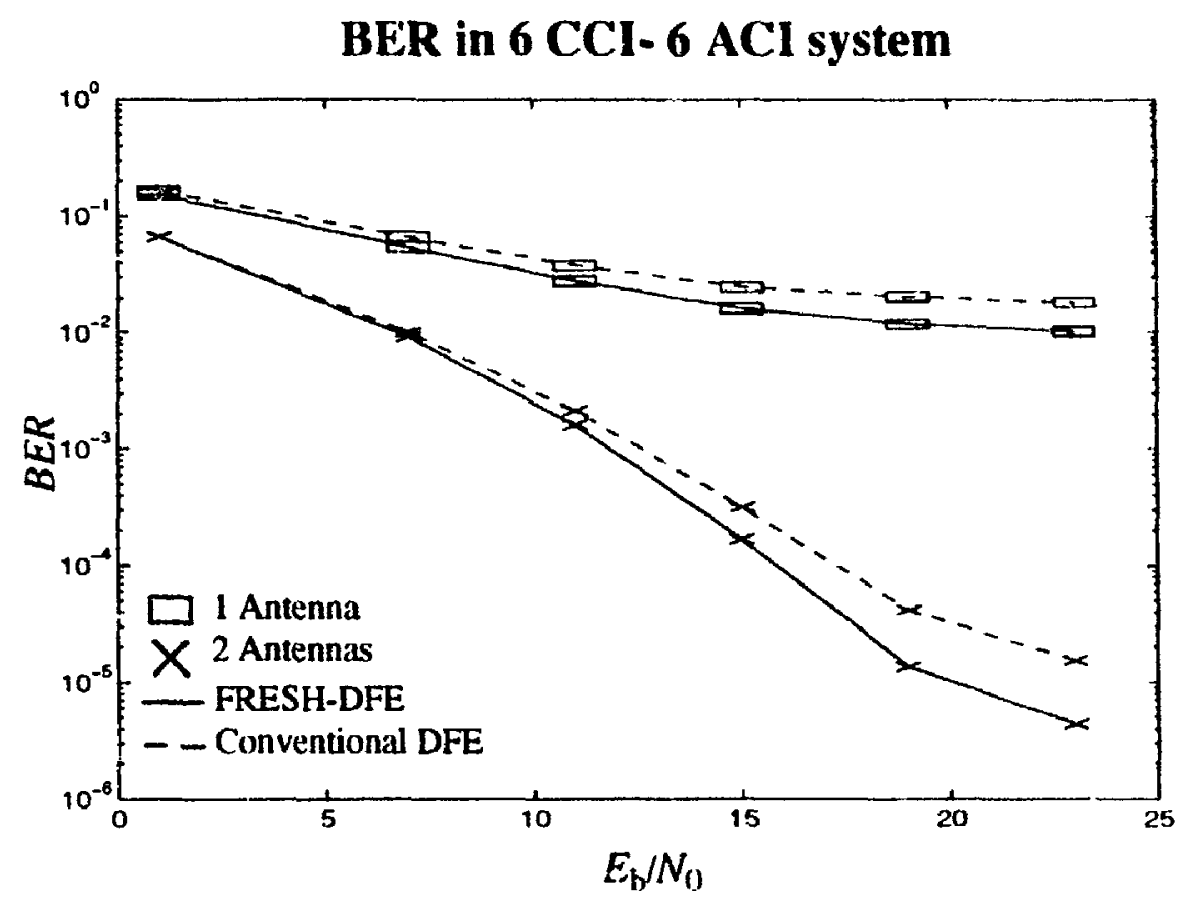

(a)

BER improvement in $6 \mathrm{CCI}-6 \mathrm{ACI}$ system

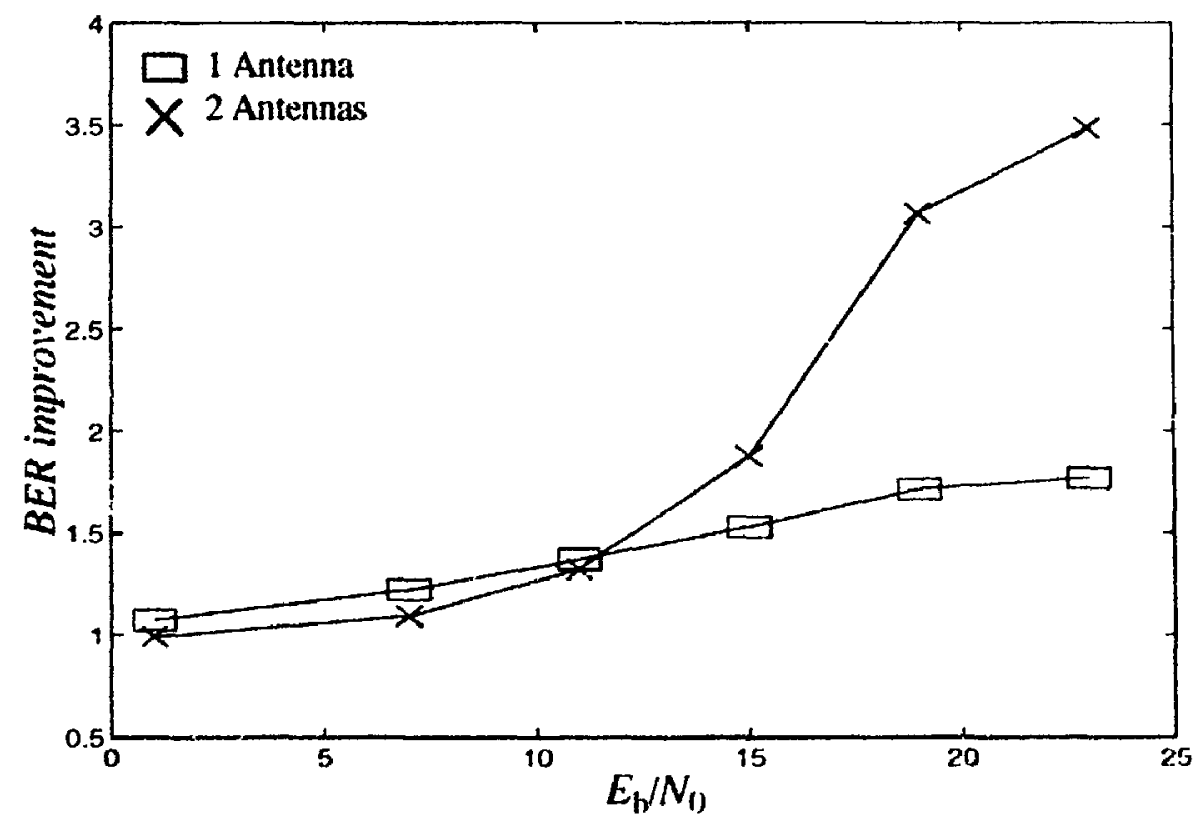

(b)

Figure 6.32 (a) The BER and (b) The BER improvement for multi-antenna receiver in $6 \mathrm{CCl}-6 \mathrm{ACI}$ BPSK system. 


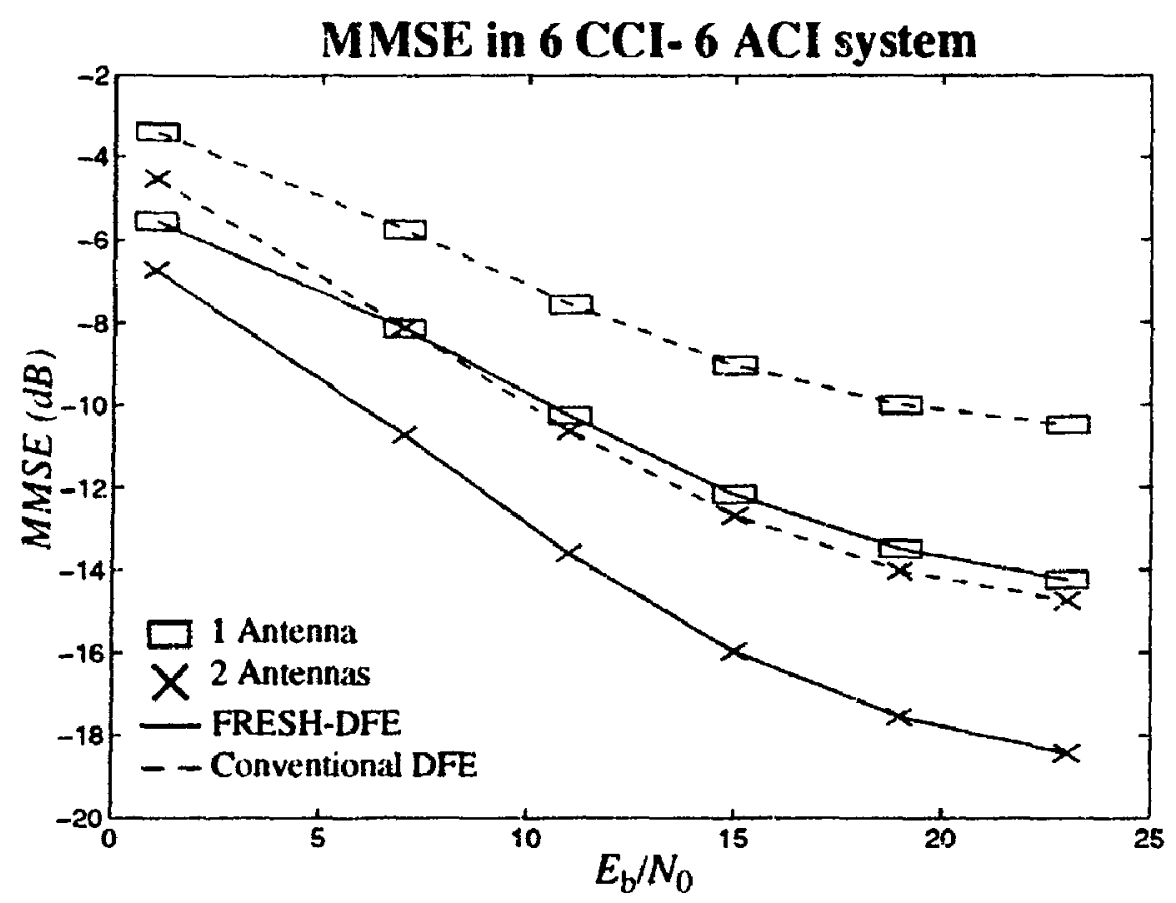

(a)

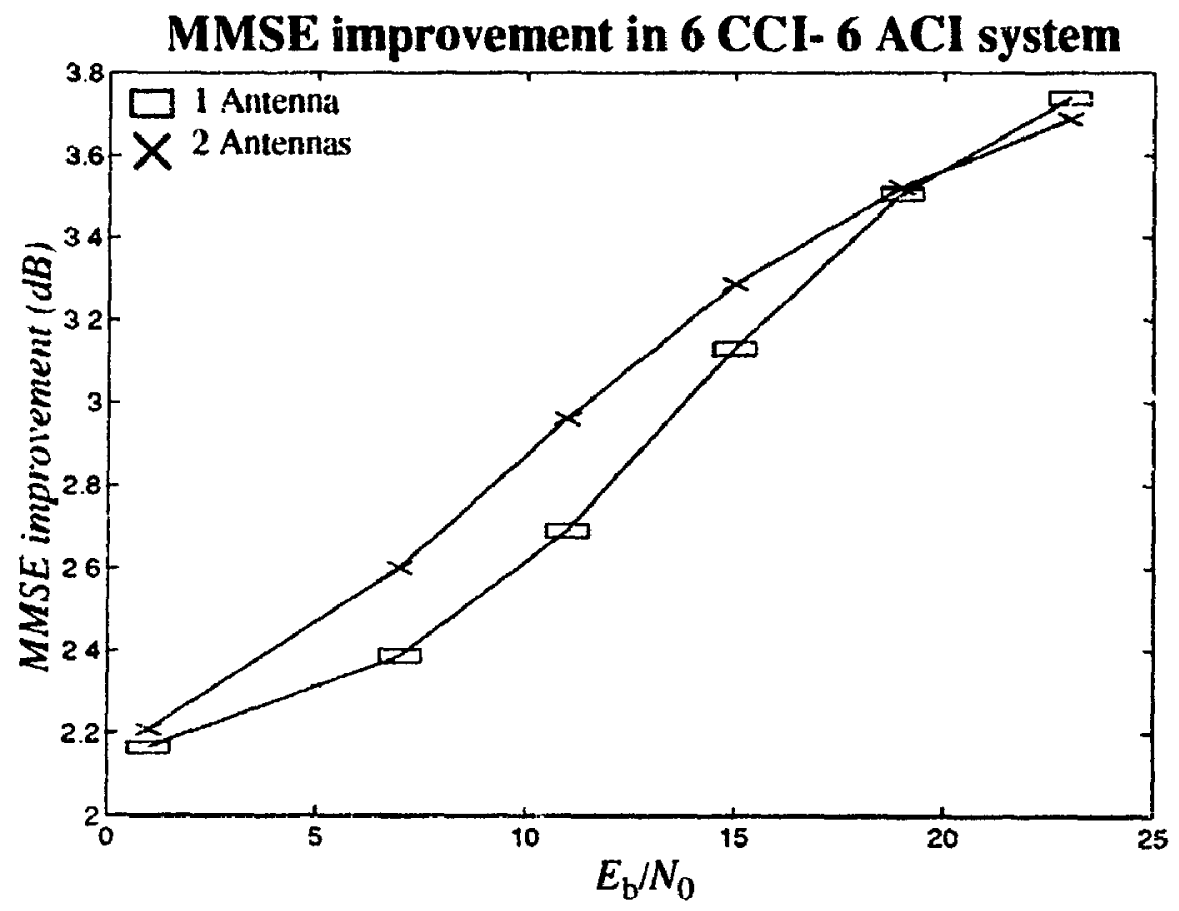

(b)

Figure 6.33 (a) The MMSE and (b) The MMSE improvement for multi-antenna receiver in $6 \mathrm{CCl}-6$ ACI BPSK systom. 
BER in 6 CCI- 6 ACI system

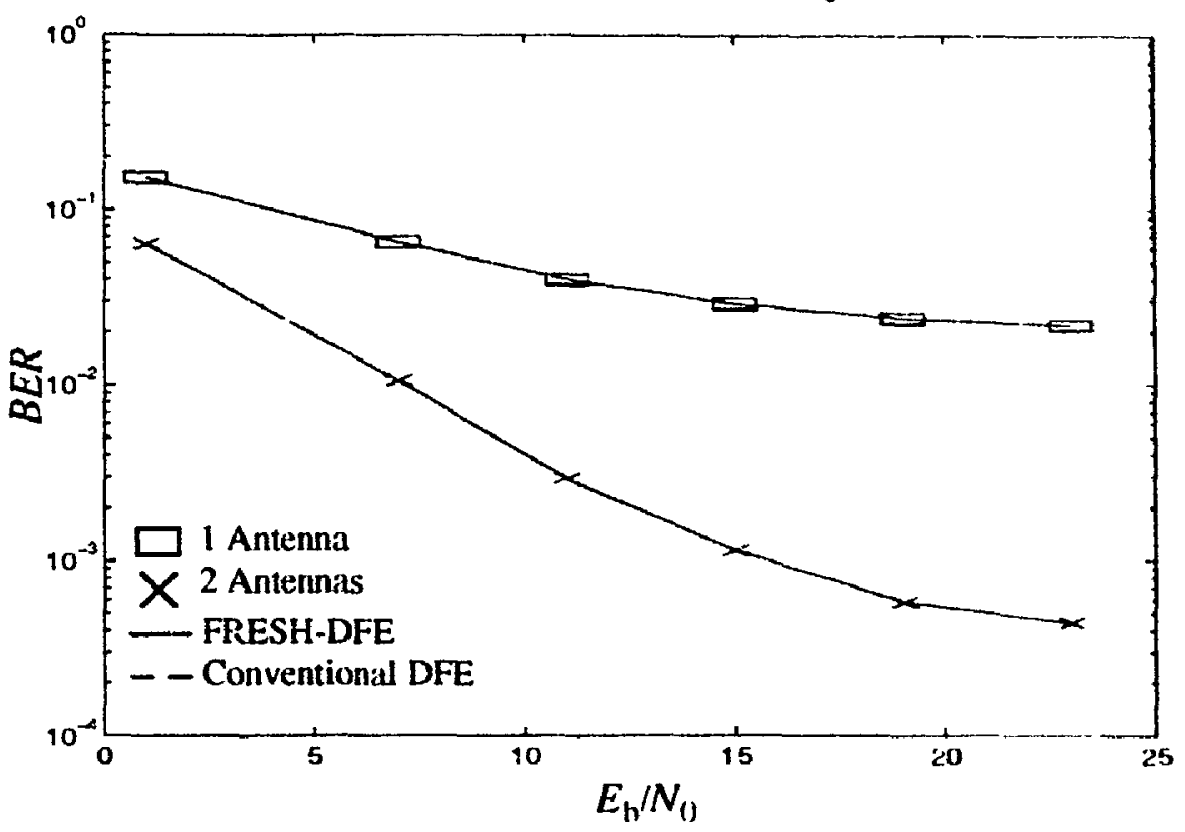

(a)

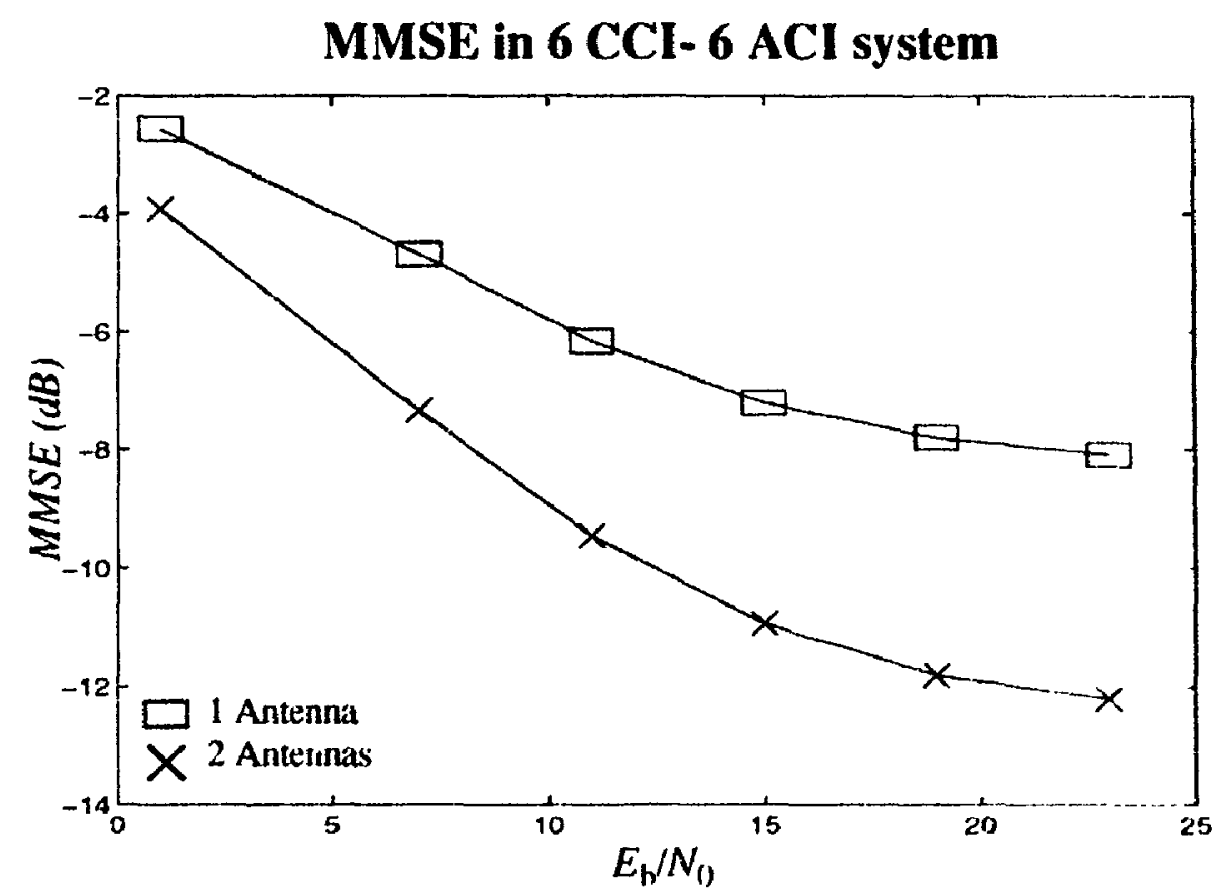

(b)

Figure 6.34 (a) The BER and (b) The MMSE for multi-antunna receiver in $6 \mathrm{CCl}-6$ ACI QPSK systorn. 


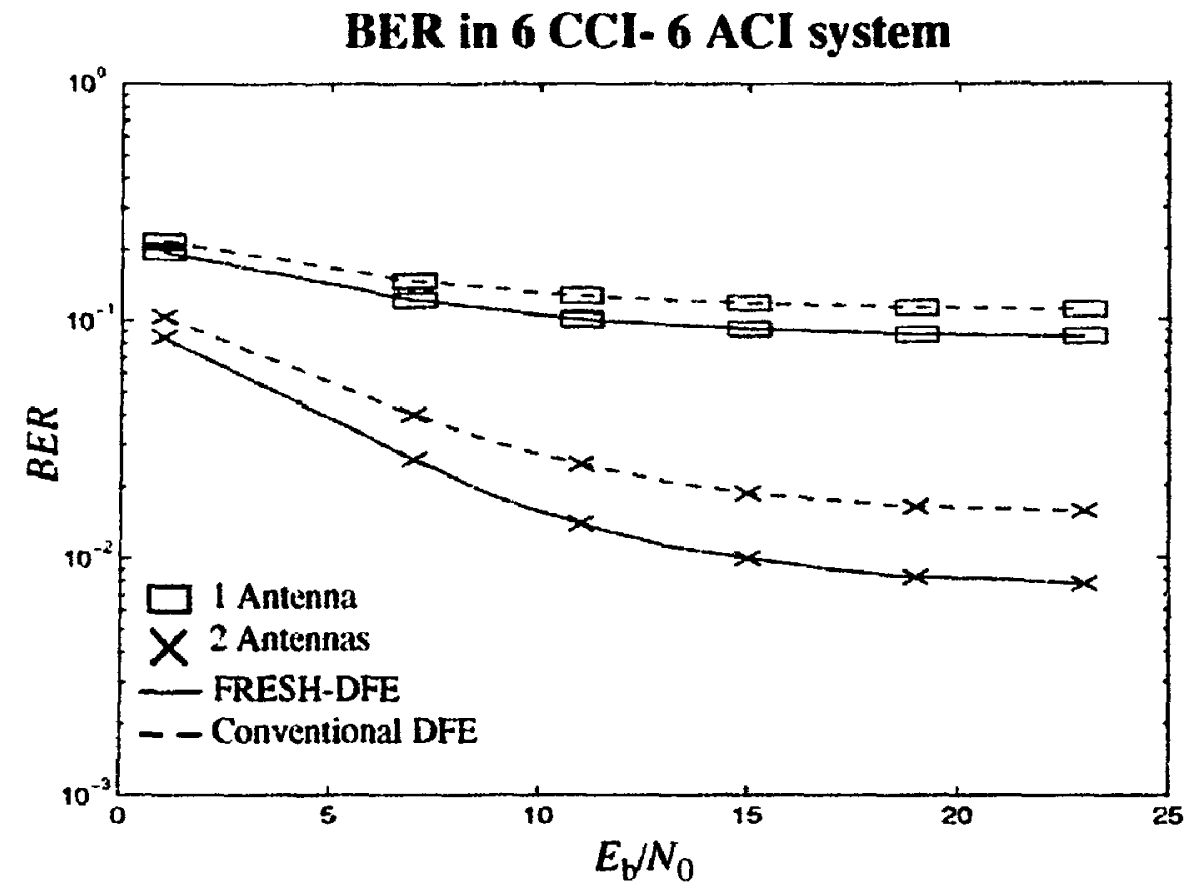

(a)

BEK improvement in 6 CCI- 6 ACI system

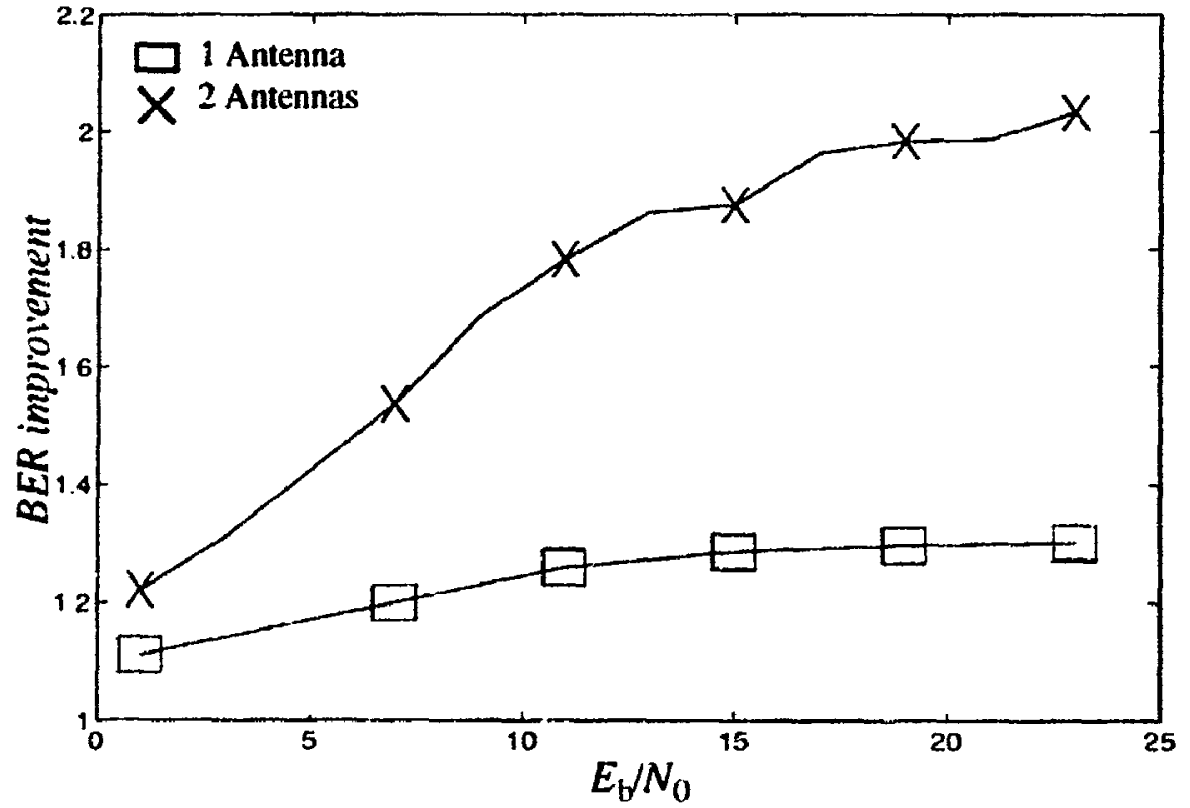

(b)

Figure 6.35 (a) The BER and (b) The BER improvement for multi-antenna receiver in 6 CCI- $6 \mathrm{ACl}$ OOPSK system when channel spacing is half the symbol rate. 


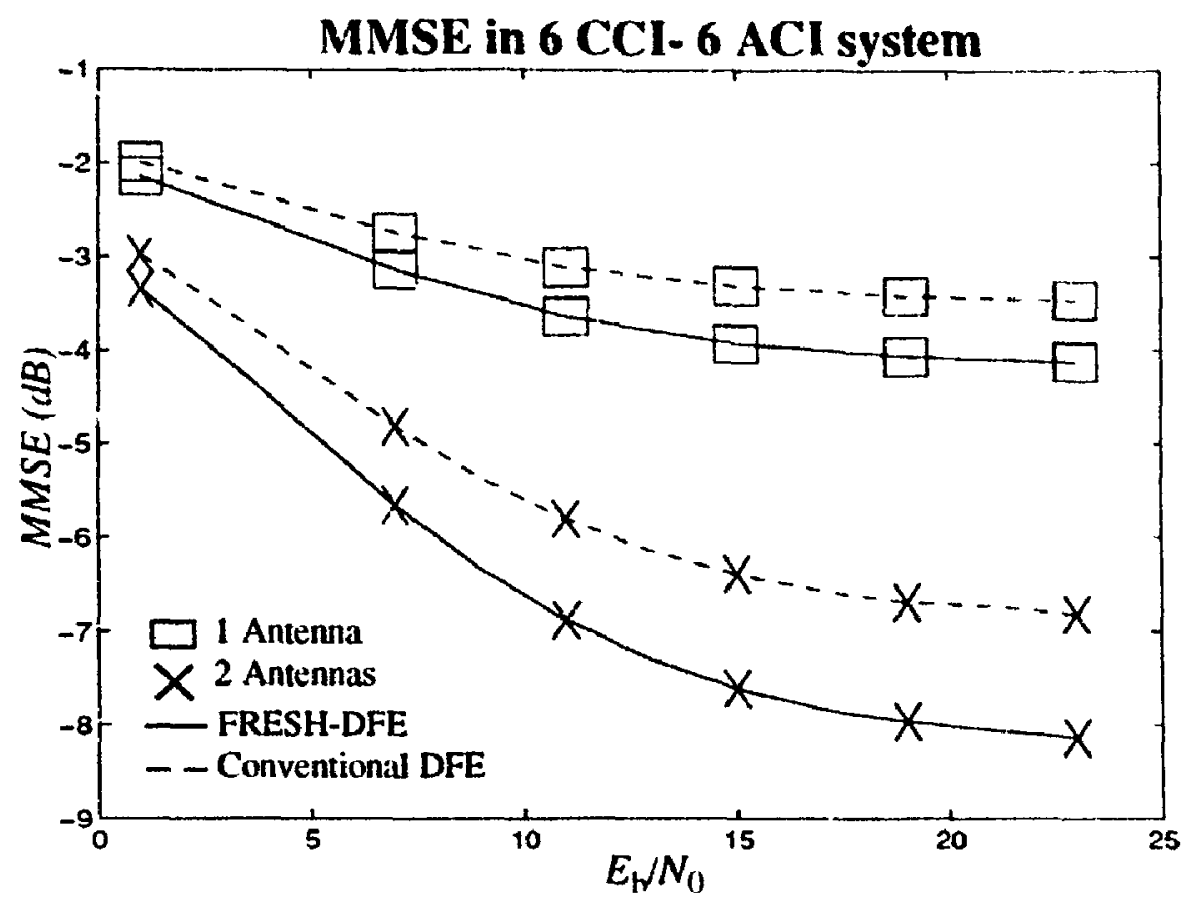

(a)

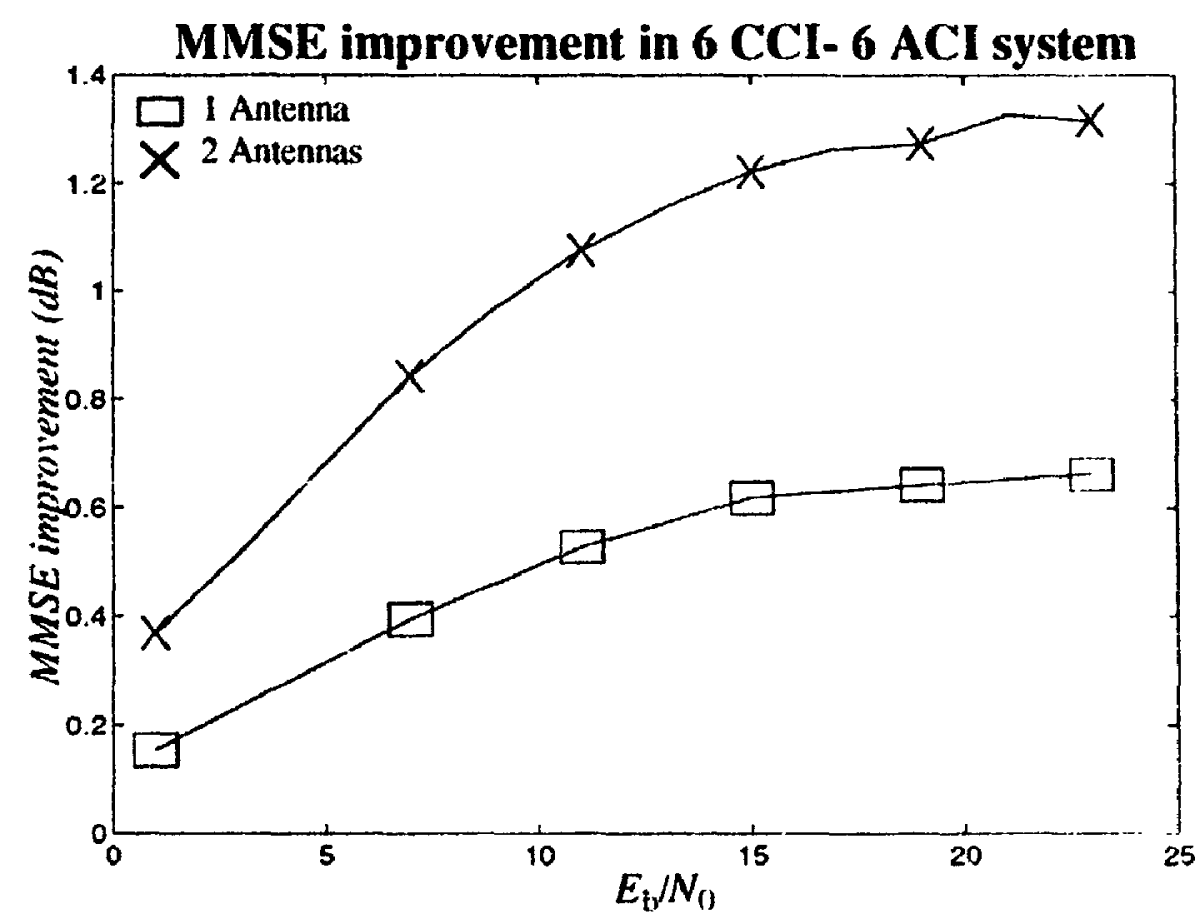

(b)

Figure 6.36 (a) The MMSE and (b) The MMSE improvement for multi-antenna recoiver in $6 \mathrm{CCl}-6$ ACI OQPSK system when channel spacing is half the symbol rate. 


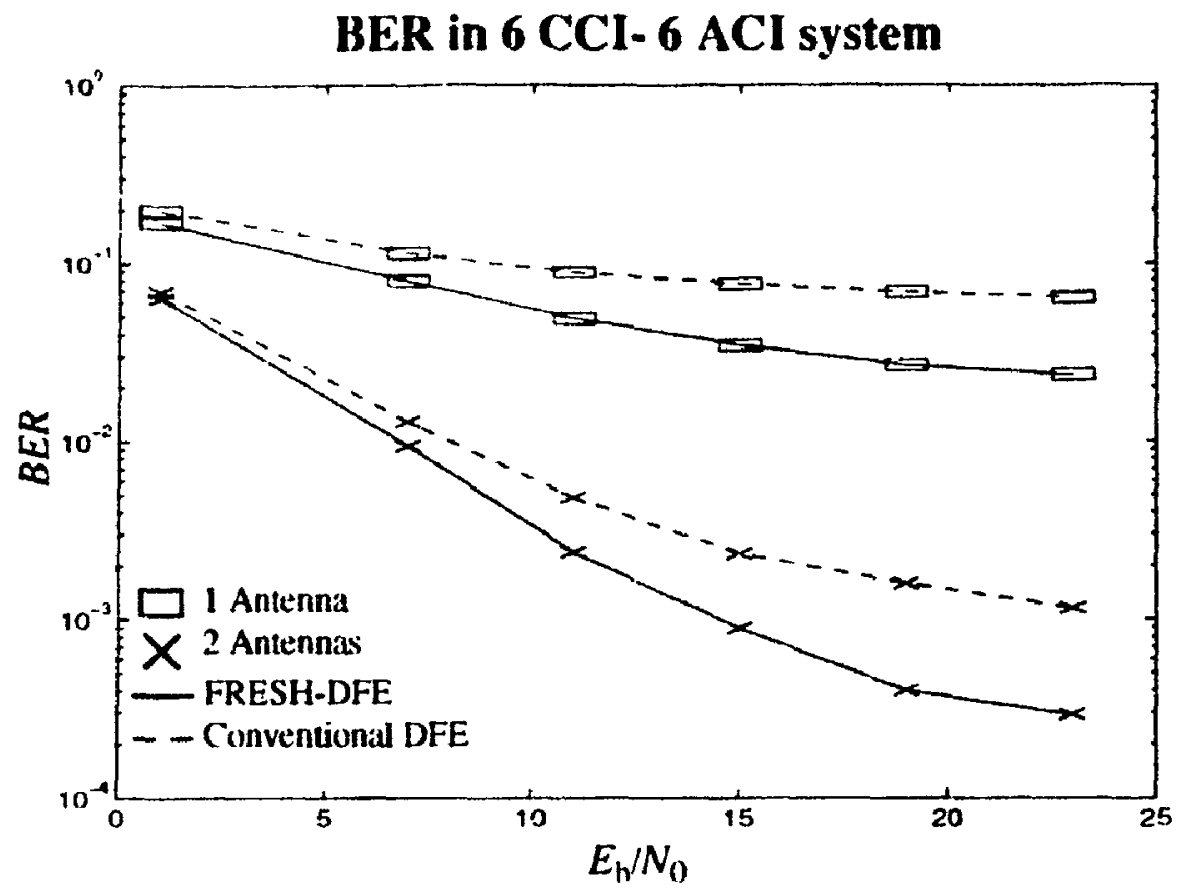

(a)

\section{BER improvement in 6 CCI- 6 ACI system}

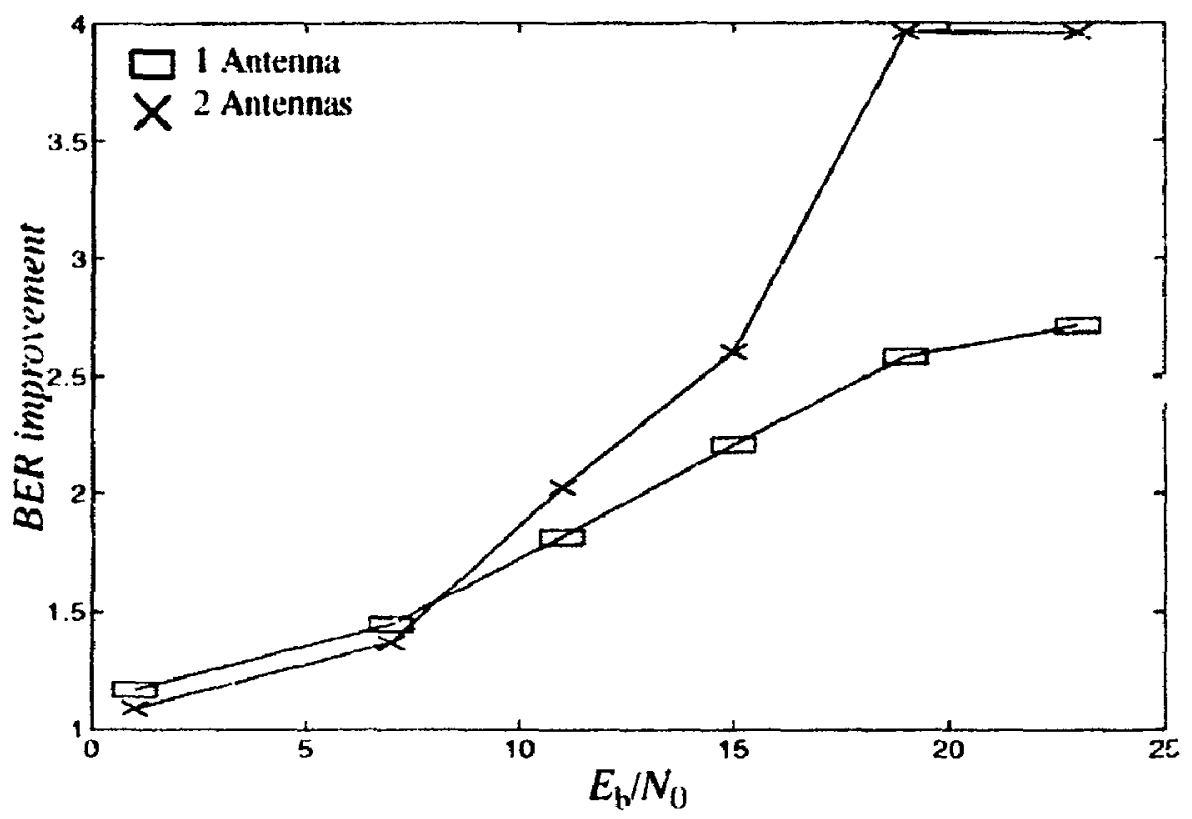

(b)

Figure 6.37 (a) The BER and (b) The SER improvement for multi-antenna receiver is $6 \mathrm{CCl} 6 \mathrm{ACI}$ BPSK system when channel spacing is half the symbil rate. 


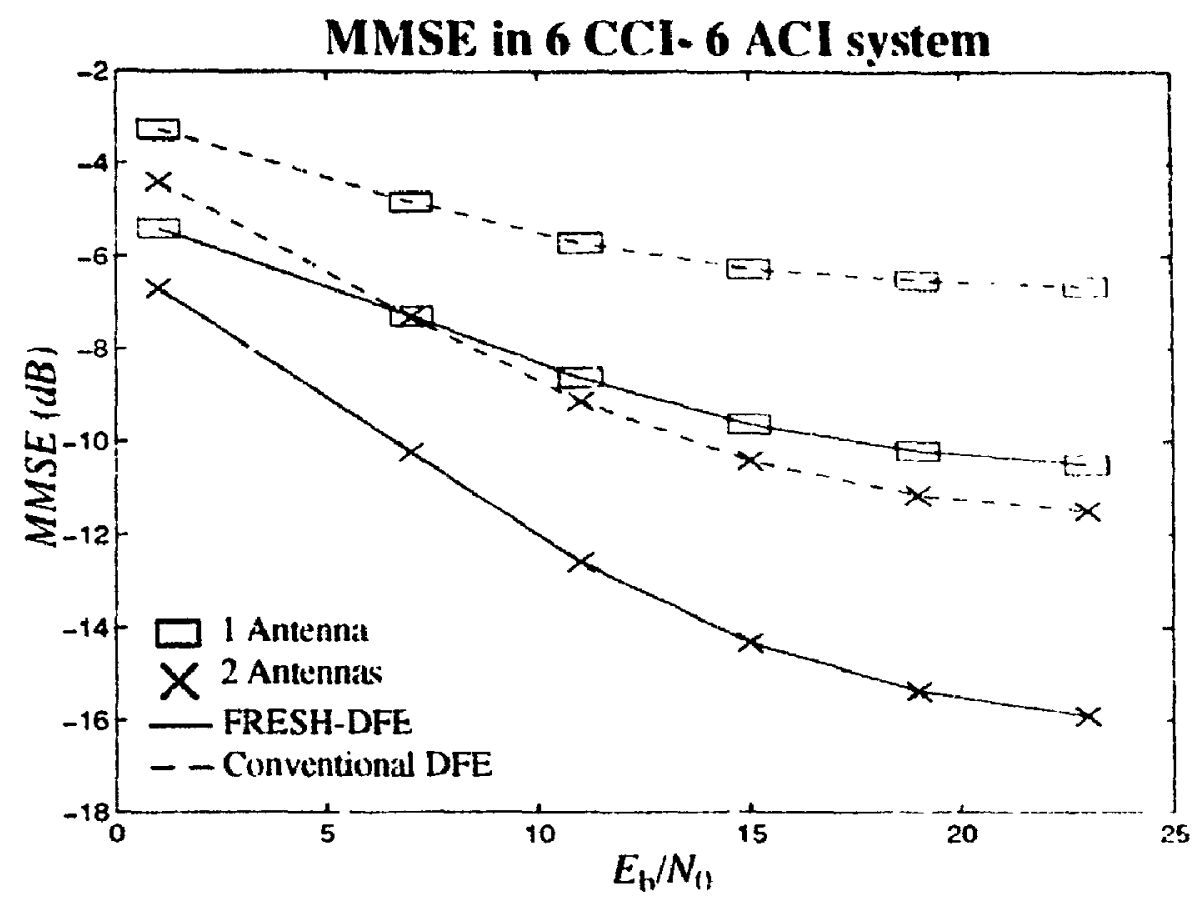

(a)

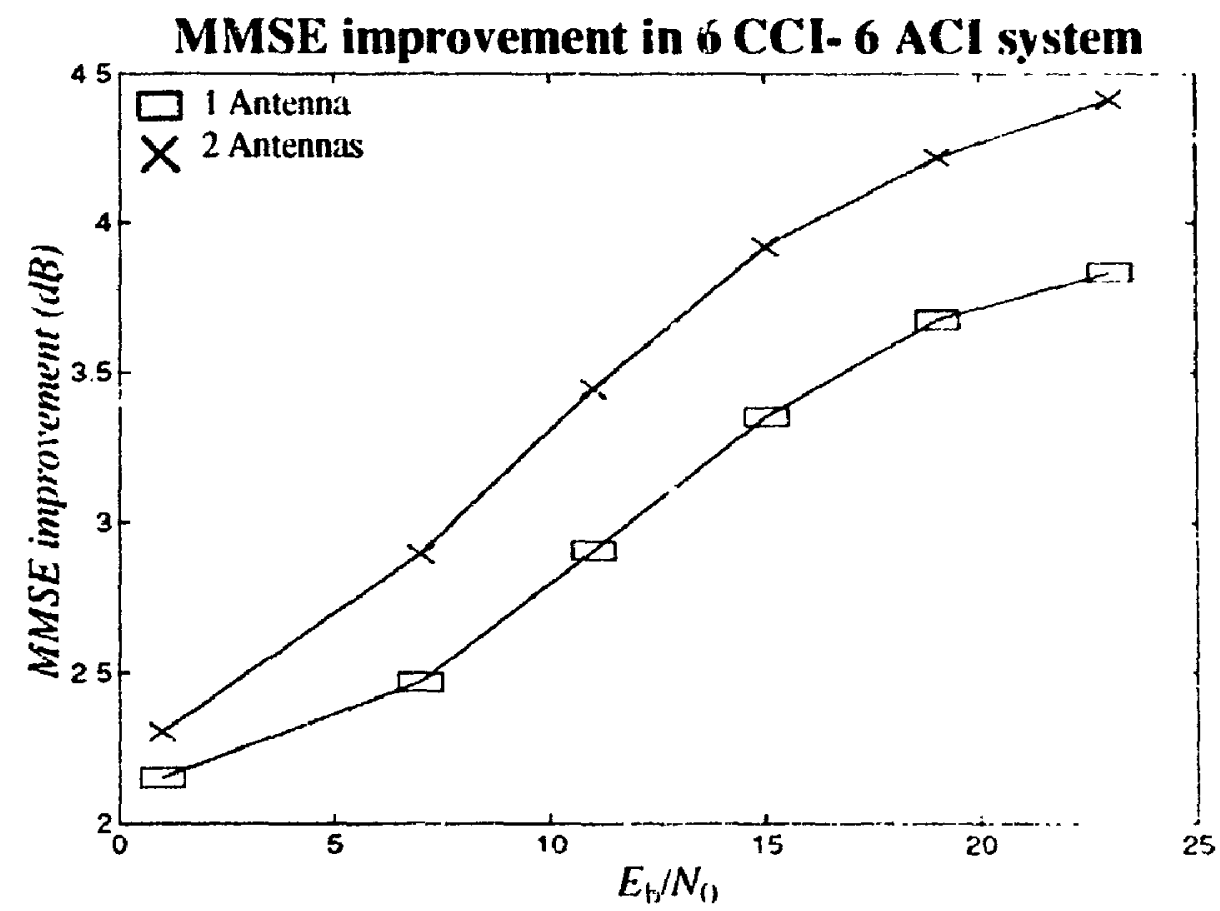

(b)

Figure 6.38 (a) The MMSE and (b) The MMSE improvement for multi-antenna receiver in $6 \mathrm{CCl}-6$ ACI BPSK system when channel spacing is half the symbol rate. 

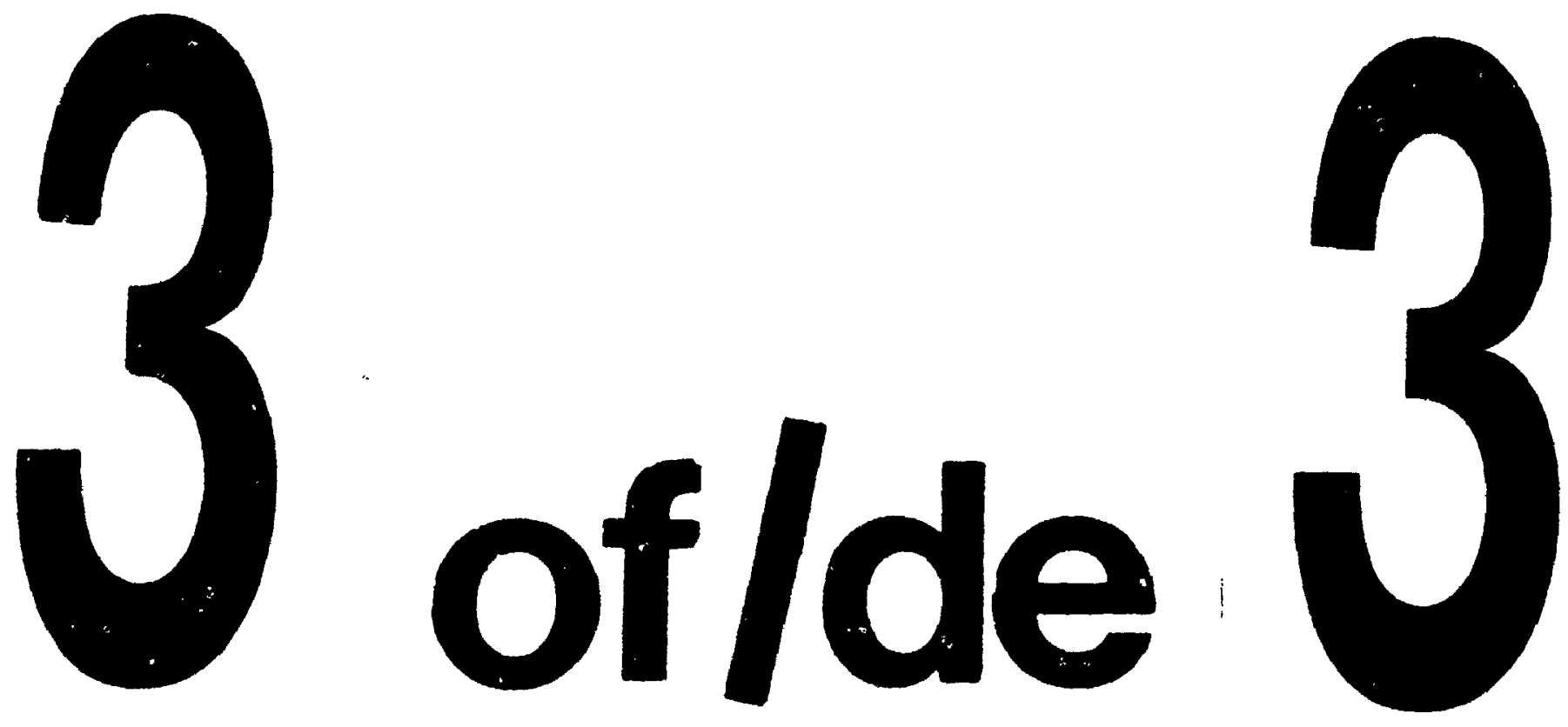

PM- $\left\{3 \frac{1}{2}\right.$ "X4" PHOTOGRAPHIC MICROCOPY TARGET NBS 1010a ANS1/ISO \#2 EOUIVALENT

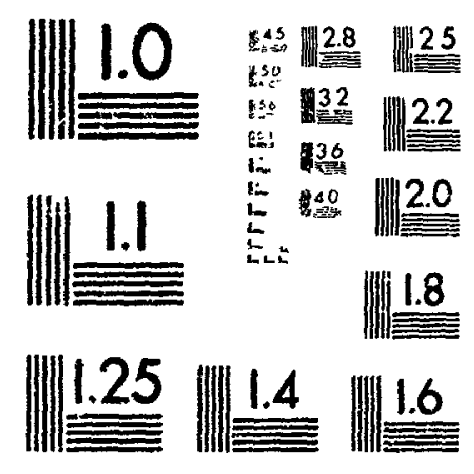

PAECISIONSM RESOLUTION TARGETS 


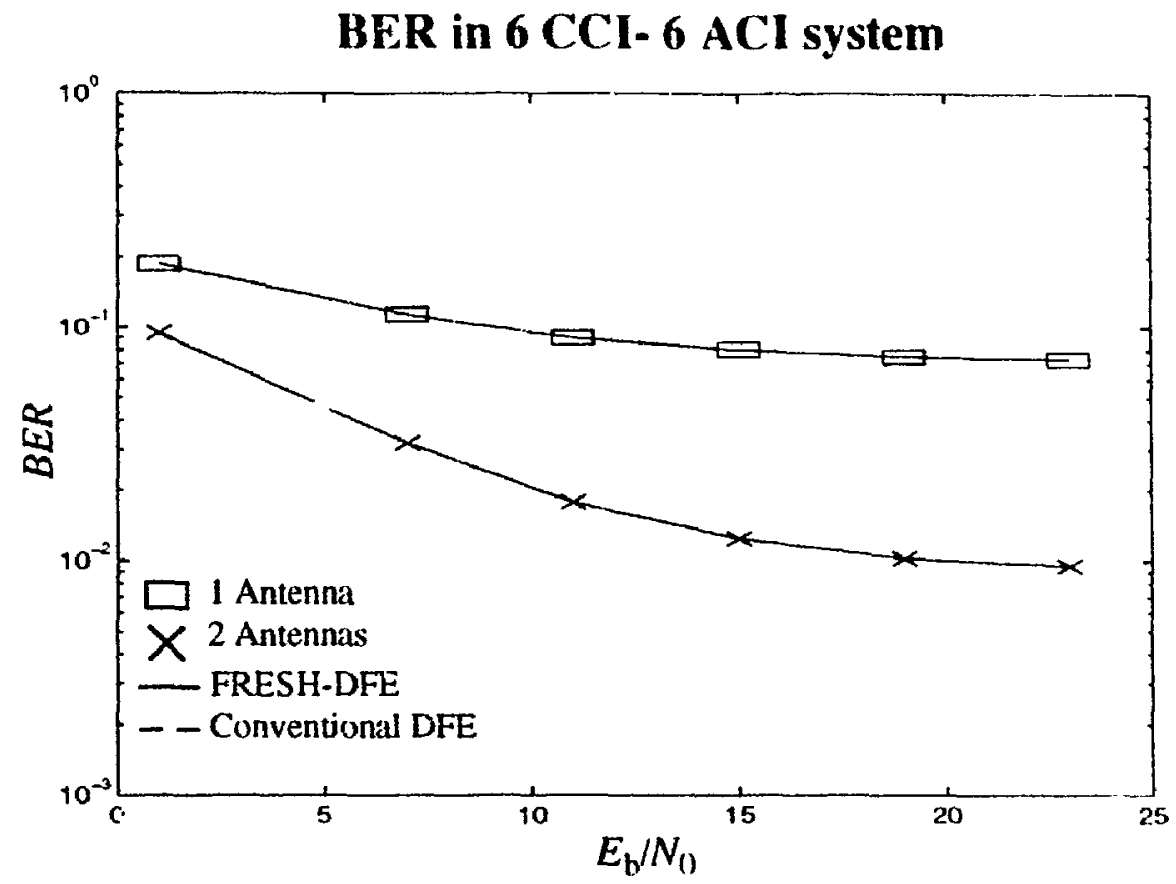

(a)

MMSE in $6 \mathrm{CCI}-6 \mathrm{ACI}$ system

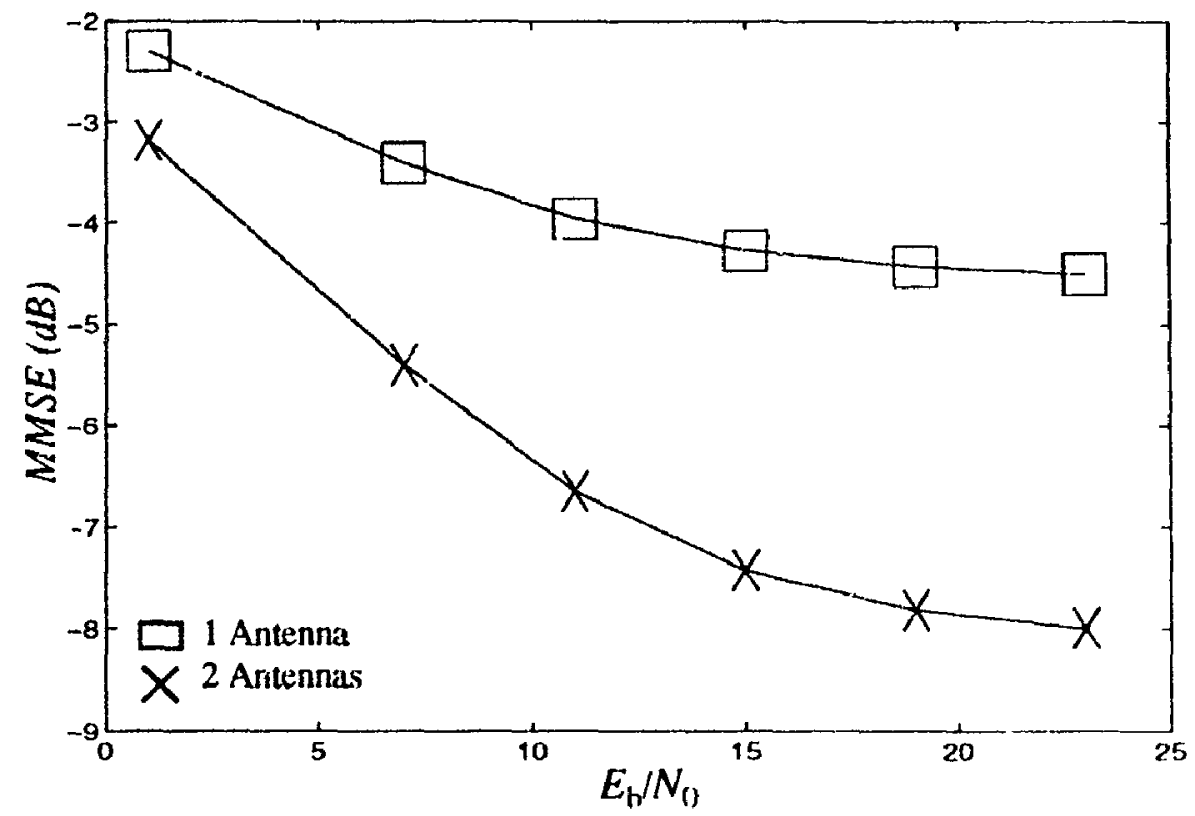

(b)

Figure 6.39 (a) The BER and (b) The MMSE for multi-antenna receiver in 6 CCI- 6 ACI QPSK system when channel spacing is half the symbol rate. 
second is $30(j$ percent. With these symbol durations and excess bandwidths, the signal bandwidths are the same. The channel spacing equal to $1 \mathrm{~Hz}$ is used. The structure of FRESH-DFE uses the following frequency shifts for this system :

$\{0,0) .5\} \mathrm{Hz}$ in Lirear path and $\{0,0.5\} \mathrm{Hz}$ in Conjugate path for BPSK.

$\{0\} \mathrm{Hz}$ in Linear path and $\{0,0.5\} \mathrm{Hz}$ in Conjugate path for OQPSK.

$\{0,0.5\} \mathrm{Hz}$ in Linear path for QPSK.

Figure 6.40 to Figure 6.45 show the simulation results for this system. Although the FRESH-DFE performance is shown to be better than the conventional DFE (Figure 6.40), the amount of improvement in BER is not significant. The reason is that the total interference at cyclic frequency equal of zero $(\alpha=0$ and $\beta=0)$ is reduced comparing with singlesymbol rate system.

If $T_{0}$ equals 1 second and $T_{1}$ is 2 seconds, the structure of FRESH-DFE is reduced and there is no need for $0.5 \mathrm{~Hz}$ frequency shifts. However, the increased total interference at $\alpha=()$ and $\beta=0$ yields worse performance compared with the case when time $T_{0}=1$ and $T_{1}=2$. Figure 6.46 to Figure 6.50 show the simulation results for two cases (1) $T_{0}=2$ and $T_{1}=1$, (2) $T_{0}=1$ and $T_{1}=2$. The results indicate that both conventional DFE and FRESHDFE deliver better performance when the SOI symbol rate is twice SNOI symbol rate. This improvement in BER is greater when two antennas branch receiver is used.

\subsection{Receivers with Limited Complexity}

In all above simulations, it is assumed that there is no concem about the complexity of the receiver. However, in real world the compleyity of the receiver is a main consideration. In equalizers, the number of taps determines complexity. It is proposed to use half the available number of taps in linear path and the other half in conjugate path for BPSK and $\mathrm{OPPSK}$. Figure 6.51 and Figure 6.52 show simulation results for 1-CCI-2 ACI system whe 19 number of available taps is limited. In this simulation, the conventional DFE uses 32 taps (which is equivalent to 8 symbols) for forward filter and 3 taps for feedback filter while ODFE uses 16 taps (4 symbols) in Linear path ( $\alpha=0), 16$ taps in Conjugate path $(\beta=0)$ and 3 taps in feed back filter. Therefore, the complexity of conventional DFE and ODFE are the same. As it is clear from the figures, the ODFE performance is still superior to the conventional DFE performance. 


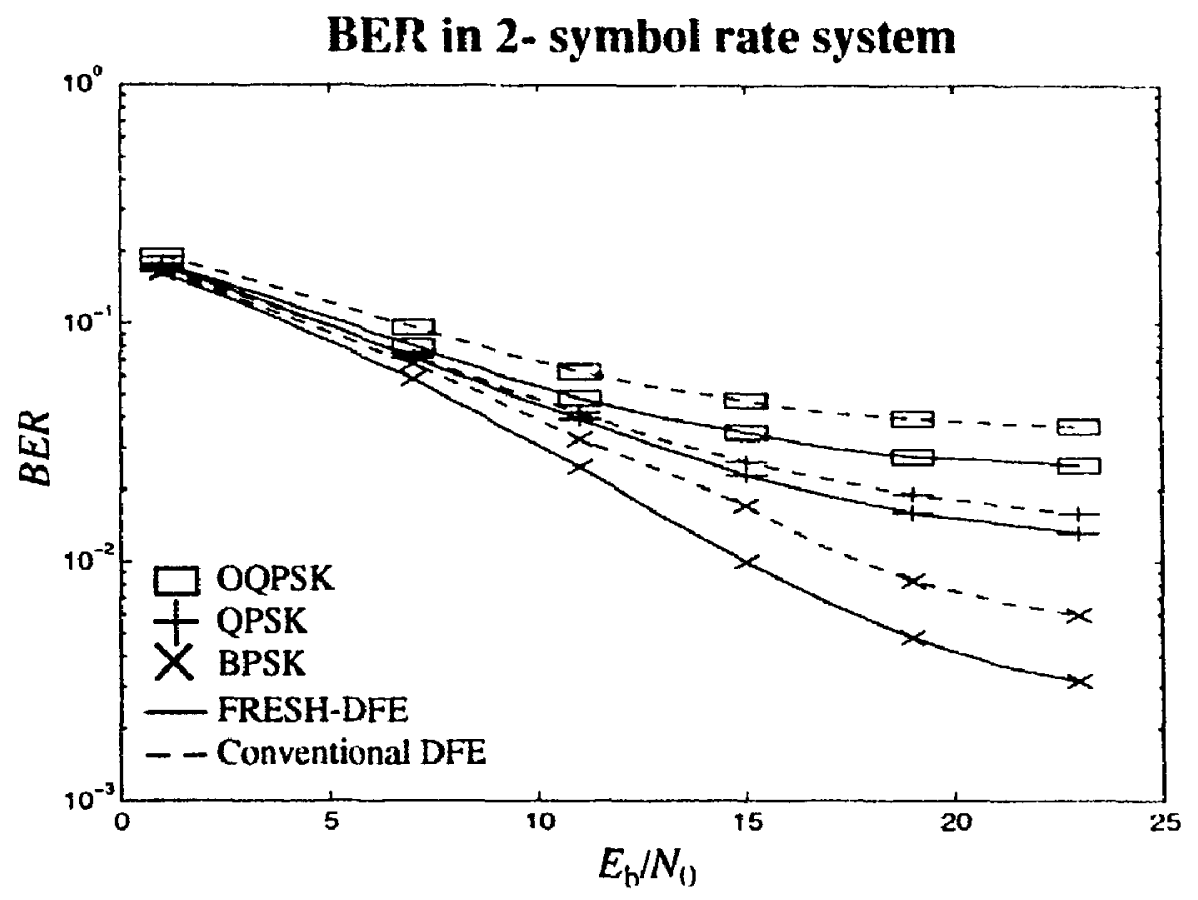

(a)

\section{BER improvement in 2- symbol rate system}

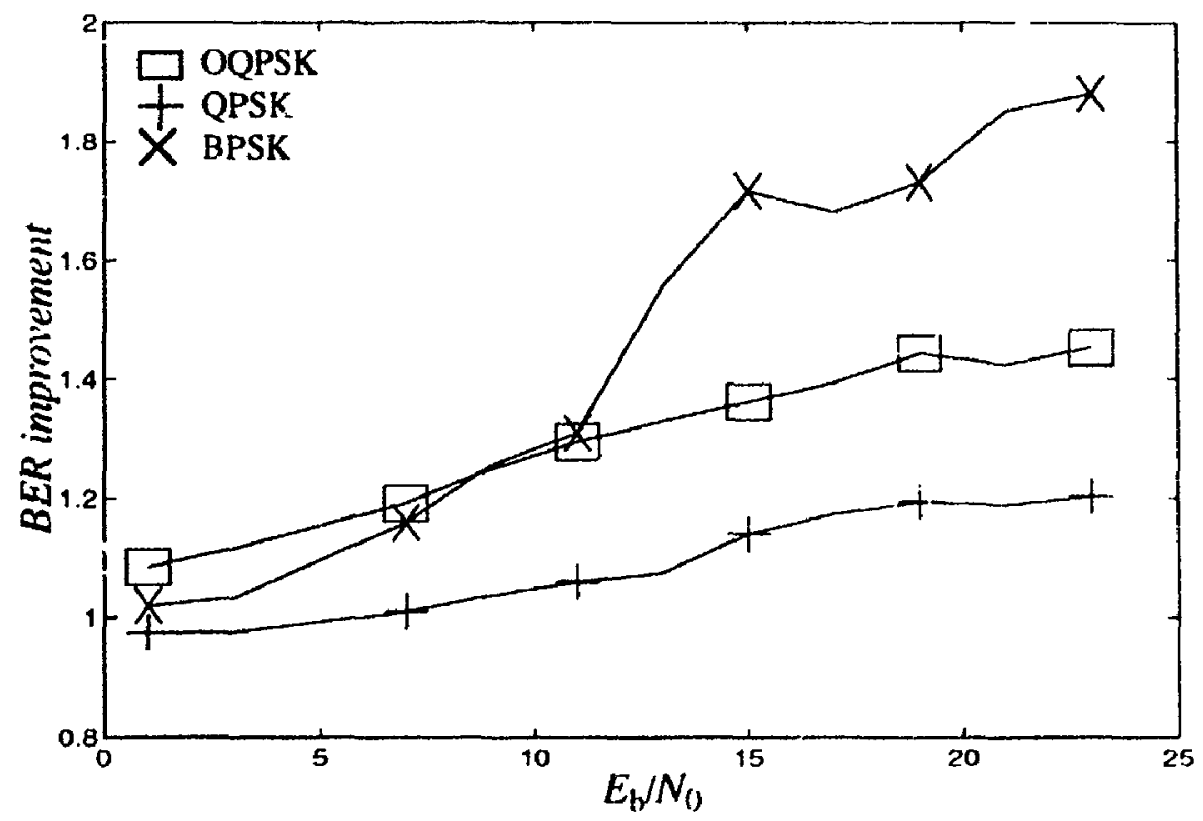

(b)

Figure 6.40 (a) The BEP and (b) The BER improvement for 2-symbol rate systom. 


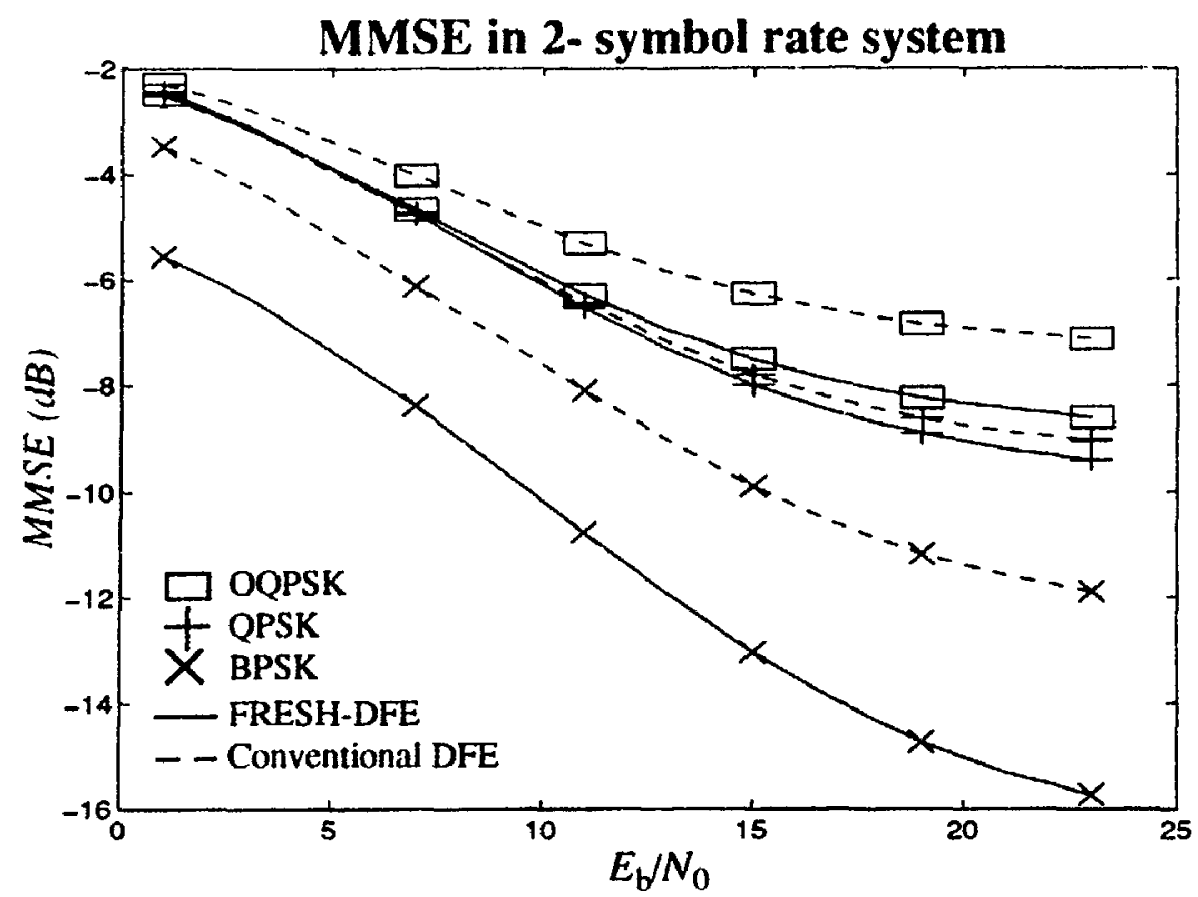

(a)

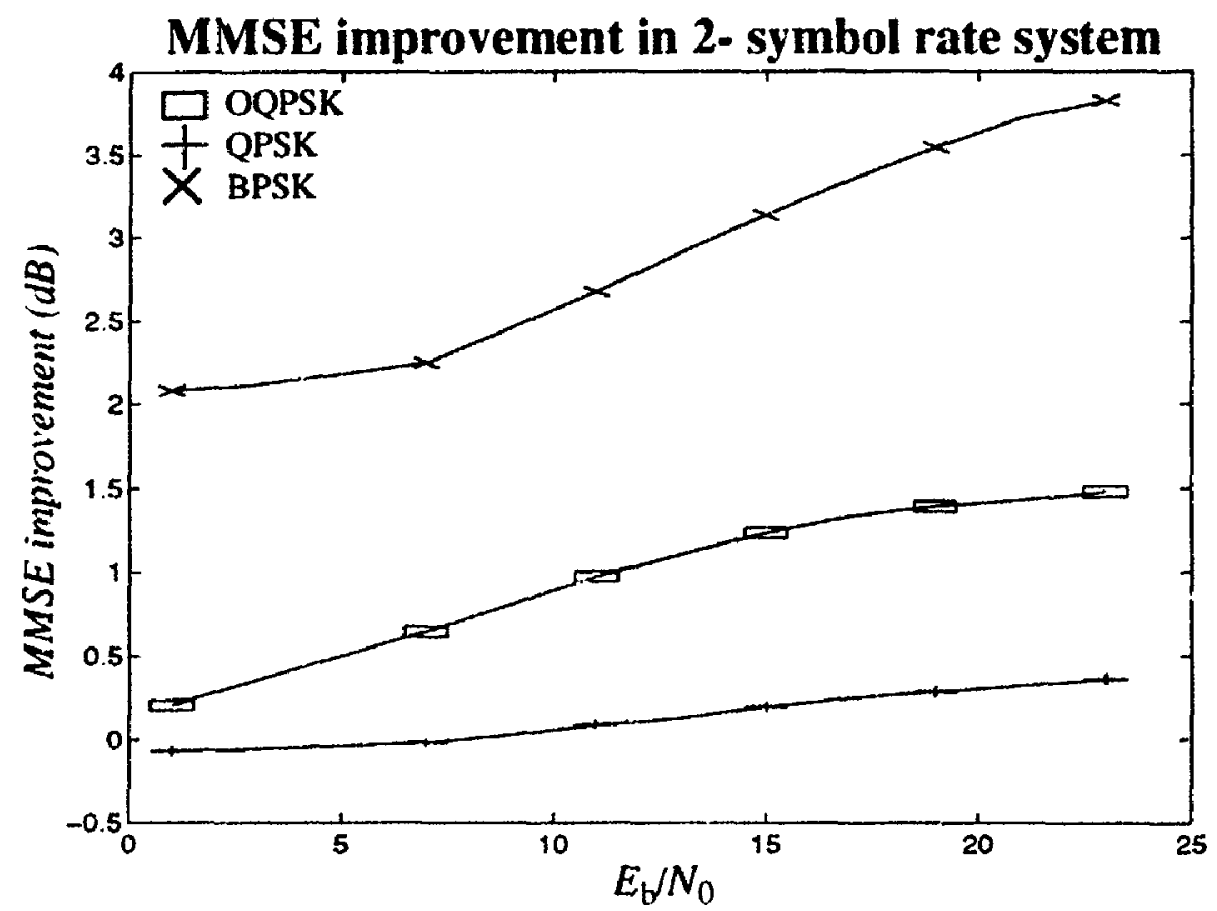

(b)

Figure 6.41 (a) The MMSE and (b) The MMSE improvement for 2-symbol rate system. 


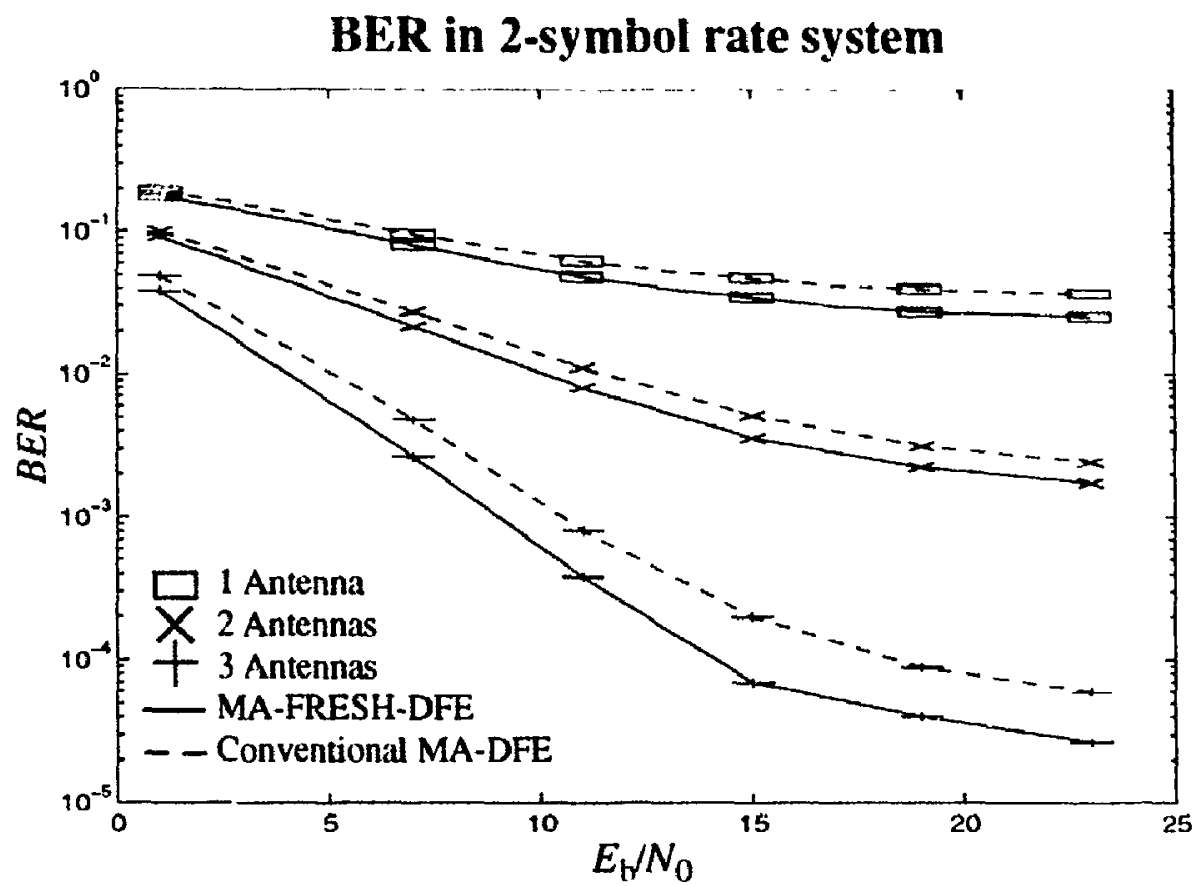

(a)

BER improvement in 2- symbol rate system

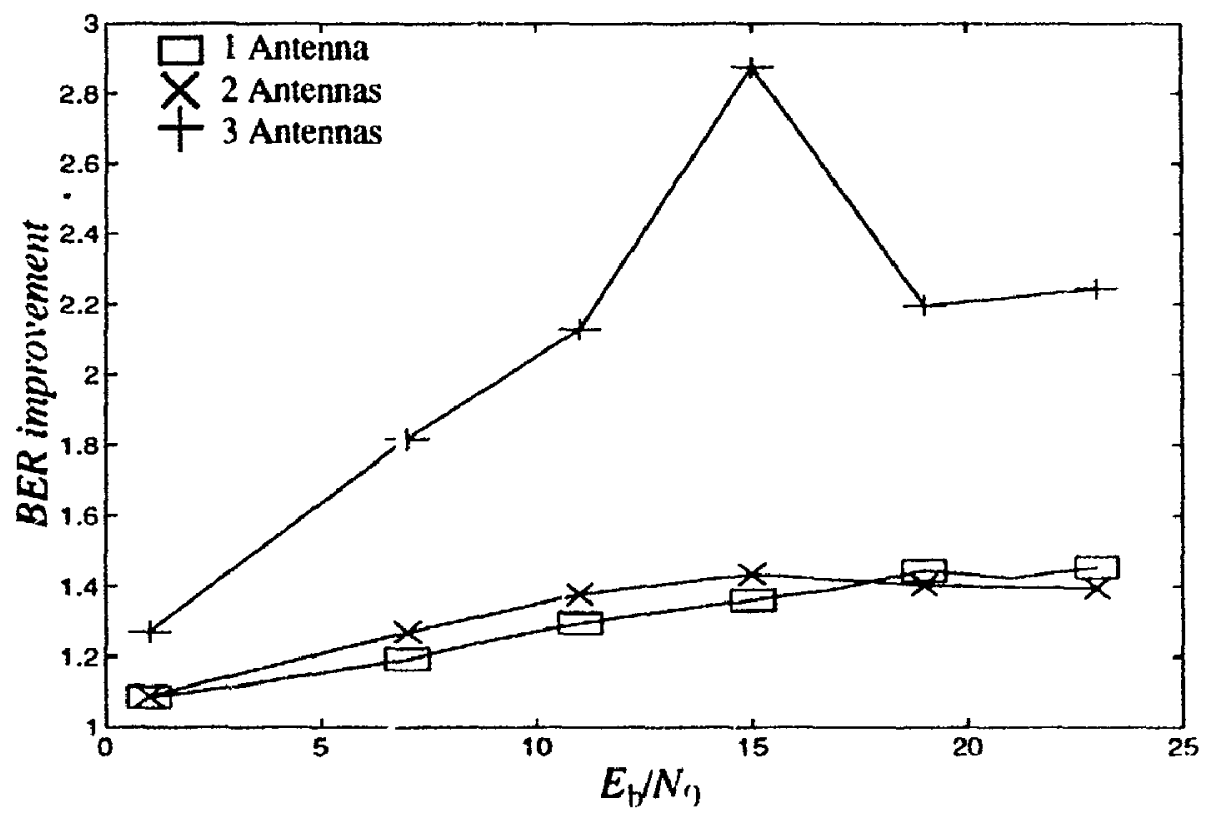

(b)

Figure 6.42 (a) The BER and (b) The BER improvement for 2-8ymbol rate OQPSK system. 


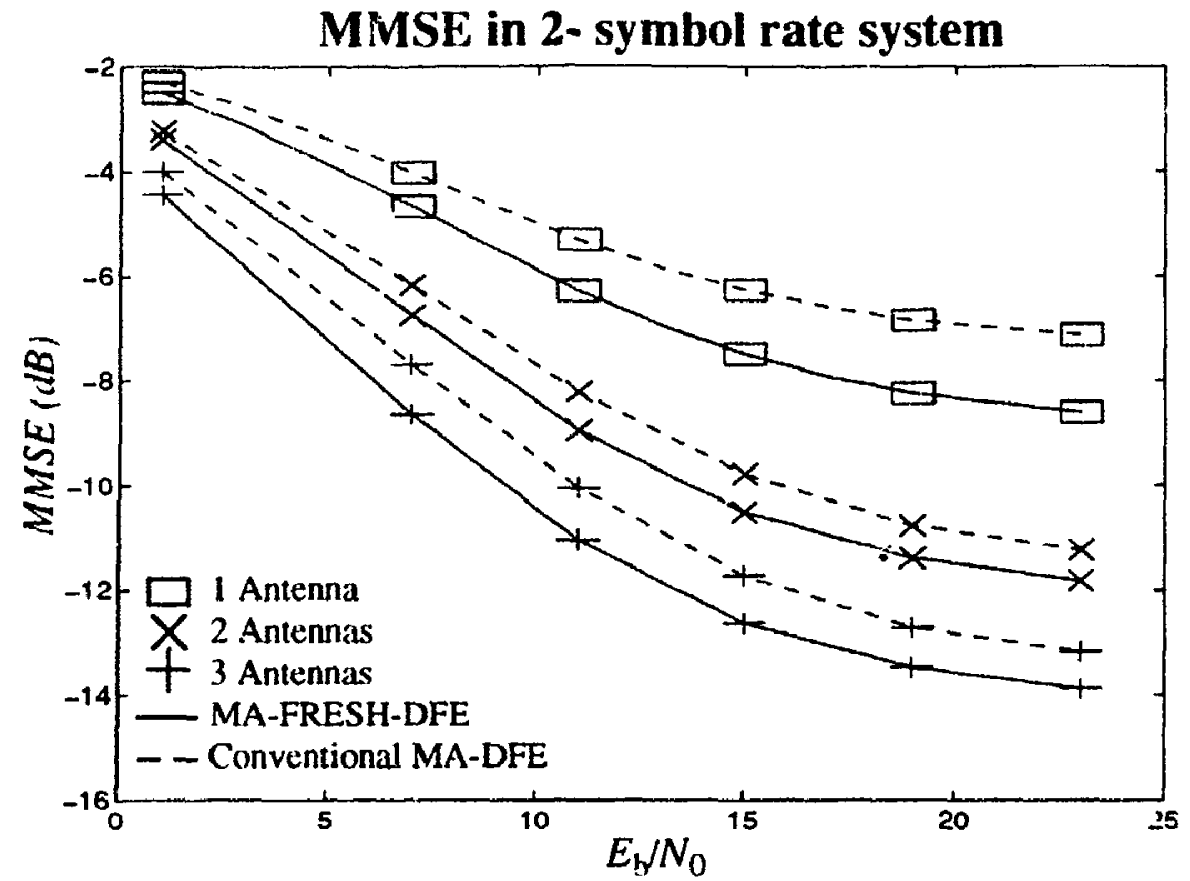

(a)

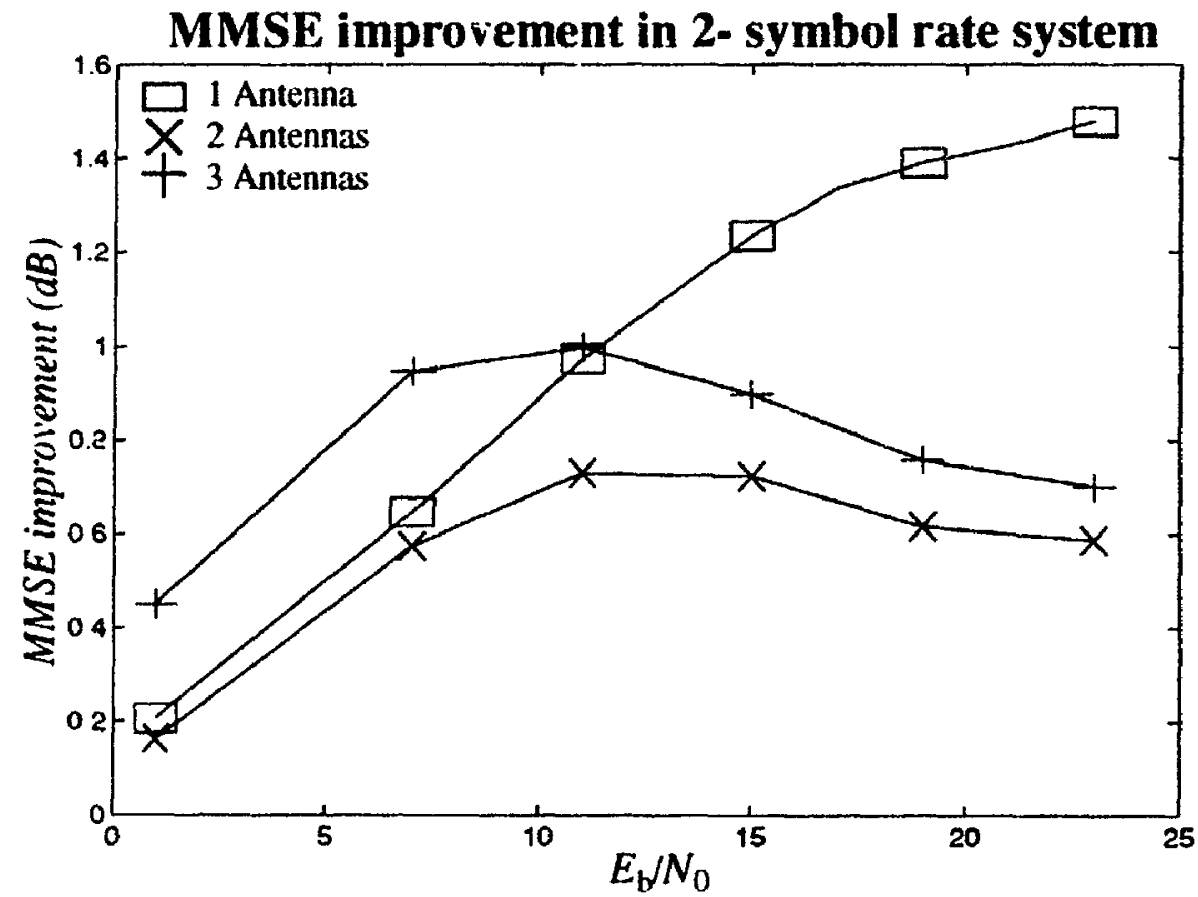

(b)

Figure 6.43 (a) The MMSE and (b) The MMSE improvement for 2-symbol rate OQPSK system. 


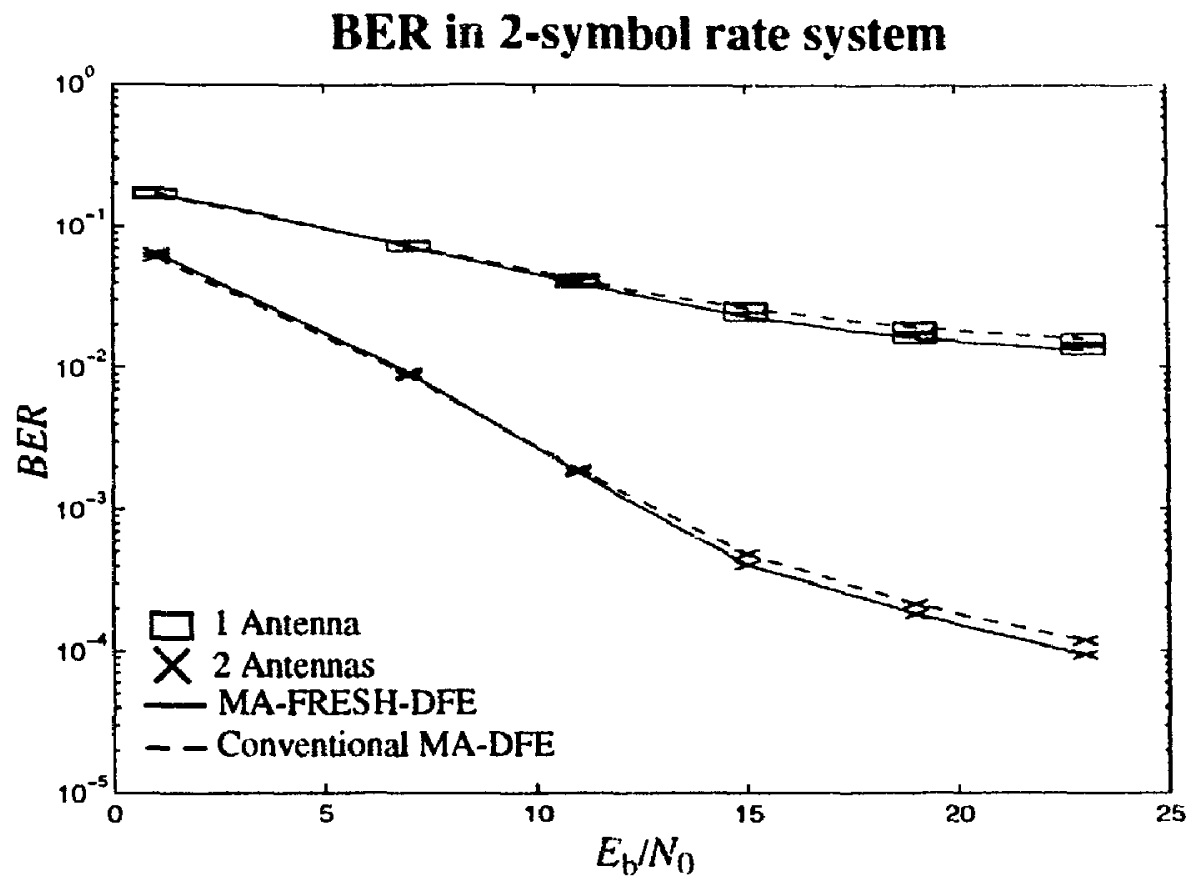

(a)

\section{BER improvement in 2- symbol rate system}

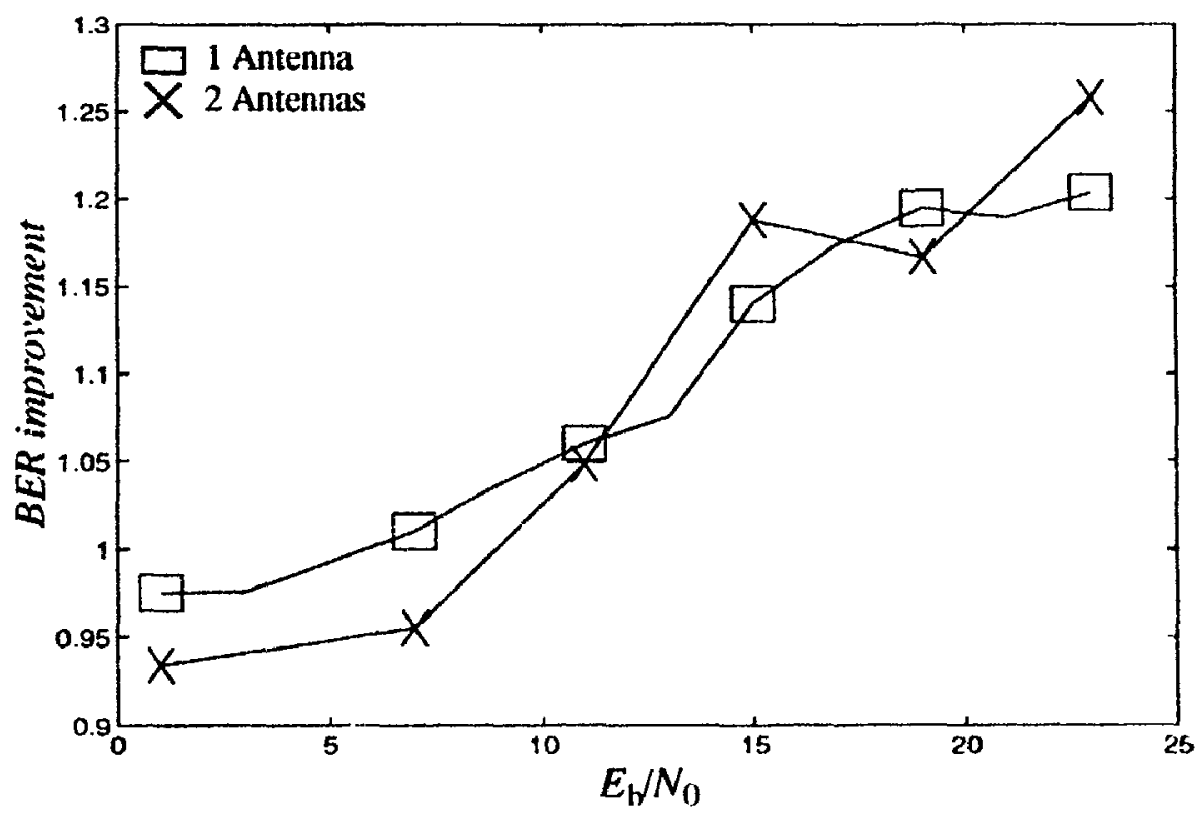

(b)

Figure 6.44 (a) The BER and (b) The BER improvement for 2-symbol rate OPSK system. 


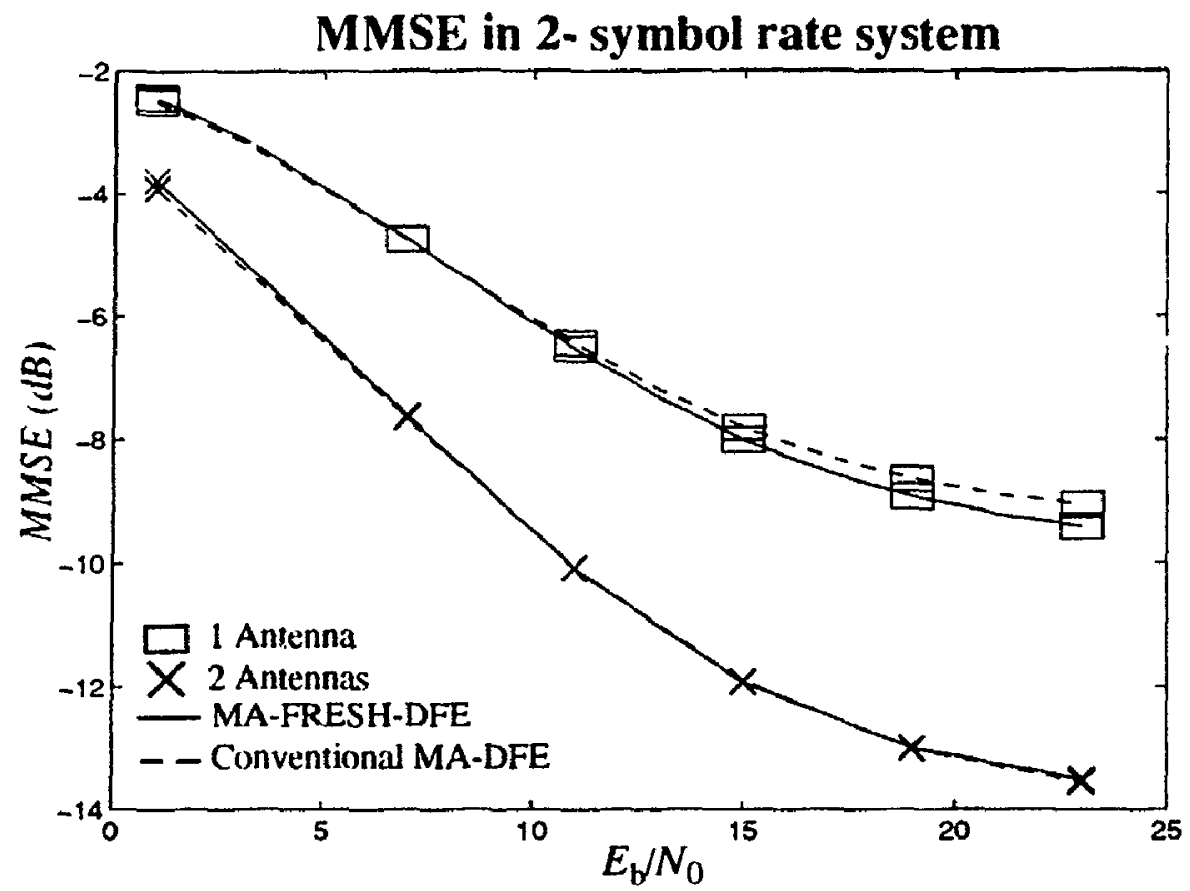

(a)

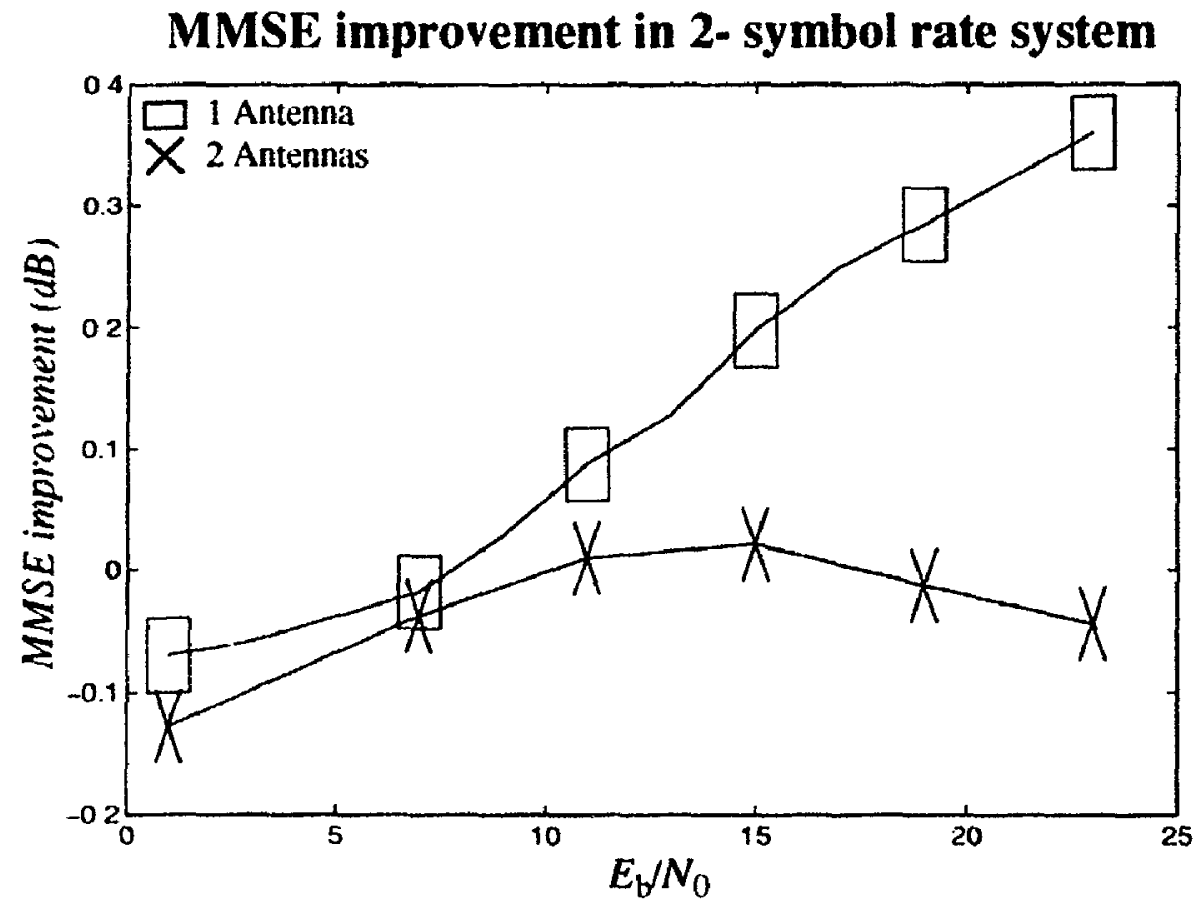

(b)

Figure 6.45 (a) The MMSE and (b) The MMSE improvement for 2-symbol rate QPSK system. 


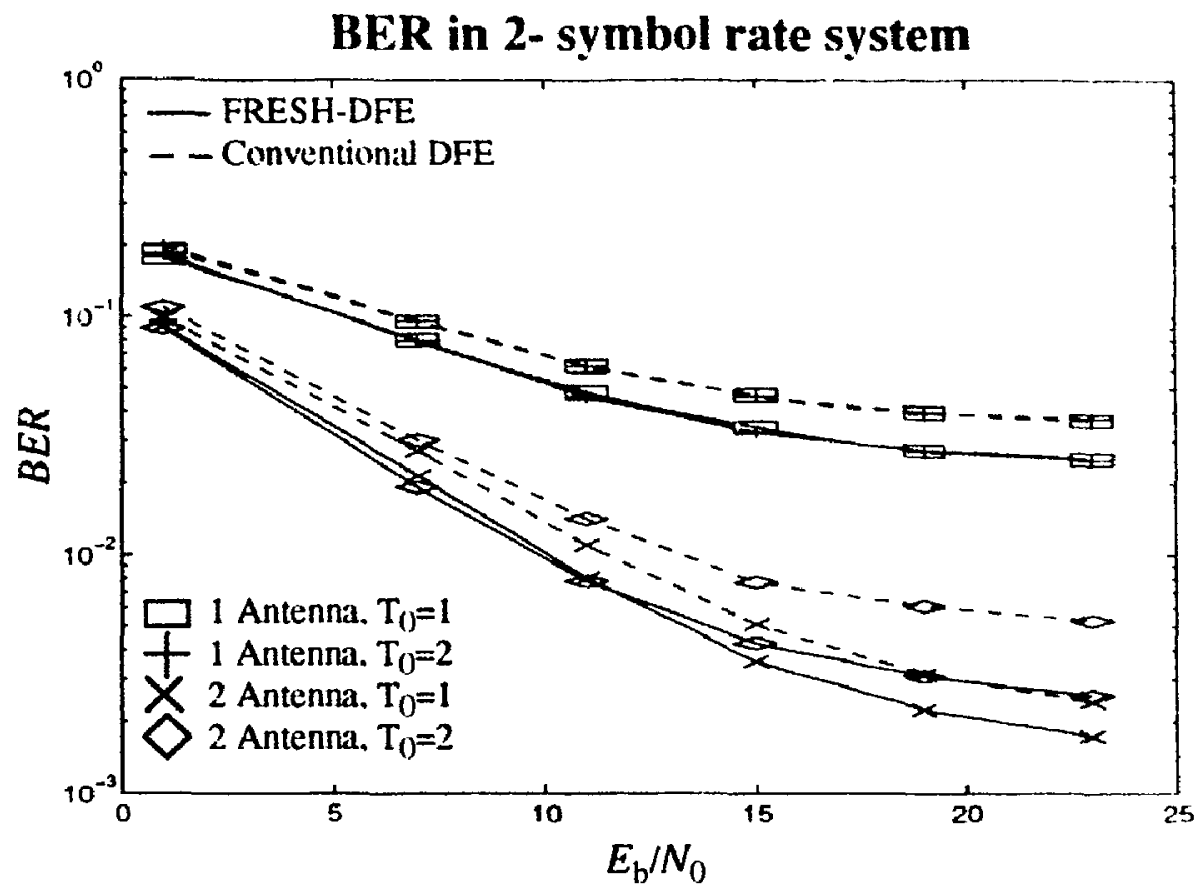

(a)

\section{BER improvement in 2-symbol rate system}

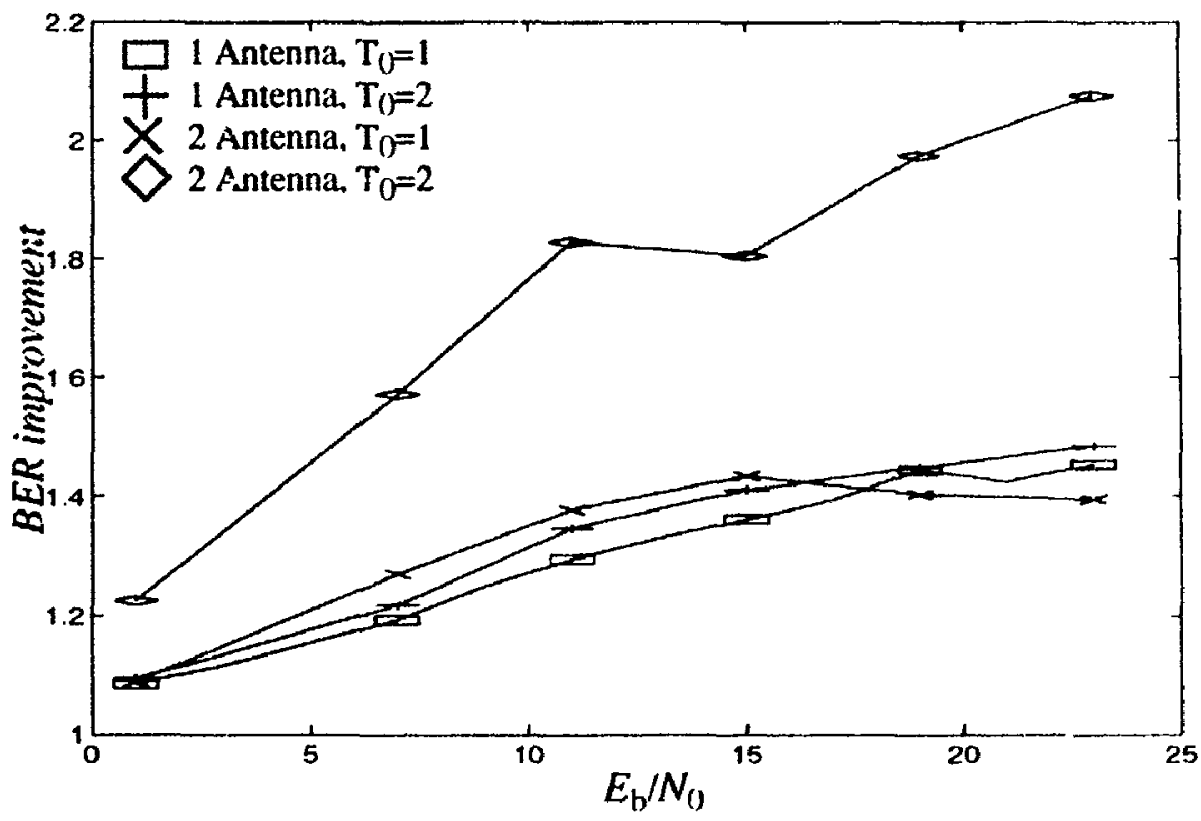

(b)

Figure 6.46 (a) The BER and (b) The BER improvement for 2-symbol rate system and when $T_{(j)}=1$ and 2 seconds. 


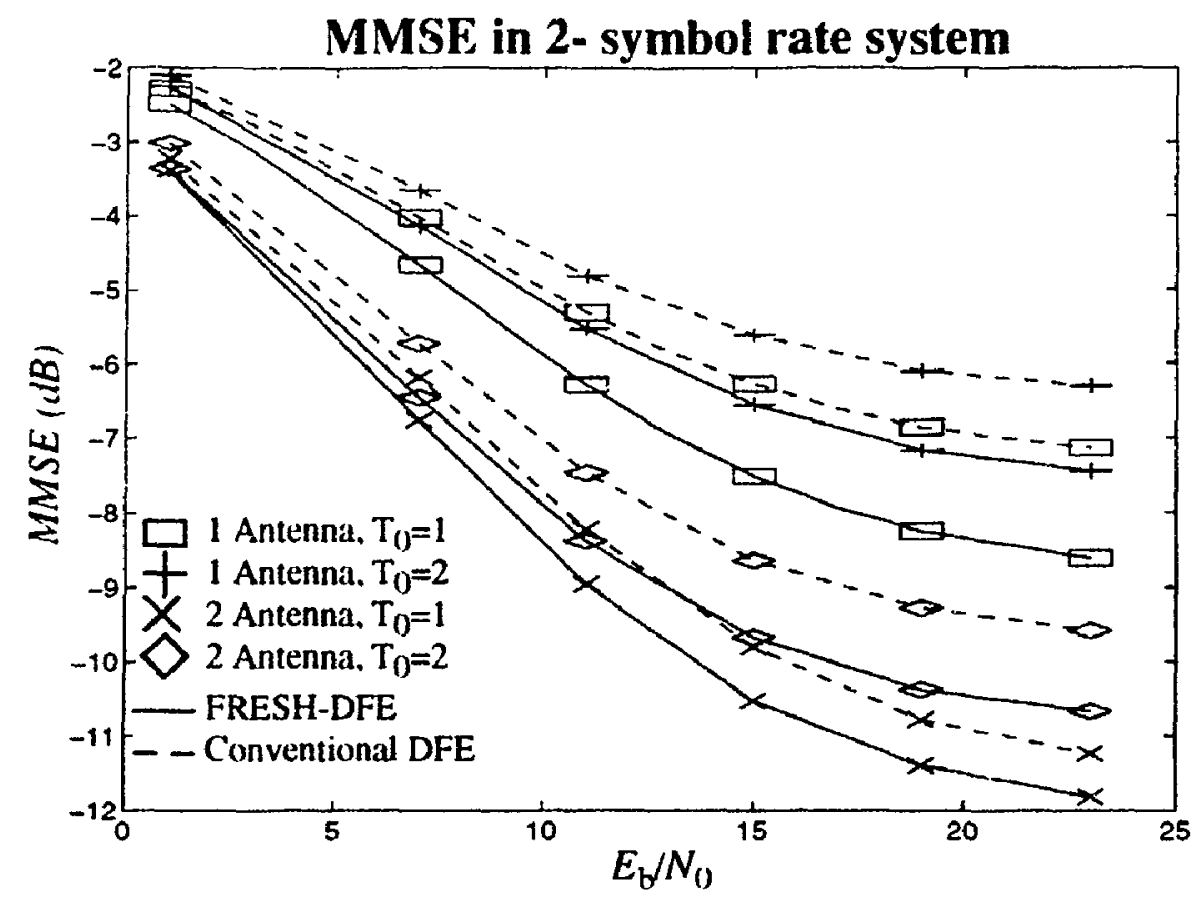

(a)

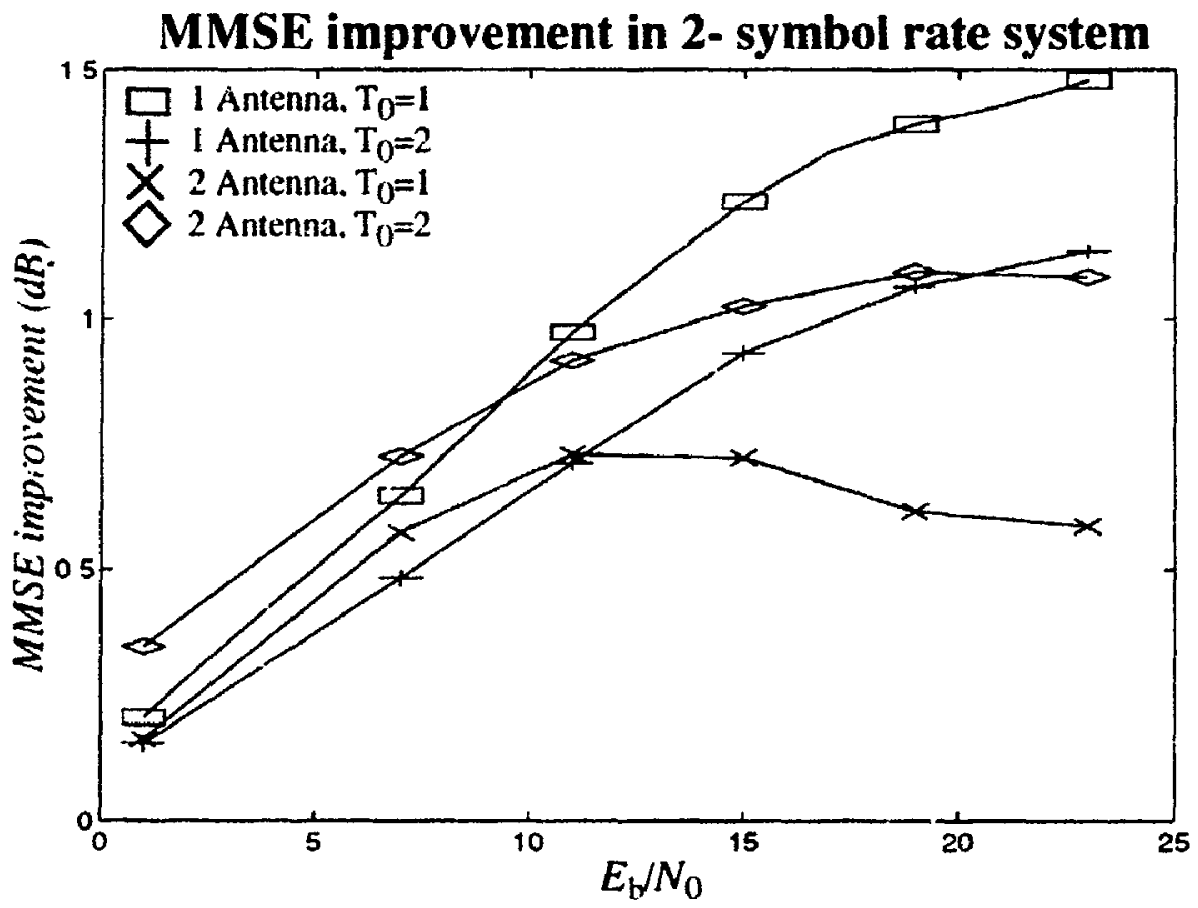

(b)

Figure 6.47 (a) The MMSE and (b) The MMSE improvement for 2-symbol rate system when $T_{0}=1$ and 2 seconds. 
BER in 2-symbol rate system

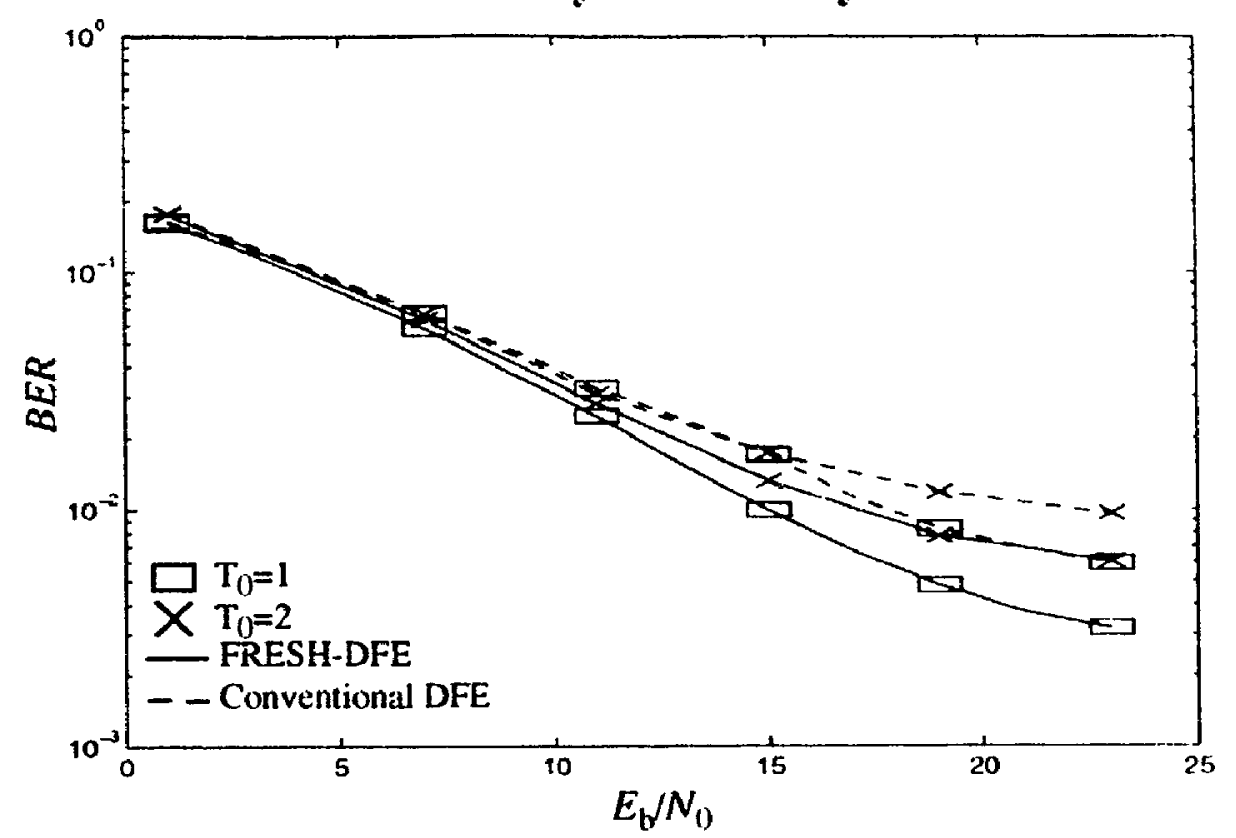

(a)

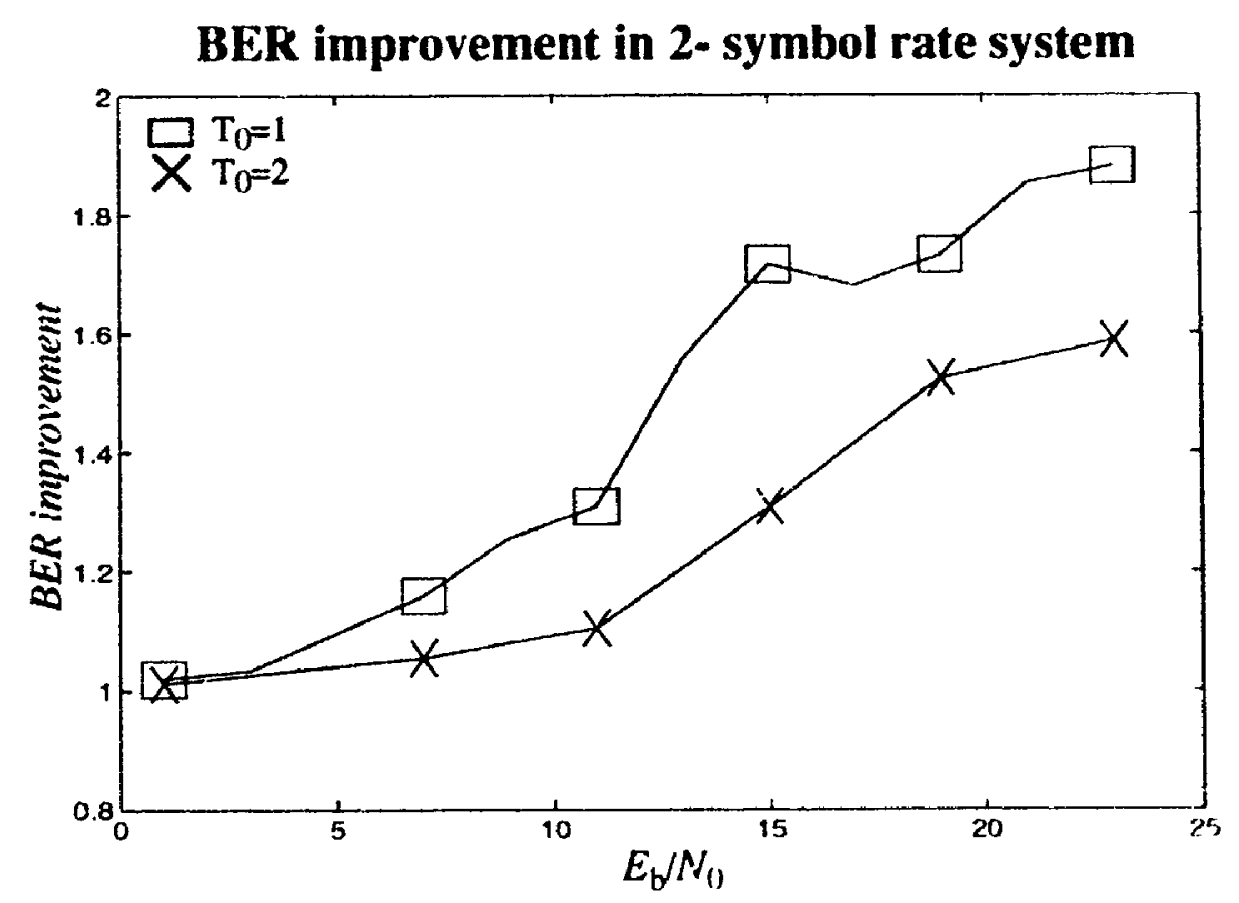

(b)

Figure 6.48 (a) The BER and (b) The BER improvement for 2-symbol rate BPSK system when $T_{1}=1$ and 2 seconds. 


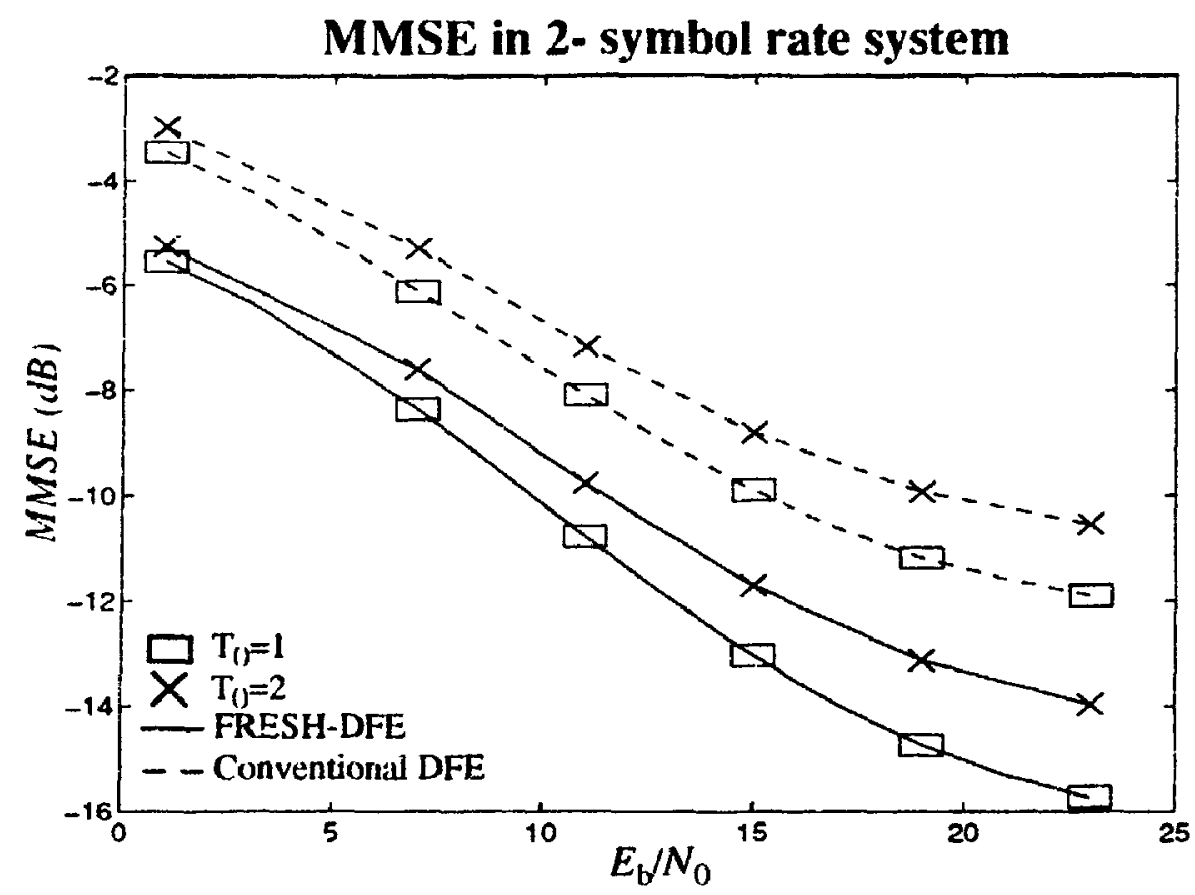

(a)

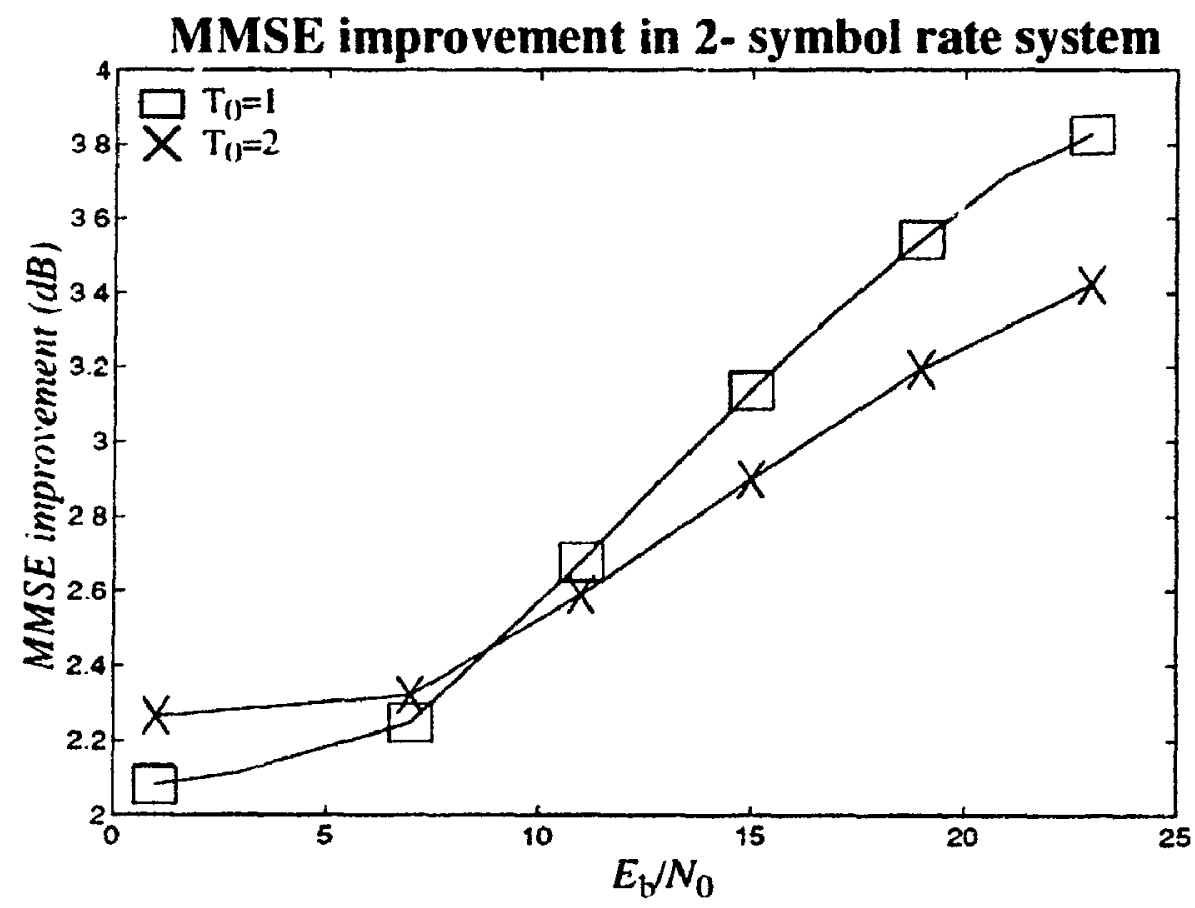

(b)

Figure 6.49 (a) The MMSE and (b) The MMSE improvement for 2-symbol rate BPSK system when $T_{0}=1$ and 2 seconds. 


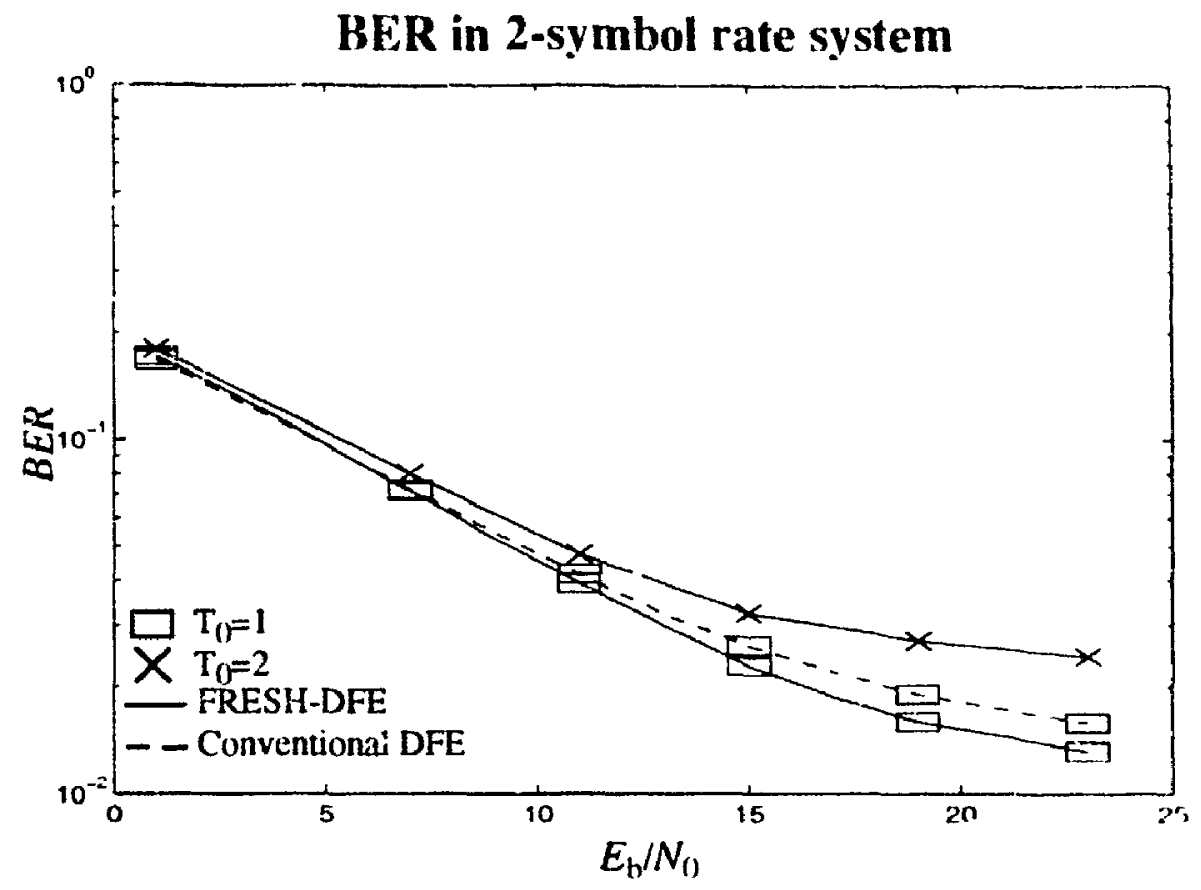

(a)

MMSE in 2- symbol rate system

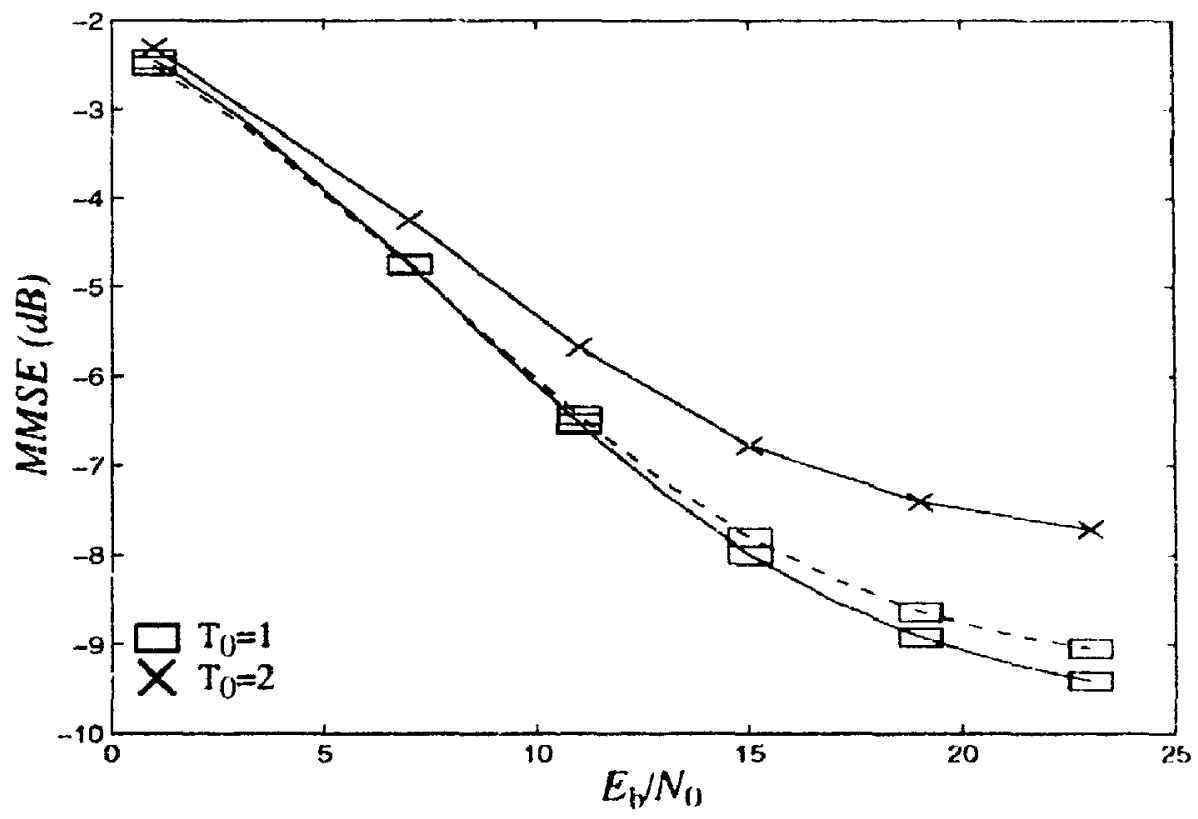

(b)

Figure 6.50 (a) The BER and (b) The BEA improvement for 2-symbol rate QPSK system when $T_{1}=1$ and 2 seconds. 


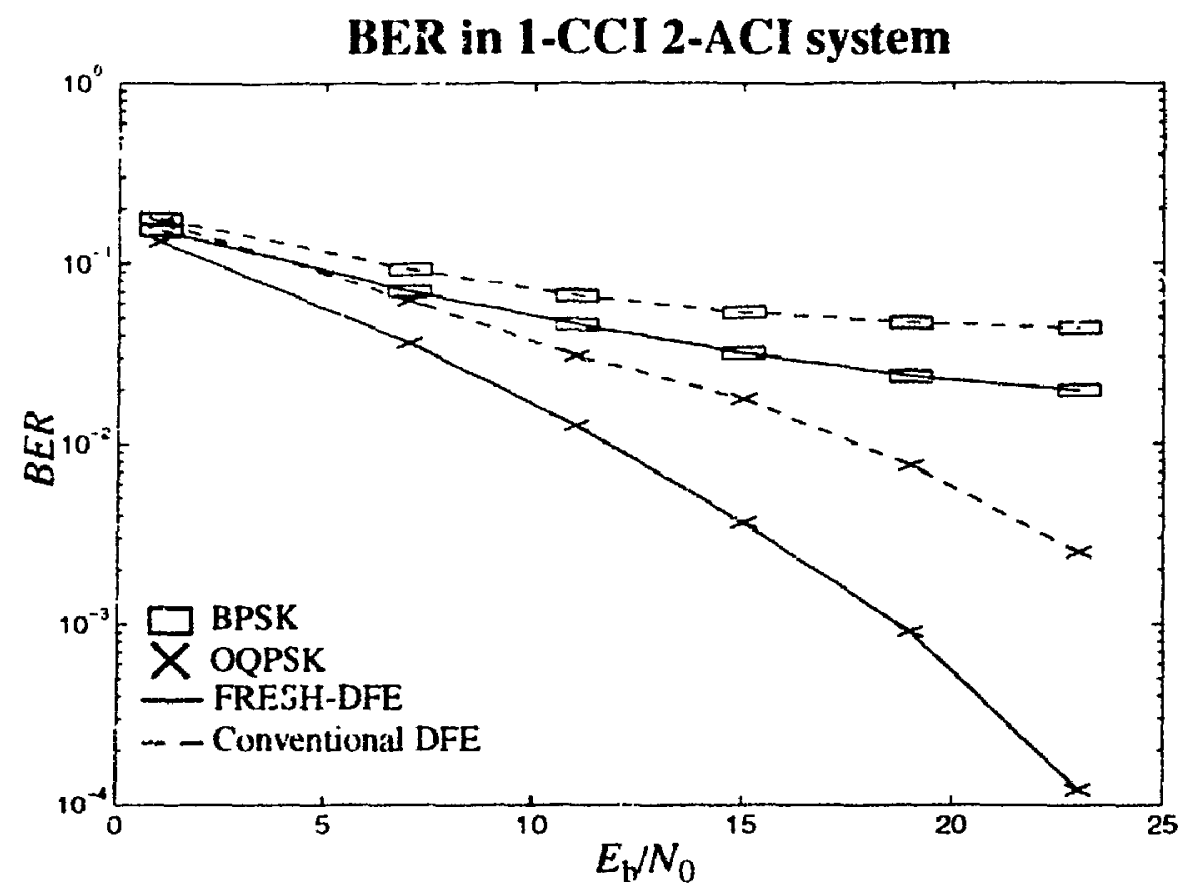

(a)

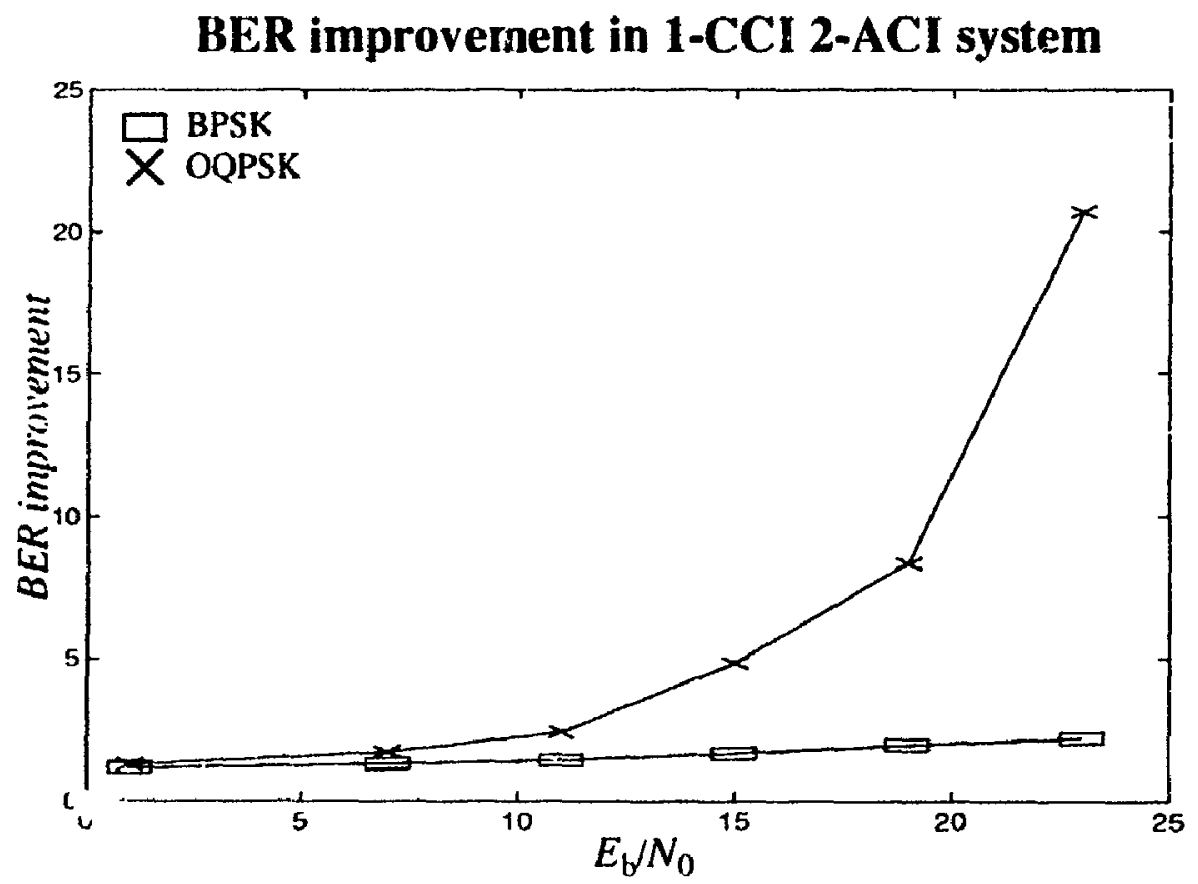

(b)

Figure 6.51 (a) The BER and (b) The BER improvement for $1 \mathrm{CCl}-2 \mathrm{ACl}$ system when the complexity of DFE and ODFE are the same. 


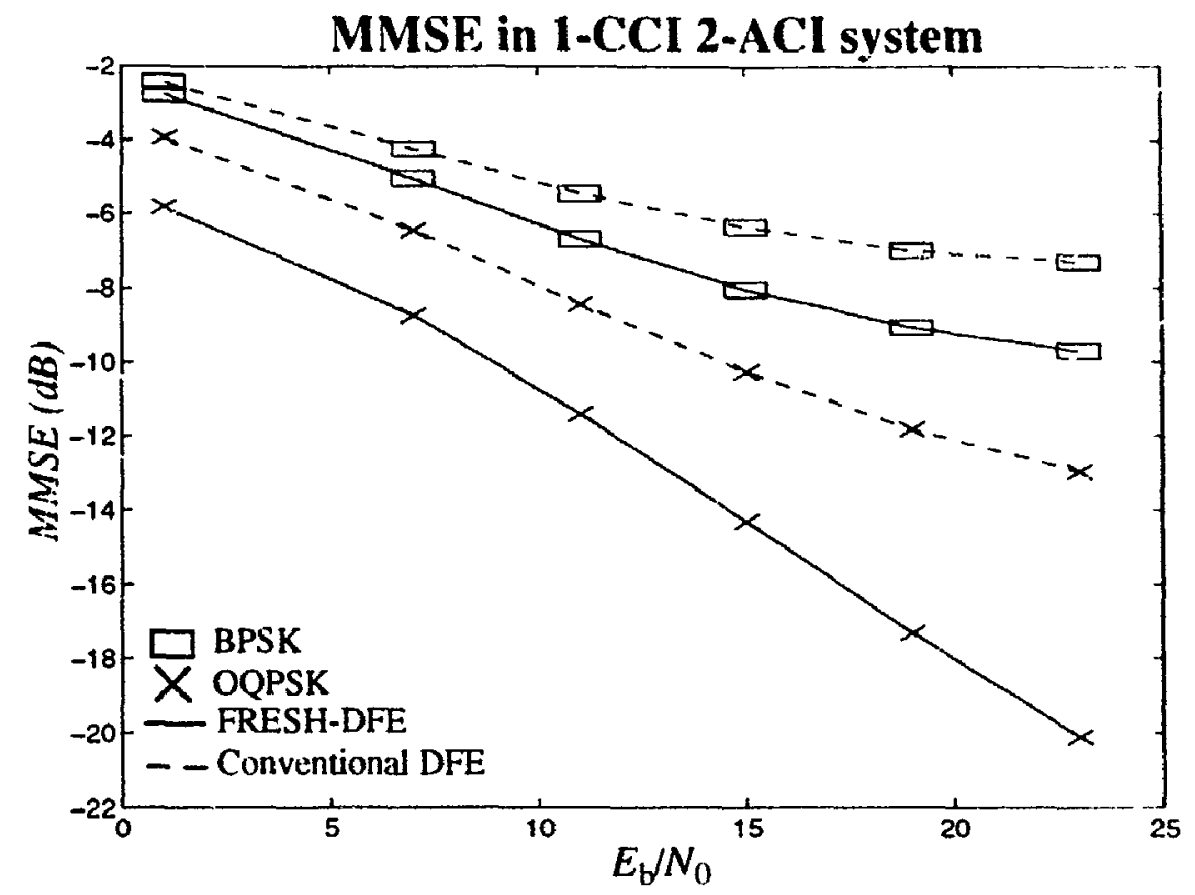

(a)

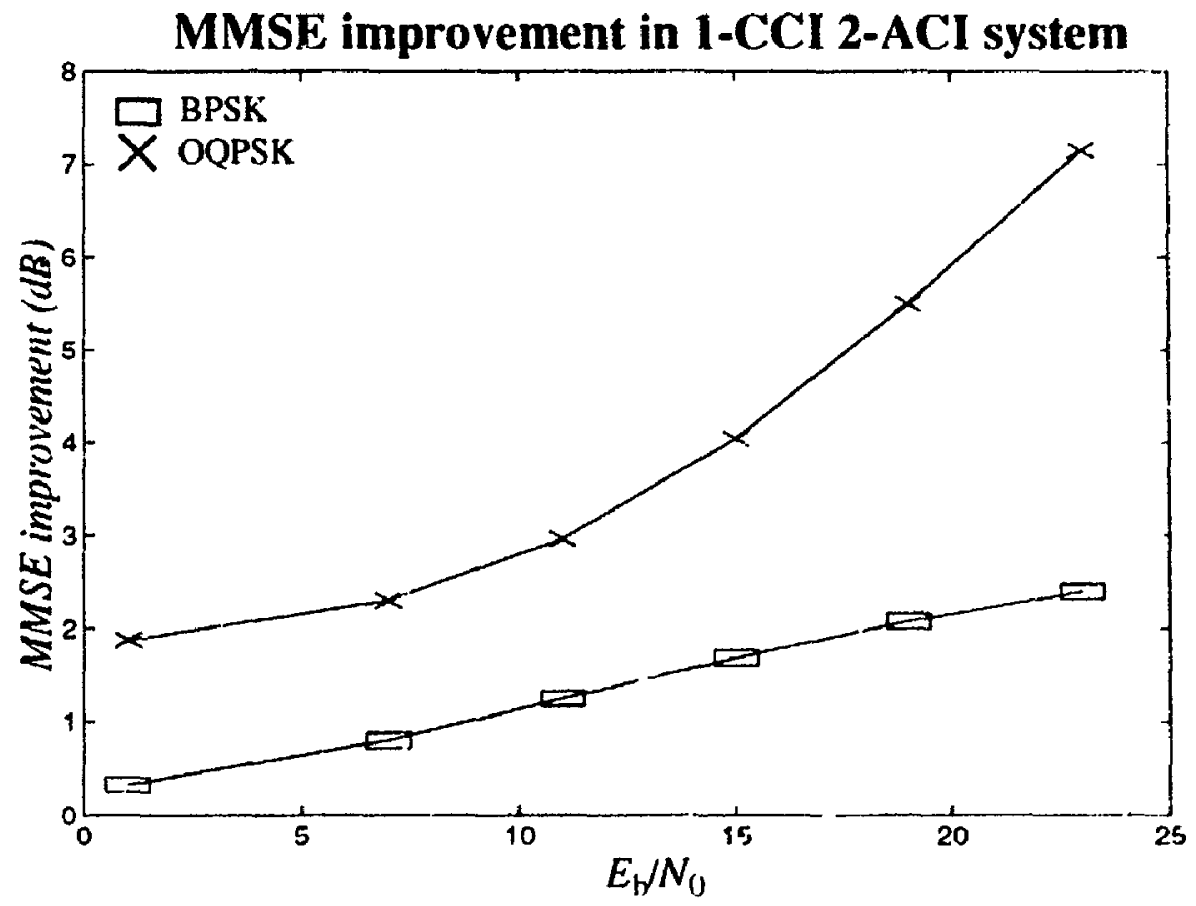

(b)

Figure 6.52 (a) The MMSE and (b) The MMSE improvement for $1 \mathrm{CCl} 2 \mathrm{ACl}$ system when the complexity of DFE and UDFE are the same. 


\subsection{Conclusion}

A cluster 7 cellular system was simulated and following concludes are drawn:

1- The performance of FRESH-DFE is superior to that of conventional DFE.

2. The superiority of FRESH-DFE over conventional DFE increases when signals with higher redundancy are used. The greater spectral redundancy yields greater superiority of FRESH-DFE over DFE.

3- Multi-antenna receiver can achieve very low bit error rate even if the channel spacing is less than the symbol rate.

4- The superiority of FRESH-DFE over conventional DFE increases when multiantenna receiver is used.

5- If the optimum receiver is used, the capacity and BER of a system using BPSK with channel spacing equals to half the symbol rate and the capacity and BER of a system using QPSK with channel spacing equals to the symbol rate are the same.

6- The performances of FRESH-DFE and conventional DFE in the 2-symbol rate system shown in Figure 5.14 are better when the SOI symbol rate is twice the SNOI symbol rate.

7- With same complexity, ODFE can achieve better performance than conventional DFE. 


\section{Chapter 7}

\section{Conclusions}

\subsection{Conclusions}

This thesis studied digital communication in high interference multipath fading environment and obtained the structure of the optimum receiver with an acceptable pertormance. The receiver is optimum in the sense that it is able to exploit all the spectral redundancies present in the received signal due to modulation technique. It has been shown that the structure of optimum receiver changes from one system to another as it is related to: (1) channel spacing, (2) signal excess bandwidth, (3) number of available receiver antennas (4) modulation scheme and (5) users' symbol rates.

It has been shown that the modulation schemes can be classinied into three categories in the sense of cyclostationarity. BPSK, QPSK, and OQPSK have been used to represent these three categories. An optimum receiver can be designed and analyzed for each of these three modulations without a loss of generality.

The CCI and ACl signals have been considered separately and it has been shown that the interfering effect of $\mathrm{ACl}$ can be removed despite its considerable spectral overlap with SOI. In addition to overlapping $\mathrm{ACl}$, multi-symbol rates systems have also been considered and the structure of the optimum receiver in such a case was obtained.

The conventional DFE was fully analyzed in cyclostationary environment by using MTAMSE criterion. It has been shown that the DFE is not an optimum receiver for BPSK and OQPSK signals and it is optimum for QPSK signals if and only if all users' symbol rates are identical.

Two different structures for DFE based on two different stationary representations of a CRP, i.e. HSR and TSR, were introduced. FRESH-DFE was built on HSR and GDFE was built on TSR. It has been shown that FRESH-DFE and GDFE are identical for single symbol rates systems. We referred FRESH-DFE (or GDFE) in single symbol rate systems to as ODFE. The results of analysis and simulation presented in this thesis has shown that the ODFE has better performance than the conventional DFE specially in high interfer- 
ence environment.

Digital cellular radio systems were considered to evaluate the performance of designed receiver. The SNR, SCCIR and SIR of a digital cellular radio system has been studied. A definition for the capacity of a digital cellular radio system has been presented and it has been shown that the capacity can be increased if not very low BER is needed.

The simulation results have verified the analytical results. The results have been shown that the performance of FRESH-DFE always is better than that of conventional DFE. It has been also shown that the multi-antenna receiver can achieve low BER even though the channel spacing is smaller than the symbol rate. A 2-symbol rate cellular system has been simulated and it has been shown that the performance of FRESH-DFE and the conventional DFE are better when the SOI symbol rate is twice that of SNOI. It has been also shown that for the same receiver complexity, the ODFE has better performance than the convention DFE.

\subsection{Recommendations for Future Research}

The followings are some suggestions for future research related to this thesis work:

\subsubsection{Using Recursive Least Squares (RLS) Algorithm in Adaptive FRESH-DFE}

It is well known that the RLS algorithm provides much faster convergence and therefore it need a fewer number of training data. The frame length can be reduced when the header is reduced. In this case the assumption of time invariance of the channel in one frame time is more closer to reality.

\subsubsection{Considering The Effect of Sampling Phase on The Performance of The FRESH-DFE}

It is well known that the sampling phase is important when a strong cyclostationary signal is present [1]. However, the importance of sampling phase is reduced when the received signal is more close to stationary [1]. It is strongly recommended to study the effect of sampling phase on the performance of the FRESH-DFE.

\subsubsection{Considering The Effect of The Doppler Spread on The FRESH-DFE}

It has been described the Doppler effect on multipath channel in chapter 5 . The 
behavior of channel is more harsh when Doppler frequency increases. It is strongly recommended to study the effect of the Doppler spread on the FRESH-DFE. Since Doppler spread is created by simultaneously frequency shifting of the spectrum with set of tre quencies, it seems one can cancel this effect if he can find the best frequency shifts set.

\subsubsection{Investigating The Performance and Perhaps New Structure of Optimum DFE in Fast Fading Environment.}

A time variant channel in one frame time can be objective of another study. In this case, a new structure for FRESH-DFE can be found which perhaps uses time variant sampler to sample at appropriate times.

\subsubsection{Using Channel Estimator Besides ODFE}

Another objective of study many be to use a channel estimator with ODFE to reduce the fading effect and consequently reduce the BER.

\subsubsection{Investigating The Performance of The FRESH-DFE in Code Division Multiple Access (CDMA) Systems}

The performance and perhaps new structure for the FRESH-DFE can be studied when CDMA is used. It is well known that CDMA signals are highly spectral redundant. Therefore, the performance of FRESH-DFE should be much better when CDMA is used. 


\section{Appendix A}

\section{Some Useful Cyclic Relations}

Followings are some useful cyclic relations which used in this thesis :

- The cyclic relations for two complex CRPs $x(t)$ and $y(t)$ are : [10]

$$
\begin{gathered}
R_{x y}^{\alpha}(\tau)=R_{r y}^{-\alpha}(-\tau)^{*} \\
R_{x y^{*}}^{\alpha}(\tau)=R_{y x^{*}}^{\alpha}(-\tau)=R_{x^{*} y}^{-\alpha}(\tau)^{*} \\
R_{x^{*} y^{*}}^{\alpha}(\tau)=R_{x y}^{-\alpha}(\tau)^{*} \\
R_{x}^{\alpha}(\tau)=R_{x}^{-\alpha}(-\tau)^{*} \\
R_{x x^{*}}^{\alpha}(\tau)=R_{x x^{*}}^{\alpha}(-\tau)=R_{x^{*} x}^{-\alpha}(\tau)^{*} \\
S_{y x}^{\alpha}(f)=S_{x y}^{-\alpha}(f)^{*} \\
S_{y x^{*}}^{\alpha}(f)=S_{x y^{*}}^{\alpha}(-f)=S_{x^{*} y}^{-\alpha}(-f) \\
S_{x^{*} y^{*}}^{\alpha}(f)=S_{x y}^{-\alpha}(-f)^{*}=S_{y x}^{\alpha}(-f) \\
S_{x}^{\alpha}(f)=S_{x}^{-\alpha}(f){ }^{*} \\
S_{x x^{*}}^{\alpha}(f)=S_{x x^{*}}^{\alpha}(-f)=S_{x^{*} x}^{-\alpha}(-f)^{*} \\
S_{x^{*} x^{*}}^{\alpha}(f)=S_{x}^{-\alpha}(-f){ }^{*}=S_{x}^{\alpha}(-f)
\end{gathered}
$$

- The CA, CCA, CSD, CCSD of a time delayed version of CRP $x(t)$, i.e. $x(t-\tau)$, are found by multiplying the CA, CCA, CSD, and CCSD of $x(t)$ by $e^{-j 2 \pi \alpha \tau}$ respectively. [10]

- The following equations shows the cyclic relations between CRP $x(t)$ and $y(t)=x(t) q(t)$ where $q(t)$ is another CRP. 


$$
\begin{aligned}
& R_{y}^{\alpha_{k}}(\tau)=\sum_{m} R_{x}^{\alpha_{k}-\alpha_{m}}(\tau) R_{q}^{\alpha_{m}}(\tau) \\
& S_{y}^{\alpha_{k}}(f)=\sum_{m} S_{x}^{\alpha_{k}-\alpha_{m}}(f) \otimes S_{q}^{\alpha_{m}}(f)
\end{aligned}
$$

- The input-output cyclic relations for linear almost periodic system $h(t, u)=\sum_{\mu} g_{\mu}(t-u) e^{j 2 \pi \mu u}$ are: $[10]$

$$
\begin{aligned}
S_{y}^{\alpha}(f)= & \sum_{\lambda, \mu} H_{\mu}\left(f+\frac{\alpha}{2}\right) S_{x}^{\alpha+\lambda-\mu}\left(f-\frac{\mu+\lambda}{2}\right) H_{\lambda}\left(f-\frac{\alpha}{2}\right) \\
S_{y y^{*}}^{\alpha}(f)= & \sum_{\lambda, \mu} H_{\mu}\left(f+\frac{\alpha}{2}\right) S_{x x^{*}}^{\alpha+\lambda-\mu}\left(f-\frac{\mu+\lambda}{2}\right) H_{-\lambda}\left(-f+\frac{\alpha}{2}\right) \\
S_{y x}^{\alpha}(f) & =\sum_{\mu} H_{\mu}\left(f+\frac{\alpha}{2}\right) S_{x}^{\alpha-\mu}\left(f-\frac{\mu}{2}\right) \\
S_{y x^{*}}^{\alpha}(f) & =\sum_{\mu} H_{\mu}\left(f+\frac{\alpha}{2}\right) S_{x x^{*}}^{\alpha-\mu}\left(f-\frac{\mu}{2}\right) \\
S_{x y}^{\alpha}(f) & =\sum_{\mu} s_{x}^{\alpha+\mu}\left(f-\frac{\mu}{2}\right) H_{\mu}\left(f-\frac{\alpha}{2}\right) \\
s_{x y^{*}}^{\alpha}(f) & =\sum_{\mu} s_{x x^{*}}^{\alpha-\mu}\left(f+\frac{\mu}{2}\right) H_{\mu}\left(-f+\frac{\alpha}{2}\right)
\end{aligned}
$$

where $x(t)$ and $y(t)$ are the input and output of the system, respectively. 


\section{Appendix B}

\section{Analysis of The FRESH-DFE}

Same as the conventional DFE, FRESH-DFE minimizes the TAMSE too. Taking the gradient of TAMSE according to the backward filter coefficients and setting them to zero, yields:

$$
\nabla_{h_{h}}^{T A M S E}(m)=\sigma_{d_{0}}^{2} h_{b}(m)+\left\langle E\left\{\tilde{y}(n) d_{0}^{*}(n-D-m)\right\}\right\rangle
$$

Replacing $\tilde{y}(n)$ by:

$$
\begin{aligned}
& \tilde{y}(n)=\sum_{k} \int_{-\infty}^{\infty} a_{k}(u) x\left(n T_{0}-u\right) e^{j 2 \pi\left[\alpha_{k}\right]} T_{T^{-1}}^{\left(n T_{0}-u\right)} d(u)+ \\
& \sum_{i} \int_{-\infty}^{\infty} b_{i}(u) x^{*}\left(n T_{0}-u\right) e^{j 2 \pi \mid \beta, 1} r_{r_{0}^{-1}}^{\left(n T_{0}-u\right)} d(u)
\end{aligned}
$$

yields:

$$
\begin{aligned}
& \nabla_{h_{b}}^{T M S E}(m)=\sigma_{a_{0}}^{2} h_{b}(m)-
\end{aligned}
$$

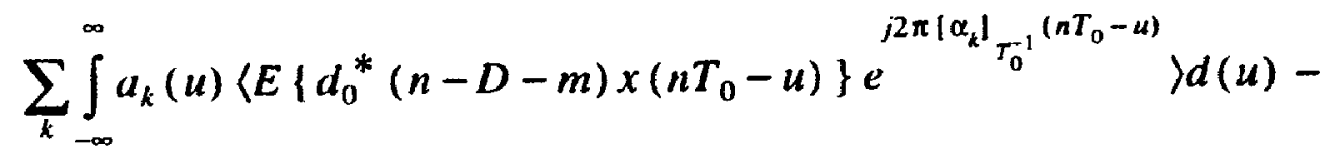

$$
\begin{aligned}
& \sum_{l} \int_{-\infty}^{\infty} b_{i}(u)\left\langle E\left\{d_{0}^{*}(n-D-m)\left(x^{*}\left(n T_{0}-u\right)\right)\right\} e^{j 2 \pi\left\{\beta_{1}\right\}_{T_{0}^{-1}}\left(n T_{0}-u\right)}\right\rangle d(u)
\end{aligned}
$$

Using (4.1) to (4.5):

$$
\begin{gathered}
E\left\{d_{0}^{*}(n-D-m) x\left(n T_{0}-u\right)\right\}=\sigma_{d_{0}^{(r)}}^{2} z_{0}^{(1)}\left((D+m) T_{0}-t_{0}^{(r)}-u\right) e^{j 2 \pi \phi_{0}^{(r)}}+ \\
\sigma_{d_{0}^{(1)}}^{2} z_{0}^{(2)}\left((D+m) T_{0}-t_{0}^{(i)}-u\right) e^{j 2 \pi \phi_{0}^{(1)}} \\
E\left\{d_{0}^{*}(n-D-m) x^{*}\left(n T_{0}-u\right)\right\}=\sigma_{d_{0}^{(r)}}^{2} z_{0}^{(1) *}\left((D+m) T_{0}-t_{0}^{(r)}-u\right) e^{-j 2 \pi \phi_{0}^{(r)}}- \\
\sigma_{d_{0}^{(1)}}^{2} z_{0}^{(2) *}\left((D+m) T_{0}-t_{0}^{(i)}-u\right) e^{-j 2 \pi \varphi_{0}^{(1)}}
\end{gathered}
$$


Replacing (B.4) in (B.3) and considering

$$
\begin{aligned}
& \left\langle e^{j 2 \pi\left|\alpha_{k}\right|_{r_{1}^{\prime}{ }^{\prime}}\left(n T_{0}-w\right)}\right\rangle= \begin{cases}\left.1 \text { for } \mid \alpha_{k}\right]_{r_{0}{ }^{\prime}}=0 \\
0 & \text { othcrwise }\end{cases} \\
& \left\langle e^{j 2 \pi\left[\beta_{1}\right]_{r_{0}^{-1}}\left(n T_{0}-u\right)}\right\rangle= \begin{cases}1 \text { for }\left|\beta_{i}\right|_{T_{0}{ }^{\prime}}=0 \\
0 & \text { otherwise }\end{cases}
\end{aligned}
$$

yields:

$$
\begin{gathered}
\nabla_{h_{b}}^{T M S E}(m)=\sigma_{d_{0}}^{2} h_{b}(m)- \\
\sigma_{d_{0}^{(j)}}^{2} \int_{-\infty}^{\infty}\left(a_{0}(u) z_{0}^{(1)}\left((D+m) T_{0}-t_{0}^{(r)}-u\right) e^{j 2 \pi \phi_{0}^{(r)}}+\right. \\
\left.b_{0}(u) z_{0}^{(1)^{*}}\left((D+m) T_{0}-t_{0}^{(r)}-u\right) e^{-j 2 \pi \phi_{0}^{(r)}}\right) d(u)- \\
\sigma_{d_{0}^{(1)}}^{2} \int_{-\infty}^{\infty}\left(a_{0}(u) z_{0}^{(2)}\left((D+m) T_{0}-t_{0}^{(t)}-u\right) e^{j 2 \pi \phi_{0}^{(\prime)}}-\right. \\
\left.b_{0}(u) z_{0}^{(2)}\left((D+m) T_{0}-t_{0}^{(i)}-u\right) e^{-j 2 \pi \phi_{11}^{(1)}}\right) d(u)
\end{gathered}
$$

Optimum coefficient will be achieved by tending gradient to zero. Therefore the optimum coefficients for the feedback filter are:

$$
\begin{gathered}
h_{b}(m)= \\
\frac{\sigma_{d_{0}^{(\prime)}}^{2}}{\sigma_{d_{0}}^{2}} \int_{-\infty}^{\infty}\left(a_{0}(u) z_{0}^{(1)}\left((D+m) T_{0}-t_{0}^{(r)}-u\right) e^{j 2 \pi \phi_{0}^{(r)}}+\right. \\
\left.b_{0}(u) z_{0}^{(1) *}\left((D+m) T_{0}-t_{0}^{(r)}-u\right) e^{-j 2 \pi \phi_{0}^{(r)}}\right) d(u)+ \\
\frac{\sigma_{d_{0}^{(1)}}^{2}}{\sigma_{d_{0}}^{2}} \int_{-\infty}\left(a_{0}(u) z_{0}^{(2)}\left((D+m) T_{0}-t_{0}^{(i)}-u\right) e^{j 2 \pi \phi_{0}^{(1)}}-\right. \\
\left.b_{0}(u) z_{0}^{(2) *}\left((D+m) T_{0}-t_{0}^{(i)}-u\right) e^{-\jmath 2 \pi \phi_{0}^{(1)}}\right) d(u)
\end{gathered}
$$




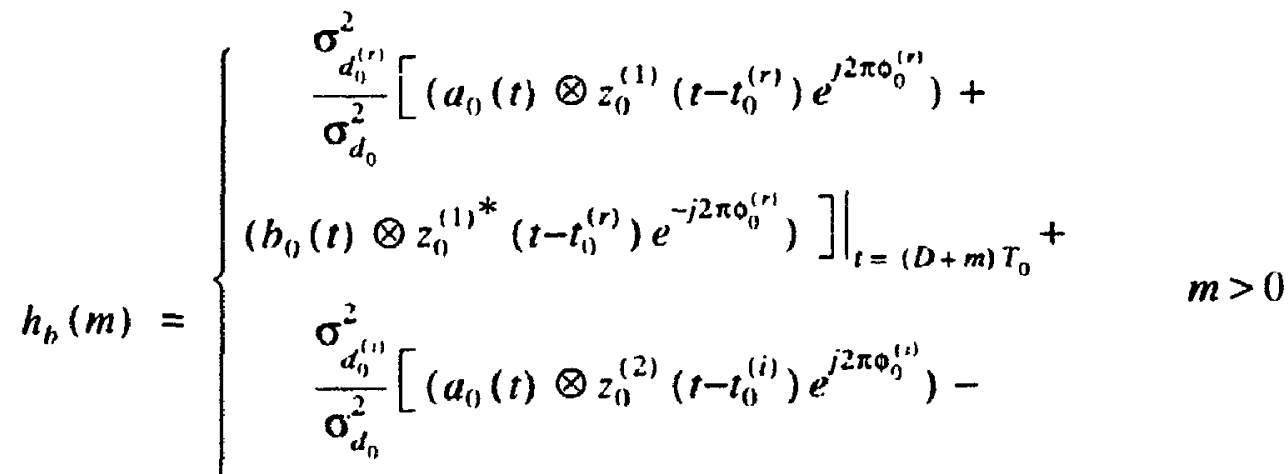

$$
\begin{aligned}
& \left.\left(b_{0}(t) \otimes z_{0}^{(2)^{*}}\left(t-t_{0}^{(i)}\right) e^{-j 2 \pi \phi_{0}^{(1)}}\right)\right]\left.\right|_{s=(D+m) T_{0}} \\
& 0 \quad m \leq 0
\end{aligned}
$$

Equation (B.8) is reduced to:

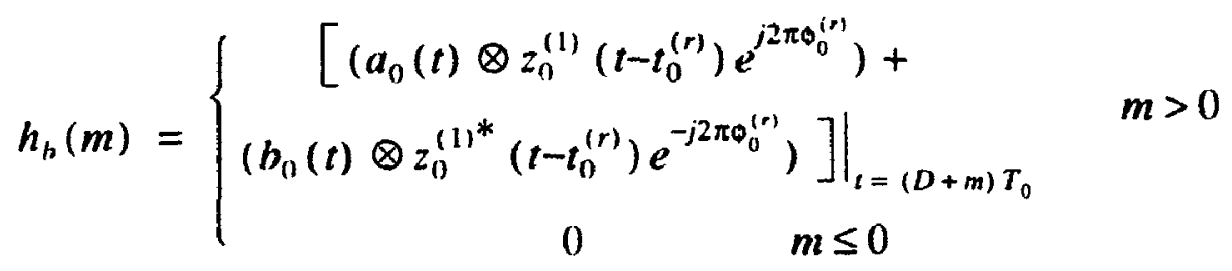

for BPSK systems, to:

$$
h_{b}(m)=\left\{\begin{array}{c}
\frac{1}{2}\left[\left(a_{0}(t) \otimes\left(z_{0}^{(1)}\left(t-t_{0}^{(r)}\right)+z_{0}^{(1)}\left(t-t_{0}^{(i)}\right)\right) e^{j 2 \pi \varphi_{0}^{(r)}}\right)+\right. \\
\left.\left(b_{0}(t) \otimes\left(z_{0}^{(1)^{*}}\left(t-t_{0}^{(r)}\right)-z_{0}^{(1)^{*}}\left(t-t_{0}^{(i)}\right) j e^{-j 2 \pi \varphi_{0}^{(r)}}\right)\right]\right|_{t=(D+m) T_{0}} \\
0 \\
m>0 \\
m \leq 0
\end{array}\right.
$$

for OQPSK systems, and 20 :

$$
h_{b}(m)=\left[\begin{array}{ccc}
{\left.\left[a_{0}(t) \otimes z_{0}^{(1)}\left(t-t_{0}^{(n)}\right) e^{j 2 \pi 0_{0}^{(r)}}\right]\right|_{t=(D+m) T_{0}}} & m>0 \\
0 & m \leq 0 &
\end{array}\right.
$$

for balanced QPSK.

Same procedure has to be used to find the other optimum filers coefficients. The gradient of TAMSE according to the $a_{k}(t)$ in the time $u$ is:

$$
\nabla_{a_{k}}^{T M S E}(u)=\left\langle E\left\{\left(\tilde{y}(n)-\left(d_{0}(n-D)+f(n)\right)\right) \nabla_{a_{k}}^{\tilde{y}^{*}(n)}(u)\right\}\right\rangle
$$


where

$$
\nabla_{i_{k}}^{*}(n)(u)=x^{*}\left(n T_{0}-u\right) e^{-22 \pi\left[\left(\left.\alpha_{k}\right|_{r_{0}}\left(n T_{0}-u\right)\right.\right.}
$$

Replacing (B.13) in (B.12) and also considering (4.1) to (4.5), 2nd and 3th term of (B.12) will be

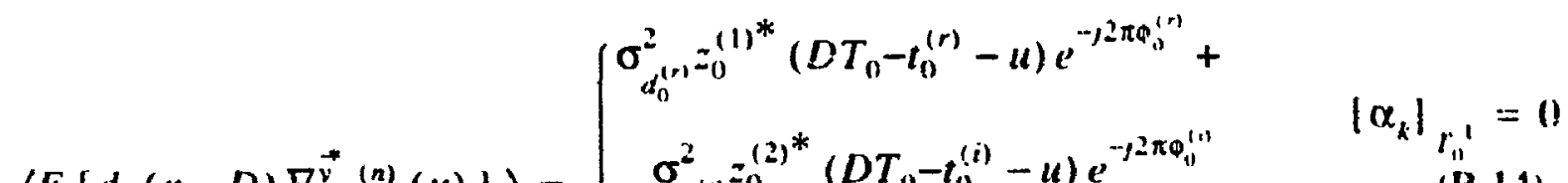

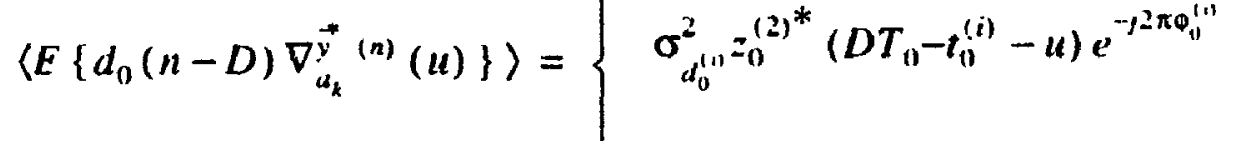

$0 \quad\left|\alpha_{k}\right|_{r_{1}{ }^{1}} \neq 0$

$\left\langle E\left\{f(n) \nabla_{a_{k}}^{*}(n)(u)\right\}\right\rangle=\left\{\begin{array}{c}\sigma_{d_{0}^{(n)}}^{2} e^{-j 2 \pi \theta_{0}^{(n)}} \sum_{m=1}^{\infty} z_{0}^{(1)^{*}}\left((D+m) T_{0}-u-t_{0}^{(n)}\right) h_{b}(m)+ \\ \sigma_{d_{0}^{(1)}}^{2} e^{-j 2 \pi \phi_{0}^{(1)}} \sum_{m=1}^{\infty} z_{0}^{(2)^{*}}\left((D+m) T_{0}-u-t_{0}^{(1)}\right) h_{b}(m\}_{(\mathrm{B} .15)} \\ \left.0 \quad \mid \alpha_{k}\right]_{T_{0}{ }^{\prime}}=0 \\ \left|\alpha_{k}\right|_{T_{0}{ }^{1} \neq 0}\end{array}\right.$

The first term of (B.12) is:

$$
\begin{aligned}
& \left\langle E\left\{\tilde{y}(n) \nabla_{a_{k}}^{\vec{y}^{*}(n)}(u)\right\}\right\rangle \\
& =\sum_{m} \int_{-\infty}^{\infty} a_{m}(v)\left\langle E\left\{x\left(n T_{0}-v\right) x^{*}\left(n T_{0}-u\right)\right\} e^{j 2 \pi n T_{0}\left|\alpha_{m}-\alpha_{k}\right|} r^{\prime}{ }^{\prime}\right\} e^{j 2 \pi\left\{u \left(x_{k}-v \alpha_{m} \mid\right.\right.}{ }^{\prime{ }^{\prime}{ }^{\prime}} d v+
\end{aligned}
$$

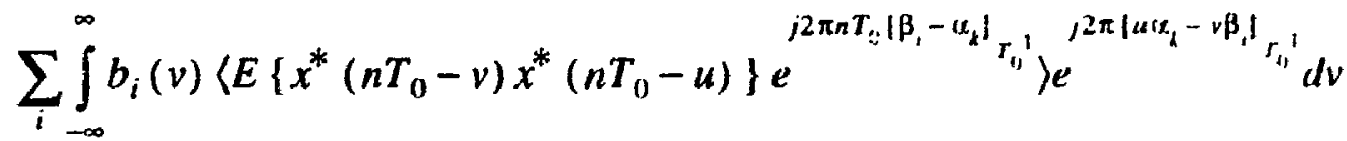

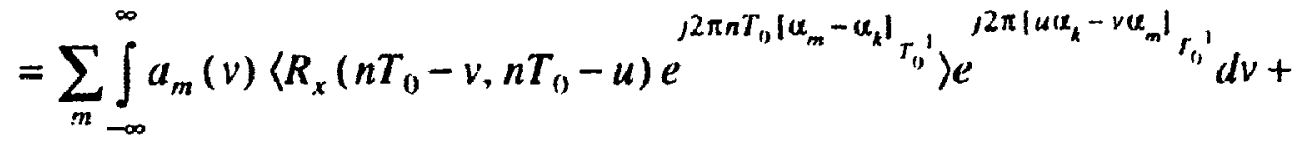

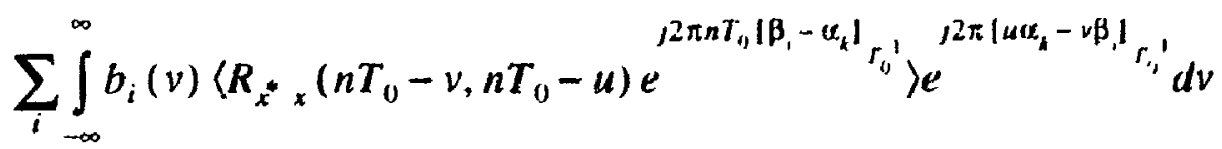


Using (2.58) yields:

$$
\begin{aligned}
& \left\langle R_{x}\left(n T_{0}-v, n T_{11}-u\right) e^{j 2 \pi n T_{0}\left|\alpha_{m}-\alpha_{k}\right|} r_{0}^{\prime}\right\rangle=
\end{aligned}
$$

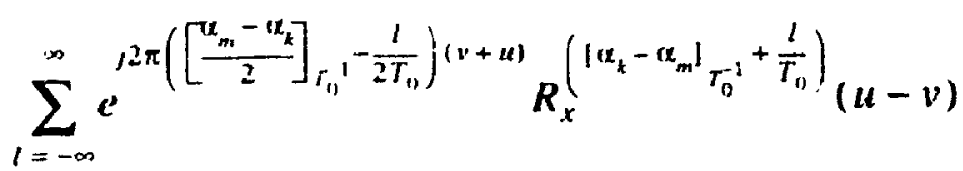

$$
\begin{aligned}
& \left\langle R_{x^{*}}\left(n T_{0}-v, n T_{0}-u\right) e^{\left.j 2 \pi n T_{0} \mid \beta_{1}-\alpha_{k}\right]} T_{0}^{-1}\right\rangle= \\
& \sum_{l=-\infty}^{\infty} e^{j ? \pi\left(\left[\frac{\beta_{1}-\alpha_{k}}{2}\right]_{T_{0}}{ }^{i-\frac{l}{2 T_{0}}}\right)(v+u)} R_{x^{2} x}^{\left.\left(\mid \alpha_{k}-\beta_{t}\right]_{T_{0}^{-1}}+\frac{l}{T_{0}}\right)}(u-v)
\end{aligned}
$$

Replacing (B.17) in (B.16) yields:

$$
\begin{aligned}
& \left\langle E\left\{\tilde{y}(n) \nabla_{a_{k}}^{*}(n)(u)\right\}\right\rangle
\end{aligned}
$$

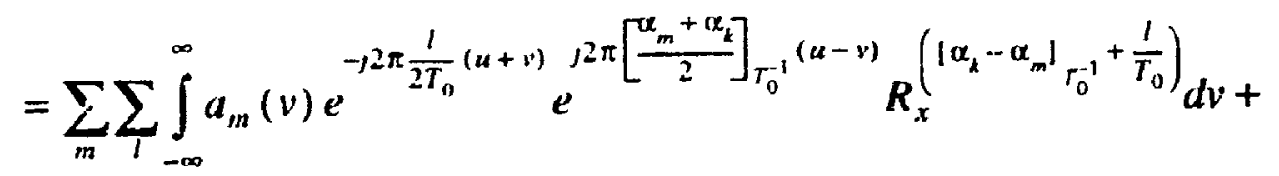

$$
\begin{aligned}
& \sum \sum_{l} \int_{-\infty}^{\infty} b_{l}(v) e^{-j 2 \pi \frac{l}{2 T_{0}}(u+v)} e^{j 2 \pi\left[\frac{\beta_{1}+\alpha_{k}}{2}\right]_{T_{0}{ }^{1}}(u-v)} R_{x^{*} x}^{\left(\left|\alpha_{k}-\beta_{1}\right|{ }_{r_{0}^{-1}}+\frac{l}{T_{0}}\right)}(u-v) d v
\end{aligned}
$$

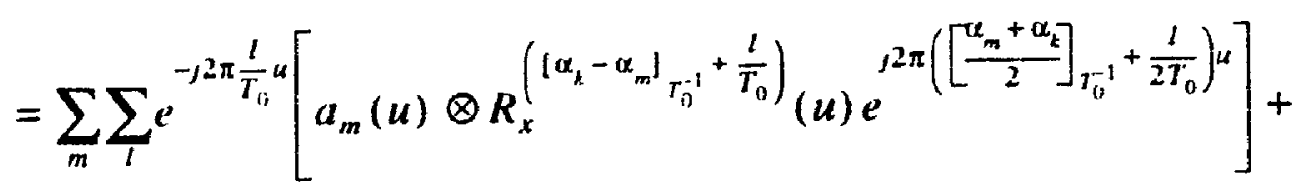

$$
\begin{aligned}
& \sum_{i} \sum_{i} e^{-12 \pi \frac{1}{T_{i}} u}\left[b_{i}(u) \otimes R_{x}^{\left(\left|\alpha_{k}-\beta_{1}\right| T_{0}^{-1}+\frac{l}{T_{0}}\right)}(u) e^{j 2 \pi\left(\left[\frac{\beta_{1}+\alpha_{k}}{2}\right]_{T_{0}^{-1}}+\frac{!}{2 T_{0}}\right) \mu}\right]
\end{aligned}
$$

Changing the variable from $l$ to $l+p$ where either $p=\left\lceil\frac{\alpha_{k}-\alpha_{m}}{T_{0}}\right\rceil$ or $p=\left\lceil\frac{\alpha_{k}-\beta_{i}}{T_{0}}\right\rceil$ yields: 


$$
\begin{aligned}
& \left\langle E\left\{\tilde{y}(n) \nabla_{a_{k}}^{*}(n)(u)\right\}\right\rangle \\
& =\sum_{m} \sum_{l} e^{-j 2 \pi \frac{1}{T_{s 1}} u}\left[a_{m}(u) \otimes R_{x}^{\left(\alpha_{k}-\alpha_{m}+\frac{1}{\Gamma_{1}}\right)}(u) e^{j 2 \pi\left(\frac{u_{m}+u_{k}}{2}+\frac{1}{2 T_{11}}\right)}\right]+
\end{aligned}
$$

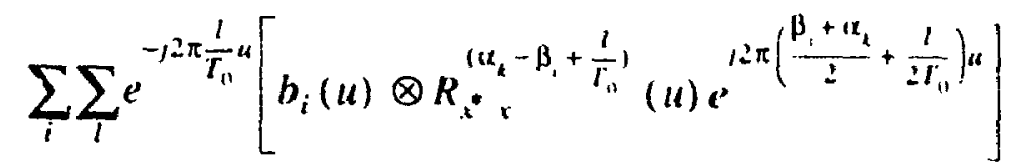

Substituting (B.14), (B.15) and (B.2()) in :B.12) and tending the gradient to zero yields the following equation for $\left[\alpha_{k}\right]_{T_{0}^{-1}}=0$ :

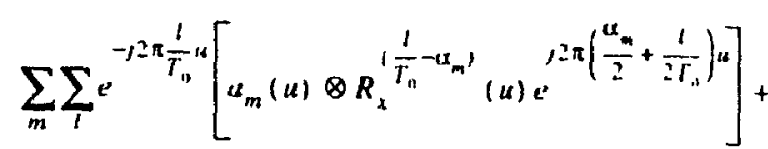

$$
\begin{aligned}
& \sum_{1} \sum_{l} e^{-j 2 \pi \frac{1}{T_{n}} u}\left[b_{2}(u) \otimes R_{x^{2}}^{\left.1 \frac{1}{T_{n}}-\beta_{1}\right)}(u) e^{j 2 \pi\left(\frac{\beta_{1}}{2}+\frac{1}{2 T_{n}}\right) \mu}\right]
\end{aligned}
$$

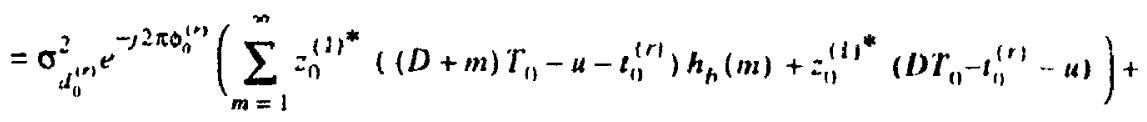

$$
\begin{aligned}
& \left.\sigma_{u_{n}^{(1)}}^{2} e^{-j 2 \pi 0_{n}^{(1)}}\left(\sum_{m=1}^{\infty} z_{11}^{(2)^{*}}\left((D+m) T_{19}-u-t_{11}^{(t)}\right) h_{h}(m)+z_{11}^{(2)^{*}}(D) T_{11}-t_{11}^{(1)}-u\right)\right)
\end{aligned}
$$

and the following equation for $\left[\alpha_{k}\right]_{T_{n}{ }^{1}} \neq 0$ :

$$
\begin{aligned}
& \sum_{m} \sum_{l} e^{-j 2 \pi \frac{1}{T_{n}} u}\left[u_{m}(u) \otimes R_{x}^{\left(\alpha_{k}-\alpha_{m}+\frac{i}{T_{11}}\right)}\left\{(\mu) e^{j 2 \pi\left(\frac{\alpha_{k}+u_{m}}{2}+\frac{t}{\left.2 T_{n}\right)}\right)}\right]+\right.
\end{aligned}
$$

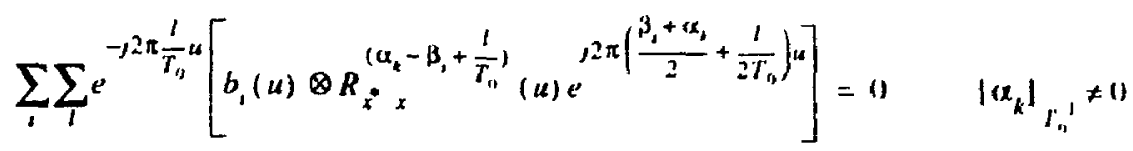

Taking the Fourier transform of (B.2l) and (B.22) and using $R_{i}^{u}(\tau)=R_{i}^{-1 t}(-\tau)$ |Appendix A] yields:

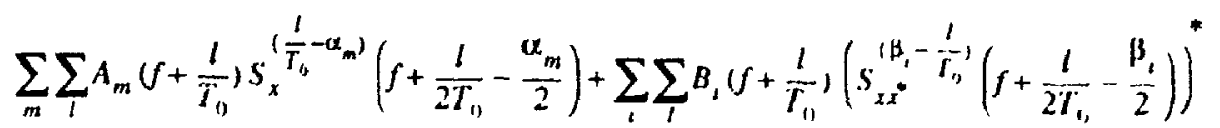

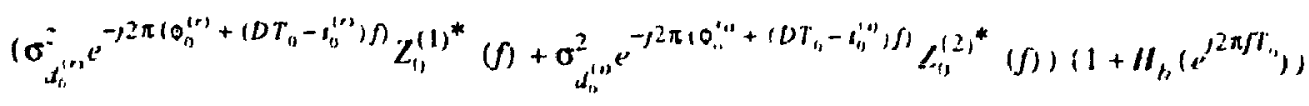




$$
\begin{gathered}
\sum_{m} \sum_{l} A_{m}\left(f+\frac{l}{T_{i 1}}\right) s_{k}^{\left(\frac{l}{T_{n}}+\alpha_{k}-\alpha_{m}\right)}\left(f+\frac{l}{\left.2 T_{1}\right)}-\frac{\alpha_{m}+\alpha_{k}}{2}\right)+ \\
\sum_{i} \sum_{l} B_{t}\left(f+\frac{l}{T_{1 k}}\right)\left(s_{x x^{*}}^{\left(\beta_{1}-\alpha_{k}-\frac{l}{T_{n}}\right)}\left(f+\frac{l}{2 T_{t r}}-\frac{\beta_{t}+\alpha_{k}}{2}\right)\right)^{*}=0 \quad\left[\alpha_{k}\right]_{T_{n}^{-1}} \neq 0
\end{gathered}
$$

The gradient of TAMSE according to the $b_{i}(t)$ in the time $u$ is:

$$
\nabla_{h_{1}}^{T M S E}(u)=\left\langle E\left\{\left(\tilde{y}(n)-\left(d_{0}(n-D)+f(n)\right)\right) \nabla_{b_{1}}^{\dot{y}^{(n)}}(u)\right\}\right\rangle
$$

where

$$
\nabla_{h_{1}}^{\vec{y}^{(n)}}(u)=x\left(n T_{0}-u\right) e^{\left.-ر 2 \pi \mid \beta_{1}\right]} T_{T^{\prime}}{ }^{\left(n T_{0}-u\right)}
$$

Replacing (B.26) in (B.25) and also considering (4.1) to (4.5), 2nd and 3th term of (B.25) will be

$$
\begin{aligned}
& \left\langle E\left\{d_{0}(n-D) \nabla_{b_{1}}^{v^{*}(n)}(u)\right\}\right\rangle=\left\{\begin{array}{cc}
\sigma_{d_{0}^{(n)} z_{0}^{(1)}\left(D T_{0}-t_{0}^{(r)}-u\right) e^{j 2 \pi \phi_{0}^{(r)}}-} & {\left[\beta_{i}\right]_{T_{0}^{-1}}=0} \\
\sigma_{d_{0}^{(n)}}^{2} z_{0}^{(2)}\left(D T_{0}-t_{0}^{(i)}-u\right) e^{j 2 \pi \phi_{0}^{(3)}} & (\mathrm{B} .27) \\
0 & {\left[\beta_{i}\right]_{T_{0}^{-1}} \neq 0}
\end{array}\right. \\
& \left\langle E\left\{f(n) \nabla_{h_{1}^{*}(n)}^{*}(u)\right\}\right\rangle=\left\{\begin{array}{cc}
\sigma_{d_{0}^{(r)}}^{2} e^{j 2 \pi \theta_{0}^{(r)}} \sum_{m=1}^{\infty} z_{0}^{(1)}\left((D+m) T_{0}-u-t_{0}^{(r)}\right) h_{b}(m)+ \\
\sigma_{d_{0}^{(1)}}^{2} e^{j \pi \theta_{0}^{(n)}} \sum_{m=1}^{\infty} z_{0}^{(2)}\left((D+m) T_{0}-u-t_{0}^{(i)}\right) h_{b}(m)(\mathrm{B} .28) \\
0 & {\left[\beta_{i}\right]_{T_{0}^{-1}}=0} \\
0 & {\left[\beta_{i}\right]_{T_{0}^{-1}} \neq 0}
\end{array}\right.
\end{aligned}
$$

The first term of (B.25) is : 


$$
\begin{gathered}
\left\langle E\left\{j(n) \nabla_{b_{i}}^{*}(n)(u)\right\}\right\rangle \\
=\sum_{k} \sum_{l} e^{-12 \pi \frac{l}{T_{0}} u}\left[a_{m}(u) \otimes R_{x x^{*}}^{\left(\beta_{1}-\alpha_{k}+\frac{l}{T_{0}}\right)}(u) e^{j 2 \pi\left(\frac{\alpha_{k}+\beta_{1}}{2}+\frac{l}{2 T_{0}}\right) u}\right]+ \\
\sum_{m} \sum_{l} e^{-j 2 \pi \frac{l}{T_{0}} u}\left[b_{i}(u) \otimes R_{x^{*} x^{*}}^{\left(\beta_{3}-\beta_{m}+\frac{l}{T_{i}}\right)}(u) e^{j 2 \pi\left(\frac{\beta_{m}+\beta_{i}}{2}+\frac{l}{2 T_{n}}\right) u}\right]
\end{gathered}
$$

Substituting (B.27), (B.28) and (B.29) in (B.25) and tending the gradient to zero. yields the following equation for $\left[\beta_{i}\right]_{T_{0}^{-1}}=0$ :

$$
\begin{aligned}
& \sum_{k} \sum_{i} e^{-22 \pi \frac{1}{T_{n}} u}\left[a_{m}(u) \otimes R_{x x^{\prime}}^{\left(\frac{l}{T_{n}}-\alpha_{k}\right)}(u) e^{j 2 \pi\left(\frac{\alpha_{k}}{2}+\frac{l}{3 T_{n}}\right) u}\right]+ \\
& \sum_{m} \sum_{i} e^{-ر 2 \pi \frac{1}{T_{0}} u[}\left[b_{1}(u) \otimes R_{x^{*}}^{\left(\frac{1}{T_{n}}-\beta_{m}\right)}(u) e^{\rho 2 \pi\left(\frac{\beta_{m}}{2}+\frac{1}{2 T_{0}}\right) u}\right] \\
& \left.=\sigma_{d_{0}^{(m)}}^{2} e^{j 2 \pi \phi_{0}^{(n)}}\left(\sum_{m=1}^{\infty} z_{0}^{(1)}\left((D+m) T_{0}-u-t_{0}^{(r)}\right) h_{h}(m)+z_{0}^{(1)}\left(D T_{0}\right)^{-t_{01}^{(r)}}-u\right)\right)- \\
& \sigma_{d_{0}^{(1)}}^{2} e^{\prime 2 \pi \theta_{0}^{(1)}}\left(\sum_{m=1}^{\infty} z_{t)}^{(2)}\left((D+m) T_{0}-u-t_{0}^{(1)}\right) h_{b}(m)+i_{01}^{(2)}\left(D T_{0}-t_{01}^{(1)}-u\right)\right)
\end{aligned}
$$

and the following equation for $\left[\beta_{i}\right]_{T_{0}^{-1}} \neq 0$ :

$$
\begin{aligned}
& \sum_{k} \sum_{t} e^{-j 2 \pi \frac{1}{T_{0}} \mu[}\left[a_{k}(\mu) \otimes R_{x x^{0}}^{\left(\frac{1}{T_{0}}+\beta_{1}-\alpha_{2}\right)}(u) e^{j 2 \pi\left(\frac{\beta_{1}+\alpha_{k}}{2}+\frac{1}{2 T_{n}}\right) \mu}\right]+ \\
& \sum_{m} \sum_{T} e^{-j 2 \pi \frac{1}{T_{0}} u}\left[b_{m}(u) \otimes R_{x^{*}}^{\left(\frac{1}{T_{0}}+\beta_{1}-\beta_{m}\right)}(u) e^{2 \pi\left(\frac{\beta_{1}+\beta_{m}}{2}+\frac{1}{2 T_{n}}\right) \mu}\right]=0 \quad\left|\beta_{1}\right|_{T_{n},} \neq 0
\end{aligned}
$$

Taking the Fourier transform of (B.30) and (B.31) yields:

$$
\begin{aligned}
& \sum_{k} \sum_{l} A_{k}\left(f+\frac{l}{T_{0}}\right) S_{x x^{0}}^{\left(\frac{l}{T_{0}}-\alpha_{k}\right)}\left(f+\frac{l}{2 T_{0}}-\frac{\alpha_{k}}{2}\right)+\sum_{m} \sum_{i} B_{m}\left(f+\frac{l}{T_{0}} ;\left(S_{x}^{\left(\frac{l}{T_{0}}-\beta_{m}{ }^{\prime}\right.}\left(-f-\frac{l}{2 T_{0}}+\frac{\beta_{m}}{2}\right)\right)=\right.
\end{aligned}
$$

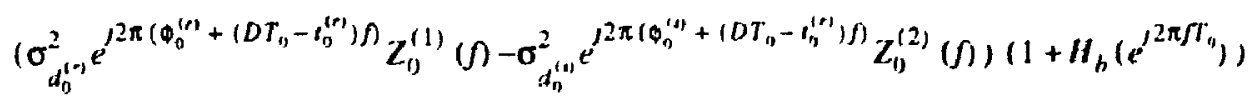

$$
\begin{aligned}
& \sum_{k} \sum_{i} A_{k}\left(f+\frac{l}{T_{0}}\right) S_{x x^{\circ}}^{\left(\frac{l}{T^{2}}+\beta_{1}-\alpha_{k}\right)}\left(f+\frac{l}{2 T_{0}}-\frac{\beta_{1}+\alpha_{k}}{2}\right)+ \\
& \left.\sum_{m} \sum_{1} B_{m}\left(f+\frac{l}{T_{11}}\right)\left(s_{x}^{\left(\beta_{1}-\alpha_{k}-\frac{l}{\left.T_{n}\right)}\right.}\left(-f-\frac{l}{2 T_{1}}+\frac{\beta_{1}+\beta_{m}}{2}\right)\right)=0 \quad\left|\beta_{1}\right|_{T_{11}{ }^{1}} \neq 0\right)
\end{aligned}
$$


Appendix B Analysis of The FRESH-DFE

195

Using (4.67) and (B.4), the MTAMSE which can be reached is equal to the following equation:

$$
\begin{aligned}
& \text { MTAMSE }=\sigma_{d_{0}}^{2}-\int_{-\infty}^{\infty} a_{0}(u)\left(\sigma_{d_{0}^{(r)}}^{2} e^{2 \pi \phi_{0}^{(r)}} z_{0}^{(1)}\left(D T_{0}-u-t_{0}^{(r)}\right)+\right. \\
& \left.\sigma_{d_{0}^{(1)}}^{2} e^{j 2 \pi \phi_{0}^{(1)}} z_{0}^{(2)}\left(D T_{0}-u-t_{0}^{(i)}\right)\right) d(u)- \\
& \int_{-\infty}^{\infty} b_{0}(u)\left(\sigma_{d_{0}^{(r)}}^{2} e^{j 2 \pi Q_{0}^{(n)}} z_{0}^{(1)}\left(D T_{0}-u-t_{0}^{(r)}\right)-\right. \\
& \left.\sigma_{d_{0}^{(1)}}^{2} e^{j 2 \pi \varphi_{0}^{(i)}} z_{0}^{(2)}\left(D T_{0}-u-t_{0}^{(i)}\right)\right) d(u) \\
& =\sigma_{d_{0}}^{2}-\left.\left[a_{0}(t) \otimes\left(\sigma_{d_{0}^{(r)}}^{2} e^{j 2 \pi \varphi_{0}^{(n)}} z_{0}^{(1)}\left(t-t_{0}^{(r)}\right)+\sigma_{d_{0}^{(1)}}^{2} e^{j 2 \pi \varphi_{0}^{(1)}} z_{0}^{(2)}\left(t-t_{0}^{(i)}\right)\right)\right]\right|_{t=D T_{0}} \\
& {\left.\left[b_{0}(t) \otimes\left(\sigma_{d_{0}^{(r)}}^{2} e^{-j 2 \pi \varphi_{0}^{(n)}} z_{0}^{(1) *}\left(t-t_{0}^{(r)}\right)-\sigma_{d_{0}^{(1)}}^{2} e^{-j 2 \pi \varphi_{0}^{(1)}} z_{0}^{(2)^{*}}\left(t-t_{0}^{(i)}\right)\right)\right]\right|_{t=D T_{0}}}
\end{aligned}
$$

Although all the forward filters does not effect (B.34) directly, their influences in $a_{0}(t)$ and $b_{0}(t)$ effect (B.34) indirectly. As it is apparent from (B.8), (B.23), (B.24), (B.32), (B.33) and (B.34), FRESH-DFE uses both CSD and CCSD of the received signal. 


\section{Appendix C}

\section{Analysis of GDFE}

The output of TSR-TVF can be found by the following equation :

$$
\begin{gathered}
\hat{s}_{0}(n)=\sum_{l=-\frac{M_{s}}{2}}^{\frac{M_{u}}{2}} \sum_{=0}^{M_{l}}\left(x(n) \otimes a_{l, m}(n)\right) \delta_{n}(l, m)+ \\
\sum_{l=1}^{M_{c}} \sum_{m=0}^{M_{0 l}}\left(x(n) \otimes a_{0 l, m}(n)\right) \delta_{n}(0 l, m)+ \\
\sum_{l=-\frac{M_{u}}{2}} \sum_{m=0}^{M_{t}}\left(x^{*}(n) e^{j 2 \pi\left(2 f_{l}\right) n} \otimes b_{l, m}(n)\right) \delta_{n}(l, m)+ \\
\sum_{l=1}^{M_{c}} \sum_{m=0}^{M_{0 l}}\left(x^{*}(n) \otimes b_{0 l, m}(n)\right) \delta_{n}(0 l, m)
\end{gathered}
$$

where

$$
\delta_{n}(l, m)=\left\{\begin{array}{cc}
1 & n=m, m \pm M_{l}, m \pm 2 M_{l}, \ldots \\
0 & \text { Otherwise }
\end{array}\right.
$$

Defining :

$$
\begin{aligned}
l+\frac{M_{u}}{2} & =0 l \\
M_{1+\frac{M_{u}}{2}} & =M_{0 l} \\
a_{1+\frac{M_{u}}{2} \cdot m}(n) & =a_{f l, m}(n) \\
b_{l+\frac{M_{u}}{2} \cdot m}(n) & =b_{n l, m}(n)
\end{aligned}
$$

yields : 


$$
\begin{aligned}
& \hat{s}_{0}(n)=\sum_{l=-\frac{M_{u}}{2}} \sum_{m=0}^{\frac{M_{u}}{2}+M_{c}}\left(x(n) \otimes a_{l, m}(n)\right) \delta_{n}(l, m)+ \\
& \sum_{l=-\frac{M_{u}}{2}}^{\frac{M_{u}}{2}+M_{r}} \sum_{m=0}^{M_{l}}\left(x^{*}(n) e^{j 2 \pi\left(2 f_{l}\right) n} \otimes b_{l, m}(n)\right) \delta_{n}(l, m)
\end{aligned}
$$

Since $\delta_{n}(l, m)$ is periodic with period $M_{l}$, it can be represented by the following Fourier series :

$$
\delta_{n}(l, m)=\frac{1}{M_{l}} \sum_{k=0}^{M_{l}} e^{j 2 \pi k \frac{(n-m)}{M_{l}}}
$$

Substituting (C.5) in (C.4) yields :

$$
\begin{aligned}
& \hat{s}_{0}(n)=\sum_{l=-\frac{M_{a}}{2}} \sum_{m=0}^{\frac{M_{s}}{2}+M_{c}}\left(x(n) \otimes a_{l, m}(n)\right) \frac{1}{M_{l}} \sum_{k=0}^{M_{l}} e^{j 2 \pi k \frac{(n-m)}{M_{l}}}+ \\
& \sum_{l=-\frac{M_{d}}{2}}^{\frac{M_{s}}{2}+M_{c}} \sum_{m=0}^{M_{c}}\left(x^{*}(n) e^{j 2 \pi\left(2 f_{l} T_{s}\right) n} \otimes b_{l, m}(n)\right) \frac{1}{M_{l}} \sum_{k=0}^{M_{l}} e^{j 2 \pi k \frac{(n-m)}{M_{l}}} \\
& =\sum_{l=-\frac{M_{J}}{2}} \sum_{k=0}^{\frac{M_{s}}{2}+M_{c}} x(n) e^{j 2 \pi n \frac{k}{M_{l}}} \otimes\left[\frac{1}{M_{l}} \sum_{m=0}^{M_{l}} a_{l, m}(n) e^{j 2 \pi m \frac{k}{M_{l}}}\right]+ \\
& \sum_{l=-\frac{M_{s}}{2}}^{\frac{M_{s}}{2}+M_{c}} \sum_{l=0}^{M_{l}} x^{*}(n) e^{j 2 \pi n\left(\frac{k}{M_{l}}+2 f_{l}\right)} \otimes\left[\frac{1}{M_{l}} \sum_{m=0}^{M_{l}} b_{l, m}(n) e^{j 2 \pi m \frac{k}{M_{l}}}\right]
\end{aligned}
$$




$$
\begin{aligned}
& \hat{s}_{0}(n)=\sum_{l=-\frac{M_{d}}{2}}^{\bar{M}_{a}+M_{c} H_{1}} \sum^{H_{1}} x(n) e^{12 \pi t \frac{k}{M_{l}}} \otimes A_{l, k}(n)+ \\
& \sum_{l=-\frac{M_{a}}{2}}^{\frac{M_{a}}{2}+M_{c}} \sum_{k=0}^{M_{l}} x^{*}(n) e^{j 2 \pi n\left(\frac{k}{M_{l}}+2 f_{l}\right)} \otimes B_{l, k}(n)
\end{aligned}
$$

where $A_{l, k}(n)$ and $B_{l, k}(n)$ are the discrete Fourier transforms (related to the parameter $m)$ of $a_{l, m}(n)$ and $b_{l, m}(n)$ respectively. Defining $\alpha_{k, l}=\frac{k}{M_{l}}$ yields:

$$
\begin{gathered}
\hat{s}_{0}(n)=\sum_{l=-\frac{M_{a}}{2}} \sum_{k=0}^{\frac{M_{a}}{2}+M_{c} M_{l}} x(n) e^{j 2 \pi n \alpha_{k, l}} \otimes A_{l, k}(n)+ \\
\frac{M_{d}}{2}+M_{c} M_{l} \\
\sum_{l=-\frac{M_{a}}{2}} \sum_{k=0}^{*} x^{*}(n) e^{j 2 \pi n\left(\alpha_{k, l}+2 f_{l}\right)} \otimes B_{l, k}(n)
\end{gathered}
$$

Changing the variable of the summation to $m=k l$ and assuming:

$$
\begin{gathered}
\alpha_{m}=\alpha_{k_{,} l}, \quad h_{m}(n)=A_{!, k}(n) \\
\beta_{m}=\alpha_{k, l}+2 f_{l}, \quad g_{m}(n)=B_{l, k}(n)
\end{gathered}
$$

yields:

$$
\hat{\boldsymbol{S}}_{0}(n)=\sum_{m=0}^{\left(M_{a}+M_{c}\right) M_{l}} x(n) e^{i 2 \pi n \alpha_{m}} \otimes h_{m}(n)+\sum_{m=0}^{\left(M_{u}+M_{c}\right) M_{t}} x^{*}(n) e^{j 2 \pi \sigma_{m} \beta_{m}} \otimes g_{m}(n)
$$

Equation (C.10) represents the sampled version of HSR-TVF. Therefore HSR-TVF is equivalent to the TSR-TVF and its filters are the discrete Fourier transform of the TSRTVF filters. Therefore the bank of tilters in HSR-TVF and the bank of filters in TSR-TVF are time-frequency duals.

Since HSR-TVF and TSR-TVF are time-frequency duals, FSE and GFSE are time-frequency duals too. 


\section{References}

11] M. Abdulrahman and D. D. Falconer, "Cyclostationary Crosstalk Suppression by Decision Feedback Equalizer on Digital Subscriber Loops," IEEE J. Select. Areas Commun., vol. SAC-11. no. 3, pp. 640-649, Apr. 1992.

[2] A. S. Acarmpora and J. H. Winters, "System Application for Wireless Indoor Communications," IEEE. Comm. Mag., vol. 25, no. 8, pp. 11-20, Aug. 1987.

13] Y. Akawia and Y. Nagata, "Highly efficient Digital Mobile Communications with a linear Modulation Method," IEEE J. Select. Areas in commun., vol. SAC-5, no. 5, pp. 89()-895, Jun. 1987.

14] American National Standards Institute, Integrated services digital network (ISDN) hasic access interface for use on metallic loops for application on the network side of the NT (layer l specification), ANSI T1-601-1988, Sep. 1988.

[5] V. Aue, "CDMA demodulation and interference rejection using an optimal time-dependent filter," in IEEE Transactions of Communications. submitted.

[6] P. Balaban and J. Salz, "Optimum Diversity Combining and Equalization in Digital Data Transmission with Applications to Cellular Mobile Radio - Part I: Theoretical Considerations," IEEE Trans. on Commun., vol. 40, no. 5, pp. 885-894, May 1992.

17] P. Balaban and J. Salz, "Optimum Diversity Combining and Equalization in Digital Data Transmission with Applications to Cellular Mobile Radio - Part II: Numerical Results." IEEE Trans. on Commun., vol. 40, no. 5, pp. 895-907, May 1992.

[8] J. W. M. Bergmans and A. J. E. M. Janssen, "Robust Data Equalization, Fractional Tap Spacing and the Zak Transform," Phillips Journal of Research, vol. 42, no. 4, 1987.

[9] R. A. Boyles and W. A. Gardner, "Cycloergodic properties of discrete-parameter nonstationary stochastic processes," IEEE Trans. on Information Theory, vol.IT-29, no. 4, pp. 105-114, A.pr. 1983.

[10] W. A. Brown, On the theory of cyclostationary signals, Ph.D. Dissertation, Depart- 
ment of Electrical Engineering and Computer Science. University of California. Davis. September 1989.

[11] W. M. Brown, "Conjugate linear filtering," IEEE Trans. on Information The'ory. vol. IT-15, pp. 462-465, 1969.

[12] W. M. Brown, Analysis of Linear Time-Variant Systems, New York: Mc Graw-Hill. 1963.

[13] R. W. Charg and R. A. Gibby, "A theoretical Study of Performance of an Orthogonal Multiplexing Data Transmission Scheme," IEEE Trans. on Commun. Technology, vol. COM-16, no. 4, pp. 529-540, Aug. 1968.

[14] S. Chennakeshu and G. J. Saulnier, "Differential Detection of p /4-Shifted-DQPSK for Digital Cellular Radio," IEEE Trans. on Vehicular Technology, vol. 42, no. 1. Feb. 1993.

[15] C. Corduneanu, Almost Periodic Functions. New York: Wiley 1961.

[16] D. C. Cox, "Universal Digital Portable Radio Communications," Proc. IEEE, vol 75, no.4, pp.436-477, Apr. 1987.

[17] D. C. Cox, "Cochan. el interference considerations in frequency reuse small coverage-area radio systems," IEEE Trans. on Commun., vol. COM-30, no. 1, pp. 135-142, Jan. 1982.

[18] R. E. Crochiere and L. R. Rabiner, Multirate Digital Signal Processing, Englewood cliffs, Nj: Prentice-Hall, 1983.

[19] G. W. Davidson, An Investigation of $B L_{-\alpha}$ Adaptive Equalization on a Fading $H F$ Channel, M. Eng. thesis, Dept. of Systems and Computer Engineering, Carleton University, Ottawa, ON., Canada, Aug. 1986.

[20] C. L. Despins, Coding and Optimum Baseband Combining for Wideband TDMA Indoor Wireless Channels, Ph. D. thesis, Dept. of systems and computer Engineering, Carleton University, Ottawa, ON., Canada, Aug. 1991.

[21] D. D. Falconer, "Application of Passband Decision Feedback Equalization in Two Dimensional Data Communication Systems," IEEE Trans. Commun., vol. COM-24, no. 10, pp. 1159-1166, Oct. 1976. 
22] D. D. Falconer, M. Abdulrahman, N. W. K. Lo, B. R. Petersen, and A. U. H. Sheikh, "Advances in equalization and diversity for portable wireless systems," Digital Signal Processing 3. pp. 148-162, 1993.

|23| L. E. Franks and W. A. Gardner, "Estimation for cyclostationary random processes," in Proc. 9th Annu. Allerton Conf. Circuit and System Theory, pp. 222-231, 1971.

[24] L. E. Franks, Signal Theory, Prentice-Hall, Englewood-Cliffs, NJ, 1969.

125] C. A. French and W. A. Gardner, "Spread-Spectrum Lispreading Without the Code," IEEE Trans. on Communications, vol.COM-34, no. 4, pp. 404-407, Apr. 1986.

[26] W. A. Gardner, Statistical Spectral Analysis: A Nonprobabilistic Theory. Englewood Cliffs, NJ: Prentice-Hall, 1987.

[27] W. A. Gardner, Introduction to Random Processes with Application to Signals and Systems. New York: Mcmillan, 1985; second ed. Mc Graw-Hill, 1990.

[28] W. A. Gardner, "Spectral correlation of modulated signal-Part I: Analog modulation," IEEE Trans. on Commun., vol. COM-35, no. 6, pp. 97-115, Jan. 1987.

[29] W. A. Gardner, W. A. Brown, III, and C. K. Chen, "Spectral correlation of modulated signals-Part II: Digital modulation," IEEE Trans. on Commun., vol. COM-35, no. 6, pp. 595-601. June 1987.

[30| W. A. Gardner, "Exploitation of spectral redundancy in cyclostationary signals," IEEE SP Magazine, pp. 14-37, April 1991.

[31] W. A. Gardner, "Common pitfalls in the application of stationary process theory to time-sampled and modulated signals," EEE Trans. on Commun., vol. COM-35, no. 5, pp. 529-534, May 1987.

132] W. A. Gardner, "Cyclic Wiener filtering: Theory and method," EEE Trans. on Commun.. 1992.

[33] W. A. Gardner, "Signal interception: A unifying theoretical framework for feature detection." EEE Trans. on Commun., vol. 36, no. 8, pp. 897-906, Aug. 1988.

[34] W. A. Gardner, "Rice's representation for cyclostationary processes," IEEE Trans. on Commun.. vol. COM-35. no. 1, pp. 74-78, Jaı. 1987. 
[35] W. A. Gardner, "Measurement of spectral correlation," IEEE Trans. on Alomsicis. Speech, and Signal Processing., vol. ASSP.34, no. 5, pp. 1111-1123, Jan. 1986.

[36] W. A. Gardner and L. E. Franks. "Characterization of cyclostationary random signal processes," EEE Trans. on Information thery, vol. IT-21, no. 1, pp. 4-14, Jan. 1975.

[37] W. A. Gardner and W. A. Brown, "Frequency-shift filtering theory for adaptive cochannel interference removal," Proceedings of the Twenty-Third Annual Asilomar Conference on Signals, Systems, and Computers, Pacific Grove, CA, Oct. 30-Nov. 1, 1984, pp. $562-567$.

[38] W. A. Gardner and S. Venkataraman. "Performance of optimum and adaptive frequency - shift filters for cochannel interference and fading." Proceedings of the TwentyFourthd Annual Asilomar Conference on Signals, Systems, and Computers, Pacific Grove. CA, Nov. 5-7, 1990.

[39] W. A. Gardner, "The Role of Spectral Correlation in Design and Performance Analysis of Synchronizers," EEE Trans. on Communications, vol. COM.-34, no. 11, pp. 1(1891095, Nov. 1986.

[40] W. A. Gardner, "On the Spectral Coherence of Nonstationary Processes," IEEE Trans. on Signal Processing, vol. 39, no.2, pp. 424-429. Feb. 1991.

[41] W. A. Gardner, "The structure of linear least-mean-square estimators for synchronous M-array signals," IEEE Trans. on Information Theory, vol.IT-19, pp. 24()-243, Mar. 1973.

[42] W. A. Gardner and C. M. Spooner, "Detection and Source Location of Weak Cyclostationary Signals: Simplifications of the Maximum-Likelihood Receiver," IEEETrans. on Commun., vol. 41 , no. 6, Jun. 1993.

[43] K. S. Gilhousen, I. M. Jacobs, R. Padovani, A. J. Viterbi, L. A. Weaver Jr. and C: E. Wheatley III, “On the Capacity of a Cellular CDMA System," IEEE Trans. Vehic. Tech., vol. VT-40, no. 2, pp. 303-312, May 1991.

[44] E. G. Gladyshev, "Periodically and almost periodically correlated random processes with continuous time parameter," Theory Prob. appl., (USSR), PP. 173-177, 1963. 
Vehic. Tech., vol. VT-40, no. 2, pp. 3666-374, May 1991.

B. Glance and L. J. Green stein, "Frequency-Selective Fading Effects in Digital Mobile Channels," IEEE Trans. on Comin., vol. COM-31, no. 9. pp. 1085-1094, Sep. 1983.

147] Y. Grenier, "Time-dependent ARMA modeling of non-stationary signals," IEEE Trans. oll Acoustics Speech and Signal Processing, vol. ASSP-31, PP. 899-911, 1983.

[48] M. Hajivandi and W. A. Gardner, “Measures of Tracking Performance for the LMS Algorithm," IEEE Trans. on Acoustics Speech and Signal Processing, vol. 38, no.11. pp. 1953-1958, Nov. 1990.

[49] H. Hammuda, J. P. McGeehan and A. Bateman, "Spectral efficiency of cellular land mobile radio systems." VTC 1988.

[50] D. N. Hatfield, "Measures of spectral efficiency in land mobile radio", IEEE Trans. Electromgn. Compat., vol., EMC-19, Aug. 1977.

[51] S. Haykin, Digital Communications, John Wiley \& Sons, 1988.

[52] S. S Haykin, Adaptive Filter Theory, NJ: Prentice-Hall, 1986.

153] F. Hendessi, H. M. Hafez, A. U. H. Sheikh, "The Structure and Performance of FRESH-Decision Feedback Equalizer in the Presence of Adjacent Channel Interference," in Proc. VTC 93. pp. 641-644, May 1993.

154| L. Herbst, "Almost periodic variances," Ann. Math. Stat., vol. 34, pp. 1549-1557. 196.3.

[55] B. Hirosaki, S. Hasegawa, and A. Sabato, "Advanced Groupband Data Modem Using Orthogonally Multiplexed QAM technique," IEEE Trans. on commun. vol. COM-34 no. 6. pp.587-592,Jun. 1986.

[56] B. Hirosaki, “An Analysis of Automatic Equalizers for Orthogonally Multiplexed QAM Systems." IEEE Trans. on commun. vol. COM-28 no. 1, pp. 73,83, Jan. 1980.

[57] J. Y. Hui, "Bit-Rate Limitations of the Digital Radio Channel Due to Multipath Intersymbol Interference and Fading." in Proc. Globecom. 87, pp. 48.4.1-7, May 1987.

[58] K. Jacobs. "Almost periodic sources and channels," Z. Wahrschein-Lichkeitstheo- 
rie verw, Geb. 9. pp. 65-84, 1967.

[59] W. C. Jakes, Micron'ave Mobile Communications, John Wiley and Sons, New York, NY, 1973.

[60] N. S. Jayant and P. Noll, Digital Coding of W'an'eforms. Englewood Clitfs, NJ: Prentice-Hall, 1984.

[61] M. Kavehrad and J. Salz. "Cross-polarization Cancellation and Eyualization in Digital Transmission over Sually Polarized Multipath Fading Channels," AT\&T Tech. I., vol. 64, no. 10. pp. $2211-2245$. Dec. 1985.

[62] L. H. Koopmans, The Spectral Analysis of Time Series. New York: Academic. 1974.

[63] W. C. Y. Lee, Mobile Cellular Telecommunications Systems, McGraw-Hill, 1989.

[64] W. C. Lee, "Spectral efficiency and digital cellular", VTC 1988.

[65] W. C. Y. Lee, Mobile Communications Design Fundumentals, Howard W. Sams \& Co., 1986.

[66] W. C. Y. Lee, Mobile Communications Engineering, New York, NY: McGiraw-Hill, 1982.

[67] Norm W. K. Lo, Adaptive Equalization for a Multipath Fading Channel in the'Presence of Interference, Ph.D. Thesis proposal, Dept. of systems and computer Engineering. Carleton University, Ottawa, ON., Canada, Aug. 1991.

[68] R. W. Lorenz, J. deWeck, and P. Merki, "Power Delay Profiles Measured in Mountainous Terrain," in Proc. VTC 88, pp. 105-112, 1988.

[69] R. M. Loynes, "On the concept of the spectrum for non-stationary processes," J. Roy. Statist. Soc., Vol. 30, Ser. B, pp. 1-30, 1968.

[70] R. W. Lucky, "Techniqu ., for adaptive equalization of digital communications systems," Bell Syst. Tech. J., vol. 45, pp. 255-286, Feb. 1966.

171) E. Miki, I. Horikawa, "Combined Effects of Compact Spectrum Modulation And Adaptive Channel Assignment in Micro cellular Systems." pp. 509-518. 
172] P. Monsen, "Feedback equalization for fading dispersive channels," IEEE Trans. on Inform. Theory, vol, IT-17, pp. 56-64, Jan. 1971.

173] P. Monsen, "MMSE Equalization of Interference on Fading Diversity Channels," IEEE Truns. on Comm., vol. COM-32, no. 1, pp. 5-12, Jan. 1984.

1741 C. L. Nikias and J. Mendel, "Signal Processing with High-Order Spectra," IEEE Signal Processing Magazine, pp. 10-37, July 1993.

175] C. L. Nikias and A. P. Petropulu, Higher-order Spectral Analysis: A Nonlinear Signal Processing Framework, Prentice-Hall Inc., 1993.

1761 H. Nyquist, "Certain Topics in Telegraph Transmission Theory," Trans. AJEE, vol. 47. pp. 617-664, Feb. 1928.

177] A. Papoulis, Probability, Random Variables and Stochastic Processes, 2nd Ed., Mc Graw-Hill Book Company, New York, NY, 1984.

[78] A. Papoulis, “Random Modulation: A Review," IEEE Trans. on Acoustics, Speech, and Signal Processing, vol. ASSP-31, no. 1, pp. 96-105, Feb. 1983.

179] E. Parzen, "Spectral analysis of asymtotically stationary time series," Bull. Inst. Int. Statist., Vol. 39, Livraison 2, pp. 87-103, 1962.

[X()] B. R. Peterson, Equalization in Cyclostationary Interference, Ph.D. Dissertation, Dept. of systems and computer Engineering, Carleton University, Ottawa, ON., Canada, Aug. 1992.

[81] B. R. Peterson and D. D. Falconer, "Minimum Mean Square Equalization in Cyclostationary and stationary Inter rerence-Analysis and Subscriber Line Calculations," IEEE J. Select. Areas Commun., vol. SAC-9, no. 6. pp. 931-940, Aug. 1991.

|82| J. G. Proakis, "Adaptive receivers for digital signaling over random or unknown channels," Ph. D. disscrtation. Harvard Univ.. Cambridge, MA, 1966.

[83] J. G. Proakis, “Adaptive Equalization for TDMA Digital Mobile Radio," IEEE Trans. Vehic: Tech., vol. VT-40,no. 2. pp. 333-341. May 1991.

[84] J. G. Proakis. Digital Communications, 2 nd Ed., McGraw-Hill, New York, NY, 1989. 
[85] S. U. H. Qurcshi. "Adaptive Equalization," Proc. of the IEEE. vol. 73, no. ". pp. 1349-1387, Sep. 1985.

[86] T. S. Rao. "The fitting of non-stationary time-series models with time-dependent parameters,“ J. Roy. Statist. Soc. Ser. B, Vol. 32, pp. 312-322, 1970.

[87] J. H. Reed and T. C. Hsia, "The performance of time dependent adaptive filters for interference rejection," IEEE Trans. on Acoustics, Speech. and Signal Processing. vol. 38. pp. 1373-1385. Aug. 1990.

[88] J. H. Reed, Time-dependent adaptive filters for interferenc' reje'tion. Ph. D. Dissertation, Department of Electrical Engineering and Computer Science, University of California, Davis, Dec. 1987.

[89] R. S. Roberts, W. A. Brown, and H. H. Loomis, JR.. "Computationally efficient algorithms for cyclic spectral analysis," IEEE SP Magazine, pp. 38-48. April 1991.

[90] J. Ruprecht, F. D. Neeser and M. Hufschmid, “Code Time Division Multiple Access: An Indoor Cellular System," IEEE Proc. VTC, Denver. CO. USA, May 1492.

[91] A. Salmasi and K. S. Gilhousen, "On the system design aspects of code division multip!e access (CDMA) applied to digital cellular and personal communications networks,' in IEEE 41st Vehicular Tech. Conf:rence, pp. 57-62. 1991.

[92] S. Sampei and M. Yokoyama, "Rejection Method of Adjacent Channel Interference for Digital Land Mobile Communications," The Trans. of the IECE of Jalpan, vol. E 69, no. 5. pp. 578-580.May 1986.

[93] J. Salz, "Optimum Mean-Square Decision Feedback Equalization," Bell System Technical Journal, vol. 52, no. 8, pp. 1341-1373, Oct. 1973.

[94] D. L. Schilling, L. B. Milstein, R. L. Pickholtz, M. Kullback and F. Miller, "Spread Spectrum for Commercial Communications," IEEE Commun. Mag., vol. 29, no. 4, nn. 6679. Apr. 1991.

[95] M. R. Schroeder and B. S. Atal, "Code-Excited Linear Prediction (C'ELP): HighQuality speech at very Low bit rates," in Proc. ICASSP 85, pp. 937-940. Aug. 1985.

[96] K. C. Sharman and B. Friedlander, "Time Varying modeling of a class of nonsta- 
tionary signals," Proc. Internal Conf. Acoustics Speech and Signal Processing, San Diego, pp. 22.2.1-22.2.4, 1984.

[97] S. Stein, "Fading Channel Issues in System Engineering," IEEE J. Sel. Areas in Comm., vol. SAC-5, no. 2. pp. 68-89, Feb. 1987.

[981 L. Tong, G. Xu and T. Kailath, "Blind Identification and Equalization of Multipath Channels," ICC'92, pp. 1513-1517.

[99] M. Uesugi, K. Honma, and K. Tsubaki, “Adaptive Equalizarion in TDMA Digital Mobile Radio," in Proc. Globecom 89, pp. 95-101. Dec. 1989.

[100] P. P. Vaidyanathan, "Quadrature Mirror Filter Banks, M-Band Extensions and Perfect-Reconstruction Techniques," IEEE ASSP Magazine, pp. 4-20, July 1987.

[101] P. Vary, K. Hellwig, R. Hofmann, R.J. Sluyter, C. Galand, and M. Rosso, "Speech Coder for the European Mobile Radio System," in Proc. ICASSP 88, pp. 227-230, Aug. 1988.

[1(12] R. G. Vaughan and J. B. Anderson, “Antenna Diversity in Mobile Communications," IEEE Trans. on Vech. Tech., vol. VT-36, no. 4, pp. 149-156. Nov. 1987.

[103] S. Verdu, "Optimum Multiuser Asymptotic Efficiency," IEEE Trans. on Commun., vol. COM-34, no. 9, pp. 890-897,Sep. 1986.

[104] S. K. Wilson and J. M. Cioffı, "Multi-dimensional Equalization for Adjacent Channel Interference," in Proc: of ICC'91.

[1105] G. D. Zivanovic, W. A. Gardner, "Degree of cyclostationarity and their application to signal detection and estimation," Signal Processing 22, pp. 287-297, 1991. 

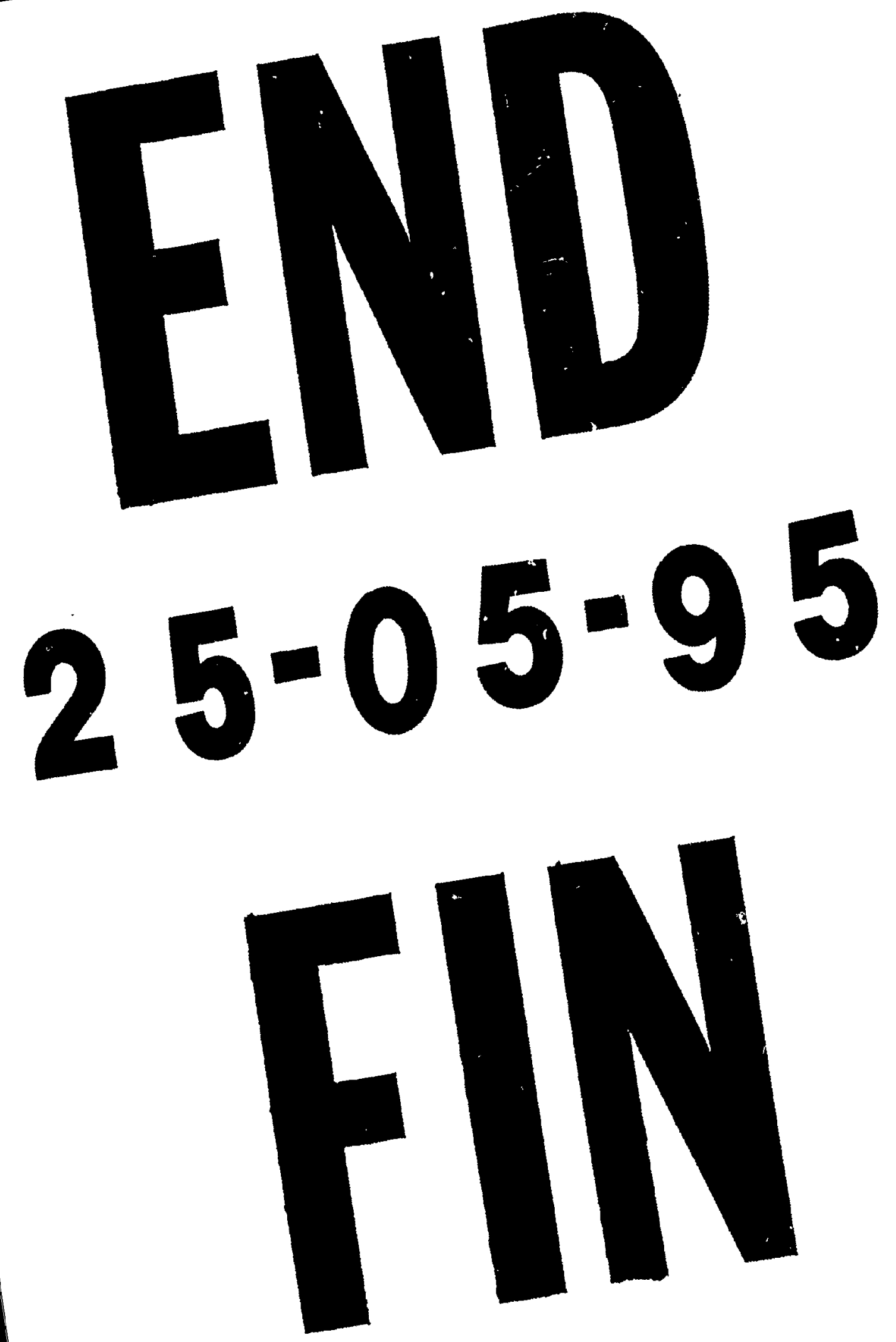